
and Transport Properties of Hydrogen (Tabcode-II)

\section{U.S. ARTMENT OF DMMERCE National Bureau of Standards}


The National Bureau of Standards ${ }^{1}$ was established by an act of Congress March 3, 1901. The Bureau's overall goal is to strengthen and advance the Nation's science and technology and facilitate their effective application for public benefit. To this end, the Bureau conducts research and provides: (1) a basis for the Nation's physical measurement system, (2) scientific and technological services for industry and government, (3) a technical basis for equity in trade, and (4) technical services to promote public safety. The Bureau consists of the Institute for Basic Standards, the Institute for Materials Research, the Institute for Applied Technology, the Center for Computer Sciences and Technology, and the Office for Information Programs.

THE INSTITUTE FOR BASIC STANDARDS provides the central basis within the United States of a complete and consistent system of physical measurement; coordinates that system with measurement systems of other nations; and furnishes essential services leading to accurate and uniform physical measurements throughout the Nation's scientific community, industry, and commerce. The Institute consists of a Center for Radiation Research, an Office of Measurement Services and the following divisions:

Applied Mathematics-Electricity-Heat-Mechanics-Optical Physics-Linac Radiation ${ }^{2}$ - Nuclear Radiation ${ }^{2}$ - Applied Radiation ${ }^{2}$ - Quantum Electronics ${ }^{3}$ Electromagnetics ${ }^{3}$ - Time and Frequency ${ }^{3}$-Laboratory Astrophysics ${ }^{3}$-Cryogenics $^{3}$.

THE INSTITUTE FOR MATERIALS RESEARCH conducts materials research leading to improved methods of measurement, standards, and data on the properties of well-characterized materials needed by industry, commerce, educational institutions, and Government; provides advisory and research services to other Government agencies; and develops, produces, and distributes standard reference materials. The Institute consists of the Office of Standard Reference Materials and the following divisions:

Analytical Chemistry-Polymers-Metallurgy-Inorganic Materials-Reactor Radiation-Physical Chemistry.

THE INSTITUTE FOR APPLIED TECHNOLOGY provides technical services to promote the use of available technology and to facilitate technological innovation in industry and Government; cooperates with public and private organizations leading to the development of technological standards (including mandatory safety standards), codes and methods of test; and provides technical advice and services to Government agencies upon request. The Institute also monitors NBS engineering standards activities and provides liaison between NBS and national and international engineering standards bodies. The Institute consists of the following divisions and offices:

Engineering Standards Services-Weights and Measures-Invention and Innovation-Product Evaluation Technology-Building Research-Electronic Technology-Technical Analysis-Measurement Engineering-Office of Fire Programs.

THE CENTER FOR COMPUTER SCIENCES AND TECHNOLOGY conducts research and provides technical services designed to aid Government agencies in improving cost effectiveness in the conduct of their programs through the selection, acquisition, and effective utilization of automatic data processing equipment; and serves as the principal focus within the executive branch for the development of Federal standards for automatic data processing equipment, techniques, and computer languages. The Center consists of the following offices and divisions:

Information Processing Standards-Computer Information-Computer Services - Systems Development-Information Processing Technology.

THE OFFICE FOR INFORMATION PROGRAMS promotes optimum dissemination and accessibility of scientific information generated within NBS and other agencies of the Federal Government; promotes the development of the National Standard Reference Data System and a system of information analysis centers dealing with the broader aspects of the National Measurement System; provides appropriate services to ensure that the NBS staff has optimum accessibility to the scientific information of the world, and directs the public information activities of the Bureau. The Office consists of the following organizational units:

Office of Standard Reference Data-Office of Technical Information and Publications-Library-Office of International Relations.

\footnotetext{
${ }_{1}$ Headquarters and Laboratories at Gaithersburg, Maryland, unless otherwise noted; mailing address Washington, D.C. 20234.

2 Part of the Center for Radiation Research.

${ }^{3}$ Located at Boulder, Colorado 80302.
} 


\title{
Computer Programs for Thermodynamic and Transport Properties of Hydrogen (Tabcode-II)
}

\author{
H. M. Roder \\ R. D. McCarty \\ W. J. Hall
}

Cryogenics Division

Institute for Basic Standards

National Bureau of Standards

Boulder, Colorado 80302

NBS Technical Notes are designed to supplement the Bureau's regular publications program. They provide a means for making available scientific data that are of transient or limited interest. Technical Notes may be listed or referred to in the open literature.

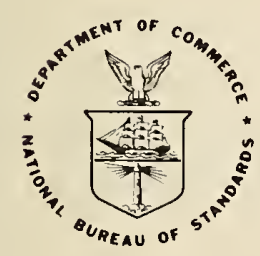

U.S. DePARTMENT OF COMMERCE, Peter G. Peterson, Secrefary NATIONAL BUREAU OF STANDARDS, Lowrence M. Kushner, Acting Director 
National Bureau of Standards Technical Note 625

Nat. Bur. Stand. (U.S.), Tech. Note 625, 226 pages (October 1972) CODEN: NBTNAE 
1. Introduction . . . . . . . . . . . . . . . . . . . . 1

1.1 Symbols and Units Used ..................... . . 2

2. Hydrogen Modifications, Property Differences, and Phase Diagrams ...... 4

2.1 Hydrogen Modifications ................... . . 4

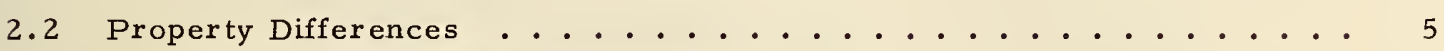

2.3 Range of the Programs and Phase Diagrams ........... 6

2.4 The Phase Designator, $\mathrm{C} \ldots \ldots . \ldots . \ldots$

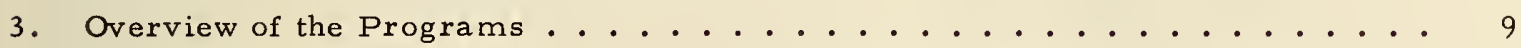

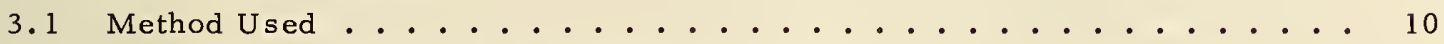

3.2 Peculiarities of the P-T Programs ............... 10

3.3 Peculiarities of the P-H Programs .............. 10

3.4 The Saturation Routines ...................... 11

4. Programming Information .................... 12

4.1 Deck Description . . . . . . . . . . . . . . . . 12

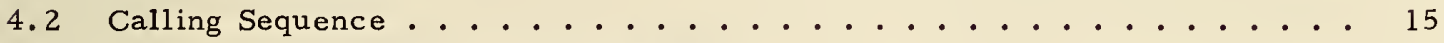

4.3 Exceeding the Range of the Programs ............. 16

4.4 Conversion to Other Systems or Installations . . . . . . . . 16

4.5 Pitfalls, Common Errors in Applications . . . . . . . . . 17

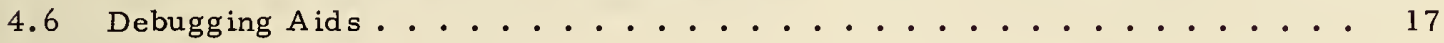

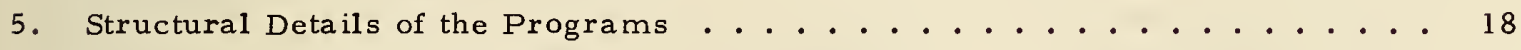

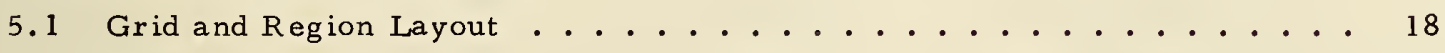

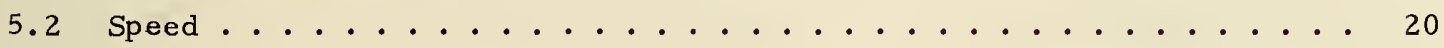

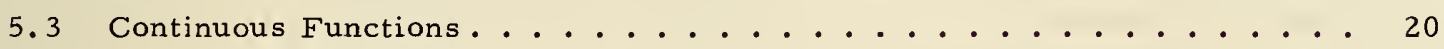

5.4 Modifications, Future Developments ............. 21

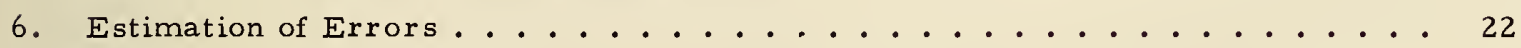

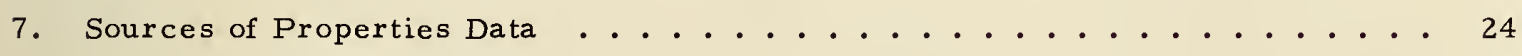

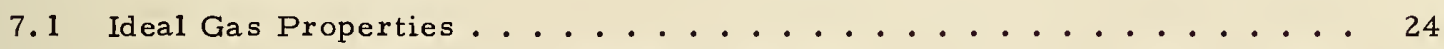

7.2 The PVT Surface at Low Temperatures ............. 26

7.3 The PVT Surface at Intermediate Temperatures . . . . . . . . 26

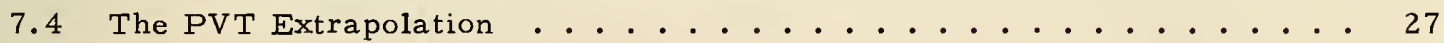

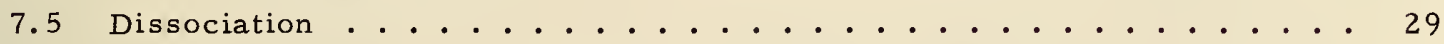

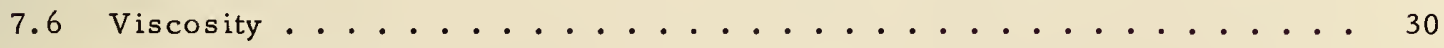

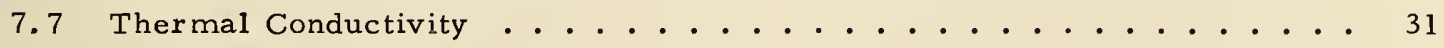

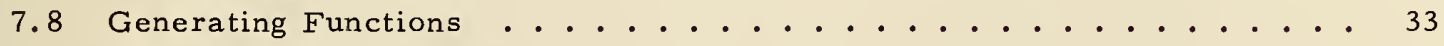

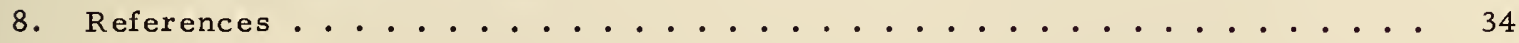


Appendix A. Tables of Values for Selected Isobars . . . . . . . . . . 37

Appendix B. Program Listings: CDC and IBM . . . . . . . . . . . 52

Appendix C. Plots of Maximum Interpolation Error and Estimated

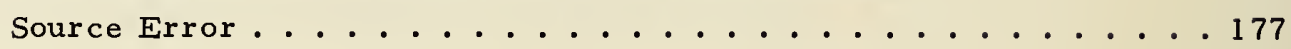

\section{LIST OF TABLES}

1. Values of the Phase Designator, Q ................. 8

2. List of Available Program Decks . . . . . . . . . . . . . . 9

3. Returns from the Liquid-Vapor Two Phase Region . . . . . . . . . 12

4. Deck Description, p-hydrogen ... . . . . . . . . . . . . 13

5. Deck Description, e-hydrogen .................... . . 14

6. Low Temperature Error Grid, Pressures, psia . . . . . . . . . . 23

7. Low Temperature Error Grid, Temperature, ${ }^{\circ} \mathrm{R} . . . . . . . . . .24$

8. Coefficients of the Equation of State . . . . . . . . . . . . 27

9. Generating Functions ..................... 33

\section{LIST OF FIGURES}

1. Hydrogen Composition at Equilibrium . . . . . . . . . . . . . . 4

2. Ideal Gas Specific Heat of Para and Equilibrium Hydrogen . . . . . . . . 6

3. The P-T Phase Diagram for Hydrogen . . . . . . . . . . . 7

4. The P-H Phase Diagram for Parahydrogen . . . . . . . . . . . 7

5. Region Layout fór $\mathrm{C}_{\mathrm{p}}$ with Entry Variables $\mathrm{P}-\mathrm{H}$, p-hydrogen . . . . . . . 18

6. Partial Grid Layout for Program PTENTH . . . . . . . . . . . 19

7. Matching of Values at Boundaries ................. 21

8. Primary Sources of Data . . . . . . . . . . . . . . . 25

9. Comparison of PVT and Derived Properties . . . . . . . . . . . 28

10. The Effect of Dissociation on Enthalpy . . . . . . . . . . . 30

11. The Viscosity of Dissociating Hydrogen at Various Pressures . . . . . . 31

12. The Thermal Conductivity of Dissociating Hydrogen at Various Pressures . . . 32 


\title{
COMPUTER PR OGRAMS FOR THER MODYNAMIC
}

\section{AND TRANSPORT PROPER TIES OF HYDROGEN}

(Tabcode-II)

\author{
H. M. Roder, R. D. McCarty, and W. J. Hall
}

The thermodynamic and transport properties of para and equilibrium hydrogen have been programmed into a series of computer routines. Input variables are the pair's pressure-temperature and pressure-enthalpy. The programs cover the range from 1 to $5000 \mathrm{psia}\left(34 \mathrm{MN} / \mathrm{m}^{2}\right)$ with temperatures from the triple point to $6000^{\circ} \mathrm{R}(3300 \mathrm{~K})$ or enthalpies from $-130 \mathrm{BTU} / \mathrm{lb}(-623 \mathrm{~J} / \mathrm{mol})$ to $25,000 \mathrm{BTU} / \mathrm{lb}(117000 \mathrm{~J} / \mathrm{mol})$. Output variables are enthalpy or temperature, density, entropy, thermal conductivity, viscosity, velocity of sound, heat capacity at constant pressure, heat capacity at constant volume, the heat capacity ratio, and a heat transfer parameter. Property values on the liquid and vapor boundaries are conveniently obtained through two small routines. The programs achieve high speed by using linear interpolation in a grid of precomputed points which define the surface of the property returned. The maximum errors arising from the linear interpolation are shown on individual deviation plots for each combination of variables. Error estimates for the sources of data are similarly displayed.

Key Words: Computer programs; density; enthalpy; entropy; heat capacity at constant pressure; heat capacity at constant volume; heat capacity ratio; heat transfer coefficient; hydrogen; pressure; saturation boundary; temperature; thermal conductivity; velocity of sound; viscosity.

\section{INTR ODUCTION}

Extensive use of hydrogen in the chemical industry and as a propellant in the U. S. space program requires that the engineer be provided values of thermodynamic and transport properties of this fluid in useful form. In many problems the properties have to be evaluated at numerous thermodynamic state points. The problems are often handled on a computer and they involve iterative computational methods. In the present report we describe a series of computer routines which are fast, moderately accurate, and operate over wide ranges of input variables. They are based on selected source data. The input (entry) and output (returned) variables are:

Input

pressuretemperature
Output

enthalpy
density
entropy
thermal conductivity
viscosity
velocity of sound
heat capacity, C
heat capacity, $\mathrm{C}_{\mathrm{p}}$
heat capacity ratio
heat transfer parameter

Input

pressure- temperature, and phase or state enthalpy density

entropy

thermal conductivity

viscosity

velocity of sound

heat capacity, C

heat capacity, $C^{p}$

heat capacity ratio

heat transfer parameter 
In addition there are two small saturation routines which return values on the saturation boundaries. The computer routines were developed primarily for nuclear rocket engine design. The programs cover a wide range of conditions as follows: for temperatures from the triple point $\left(24.845^{\circ} \mathrm{R}, 13.803 \mathrm{~K}\right)$ to about $6000^{\circ} \mathrm{R}(3300 \mathrm{~K})$, for pressures from 1 to $5000 \mathrm{psia}\left(34 \mathrm{MN} / \mathrm{m}^{2}\right)$, and for enthalpies from -130 to $25,000 \mathrm{BTU} / 1 \mathrm{~b}(-623$ to $117000 \mathrm{~J} / \mathrm{mol})$. In addition, the thermodynamic and transport properties of both para hydrogen and equilibrium hydrogen are available through these programs. An important criterion for many applications is computational speed, as some process control calculations have to be made in real time. The linear interpolation employed achieves speed but sacrifices accuracy. As an aid to the user, we have determined the maximum error due to the interpolation scheme and estimated the error in the source data. These errors are displayed in departure graphs for each program.

An older version of the para hydrogen programs, which has been distributed widely, should now be considered obsolete (Tabcode-I, Hall, et al., 1967, and McCarty 1968). The changes and improvements over the older version are as follows:

1. The temperature range has been extended to $6000^{\circ} \mathrm{R}$, the enthalpy range to 25,000 $\mathrm{BTU} / \mathrm{lb}$.

2. New measurements of thermal conductivity between $30^{\circ}$ and $275^{\circ} \mathrm{R}$ are now included.

3. Improved values for both thermal conductivity and viscosity have been inserted for temperatures above $1200^{\circ} \mathrm{R}$.

4. The saturation subroutines are now included in the package.

5. For equilibrium hydrogen, which was not treated previously, an entire set of programs is now provided.

6. A combination of variables is now included which describe a commonly used heat transfer parameter, designated herein as L-factor (see Section 1.1).

The main divisions of the report are text, program listings, and error graphs. The text contains all the normal description given for computer programs such as deck size, calling sequence, etc. However, for this problem the selection of source data and the computation of the property values placed in data arrays are important; they are, therefore, described in some detail.

\subsection{Symbols and Units Used}

The programs were developed for engineering applications; therefore, the units of this report are those commonly used in engineering, i.e., psia, BTU, etc. The sources of data, however, normally employ some version of metric units. In the discussion of the equation of state, the virial $B$, and in figures 11 and 12 , the units of the original sources had to be retained. Conversion factors for changes from source papers to generating functions to the present program have all been taken from NASA Special Publication 7012 (Mechtly 1969). 


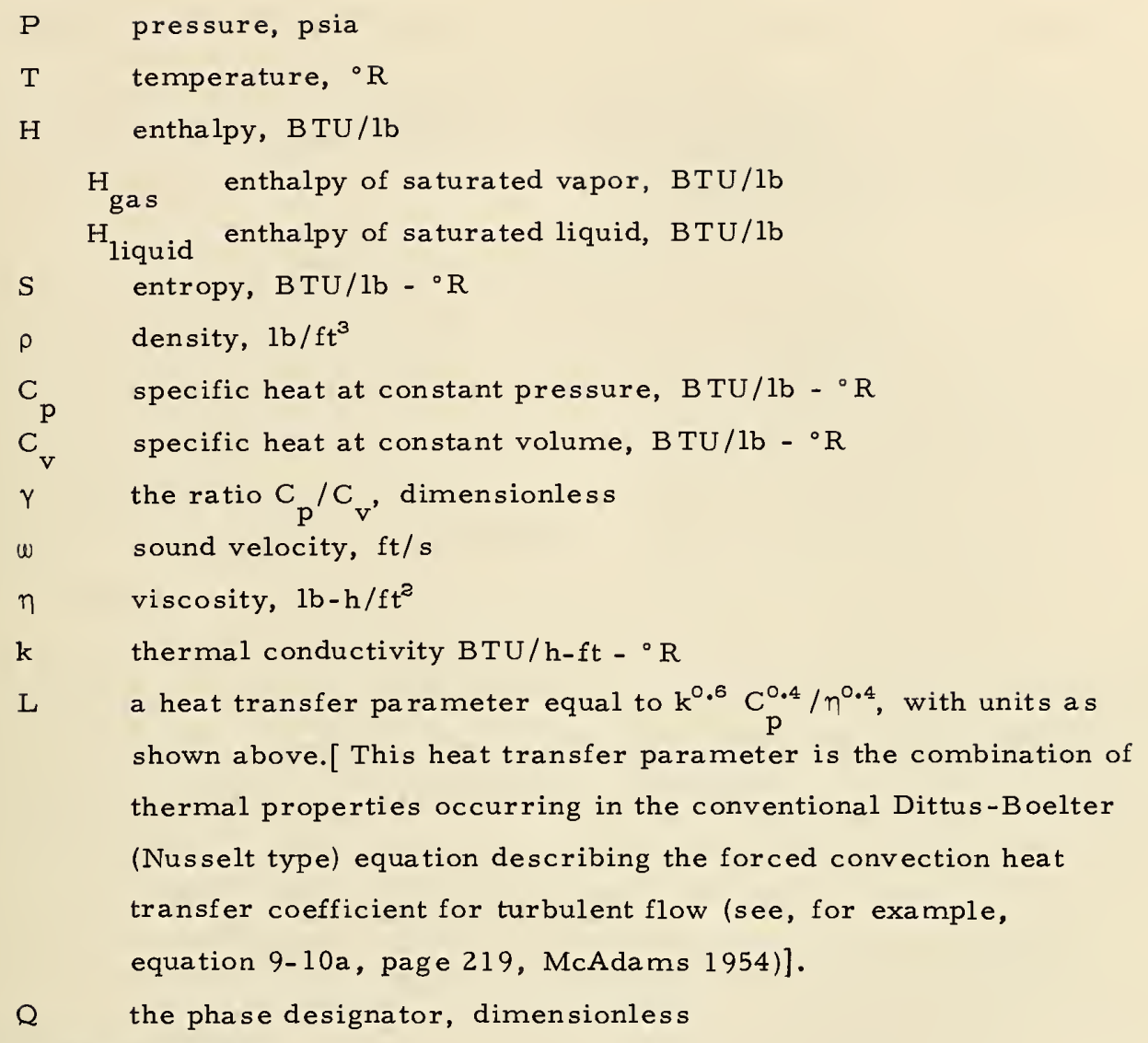

Additional symbols used in text:

$\mathrm{B}$ or $\mathrm{B}(\mathrm{T})$ the second virial coefficient
$E_{0}^{0} \quad$ ideal gas internal energy at absolute zero, the reference state, taken to be zero
$\pi \quad$ general symbol for property
$\mathrm{P}_{\mathrm{c}} \quad$ critical pressure
$\mathrm{R}$ gas constant

RE general symbol for the return from the liquid-vapor two phase region superscript ${ }^{\circ} \quad$ ideal gas thermodynamic properties, i. e., $\mathrm{C}_{\mathrm{p}}^{\circ}, \mathrm{C}_{\mathrm{v}}^{\circ}, \mathrm{H}^{\circ}$

U internal energy

V volume

$\mathrm{Z} \quad \mathrm{PV} / \mathrm{RT}$

Important state points for para and equilibrium hydrogen:

$\begin{array}{lll}\text { triple point } & \text { normal boiling point } & \text { critical point } \\ \mathrm{P}=1.022 \mathrm{psia} & \mathrm{P}=14.696 \mathrm{psia} & \mathrm{P}=187.506 \text { psia } \\ \mathrm{T}=24.845^{\circ} \mathrm{R} & \mathrm{T}=36.482^{\circ} \mathrm{R} & \mathrm{T}=59.356^{\circ} \mathrm{R}\end{array}$




\section{HYDR OGEN MODIFICATIONS, PROPERTY DIFFERENCES, AND PHASE DIAGRAMS}

A brief review of the more important features in the physics of hydrogen is essential if we are to understand what properties the programs return, and why some of the peculiarities of the programs occur.

\section{1 Hydrogen Modifications}

Hydrogen molecules occur in two natural modifications, parahydrogen and or thohydrogen. In the parahydrogen molecule the two nuclear spins are opposed while in orthohydrogen they are alined. Under normal conditions, the transition probability between states is practically zero; a catalyst, a sharp gradient in magnetic field, or external radiation (e.g., nuclear radiation, x-rays, ultraviolet, etc.) is required to let the transition proceed. Conversion between these two modifications is temperature dependent, as shown in figure 1, which is based on Woolley, et al., (1948). At low temperatures in the presence of an appropriate catalyst, the thermal equilibrium favors the para modification. At the normal boiling point $\left(36.482^{\circ} \mathrm{R}\right)$, the equilibrium composition is $99.79 \%$ para and only $0.21 \%$ orthohydrogen. This composition is often simply referred to as parahydrogen or p-hydrogen.

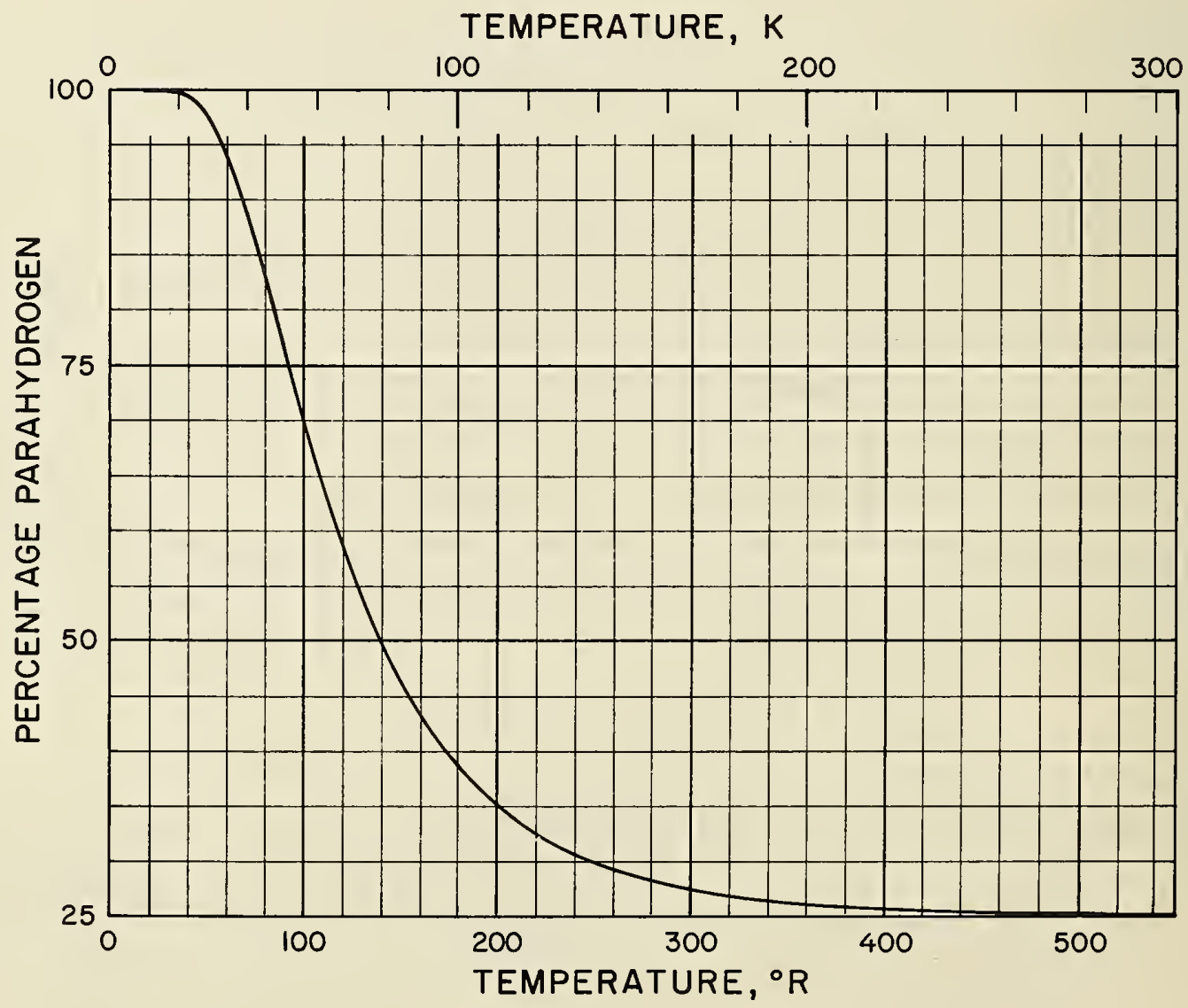

Figure 1. Hydrogen Composition at Equilibrium 
Conversely, the equilibrium composition near ambient temperature is $75 \%$ or tho and $25 \%$ para. This latter composition is usually designated normal or n-hydrogen. When conversion occurs between the normal boiling point and room temperature, then the thermodynamic equilibrium mixture of para and ortho hydrogen is called "equilibrium" or e-hydrogen and the composition at intermediate temperatures varies as shown in figure 1 . In this report we describe programs for both p-hydrogen and e-hydrogen.

At temperatures above $2700^{\circ} \mathrm{R}(1500 \mathrm{~K})$ the hydrogen molecule dissociates into hydrogen atoms. This dissociation is both temperature and pressure dependent. Assumptions made in this report are that dissociation occurs according to the equilibrium constant for normal hydrogen, and that the property values can be obtained by adding the dissociation effects calculated for the ideal gas to the extrapolated properties of the real gas.

\subsection{Property Differences}

At comparable state points, property values of the various modifications of hydrogen may differ, that is, some of the properties depend on ortho-para composition. Experimentally these effects are observed in different vapor pressures and in different critical parameters. Most important to this discussion is the fact that the properties of the ideal gas for para and equilibrium hydrogen as calculated from statistical mechanics differ, and further, since the composition change depends only on temperature, that the property differences depend only on temperature. Thus, if a difference exists for an ideal gas property at low pressures, then the same difference exists at all other pressure levels for a given temperature. Stated another way, the statistical calculation, by including the temperature dependent composition change, automatically includes the reaction occurring as paratransforms to equilibrium-hydrogen - i.e., the sensible heat of e-hydrogen includes the appropriate part of the heat of chemical reaction. Not all properties are composition dependent; density and viscosity, for example, are nearly independent of composition. On the other hand, the properties with significant ortho-para dependency are specific heat, and properties related to specific heat such as velocity of sound, entropy, enthalpy, and thermal conductivity. When the properties of $\mathrm{p}$ - and e-hydrogen differ, they may differ considerably. For example, the value of enthalpy near the critical point for p-hydrogen is about $16 \mathrm{BTU} / 1 \mathrm{~b}$, whereas the value for equilibrium hydrogen is about $31 \mathrm{BTU} / \mathrm{lb}$. The difference in properties for the ideal gas specific heats, $\mathrm{C}_{\mathrm{p}}{ }^{0}$, is illustrated in figure 2. Differences for the other properties are best expressed in terms of a table; approximate differences can be obtained by comparing corresponding entries for a pressure of 1 psia in Appendix A (pages 38 and 45$)$. 


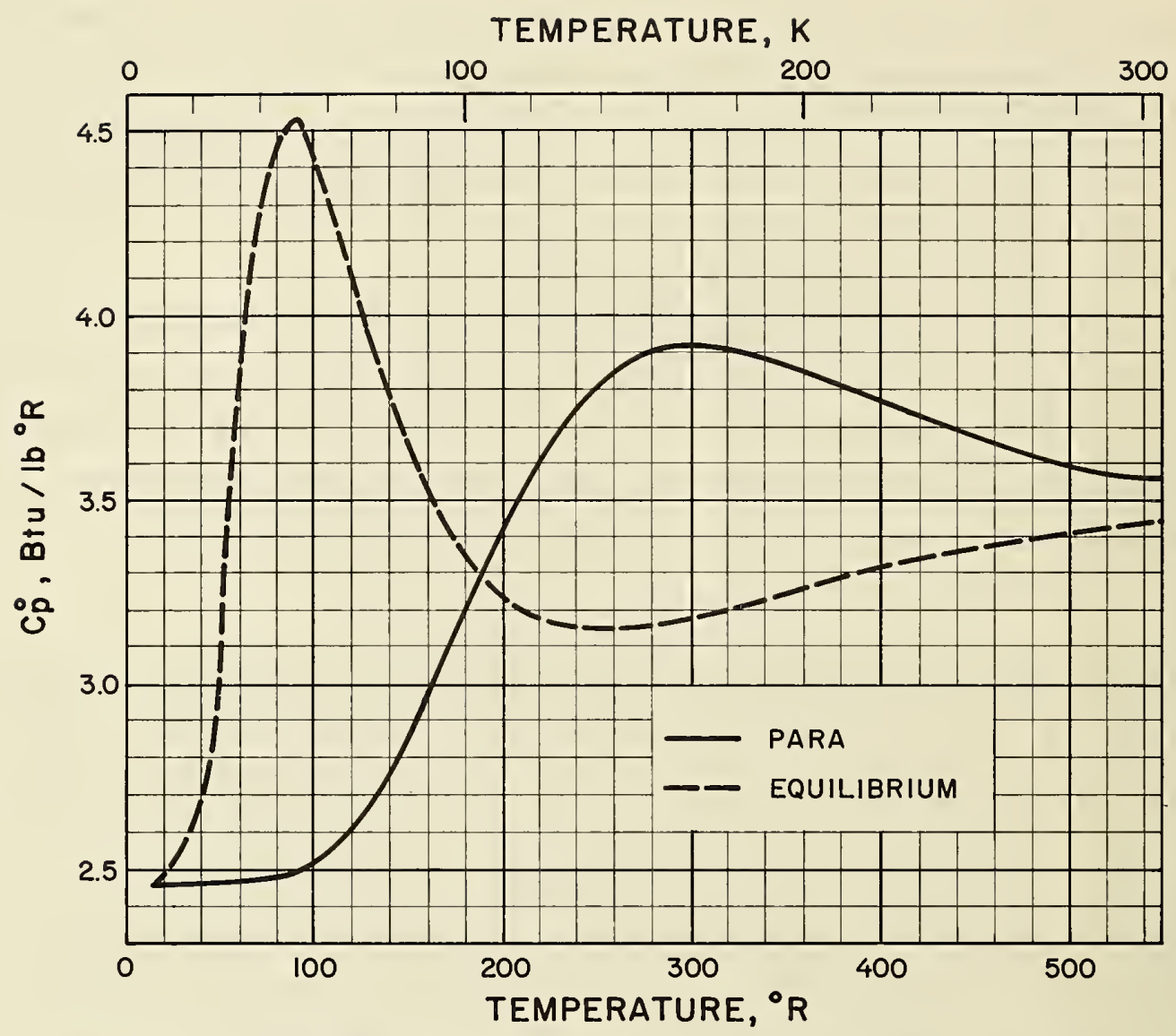

Figure 2. Ideal Gas Specific Heat of Para and Equilibrium Hydrogen

\subsection{Range of the Programs and Phase Diagrams}

For programs which use pressure and temperature as entry variables, the pressure range is 1 to $5000 \mathrm{psia}$ and the temperature range is from the triple point $\left(24.845^{\circ} \mathrm{R}\right)$ to $6000^{\circ} \mathrm{R}$. The P-T range of the programs for both para and equilibrium hydrogen is illustrated by the shaded area in figure 3. The programs cover property values for liquid, gas or vapor states, and dissociation. Note that the programs do not cover the solid phase nor values at pressures less than 1 psia. The low pressure limit is arbitrary; it arises from the behavior of entropy as follows. At any given temperature, the value of entropy approaches infinity as the pressure approaches zero. Since we cannot represent an infinity with linear interpolation between fixed points, a cutoff, 1 psia, was chosen.

For the programs which use pressure and enthalpy as entry variables, the pressure range is the same, 1 psia to $5000 \mathrm{psia}$. The range of enthalpy is $-130 \mathrm{BTU} / 1 \mathrm{~b}$ to 25,000 $\mathrm{BTU} / \mathrm{lb}$. The enthalpy limits correspond to the enthalpy of the liquid at the triple point, and the ideal gas enthalpy at the highest temperature, $6000^{\circ} \mathrm{R}$. The phase diagram of parahydrogen in pressure-enthalpy coordinates is shown in figure 4. The phase diagram for 


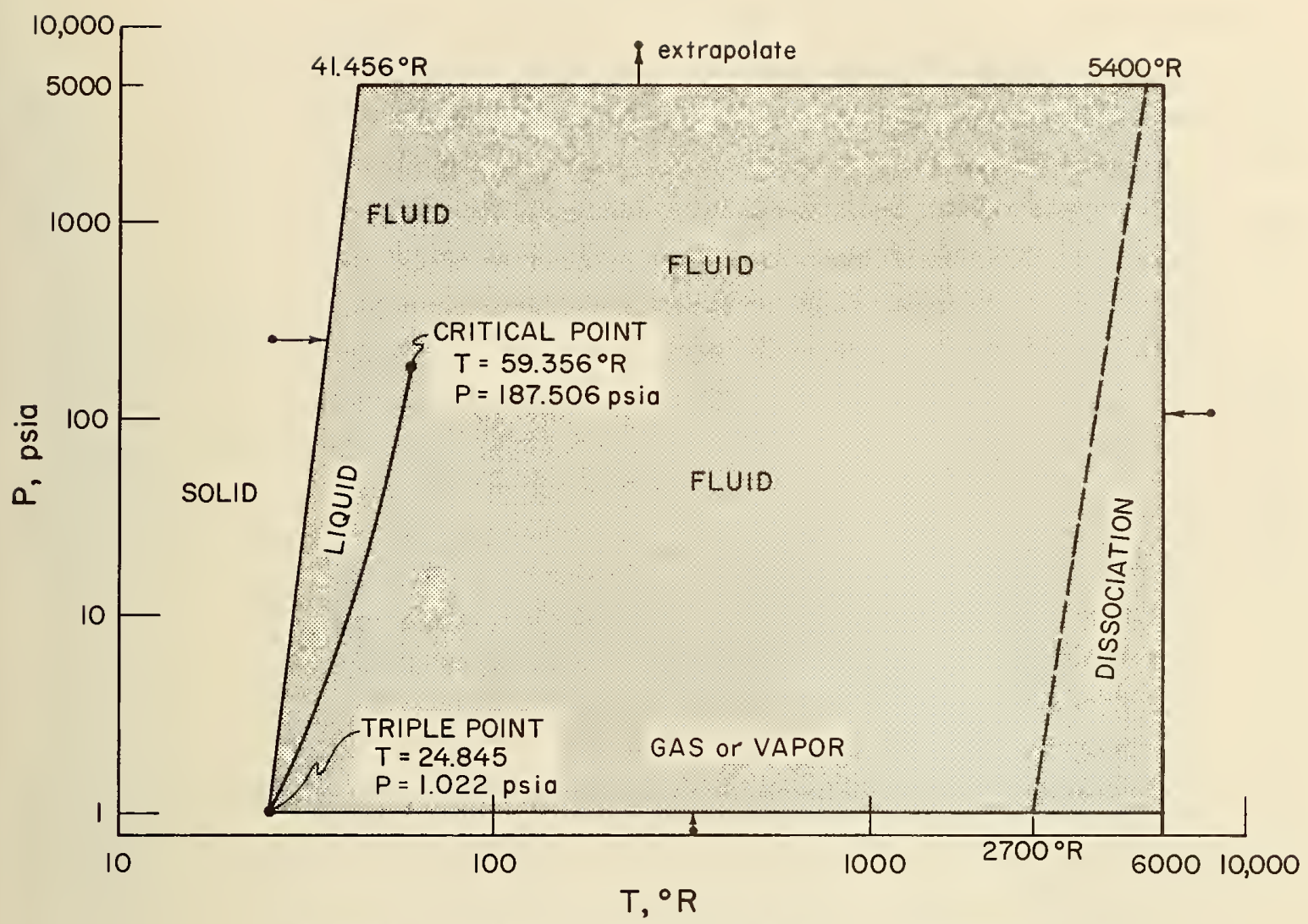

Figure 3. The P-T Phase Diagram for Hydrogen

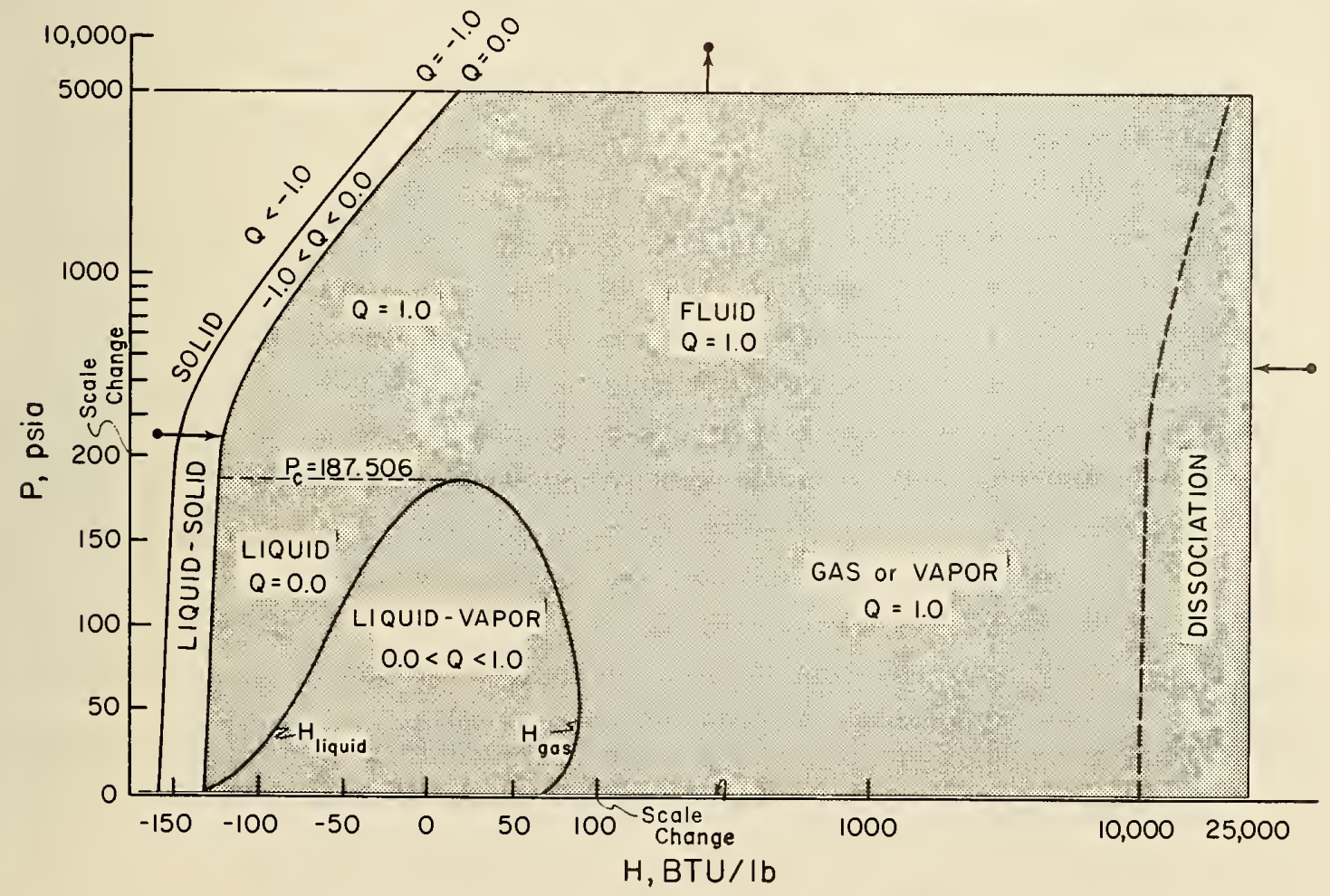

Figure 4. The P-H Phase Diagram for Parahydrogen 
e-hydrogen would look quite similar to figure 4 except that the liquid-vapor two phase boundary is gradually displaced to higher values of enthalpy. For example, the enthalpy of e-hydrogen at the critical pressure is about $31 \mathrm{BTU} / \mathrm{lb}$ while the maximum value of enthalpy along the two-phase boundary is about $110 \mathrm{BTU} / 1 \mathrm{~b}$. The programs cover property values for liquid, gas or vapor states, and dissociation as before. They do not cover the solid phase, pressures below 1 psia, or the two phase liquid-solid ("slush") conditions.

A comparison of figures 3 and 4 discloses that the conditions, the phases or states of the hydrogen, covered by the P-T and the P-H programs are similar, except that the P-H programs cover the range of states where liquid and gas coexist while the P-T programs do not. A concise designation of the phase or state of any given point is therefor available only by reference to $\mathrm{P}-\mathrm{H}$ coordinates. Since the description of phase is important in many problems, a scheme to designate the phase was devised and is presented in the next section.

\subsection{The Phase Designator, Q}

The various physical states possible in the P-H phase diagram are described by the phase designator, $Q$, available only from program PHTEMP (see also page 11). Values of $Q$ are shown in figure 4 and in table 1 . Note that figure 4 includes scale changes at $100 \mathrm{BTU} / 1 \mathrm{~b}$ and at $200 \mathrm{psia}$. For the special case of the two phase liquid-vapor region, Q corresponds to the conventional quality, as defined on page 11 .

Table 1. Values of the Phase Designator, $Q$

\begin{tabular}{|c|c|}
\hline Value of $Q$ & Applicable portions of the phase diagram, $\mathrm{P}_{\mathrm{c}}=187.506$ psia \\
\hline 1.0 & $\begin{array}{l}\text { single phase gas or vapor } \\
\text { single phase compressed fluid at pressures above } P_{C} \\
\text { saturated vapor line }\end{array}$ \\
\hline $0.0<Q<1.0$ & two phase liquid-vapor region \\
\hline 0.0 & $\begin{array}{l}\text { saturated liquid line } \\
\text { compressed liquid (fluid) states at pressures below } \mathrm{P}_{\mathrm{C}} \\
\text { liquid-solid boundary line }\end{array}$ \\
\hline$-1.0<Q<0.0$ & two phase liquid-solid region \\
\hline-1.0 & solid - compressed solid boundary \\
\hline$Q<-1.0$ & compressed solid states \\
\hline
\end{tabular}




\section{OVERVIEW OF THE PROGRAMS}

The decks available and described in this report are listed in table 2.

Table 2. List of Available Program Decks

\begin{tabular}{|c|c|c|c|}
\hline Input & Output & p-hydrogen & e-hydrogen \\
\hline P- T & $\begin{array}{l}\text { enthalpy } \\
\text { entropy } \\
\text { density } \\
\qquad \mathrm{Cp}_{\mathrm{p}} \\
\qquad \mathrm{C}_{\mathrm{v}} \\
\text { gamma } \\
\text { sound velocity } \\
\text { viscosity } \\
\text { thermal conductivity } \\
\text { heat transfer parameter }\end{array}$ & $\begin{array}{l}\text { PTENTH } \\
\text { TPENTR } \\
\text { PTDENS } \\
\text { PTCP } \\
\text { entry PTCV } \\
\text { entry PTGAMM } \\
\text { PTSOUN } \\
\text { PTVISC } \\
\text { PTCOND } \\
\text { PTLFAC }\end{array}$ & $\begin{array}{l}\text { ETENTH } \\
\text { ETENTR } \\
\text { ETDENS } \\
\text { ETCP } \\
\text { entry ETCV } \\
\text { entry ETGAMM } \\
\text { ETSOUN } \\
\text { ETVISC } \\
\text { ETCOND } \\
\text { ETLFAC }\end{array}$ \\
\hline P-H & $\begin{array}{l}\text { temperature, and } Q \\
\text { entropy } \\
\text { density } \\
\qquad C_{p} \\
\qquad C_{v} \\
\text { gamma } \\
\text { sound velocity } \\
\text { viscosity } \\
\text { thermal conductivity } \\
\text { heat transfer parameter }\end{array}$ & $\begin{array}{l}\text { PHTEMP } \\
\text { PHENTR } \\
\text { PHDENS } \\
\text { PHCP } \\
\text { entry PHCV } \\
\text { entry PHGAMM } \\
\text { PHSOUN } \\
\text { PHVISC } \\
\text { PHCOND } \\
\text { PHLFAC }\end{array}$ & $\begin{array}{l}\text { EHTEMP } \\
\text { EHENTR } \\
\text { EHDENS } \\
\text { EHCP } \\
\text { entry EHCV } \\
\text { entry EHGAMM } \\
\text { EHSOUN } \\
\text { EHVISC } \\
\text { EHCOND } \\
\text { EHLFAC }\end{array}$ \\
\hline \multicolumn{4}{|c|}{ Saturation Routines } \\
\hline $\begin{array}{l}P_{\text {vap }} \\
T_{\text {vap }}\end{array}$ & $\begin{array}{l}\mathrm{T}_{\text {vap }}, \mathrm{H}_{\text {gas }}, \mathrm{H}_{\text {liquid }} \\
\mathrm{P}_{\text {vap }}, \mathrm{H}_{\text {gas }}, \mathrm{H}_{\text {liquid }}\end{array}$ & $\begin{array}{l}\text { PSATH } \\
\text { TSATH }\end{array}$ & $\begin{array}{l}\text { EPSATH } \\
\text { ETSATH }\end{array}$ \\
\hline
\end{tabular}

Including the small saturation routines there are 36 decks (the output options $\mathrm{C}_{\mathrm{p}}, \mathrm{C}_{\mathrm{v}}$, and the ratio $\mathrm{C}_{\mathrm{p}} / \mathrm{C}_{\mathrm{v}}$ are combined into one deck). Each deck exists in a CDC and an IBM version. Program listings are given in Appendix B. 


\section{1 Method Used}

The conceptual idea of the computer programs follows from the use of a T-S or Mollier diagram. Using such a chart to obtain property data one visually or graphically interpolates between constant property lines. The computer programs imitate this procedure on the computer. However, in contrast to the visual procedure where any combination of existing variables can be used as entries to the chart, the computer programs are restricted to two entry possibilities, namely pressure-temperature and pressure-enthalpy.

Each property forms a surface with the two entry variables. A pre-determined number of points on this surface are calculated and form the array of data. The surface is divided into regions, the boundaries of which depend upon the relative linearity of the particular property surface. The $P, T$, or $H$ spacings vary from region to region to compensate for the non-linearity of the property surface. In general, spacings will be small where the property is varying quite rapidly and they will be large where the property is varying more slowly. Each program consists of an array of data and an interpolation package. The interpolation package finds the four points which bracket the entry values and then performs a two dimensional linear interpolation to obta in the value of the property to be returned.

The arrays of data are necessarily limited. When coupled with linear interpolation, errors in the values returned are possible. We have, therefore, determined the error envelope for each program and these are displayed as departure graphs.

\subsection{Peculiarities of the P-T Programs}

One prominent feature shown in the appropriate phase diagram, figure 3, is the vapor pressure curve. In all of the P-T programs this line is mathematically thin, that is, the property value returned corresponds to either the liquid or the vapor side. In following an isobar, an isotherm, or any other path that crosses the vapor pressure curve, one will encounter sizeable changes in the different variables. In addition, for many variables these large changes also exist for conditions near or just above the critical point. Re-stating the peculiarity a different way: it is impossible to pick a P-T input which will place the coordinates inside a two phase region. Since it is often necessary to obtain property values for conditions of saturation, saturated liquid, saturated vapor, or both, we have provided a way of obtaining these values. The saturation routines listed in table 2 are designed to fill this need. They are described in Section 3.4.

\section{3 Peculiarities of the P-H Programs}

In contrast to the $\mathrm{P}-\mathrm{T}$ programs it is quite possible to choose input values of $\mathrm{P}$ and $\mathrm{H}$ which place a point in question into a two phase region. From figure 4 it is seen that the boundaries of the programs are set specifically to omit returns from the liquid-solid (slush) two phase region. Returns from the liquid-vapor two phase region are, however, possible. 
Three distinct possibilities or problems connected with these returns are discussed in some detail below.

The Phase Designator, Q, from PHTEMP - One problem often encountered is that we need to know where we are on the phase diagram. To solve this problem the program PHTEMP (EHTEMP) is structured slightly different than all of the other programs. The other P-H programs carry the two entry variables $\mathrm{P}$ and $\mathrm{H}$ in the call list. PHTEMP carries an additional variable, $Q$, in its call list. A call to $\operatorname{PHTEMP}(P, H, Q)$ and a check of the $Q$ that is returned against table 1 will locate the point on the phase diagram. For example, if $0<Q<1$, then the $\mathrm{P}-\mathrm{H}$ input is in the liquid-vapor coexistence region. For this region $Q$ corresponds to the conventional quality and is defined as the percent (by mass) of the vapor phase present, or

$$
\mathrm{Q}=\left(\mathrm{H}-\mathrm{H}_{\text {liquid }}\right) /\left(\mathrm{H}_{\text {gas }}-\mathrm{H}_{\text {liquid }}\right)
$$

where $\mathrm{H}_{\text {liquid }}$ and $\mathrm{H}_{\text {gas }}$ are the enthalpy values at the respective pha se boundary appropriate to the entry pressure (see also fig. 4). As noted before, values of $Q$ for other parts of the phase diagram are given in table 1.

Returns from the Liquid-Vapor Two Phase Region - Assume that the input values of $\mathrm{P}$ and $\mathrm{H}$ place the point in question into the liquid-vapor two phase region. For this region the entry enthalpy $\mathrm{H}$ is used in all $\mathrm{PH}(\mathrm{EH})$ programs to find the phase designator or quality as defined above, and the phase designator or quality is then used to define the return, RE. For temperature, density, and entropy, these returns have the conventional meaning which is given in table 3. Other variables, that is $k, \eta, w, C_{v}$, and $L$, are not defined for this region in the sense of thermodynamics. However, the engineer often uses these properties in this region, and then defines them according to the specific application. Therefore, the returns given are artificial involving $Q$ and property values corresponding to the saturated liquid and saturated vapor as defined in table $3, \mathrm{C}_{\mathrm{p}}$ and $\gamma$ are infinite in this two phase region; thus the largest value available to the machine is returned. The returns are summarized in table 3 .

Property Values on the Saturation Boundaries - Property values of saturation are required in many applications. From the preceding discussions it is clear that these values are simply a special case of returns from the liquid-vapor two phase region. A property value on a phase boundary is obtained from the appropriate $\mathrm{PH}(\mathrm{EH})$ program by using pressure and the saturation enthalpy, liquid or gas, as entries. The saturation enthalpies can be obtained from the two small saturation routines.

\subsection{The Saturation Routines}

For points on the saturation boundary, i.e., on the vapor pressure curve of figure 3 , either $\mathrm{P}$ or $\mathrm{T}$ is sufficient to specify the conditions of the point in question (phase rule). Obviously, the pressures have to be between 1.022 and 187.506 psia and the temperatures 
Table 3. Returns from the Liquid-Vapor Two Phase Region

\begin{tabular}{|c|c|c|c|}
\hline \multicolumn{2}{|c|}{ Program } & \multirow{2}{*}{$\frac{\text { return mode }}{\text { conventional }}$} & \multirow{2}{*}{$\begin{array}{l}\text { description of return } \\
\text { RE = the temperature corresponding } \\
\text { to the input pressure (vapor pressure). } \\
\text { Additional output: the phase designator } \\
\text { Q. }\end{array}$} \\
\hline PHTEMP & EHTEMP & & \\
\hline PHDENS & EHDENS & conventional & $\begin{aligned} \mathrm{RE} & =\text { average density of mixture } \\
& =1.0 /\left[Q / \rho_{\text {vapor }}+(1-Q) / \rho_{\text {liquid }}\right]\end{aligned}$ \\
\hline PHENTR & EHENTR & conventional & RE proportional to quantities of \\
\hline PHCOND & EHCOND & artificial & liquid and vapor present according \\
\hline PHVISC & EHVISC & artificial & to the equation \\
\hline PHSOUN & EHSOUN & artificial & \\
\hline PHCV & EHCV & artificial & $R E=Q\left(\pi_{\text {vapor }}-\pi_{\text {liquid }}\right)+\pi_{\text {liquid }}$ \\
\hline PHLFAC & EHLFAC & artificial & $\begin{array}{l}\text { where } \pi \text { stands for the property } \\
\text { in question }\end{array}$ \\
\hline PHCP & $\mathrm{EHCP}$ & infinite & $\mathrm{RE}=$ the largest value available to \\
\hline PHGAMM & EHGAMM & infinite & the machine \\
\hline
\end{tabular}

between 24.845 and $59.356^{\circ} \mathrm{R}$. The saturation routines need to be entered with only one variable; the corresponding other variable on the vapor pressure curve is returned. In addition, the enthalpies of the saturated liquid, $\mathrm{H}_{\text {liquid }}$, and the saturated vapor, $\mathrm{H}_{\text {gas }}$, are returned corresponding to the two phase boundaries of figure 4. Saturation values for all other properties can then be obtained by using pressure and the appropriate saturation enthalpy as input to the desired P-H program, as shown in the example on page 16.

\section{PROGRAMMING INF ORMA TION}

\section{1 Deck Description}

All of the decks are coded as FORTRAN functions. The decks are completely self-contained, including the data arrays which are used for interpolation. Each deck may be used separately or in conjunction with others.

A prefix $\mathrm{PT}$ (ET) on a subprogram name means that it requires pressure-temperature as input variables while the prefix $\mathrm{PH}(\mathrm{EH})$ indicates that the input variables are pressureenthalpy. The remainder of the deck name designates the variable returned. The names of the decks and units for input and output are given in table 4 for p-hydrogen and in table 5 for e-hydrogen. Also shown in these tables are data loading routines, storage requirements, and number of cards in each deck. 
Table 4, Deck Description, p-hydrogen

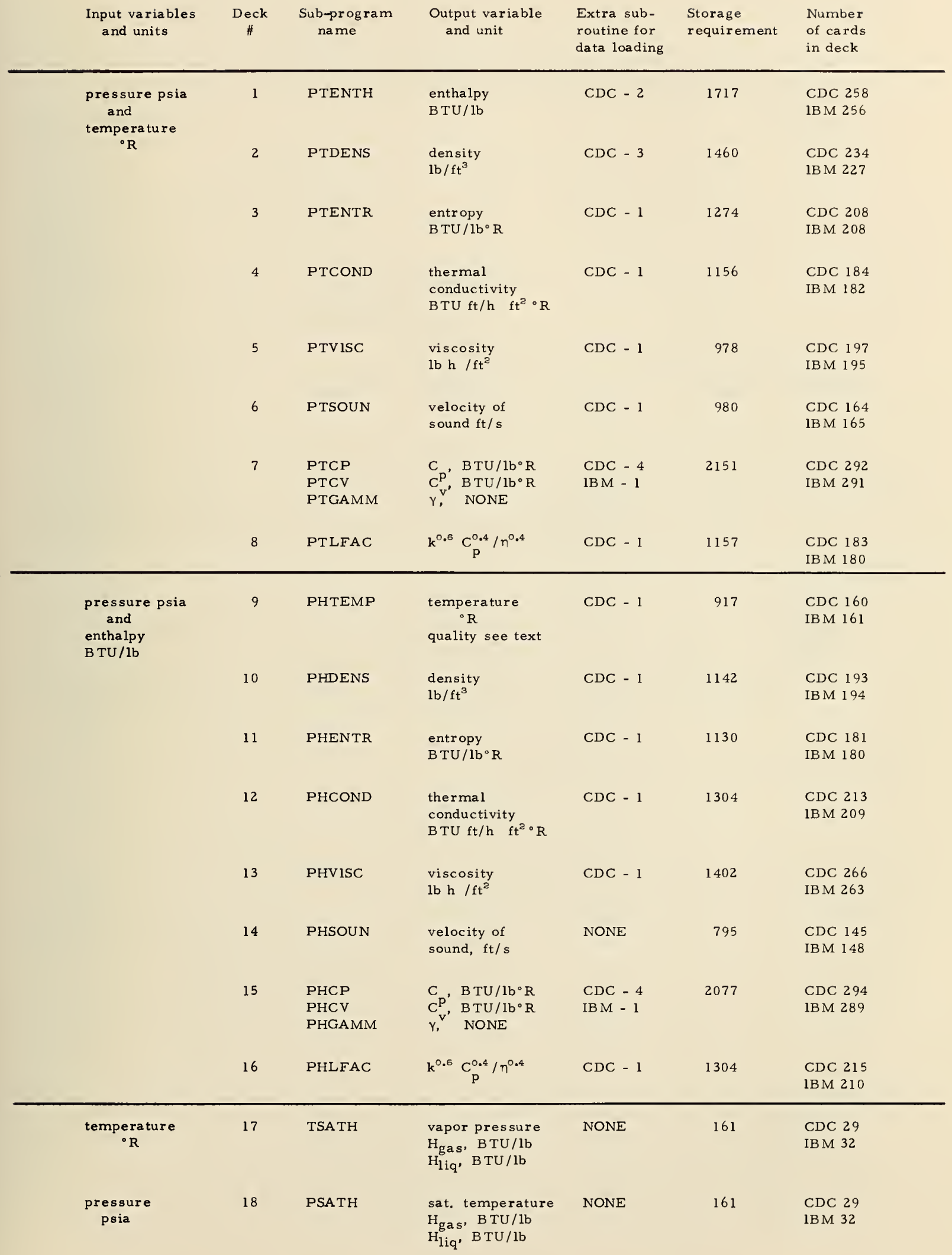


Table 5. Deck Description, e-hydrogen

\begin{tabular}{|c|c|c|c|c|c|c|}
\hline $\begin{array}{l}\text { Input variables } \\
\text { and units }\end{array}$ & $\begin{array}{c}\text { Deck } \\
\#\end{array}$ & $\begin{array}{l}\text { Sub-program } \\
\text { name }\end{array}$ & $\begin{array}{l}\text { Output variable } \\
\text { and unit }\end{array}$ & $\begin{array}{l}\text { Extra sub- } \\
\text { routine for } \\
\text { data loading }\end{array}$ & $\begin{array}{l}\text { Storage } \\
\text { requirement }\end{array}$ & $\begin{array}{l}\text { Number } \\
\text { of cards } \\
\text { in deck }\end{array}$ \\
\hline \multirow{8}{*}{$\begin{array}{l}\text { pressure psia } \\
\text { and } \\
\text { temperature } \\
{ }^{\circ} R\end{array}$} & 1 & ETENTH & $\begin{array}{l}\text { enthalpy } \\
\text { BTU/lb }\end{array}$ & CDC - 1 & 1717 & $\begin{array}{l}\text { CDC } 279 \\
\text { IBM } 255\end{array}$ \\
\hline & 2 & ETDENS & $\begin{array}{l}\text { density } \\
\mathrm{lb} / \mathrm{ft}^{3}\end{array}$ & $C D C-2$ & 1460 & $\begin{array}{l}\text { CDC } 229 \\
\text { IBM } 225\end{array}$ \\
\hline & 3 & ETENTR & $\begin{array}{l}\text { entropy } \\
\text { BTU } / 1 b^{\circ} R\end{array}$ & $C D C-1$ & 1274 & $\begin{array}{l}\text { CDC } 209 \\
\text { IBM } 206\end{array}$ \\
\hline & 4 & ETCOND & $\begin{array}{l}\text { thermal } \\
\text { conductivity } \\
\text { BTU } \mathrm{ft} / \mathrm{h} \quad \mathrm{ft}^{2}{ }^{\circ} \mathrm{R}\end{array}$ & CDC - 1 & 1156 & $\begin{array}{l}\text { CDC } 184 \\
\text { IBM } 182\end{array}$ \\
\hline & 5 & ETVISC & $\begin{array}{l}\text { viscosity } \\
\mathrm{lb} h / \mathrm{ft}^{2}\end{array}$ & $C D C-1$ & 978 & $\begin{array}{l}\text { CDC } 197 \\
\text { IBM } 195\end{array}$ \\
\hline & 6 & ETSOUN & $\begin{array}{l}\text { velocity of } \\
\text { sound } \mathrm{ft} / \mathrm{s}\end{array}$ & $C D C-1$ & 980 & $\begin{array}{l}\text { CDC } 164 \\
\text { IBM } 162\end{array}$ \\
\hline & 7 & $\begin{array}{l}\text { ETCP } \\
\text { ETCV } \\
\text { ETGAMM }\end{array}$ & $\begin{array}{ll}\mathrm{C}_{\mathrm{p}}, & \mathrm{BTU} / \mathrm{lb}^{\circ} \mathrm{R} \\
\mathrm{C}_{\mathrm{v}}, & \mathrm{BTU} / \mathrm{lb} \mathrm{b}^{\circ} \mathrm{R} \\
\mathrm{y}, & \text { NONE }\end{array}$ & $\begin{array}{l}C D C-3 \\
I B M-1\end{array}$ & 2151 & $\begin{array}{c}\text { CDC } 291 \\
\text { IBM } 293 \\
-\end{array}$ \\
\hline & 8 & ETLFAC & $\mathrm{k}^{0.6} \mathrm{C}_{\mathrm{p}}^{0.4 / \mathrm{n}^{0.4}}$ & $\mathrm{CDC}-1$ & 1157 & $\begin{array}{l}\text { CDC } 191 \\
\text { IBM } 180\end{array}$ \\
\hline \multirow{8}{*}{$\begin{array}{l}\text { pressure psia } \\
\quad \text { and } \\
\text { enthalpy } \\
\text { BTU/1b }\end{array}$} & 9 & EHTEMP & $\begin{array}{l}\text { temperature } \\
{ }^{\circ} \mathrm{R} \\
\text { quality see text }\end{array}$ & $C D C-1$ & 917 & $\begin{array}{ll}\text { CDC } & 161 \\
\text { IBM } & 160\end{array}$ \\
\hline & 10 & EHDENS & $\begin{array}{l}\text { density } \\
\mathrm{lb} / \mathrm{ft}^{3}\end{array}$ & $\mathrm{CDC}-1$ & 1142 & $\begin{array}{l}\text { CDC } 195 \\
\text { IBM } 194\end{array}$ \\
\hline & 11 & EHENTR & $\begin{array}{l}\text { entropy } \\
\text { BTU } / 1 b^{\circ} R\end{array}$ & $C D C-1$ & 1130 & $\begin{array}{l}\text { CDC } 182 \\
\text { IBM } 180\end{array}$ \\
\hline & 12 & EHCOND & $\begin{array}{l}\text { thermal } \\
\text { conductivity } \\
\text { BTU } \mathrm{ft} / \mathrm{h} \quad \mathrm{ft}^{2}{ }^{\circ} \mathrm{R}\end{array}$ & $C D C-1$ & 1304 & $\begin{array}{l}\text { CDC } 213 \\
\text { IBM } 211\end{array}$ \\
\hline & 13 & EHVISC & $\begin{array}{l}\text { viscosity } \\
\mathrm{lb} h / \mathrm{ft}^{2}\end{array}$ & $C D C-1$ & 1402 & $\begin{array}{l}\text { CDC } 267 \\
\text { IBM } 267\end{array}$ \\
\hline & 14 & EHSOUN & $\begin{array}{l}\text { velocity of } \\
\text { sound, ft/s }\end{array}$ & NONE & 798 & $\begin{array}{l}\text { CDC } 141 \\
\text { IBM } 141\end{array}$ \\
\hline & 15 & $\begin{array}{l}\text { EHCP } \\
\text { EHCV } \\
\text { EHGAMM }\end{array}$ & $\begin{array}{ll}\mathrm{C}_{\mathrm{p}}, & \mathrm{BTU} / 1 \mathrm{~b}^{\circ} \mathrm{R} \\
\mathrm{C}_{\mathrm{v}}, & \mathrm{BTU} / \mathrm{lb} \mathrm{b}^{\circ} \mathrm{R} \\
\gamma, & \text { NONE }\end{array}$ & $\begin{array}{l}\text { CDC - } 2 \\
\text { IBM }-1\end{array}$ & 2077 & $\begin{array}{l}\text { CDC } 289 \\
\text { IBM } 291\end{array}$ \\
\hline & 16 & EHLFAC & $\mathrm{k}^{0.6} \mathrm{C}_{\mathrm{p}}^{0.4} / \mathrm{n}^{0.4}$ & $C D C-1$ & 1304 & $\begin{array}{l}\text { CDC } 227 \\
\text { IBM } 210\end{array}$ \\
\hline $\begin{array}{c}\text { temperature } \\
{ }^{\circ} R\end{array}$ & 17 & ETSATH & $\begin{array}{l}\text { vapor pressure } \\
\mathrm{H}_{\text {gas }}, \mathrm{BTU} / \mathrm{lb} \\
\mathrm{H}_{\text {liq, }} \mathrm{BTU} / \mathrm{lb}\end{array}$ & NONE & 161 & $\begin{array}{l}\text { CDC } 30 \\
\text { IBM } 30\end{array}$ \\
\hline $\begin{array}{l}\text { pressure } \\
\text { psia }\end{array}$ & 18 & EPSATH & $\begin{array}{l}\text { sat. temperature } \\
\mathrm{H}_{\text {gas }}, \mathrm{BTU} / \mathrm{lb} \\
\mathrm{H}_{\text {liq, }} \mathrm{BTU} / \mathrm{lb}\end{array}$ & NONE & 161 & $\begin{array}{l}\text { CDC } 30 \\
\text { IBM } 30\end{array}$ \\
\hline
\end{tabular}


Since nearly every computer has available some type of FORTRAN processing language, the programs were written in FORTRAN IV. Program listings for both the CDC and the IBM versions of all decks are given in Appendix $B$.

\section{2 Calling Sequence}

Since the decks are individual FORTRAN functions the calling sequences are predictably simple.

$$
\begin{aligned}
& P=x \cdot x \\
& T=y \cdot y \\
& H=z \cdot z
\end{aligned}
$$

and then

or

$$
\begin{aligned}
& \text { RHO }=\operatorname{PTDENS}(\mathrm{P}, \mathrm{T}), \\
& \text { RHOE }=\operatorname{EHDENS}(\mathrm{P}, \mathrm{H}) .
\end{aligned}
$$

Provided that the values of $\mathrm{P}, \mathrm{T}$, and $\mathrm{H}$ are valid, that is, within the program range, the density of para hydrogen is stored into RHO or the density of equilibrium hydrogen into RHOE.

The only variation on this theme occurs for the routine PHTEMP (EHTEMP) and the saturation routines. The change occurs not in the calling sequence but rather in what information is available after the call is made. Examples are given below.

$$
\begin{aligned}
& P=x . x \\
& H=z . z
\end{aligned}
$$

$\operatorname{TEMP}=\operatorname{PHTEMP}(P, H, Q)$ or TEMP $=\operatorname{EHTEMP}(P, H, Q)$.

The temperature corresponding to the input pressure is found in TEMP. In addition, the phase designator $Q$ is available through the parameter list, for example,

$$
\mathrm{QUAL}=\mathrm{Q} \text {. }
$$

Note that we did not set $Q$ prior to the call. The saturation routines use very similar logic.

$$
\mathrm{P}=\mathrm{x} . \mathrm{x}
$$

TVAP $=$ PSA TH $(P, H G, H L)$ or TVAP $=$ EPSA TH $(P, H G, H L)$

The temperature corresponding to the input pressure (vapor pressure) is found in TVAP. In addition, the enthalpy of the saturated liquid and the enthalpy of the saturated vapor are available through the parameter list; thus

$$
\begin{aligned}
& \text { HGAS }=\mathrm{HG}, \quad \text { and } \\
& \text { HLIQ }=\mathrm{HL} .
\end{aligned}
$$

The sequence with the other saturation routine is as follows:

$$
\mathrm{T}=\mathrm{y} \cdot \mathrm{y}
$$

PVAP $=$ TSATH $(T, H G, H L)$ or PVAP = ETSATH $(T, H G, H L)$.

Here the vapor pressure is returned in PVAP, and again the enthalpies of saturated liquid and vapor are available through the parameter list. Next, assume the call above has been made, but what really is required is the entropy of the saturated vapor. The 
sequence is completed by

SGAS = PHENTR (PVAP, HG) or SGASE = EHENTR (PVAP, HG).

\section{3 Exceeding the Range of the Programs}

It is, of course, easy to violate the range of the programs when setting the values of the input variables. The problem seems to occur frequently if unit conversions are involved. We have omitted error messages for cases of boundary violations because each installation handles printed error messages differently.

For the PT (ET) and PH (EH) programs, the out of bounds variable is automatically set to a value acceptable to the program, and computations proceed. The value returned is, of course, in error. Possible boundary violations are shown in the phase diagrams, figures 3 and 4 , by dots, and the arrows indicate what conditions are actually processed. A complete description is as follows:

Pressure $P$ out of bounds

If the entry pressure is less than 1 psia, the value of pressure is set to 1 .

If the entry pressure is greater than 5000 psia, the returned value is extrapolated from within the defined range.

Temperature $\mathrm{T}$ out of bounds

If the entry temperature is less than the temperature appropriate to melting conditions, the temperature is set to the melting temperature at the same pressure.

If the entry temperature is greater than $6000^{\circ} \mathrm{R}$, the value of temperature is set to $6000^{\circ} \mathrm{R}$.

Enthalpy $\mathrm{H}$ out of bounds

If the entry enthalpy is less than the enthalpy appropriate to melting conditions, the enthalpy is set to the melting enthalpy.

If the entry enthalpy is greater than $25,000 \mathrm{BTU} / 1 \mathrm{~b}$, the value of the enthalpy is set to 25, $000 \mathrm{~B} \mathrm{TU} / 1 \mathrm{~b}$.

\section{Saturation routines}

If either program is entered with a value outside the pressure or temperature range of the vapor pressure curve, the variable is set to the nearest end point of the curve (see figure 3 ).

\section{4 Conversion to Other Systems or Installations}

The programs as listed in Appendix B were written to avoid known incompatibilities. We have tested them on a CDC 3600, a CDC 3800, and an IBM 360. Other installations have run them on an 1108 Univac. Thus, conversion requirements should be minimal. Incompatibilities that we are aware of include

$\begin{array}{lll}\operatorname{DATA}(\ldots \ldots) & \text { vs. } & \operatorname{DATA}(\ldots \ldots) \\ \operatorname{ABSF}(\ldots \ldots) & \text { vs. } & \operatorname{ABS}(\ldots \ldots) \\ \operatorname{EXPF}(\ldots \ldots) & \text { vs. } & \operatorname{EXP}(\ldots \ldots)\end{array}$

Another source of incompatibilities arises from the use of different key board symbols on certain types of card punches, i. e., ()$+=$ vs. $\%<\&$ \& 
The programmer should note that the data arrays are entered via DATA statements. The upper limit on the number of such statements varies from installation to installation. The least number that we are awa re of has been used. Since this means that in many cases not all of the data can be loaded with the actual program deck, we had recourse to data loading subroutines which are listed in tables 4 and 5. If a limitation is encountered, a possible solution is as follows (Goldberg, 1967):

Problem: $\quad$ File length not sufficient to include all DATA statements of routine PTENTH.

Solution: Two segments used, 1. BLOCK DATA, 2. Function PTENTH modified. Details: 1. BLOCK DATA (new for PTENTH function) COMMON/BLKPTH/AA... AO, followed by the DATA statements for arrays AA through AO (total 1183 values equivalent to $\mathrm{H}$ array).

2. Main function PTENTH is revised to include all DATA arrays except $\mathrm{H}$ (1183). The $\mathrm{H}$ array is linked to the main function through named common: COMMON/BLKPTH/H(1183).

4. 5 Pitfalls, Common Errors in Applications

The earlier version of these decks has been available for nearly five years. Several points, where users have encountered difficulties, are described below: Storage Limitation - Each program is designed to be used independently of all others. Specify only those decks actually required. Total storage required is specified in tables 4 and 5. Storage requirements range from 795 to 2151.

Program Range - Observe the limits of the programs 1 - 5000 psia, $24.845-6000^{\circ} \mathrm{R}$, $-130-25,000 \mathrm{BTU} / \mathrm{lb}$. Valid answers will not be obtained if these bounds are violated. Liquid-Vapor Two Phase Conditions - There is no built-in warning in any of the PH(EH) decks telling the user that he is in the liquid-vapor two phase region. This means the user should check the value of $Q$ to confirm the conditions he is trying to process.

Liquid-Vapor Two Phase Returns - Returns from this region for the variables $k, \eta, \omega, C_{p}$, $\mathrm{C}_{\mathrm{v}}, \gamma$, and $L$, are artificial, as defined in table 3 . The programs will return numbers for these variables, but what usually is desired are not these returns but rather the property values for saturated liquid or saturated vapor.

Errors - The two categories of errors, interpolation error and estimated source data errors, are discussed in detail in Section 6. The user has to be aware of the limitations inherent in linear interpolation. The detailed graphs of maximum interpolation errors given in Appendix $C$ should alert the user to possible problems, and serve as an aid in solving those problems.

\section{6 Debugging Aids}

In addition to the program listings given in Appendix $B$, we have found that a set of selected values for the various properties is indispensable. In Appendix A we list values 
obtained from the PT(ET) programs. Values for all properties for a representative set of temperatures are printed for isobars of 1, 15,50,150,500,1500, and 4500 psia. These skeleton tables are to be used to establish approximate values, to make an order of magnitude check, to check units, and to debug the running of the programs at other installations.

\section{STRUCTURAL DETAILS OF THE PROGRAMS}

As mentioned before, each program can be visualized as an array of data coupled with an interpolation scheme. Each property value entered into the data array corresponds to a particular pair of $\mathrm{P}-\mathrm{T}$ or $\mathrm{P}-\mathrm{H}$. The spacing or increment for each entry variable is independent of the other and varies over the surface of the property. The total network of spacings is called the grid and a sequence of equal spacings defines a region.

\section{1 Grid and Region Layout}

The grid for each variable consists of 12 to 25 different regions. The size of each region and its relation to neighboring regions is determined by the desired interpolation error and by the relative linearity of the particular property surface. A plot of constant property lines is used as an aid in layout. An example of grid-region layout for p-hydrogen is shown in figure 5 where lines of constant $C_{p}$ (in BTU/lb- ${ }^{\circ} R$ ) are plotted for entry variables $P$ and $H$. Lines of constant $C_{p}$ and regions $G$ through $Q$ are shown. Within each

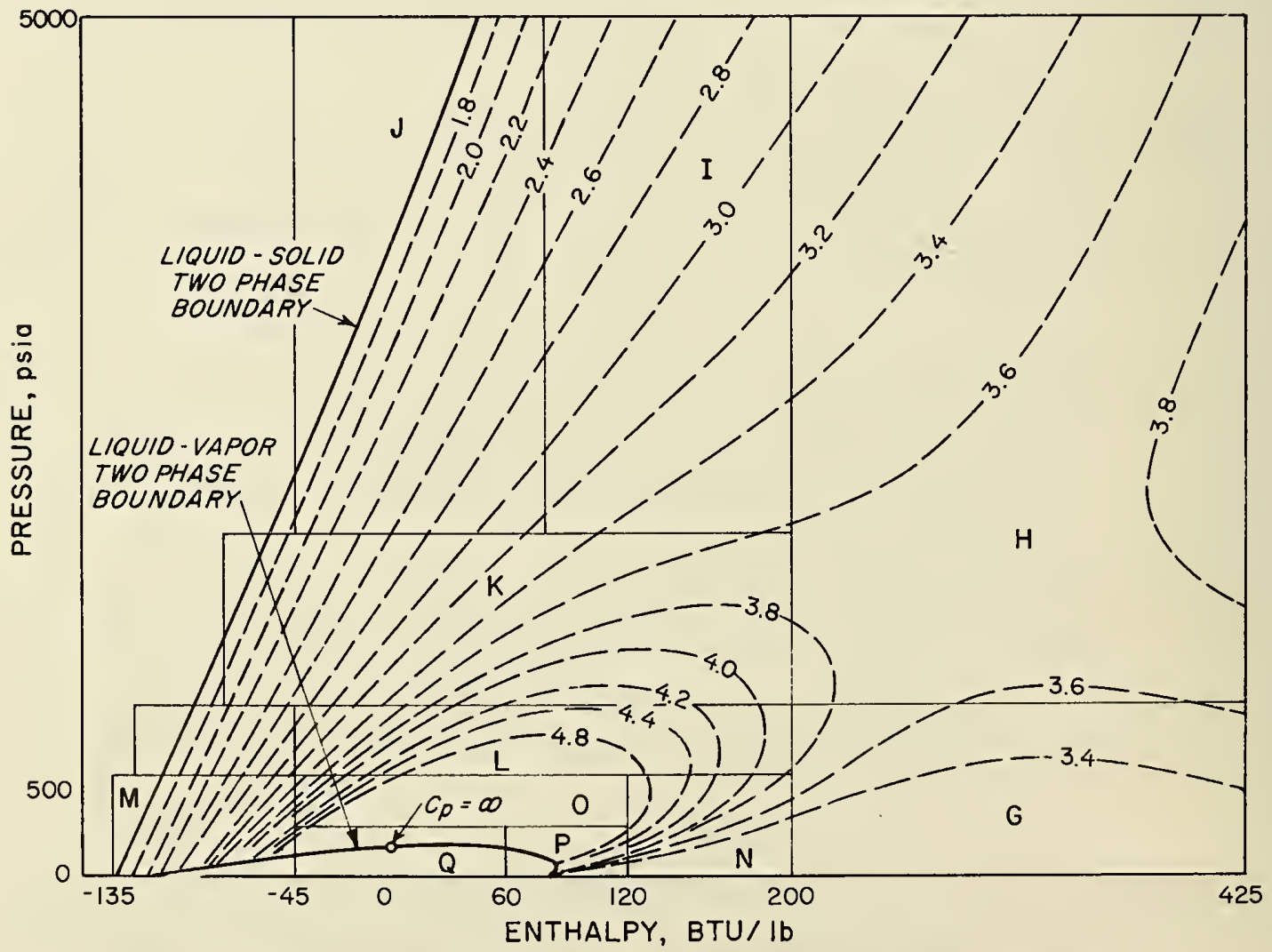

Figure 5. Region Layout for $\mathrm{C}_{\mathrm{p}}$ with Entry Variables $\mathrm{P}-\mathrm{H}$, p-hydrogen 
region increments of the entry variables, $\mathrm{P}-\mathrm{T}$ or $\mathrm{P}-\mathrm{H}$, are fixed. The spacings used for the various regions were intended to yield a $1 \%$ interpolation error; however, subsequent checks show that we have not always achieved this goal. A data array developed according to this scheme will vary from variable to variable; it will minimize the number of points required to represent the surface of the variable to be interpolated.

While in general the property values entered in the data arrays are exact, there are a few cases where these entries have been adjusted to yield better results for the interpolation. One example is entries at low pressures, that is, some regions are extended to negative pressures and the entries at these negative pressures are bogus values. They do, however, insure a more precise interpolation down to the lower limit of pressure. Region $B$ shown in the partial grid layout of figure 6 is a typical example. The lower pressure limit

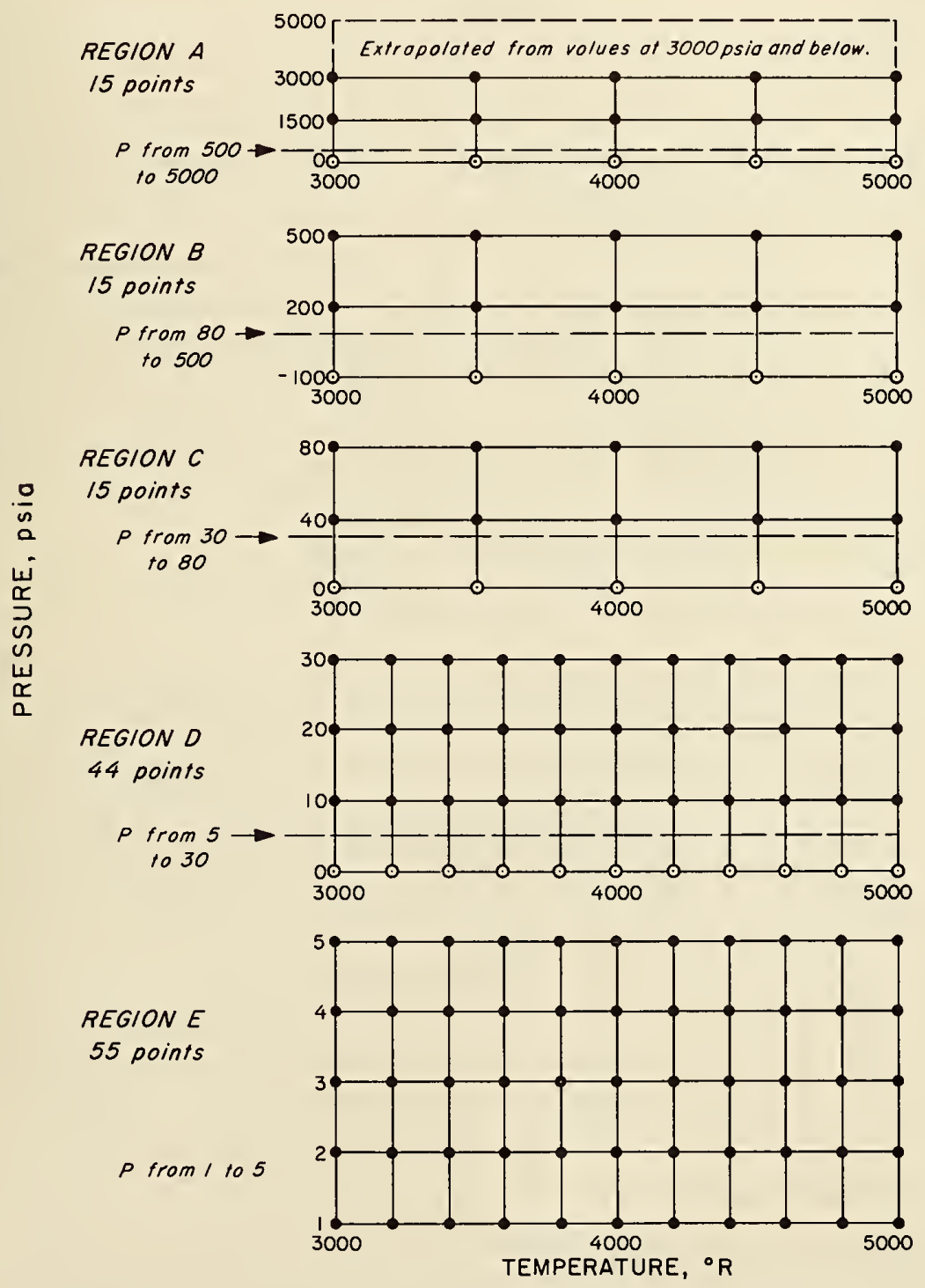

Figure 6. Partial Grid Layout for Program PTENTH 
for interpolation from this region is 80 psia and entries in the data array at - 100 psia are bogus values (shown as $\odot$ ). A similar technique better described as a partial overlap of region boundaries is used in Region A, C, and D. For best accuracy values at 15 psia are interpolated from Region $D$, not from Regions $A, B$, or $C$. Region $E$ is the only one illustrated in which bogus values are not used. The entire sequence illustrates how difficult it is to follow the large variation in enthalpy in the region of dissociation.

Interpolation along the two phase boundaries also involves the use of bogus points that have no counter part on the surface of the property in question. For the P-T programs, the points are across the two phase boundary while for the P-H programs they have been added inside the two phase boundaries. In either case, these bogus points are used only as interpolation aids to get from the single phase region right up to the two phase boundary.

\subsection{Speed}

The programs gain their operational speed from performing two dimensional linear interpolation in a fixed array of data. Actual speed of computation depends, of course, on the particular installation. At our installation, a CDC 3800, returns run from 30, 000 to 60,000 per minute. The data used for interpolation have been placed into compact DATA statements to provide rapid access. Access to any given region is just as fast as to any other region because of the way searching is arranged. The first branching divides the number of regions in half, the second in half again, and so on. Speed is reduced by about a factor of 2 if lookup of the phase boundaries is required for the point under consideration. The programs return a single answer with two variables (three for PHTEMP) going through the transfer vector.

\section{3 Continuous Functions}

In the approach taken here, continuous functions cannot be obtained because the increments of the entry variables change when crossing region boundaries. The problem is illustrated by two simple examples shown in figure 7. In case I, points $1,2,3,4$, and 5 lie on the boundary of the lower region, while points 1,3 , and 5 are points on the boundary of the adjacent upper region. Linear interpolation along the boundary for each region is shown by the straight lines. If points 2 and 4 are adjusted to $2^{\prime}$ and $4^{\prime}$, interpolation in both regions will be the same and jumps in the value of the property returned will not occur across this boundary. In case II, points $1,2,4$, and 5 are in the lower region while 1,3 , and 5 lie in the adjacent upper region. The values of 2 and 4 cannot be adjusted to avoid a jump in the property interpolated*. An actual example where boundaries cannot be made to match can be seen in the transition from 30 psia, Region D, to 30 psia, Region C, of figure 6.

* We cannot adjust the value of point 3 as the interpolation error at 3 would be larger than the desired $1 \%$. 

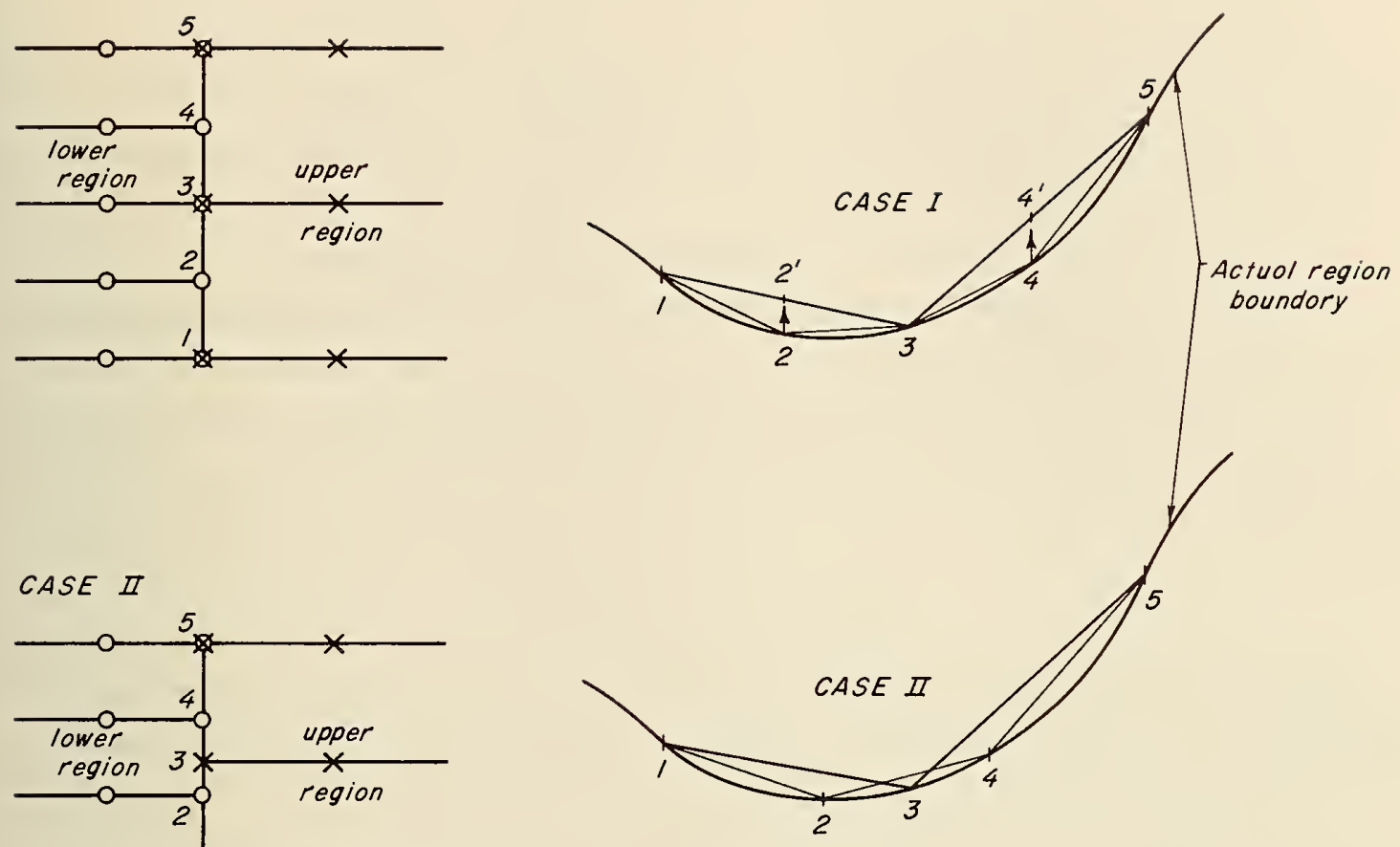

CASE II

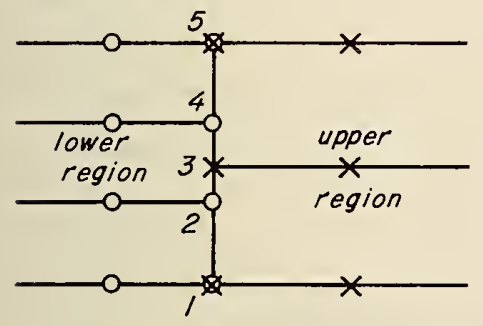

If the points on the boundary are common to both regions, then the boundaries con be mode continuous.

Figure 7. Matching of Values at Boundaries

\section{4 Modifications, Future Developments}

Changes are a way of life with computer programs. Improvements will be dictated by the user - they may arise from better source data. One possible modification is described below.

Return of Values on the Two Phase Boundary - In many applications involving the PH (EH) programs, some of the returns are from the two phase region. In this case, the property values on the respective boundaries are required. Since the programs calculate the values at the boundaries for the regular return, a slight change in program structure will yield the desired answers. The changes are illustrated by the following example for entropy:

FUNCTION PHENTR (PRES, ENTH) change to FUNCTION PHENTR (PRESS, ENTH, SGG, SLL)

and add at $25+2$

$$
\begin{aligned}
& \text { SGG }=\text { SGAS } \\
& \text { SLL }=\text { SLIQ }
\end{aligned}
$$

In other words, the new logic is similar to that used in $\operatorname{PHTEMP}(P, H, Q)$ and is accomplished at the expense of having two additional variables pass through the transfer vector.

Future Possibilities - It is interesting to speculate on improvements that might be accomplished in the future based on what we have learned about the linear interpolation scheme. An advanced scheme of interpolation would save storage, improve accuracy, and allow a large combination of variables. It would be based on the following elements: 
1. The development of a single program to include

a) an interpolation package

b) a data array of fixed size, about 350 points per property.

2. The use of higher order or, perhaps spline interpolation in the interpolation package.

3. The use of the distribution in grid entries obtained during the present error analysis (see for example tables 8 and 9) to create an optimum point distribution for the new array of data.

4. A separate program which would call the generating functions (see Section 6.8) and create the array of data desired.

\section{ESTIMATION OF ERR ORS}

Terms such as accuracy, precision, uncertainty, and error are used in scientific and engineering papers with many shades of meaning. To avoid ambiguity, the terms used in this report are defined as follows:

First, we define error as the quantitative difference which exists between the true value and the value which is measured, calculated, or otherwise determined.

Second, uncertainty is defined as an estimate of the error that may exist between reported and true values. Since usually we do not know the true value, the estimate is by nature a variable quantity. The estimate of error or uncertainty will vary according to the information available and the manner in which the estimate is obtained.

Statistical methods normally are used to arrive at estimates of error or uncertainties. However, statistical methods are valid only for random processes, and therefore uncertainties based on statistical methods are estimates based on the presence of random errors only. In contrast to statistics, the nature of thermophysical data is such that systematic errors usually are present, i.e., errors which are not random. Not only are systematic errors present but of ten they are of much greater importance than the truly random errors.

To be realistic in assigning uncertainties we have combined the estimates of both random and systematic errors, except where the uncertainties are labeled "statistical uncertainties" in which case only random errors are given. To minimize the risk of a true value being outside of the interval specified by our assigned uncertainty, the assignment of uncertainties is large enough to give a $99 \%$ chance of including the correct value, i.e., a $3 \sigma$ level. The $\sigma$ is used here in the conventional sense of standard deviation.

Differentiation is made between uncertainties in the data of the sources chosen and interpolation error, i.e., the difference between the values returned by linear interpolation and the source chosen. Maximum interpolation errors and estimated source errors for each program are displayed graphically in Appendix $C$ in three ranges of temperature: 25 to $180^{\circ} \mathrm{R}, 180$ to $1200^{\circ} \mathrm{R}$, and 1200 to $6000^{\circ} \mathrm{R}$. The ranges of enthalpy for the $\mathrm{PH}$ and EH type 
programs correspond closely to the temperature ranges just mentioned. All plots are given in percentages except for the low temperature range of enthalpy, i.e., 25 to $180^{\circ} \mathrm{R}$ for program PTENTH (ETENTH). The enthalpy in this range of temperature can assume both negative and positive values (see also fig. 4); therefore, the interpolation and source errors for this range only are plotted in $\mathrm{BTU} / \mathrm{lb}$. The symbol -..- in the plots signifies that the PVT surface for these temperatures is extrapolated.

Estimated source errors are discussed in some detail in Section 7. The estimated source errors are established by the authors of the source data, by comparison with experimental values, by noting the spread of values if several experimental sets exist, or by intercomparison of several methods of calculation if no experimental values are available. For the high temperature extrapolations we combine the estimates of Woolley (1972) for the ideal gas, our estimates of errors for the real gas contribution, and an arbitrary estimate for errors due to dissociation.

Interpolation errors are apt to intrude on most applications. These errors are nominally 3\% except for the critical region and the region of dissociation where departures from best values become large. The critical parameters are $P_{C}=187.506 \mathrm{psia}$, $\mathrm{T}_{\mathrm{c}}=59.357^{\circ} \mathrm{R}$, and $\mathrm{H}_{\mathrm{c}}=16.455 \mathrm{BTU} / 1 \mathrm{~b}$ for $\mathrm{p}$-hydrogen or $\mathrm{H}_{\mathrm{c}}=31.225 \mathrm{BTU} / 1 \mathrm{~b}$ for e-hydrogen. The onset of dissociation varies from $2700^{\circ} \mathrm{R}$ at the lower pressures to $5400^{\circ} \mathrm{R}$ at the highest pressures. The interpolation errors plotted are the envelope of maximum error in interpolation at the temperature or enthalpy in question. They are established from an intercomparison of values returned by the interpolation routine and the generating functions. The comparisons are made at a large number of temperatures and a large number of pressures for each temperature, selecting the largest deviation in a given range of temperature or enthalpy for the plot. The distribution of temperatures and pressures at which the intercomparisons were made are established from the grids of the properties by collecting values at $1 / 2$ of each normal grid increment and eliminating duplicates. As an example, each program was checked in the low temperature range at every combination of $P$ and $T$ given in tables 6 and 7 .

Table 6. Low Temperature Error Grid, Pressures, psia

$\begin{array}{rrrr}1.022 & 36.740 & 146.960 & 225.000 \\ 1.500 & 44.088 & 154.308 & 230.000 \\ 2.000 & 51.436 & 161.650 & 235.000 \\ 3.000 & 58.784 & 169.004 & 240.000 \\ 4.000 & 66.132 & 176.352 & 245.000 \\ 5.000 & 73.480 & 180.026 & 250.000 \\ 6.000 & 80.828 & 183.700 & 255.000 \\ 7.000 & 88.176 & 187.370 & 260.000 \\ 8.000 & 95.524 & 190.000 & 265.000 \\ 9.000 & 102.872 & 195.000 & 270.000 \\ 10.000 & 110.220 & 200.000 & 275.000 \\ 11.022 & 117.568 & 205.000 & 280.000 \\ 14.696 & 124.916 & 210.000 & 285.000 \\ 22.044 & 132.264 & 215.000 & 293.920 \\ 29.372 & 139.612 & 220.000 & 301.268\end{array}$

308.616

315.964

323.312

330.660

338.008

345.356

352.704

360.052

367.400

374.748

382.096

389.444

396.792

404.140

411.488
418.836

426.184

433.532

440.880

459.250

477.620

495.990

514.360

532.730

551.100

$569.47 \mathrm{~J}$

587.840

624.580

661.320

698.060
734.800

771.540

808.280

845.020

881.760

918.500

955.240

991.980

1028.720

1065.460

1102.200

1138.940

1175.680

1322.640

1469.600
1763.520

2000.000

2500.000

2645.280

3233.120

3820.960

4408.800

5000.000 
Table 7. Low Temperature Error Grid, Temperatures, ${ }^{\circ} \mathrm{R}$

$\begin{array}{lllllr}25.000 & 40.700 & 55.100 & 62.500 & 70.500 & 90.000 \\ 26.000 & 41.600 & 56.000 & 63.000 & 70.571 & 93.600 \\ 27.500 & 42.500 & 56.500 & 63.500 & 71.500 & 97.200 \\ 29.000 & 43.400 & 57.000 & 64.000 & 72.000 & 100.800 \\ 30.500 & 44.300 & 57.500 & 64.500 & 72.900 & 104.400 \\ 30.800 & 45.200 & 58.000 & 65.000 & 73.800 & 108.000 \\ 31.700 & 46.100 & 58.500 & 65.500 & 74.700 & 112.500 \\ 32.600 & 47.000 & 59.000 & 66.000 & 75.600 & 117.000 \\ 33.500 & 47.900 & 59.400 & 66.500 & 76.500 & 121.500 \\ 34.400 & 48.800 & 59.625 & 67.000 & 77.400 & 126.000 \\ 35.300 & 49.700 & 59.850 & 67.500 & 78.300 & 135.000 \\ 36.200 & 50.600 & 60.000 & 68.000 & 79.200 & 144.000 \\ 37.100 & 51.500 & 60.500 & 68.500 & 80.000 & 153.000 \\ 38.000 & 52.400 & 61.000 & 69.000 & 82.500 & 162.000 \\ 38.900 & 53.300 & 61.500 & 69.500 & 85.000 & 171.000 \\ 39.800 & 54.200 & 62.000 & 70.000 & 86.400 & 180.000\end{array}$

For the most part, users will be concerned only with the interpolation error because in most cases the uncertainties in the source data are negligible in comparison to the interpolation error. Interpolation errors can be established quickly by visual estimate from the appropriate plot. If the generating functions (Section 7.8 ) are used, only the estimated source errors need to be considered.

\section{SOURCES OF PROPERTIES DATA}

To calculate values of the various properties, we require the thermodynamic functions of the ideal gas, a description of the PVT surface, a model to calculate the effects of dissociation, and descriptions of the behavior of viscosity and thermal conductivity. The major references used are shown in figure 8 and are discussed in more detail below.

\section{1 Ideal Gas Properties}

As sources for the thermodynamic properties of the ideal gas we have considered Woolley, et al. (1948), NBS Circular 564 (Hilsenrath, et al., 1955) and NBS Monograph 20 (Haar, et a1., 1961). The first two sources are virtually identical; they differ only because Woolley includes the effect of nuclear spin in his tabulations for entropy (i.e., $2 \mathrm{R} \ln 2$ ). Normally the nuclear spin contributions are omitted; both Circular 564 and Monograph 20 are tabulated according to this convention. Slight differences in thermal properties between Circular 564 and Monograph 20 exist. They can be detected at temperatures above $1260^{\circ} \mathrm{R}$ $(700 \mathrm{~K})$; they increase as the temperature increases. For $\mathrm{C}_{\mathrm{p}}^{0}$ the difference is about $1 \%$ at $5000^{\circ} \mathrm{R}(2800 \mathrm{~K})$ while for $\mathrm{H}^{\circ}-\mathrm{E}_{0}^{\circ}$ the difference is $44.6 \mathrm{BTU} / 1 \mathrm{~b}(209 \mathrm{~J} / \mathrm{mol})$. A more recent calculation (Baehr, et al., 1966) is very close to the earlier values of Woolley, et a1. (1948). We have, therefore, elected to use the properties given by Woolley for parahydrogen up to $3600^{\circ} \mathrm{R}(2000 \mathrm{~K})$. At this temperature Woolley discontinues the entries for parahydrogen but continues the entries for normal hydrogen on up to $9000^{\circ} \mathrm{R}(5000 \mathrm{~K})$. At $3600^{\circ} \mathrm{R}$, differences between the ideal gas properties of para and normal hydrogen are 


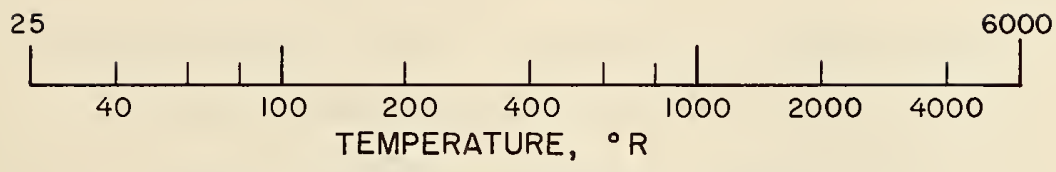

SOURCE DATA - THERMODYNAMIC PROPERTIES

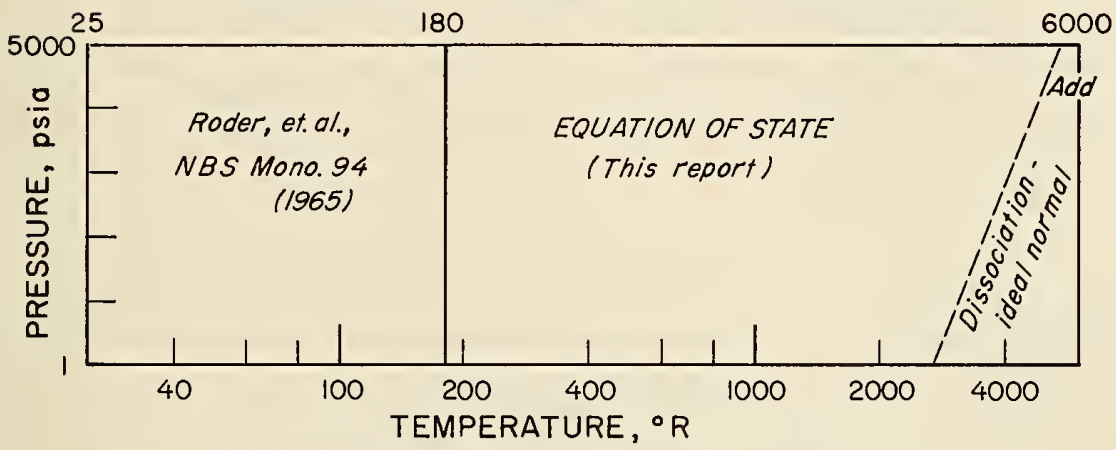

SOURCE DATA - TRANSPORT PROPERTIES

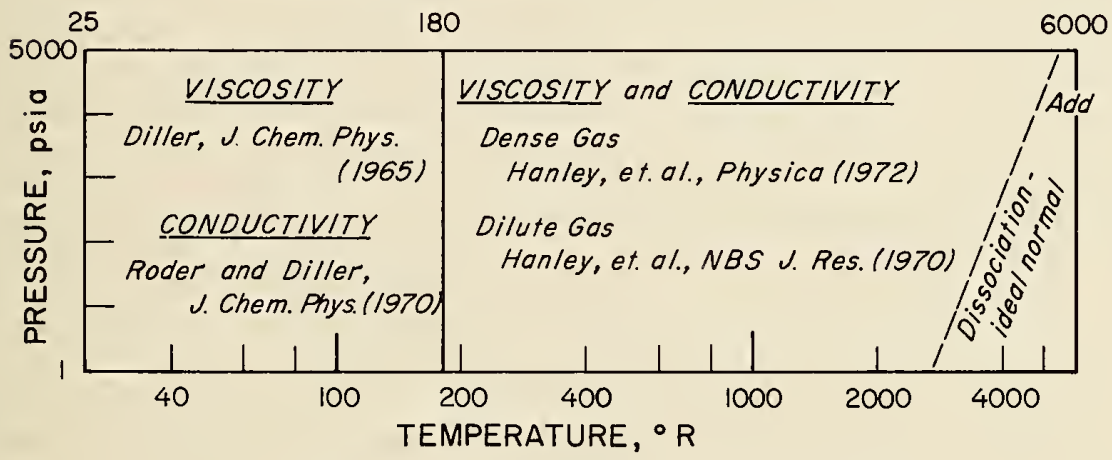

Figure 8. Primary Sources of Data

negligible except in the case of entropy where the difference is $1.367 \mathrm{BTU} / 1 \mathrm{~b}-{ }^{\circ} \mathrm{R}$. The difference represents the change in order or configuration between para and normal hydrogen. Above $3600^{\circ} \mathrm{R}$ the next temperature entry in Woolley's tables is $5400^{\circ} \mathrm{R}(3000 \mathrm{~K})$. For temperatures of $5400^{\circ} \mathrm{R}$ and above, the ideal gas properties of normal hydrogen were used for both para and equilibrium hydrogen since at these temperatures, equilibrium and normal hydrogen properties are identical.

While the entries for equilibrium hydrogen are perfectly proper, for parahydrogen this means that we as sume complete conversion to normal hydrogen between $3600^{\circ}$ and $5400^{\circ} \mathrm{R}$. A higher order interpolation insures that the transition in entropy for the parahydrogen decks is smooth and continuous. It becomes necessary to switch to the normal mixture at some point because the dissociation calculations (Section 7.5) are based on normal hydrogen. It should be noted that the present programs are oriented toward a partic- 
ular process which begins in the cryogenic range and ends at high temperatures. It would not be proper to use the parahydrogen programs on a process which begins in the cryogenic range, goes to high temperatures, and then is returned to the cryogenic range, because most likely the hydrogen would not be returned to the para form on cooling.

\subsection{The PVT Surface at Low Temperatures}

Values used in the data arrays for temperatures up to $180^{\circ} \mathrm{R}(100 \mathrm{~K})$, for all properties except viscosity and thermal conductivity, were obtained from NBS Monograph 94 (Roder, et al., 1965). The uncertainties in source data were taken from this reference and are displayed as estimated source errors in Appendix C for temperatures from 25 to $180^{\circ} \mathrm{R}$ (- 130 to $425 \mathrm{BTU} / 1 \mathrm{~b}$ or $675 \mathrm{BTU} / 1 \mathrm{~b})$.

\subsection{The PVT Surface at Intermediate Temperatures}

Initially, we considered using the exponential equation of state (Woolley, et al., 1948). The difficulties with this equation are that it cannot be integrated in closed form to find the derived properties, it has a limited range, 500 to $1200^{\circ} \mathrm{R}(273$ to $673 \mathrm{~K})$, and the uncertainties in the derived properties cannot be calculated easily.

As an alternative we considered a modified Benedict-Webb-Rubin (1940) equation of

state. The advantages of an equation of state of this type are that the functions are internally consistent and continuous. In addition, statistical uncertainties for both PVT and derived properties can be obtained from the least squares estimation of the parameters. Various modifications of this equation have been used to correlate the PVT surfaces of nitrogen (Strobridge, 1962), helium (Mann, 1962), carbon monoxide (Hust and Stewart, 1963), neon (McCarty and Stewart, 1965), argon (Gosman, et al., 1969), and oxygen (Stewart, 1966). The equation has also been used earlier on parahydrogen (Roder and Goodwin, 1961) and on an intercomparison of normal and parahydrogen (Hust and Stewart, 1965).

The PVT data used to estimate the parameters of the equation of state included the parahydrogen isotherms from 50 to $100 \mathrm{~K}$ (Goodwin, et al., 1963), all of the PVT sources surveyed by Woolley, et al. (1948), and the isotherms of normal hydrogen reported by Michels, et al. (1959). The equation is as follows:

$$
\begin{aligned}
P=\rho R T & +\rho^{2}\left(N_{1} T+N_{2}+N_{3} / T+N_{4} / T^{2}+N_{5} / T^{4}\right) \\
& +\rho^{3}\left(N_{6} T^{2}+N_{7} T+N_{8}\right)+\rho^{3}\left(N_{9} / T^{2}+N_{10} / T_{3}+N_{11} / T^{4}\right) e^{N_{17} \rho^{2}} \\
& +N_{12} \rho^{4} T+\rho^{5}\left(N_{13} / T^{2}+N_{14} / T^{3}+N_{15} / T^{4}\right) e^{N_{17} \rho^{2}} \\
& +N_{16} \rho^{6} .
\end{aligned}
$$

The coefficients are given in table 6 for $P$ in atm, $\rho$ in $\mathrm{mol} / \mathrm{l}, \mathrm{T}$ in $\mathrm{K}$, and $\mathrm{R}=0.0820535$ $\ell-\mathrm{atm} / \mathrm{mol}-\mathrm{K}$. 


$$
\begin{aligned}
& N_{1}=1.1389049685 \times 10^{-3} \\
& N_{2}=1.8072093722 \times 10^{-1} \\
& N_{3}=-5.3097164419 \times 10^{1} \\
& N_{4}=2.3690885344 \times 10^{3} \\
& N_{5}=-1.6306395805 \times 10^{6} \\
& N_{6}=3.2453595439 \times 10^{-8} \\
& N_{7}=-6.3275640592 \times 10^{-7} \\
& N_{8}=2.3115255859 \times 10^{-3} \\
& N_{9}=3.4448764044 \times 10^{1}
\end{aligned}
$$

$$
\begin{aligned}
& N_{10}=-3.7301781349 \times 10^{3} \\
& N_{11}=1.0789473341 \times 10^{5} \\
& N_{12}=8.0475741674 \times 10^{-7} \\
& N_{13}=1.6581404268 \times 10^{-1} \\
& N_{14}=-1.8158230417 \times 10^{1} \\
& N_{15}=4.9623738040 \times 10^{2} \\
& N_{16}=5.262288563 \times 10^{-8} \\
& N_{17}=-.0018
\end{aligned}
$$

The equation is valid over the range of the data and was used from 180 to $1200^{\circ} \mathrm{R}$. Unfortunately the major sources disagree considerably at the common boundary of $180^{\circ} \mathrm{R}$ (see, for example, Roder, et al., 1965) and the resulting jumps in the properties are seen clearly in the plots of maximum interpolation error of Appendix C. A comparison of calculated statistical uncertainties for PVT and derived properties with the results of Roder, et al. (1965), Woolley, et al. (1948), and Michels, et al. (1959, 1963) is shown in figure 9 where the base line is the present equation of state. The comparison confirms that the values obtained for $\sigma$ are realistic for both PVT and derived properties. For temperatures from 180 to $1200^{\circ} \mathrm{R}$ and enthalpies from 425 to $5000 \mathrm{BTU} / \mathrm{lb}$ the calculated uncertainties are shown as source errors in the plots of Appendix C.

\subsection{The PVT Extrapolation}

The highest temperature for experimental PVT values is $673 \mathrm{~K}$. This means the PVT surface from 1200 to $6000^{\circ} \mathrm{R}$ has to be extrapolated. At these temperatures the gas is nearly ideal, that is, only the lower virial coefficients contribute to the equation of state. As a first step one has to know how the virial coefficient B varies as a function of temperature, and then how much the $\mathrm{B}(\mathrm{T})$ contributes to the equation of state. The $\mathrm{B}$ for hydrogen attains its maximum value near $300 \mathrm{~K}$. Based on what is known for helium (Keesom, 1942), the B should decrease at higher temperatures. Woolley, et al. (1948) based the temperature dependence of $\mathrm{B}$ on this behavior and predicted the actual temperature dependence from the Lennard-Jones (12-6) intermolecular potential function. Considering an extreme value, that is, $5000 \mathrm{psia}(340 \mathrm{~atm})$ and $5000^{\circ} \mathrm{R}(2800 \mathrm{~K})$, the exponential equation of state (Woolley, et al., 1948) yields a density of $0.337 \mathrm{lb} / \mathrm{ft}^{3}$ (60 Amagat). In the range in which this equation is valid, 360 to $1200^{\circ} \mathrm{R}(200$ to $673 \mathrm{~K})$, the virial B contributes less than $0.2 \%$ to the density for densities less than 60 Amagat. The contribution at higher temperatures can only be less; ther efore, an extrapolation based on an equation of state which includes only the second virial coefficient is acceptable in terms of error. 

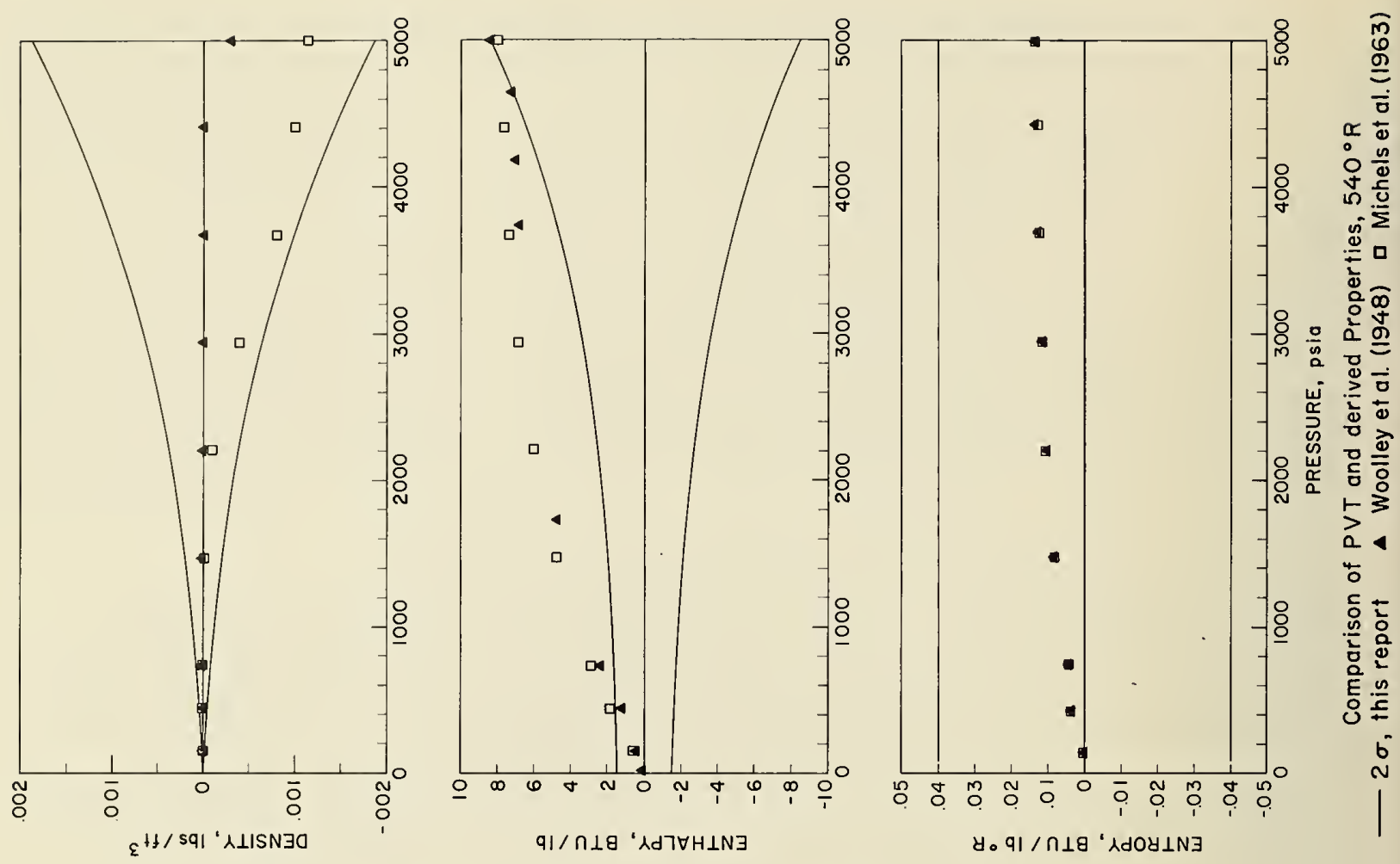

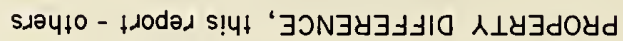
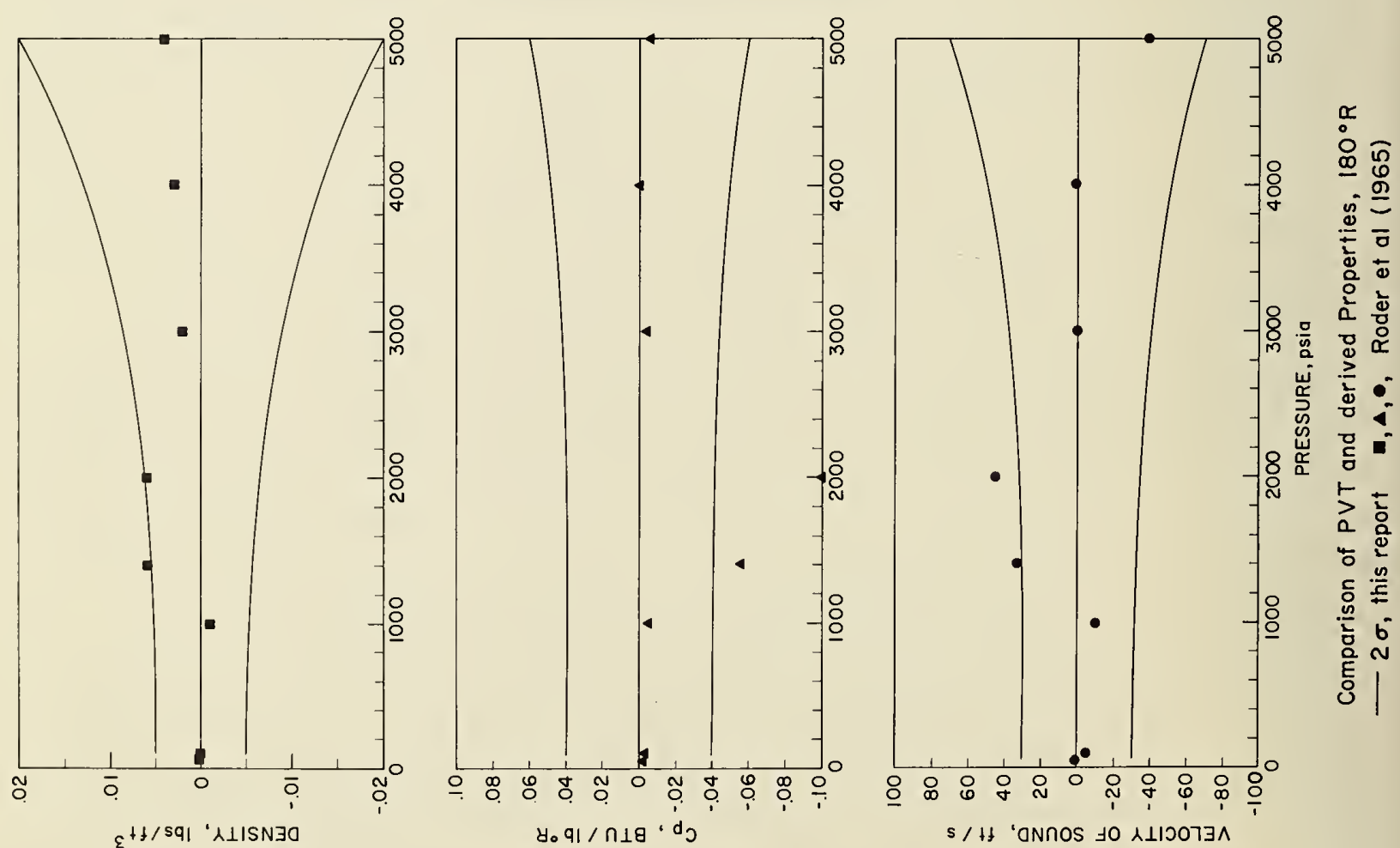

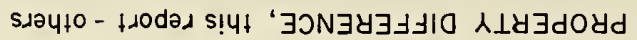

Figure 9. Comparison of PVT and Derived Properties 
Next we estimate the apparent error in the extrapolation of $\mathrm{B}(\mathrm{T})$. For $5400^{\circ} \mathrm{R}$ (3000 K), extrapolation of the Woolley, et al. (1948) calculation yields $14.55 \mathrm{~cm}^{3} / \mathrm{mol}^{\mathrm{for}} \mathrm{B}$ while Fisher (1965) obtained $12.93 \mathrm{~cm}^{3} / \mathrm{mol}$. Fisher's calculation is based on a "softer" intermolecular potential function (i.e., a 9-6) and he determines the molecular parameters from the high temperature viscosity measurements of Guevara and Wageman (1965). If hydrogen at this temperature behaved as an ideal gas, B would be zero; thus a reasonable estimate for the error in $\mathrm{B}$ at $5400^{\circ} \mathrm{R}\left(3000 \mathrm{~K} 0\right.$ is $\pm 3 \mathrm{~cm}^{3} / \mathrm{mol}$.

For the data arrays we extrapolate the equation of state (Section 7.3). Since the equation was fitted over the entire range of data up to $1200^{\circ} \mathrm{R}(673 \mathrm{~K})$, only one additional constraint (Hust and McCarty, 1967) was required to yield acceptable results. The equation was constrained to yield $14.55 \mathrm{~cm}^{3} / \mathrm{mol}$ for $\mathrm{B}(\mathrm{T})$ at $5400^{\circ} \mathrm{R}(3000 \mathrm{~K})$. Z is approximately 1.04 at $5400^{\circ} \mathrm{R}$ and $5000 \mathrm{psia}$, thus the real gas contribution is 0.04 . Woolley (1972) estimates that the real gas contribution is uncertain by no more than $10 \%$; therefore, we estimate the uncertainty in density to be $0.8 \%$ for these conditions. Uncertainties in the derived properties are estimated in a similar fashion as suming an uncertainty of no more than $10 \%$ for the real gas contribution, i. e., the contribution from non ideality. This estimate assumes virtually no error in the ideal gas thermodynamic functions and virtually no error in the dissociation calculation (Woolley, 1972).

\section{5 Dissociation}

At the highest temperatures considered in this report, molecular hydrogen will dissociate into atoms. Dissociation is both temperature and pressure dependent. To include the effects of dissociation several as sumptions are required.

1. Both ortho-para and dissociation equilibria occur instantaneously.

2. Dissociation occurs according to the equilibrium constant for normal hydrogen.

3. The property values can be obtained by adding the dissociation effects calculated for the ideal gas to the extrapolated properties of the real gas.

Fortunately, the rate of ortho-para conversion is quite high (Farkas, 1935). It is proportional to the amount of atomic hydrogen. The dissociation rates are apparently also quite high (Sutton, 1962). Assumption 1 is approximated, and thus as sumption 2 is plausible. Hence, it is not necessary to consider the "hypothetical" dissociation constant for parahydrogen (Farkas, 1935) even though the hydrogen is initially para.

The dissociation constant is calculated from the ideal gas free energies. As indicated previously, the inclusion or exclusion of nuclear spin will affect entropy as well a $\mathrm{free}$ energy. The tables of atomic hydrogen from NBS Circular 564 (Hilsenrath, et al., 1955) and the tables for molecular normal hydrogen from NBS Monograph 20 (Haar, et al., 1961) were used. These tables according to convention. do not include the effect of nuclear spin. Equation 3.5 for free energy given by Woolley, et al., (1948) is equivalent to the table for 
atomic hydrogen in Circular 564 but includes nuclear spin. The equations used to calculate dissociation effects of the ideal gas are those given by Woolley (1955).

The effects of dissociation on a variable such as enthalpy are large. They are illustrated for a limited range of temperatures and a pressure of $50 \mathrm{psia}$ in figure 10 (see also figures 11 and 12). Enthalpy differences are plotted vertically and the dissociation contribution ranges from $2 \%$ to $10 \%$ of the total enthalpy. Also shown is the linear interpolation provided by program $\operatorname{PTENTH}(\mathrm{P}, \mathrm{T})$.

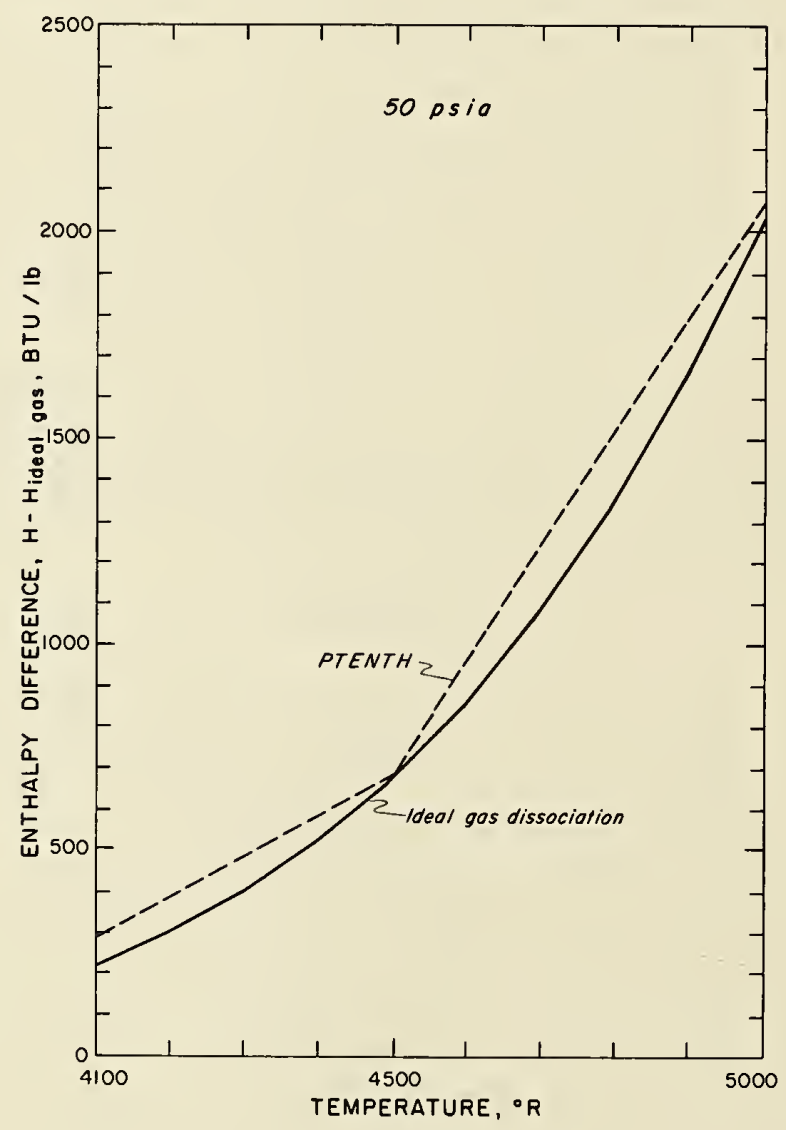

Figure 10. The Effect of Dissociation on Enthalpy

\subsection{Viscosity}

The primary experimental sources to consider are Diller (1965) for low temperatures, and Trautz and Zink (1930) and Guevara and Wageman (1965) for high temperatures. Differences in the latter two sources have now been resolved in favor of the more recent data (Guevara, et al., 1971) allowing a critical evaluation of viscosity and thermal conductivity coefficients for the dilute gas (Hanley, et al., 1970). The coefficients are represented by standard kinetic theory expressions and are based on a model intermolecular potential function. 
For temperatures up to $180^{\circ} \mathrm{R}$ for both dilute and dense gas, the analytical representation of the results by Diller (1965) was chosen as source data. At intermediate and high temperatures including dissociation, the evaluation of Hanley, et al., (1970) was chosen for the dilute gas while the dense gas contributions are calculated from the theory of Enskog (Hanley, et al., 1972). The dense gas contribution vanishes at $1000 \mathrm{~K}$, that is, above $1000 \mathrm{~K}$ the viscosity is independent of pressure or density but depends only on temperature. At yet higher temperatures with the onset of dissociation the viscosity becomes again pressure dependent as shown in figure 11 taken from Hanley, et al. (1970). We note that at the extreme temperature of $6000^{\circ} \mathrm{R}(3333 . \mathrm{K})$ the pressure dependence is still relatively small.

The uncertainty in viscosity is estimated as follows: About $2 \%$ for temperature up to $1080^{\circ} \mathrm{R}$, and then gradually increasing to about $5 \%$ at $6000^{\circ} \mathrm{R}$.

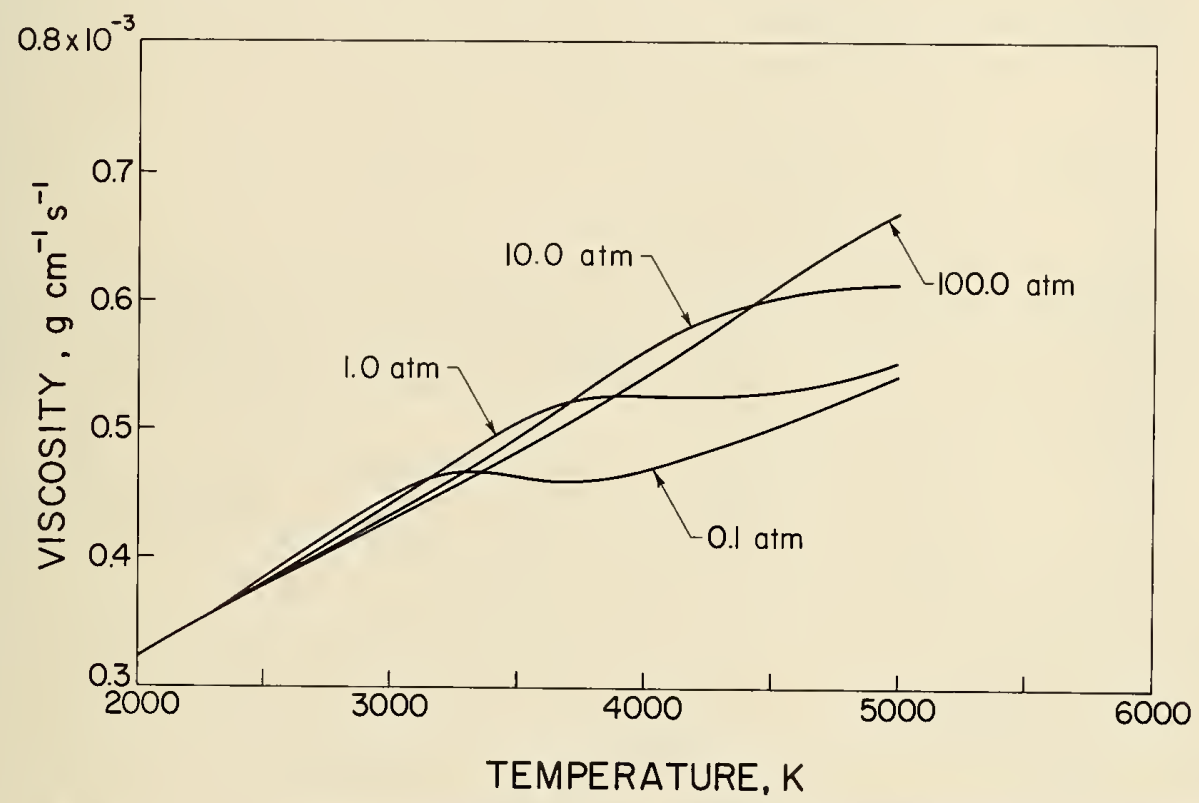

Figure 11. The Viscosity of Dissociating Hydrogen at Various Pressures

\section{7 Thermal Conductivity}

For temperatures up to $180^{\circ} \mathrm{R}$, the analytical representation of the experimental results by Roder and Diller (1970) was chosen as source data for para hydrogen for dilute gas, dense gas, and liquid states. Dilute gas values for normal and equilibrium hydrogen differ considerably from the para values and are calculated from the change in the ideal gas heat capacity, $\mathrm{C}_{\mathrm{v}}^{\circ}$. The density dependence for $\mathrm{n}$ - and e- hydrogen is taken to be the same as para.

At higher temperatures we again select the critical evaluation by Hanley, et al., (1970) for the dilute gas and the Enskog theory calculation (Hanley, et al., 1972) for the density 
(pressure) dependence. At $180^{\circ} \mathrm{R}$, taking the difference between normal and para hydrogen into account, the agreement between the experimental results by Roder and Diller (1970, para), Golubev and Kalsina (1964, normal), and the calculation by Hanley, et al. (1972) is as good as $1 \%$ for pressures up to 1500 psia and no worse than $4 \%$ at pressures of 5000 psia. Similar to viscosity, the dense gas contribution for thermal conductivity vanishes at a particular temperature $(1000 \mathrm{~K})$. At higher temperatures the potential function calculations agree reasonably well with the data of Blais and Mann (1960). At yet higher temperatures, the effects of dissociation on thermal conductivity become marked, see figure 12 . The

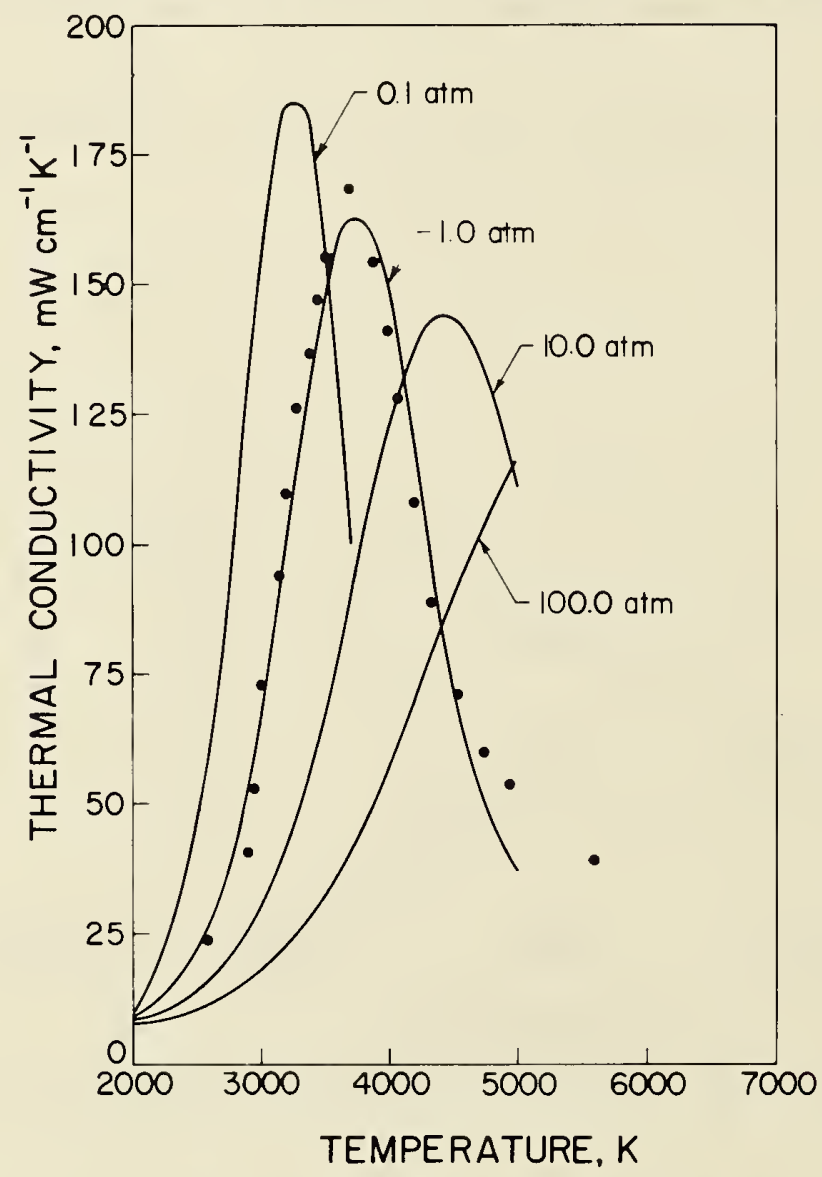

calculated, Hanley, et al. (1970)

experimental, Bethringer, et al. (1968)

Figure 12. The Thermal Conductivity of Dissociating Hydrogen at Various Pressures

calculations become sensitive to the exact model or intermolecular potential chosen, see for example, figure 9 in the paper of Grier (1962). Two sets of data exist for the thermal conductivity in the region of dissociation. The theoretical estimates selected (Hanley, et al., 1970) are in excellent agreement with the shock wave experiment of Bethringer, et al. (1968). The data of Israel, et al., (1966) differ markedly from the former experiment and from any potential function model that could be chosen. A possible explanation is that the sintered material used in this experiment enhanced dissociation. It almost appears as if the dissociation temperatures are lowered approximately $250 \mathrm{~K}$ by an apparatus effect. 
The uncertainty in thermal conductivity is estimated to be $3 \%$ for temperatures between 25 and $180^{\circ} \mathrm{R}$, increasing to $6 \%$ near $370^{\circ} \mathrm{R}$; about $6 \%$ for temperatures up to $3300^{\circ} \mathrm{R}$ and then increasing gradually to $10 \%$ at $6000^{\circ} \mathrm{R}$. Note, however, that thermal conductivity is expected to be infinite at the critical point.

\subsection{Generating Functions}

The programs described in the bulk of this report are designed to perform linear interpolations in arrays of data of the various properties. Each property value used in one of the arrays has to be computed in a separate operation and entered into the array. We have used several major computer programs to accomplish this task; to distinguish them from the linear interpolation programs, we call the major programs generating functions. An example of one such generating function is the program VALUES or the subroutine THERMO which was developed in the preparation of the tables presented in NBS Monograph 94 (Roder, et al., 1965). Since a number of users have requested copies of the generating functions, we list them below.

Table 9. Generating Functions *

\begin{tabular}{|c|c|c|c|}
\hline Temperature Range & Program Name & Input & Output \\
\hline $25-180^{\circ} \mathrm{R}$ & $\begin{array}{l}\text { program VALUES or } \\
\text { subroutine THERMO } \\
\text { function CONC } \\
\text { function VISCOS } \\
\text { program STAT }\end{array}$ & $\begin{array}{c}\mathrm{PT} \\
\mathrm{T}, \rho, \mathrm{C}_{\mathrm{p}} \\
\rho, \mathrm{T} \\
\mathrm{T}\end{array}$ & $\begin{array}{l}\rho, S, H, U, C_{p}, C_{V}, \\
\left(\frac{\partial P}{\partial T}\right)_{\rho},\left(\frac{\partial P}{\partial \rho}\right)_{T}, \omega \\
k \\
\text { o - p composition and } \\
\text { ideal gas functions, } \\
\text { thermodynamic }\end{array}$ \\
\hline $180^{\circ} \mathrm{R}$ and up & program GO & $\mathrm{P}, \mathrm{T}$ & $\begin{array}{l}\rho, \quad S, H, U, C_{p}, C_{v}, \\
\gamma, \omega, k, \eta\end{array}$ \\
\hline
\end{tabular}

* A recent revision incorporates the low temperature decks except STAT, an improved equation of state for high temperatures, and a new extrapolation to 10,000 psia into a single program HYPROP, see McCarty and Weber (1972).

\section{Acknowledgement}

The project has been supported from its inception by the Space Nuclear Propulsion Office of NASA, later the Space Nuclear Systems Office, under NASA contracts R-45 and W13,300 as a continuation of prior support of low temperature experimental mea surements on a variety of properties of hydrogen. Analysis of the transport properties at higher temperatures was supported in part by the Office of Standard Reference Data of NBS. The authors are indebted to Philip Angerhofer who prepared many of the equilibrium hydrogen routines, and Greg Hansen who prepared most of the departure graphs. 


\section{REFERENCES}

Baehr, H. D., Hartman, H., Pohl, H. C., and Schomacker, H., Thermodynamic Functions of Ideal Gases for Temperatures up to 6000 Degrees K, Springer Verlag Berlin Heidelberg New York, Volume 2 (1968).

Benedict, M., Webb, G. B., and Rubin, L. C., An Empirical Equation for Thermodynamic Properties of Light Hydrocarbons and their Mixtures I. Methane, Ethane, Propane, and n-Butane, J. Chem. Phys. $\underline{8}$, 334-45 (1940).

Bethringer, K., Kollman, W., and Mentel, J., Thermal Conductivity of Hydrogen between 2000 and 7000 K., Z. Physik 215, 127 - 151 (1968).

Blais, N. C. , and Mann, J. B., Thermal Conductivity of Helium and Hydrogen at High Temperatures, J. Chem. Phys. $32,1459-65$ (1960).

Fisher, B. B., Calculations of the Thermal Properties of Hydrogen, Los Alamos Scientific Lab., N. Mex. , Rept. No. LA-3364 (1965).

Goldberg, F. N., private communciation (1967).

Golubev, I. F., and Kal'sina, M. V., Thermoconductivity of Nitrogen and Hydrogen at Temperatures from 20 to $-195^{\circ} \mathrm{C}$ and Pressures from 1 to $500 \mathrm{Atm}, \mathrm{Gaz}$. Prom 9. No. 8. 41 - 3 (1964) (SLA Trans1. No, LA-TR-65-1).

Goodwin, R. D., Diller, D. E., Roder, H. M., and Weber, L. A., Pressure-DensityTemperature Relations of Fluid Para-Hydrogen from 15 to $100 \mathrm{~K}$ at Pressures to 350 Atmospheres, J. Res. Nat. Bur. Std. (U.S.),67A (Phys, and Chem.), No. 2, 173 - 92 (1963). Gosman, A. L., McCarty, R. D., and Hust, J. G., Thermodynamic Properties of Argon from the Triple Point to $300 \mathrm{~K}$ at Pressures to 1000 Atmospheres, Nat. Stand. Ref. Data Ser., Nat. Bur. Stand. (U.S.), 27, 146 pages (March 1969).

Grier, N. T., Calculation of Transport Properties and Heat-Transfer Parameters of Dissociationg Hydrogen, NASA (Natl. Aeron. Space Admin.) Tech. Note TN D-1406 (1962). Guevara, F. A., McInteer, B. B., Ottesen, B., and Hanley, H. J. M., A Critique of the High-Temperature Viscosity Measurements of Trautz and Zink, Los Alamos Scientific Lab., N. Mex., Rept. No. LA-4643-MS (1971).

Guevara, F. A., and Wageman, W. E., Measurement of Helium and Hydrogen Viscosities to 2340 K, Los Alamos Scientific Lab., N. Mex., Rept. No. LA-3319 (1965).

Haar, L., Friedman, A. S., and Beckett, C. W., Ideal Gas Thermodynamic Functions and Isotope Exchange Functions for the Diatomic Hydrides, Deuterides, and Tritides, Nat. Bur. Std. (U.S.), Monogr. 20 (May 1961). 
Hall, W. J., McCarty, R. D., and Roder, H. M., Computer Programs for Thermodynamic and Transport Properties of Hydrogen, Tabcode-I, unpublished (Aug. 1967).

Hanley, H. J. M. , McCarty, R. D., and Cohen, E. G. D., Analysis of the Transport Coefficients for Simple Dense Fluids: Application of the Modified Enskog Theory, Physica 60 , 322-356 (1972).

Hanley, H. J. M., McCarty, R. D., and Intemann, H., The Viscosity and Thermal Conductivity of Dilute Gaseous Hydrogen from 15 to 5000 K, J. Res. Nat. Bur. Std. (U. S.), 74A (Phys, and Chem.), No. 3 (May-June 1970).

Hilsenrath, J., et a1., Tables of Thermal Properties of Gases Comprising Tables of Thermodynamic and Transport Properties of Air, Argon, Carbon Dioxide, Carbon Monoxide, Hydrogen, Nitrogen, Oxygen, and Steam, Nat. Bur. Std. (U. S. ), Circular 564 (1955).

Hust, J. G., and McCarty, R. D., Curve-Fitting Techniques and Applications to Thermodynamics, Cryogenics 7 , No. 4, 200 - 6 (1967).

Hust, J. G., and Stewart, R. B., Thermodynamic Property Values for Gaseous and Liquid Carbon Monoxide from 70 to $300 \mathrm{~K}$ with Pressures to 300 Atmospheres, Nat. Bur. Std. (U. S. ), Tech. Note 202, 109 pages (Nov 1963).

Hust, J. G., and Stewart, R. B., A Compilation of the Property Differences of Ortho and Para Hydrogen or Mixtures of Ortho and Para Hydrogen, unpublished (1965).

Israel, S. L., Hawkins, T. D., and Hyman, S. C., Thermal Conductivity of Hydrogen from 2000 to $4700^{\circ} \mathrm{F}$, United Nuclear Corp., White Plains, N. Y., Rept. No. NASA-CR-403 (1966).

Keesom, W. H., Helium, Elsevier Pub. Co., New York (1942).

Mann, D. B., The Thermodynamic Properties of Helium from 3 to $300 \mathrm{~K}$ between 0.5 and 100 Atmospheres, Nat. Bur. Std. (U.S.), Tech. Note 154, 95 pages (Jan. 1962).

McAdams, W. H. , Heat Transmission, McGraw-Hill Book Co. Inc., New York, Toronto, London, 3rd Ed. (1954).

McCarty, R. D. , Computer Programs for Saturation Properties of Hydrogen, unpublished (1968).

McCarty, R. D., and Stewart, R B., Thermodynamic Properties of Neon from 20 to $300 \mathrm{~K}$ between 0.1 and 200 Atmospheres, Advances in Thermophysical Properties at Extreme Temperatures and Pressures, (Proc. 3rd Symp., Purdue Univ.) p. 84-97, ASME, New York (1965). 
McCarty, R. D., and Weber, L. A., Thermophysical Properties of Parahydrogen from the Freezing Liquid Line to $5000 \mathrm{R}$ for Pressures to 10,000 psia, Nat. Bur. Std. (U. S. ), Tech. Note 617, 169 pages (April 1972).

Mechtly, E. A., The International System of Units, NASA (Natl. Aeron. Space Admin.) Spec. Publ. 7012, revised, (1969).

Michels, A., de Graaff, W., Wassenaar, T., Levelt, J. M. H., and Louwerse, P., Compressibility Isotherms of Hydrogen and Deuterium at. Temperatures between $-175^{\circ} \mathrm{C}$ and $+150^{\circ} \mathrm{C}$ (at densities up to 960 Amagat), Physica 25, 25-42 (1959).

Michels, A., de Graaff, W., Wolkers, G. J., Thermodynamic Properties of Hydrogen and Deuterium at Temperatures between $-175^{\circ} \mathrm{C}$ and $150^{\circ} \mathrm{C}$ and at Pressures up to 2500 Atmospheres, Appl. Sci. Res. A12, 9 - 32 (1963).

Roder, H. M., and Diller, D. E., Thermal Conductivity of Gaseous and Liquid Hydrogen, J. Chem. Phys. 52, No. 11, 5928-49 (1970).

Roder, H. M., and Goodwin, R. D., Provisional Thermodynamic Functions for Parahydrogen, Nat. Bur. Std. (U. S. ), Tech. Note 130, 139 pages (Dec 1961).

Roder, H. M., Weber, L. A., and Goodwin, R. D., Thermodynamic and Related Properties of Parahydrogen from the Triple Point to $100 \mathrm{~K}$ at Pressures to 340 A tmospheres, Nat. Bur. Std. (U. S. ), Monogr. 94, 112 pages (Aug 1965).

Stewart, R. B., The Thermodynamic Properties of Oxygen, State Univ. of Iowa, Iowa City, $\mathrm{Ph}$. D. Thesis (1966).

Strobridge, T. R., The Thermodynamic Properties of Nitrogen from 64 to $300 \mathrm{~K}$ between 0.1 and 200 Atmospheres, Nat. Bur. Std. (U. S.), Tech. Note 129, 85 pages (Jan 1962).

Sutton, E. A., Mea surement of the Dissociation Rates of Hydrogen and Deuterium, J. Chem. Phys. 36, No. 11, 2923-31 (1962).

Trautz, M., and Zink, R., Die Reibung, Wärmeleitung, und Diffusion in Gasmischung. XII. Gasreibung bei höheren Temperaturen, Ann. Physik 7, 427-52 (1930).

Woolley, H. W., private communication (1972).

Woolley, H. W., Effect of Dissociation on Thermodynamic Properties of Pure Diatomic Gases, Nat. Advisory Comm. Aeronaut. Tech. Note 3270 (1955).

Woolley, H. W., Scott, R. B., and Brickwedde, F. G., Compilation of Thermal Properties of Hydrogen in its Various Isotopic and Ortho-para Modifications, J. Res. Nat. Bur. Std. (U. S. b $41,379-475$ (1948). 
APPENDIX A

Tables of Values for Selected Isobars

Parahydrogen (Output from PT programs) . . . . . 38

Equilibrium Hydrogen (Output from ET programs). . .45 
$\stackrel{2}{\circ}$

로은

衣这

0
1
1

เ

ㄴํㄹ

c

品

它

5

둥혀

की

恣至

20

논

골도

孚 空吉

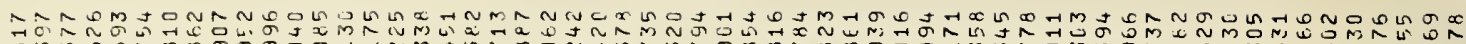

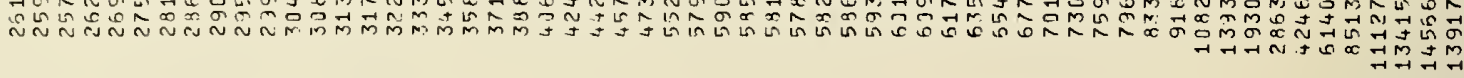
స⿻日乚

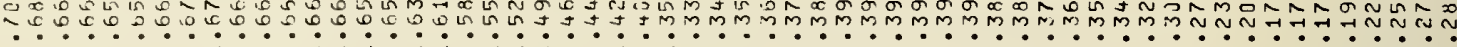

m

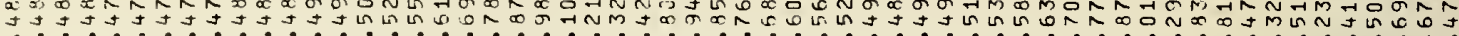

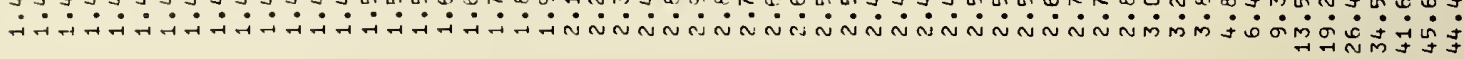

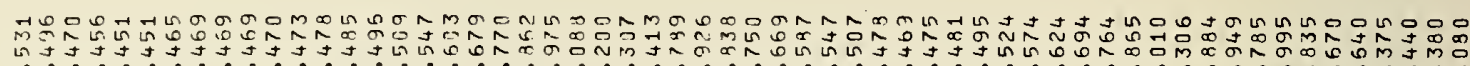

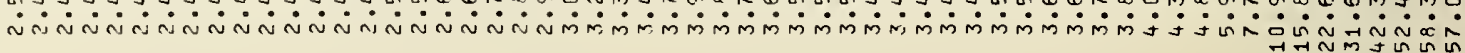

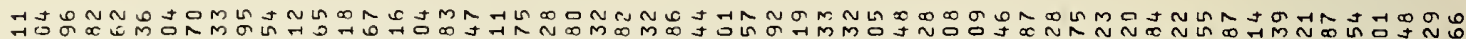
न-1

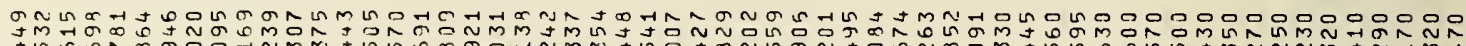

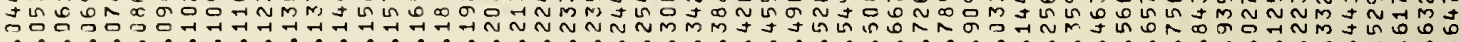

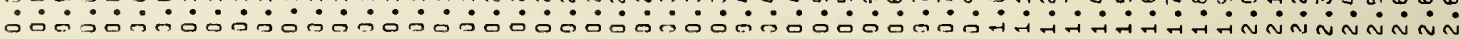

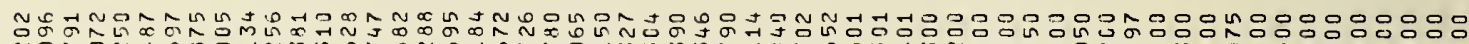
N 官品品-

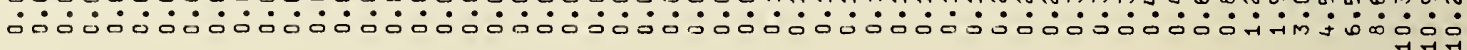

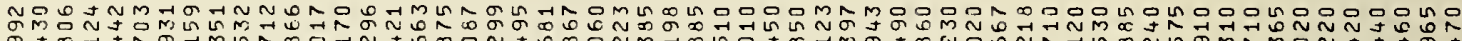
○.

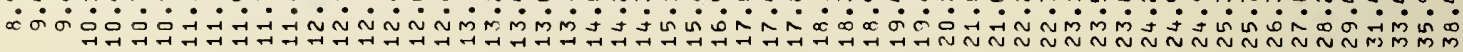

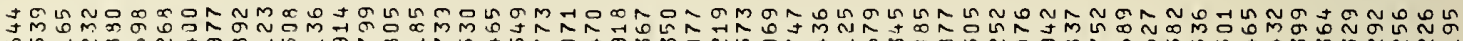

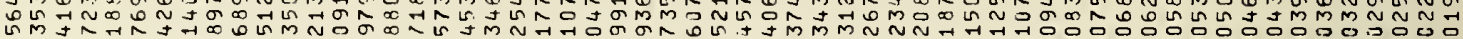

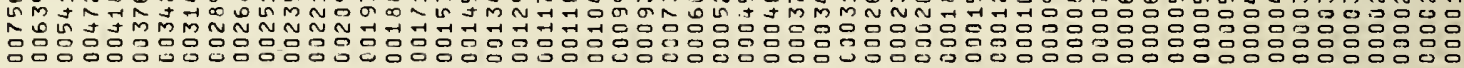

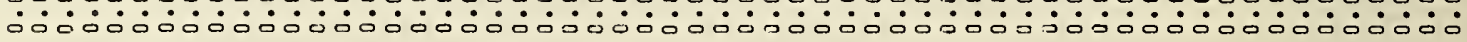

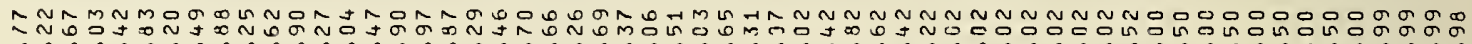

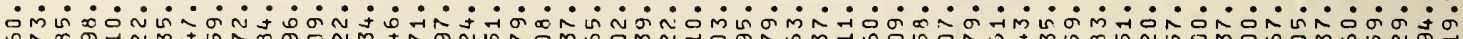

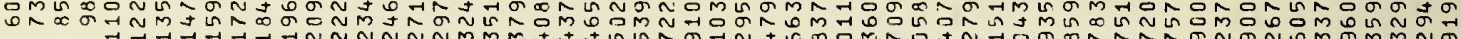

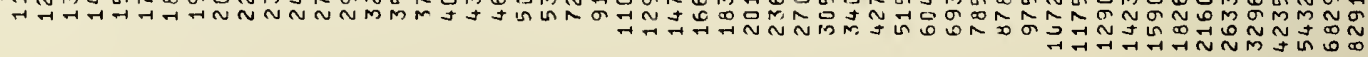

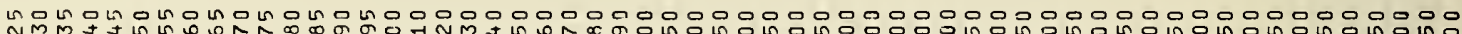

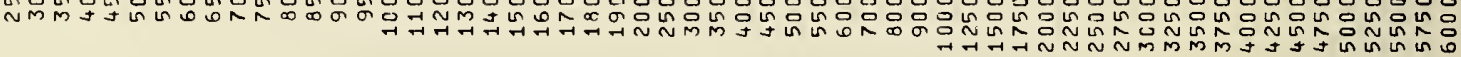

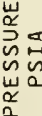




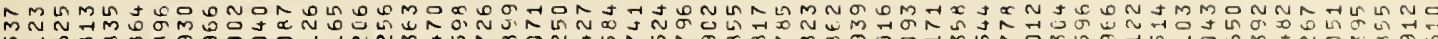

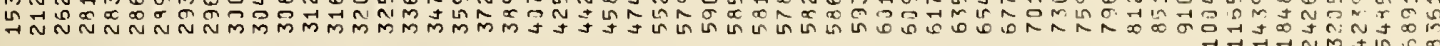

主吕

$\frac{2}{0} \approx$

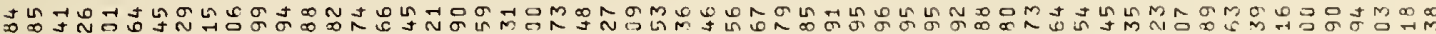

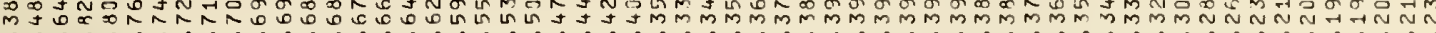

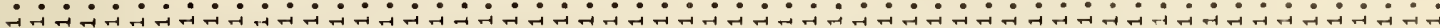

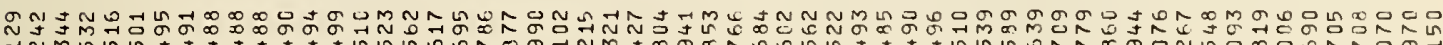
-

$\infty$

$\underset{0}{1}$

mLn in

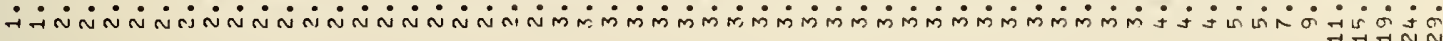

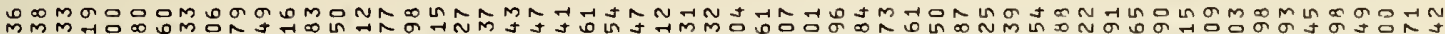

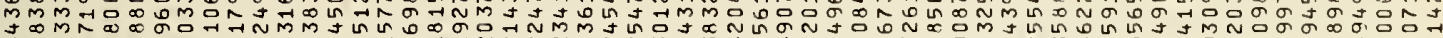

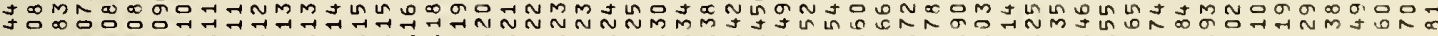

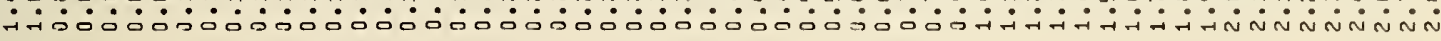

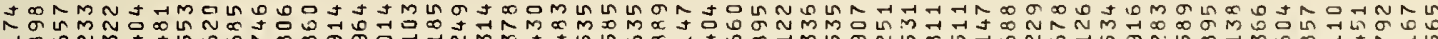

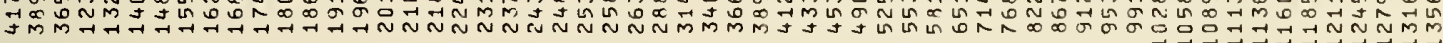

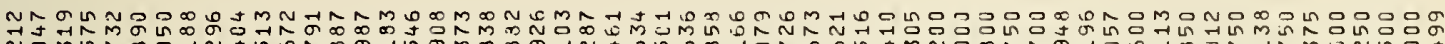

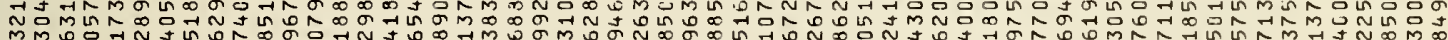

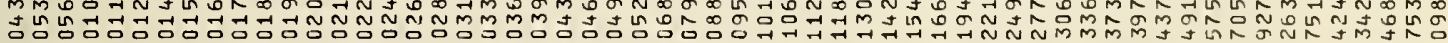

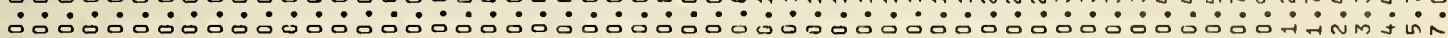

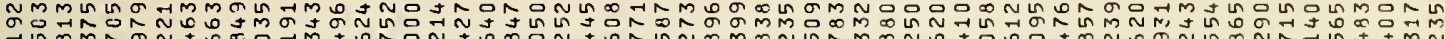
$\because$ แn

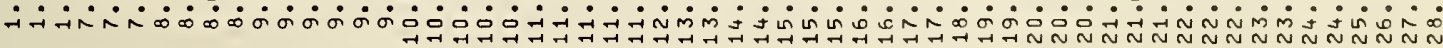

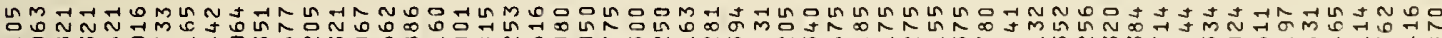

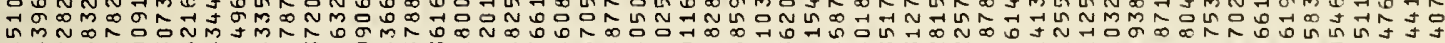

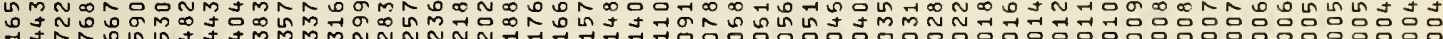

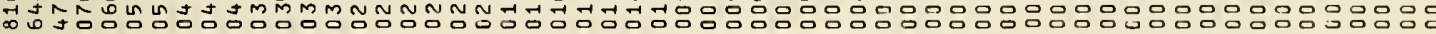

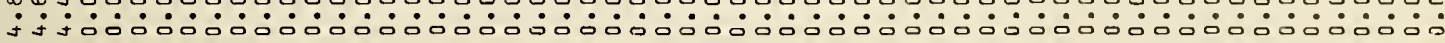

ดิ

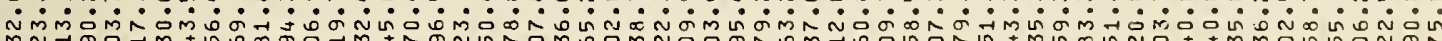
m心G

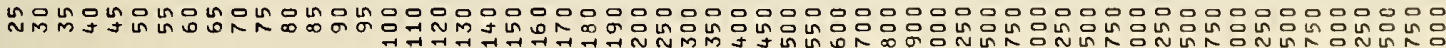
w 㟧

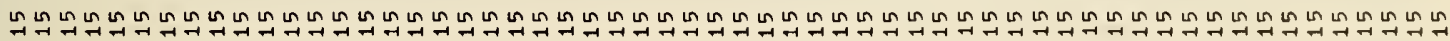




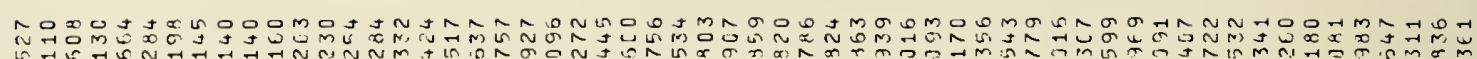

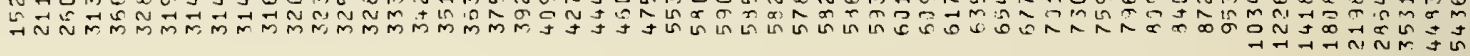

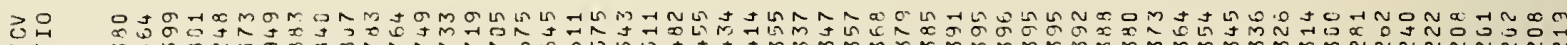
m ô

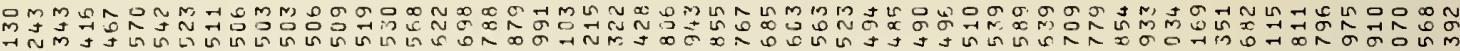
-

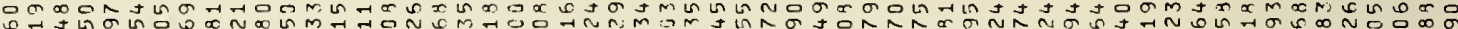

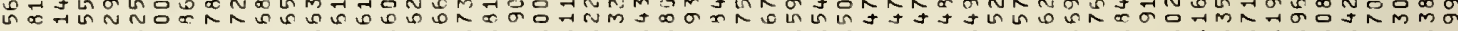

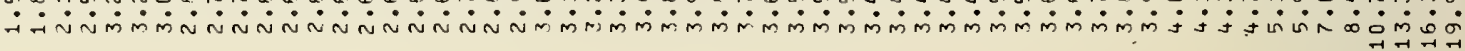

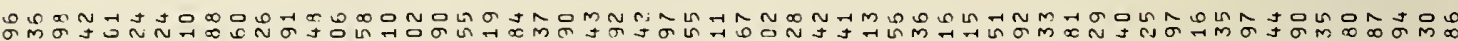

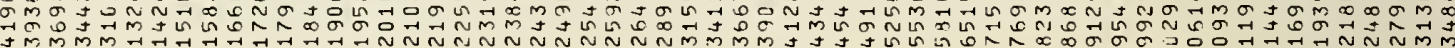

ᄂ

$2 \dot{0}:$

藏草

导沶

車

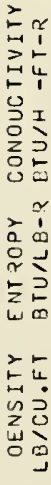

à

岸

는

u

중

壳

品㟧

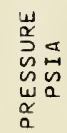

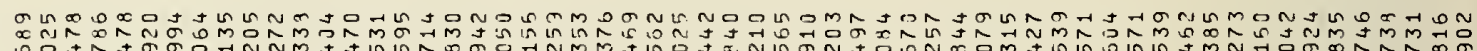

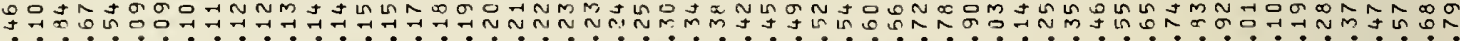

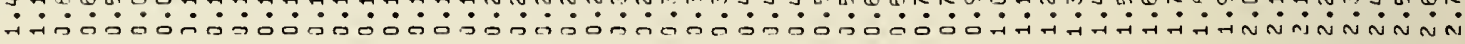

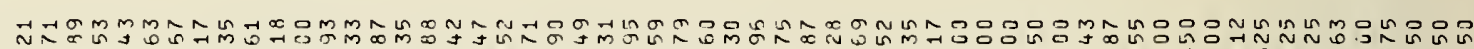
miñ

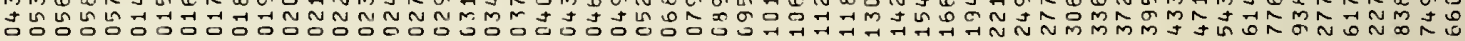

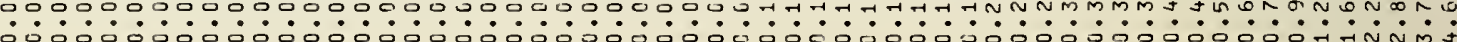

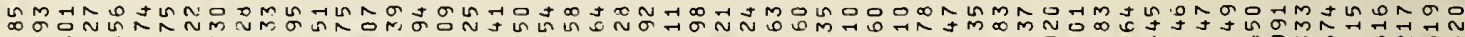

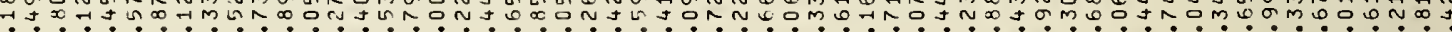

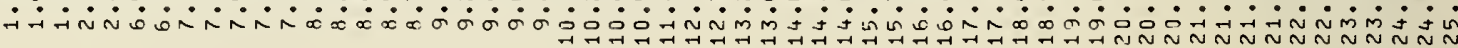

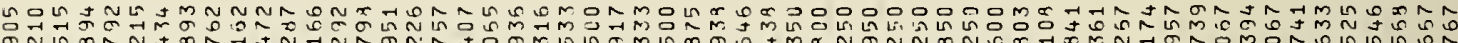
ON

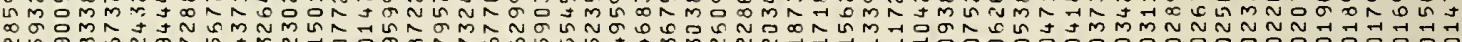

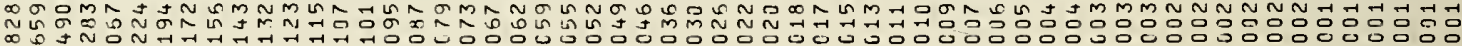

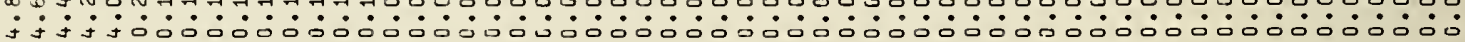

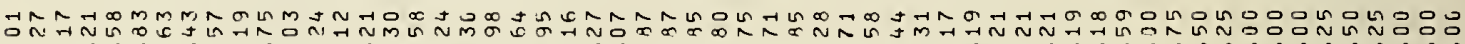

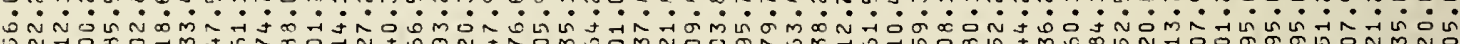

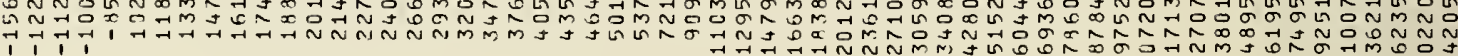
1।

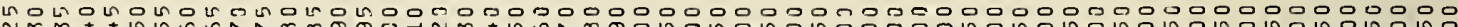

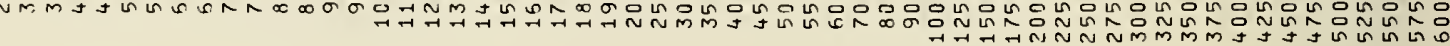

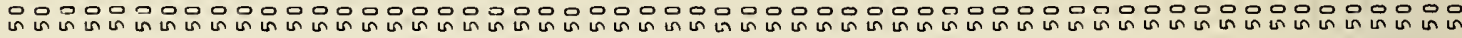




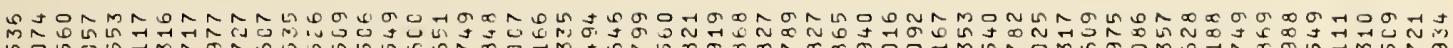

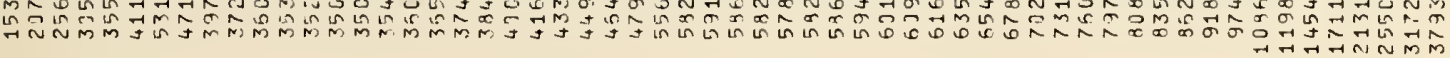

롱

恣学

is

点

ن

$\stackrel{\circ}{z}$

วิำ 는 $\stackrel{\omega}{\longrightarrow}$

足。

우

嵌是

$\underset{\substack{0 \\ 1}}{0}$

$>$

.

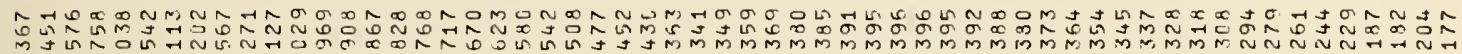
m.j

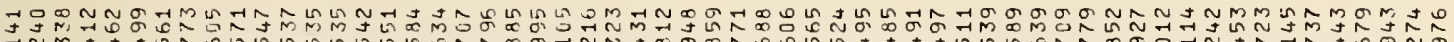
$\because N m$ s.

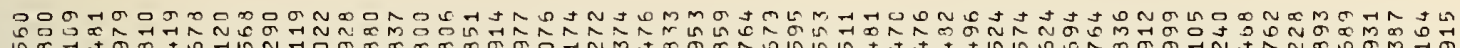

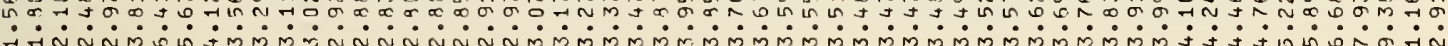

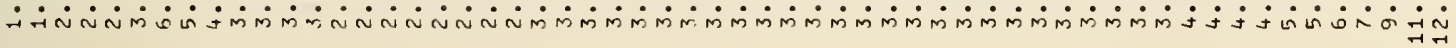

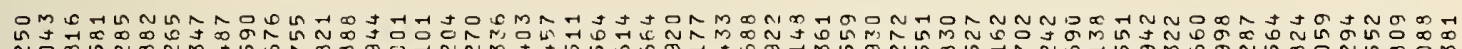

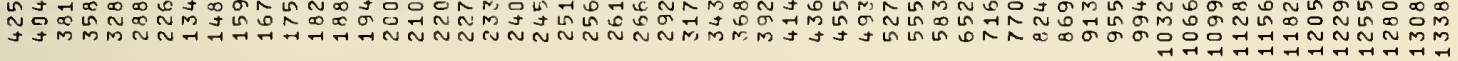

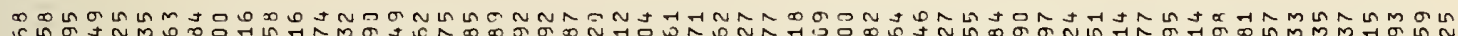

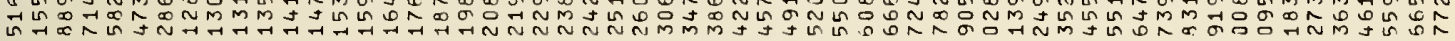

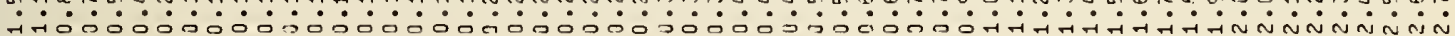

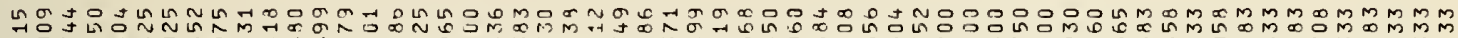

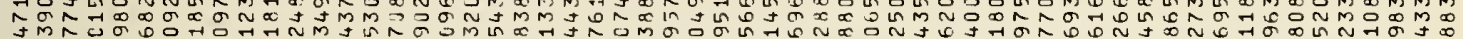

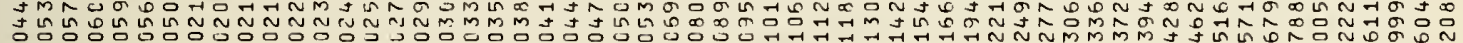

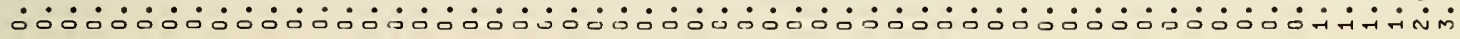

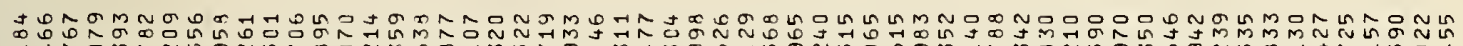

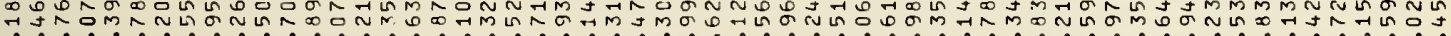

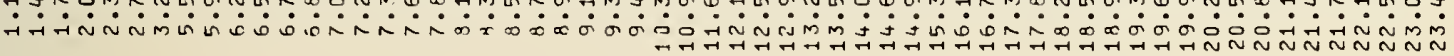

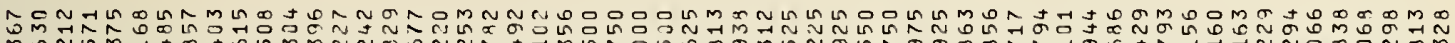

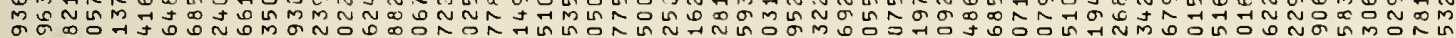

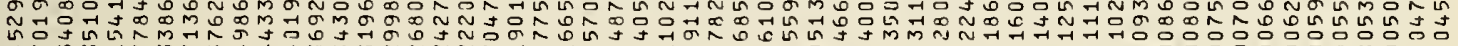

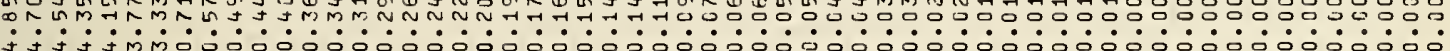

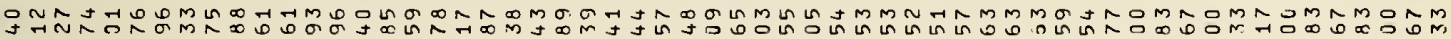

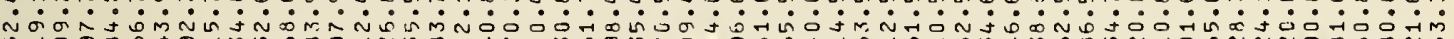

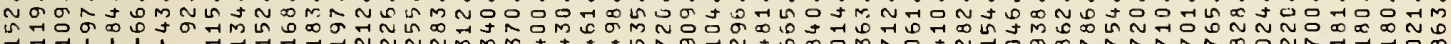
$\begin{array}{lll}T-1 & 1\end{array}$

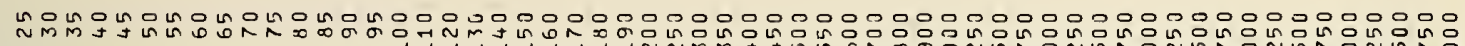
NMMง 约

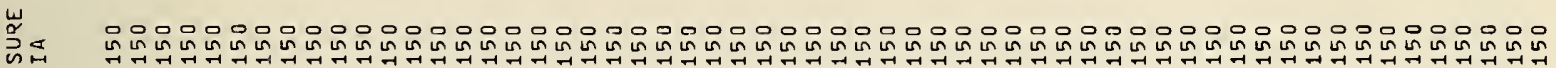




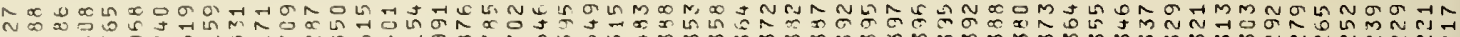

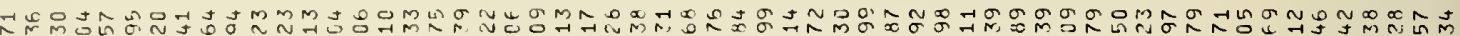

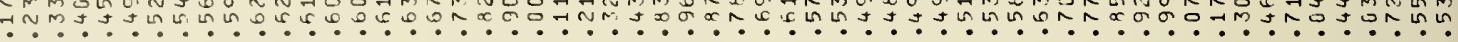

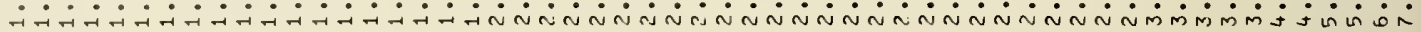

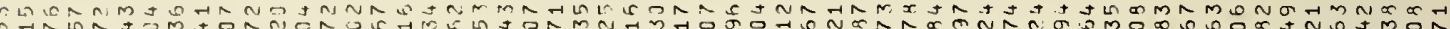

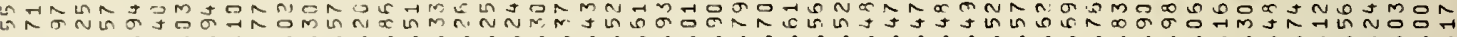

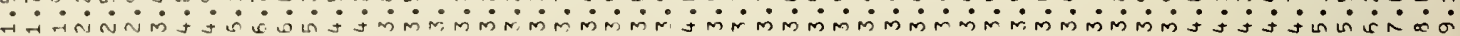

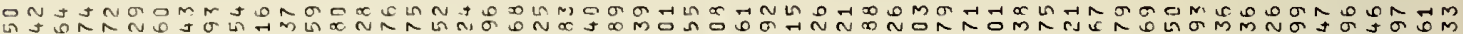
$J$
$J$
$J$

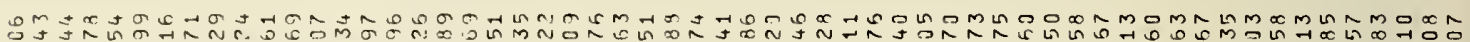

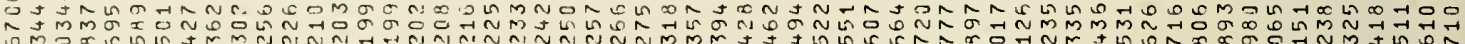

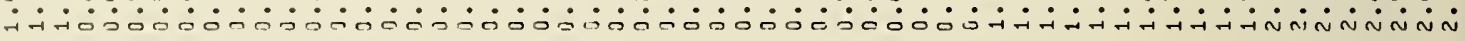

$\sum_{i=1}^{\infty}$

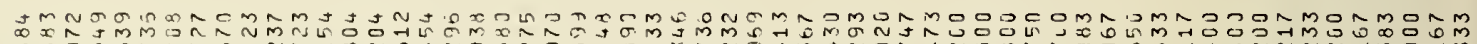

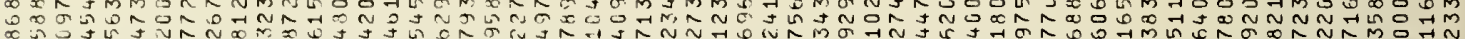

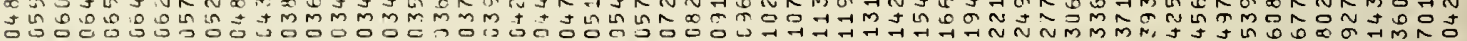

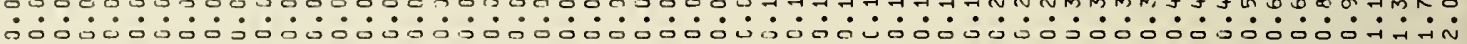

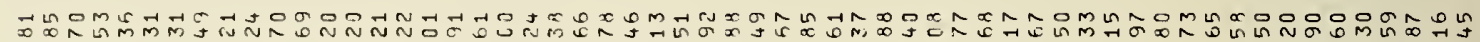

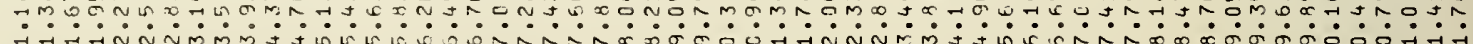

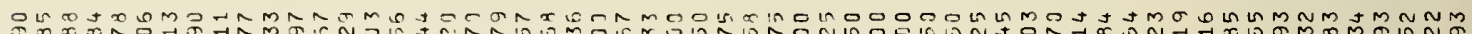

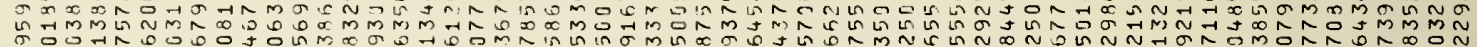

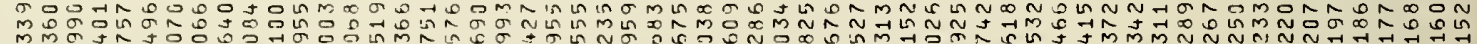

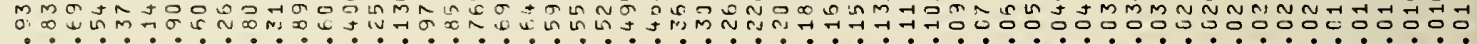

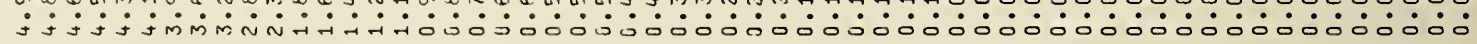

유

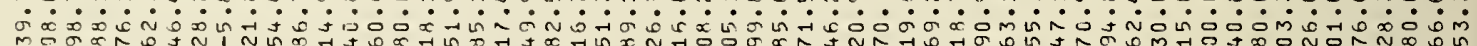

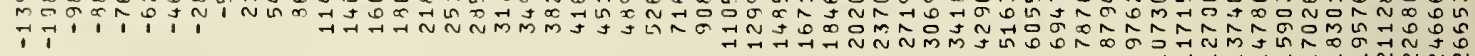

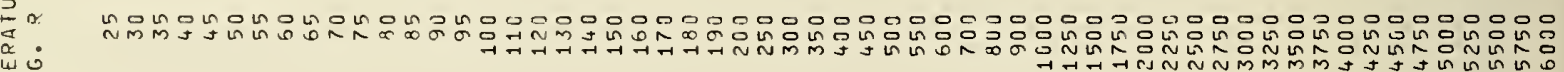




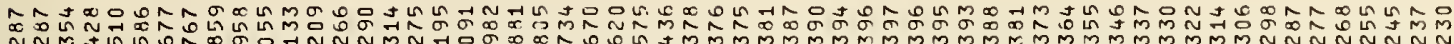

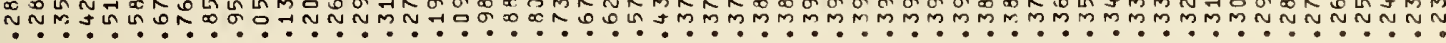

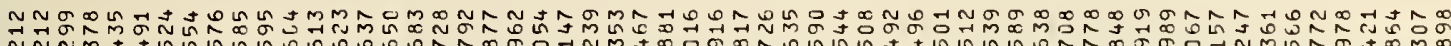

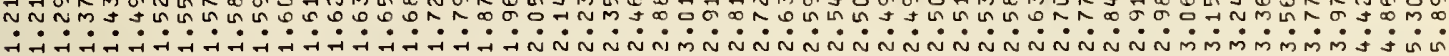

бの

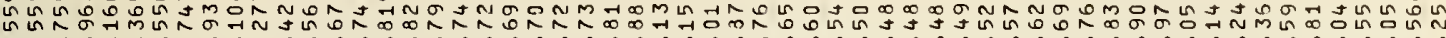

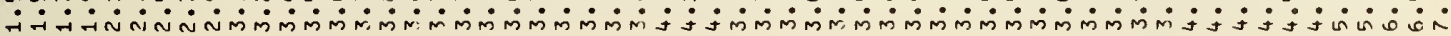

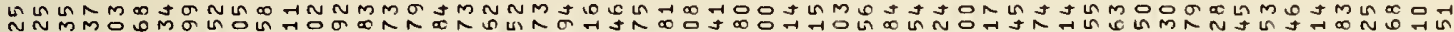

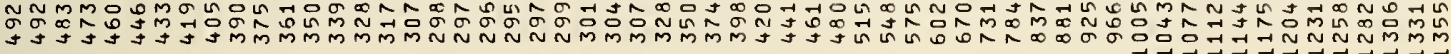

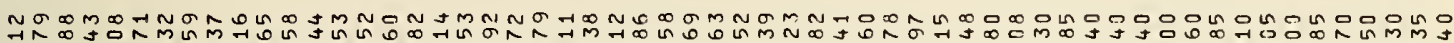

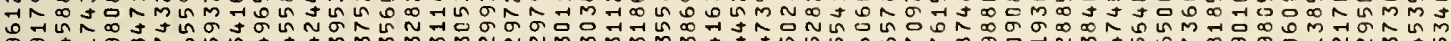

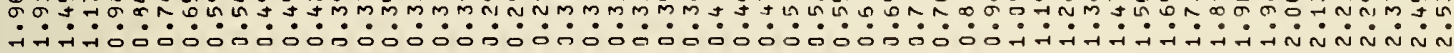

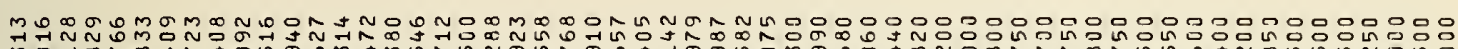

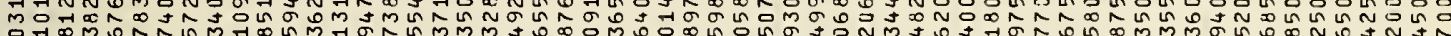

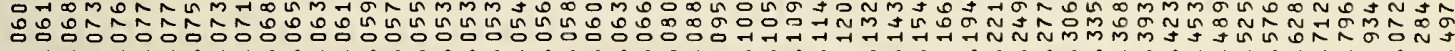

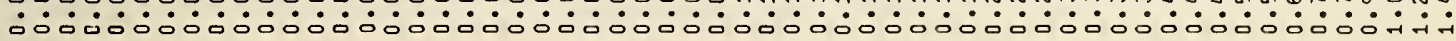

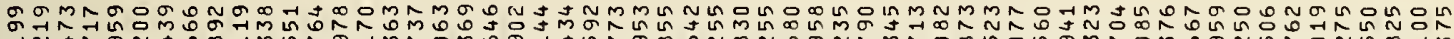

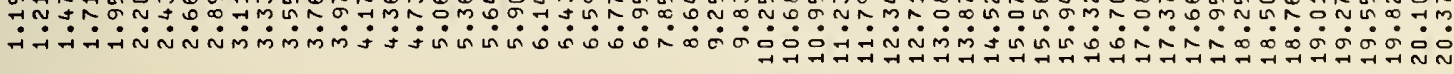

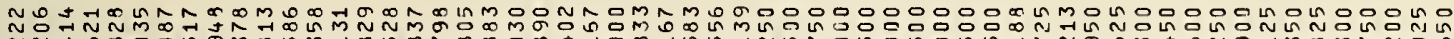

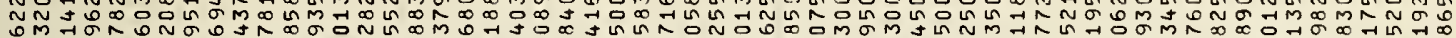

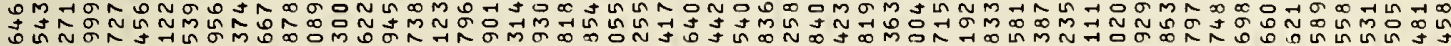
0

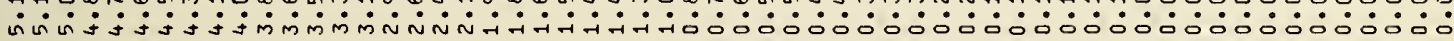

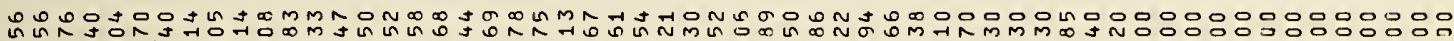

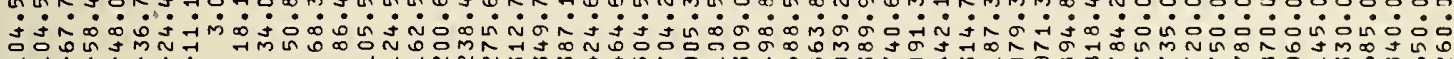

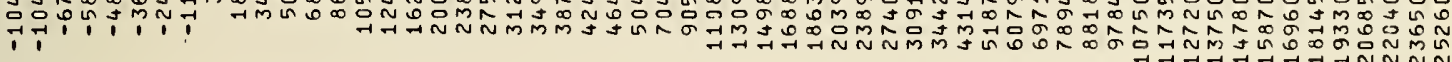

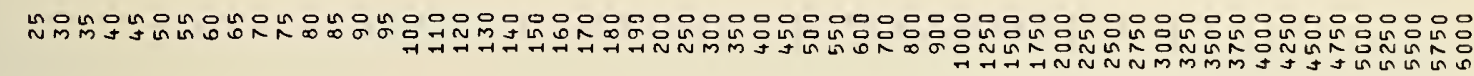

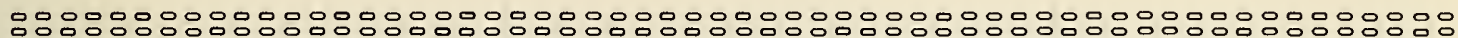

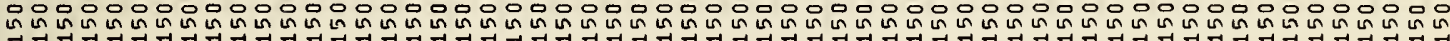




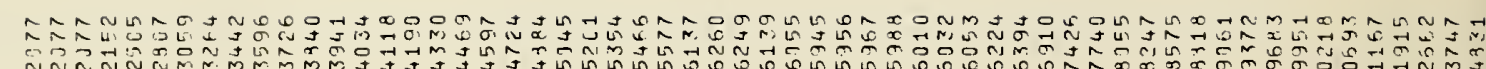

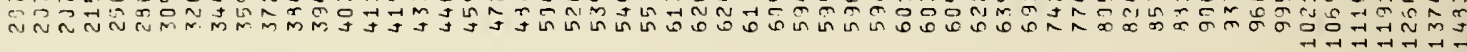

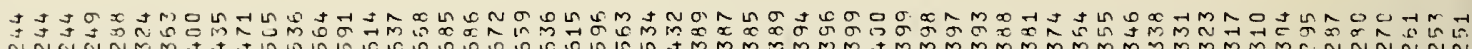

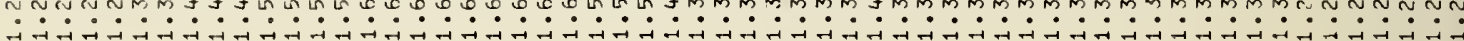

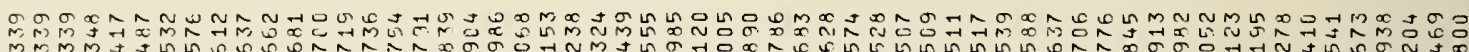

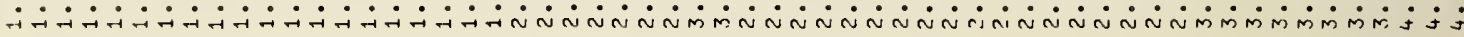

Un

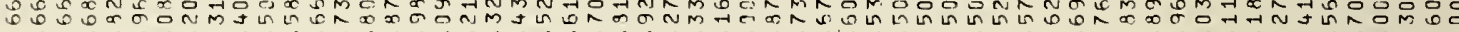

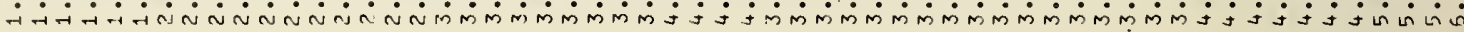

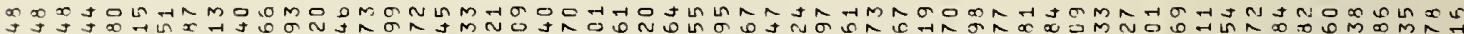

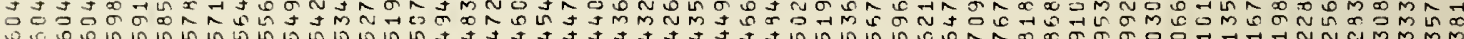

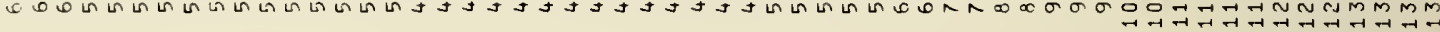

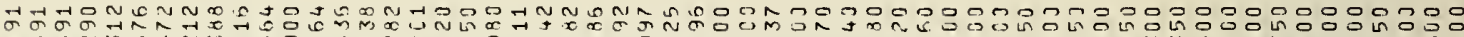

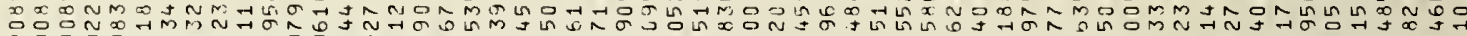
त.

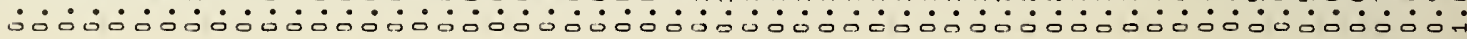

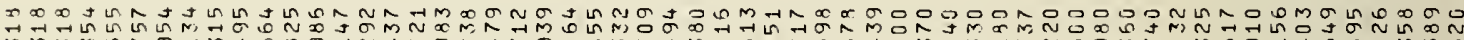
mM.

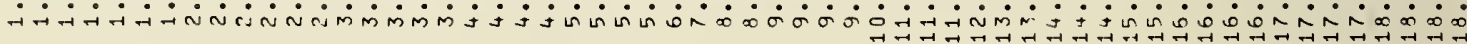

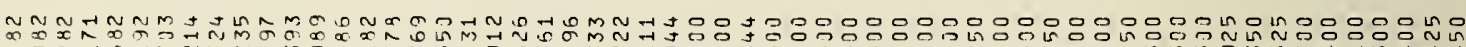
品何出

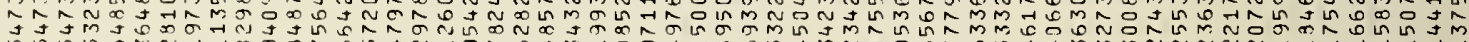

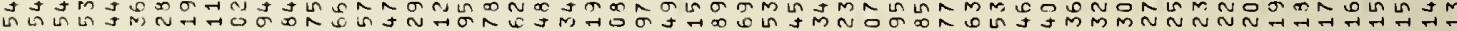

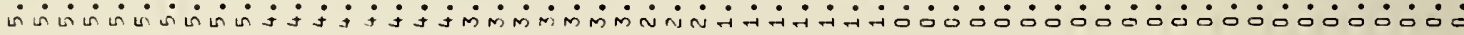

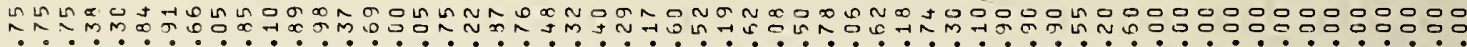

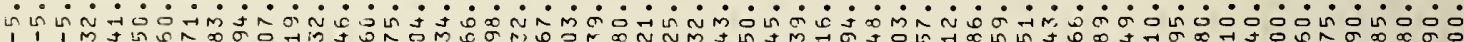
1 1 $m$ J

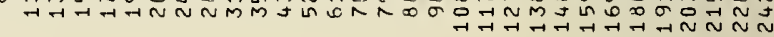

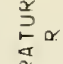

要 $\sum \mathrm{o}^{2}$

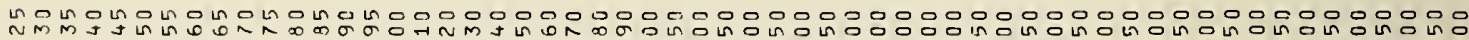

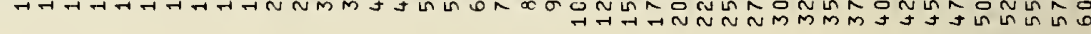

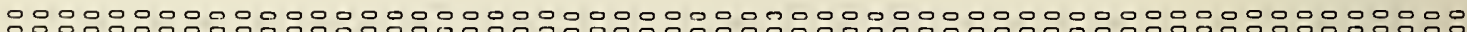

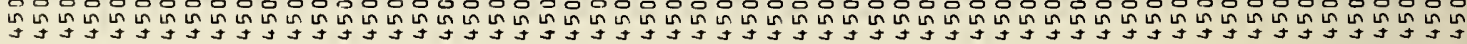




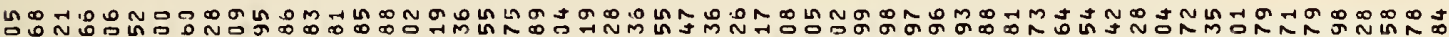

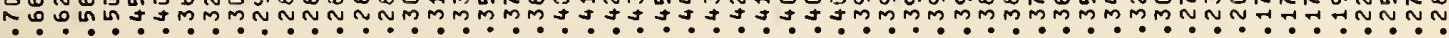

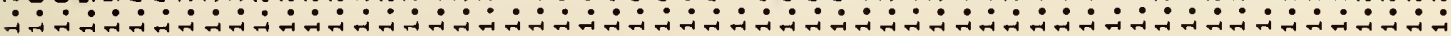

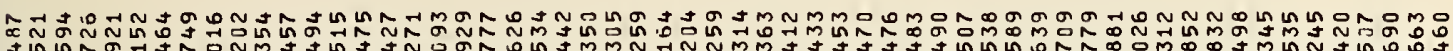

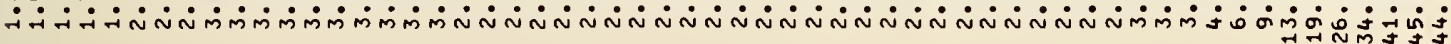

и过

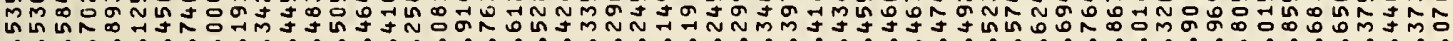

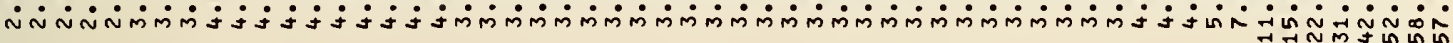

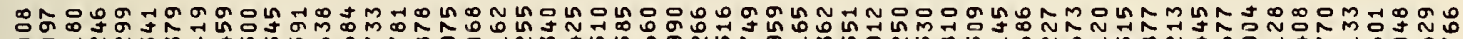

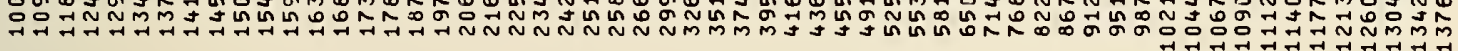

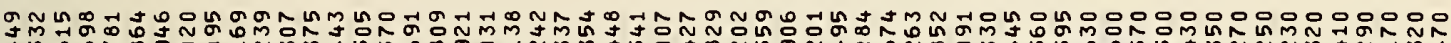
In

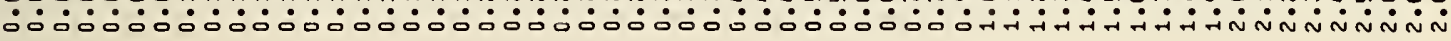

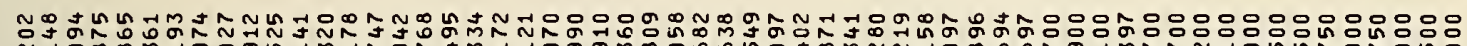
N

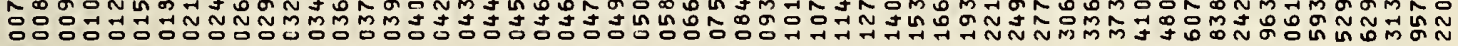

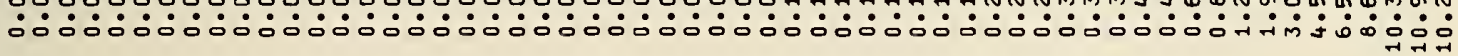

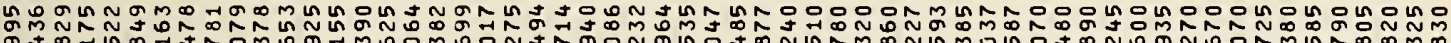
ó ป

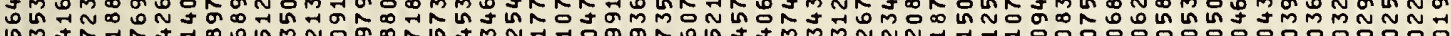

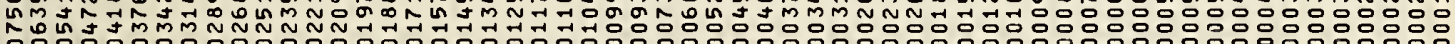

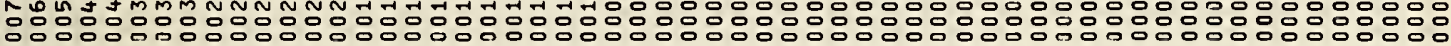

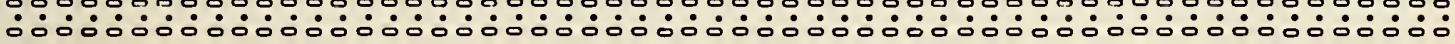

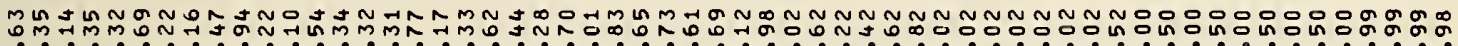

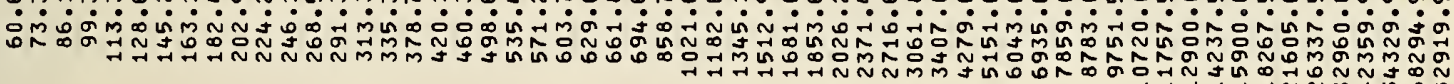

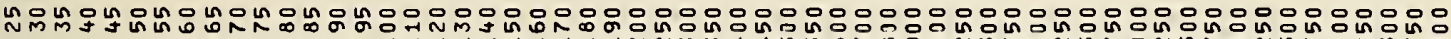

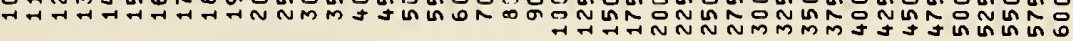




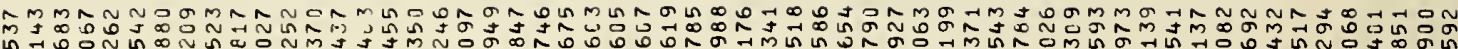

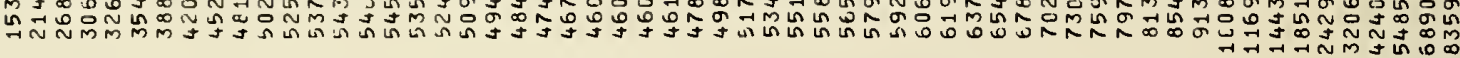

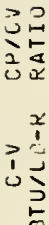

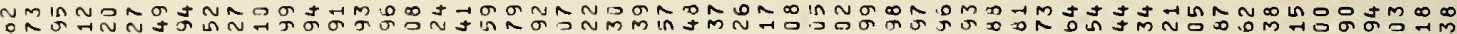
m

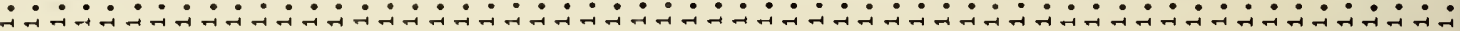

M

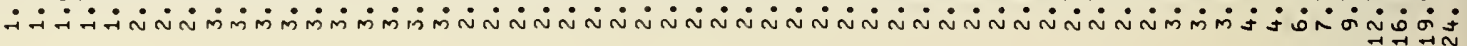

논

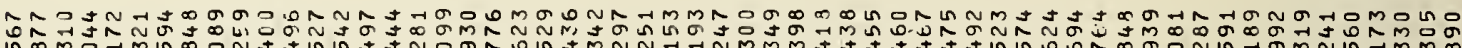

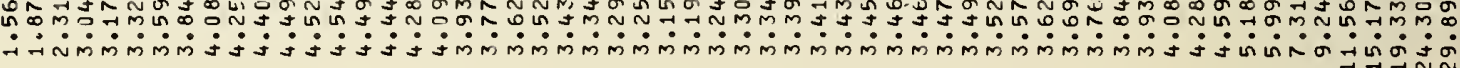

일

号

占

$\vdash$

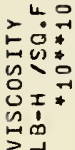

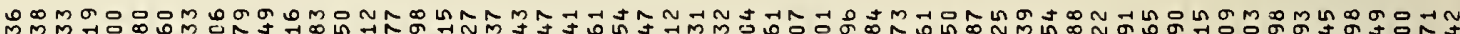

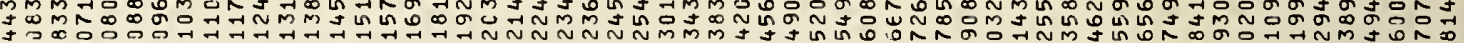

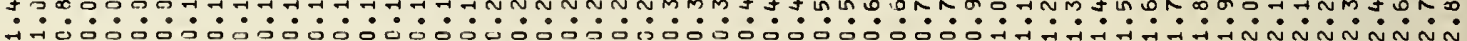

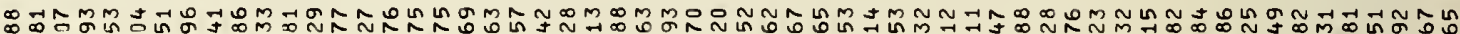

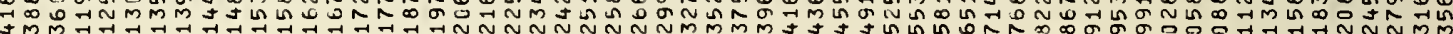

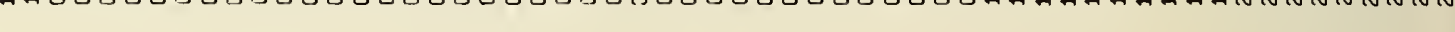

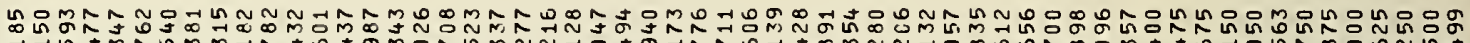

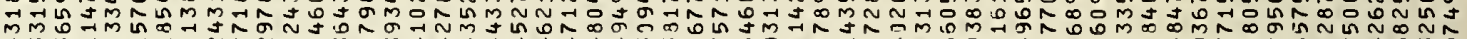

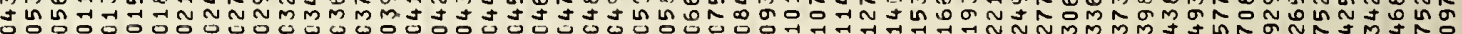

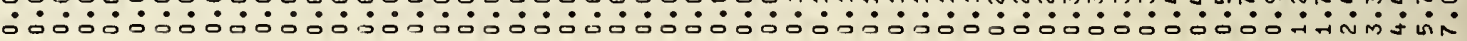

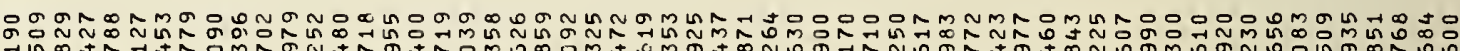

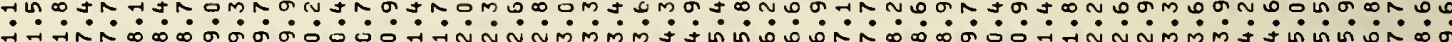
Hन

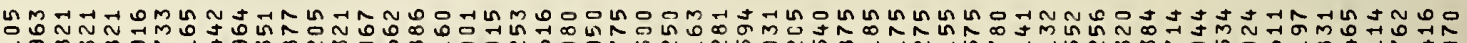
กีM $N$ N J J

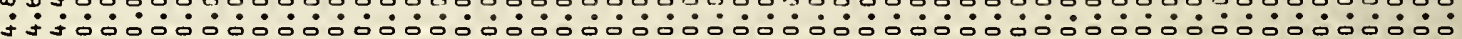

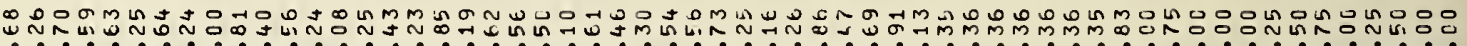

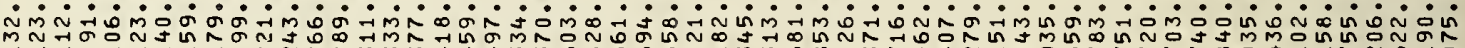

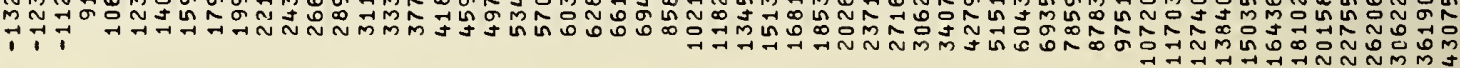
$\sum_{w}$

$\stackrel{\alpha}{\supset}$

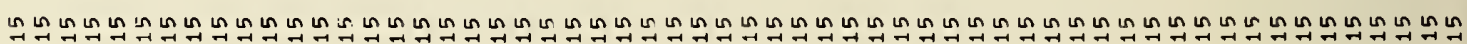




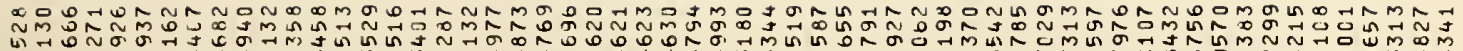

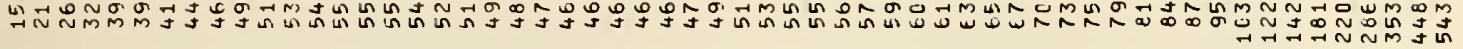

30

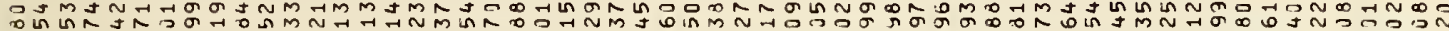
m.

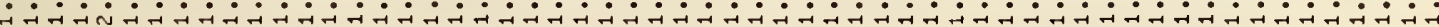

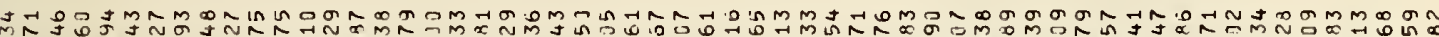

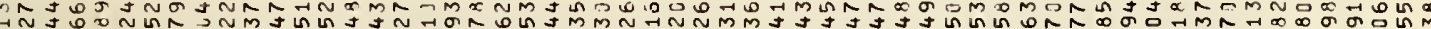

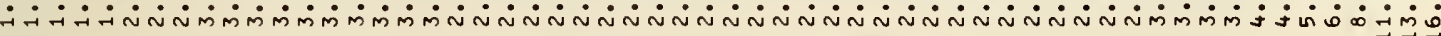

U月

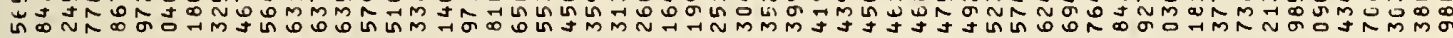

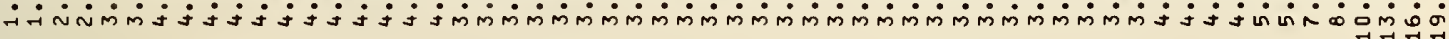

웅

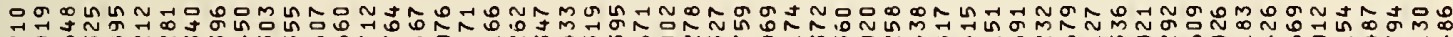

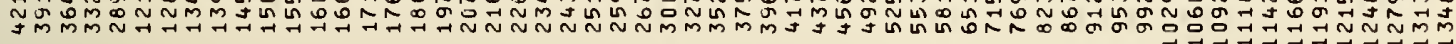

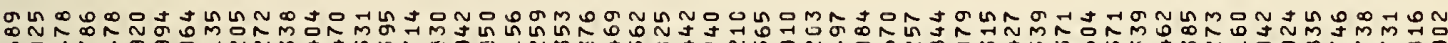

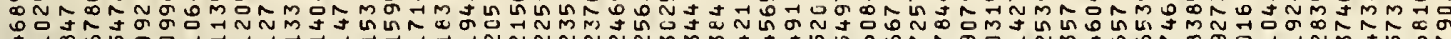
-

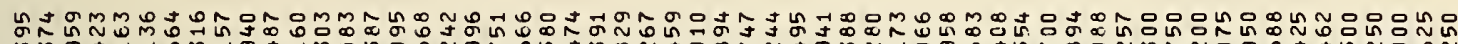

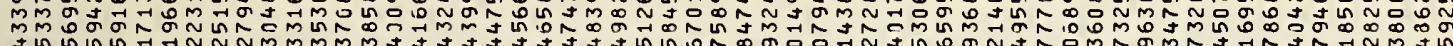

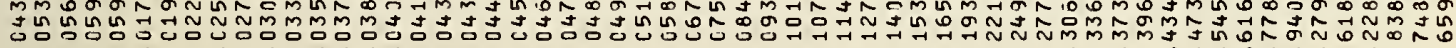

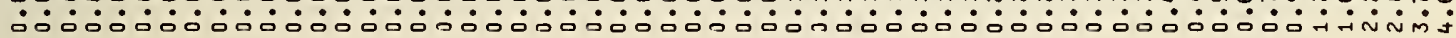

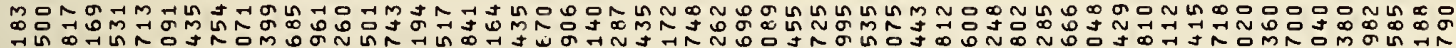

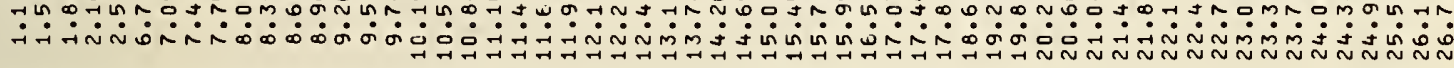

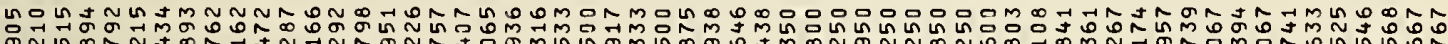

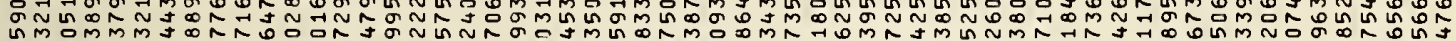

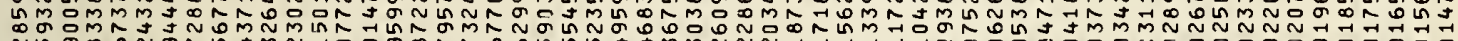

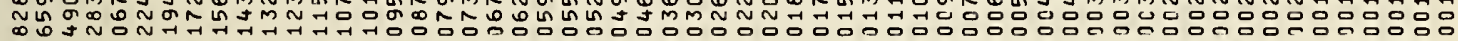

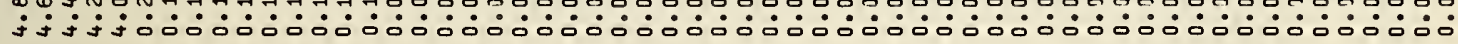

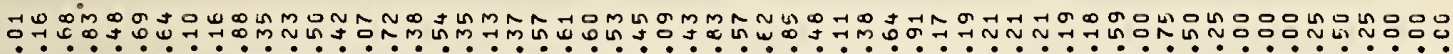

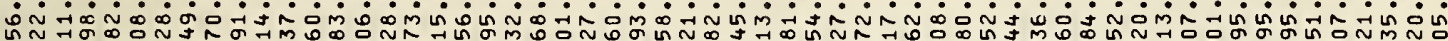
17. 1

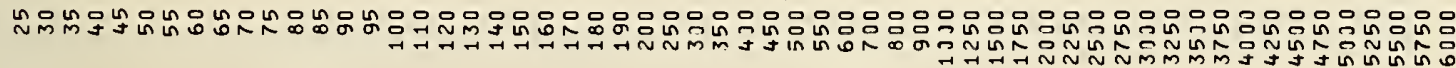

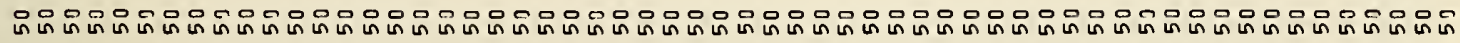




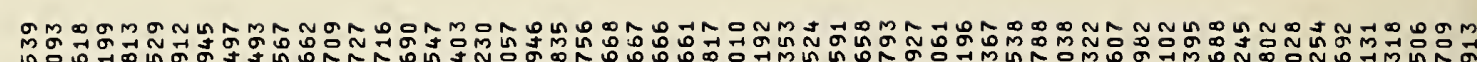

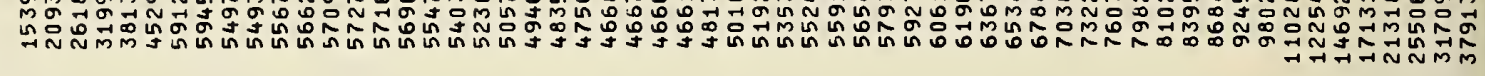

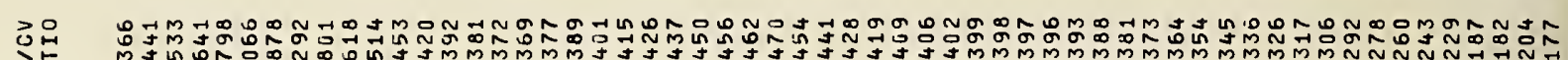
o̊

$>\frac{\alpha}{1}$

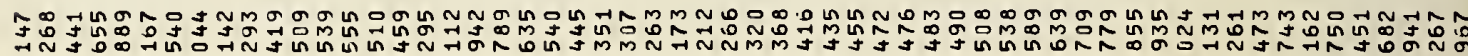

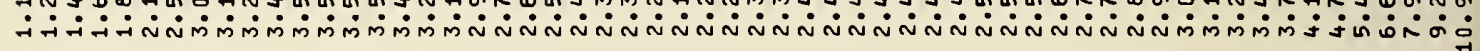

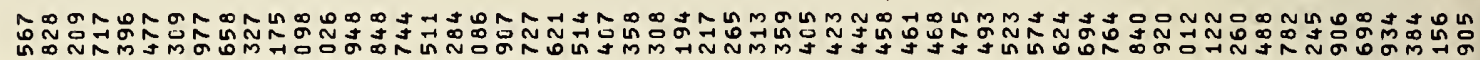

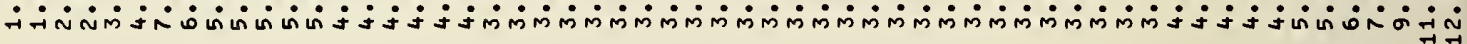

$\stackrel{0}{3}$

跑

$\dot{w}^{\stackrel{5}{L}}$

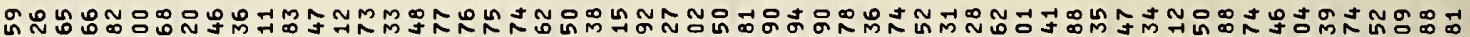

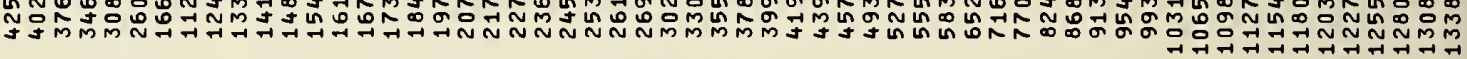

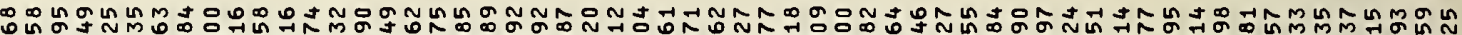

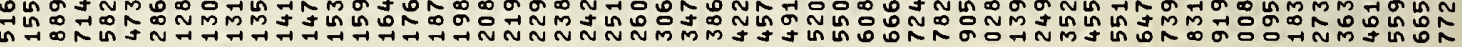

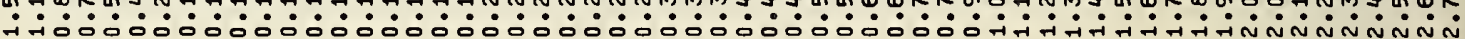

里น

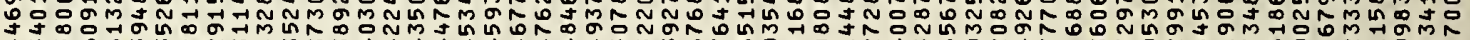

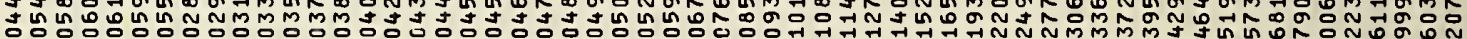

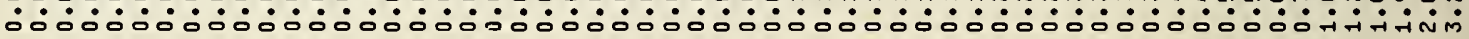

N N N N

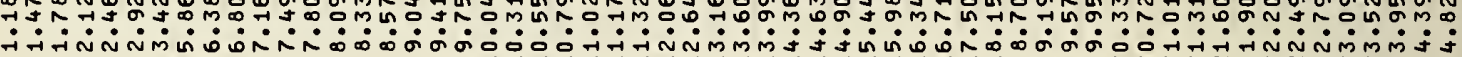

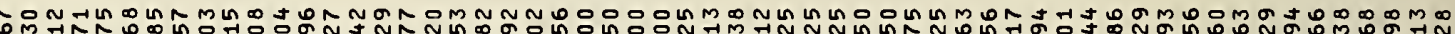

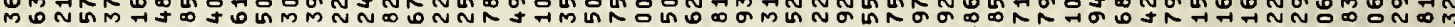
Nó. Uñ J

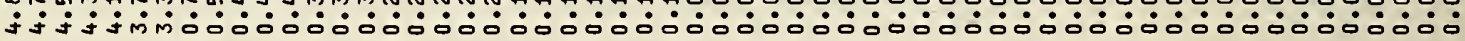

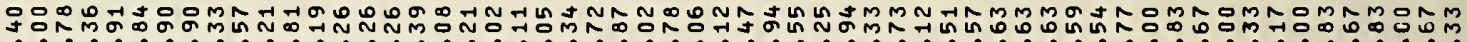

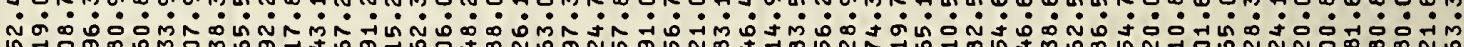

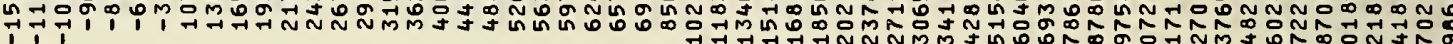

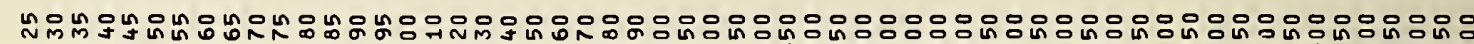

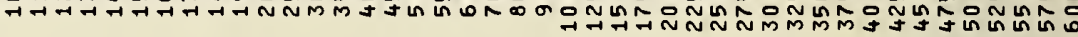

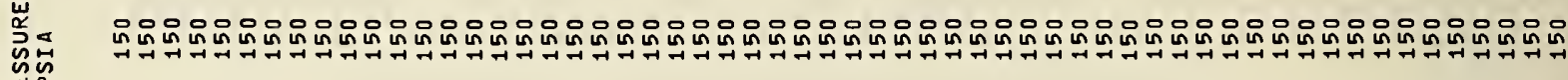
$\underset{\alpha}{\alpha} \alpha$ 


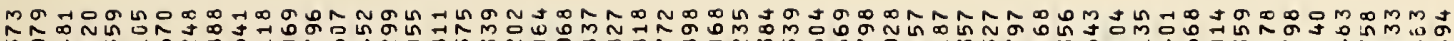

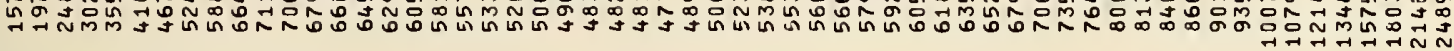

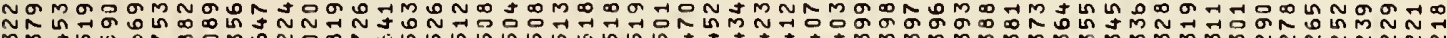

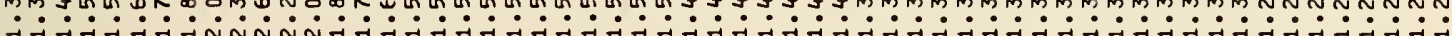

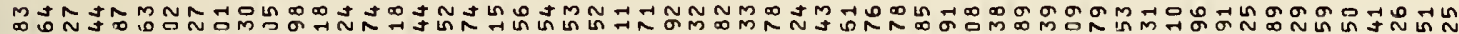

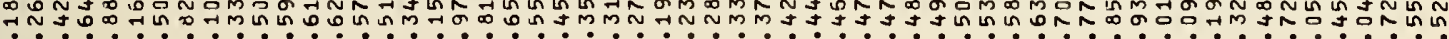

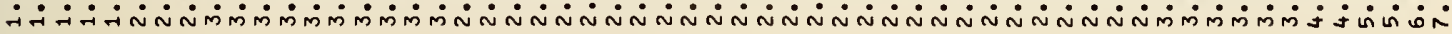

范

운

品

wh

占口

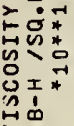



西

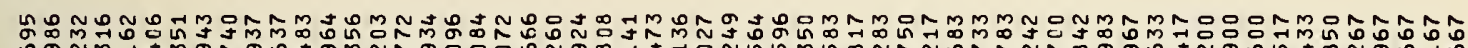

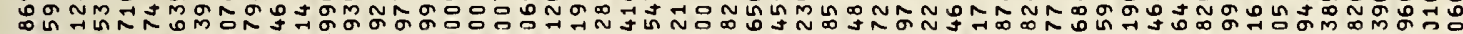

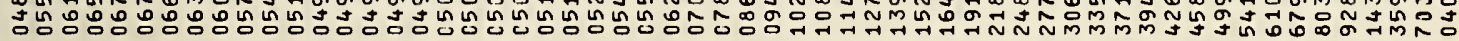

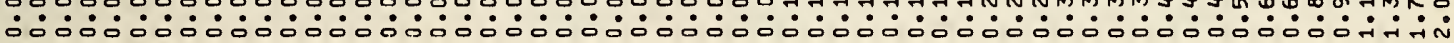

-

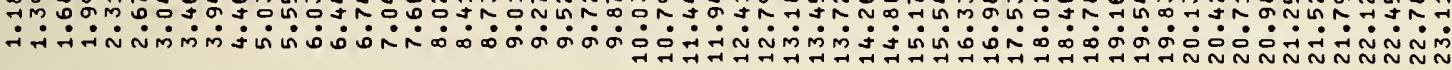

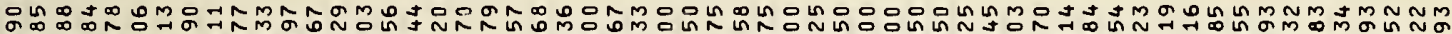

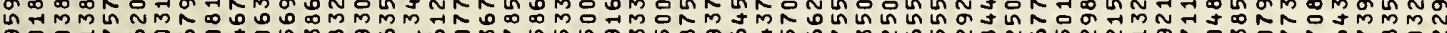

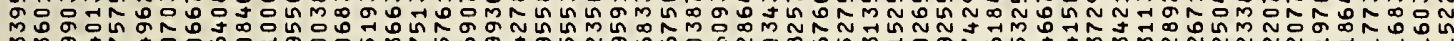

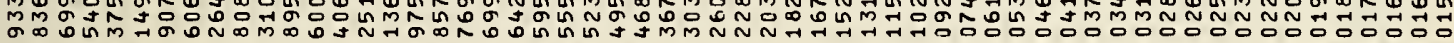

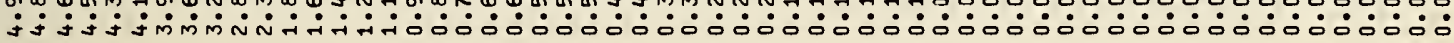

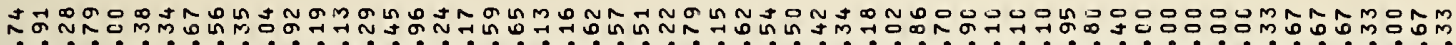

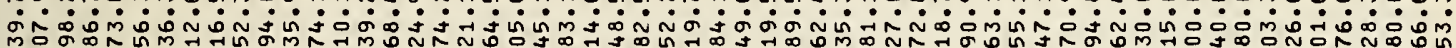

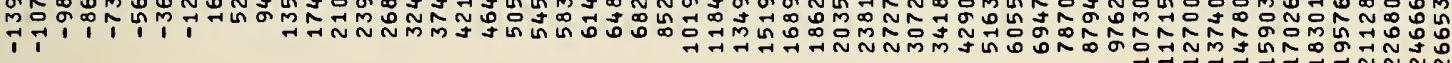

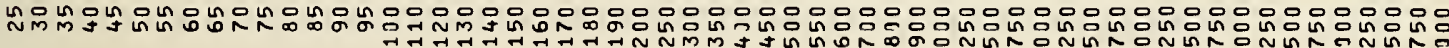

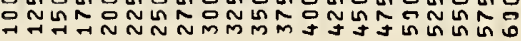

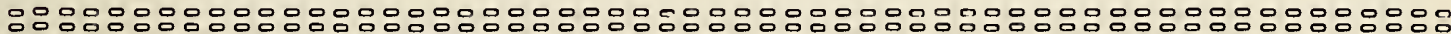

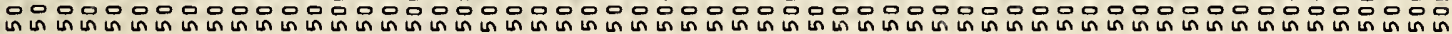


政

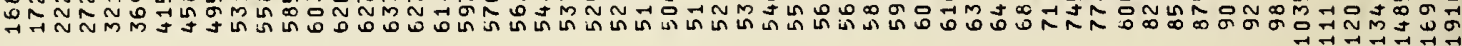

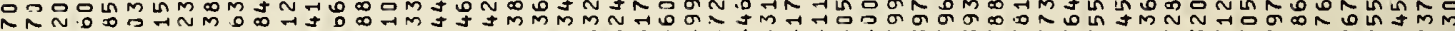

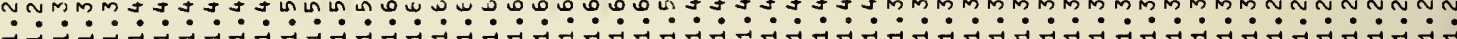

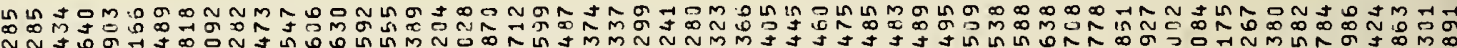

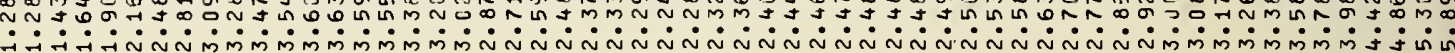

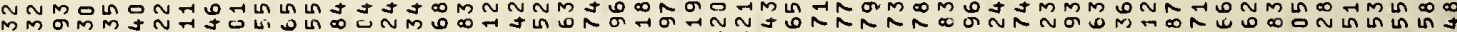
$000 N 0$ O In

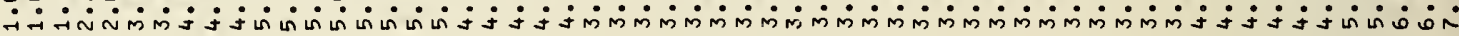

MMM

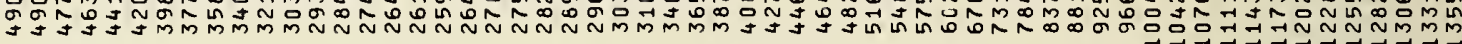

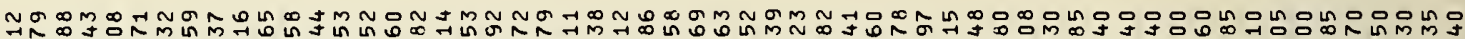
تै

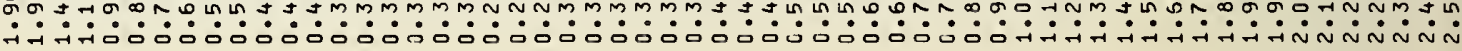

걱 MOt LN N N J J

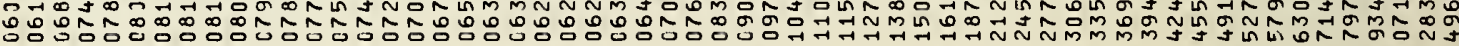

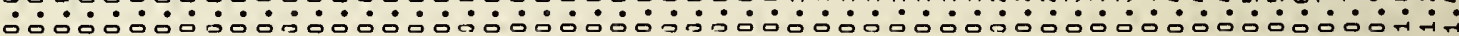

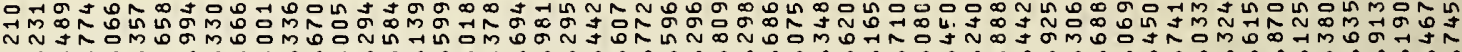

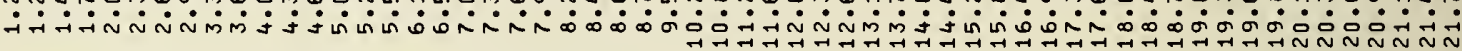

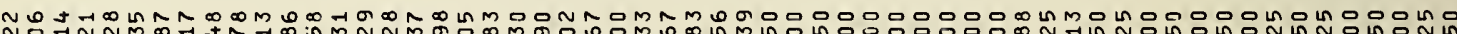
N OM J J

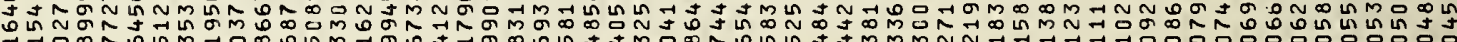
ni

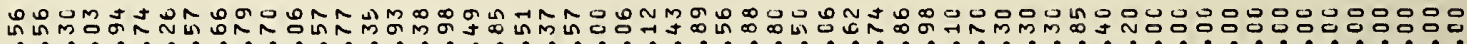

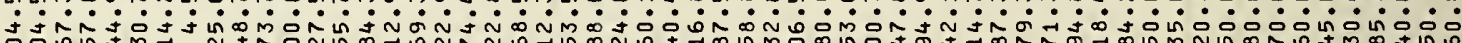

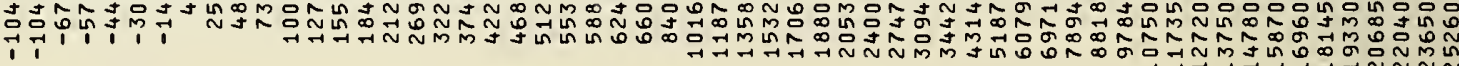

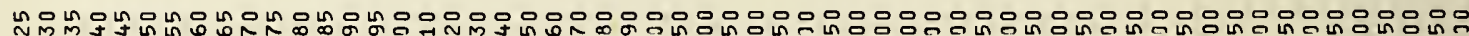

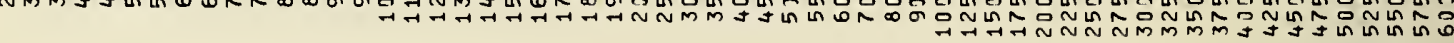

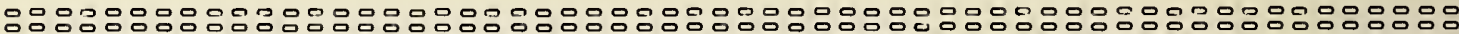

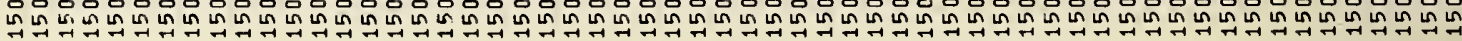




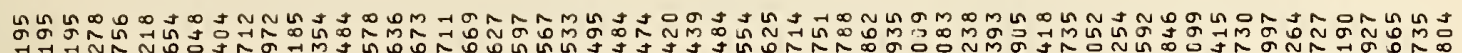

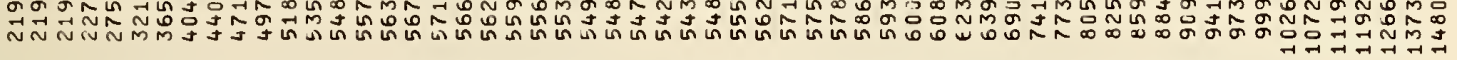

은

is

$x$
$\dot{1}$
$\dot{1}$
0

棺

$\stackrel{2}{\frac{1}{1}}$

ำ

옹

응

ษะ

崩

占。

芯品

$u x *$

虽亩

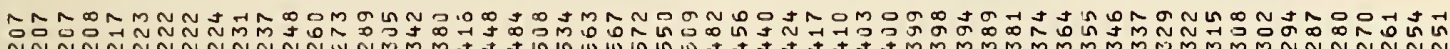

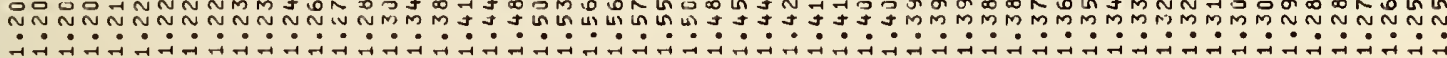

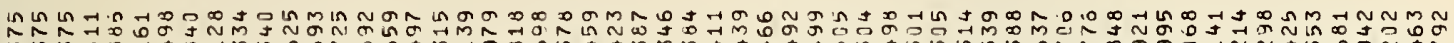
-

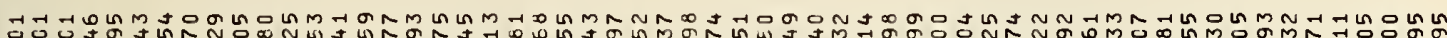

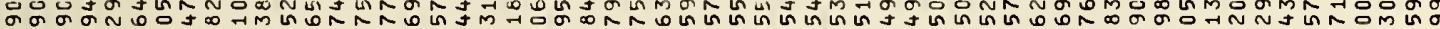

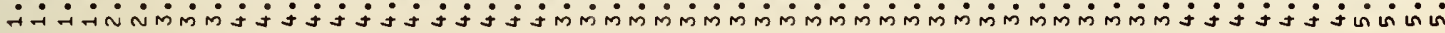

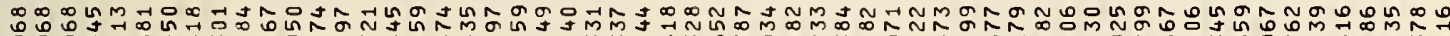

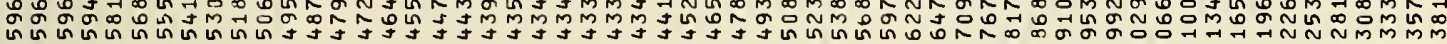

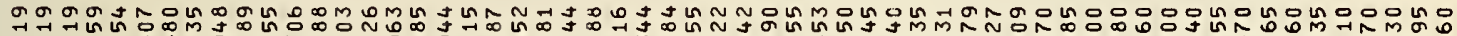

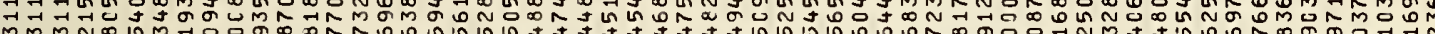

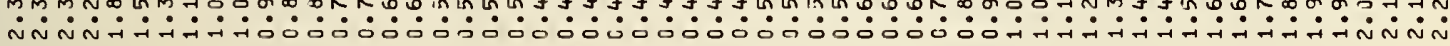

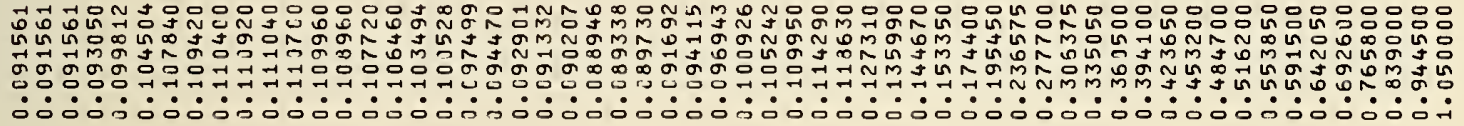

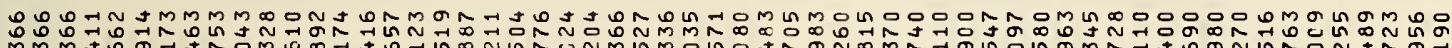

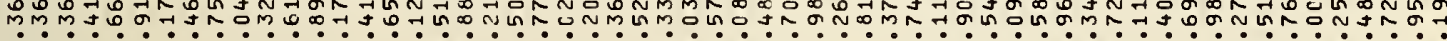

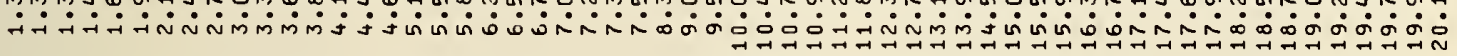
NN N N

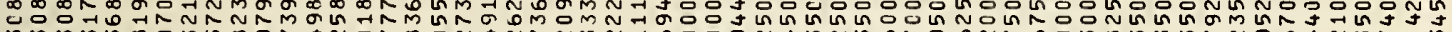
MNMMNo

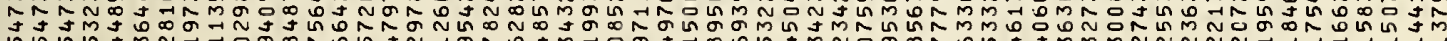

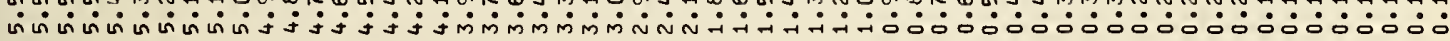
슈Nำ

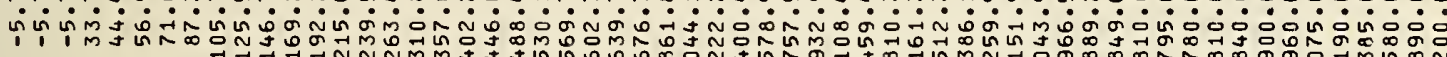
1 1 mus

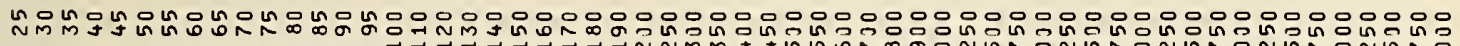

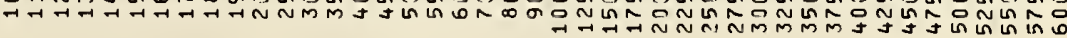

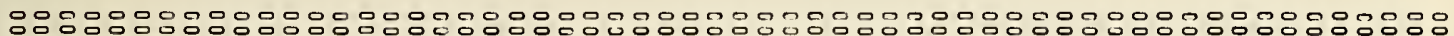

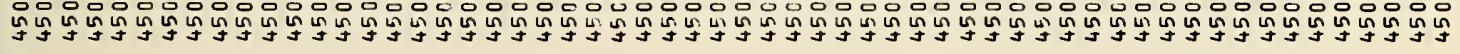


APPENDIX B. PROGRAM LISTINGS: CDC AND IBM
CDC
Page No.
IBM

Parahydrogen

PTENTH
PTDENS
PTENTR
PTCOND
PTVISC
PTSOUN
PTCP, PTCV, PTGAMM
PTLFAC
TSATH
PSATH
PHTEMP
PHDENS
PHENTR
PHCOND
PHVISC
PHSOUN
PHCP, PHCV, PHGAMM
PHLFAC

$\begin{aligned} 53 & \text { PTENTH } \\ 57 & \text { PTDENS } \\ 61 & \text { PTENTR } \\ 65 & \text { PTCOND } \\ 68 & \text { PTVISC } \\ 72 & \text { PTSOUN } \\ 75 & \text { PTCP, PTCV, PTGAMM } \\ 80 & \text { PTLFAC } \\ 83 & \text { TSATH } \\ 84 & \text { PSATH } \\ 85 & \text { PHTEMP } \\ 87 & \text { PHDENS } \\ 91 & \text { PHENTR } \\ 94 & \text { PHCOND } \\ 98 & \text { PHVISC } \\ 103 & \text { PHSOUN } \\ 105 & \text { PHCP, PHCV, PHGAMM } \\ 111 & \text { PHLFAC }\end{aligned}$

Equilibriumhydrogen

ETENTH
ETDENS
ETENTR
ETCOND
ETVISC
ETSOUN
ETCP, ETCV, ETGAMM
ETLFAC
ETSATH
EPSATH
EHTEMP
EHDENS
EHENTR
EHCOND
EHVISC
EHSOUN
EHCP, EHCV, EHGAMM
EHLFAC

115

119

124

127

131

134

137

142

146

146

147

150

153

157

160

165

168

173

\author{
ETENTH \\ ETDENS \\ ETENTR \\ ETCOND \\ ETVISC \\ ETSOUN \\ ETCP, ETCV, ETGAMM \\ ET LFAC \\ ETSATH \\ EPSA TH \\ EHT EMP \\ EHDENS \\ EHENTR \\ EHCOND \\ EHVISC \\ EHSOUN \\ EHCP, EHCV, EHGAMM \\ EHLFAC
}




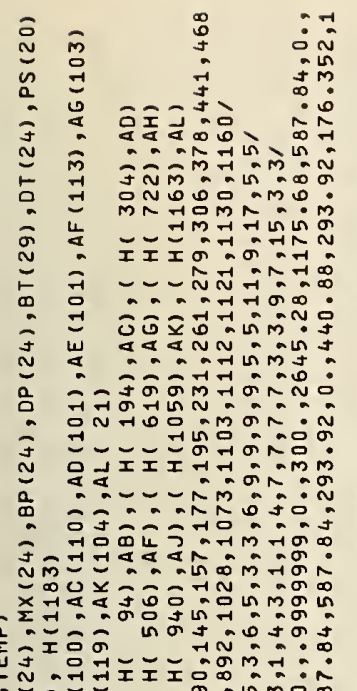

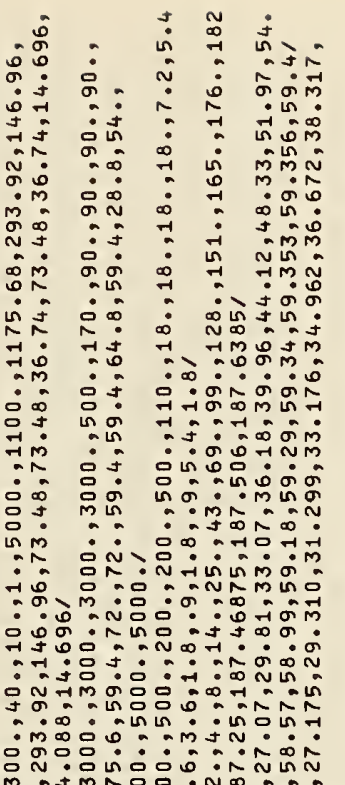

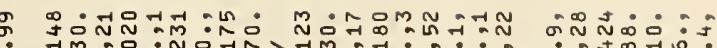

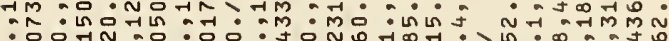

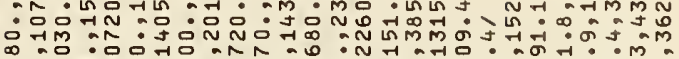

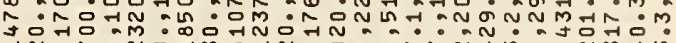

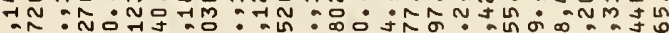

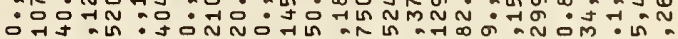

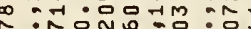

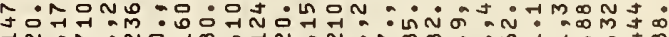
$\because N$ N - OD

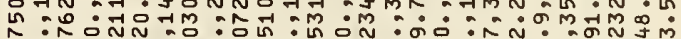
Nón

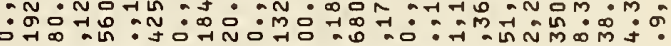
Na N NoN N

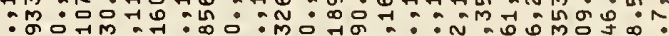
oㄴ.

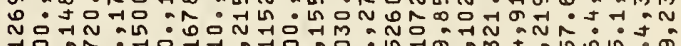

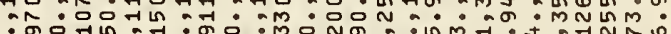

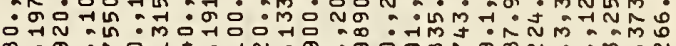

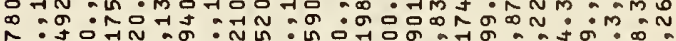

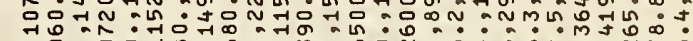

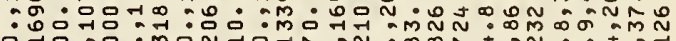
品员:

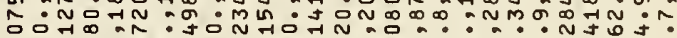

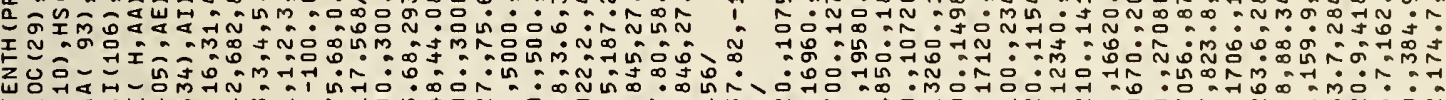

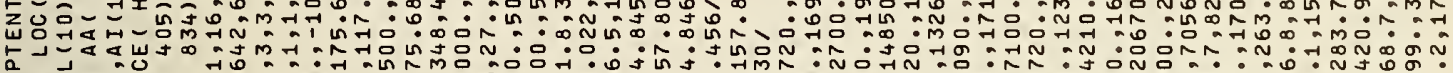

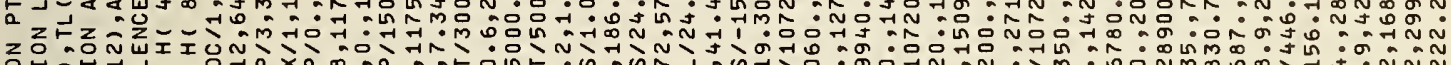
zón

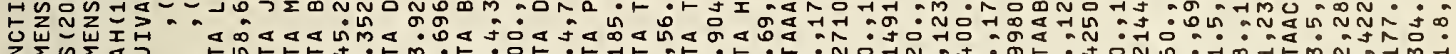

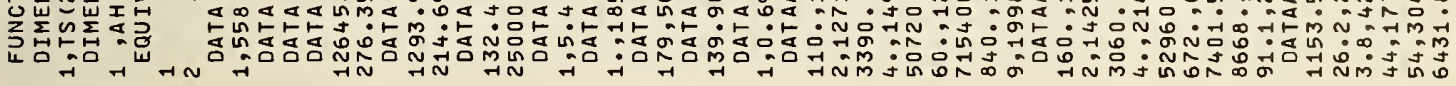

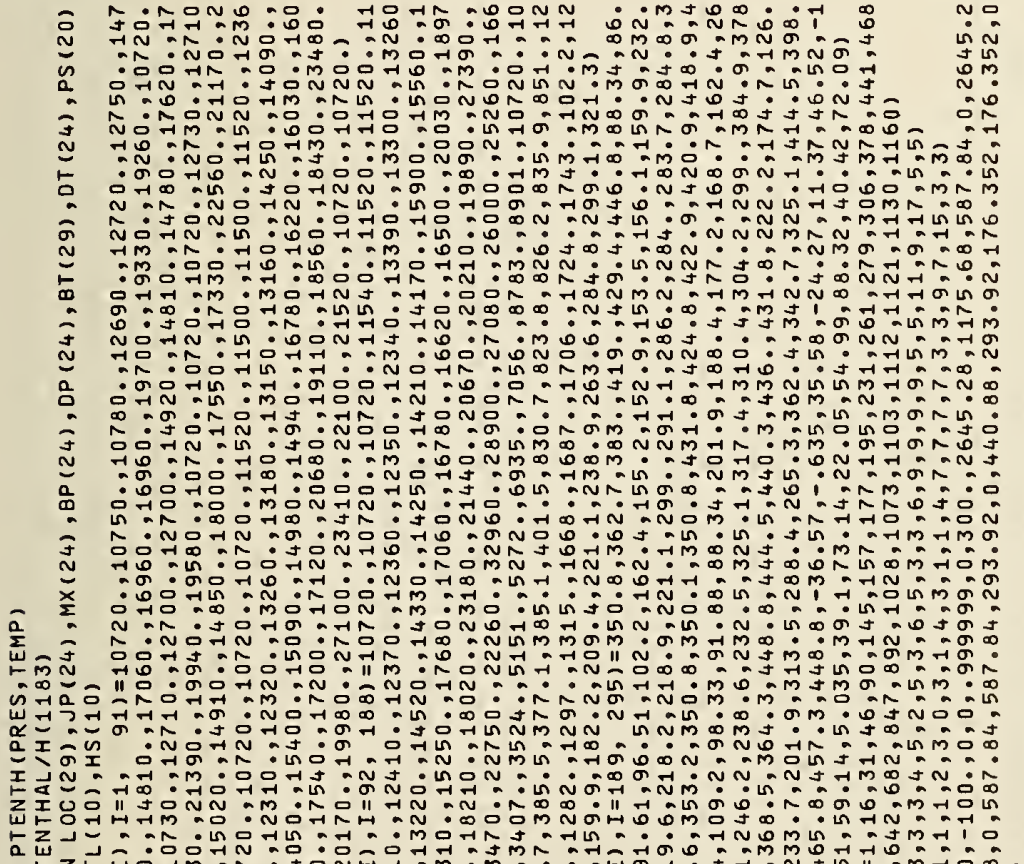
代 N T-1 约

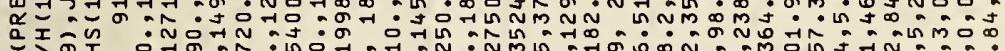
I

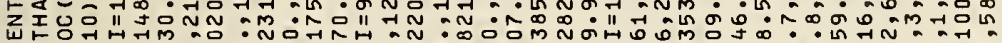

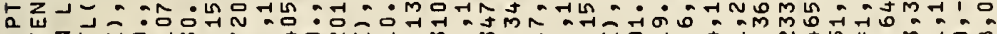

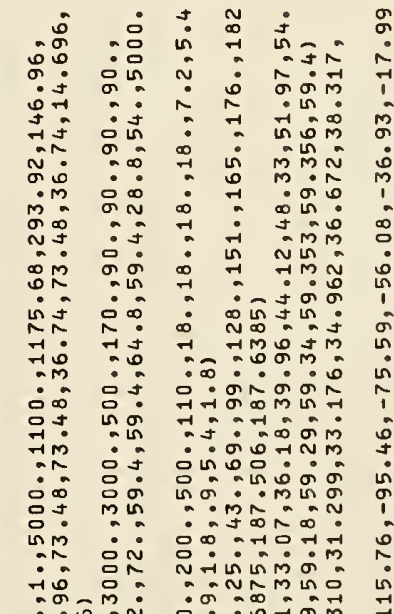

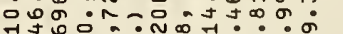

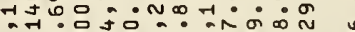

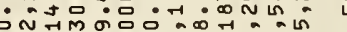
ง

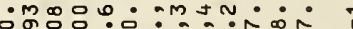

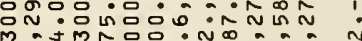
Do

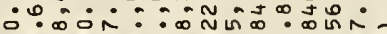

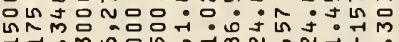

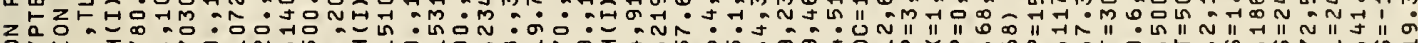

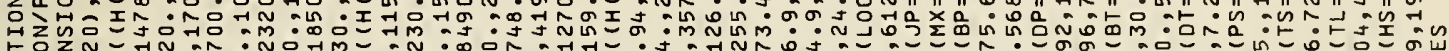

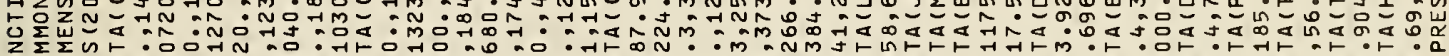

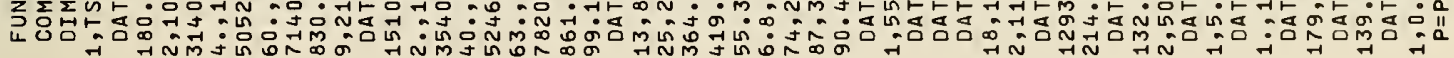




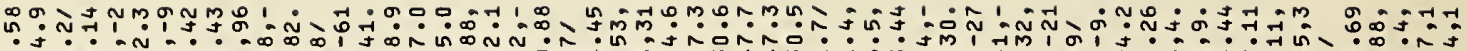
in mレT ñ

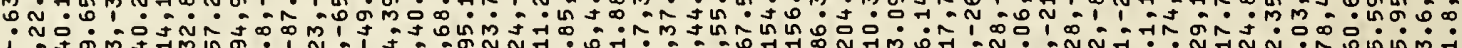
i No N on

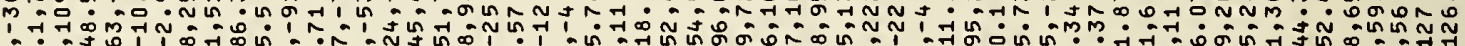
-

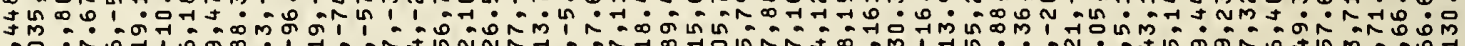
: 证

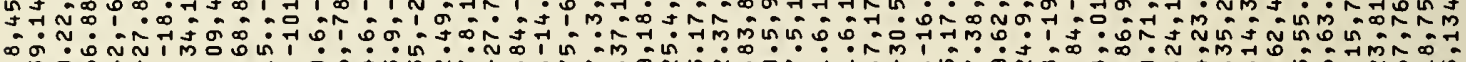

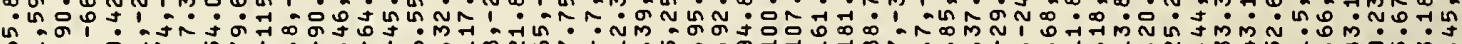
on

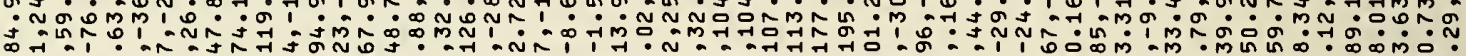
m

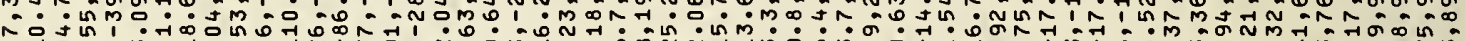
1 \%! mNa 1. ñ F. ำ

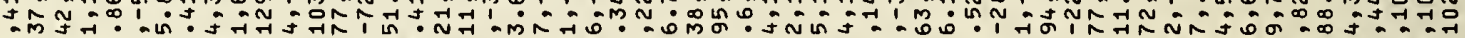

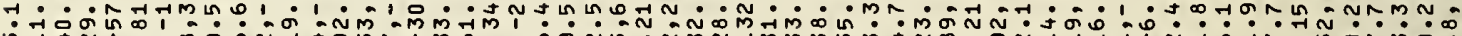

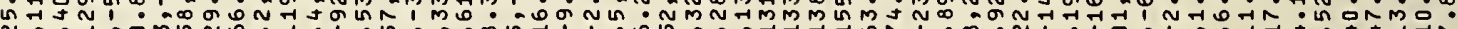

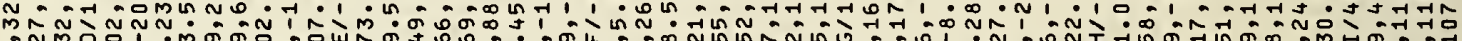
ヘ N.. ¿ N N ค.

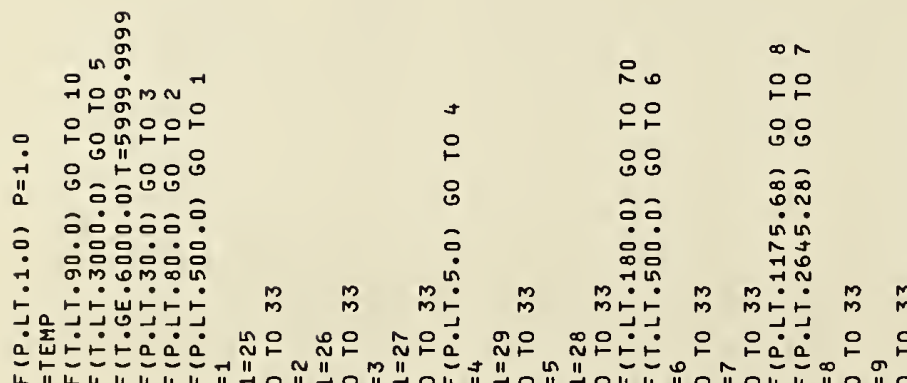
늡늡

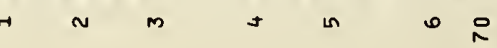

웅 or 으

न m च I 


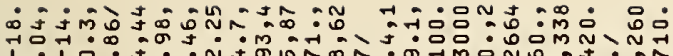
N

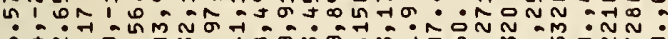

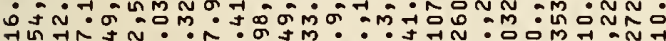

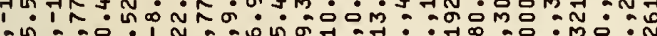

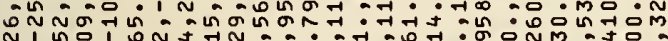

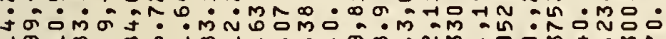
10

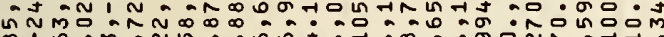

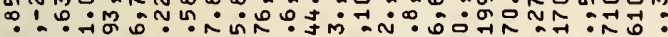

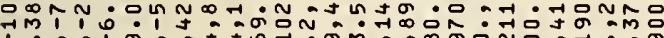
作 ก N

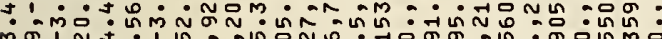

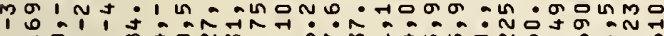

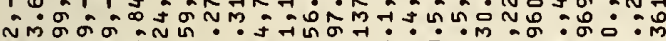

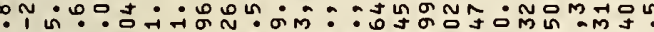

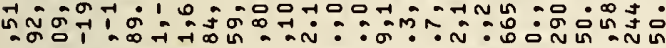

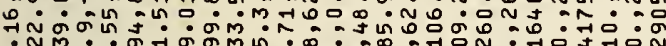
1 OfNo - $\operatorname{nin\infty } 0$ Кัง

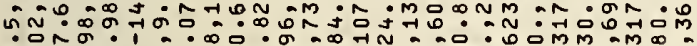
: N

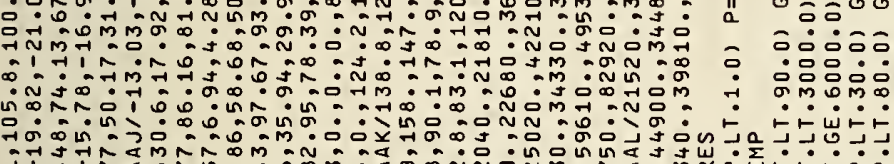

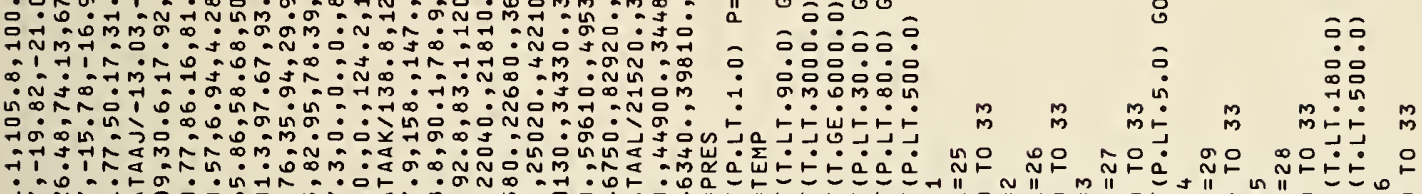

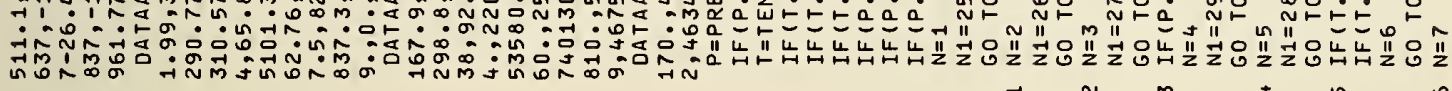

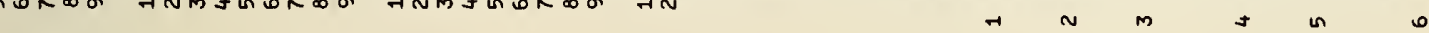

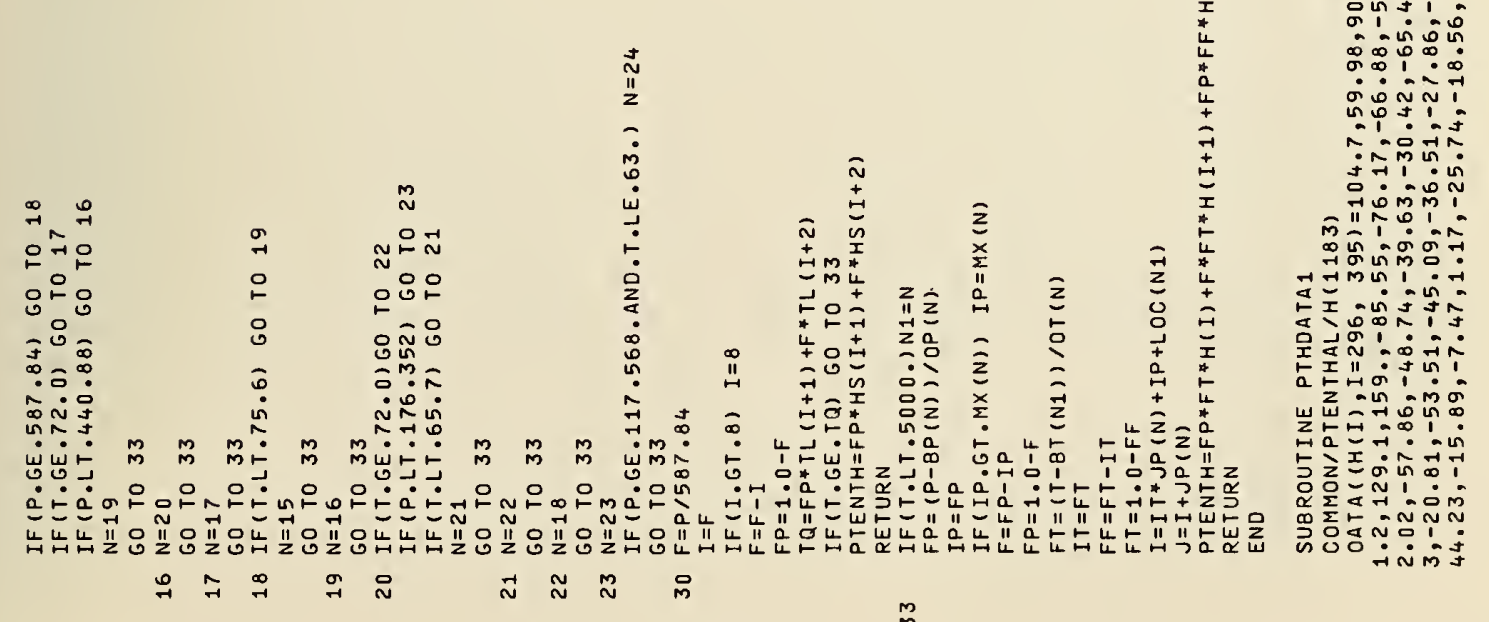

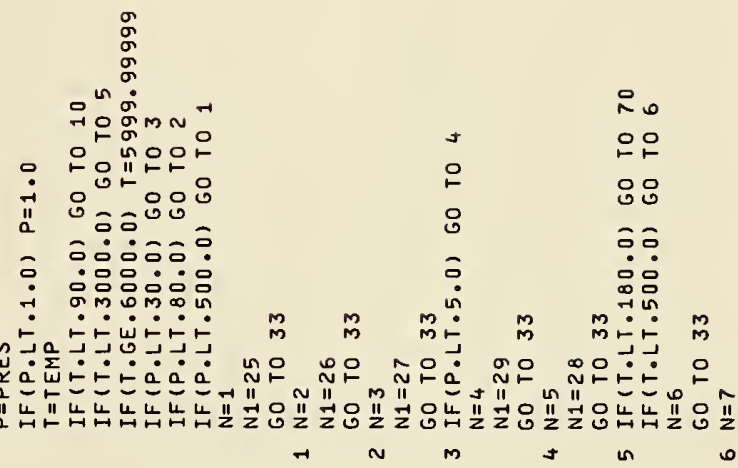

$\tilde{\square}$ $\because 1-\infty-\infty$

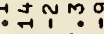

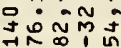
m'คูก๊ำ 迎品 01 . in 过声壬 뭉 ก บ in กิธูก: จं०न 约 0 舟管 ?. अन

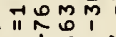
ज० नं

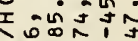

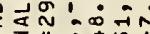
I" U一⿻上丨 这落皆 z=心必!

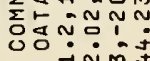




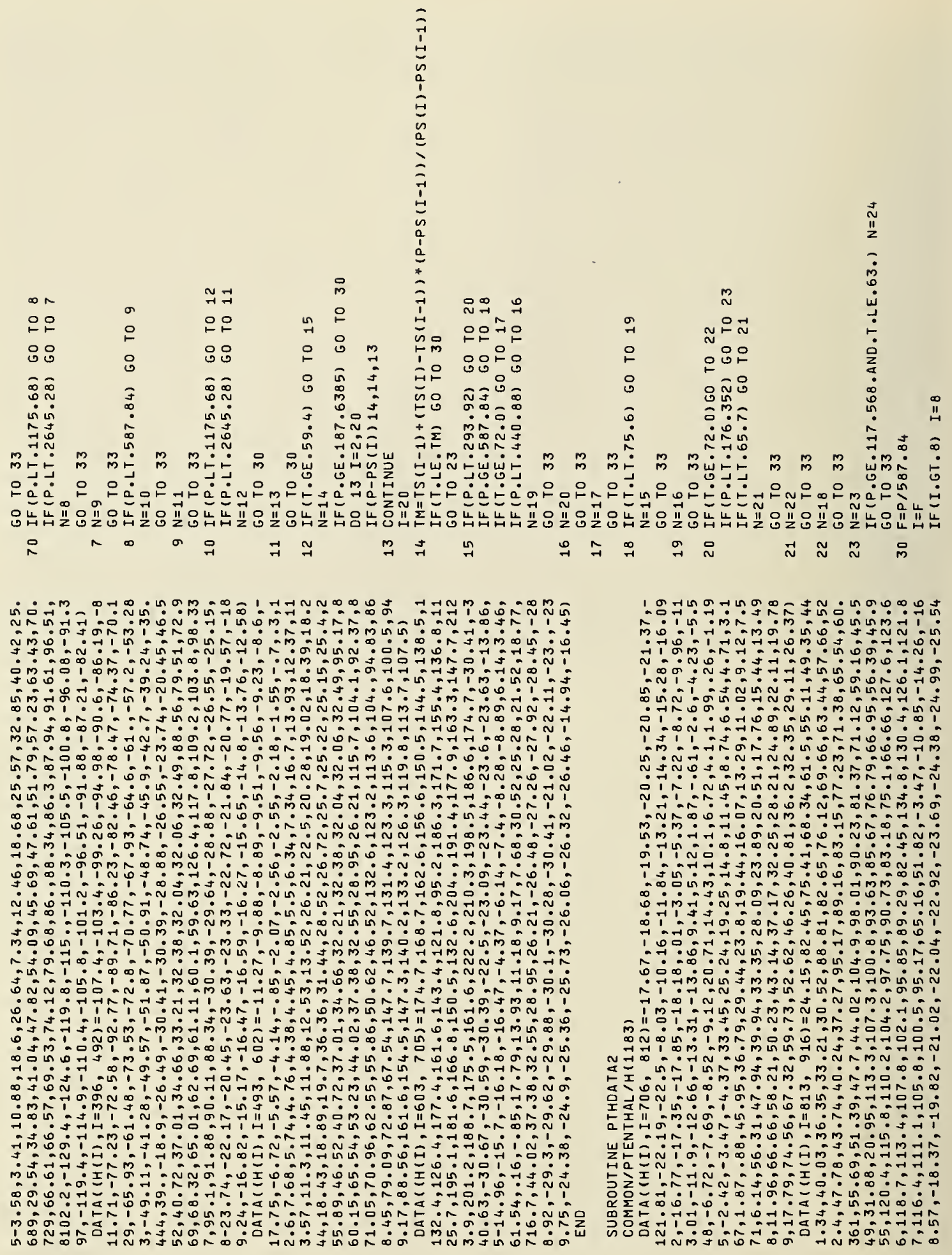




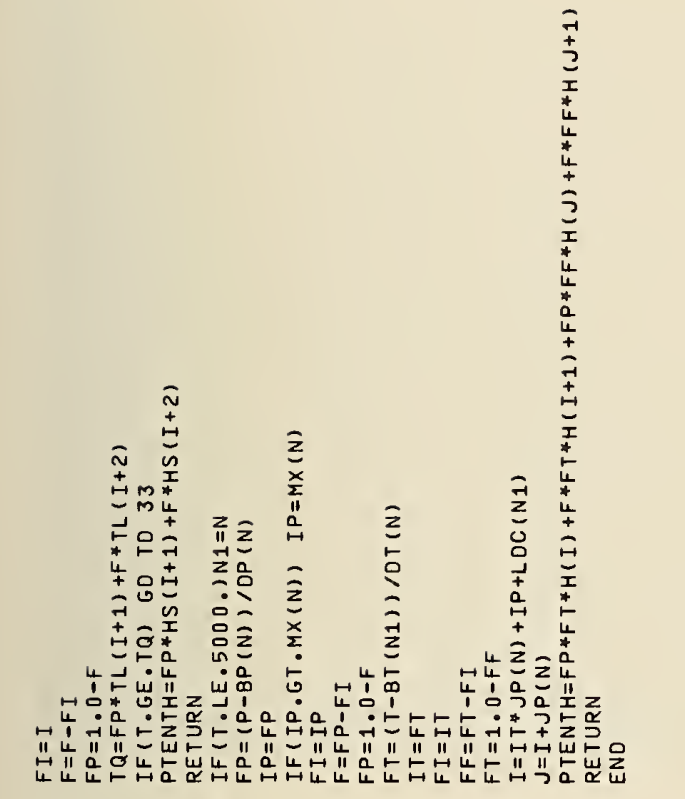
in

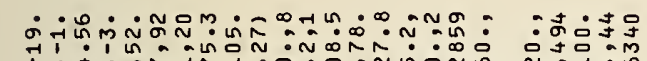
กับ ถูต

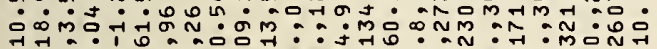
1.000

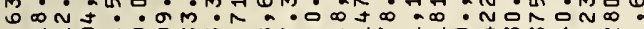
T-7 ơ

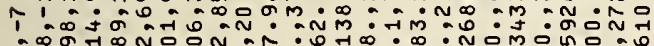

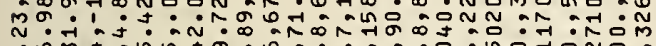
PंN Ōं कीं ONT

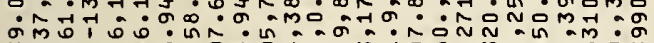
m.jo 0. 等 in б̄ं ON

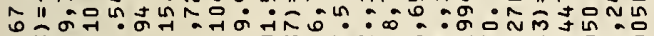

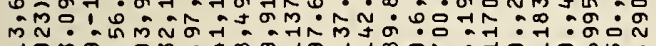

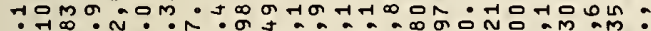
\}

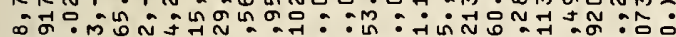

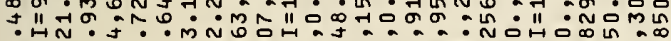
N-1

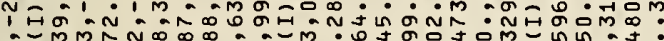
J王? ำ

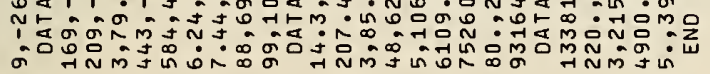
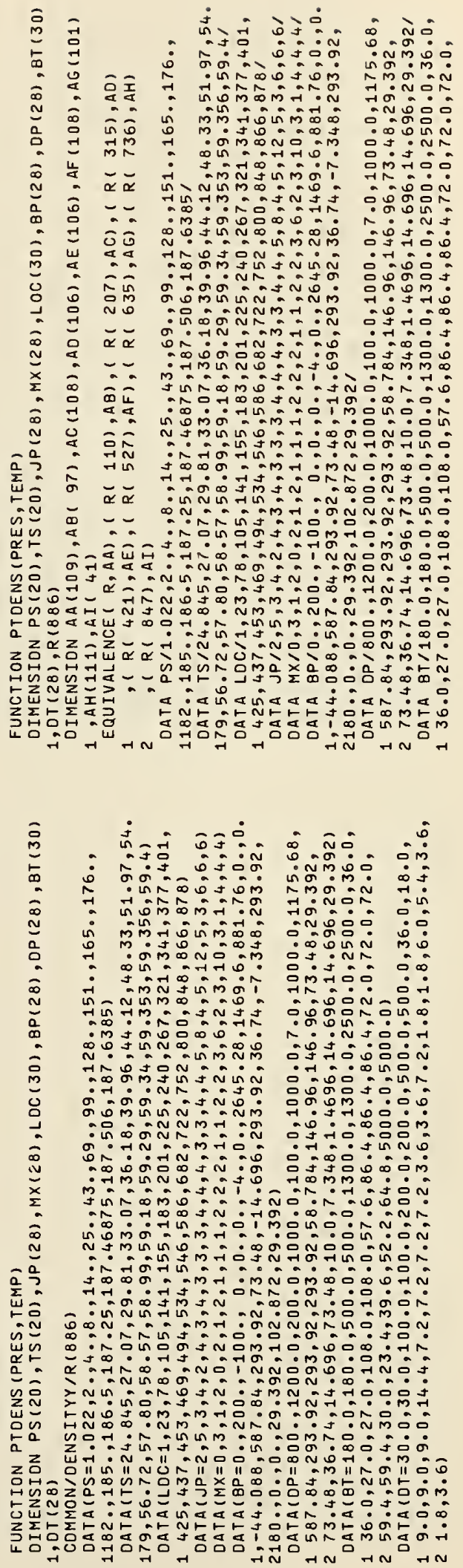

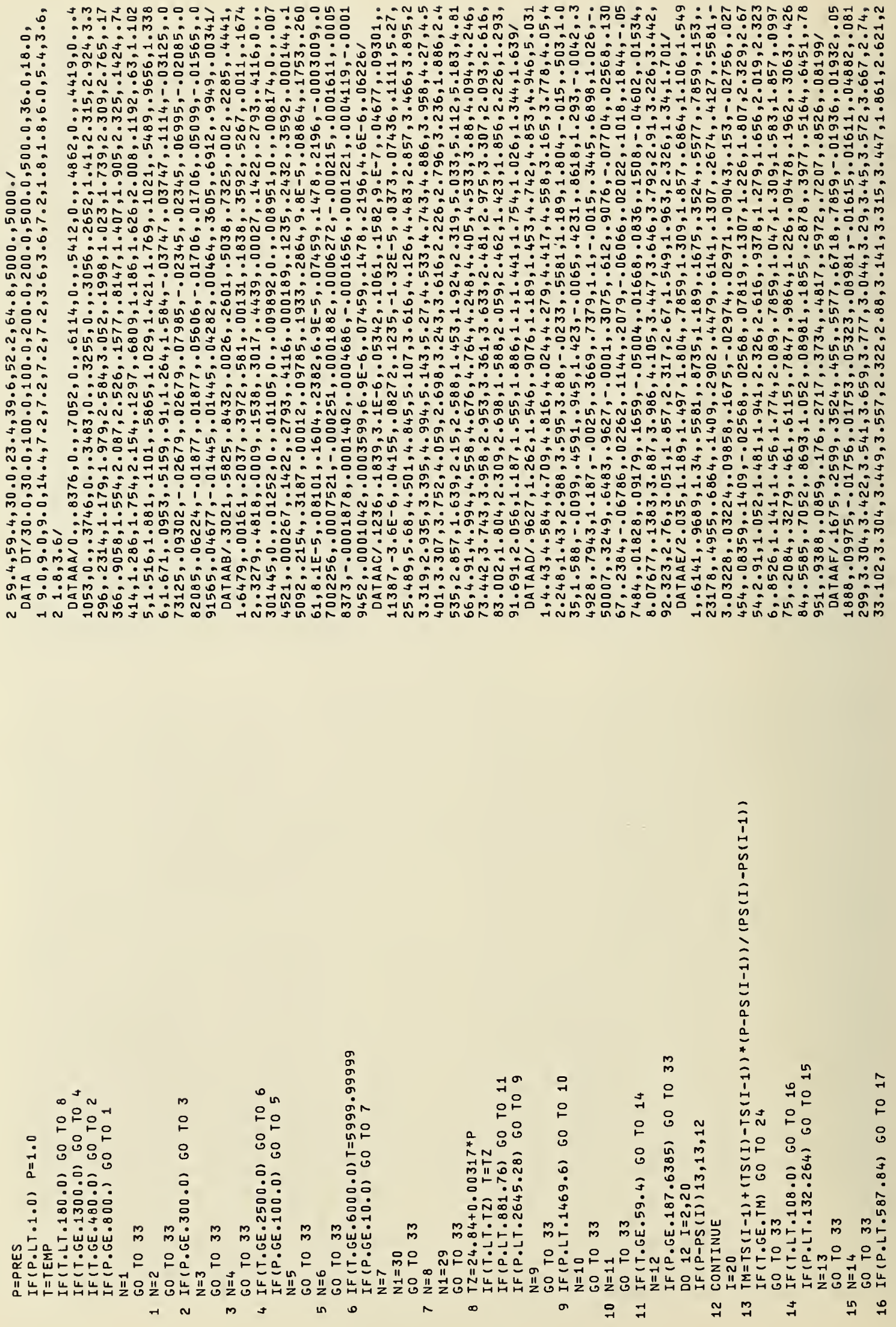


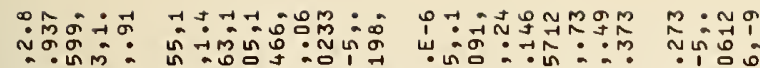

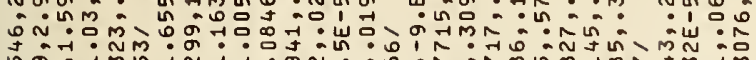

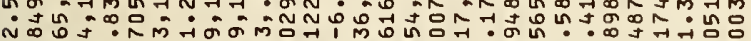

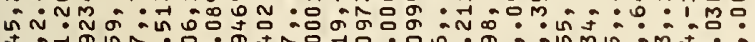
造:

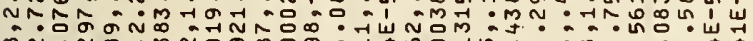

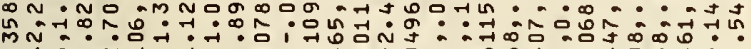

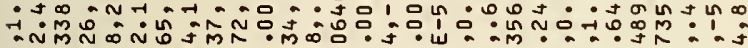

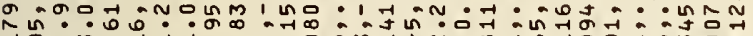

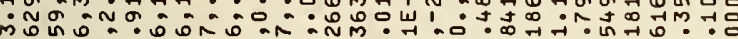
于:Nล์

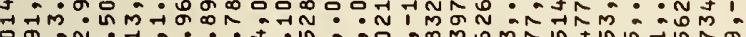

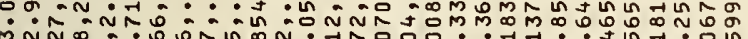
mNN

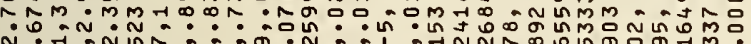
N No

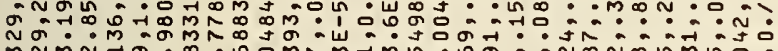

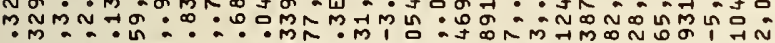
N

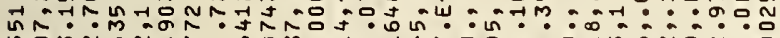

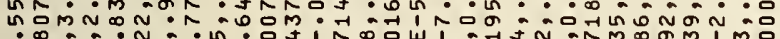

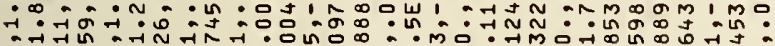

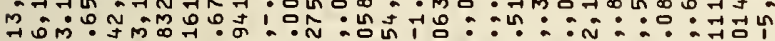

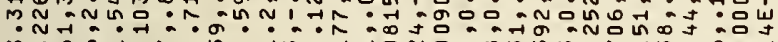

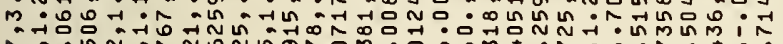

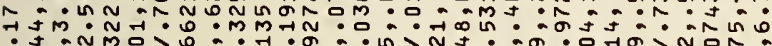

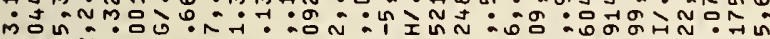

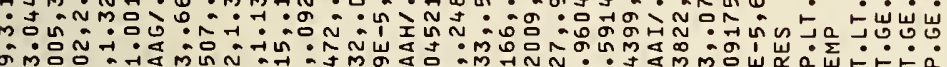

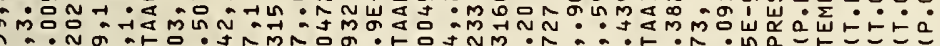

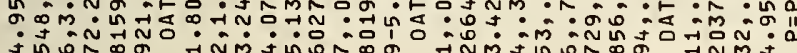

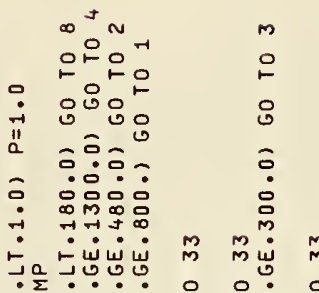

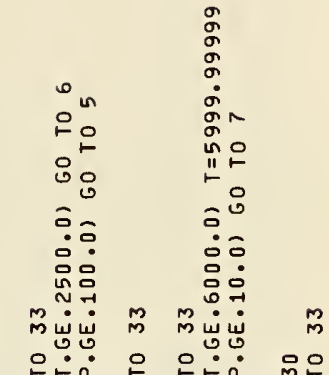

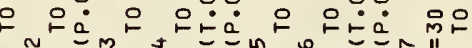

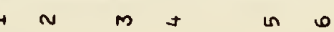

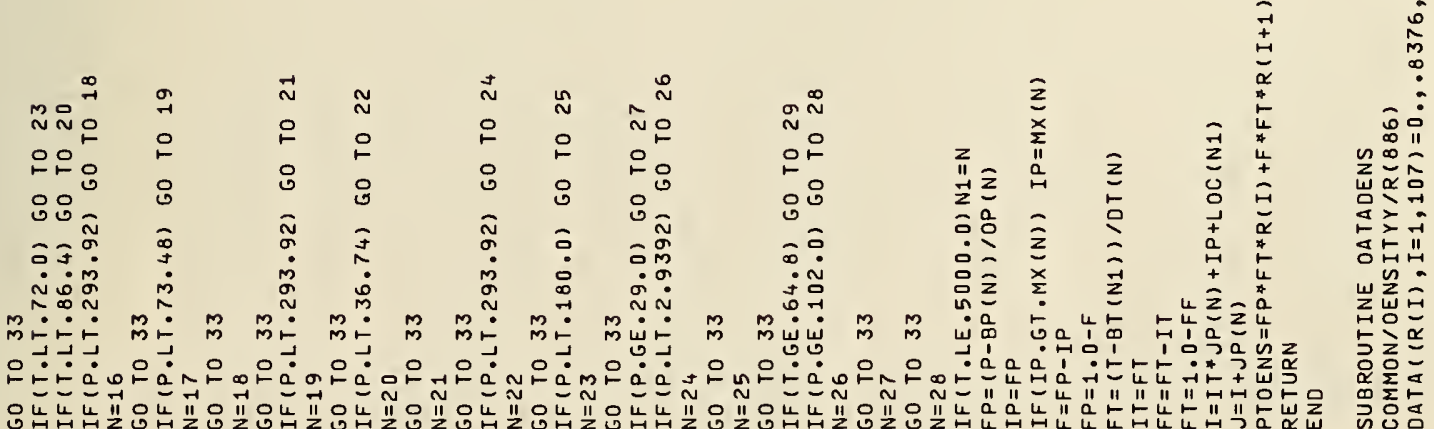

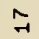

$\rightarrow$ 요

त)

$\approx \stackrel{m}{N} N$

$\stackrel{\sim}{\sim}$

$\stackrel{\infty}{\sim} \underset{m}{m}$ 


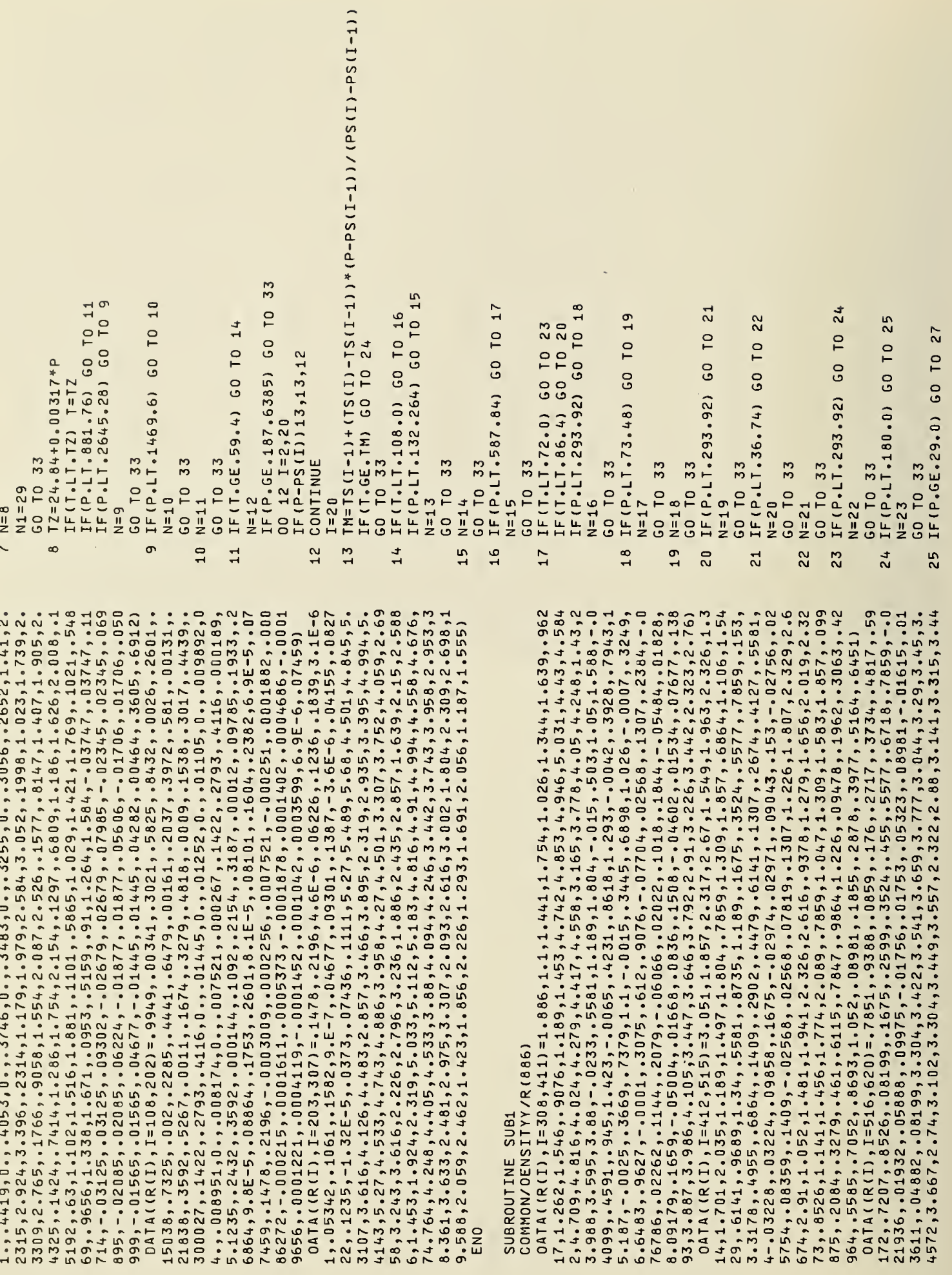




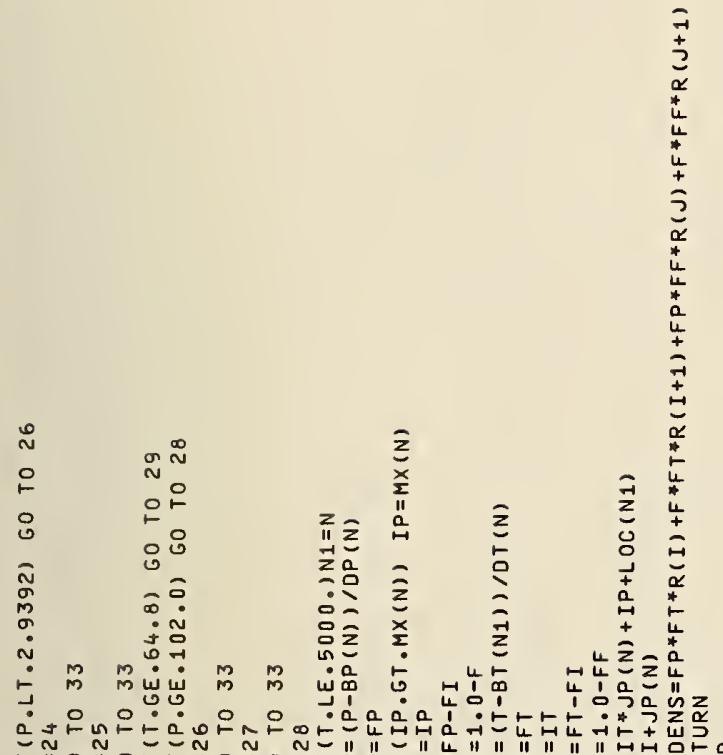

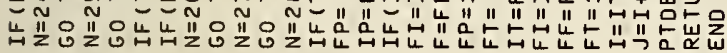
N N $\stackrel{\text { N }}{\text { NM }}$
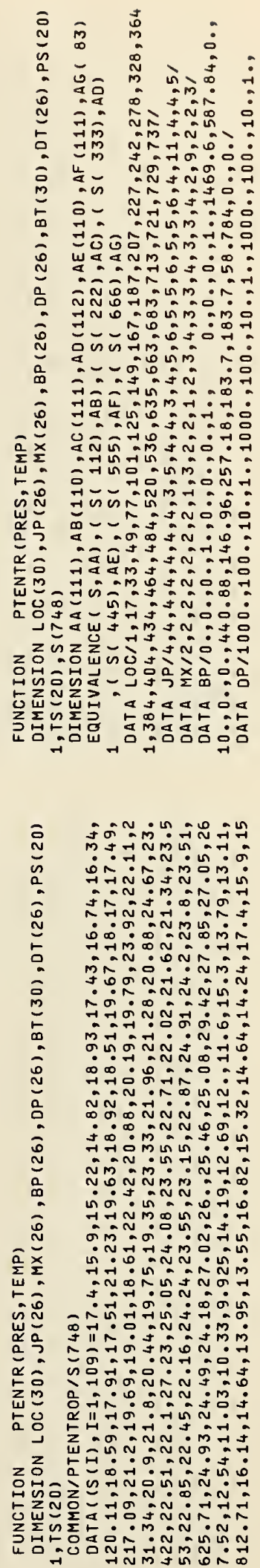

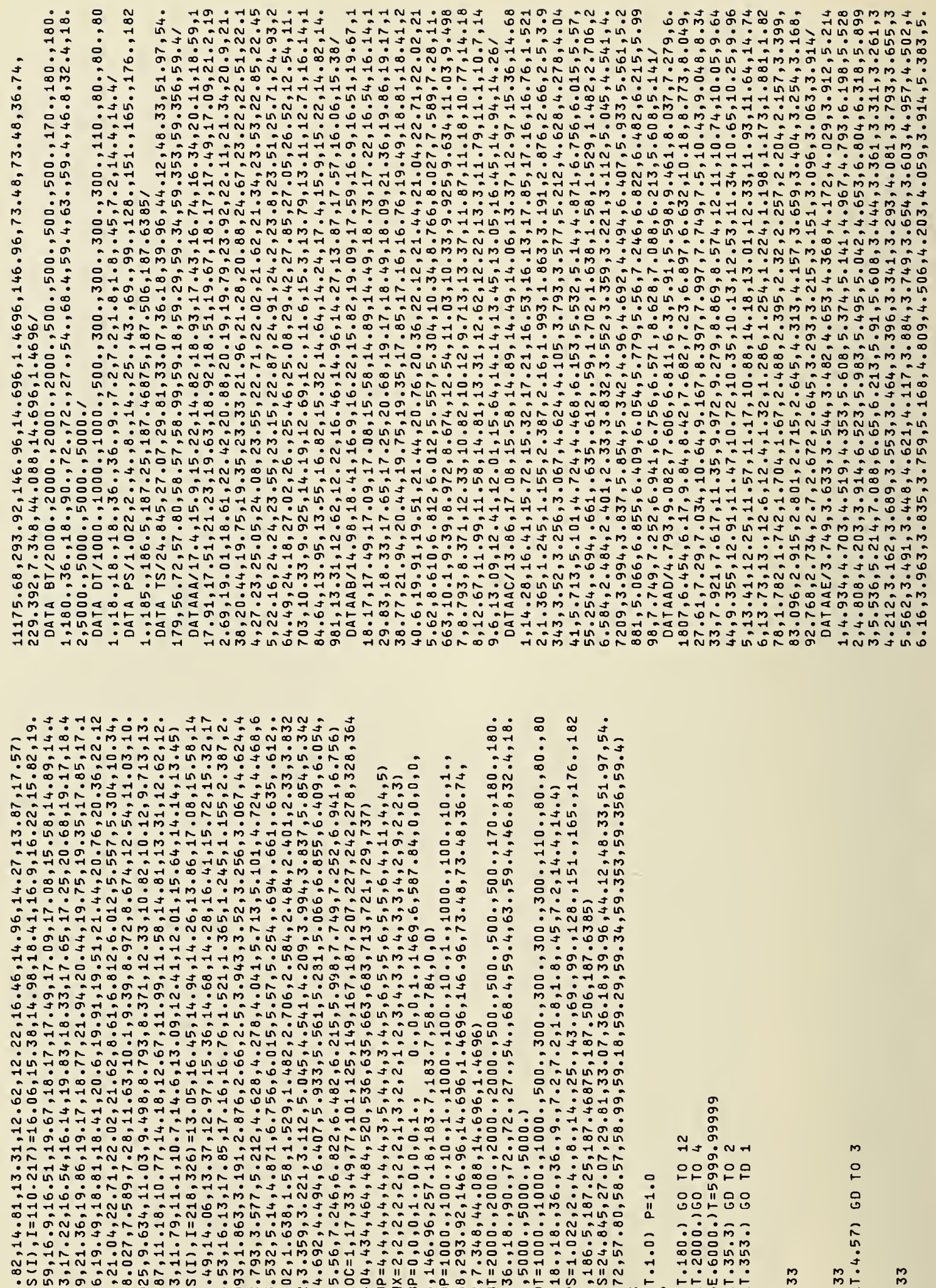

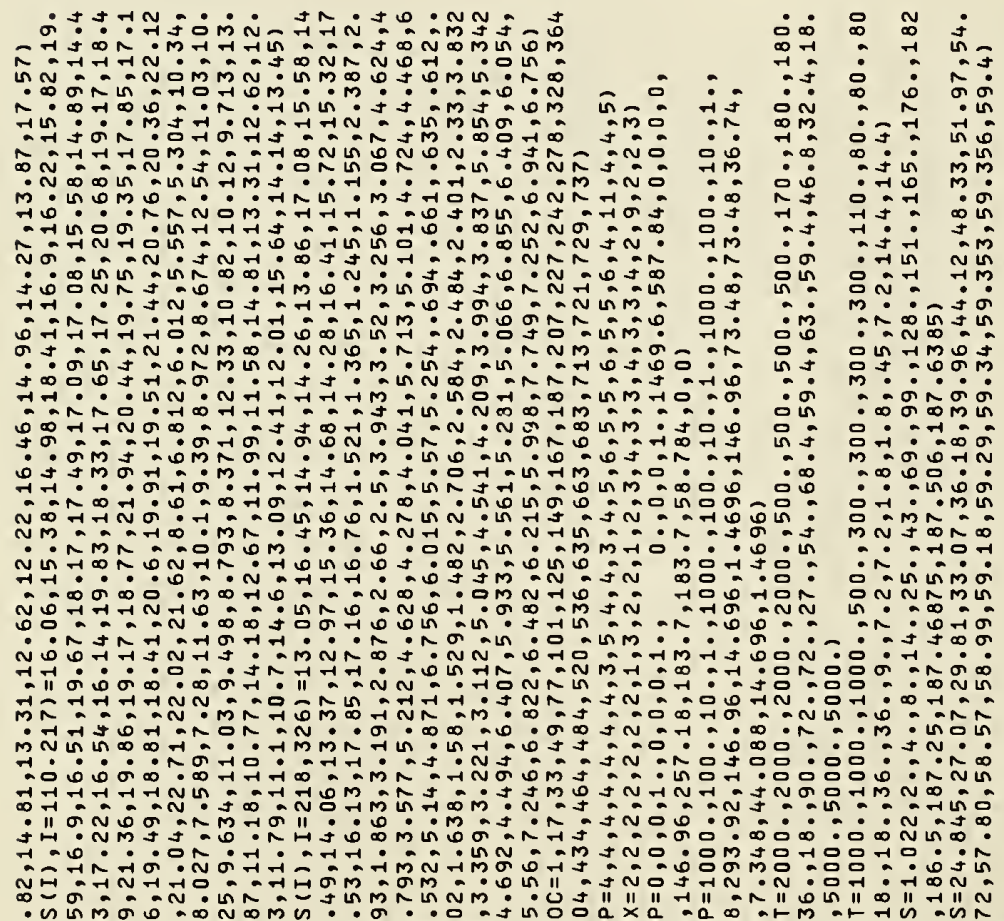

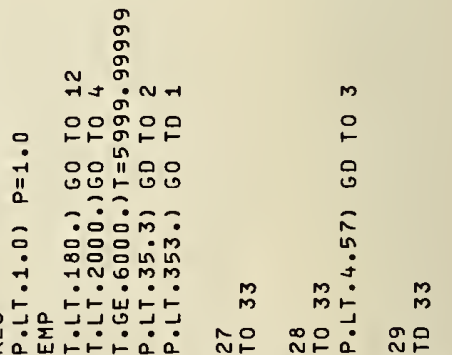

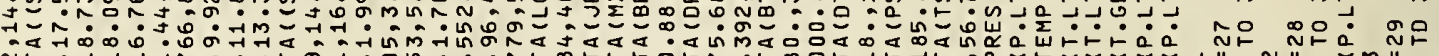

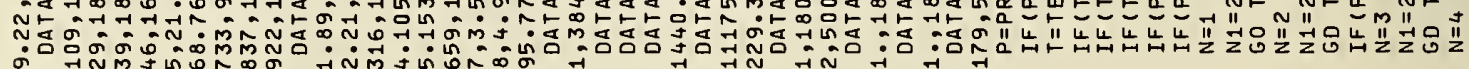




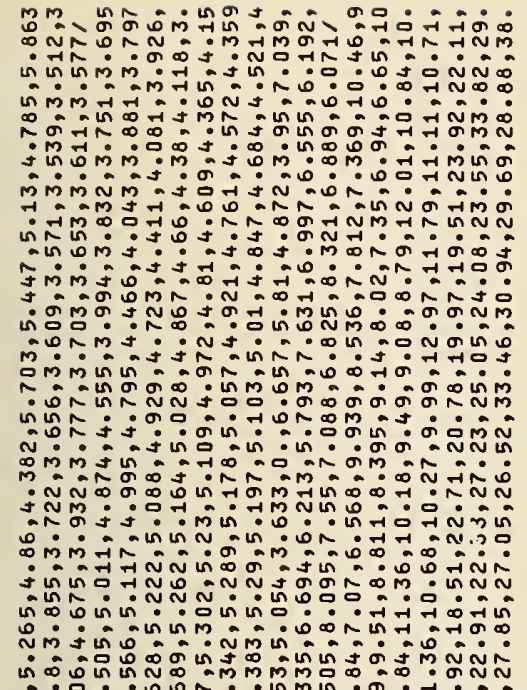

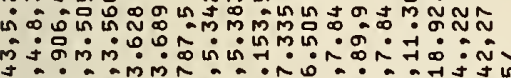

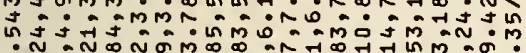

舟品

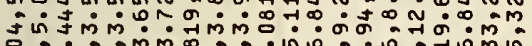

D N

मं

ó

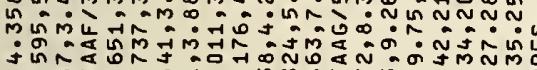

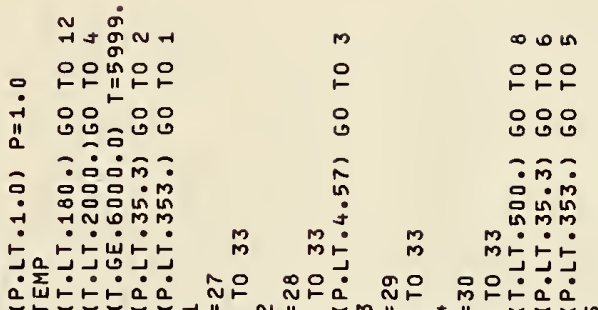

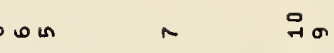

안아 아안

응영 웅응

숭.

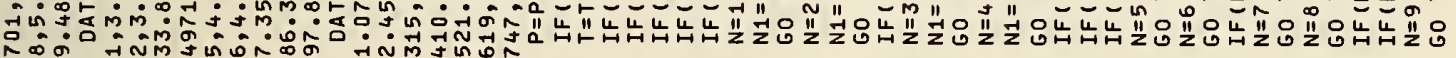

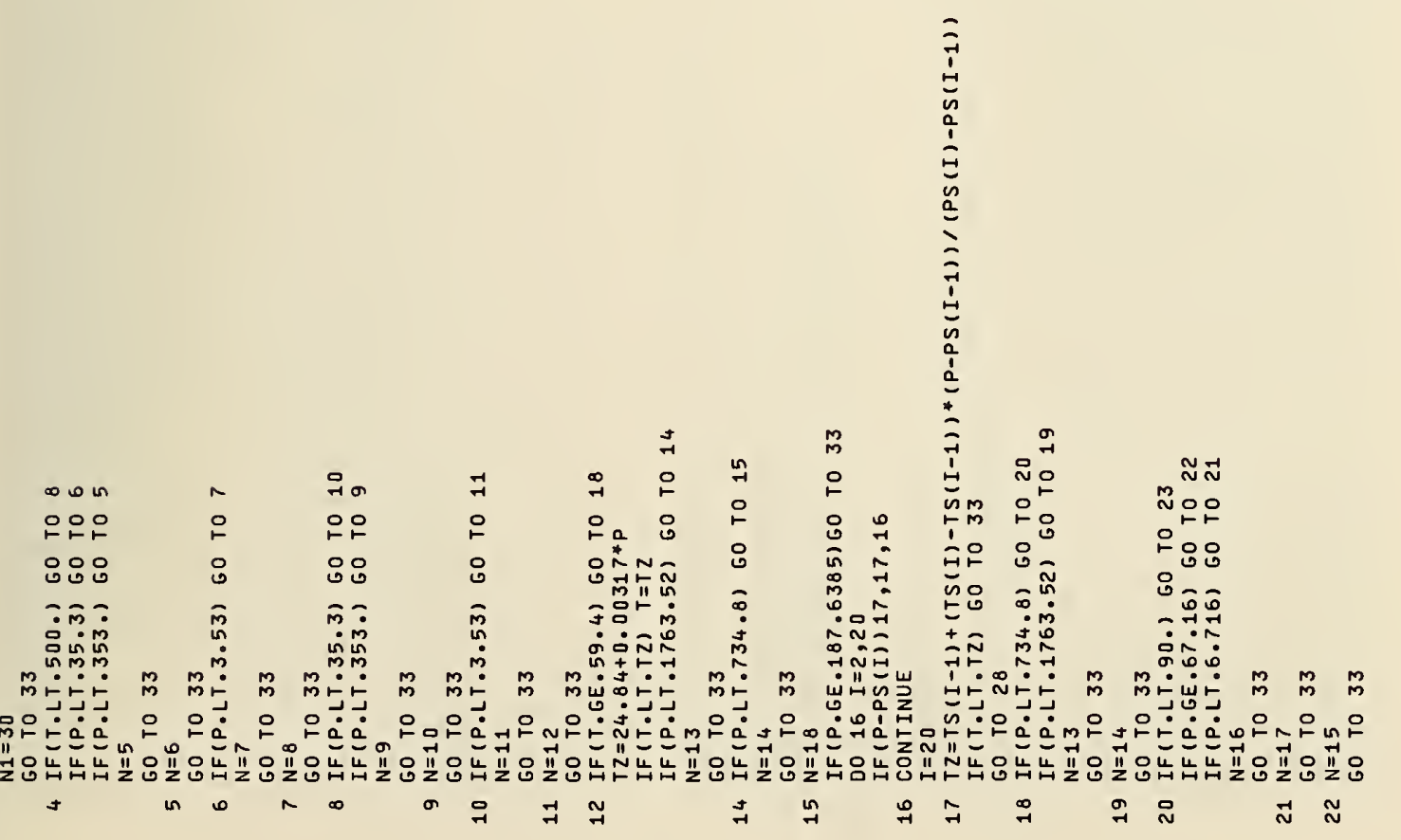




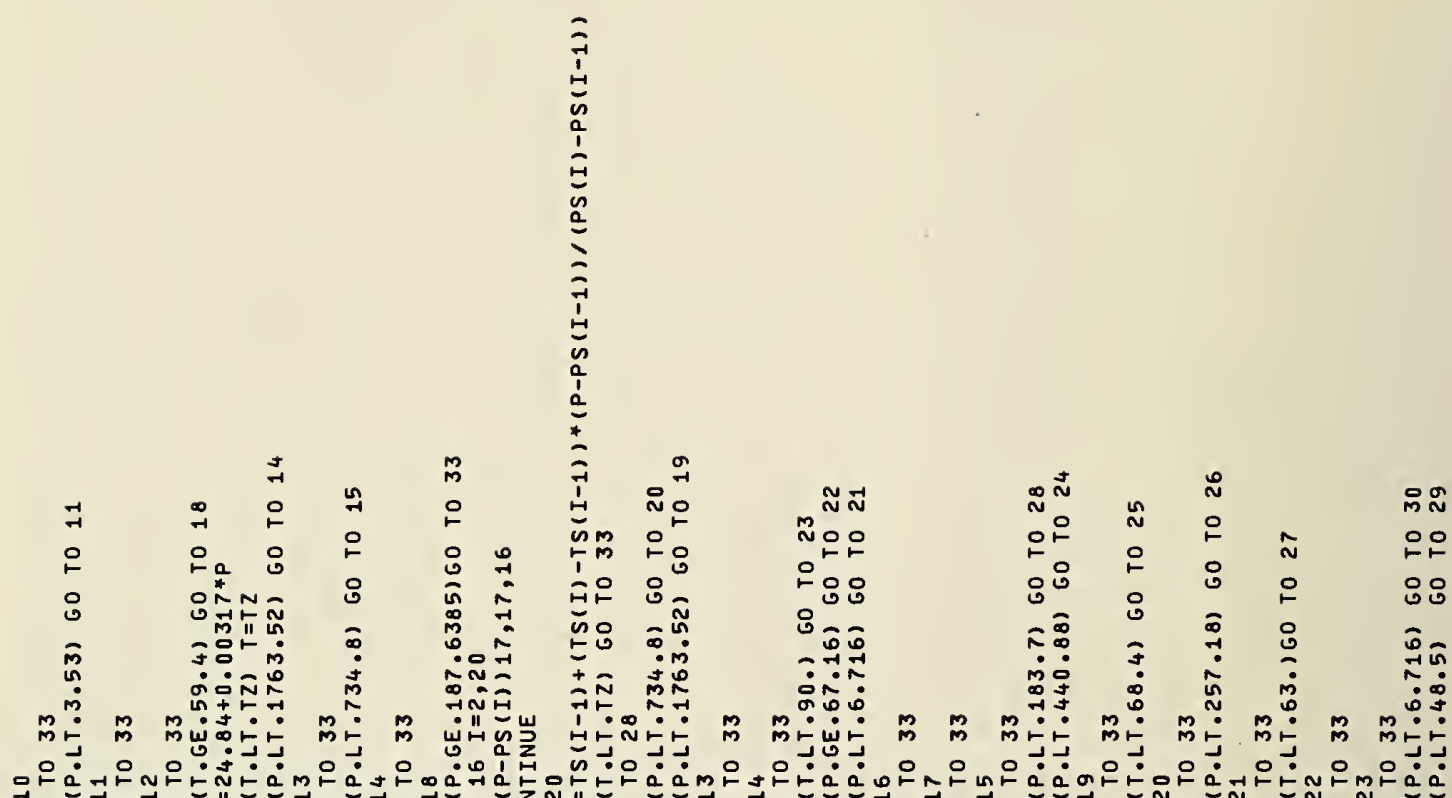

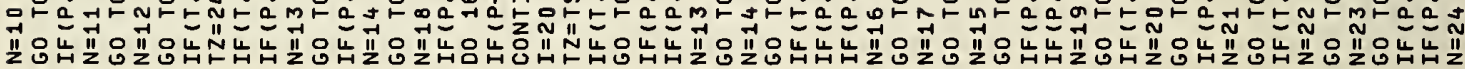
๑

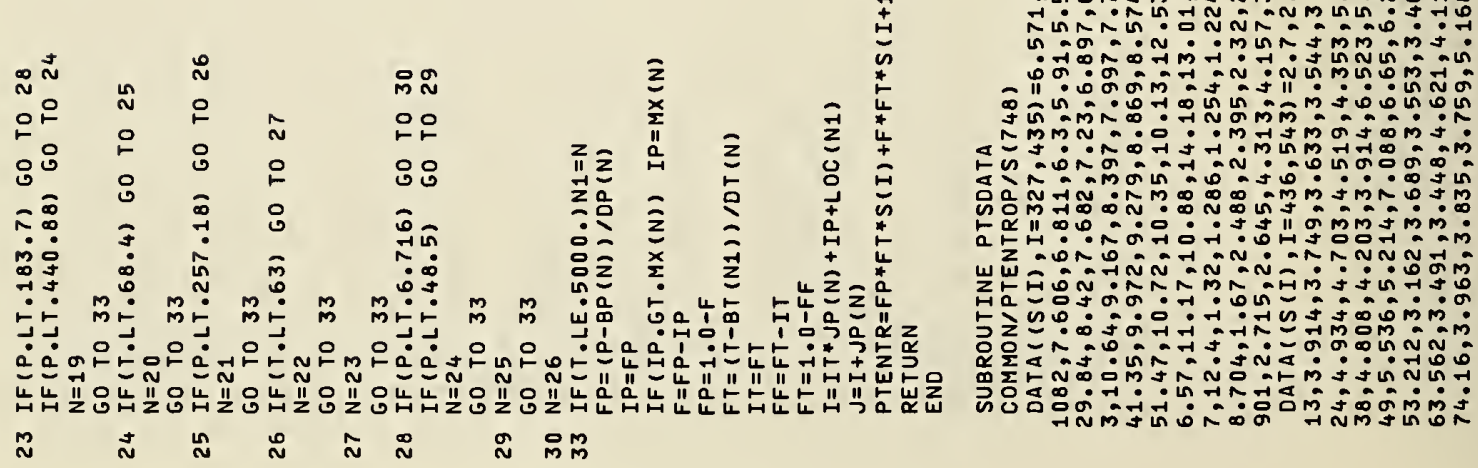




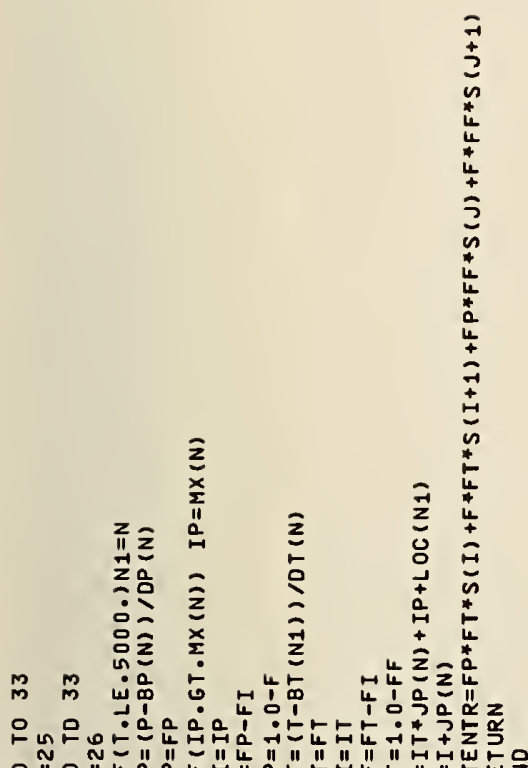

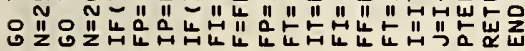
命 品

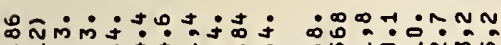

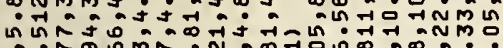

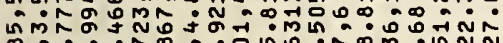
Domm

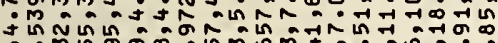
m. -10 

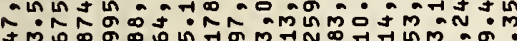

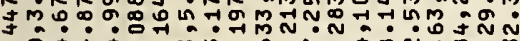
กำ

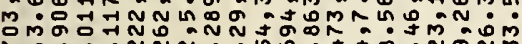

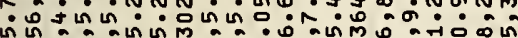

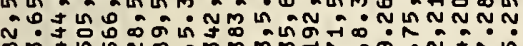
D.J TNmm món ○. 赫

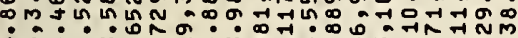

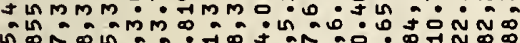
กิ๊ NM⿻ ก๊णn

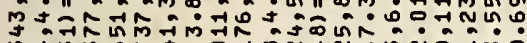

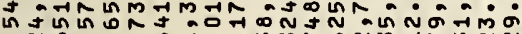

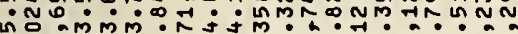
于n

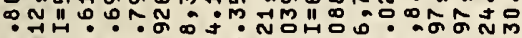
कि

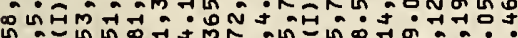

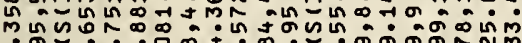
in Ann

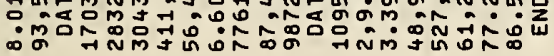

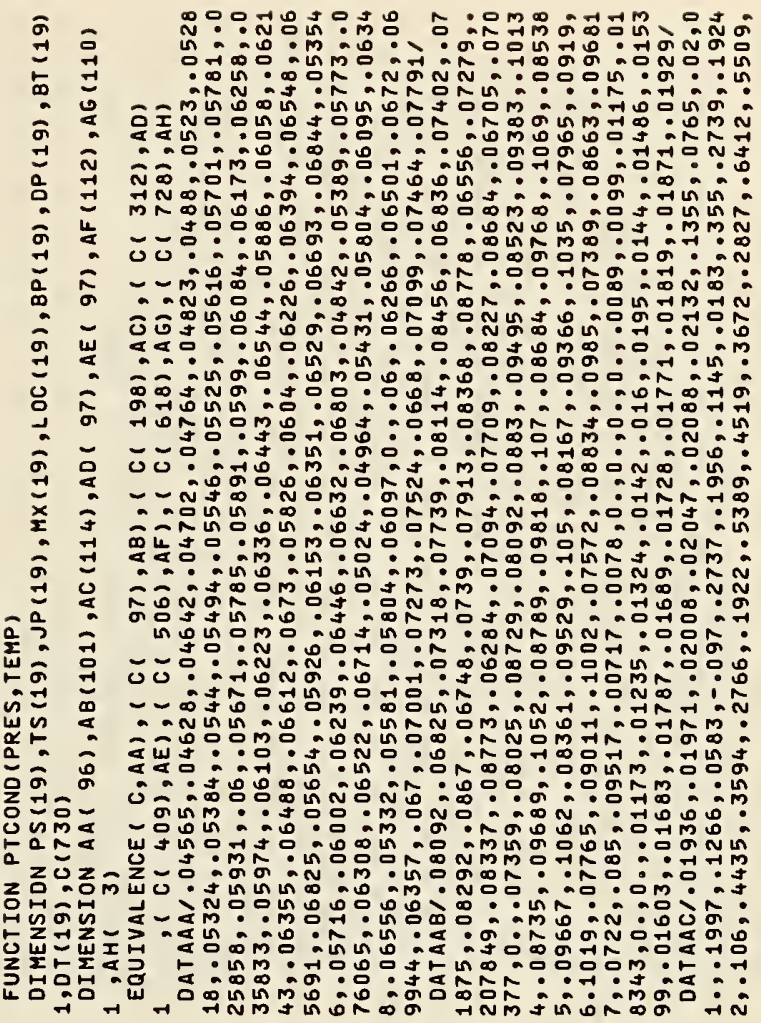




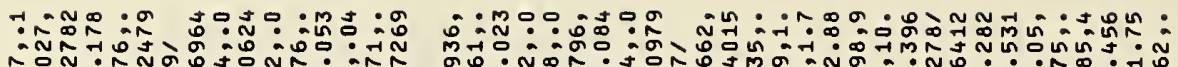

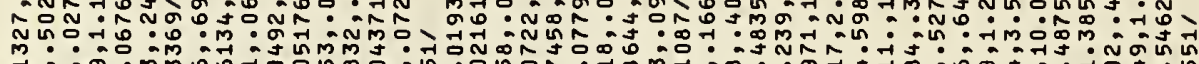
M

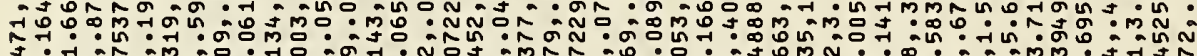

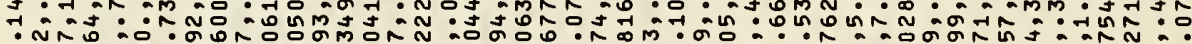

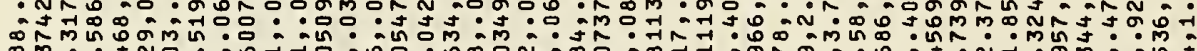

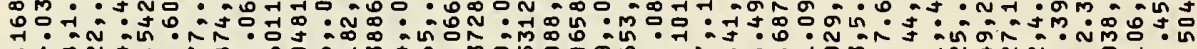
min N N

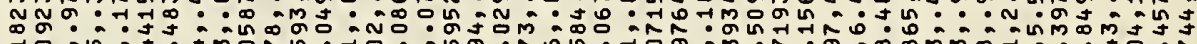
: ơ

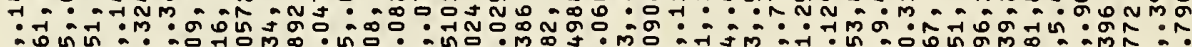

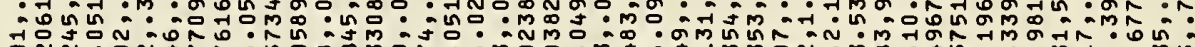

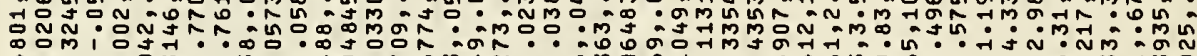
mproñ

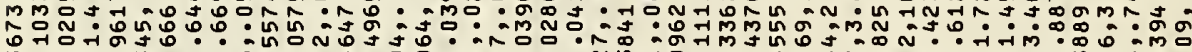

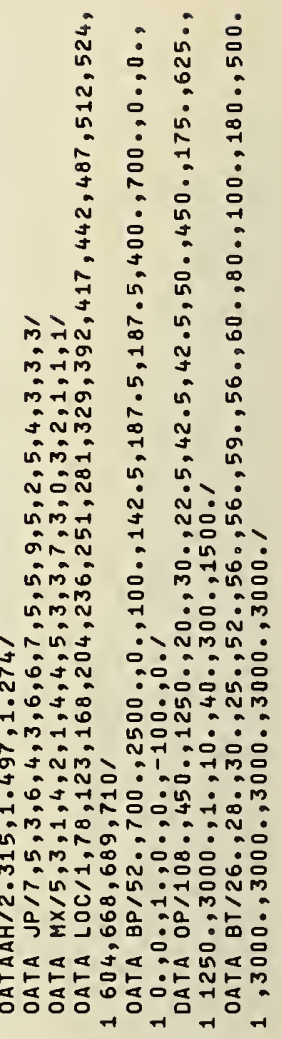

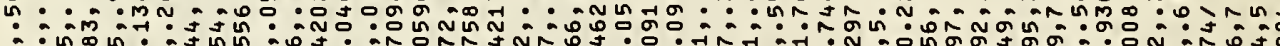

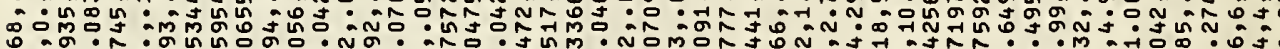
ถึำ Иิ

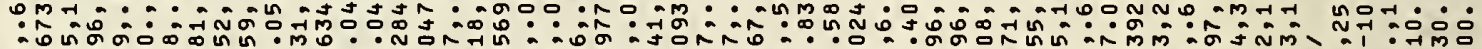
moror

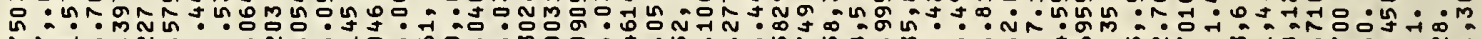
วón

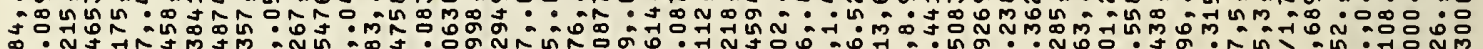
m.

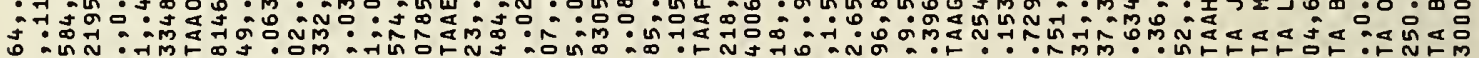

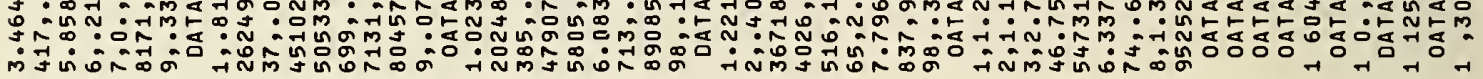

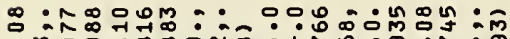
:mo

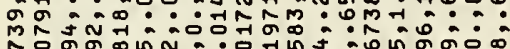
ผิ์ตัง

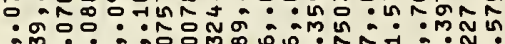
क

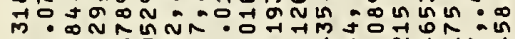
कि ง N

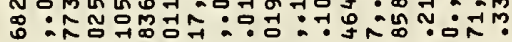

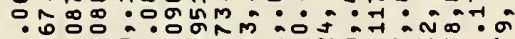
Nั:

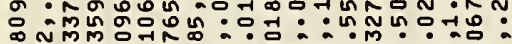
: : Noำ 范:

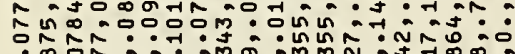

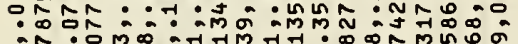

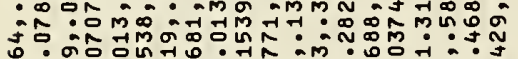

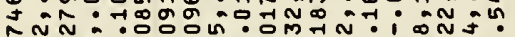

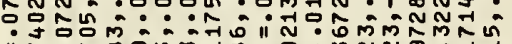

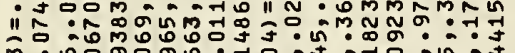

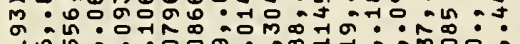

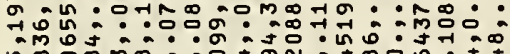

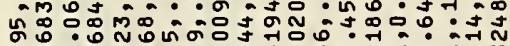
IIํ.

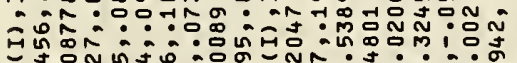
न

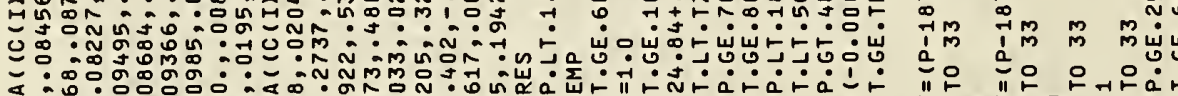

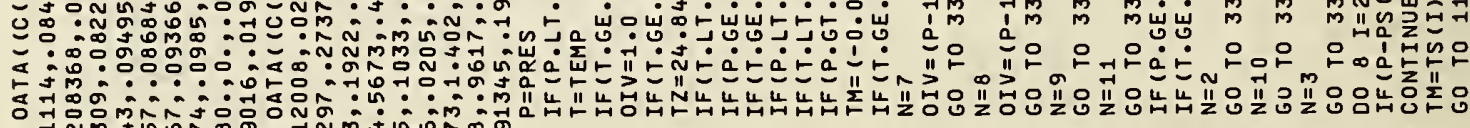



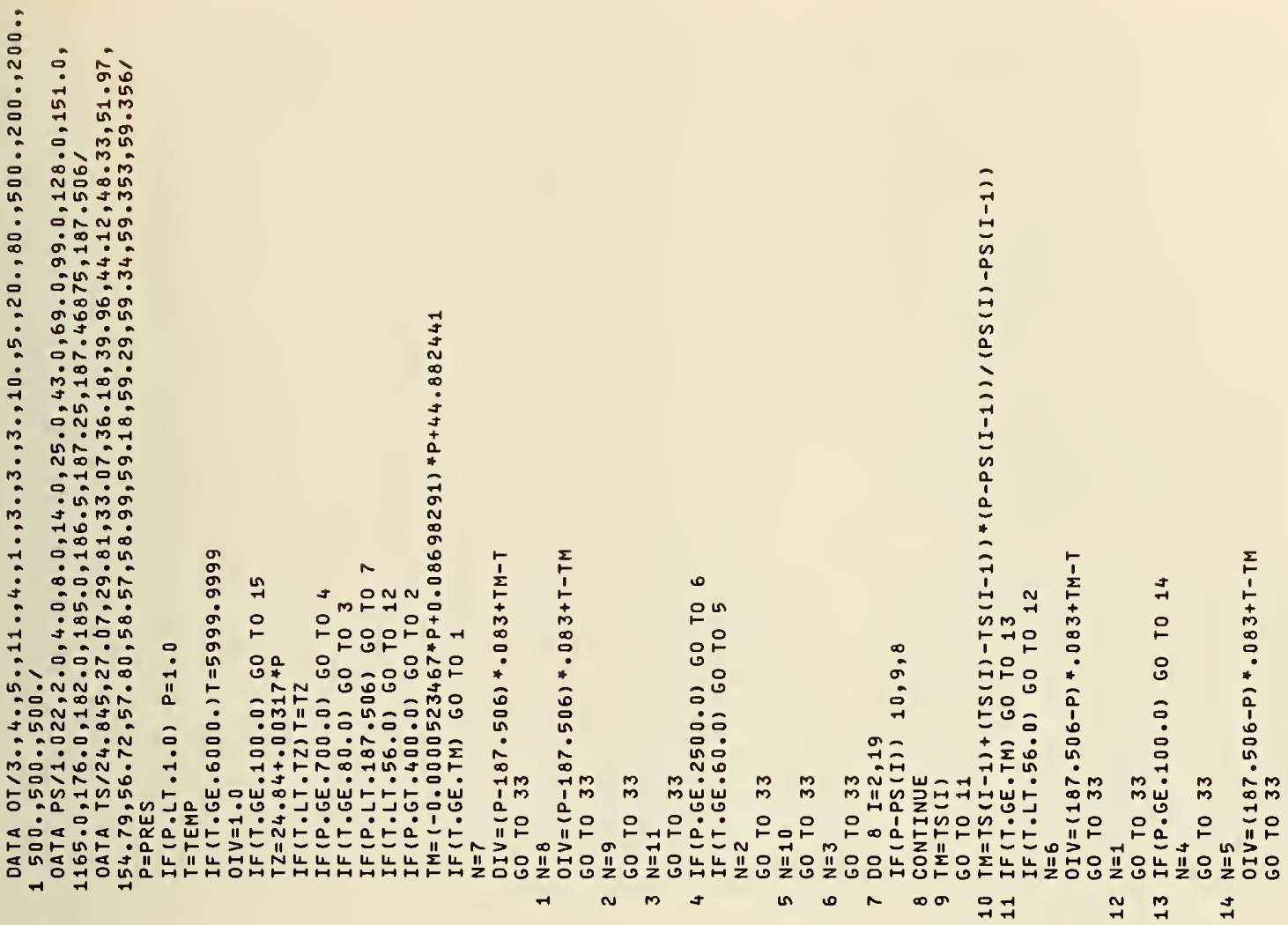

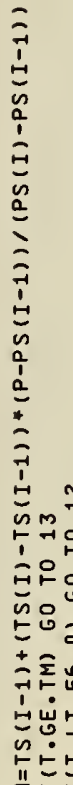

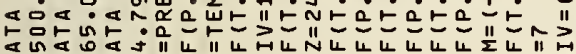

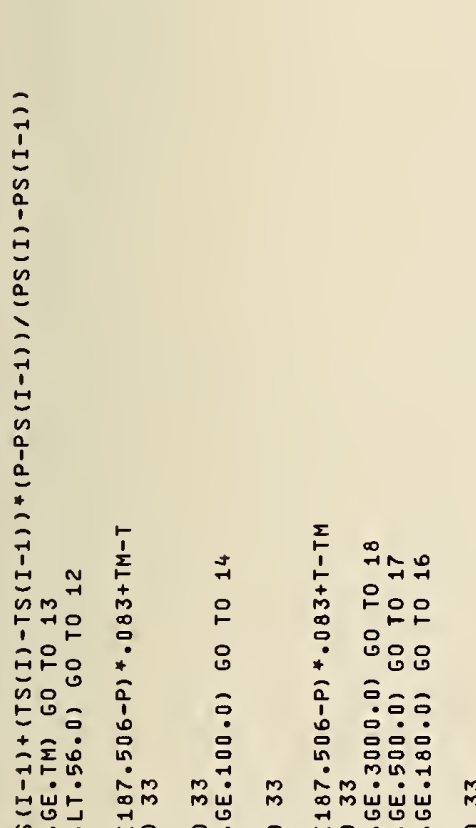

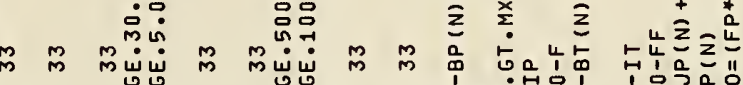

$\infty \stackrel{n}{\infty}: 0$

$\because: 0 \ln 10$ की mos is: लैด: N: :

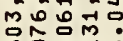
แn: 绍:

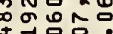
: เก : $: 0$ -

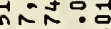
路品品 : : : 于做 mo $0: \operatorname{R}$ or

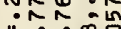

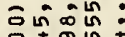

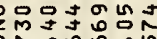
응 앙ำ 政: : : : In 代品和品?

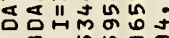

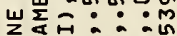

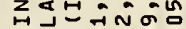

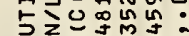

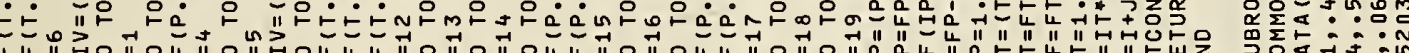

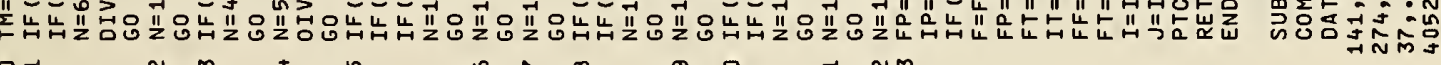




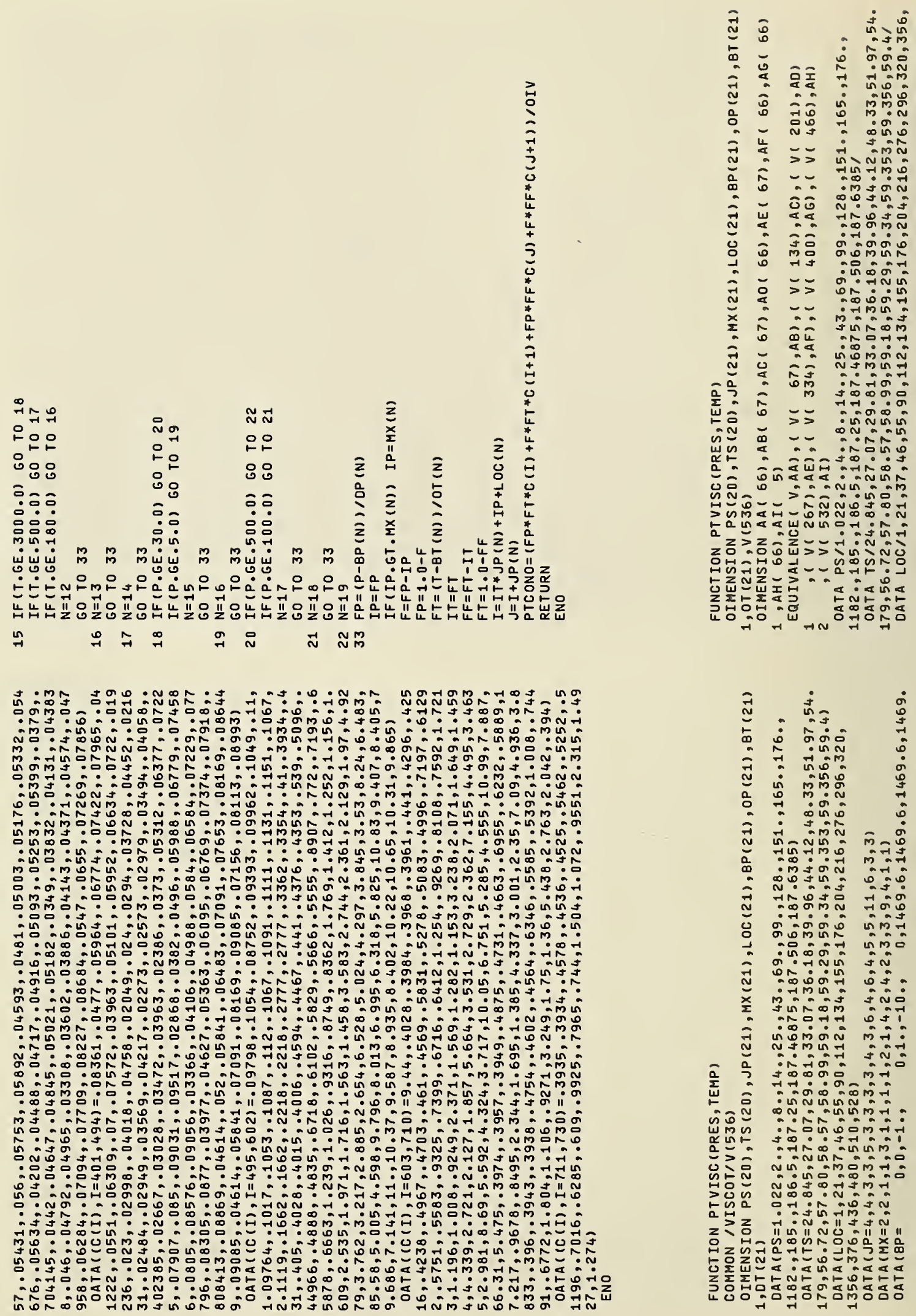




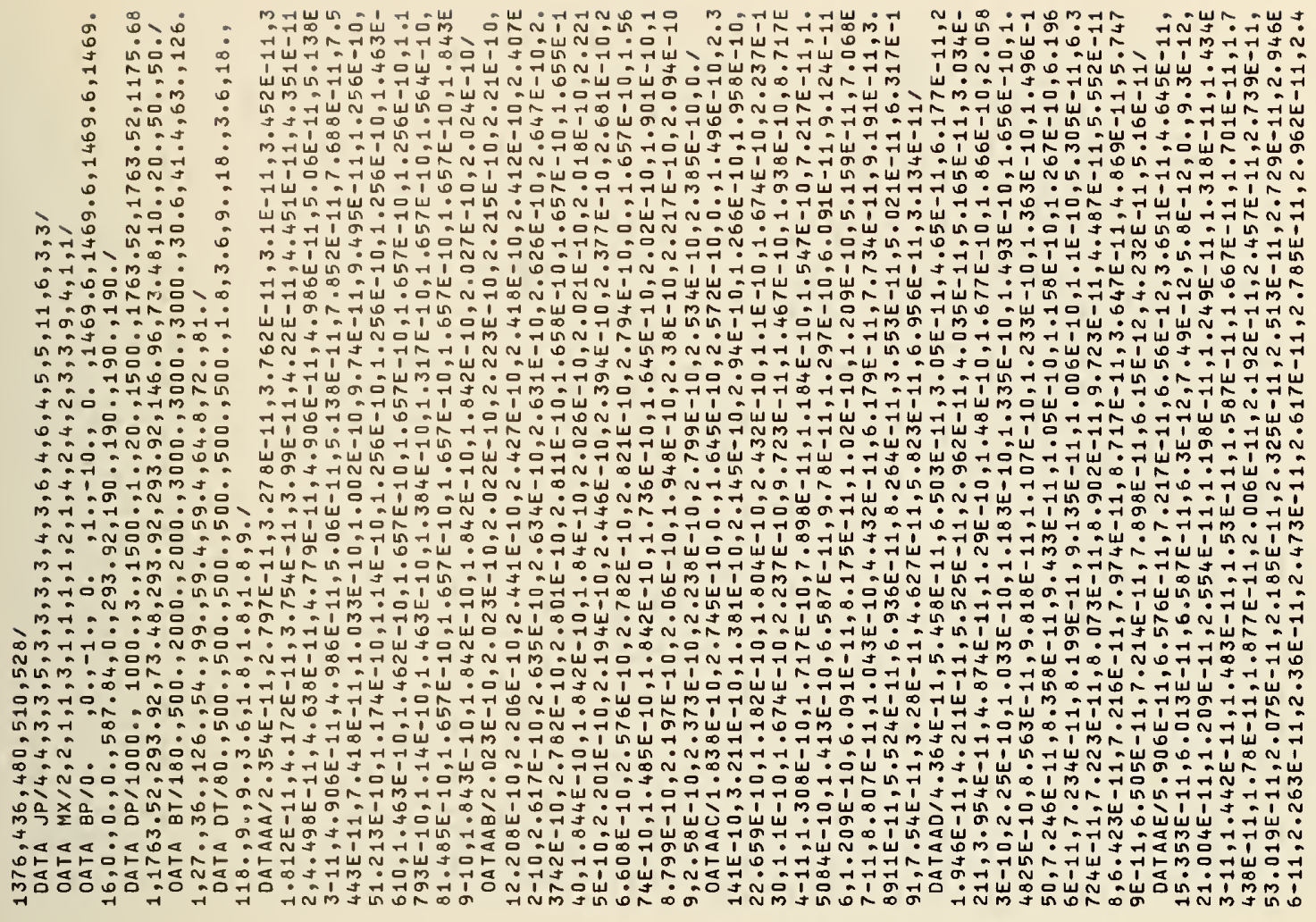

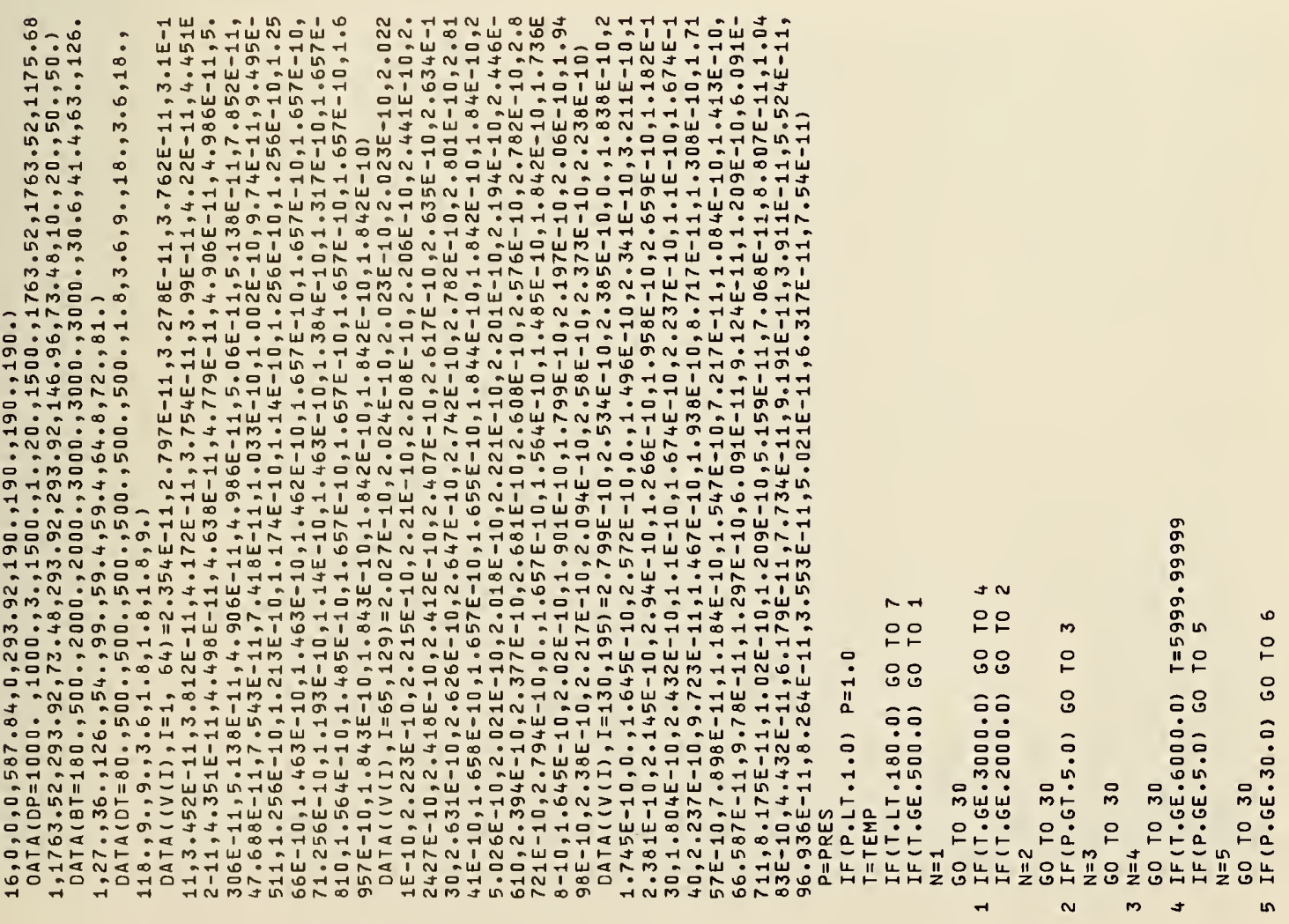



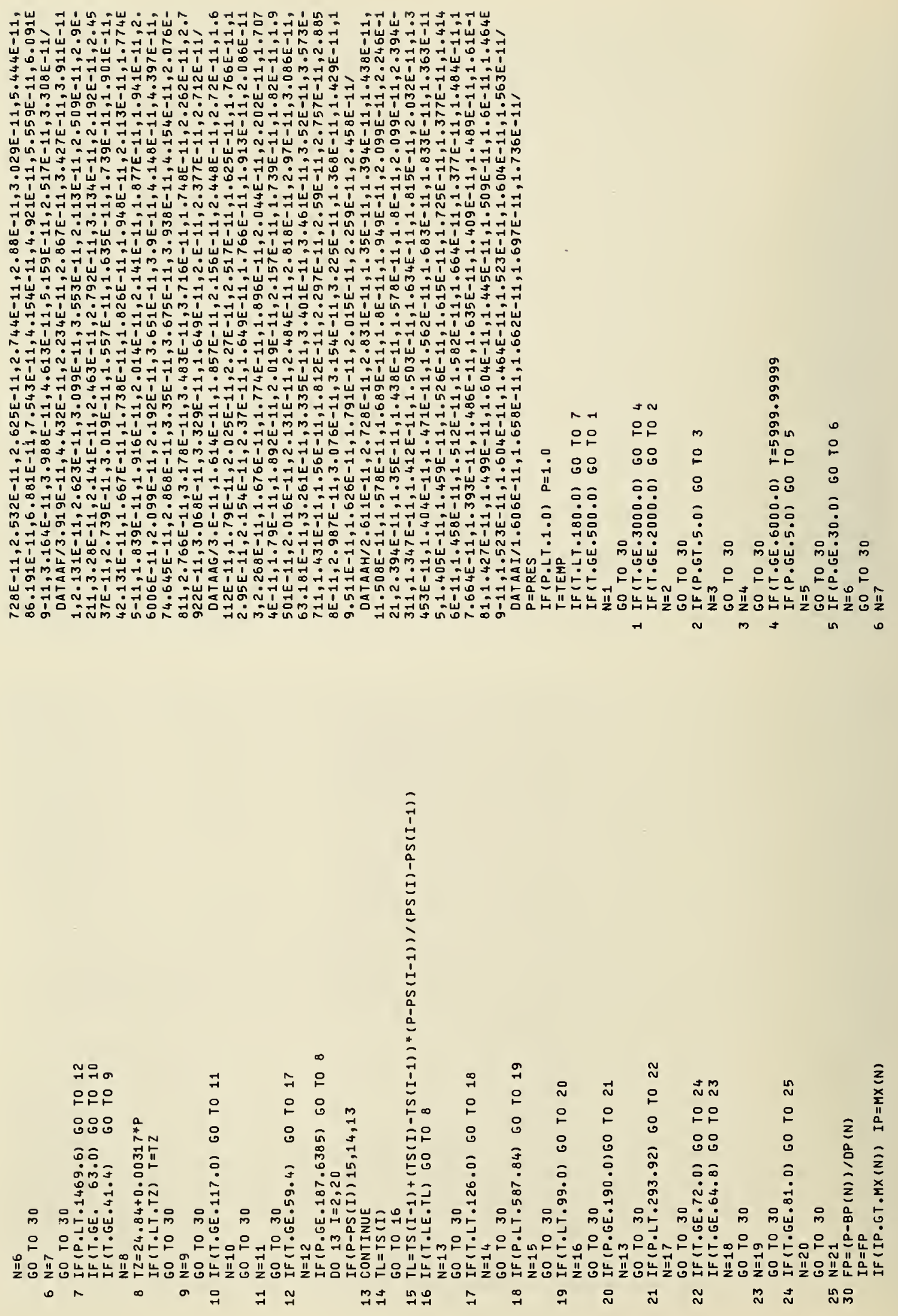


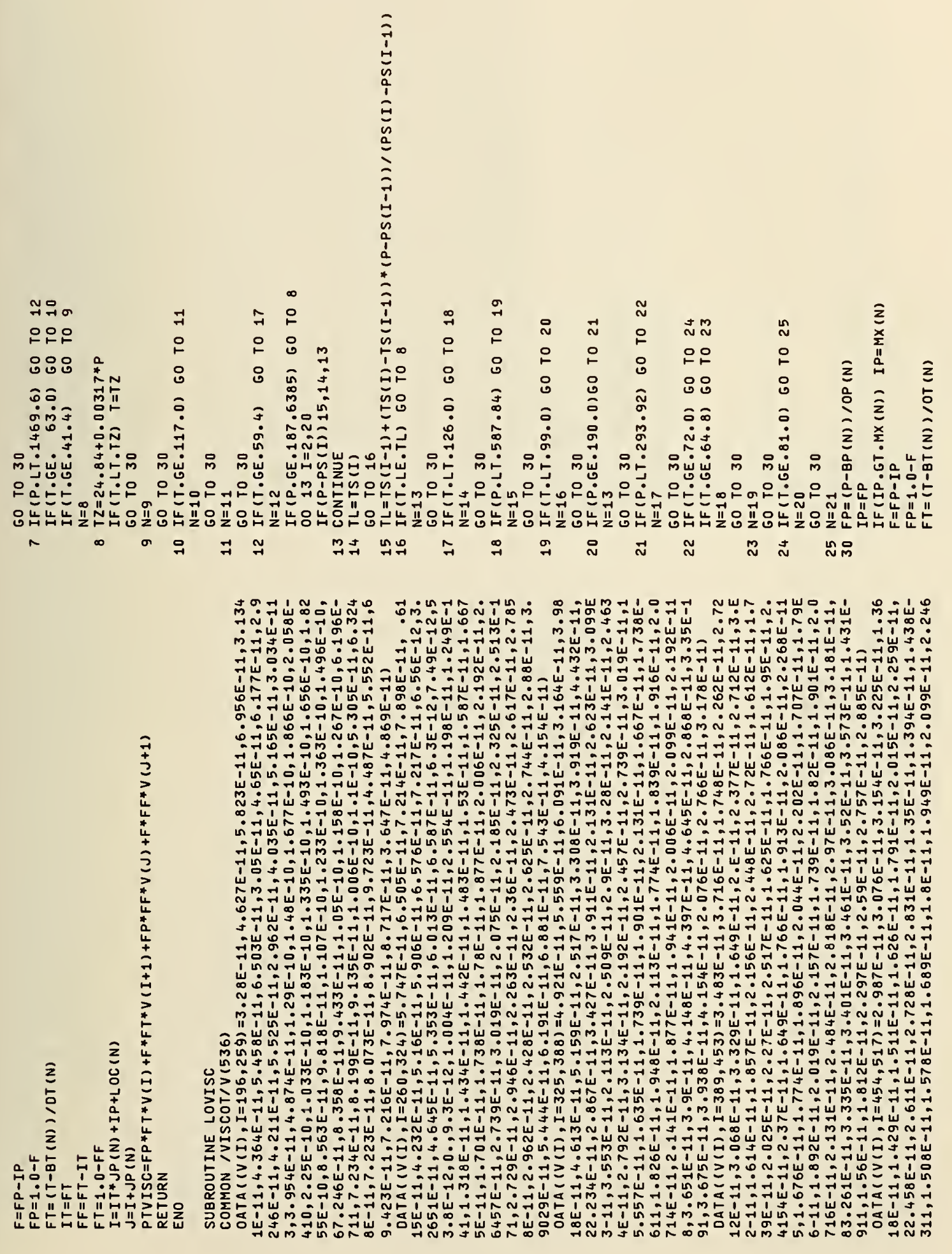



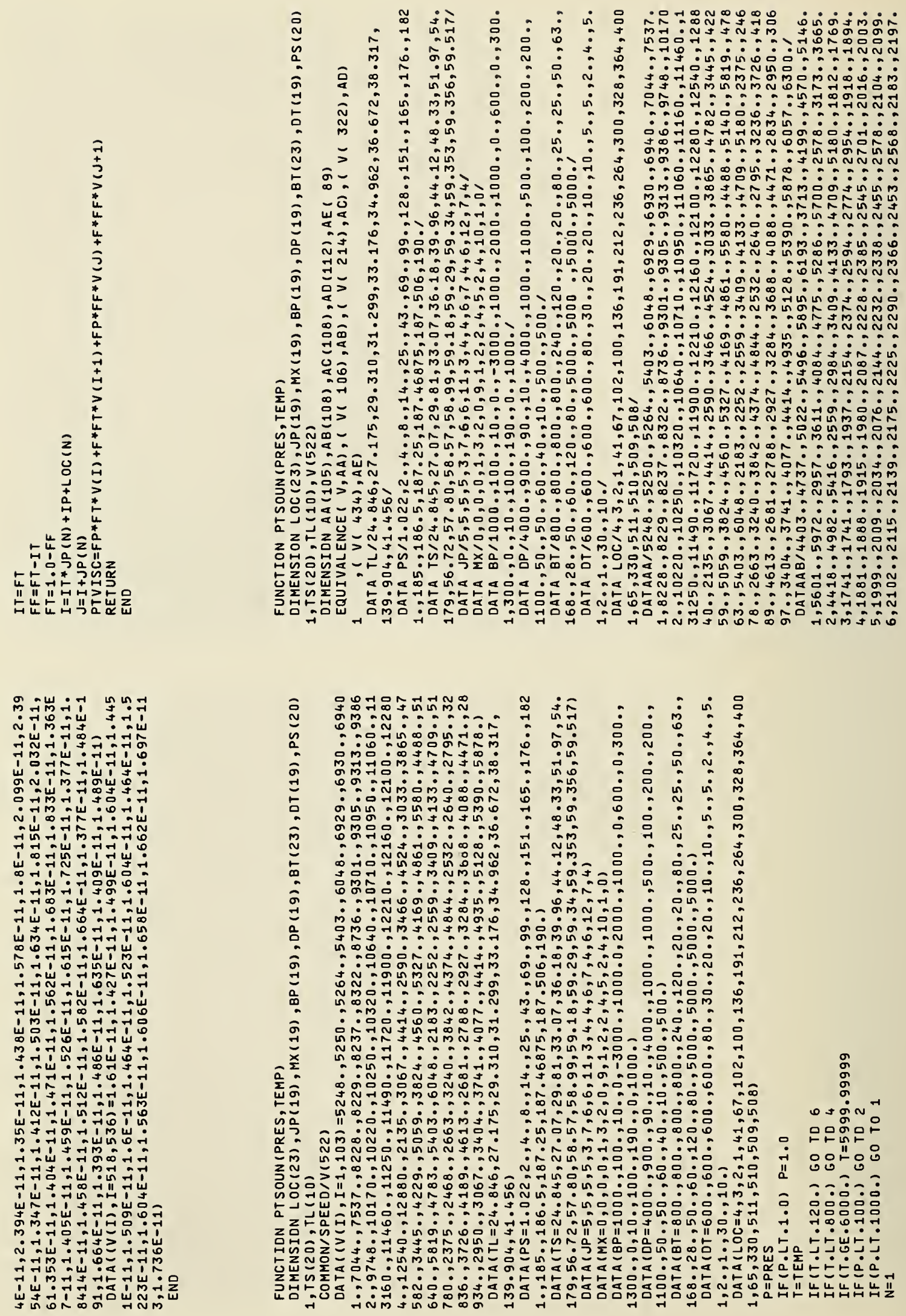


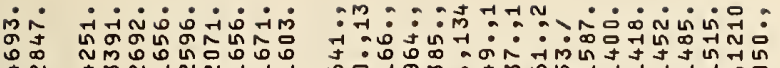

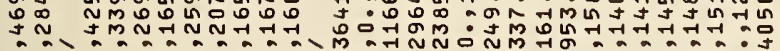

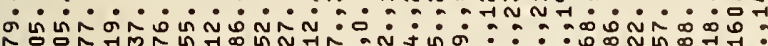

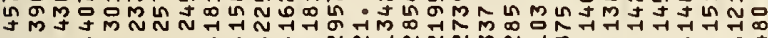
: : : : : : : : : : N N N $~ N N$ N N N

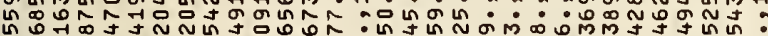
N : $\}$ ómpon A. :

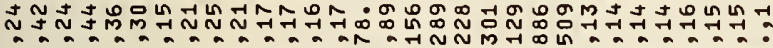

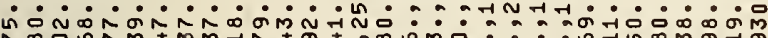

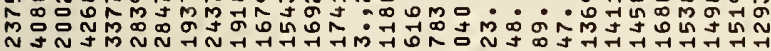

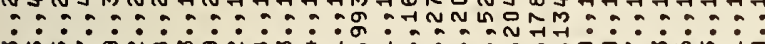
miñoñ

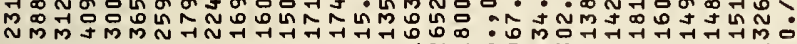
i

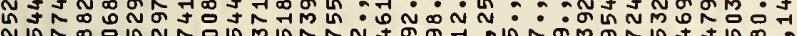

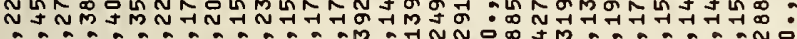
: : : :

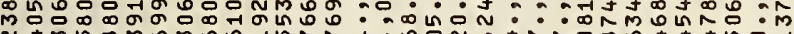

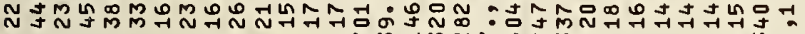

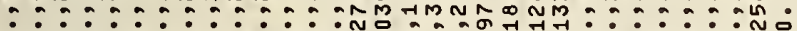
N N $\mathcal{Y}$ Tim

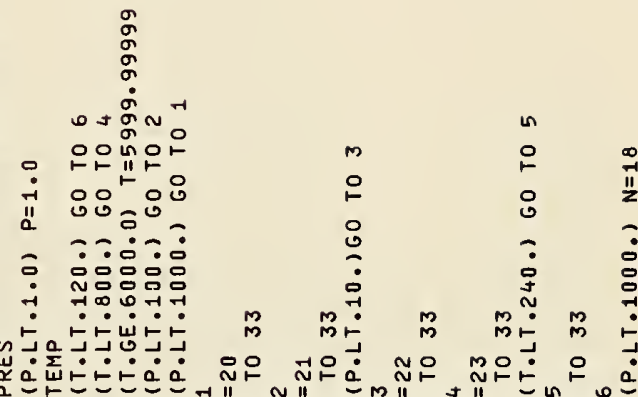

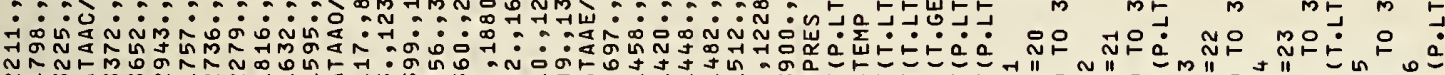
$\underset{N}{N} \mathfrak{N}$

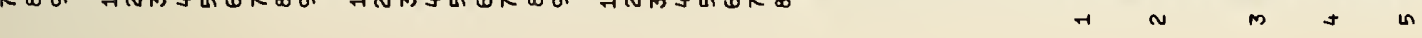

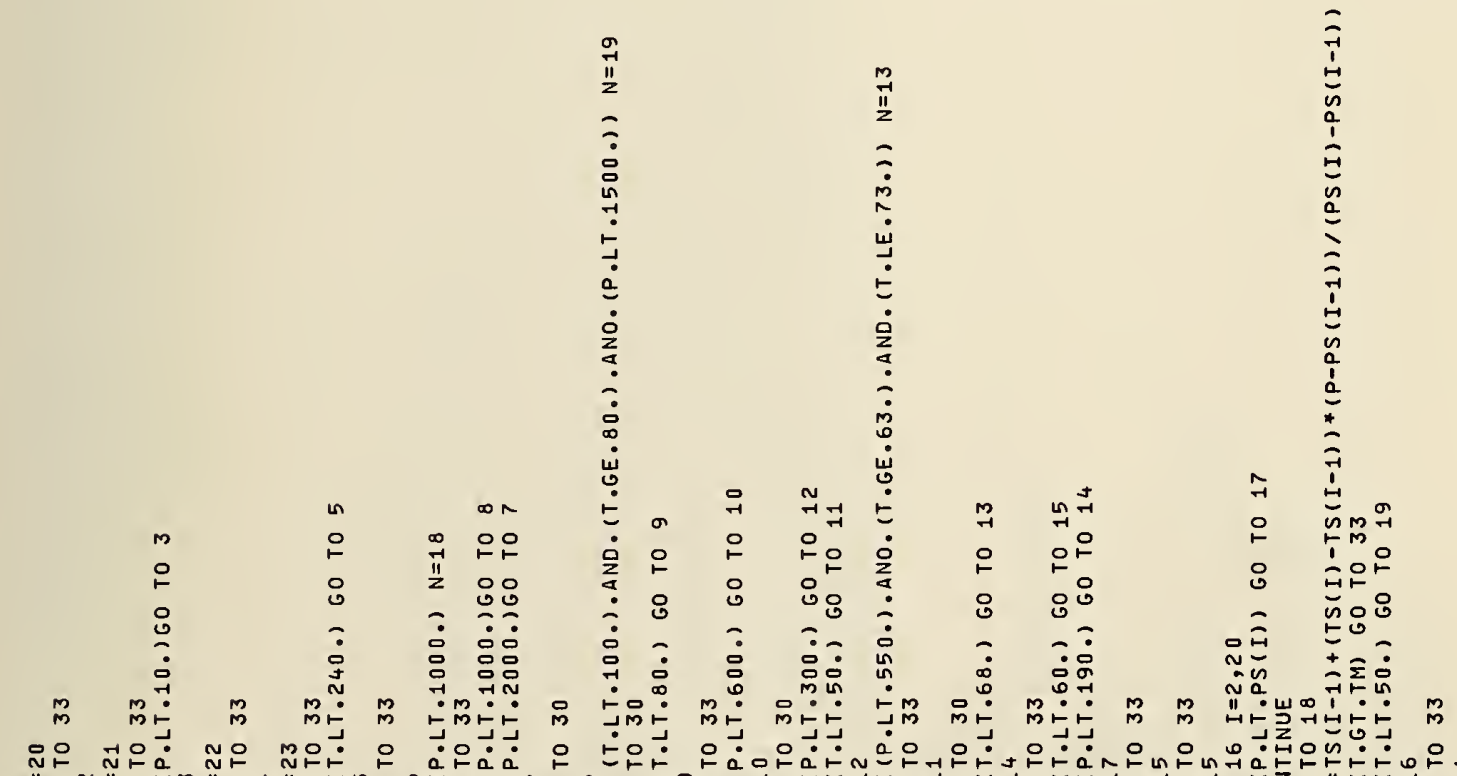

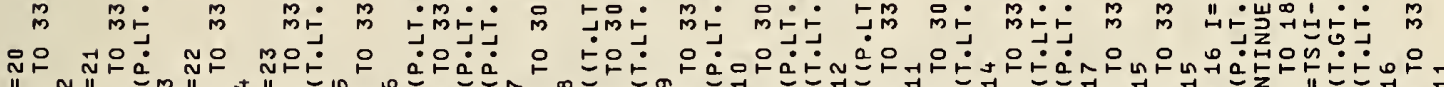

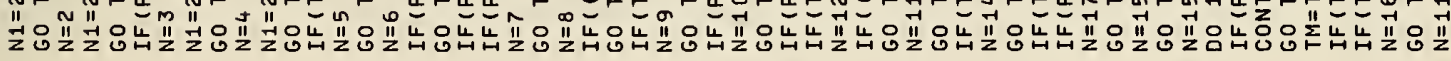
$\rightarrow N$
$m$

t in 0

$\sim \infty$ or

r $\quad \sim$

m

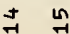

$\stackrel{7}{\rightarrow} \stackrel{\infty}{\rightarrow}$ 

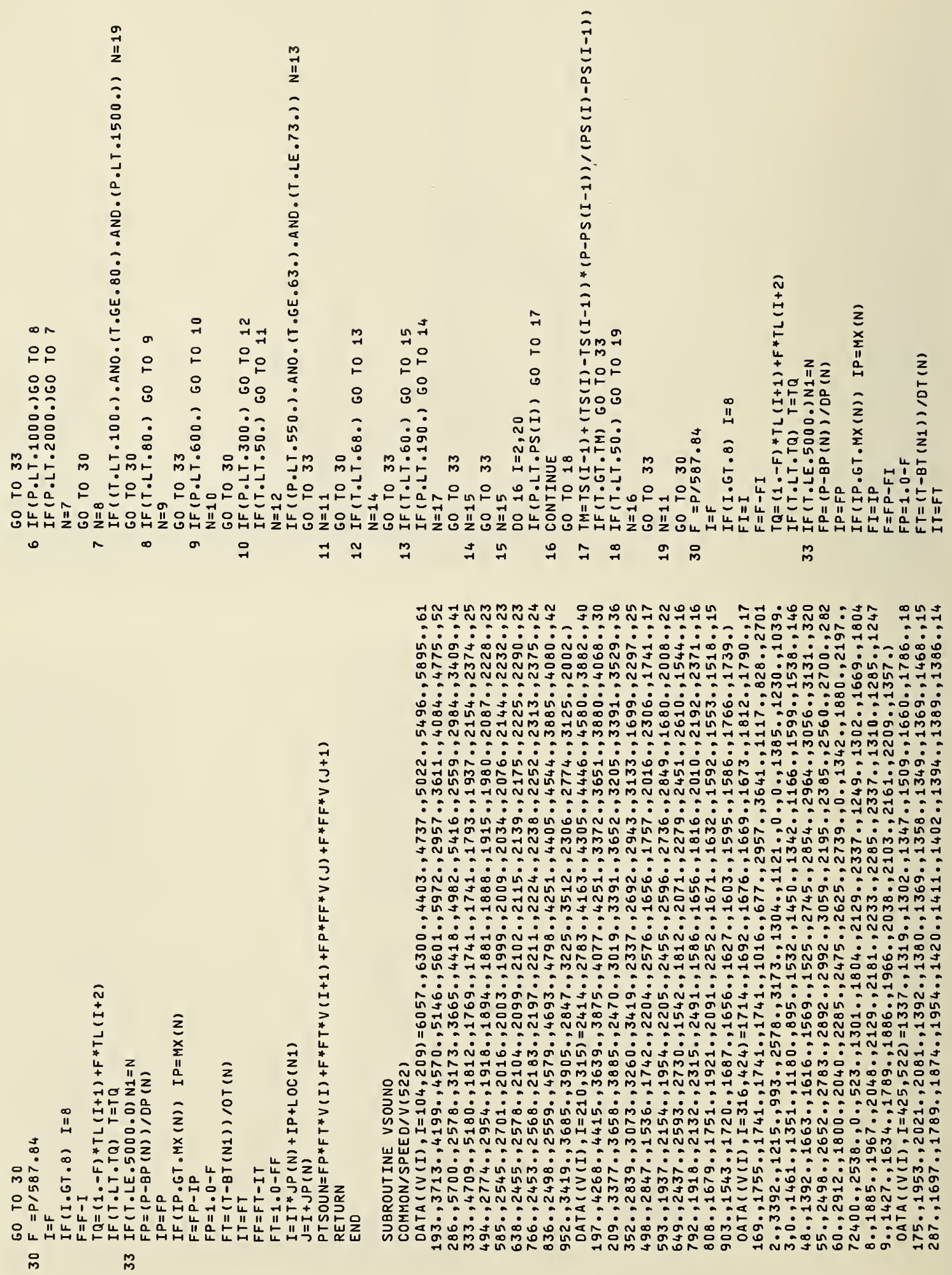


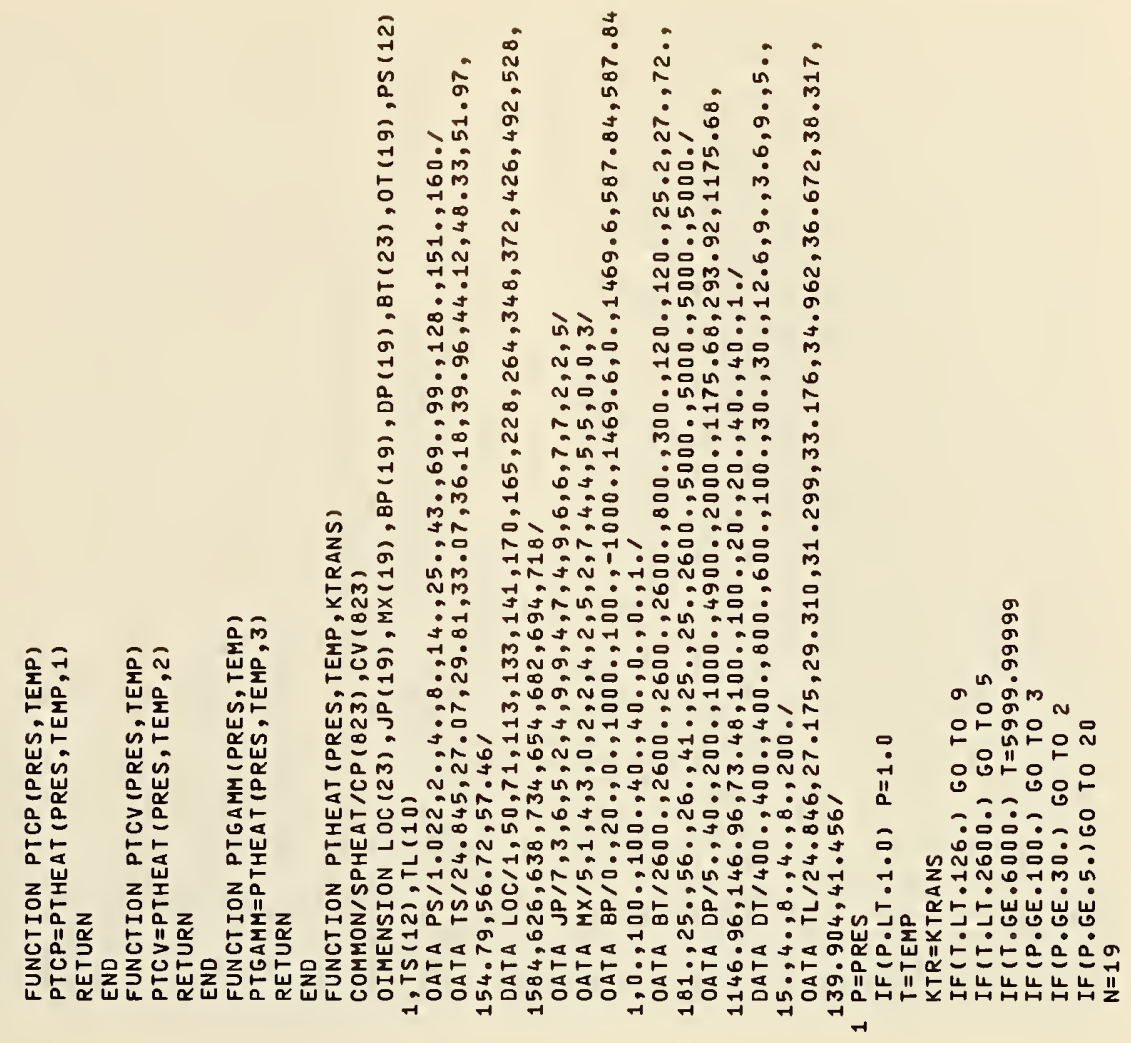

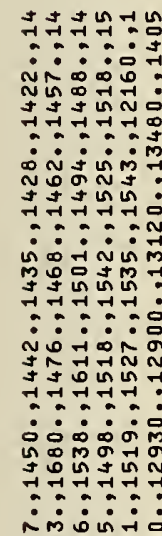

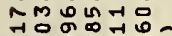

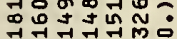

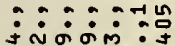

NMỡ

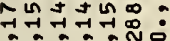

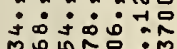

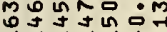

$\because 7 y$.

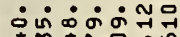

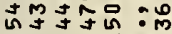

$\because 7 \div 7 \div$

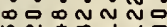

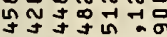

근

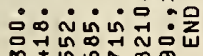

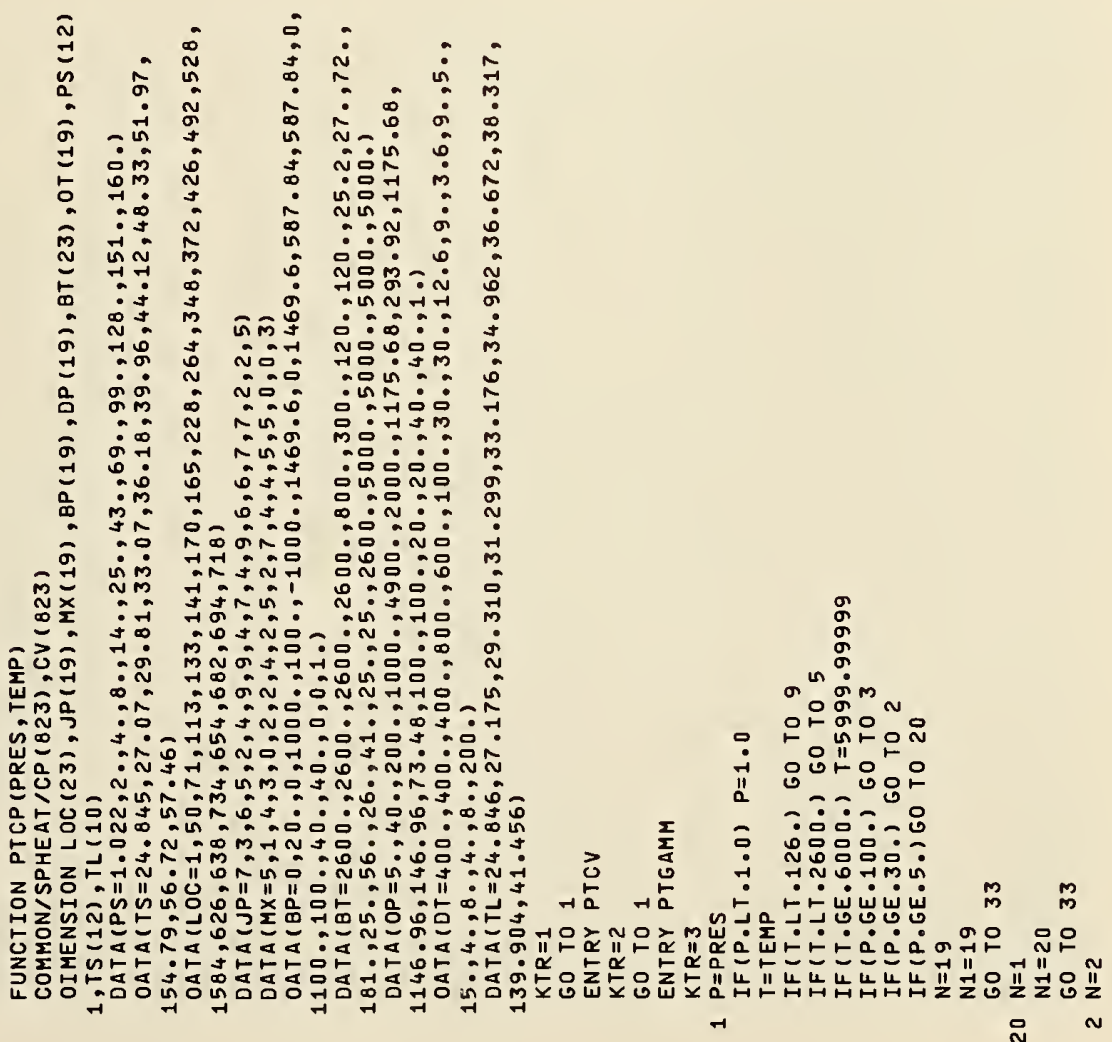



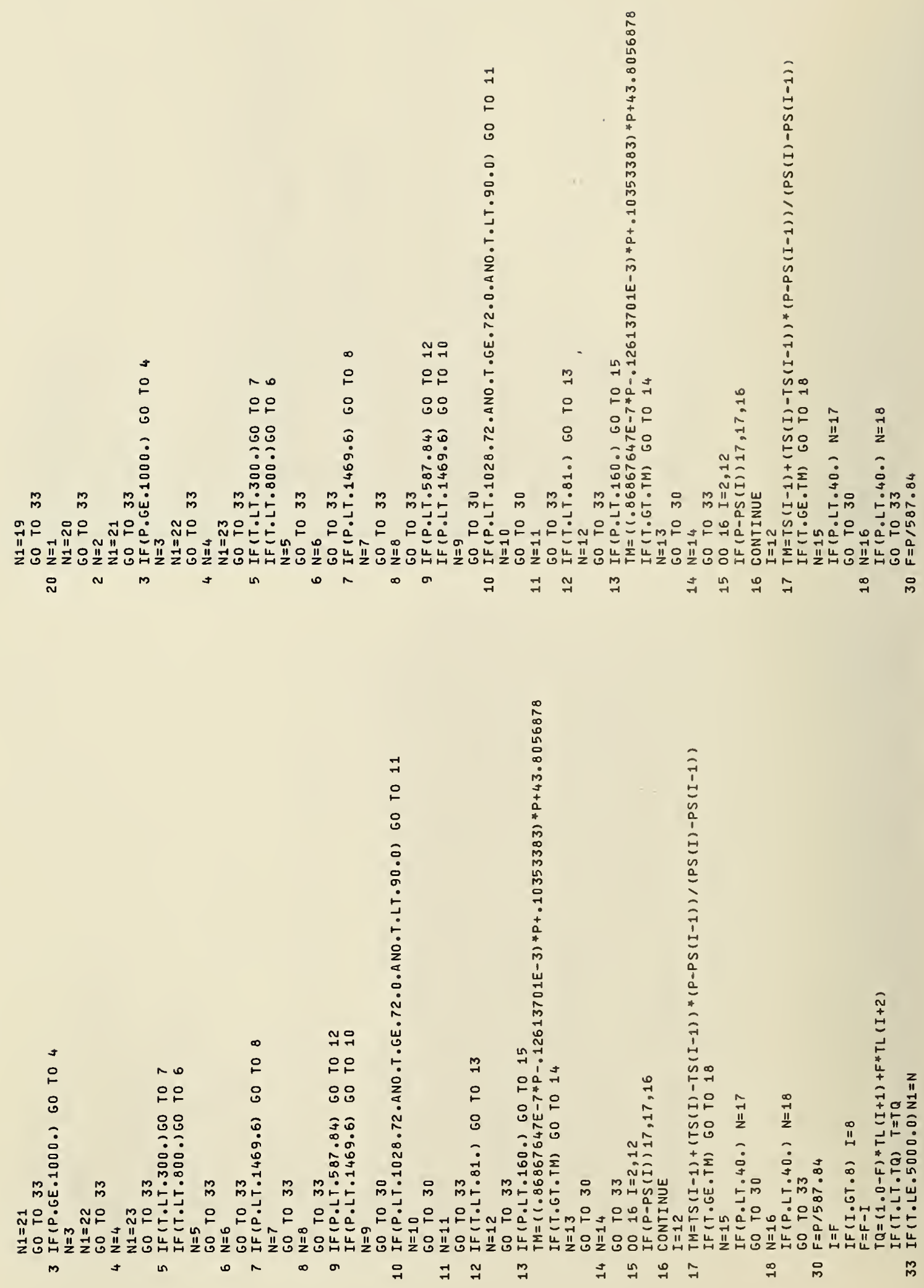


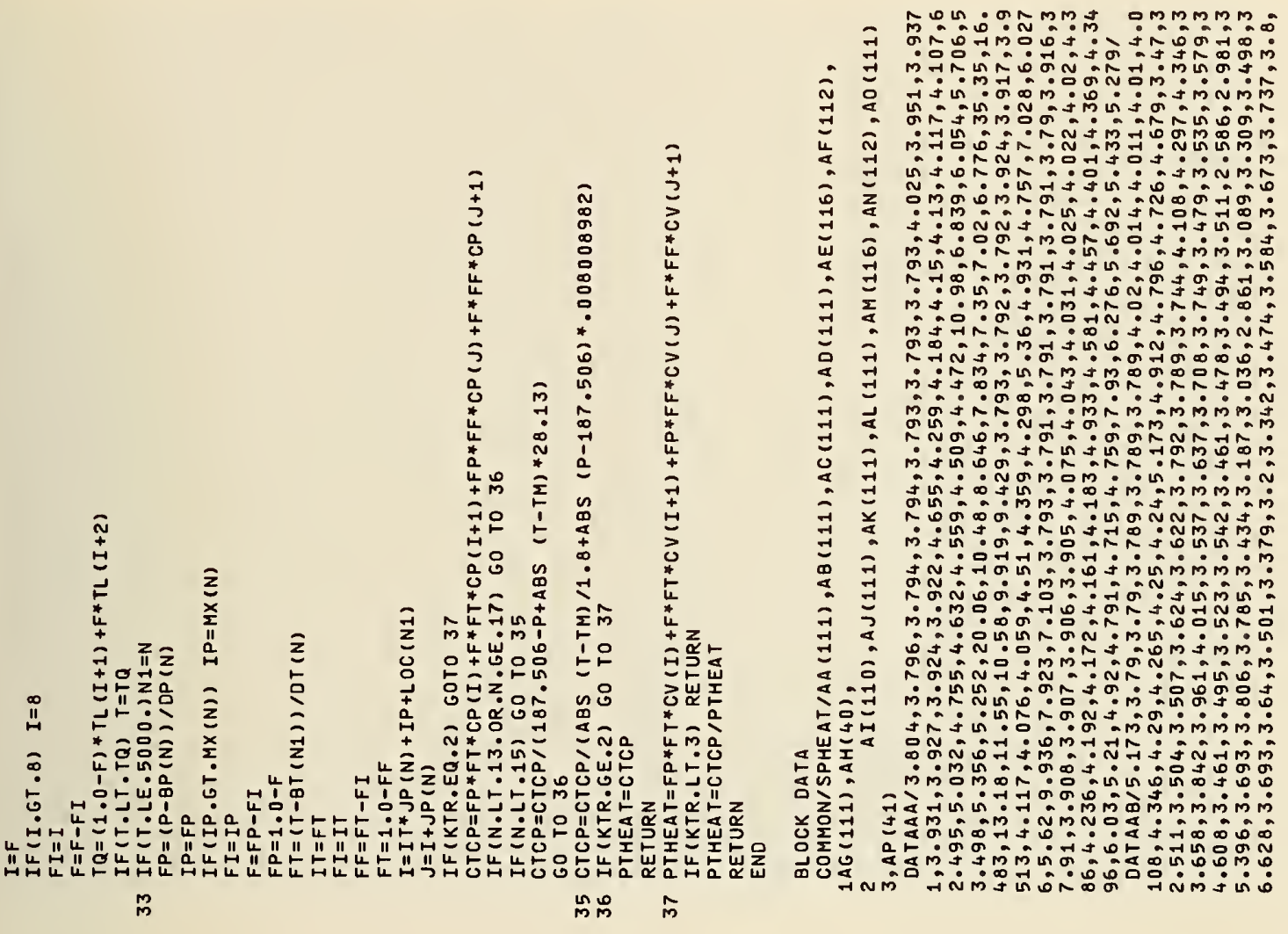

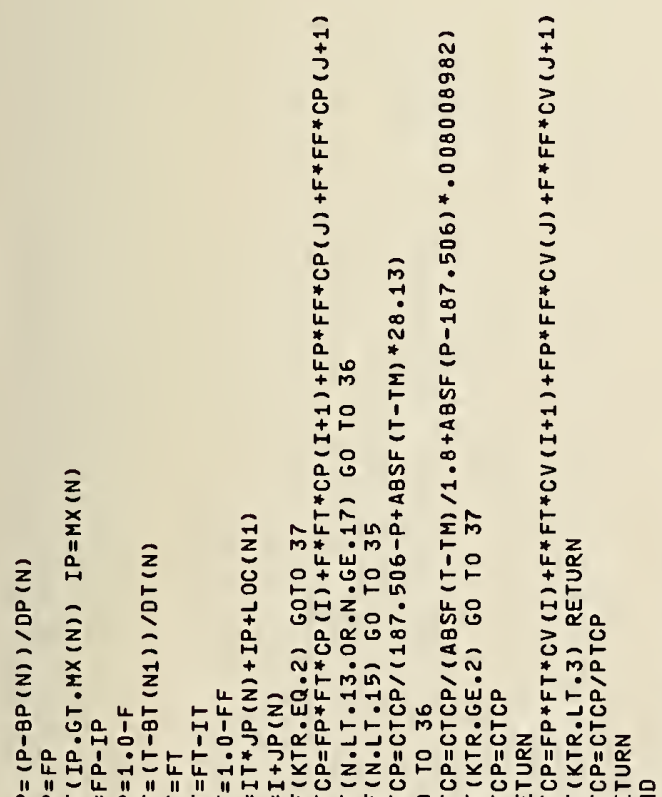

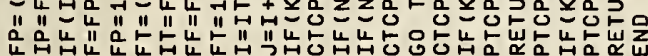

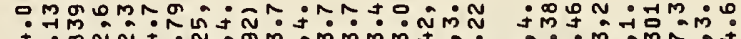

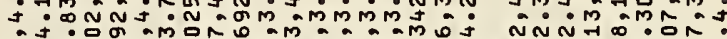
Mڤ

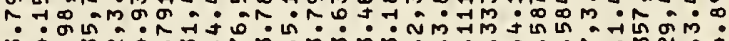

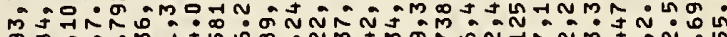
ơ

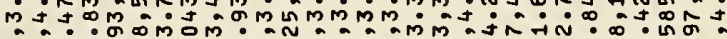
mo \%ू̆

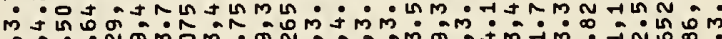

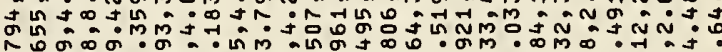

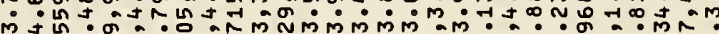

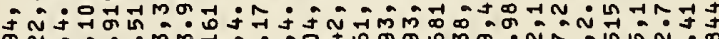
FN

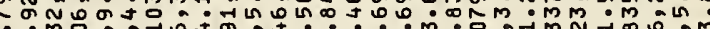

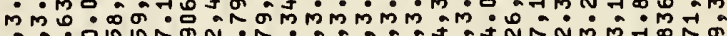
O O

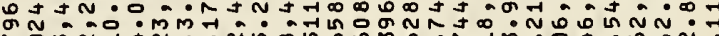
oñ

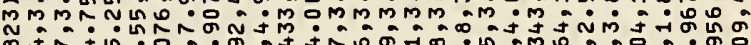
\品

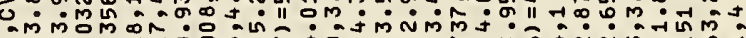
ติ Nog m ڤ仓

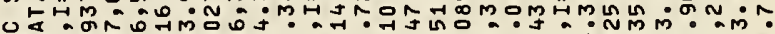

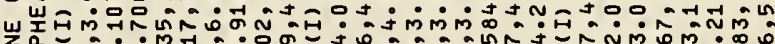
荡

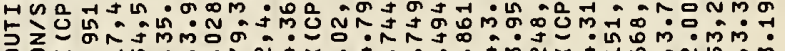
o

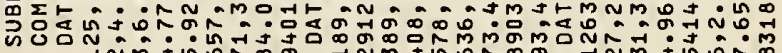




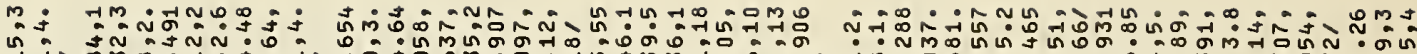

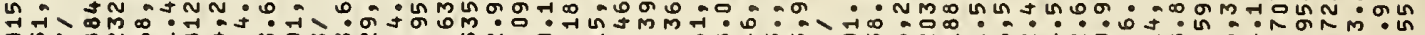

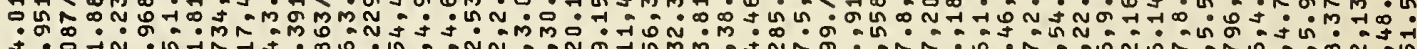
\&M⿻

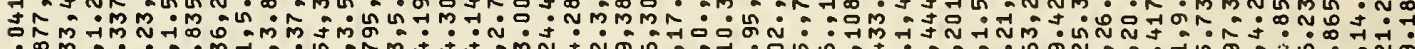

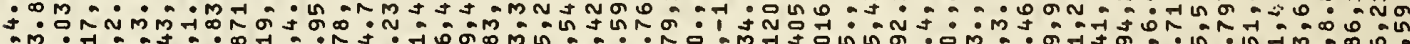
mp. ¿ Tín.

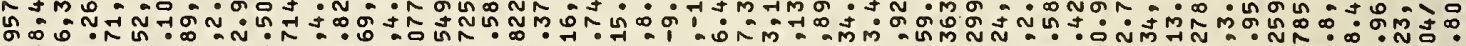

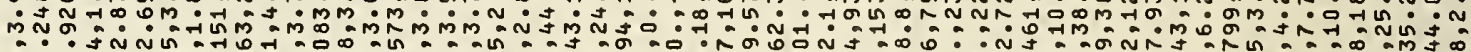
mim G以m

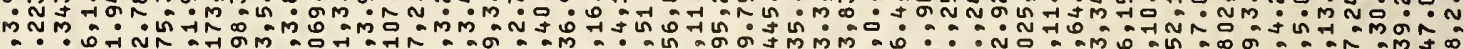
ơ m. 过

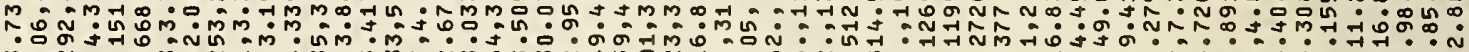

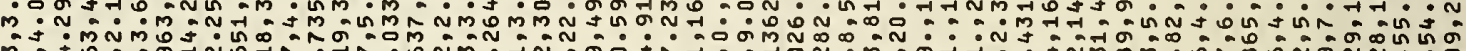
mj:

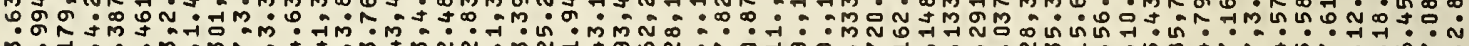
mm・ñ் OM

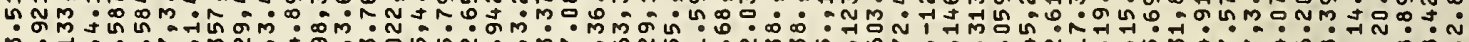
m.ं

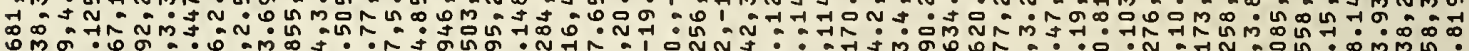

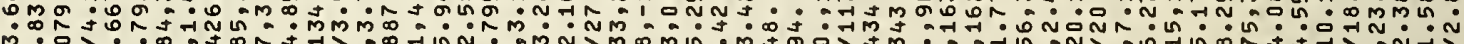

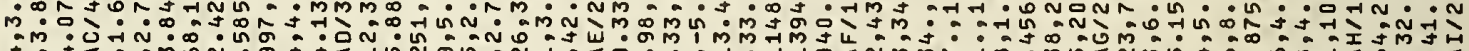
† No

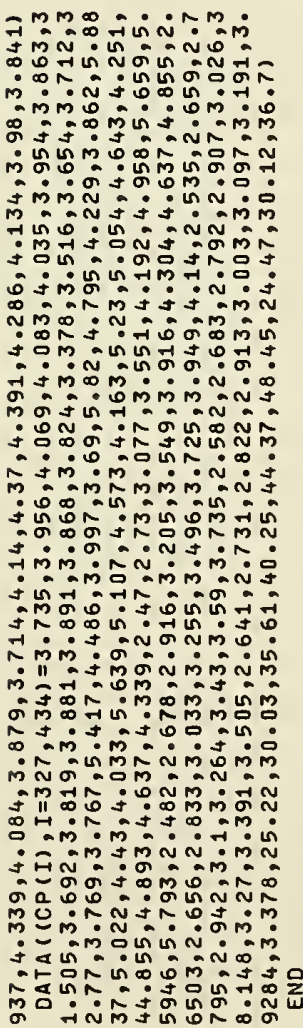

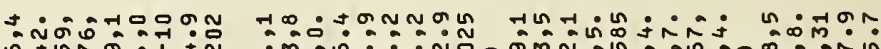

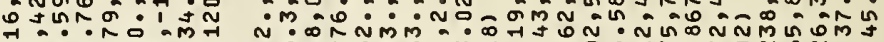

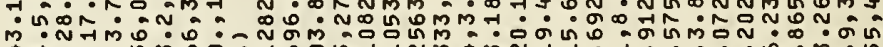
\mmळ

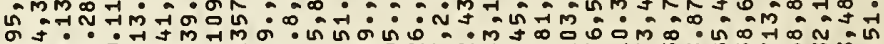

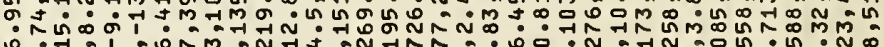
m.j:

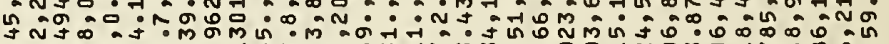
-

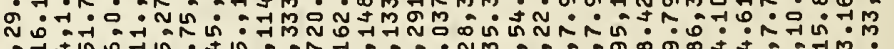

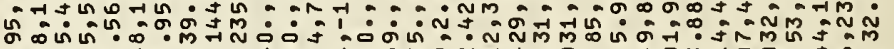
N ง o. जี óñ

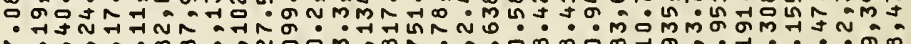
¥

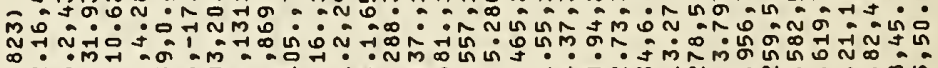
\ ㄴ.

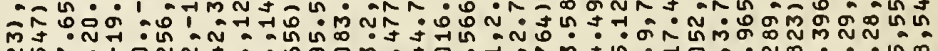

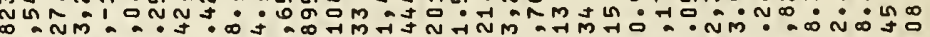
ón 원 ${ }^{\infty}$

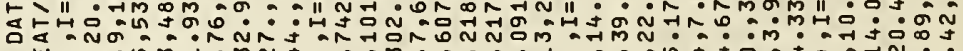

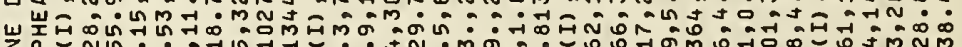
z

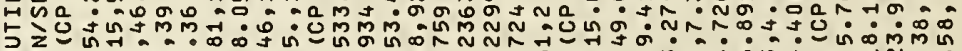
oz

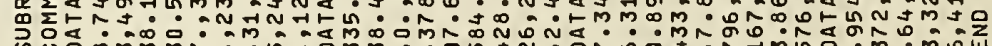
ผ $\because \stackrel{m}{\circ}$ $\dot{m} \rightarrow \overrightarrow{0}$ in : un 뒤ำ किं $\sum_{\substack{\infty \\ \infty}}^{\infty}$ N-5. ming की ำ जिए 击会 กับ

निंक

过 लूू० ज的

然文

लिजू

कै नें ल

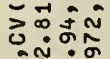

का $\cong \dot{~}$ $>$ 舟品的 कa นิํำ งเำ

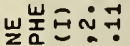
的的的 층영 웅옴ำ

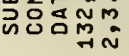




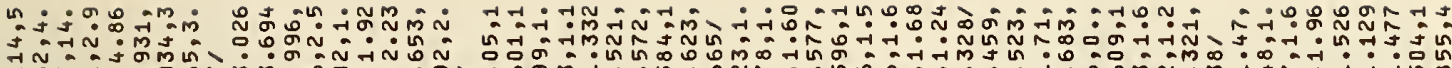

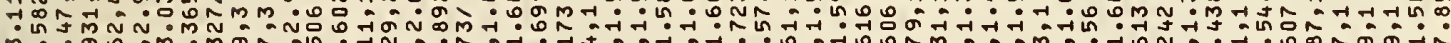
mó స̃

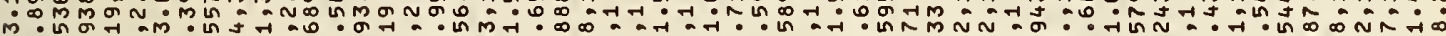
mún

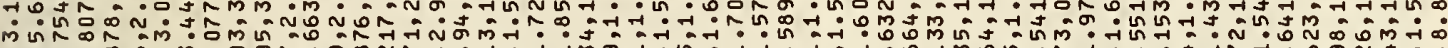
ñ்⿻一亅 Tmón

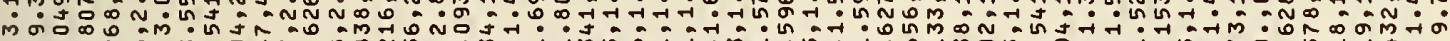

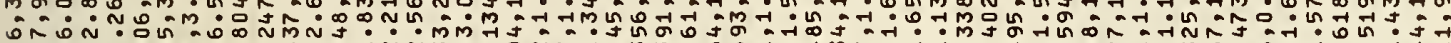

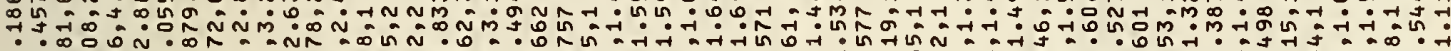

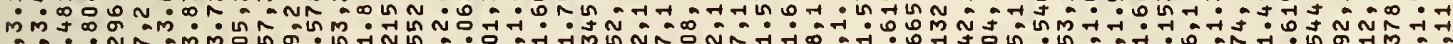
ñ

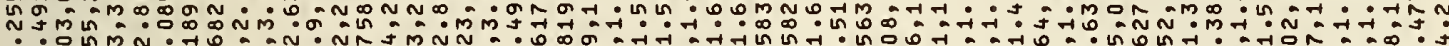

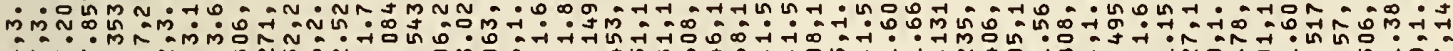

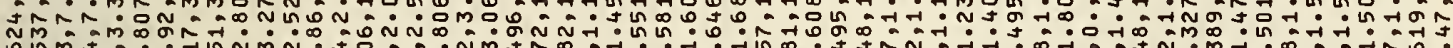
N

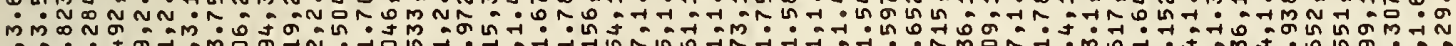

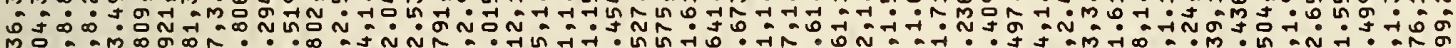
Món نm NM⿻上丨m MN MÑ

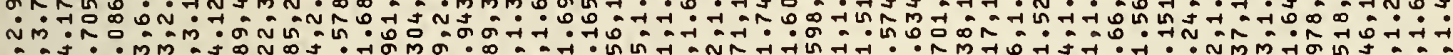

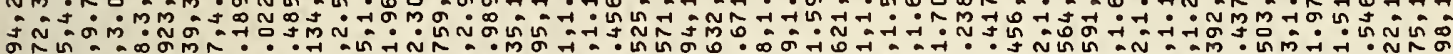

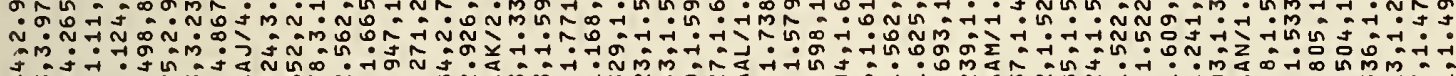
Jm

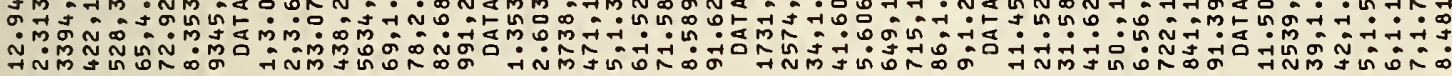

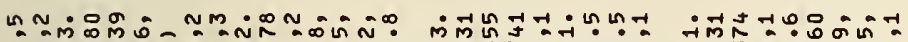

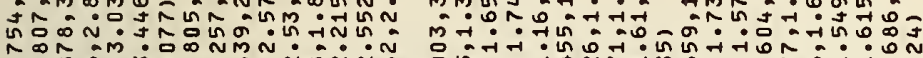

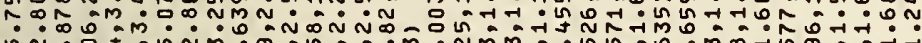
แnNm⿻.

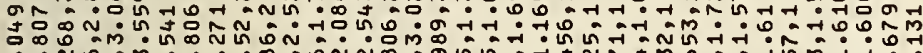

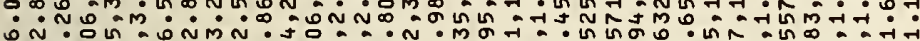

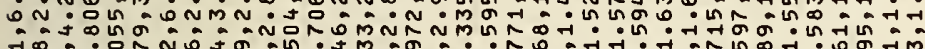

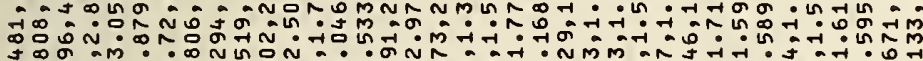

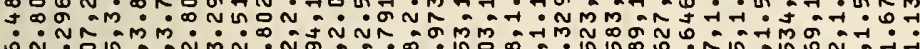

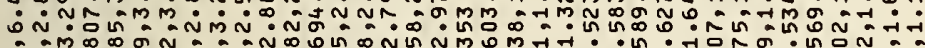

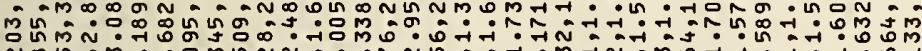

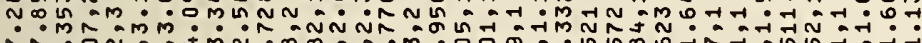

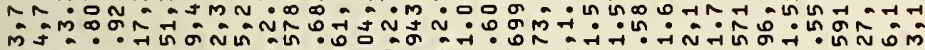

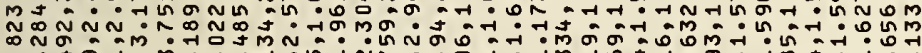

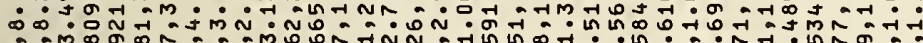
ที

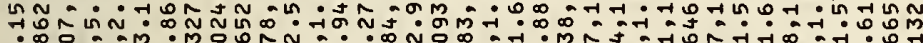

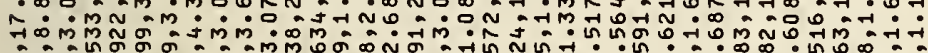

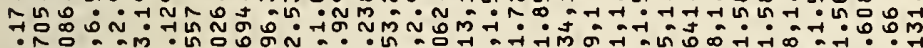
ษ? :mmm

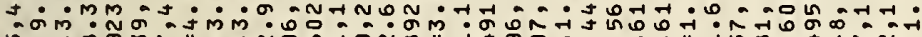

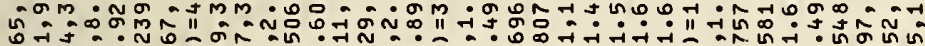

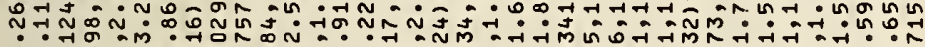

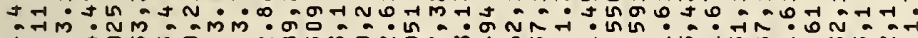

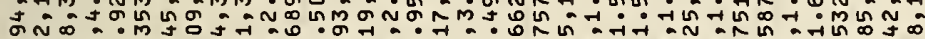

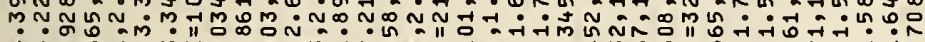

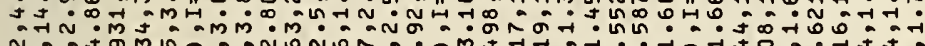

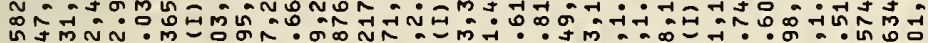

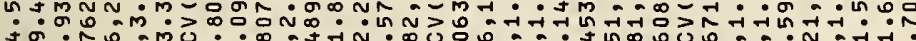
ปn ốn

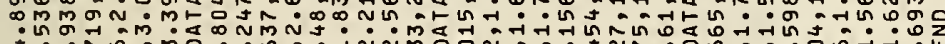

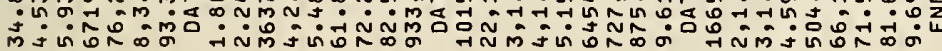

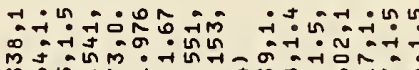

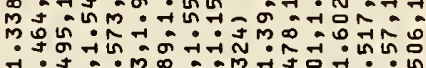
ñ mิ

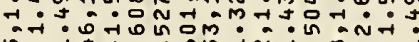

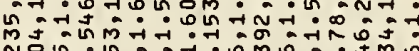

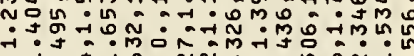

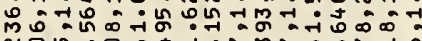

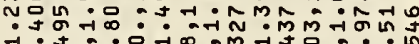

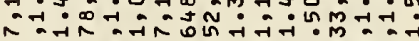

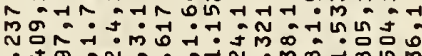
- J o क尸

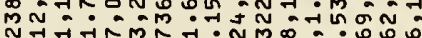
$\because$ 品 N लिके น̂न-

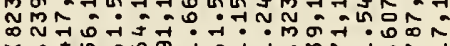

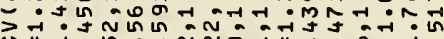
仓II -

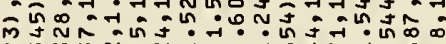

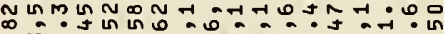
N

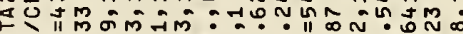

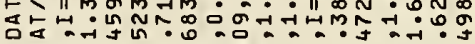

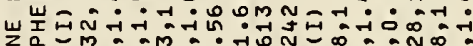

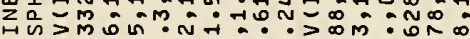

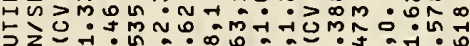
o

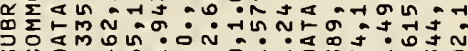

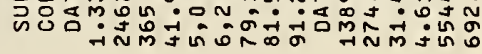




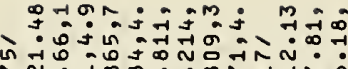

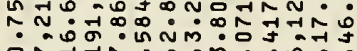

จิ

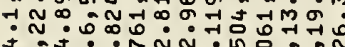

ง

Non日

o.

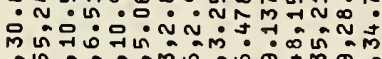

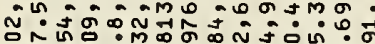

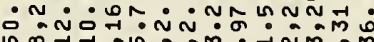

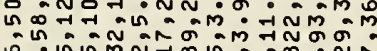

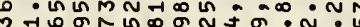

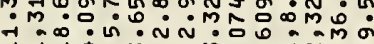

न

oำ

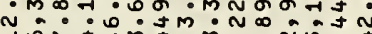

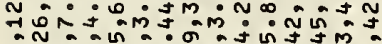

o.jỗ.

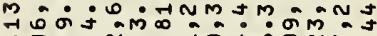

$\because \div$ Й

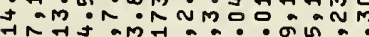

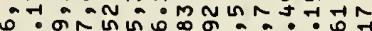

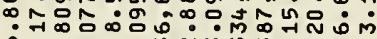
فำ

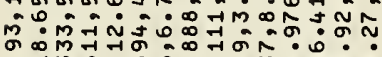

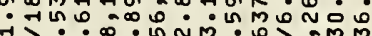

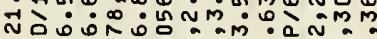

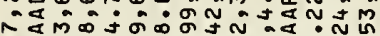

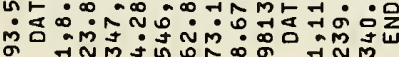

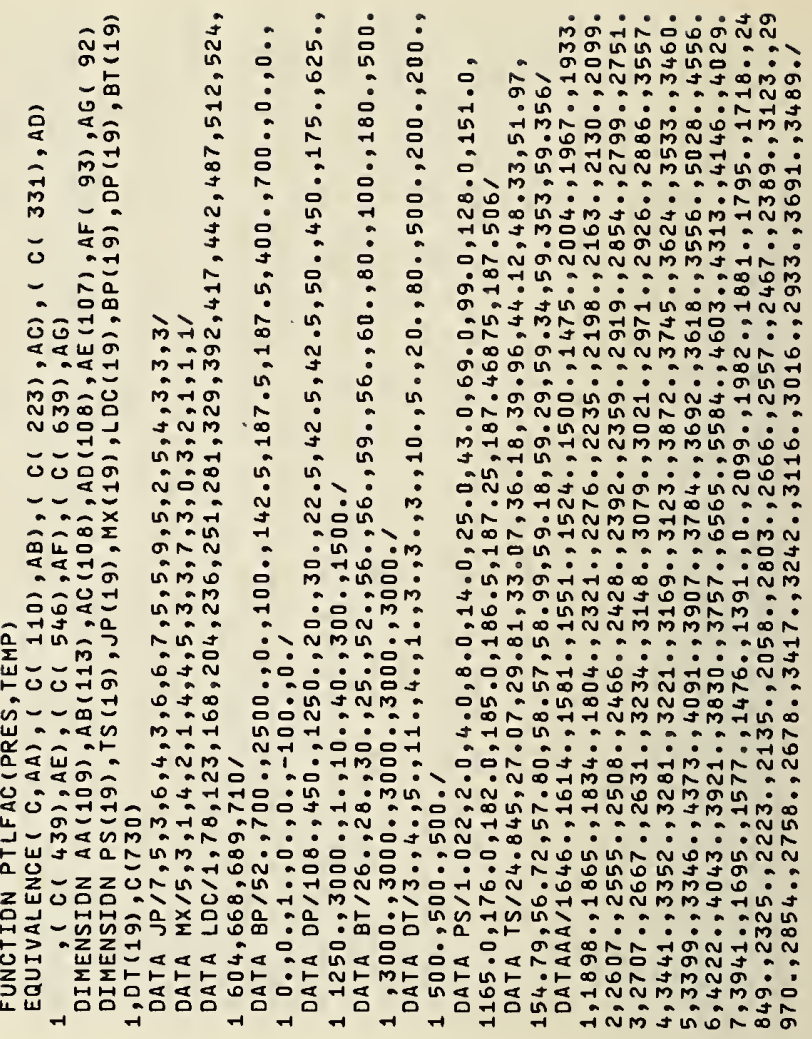

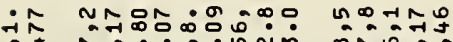

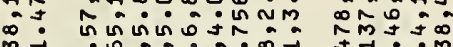
m

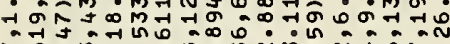
ลั.

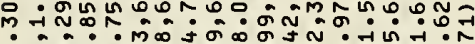

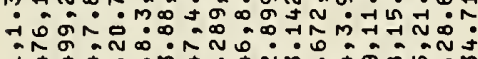

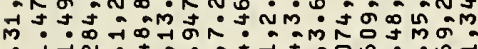

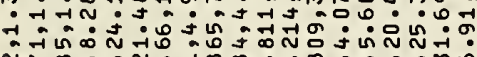

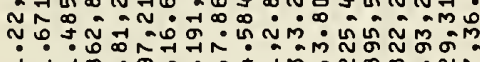
ก N N -

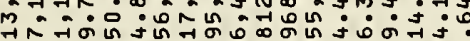
$\because$ ․ำ

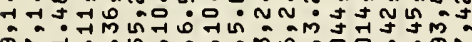

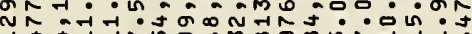

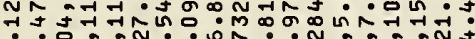
-

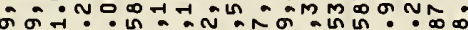
ใุ. -

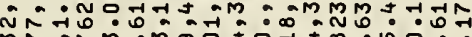
舟

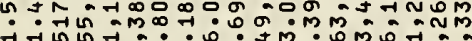

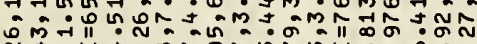
กั. -75日

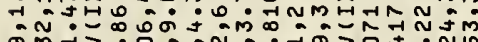
जิ -

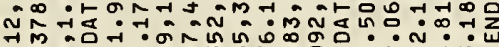

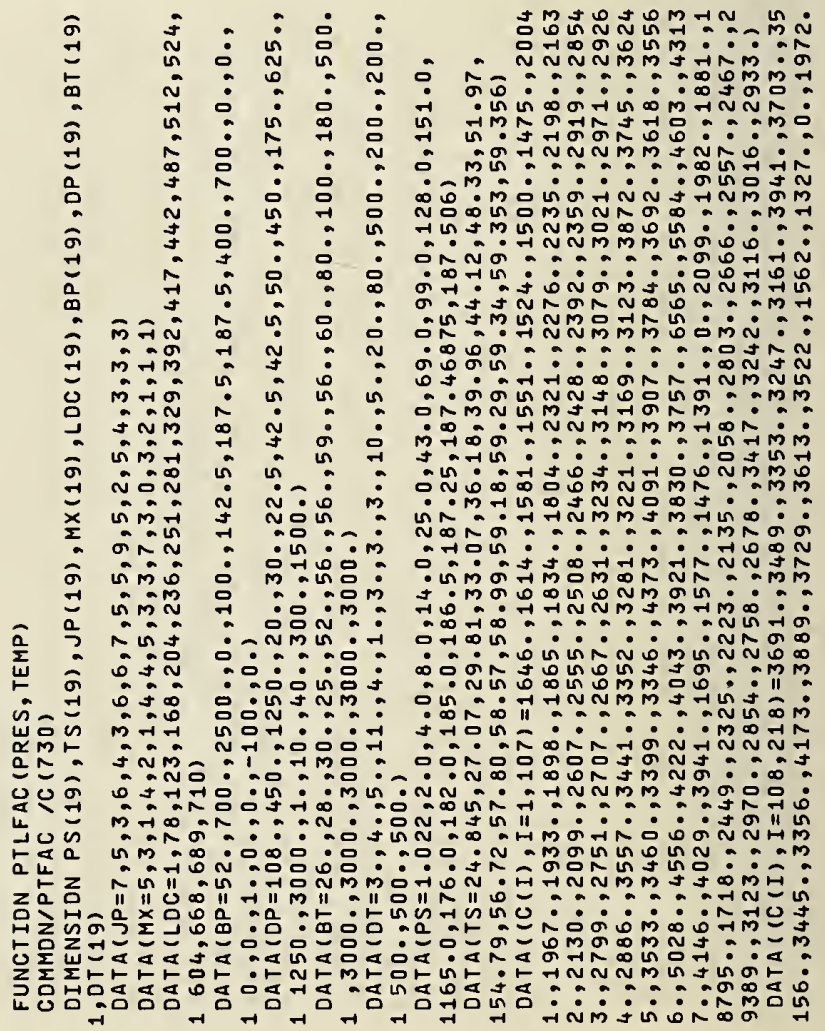




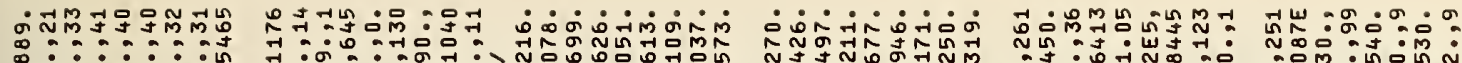

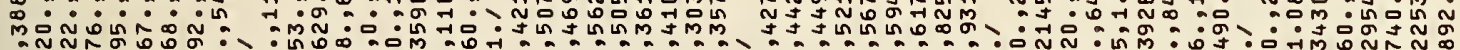

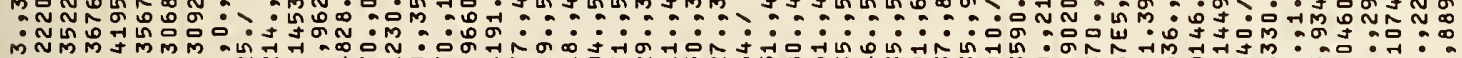

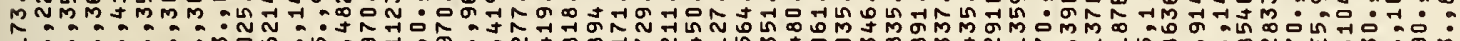
Jơ

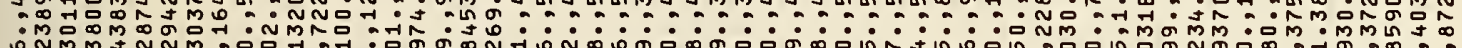

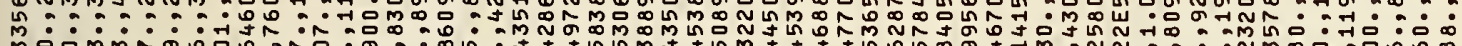
món

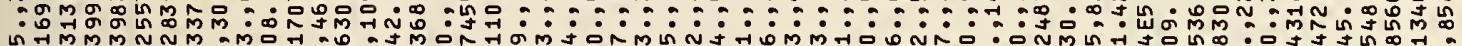

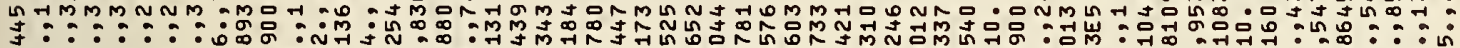
min

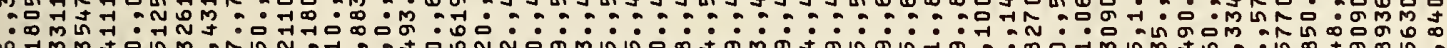

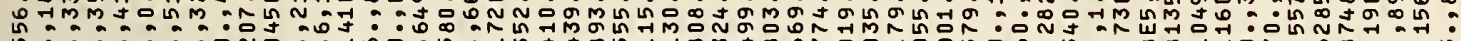

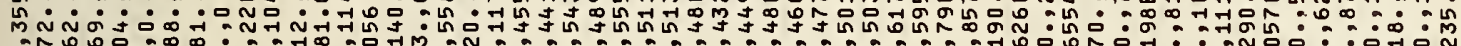

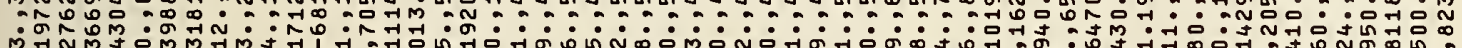

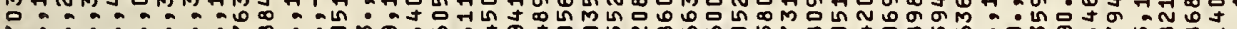

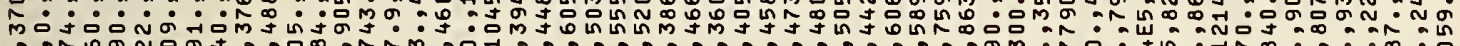

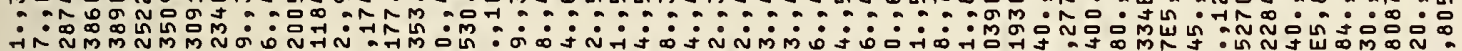

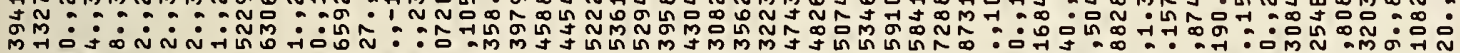

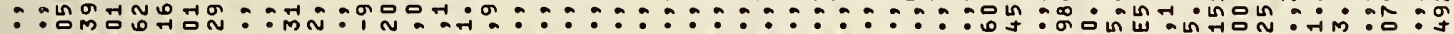

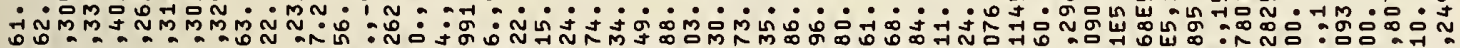

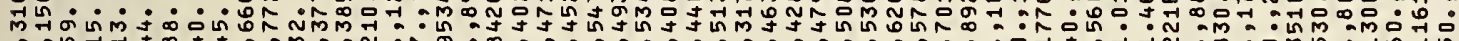

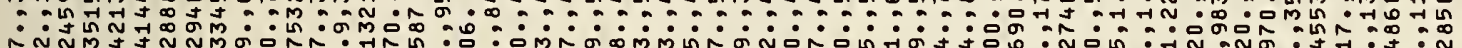
J N NMJ N NMg

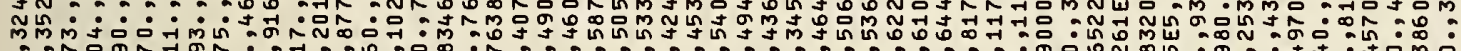

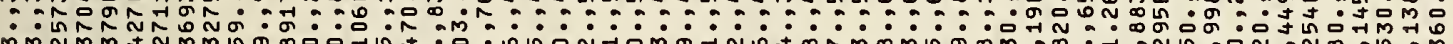

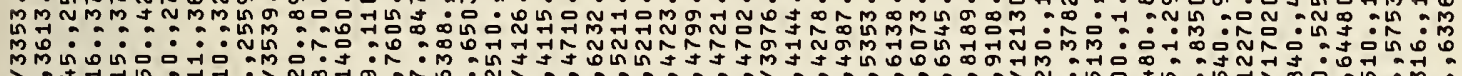

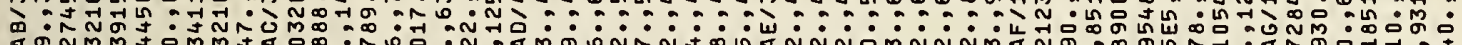

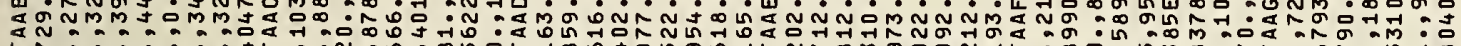

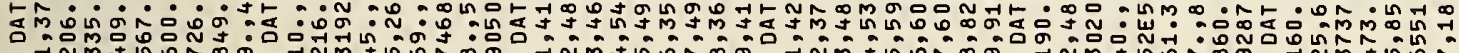
तN

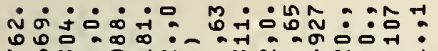

N

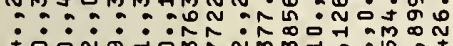

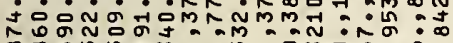
N

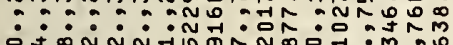

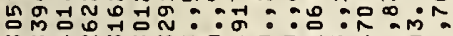
Mm N 药

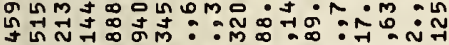
N

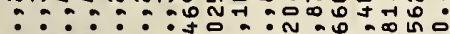

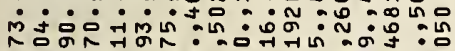

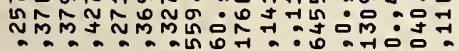

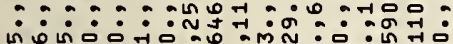

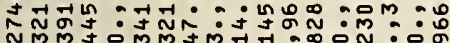

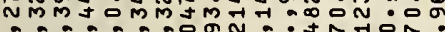
:

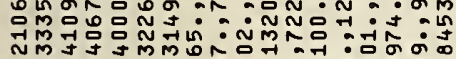
: : : : : 拈 N ลิ่ง

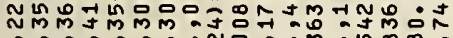
: : : :

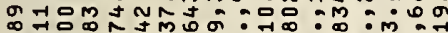

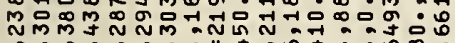

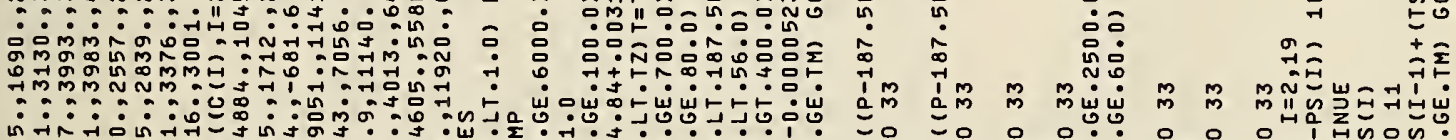

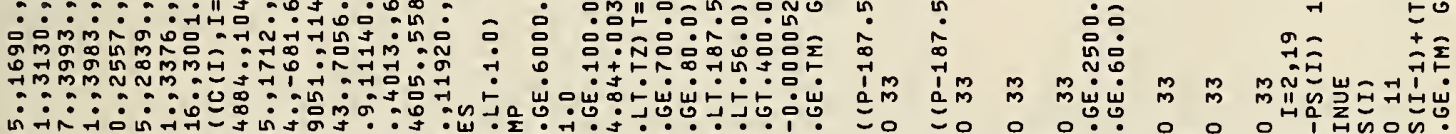

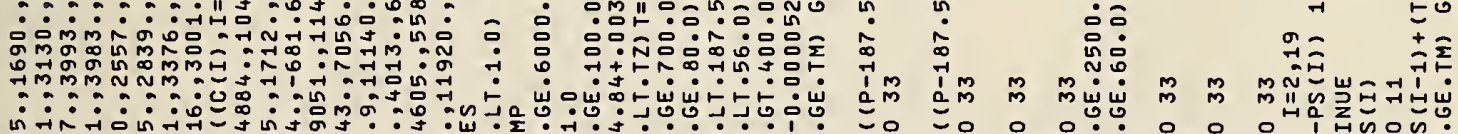

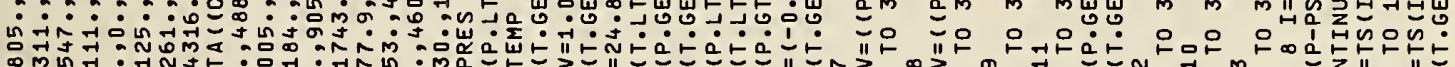

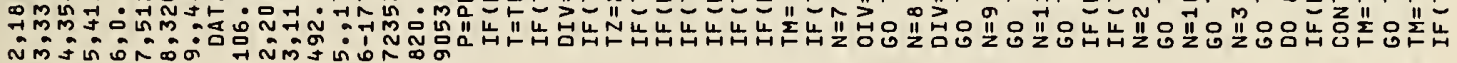

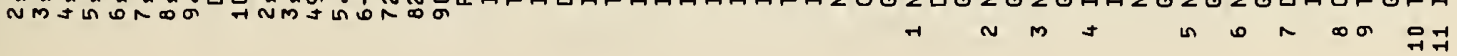



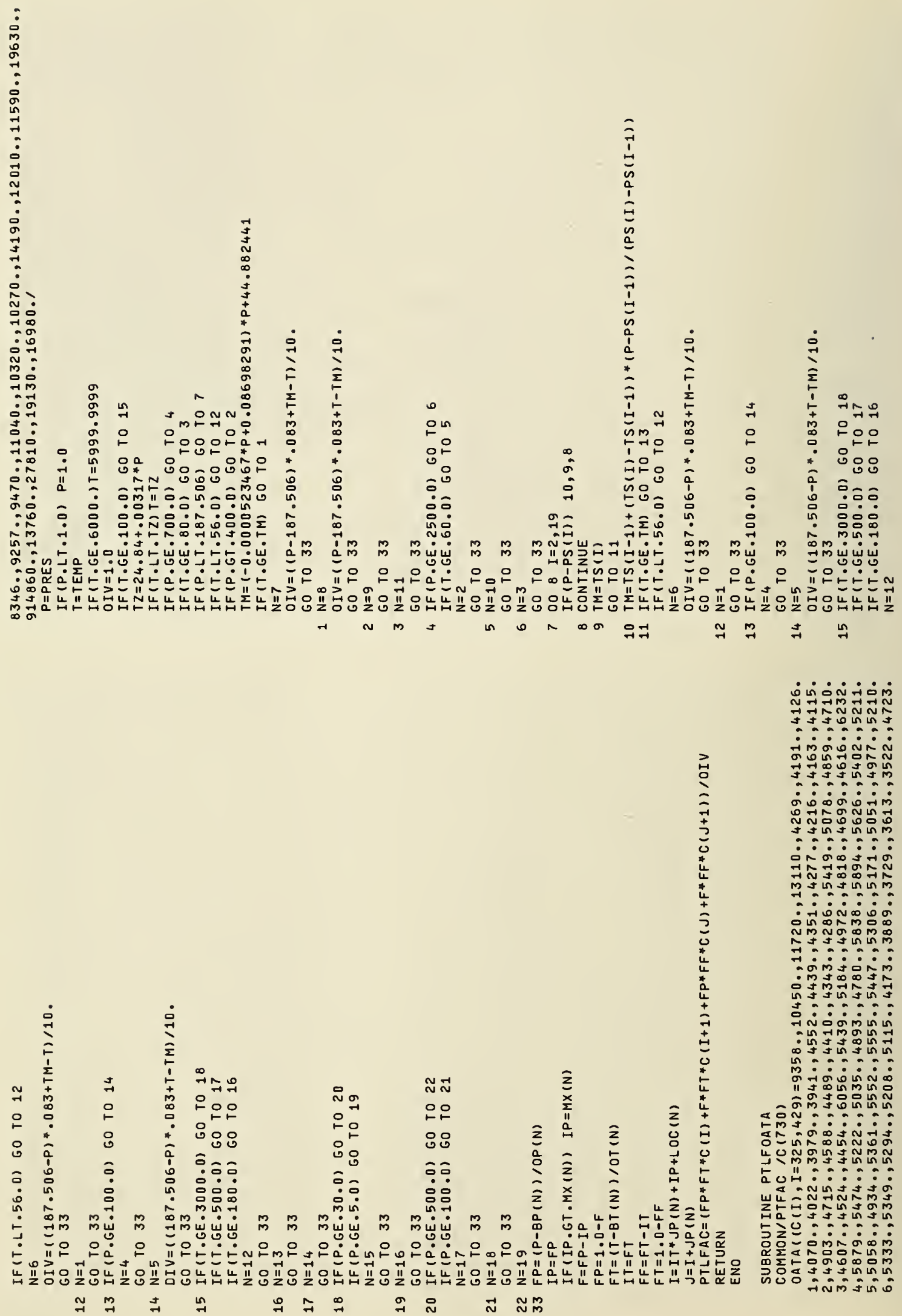


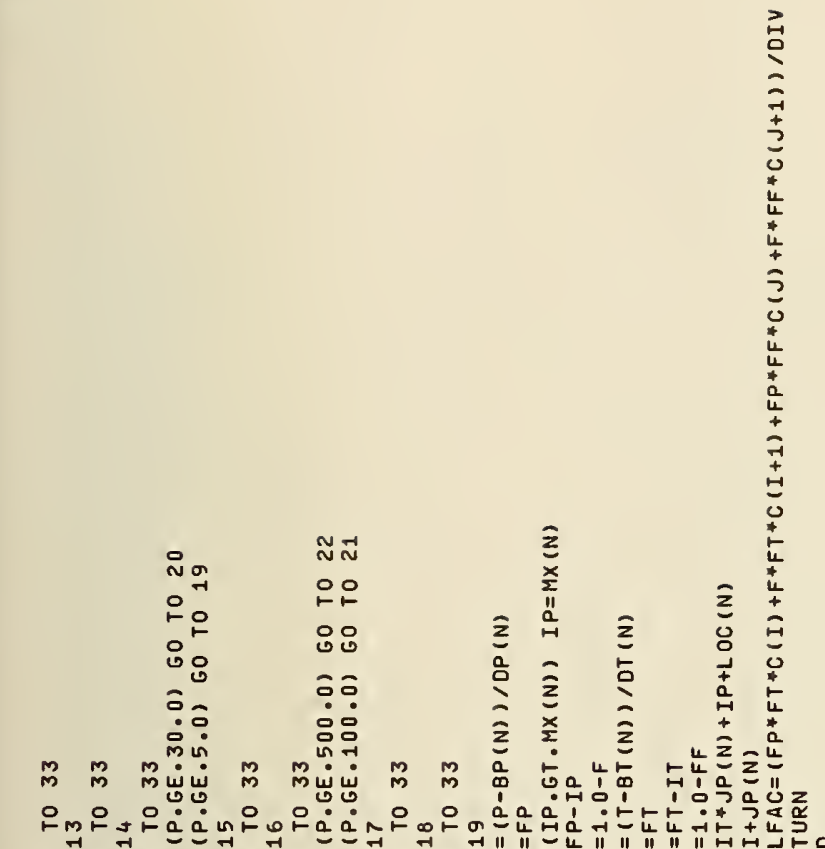

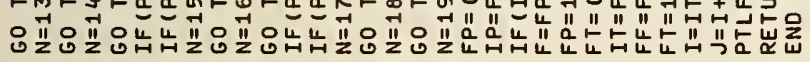

न

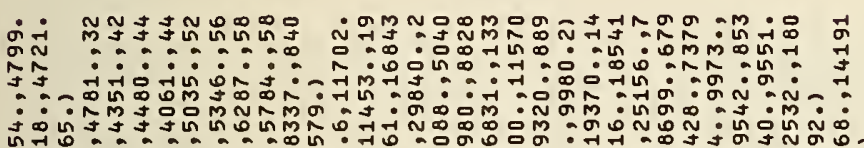
ด̂̉.

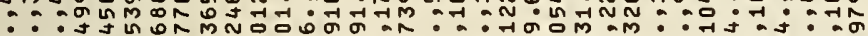

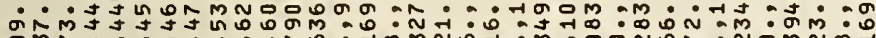

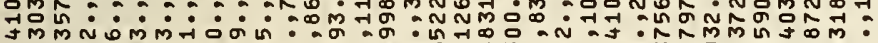

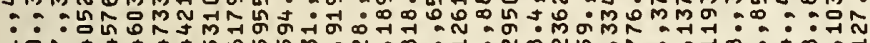

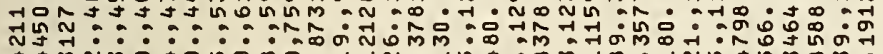
于 $\because$ :

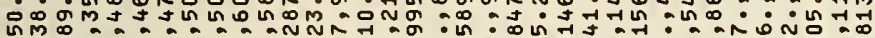

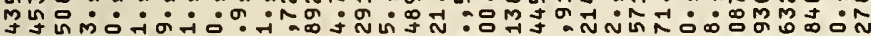
$\therefore:=1000$ nN

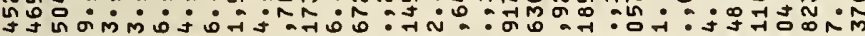
IN

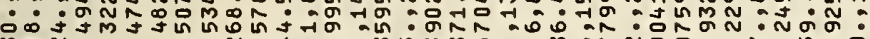

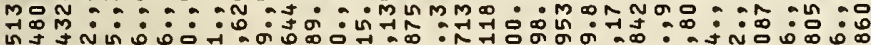

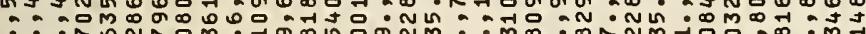

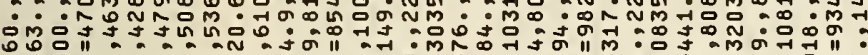

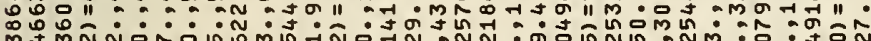

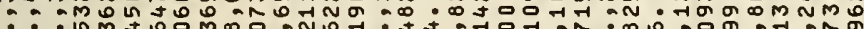

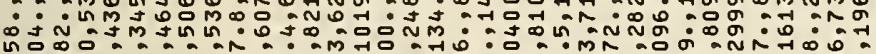

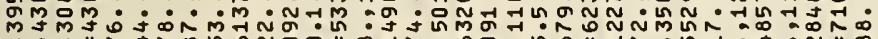

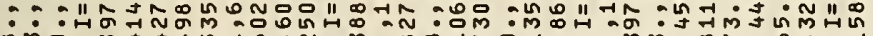
mo

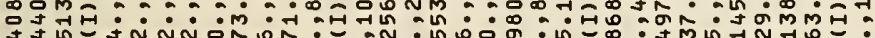

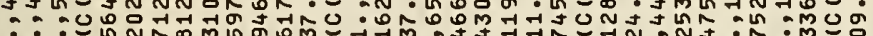
miñ

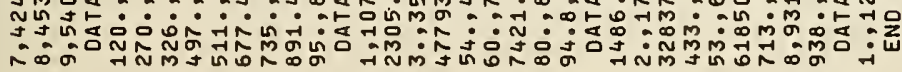
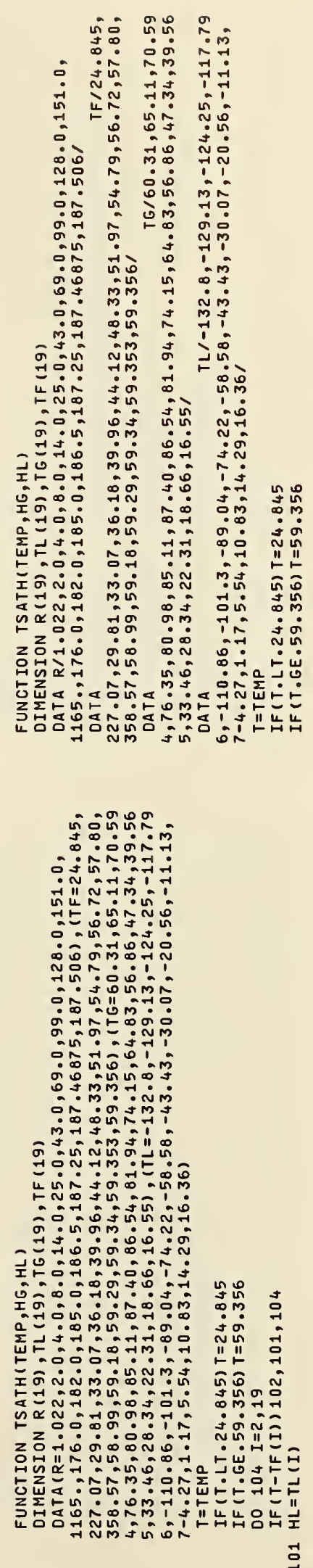

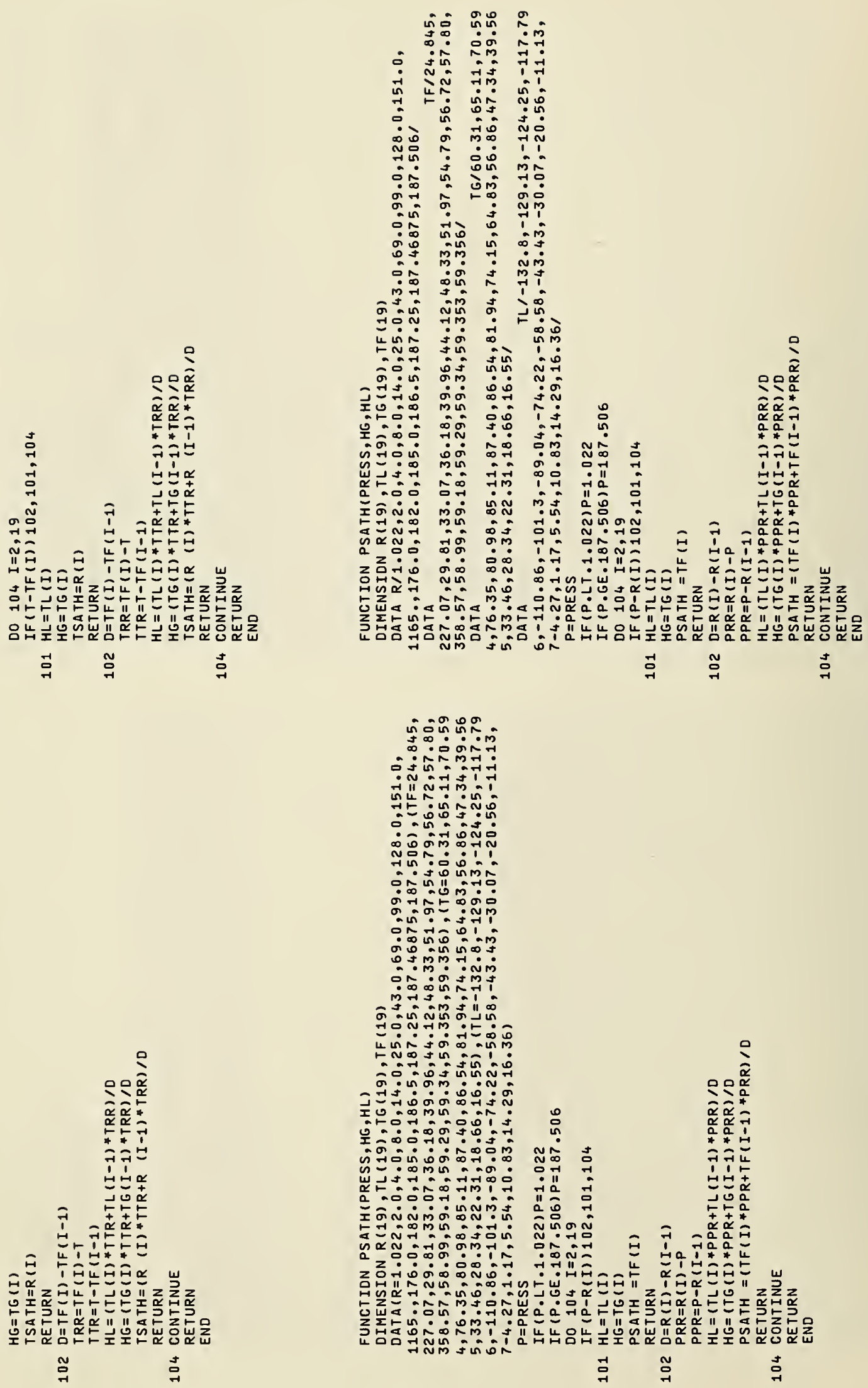


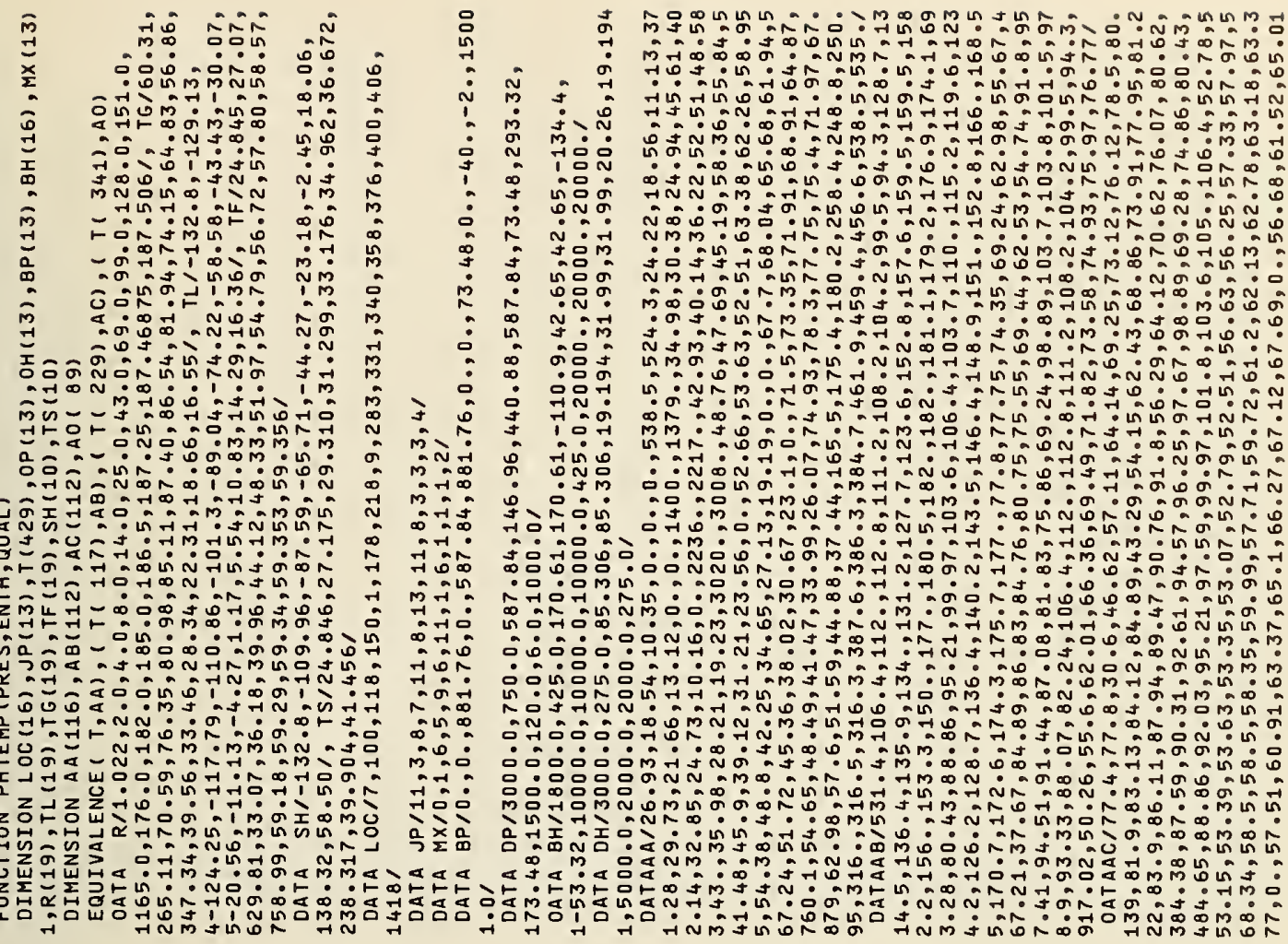

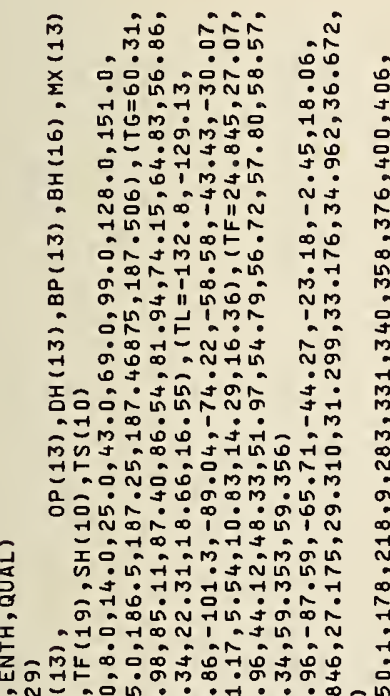

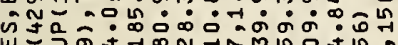
宸

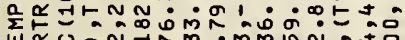

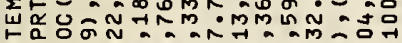

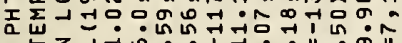

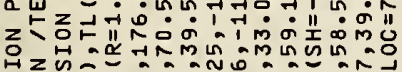

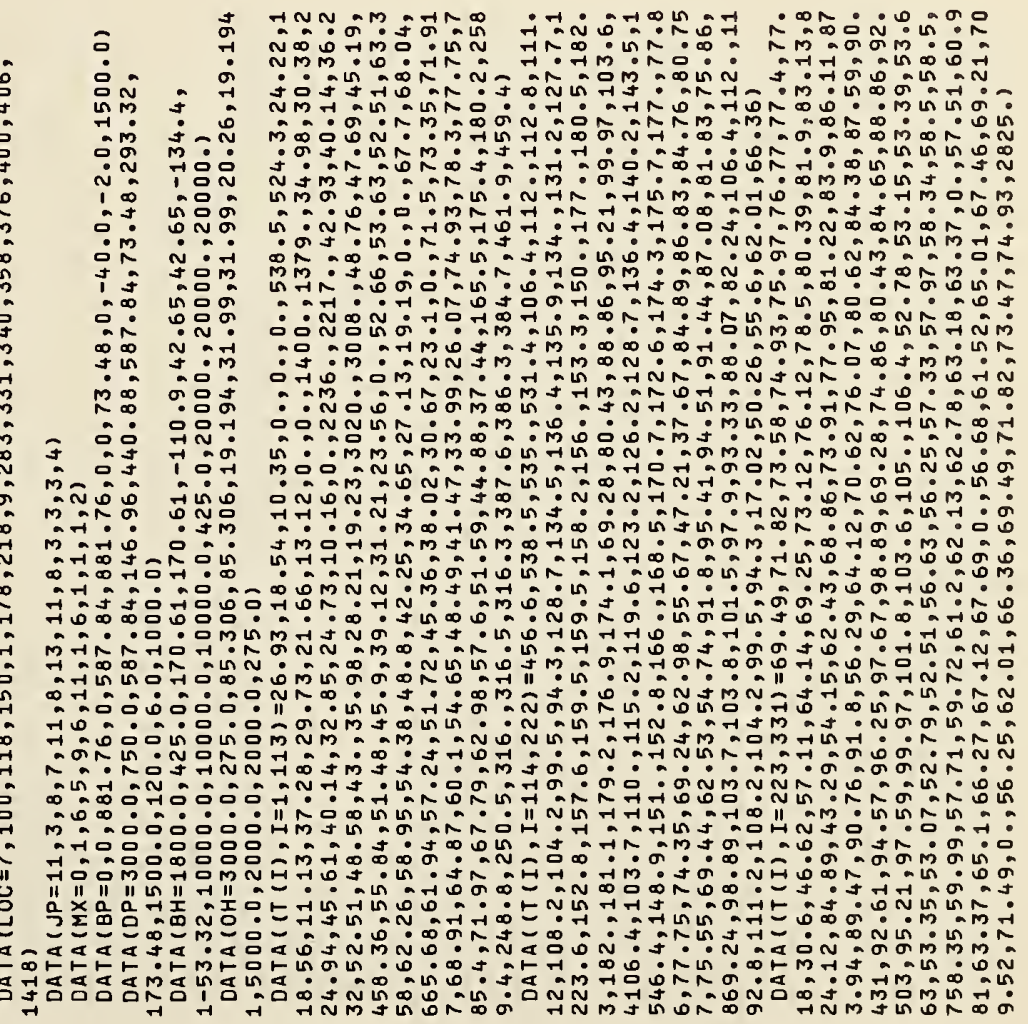



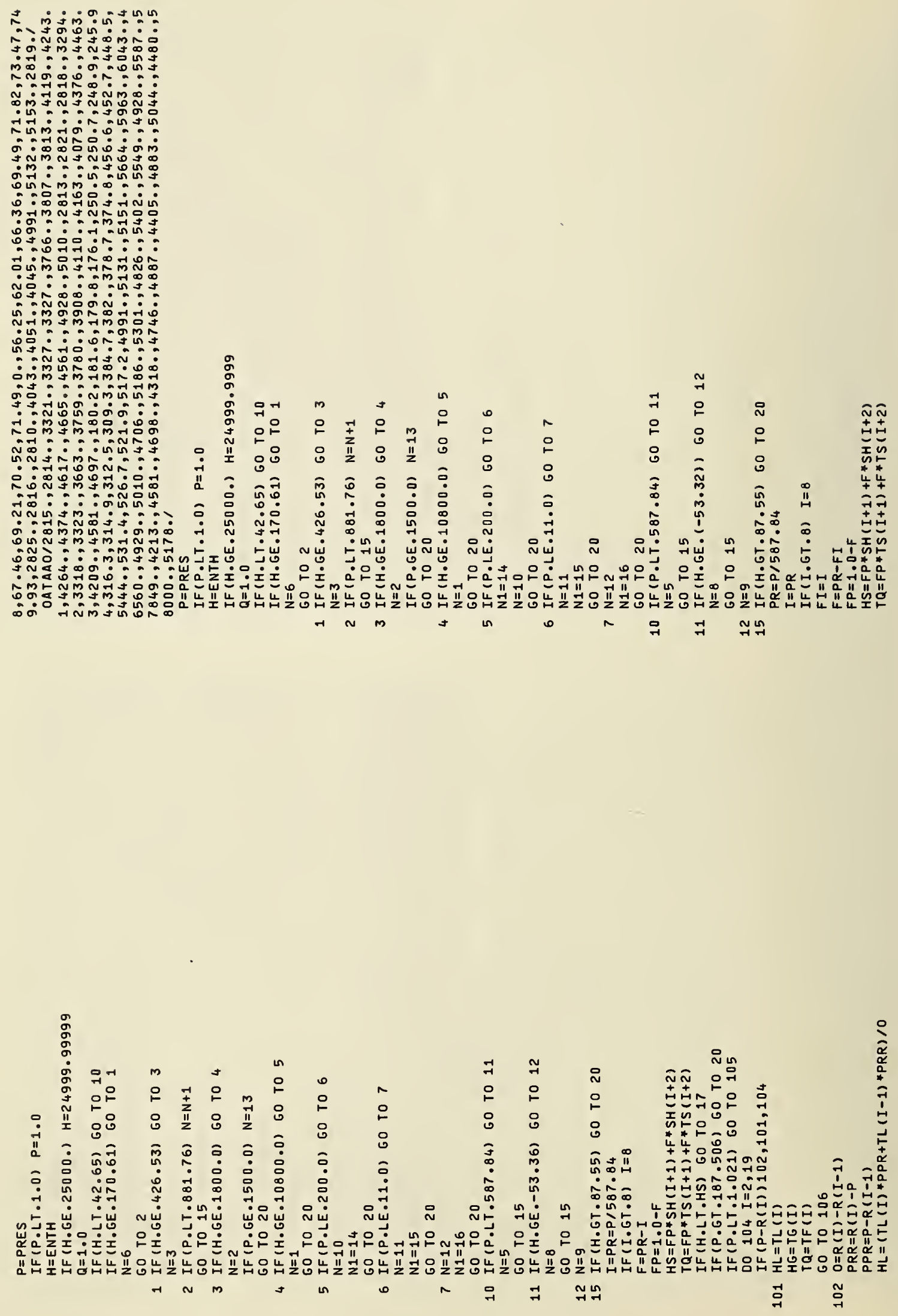


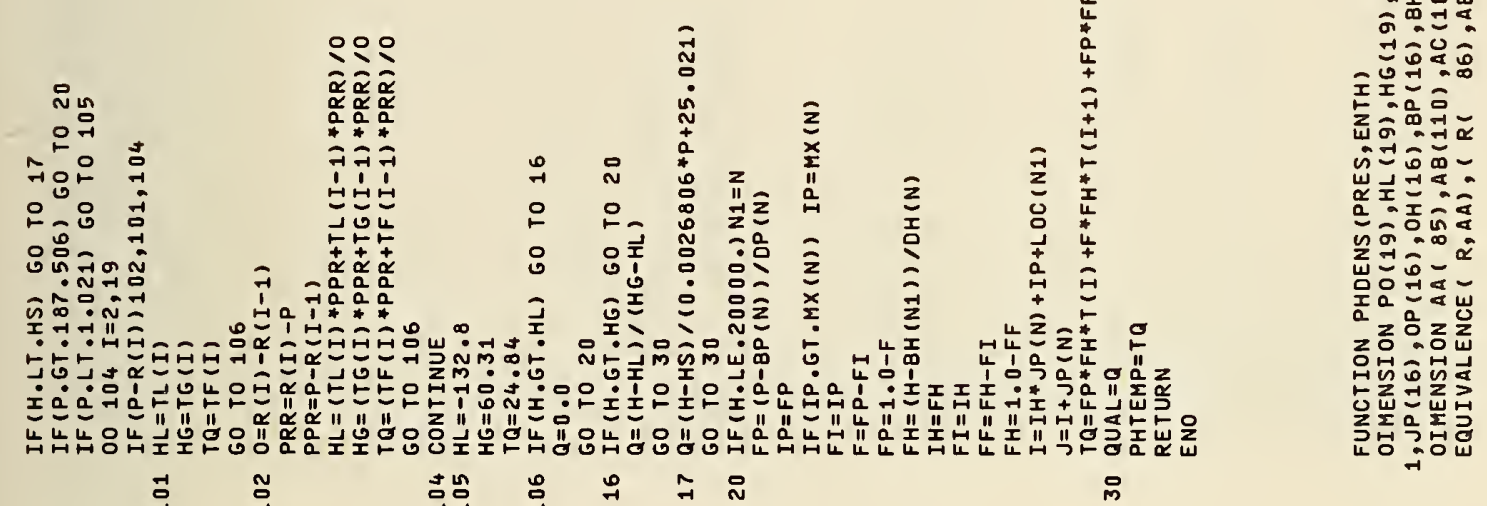

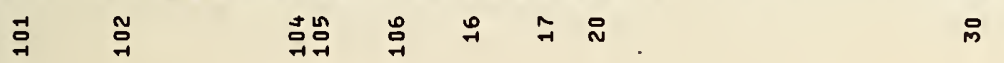
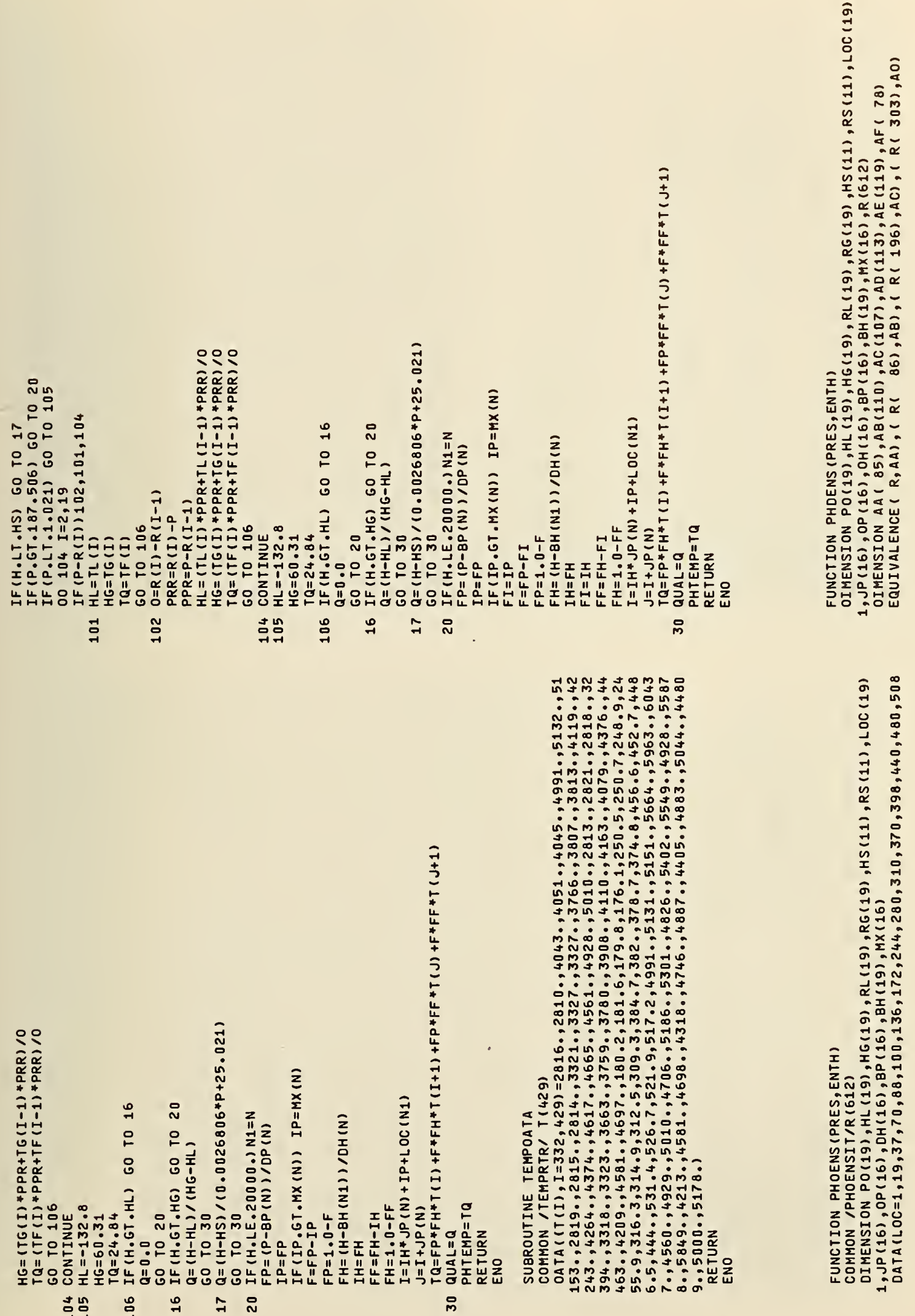


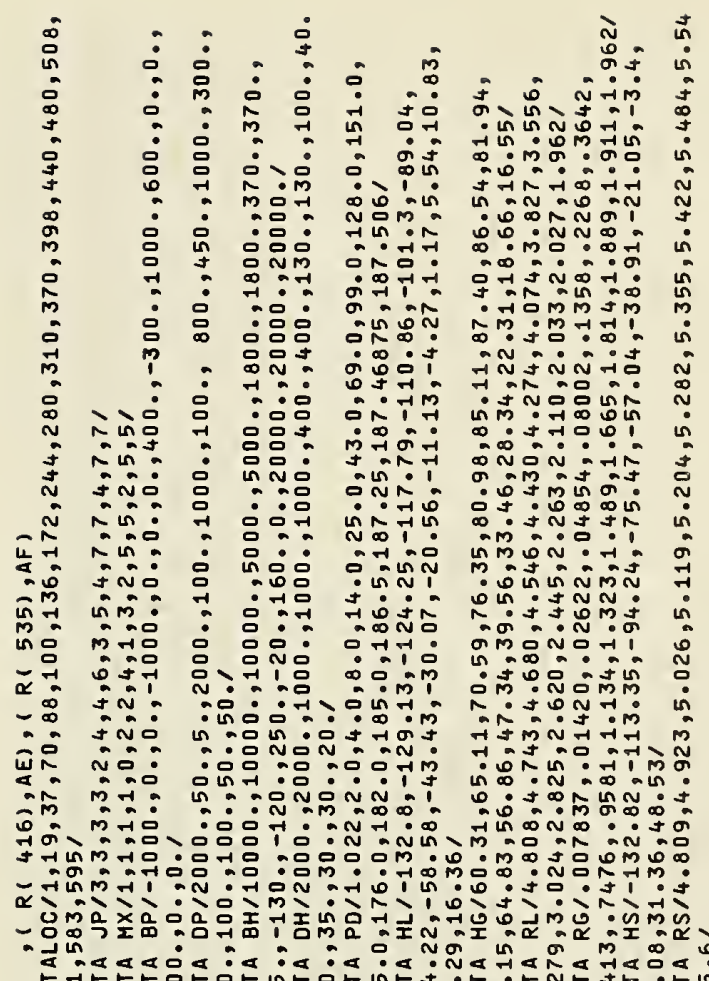

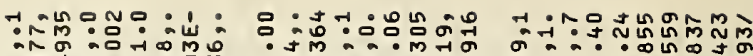

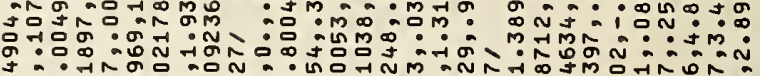

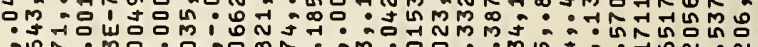

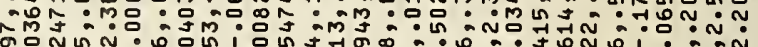
o: 견

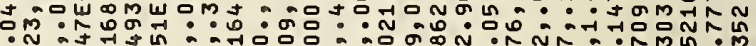

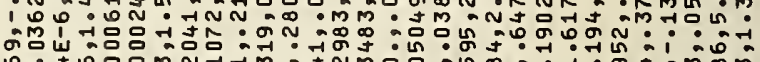

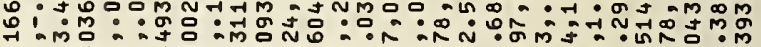
:

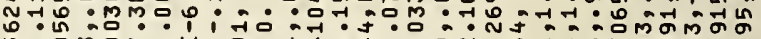

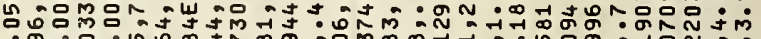

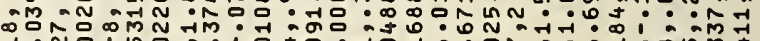

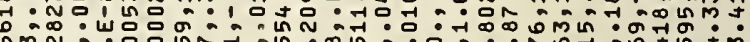
ถึm N..

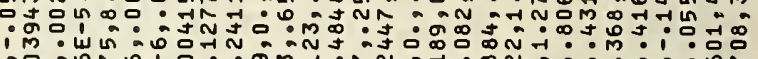
○. -

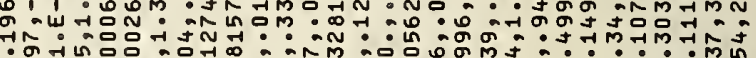
: N

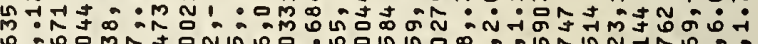

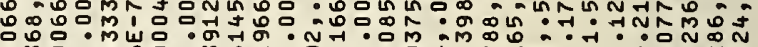

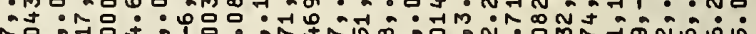
N : :

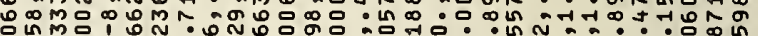

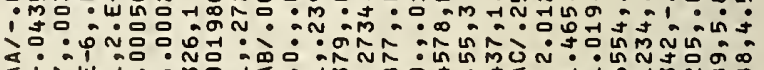

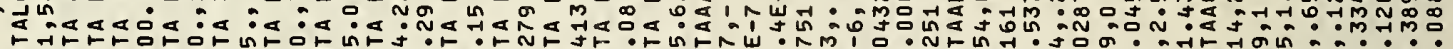

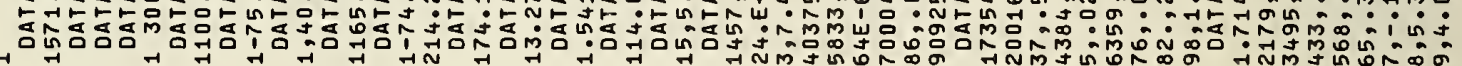

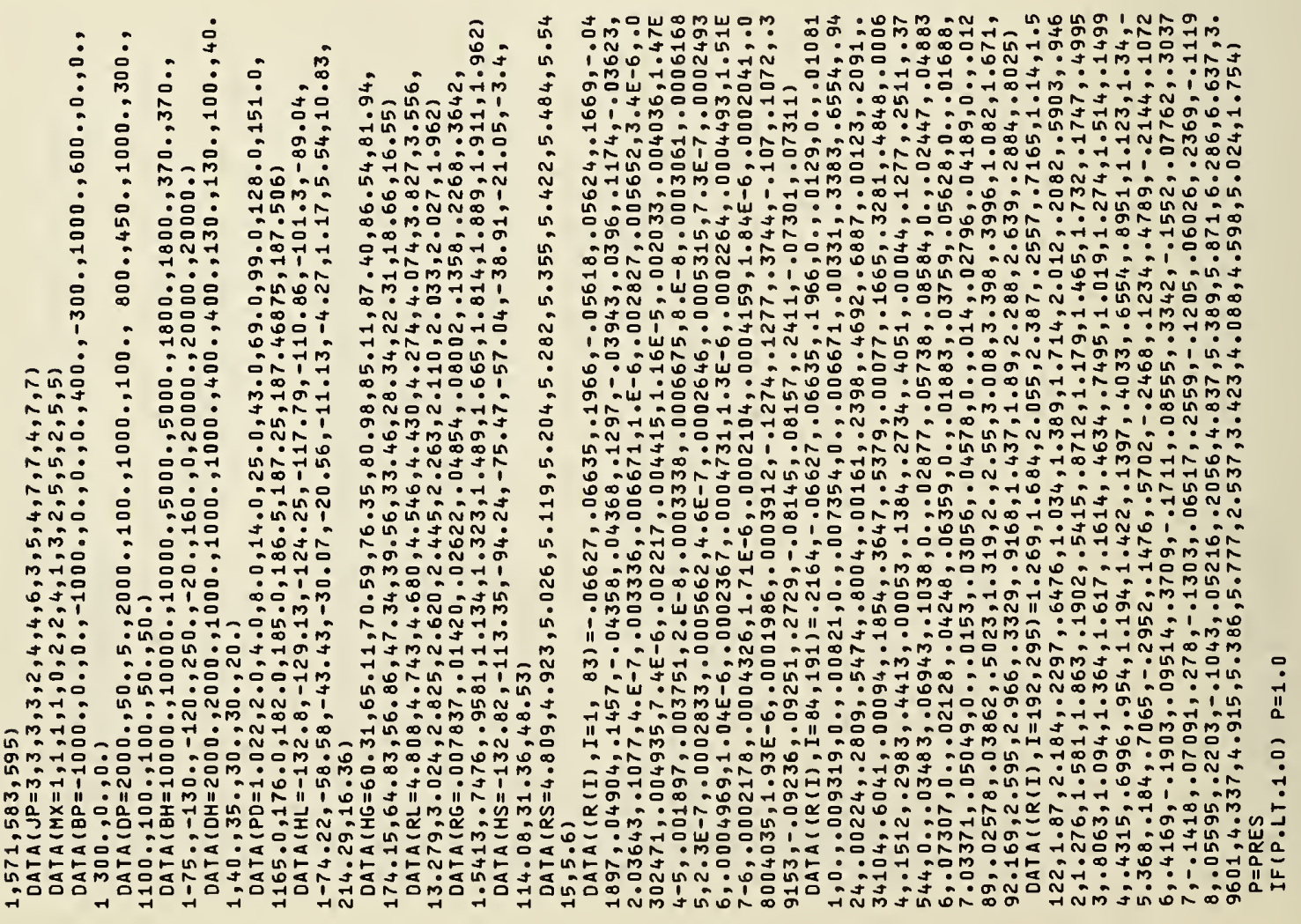




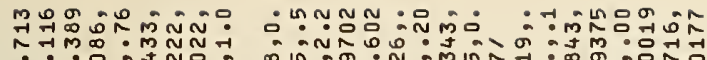

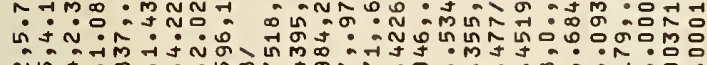

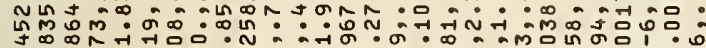
जि门कo N J T

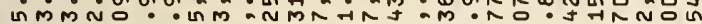

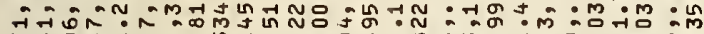
fơ m.M.

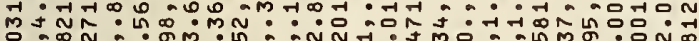
लि No

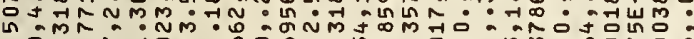

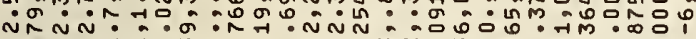

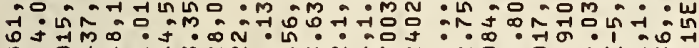

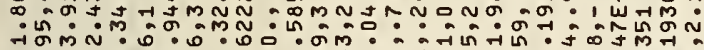
N

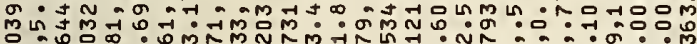

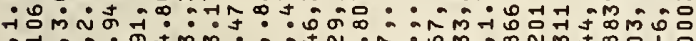
ō:

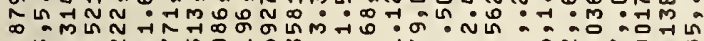

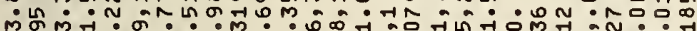

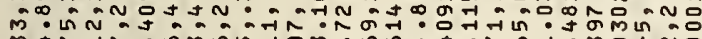

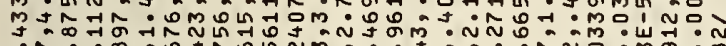

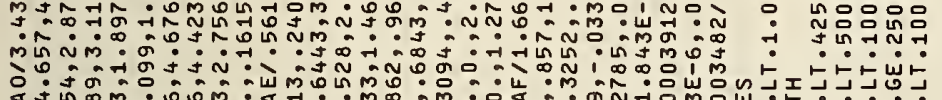

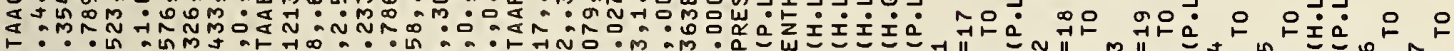

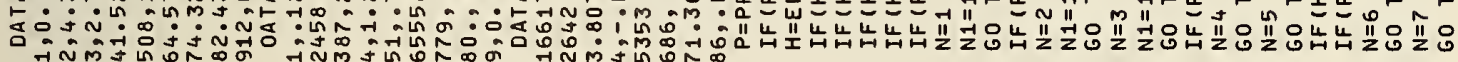

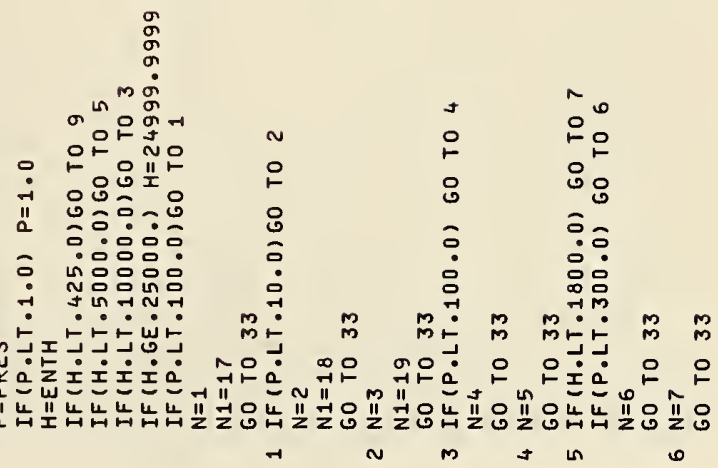

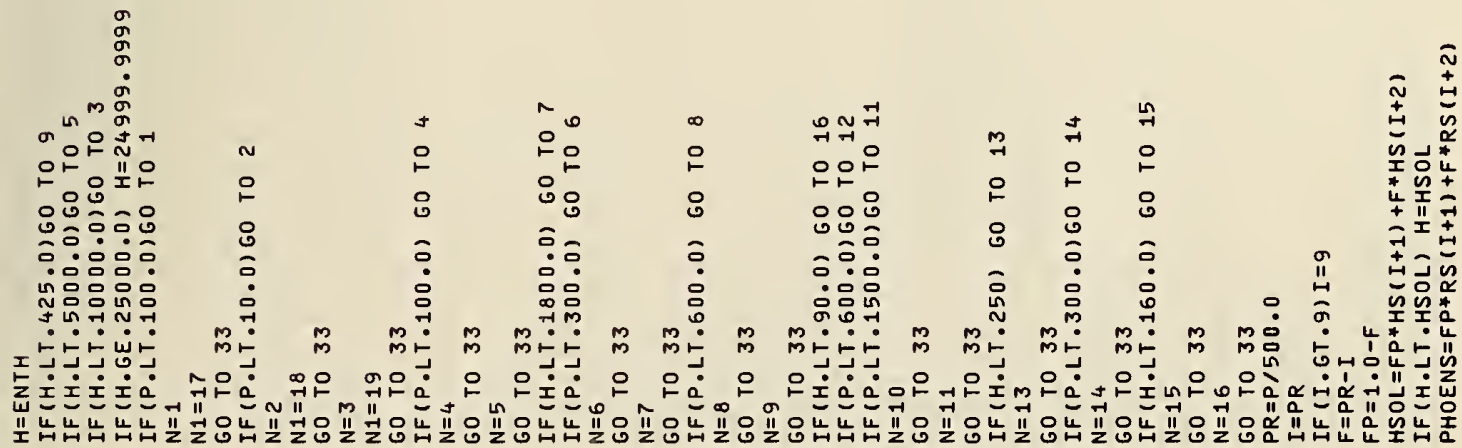

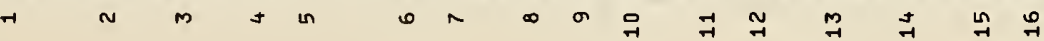



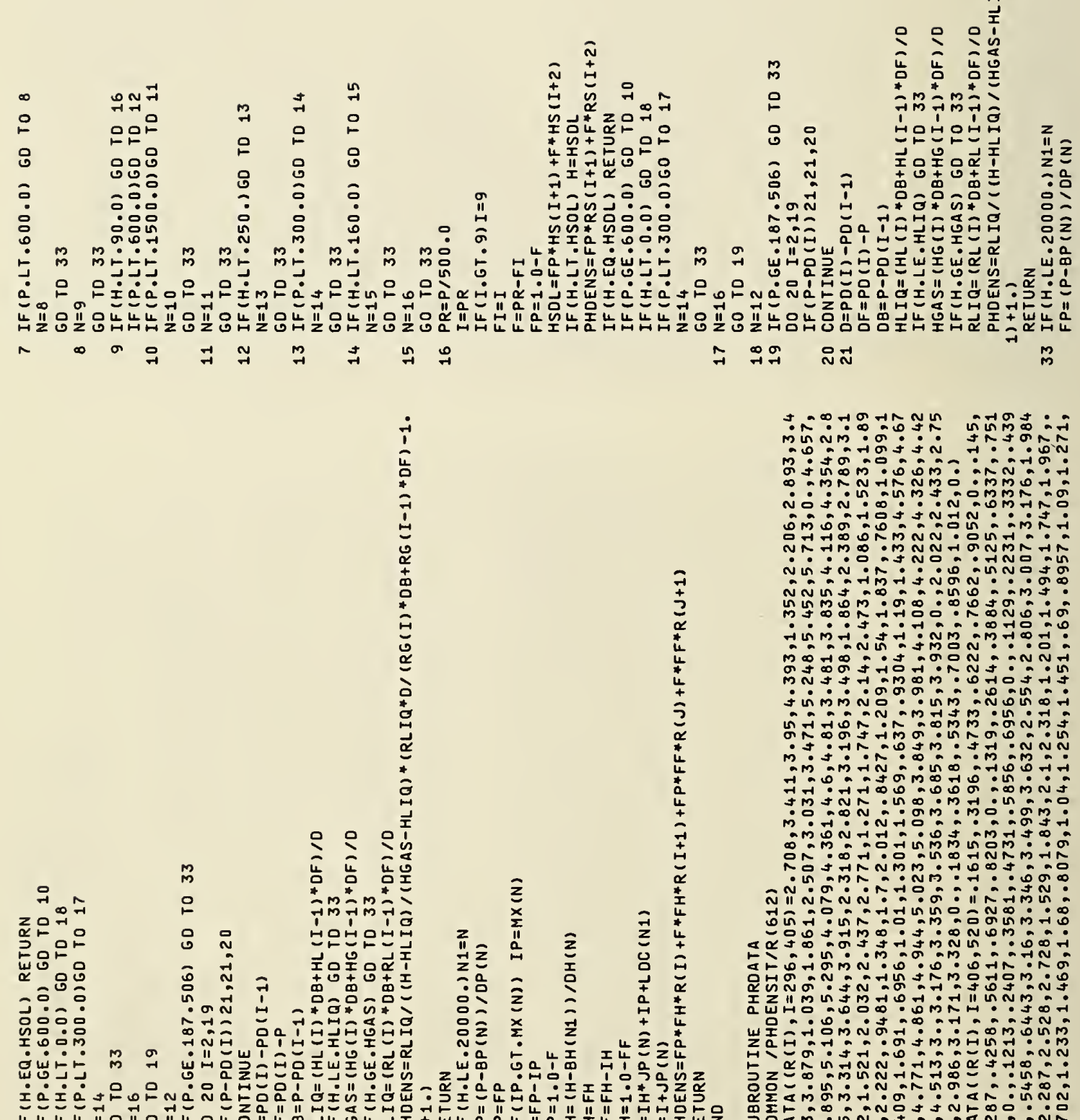

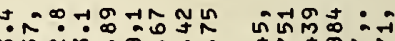
min m.t. 行战 Nanojand

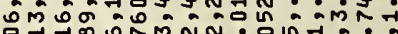
NATMOPMN

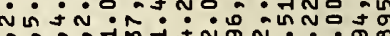

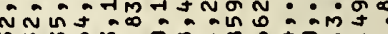

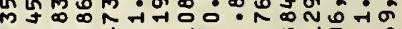

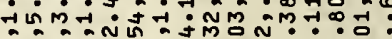

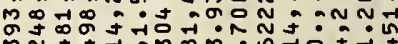

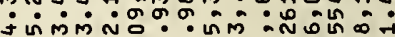

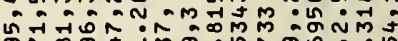
ơ $m$ m.

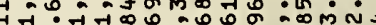
:M̃ m. न- N-10

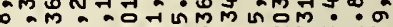

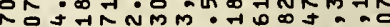

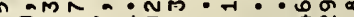
ล

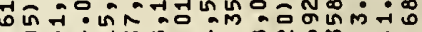

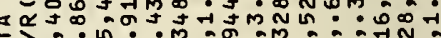

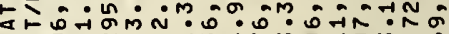

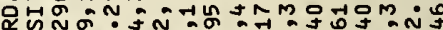

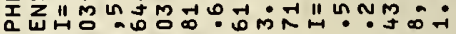

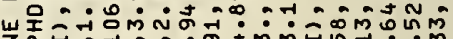

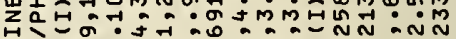

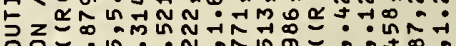

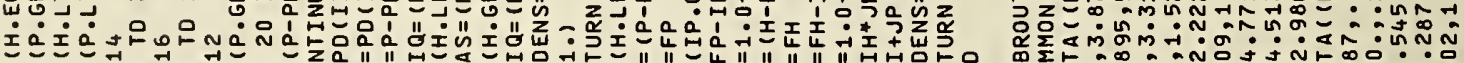

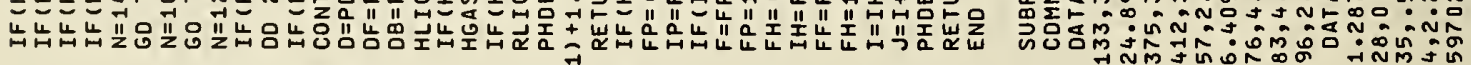
ๆ 


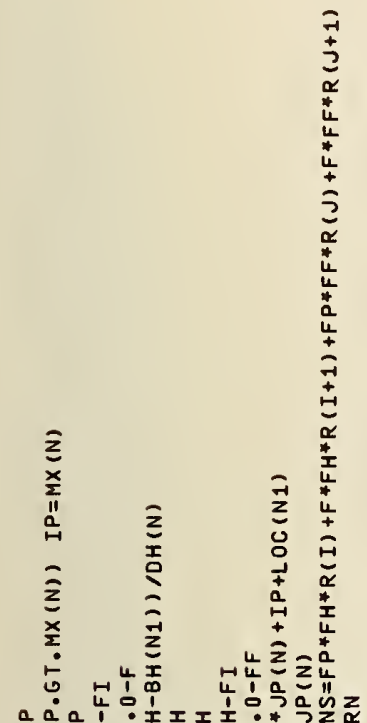

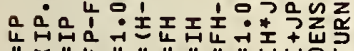

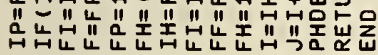

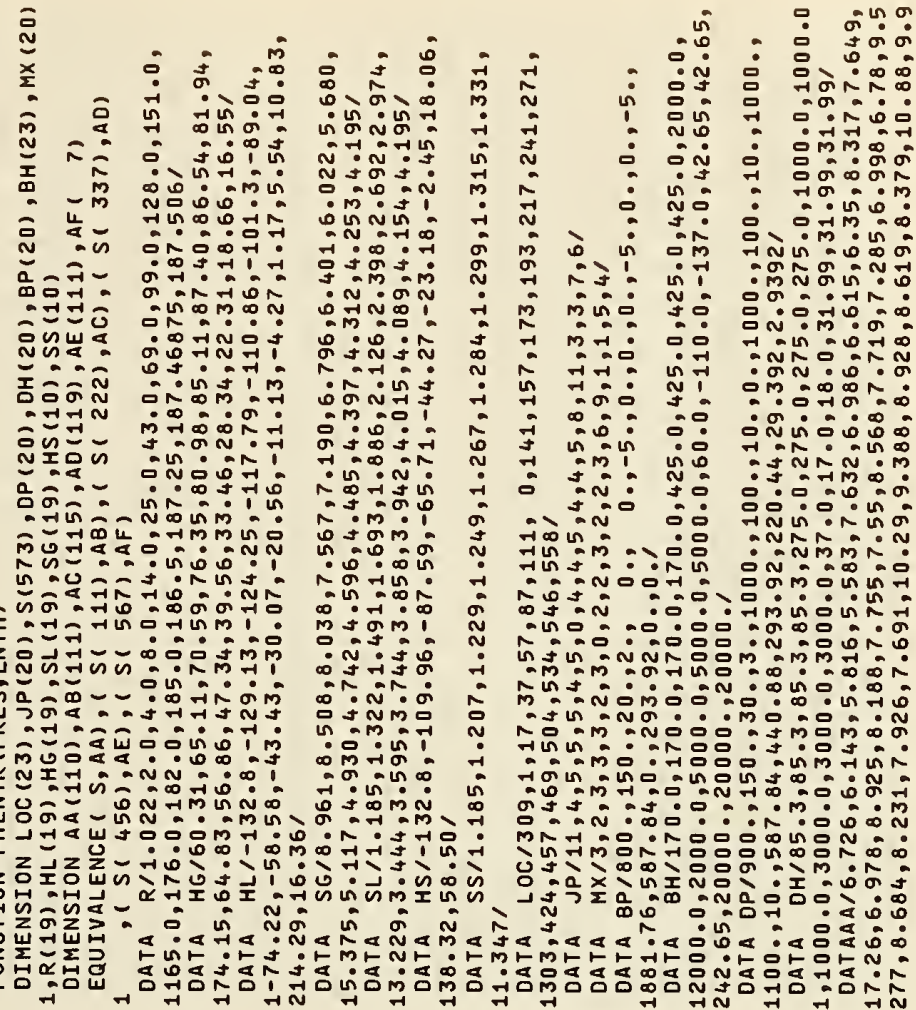

บำ :-7

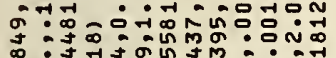
कo

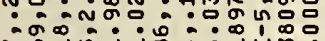

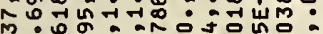

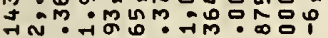

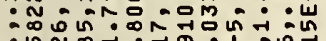
$\therefore$ : N

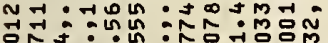

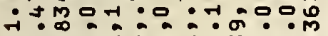
ดิล

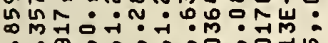
ดั mก⿻

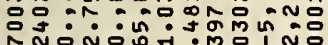
ค1

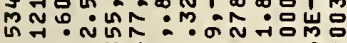
:

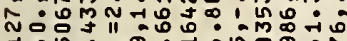

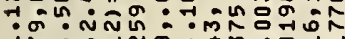

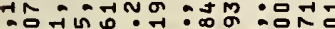
ま

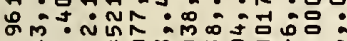
: ‘ : กัด. م : นึ์: กิก

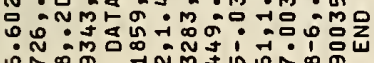

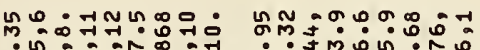

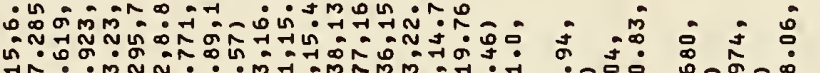

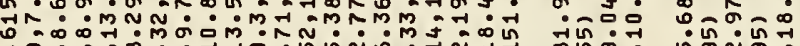
인

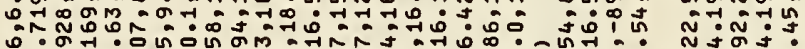

To

的

No

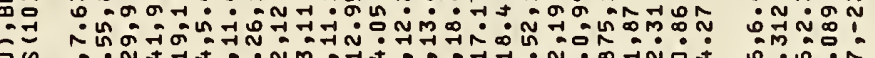

N

İ

й

त्र任

व

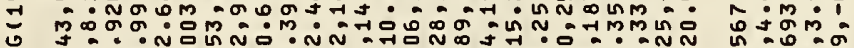

ต नผก𠃌

交

政 N

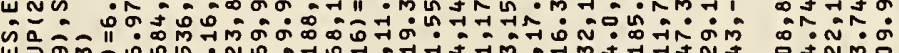
崖?

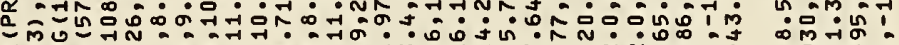

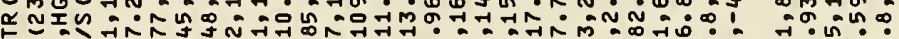

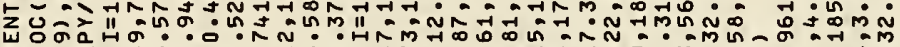

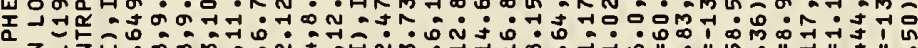

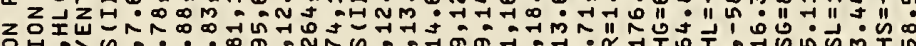
zo

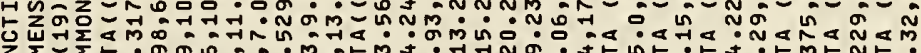

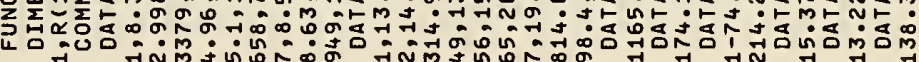




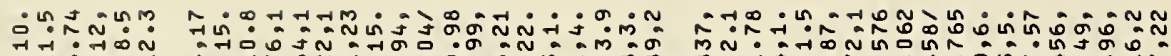

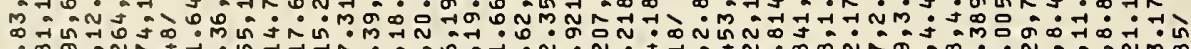
- 0 Noñ

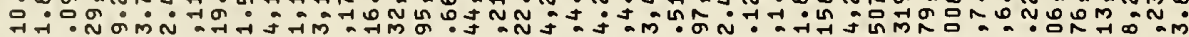

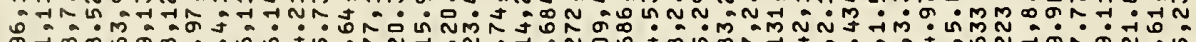

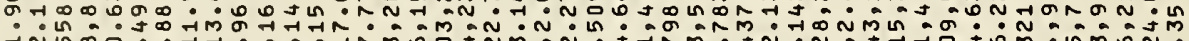
$7 N N_{0}$ m

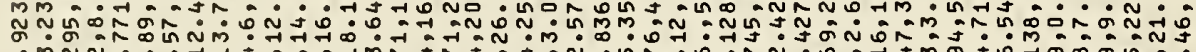

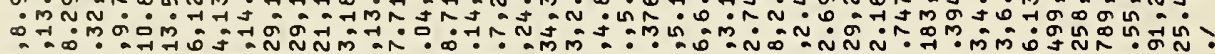

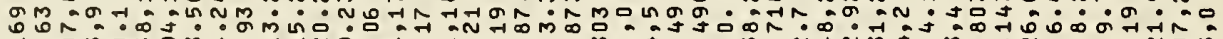

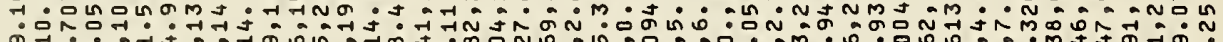

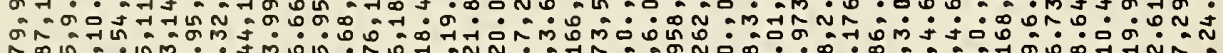
于 Oํ. J F 0.

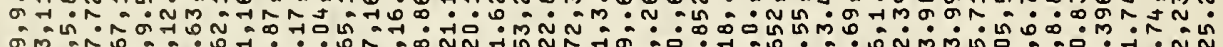
o

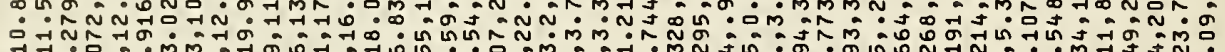
ก⿻上丨 مळ0 ๑N

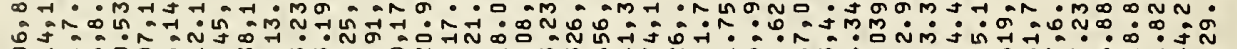

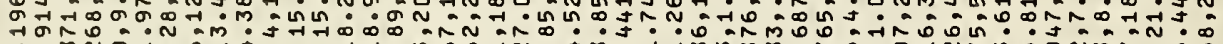
ó.m. 00

Ha. 안아유은 웅요

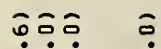

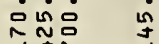

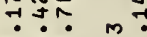

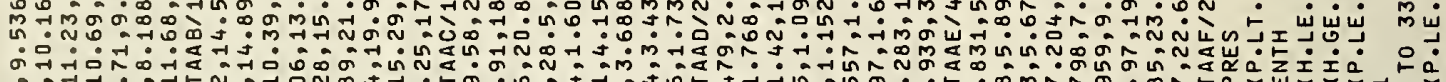

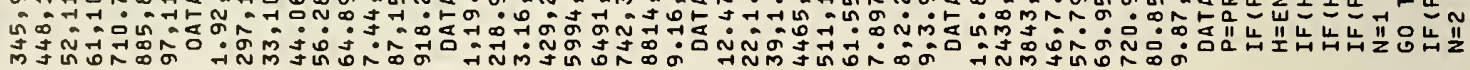

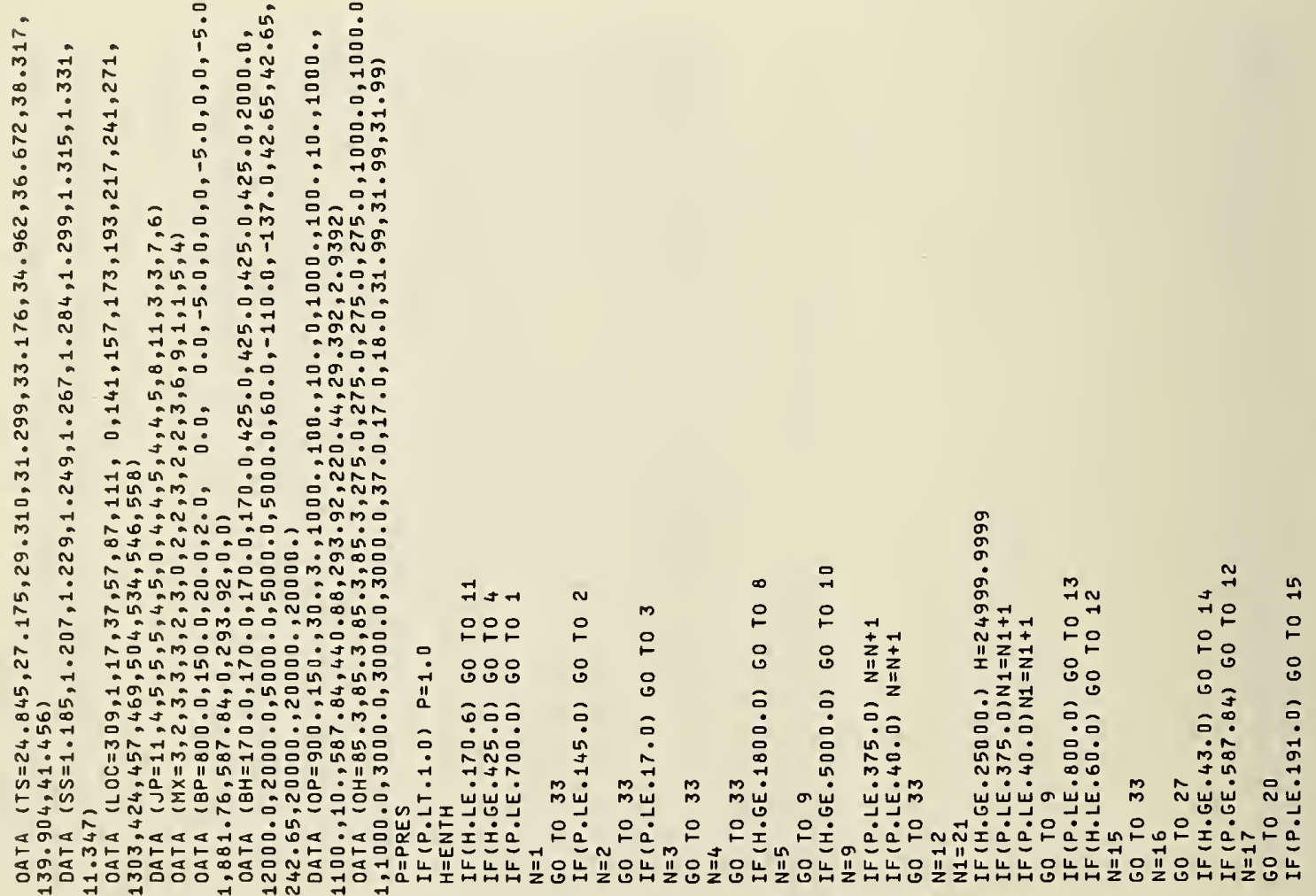



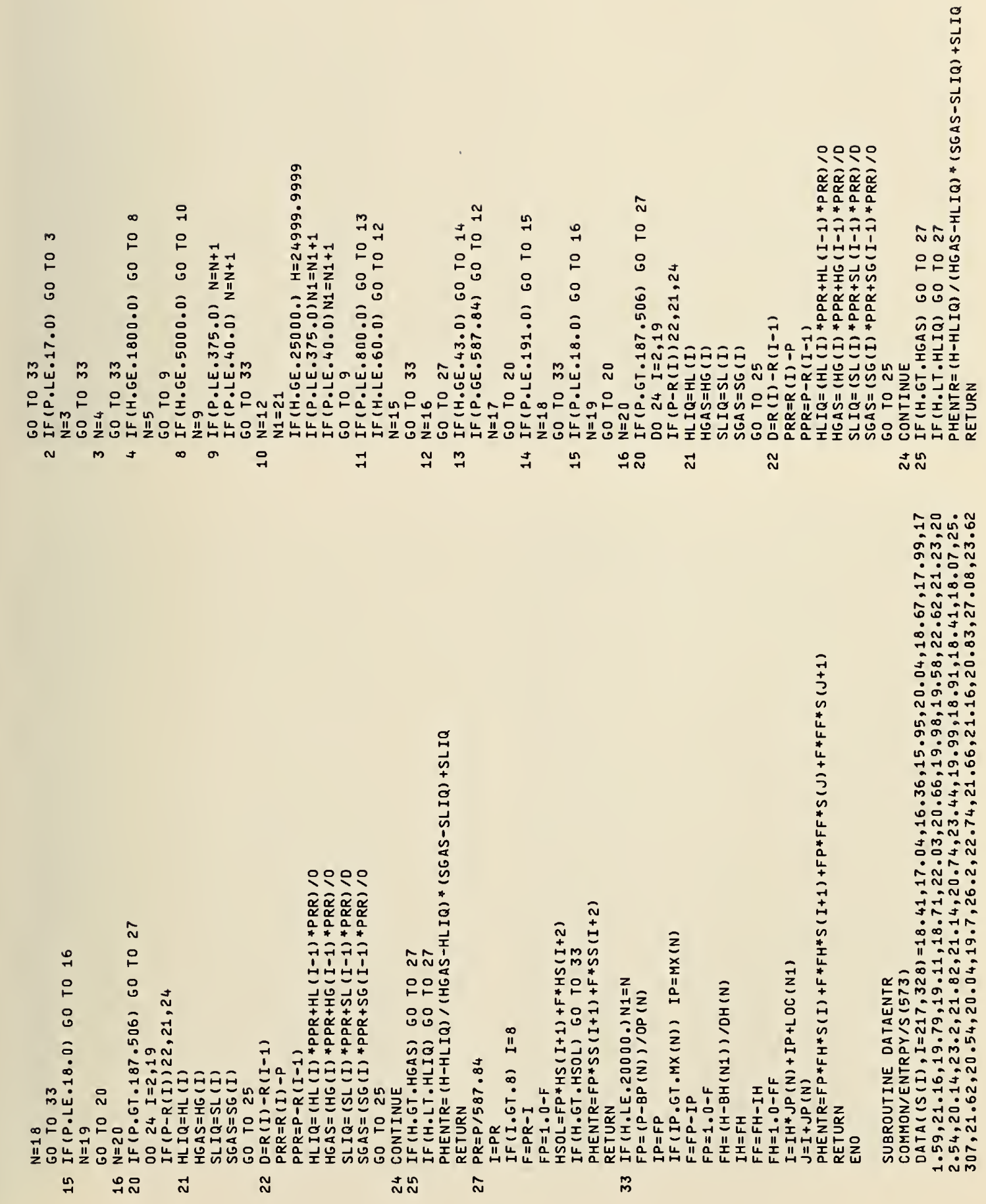


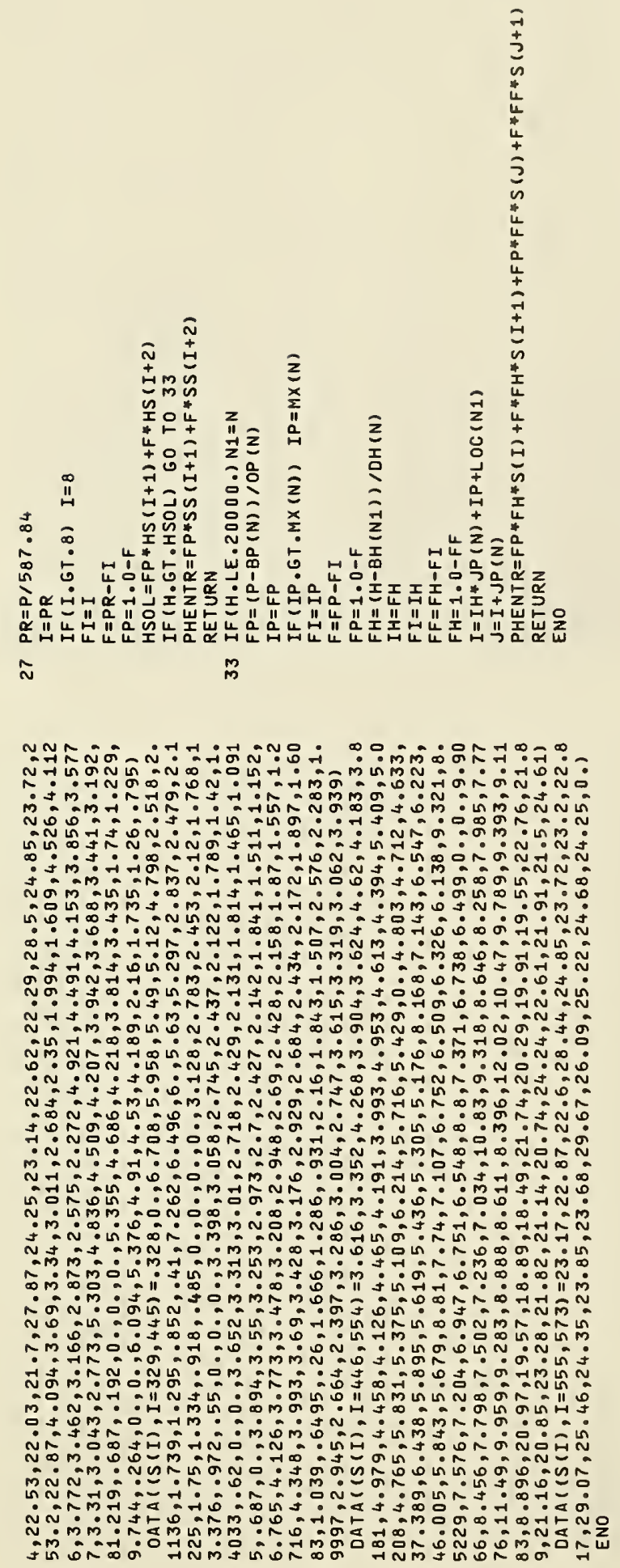


: ㅇ: :

尔

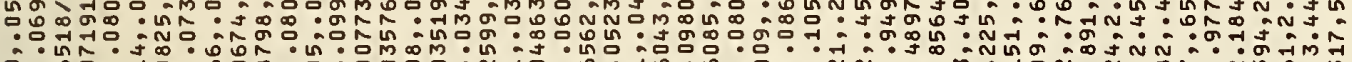
o : mo: 매: No

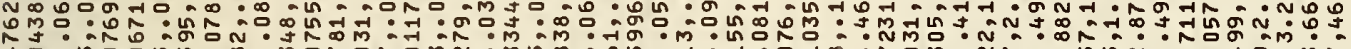
문

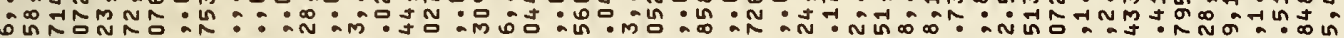

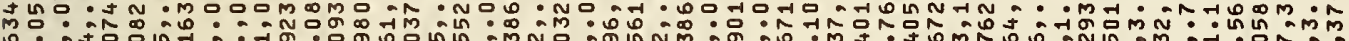

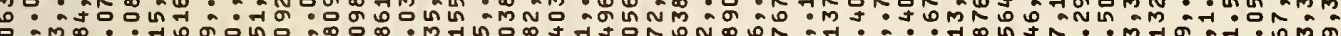
: N N n

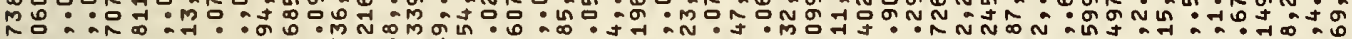

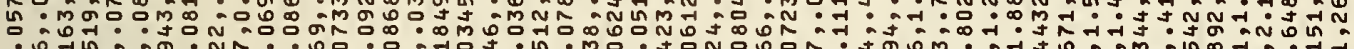

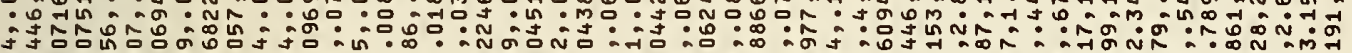
क : :

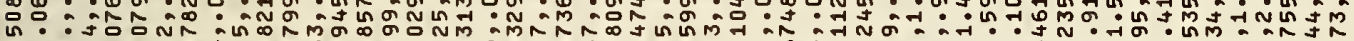

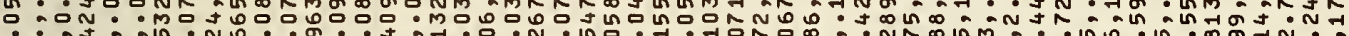

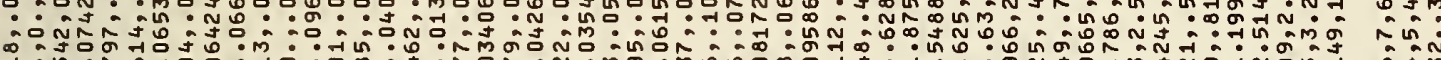

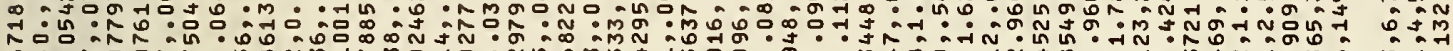
: :

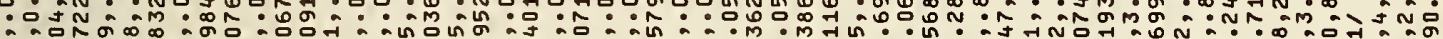

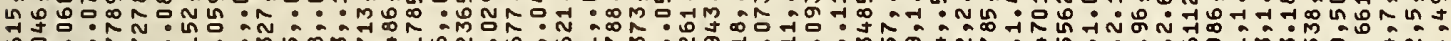

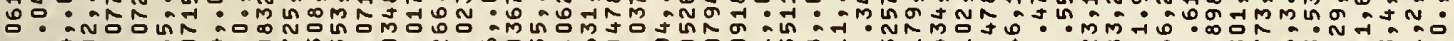
: 告

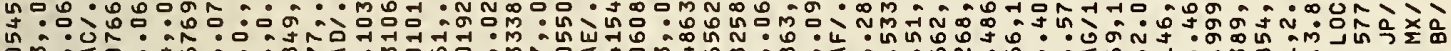

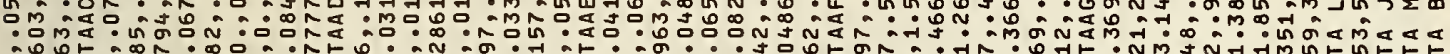

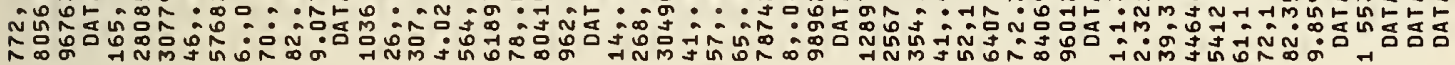

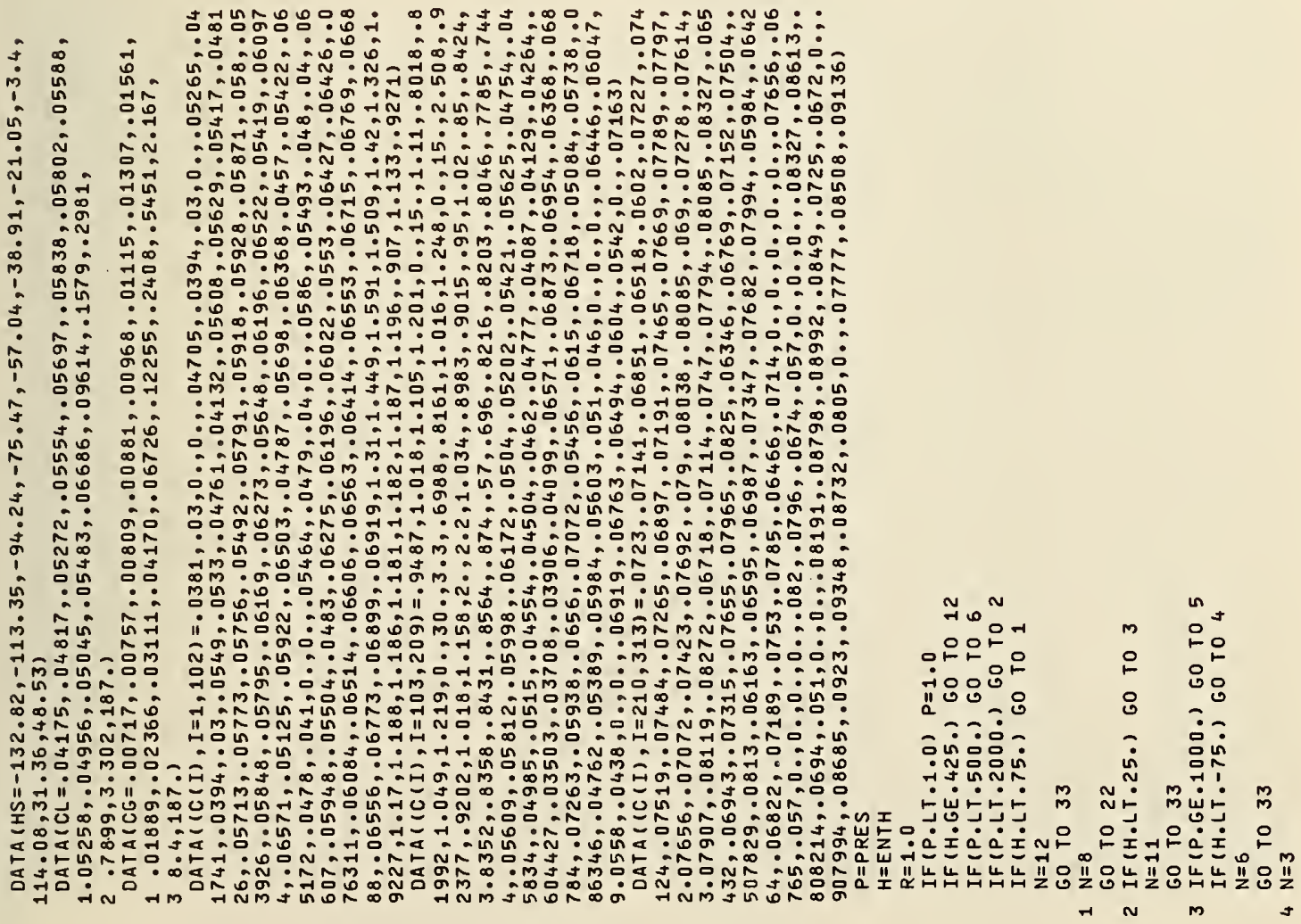




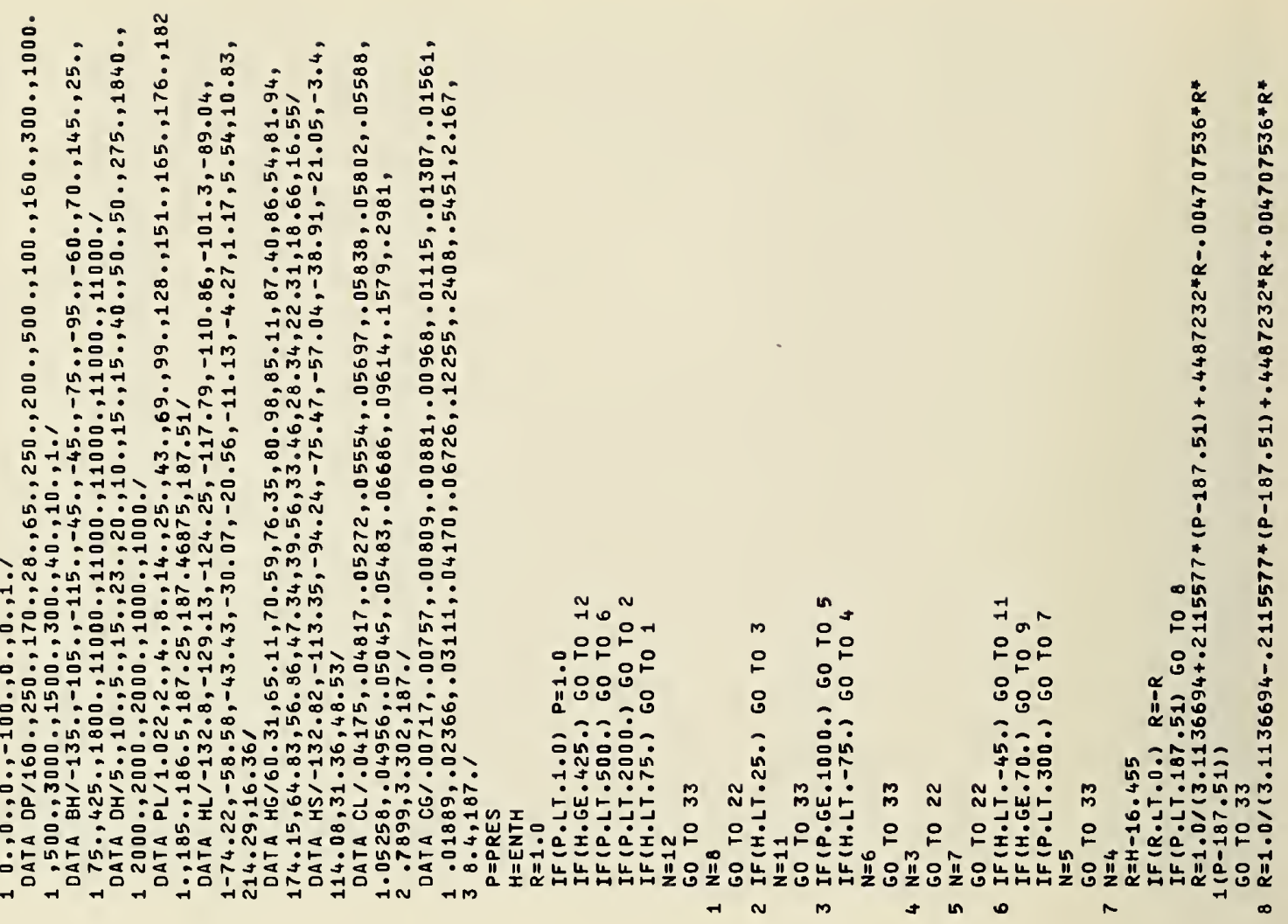

N N

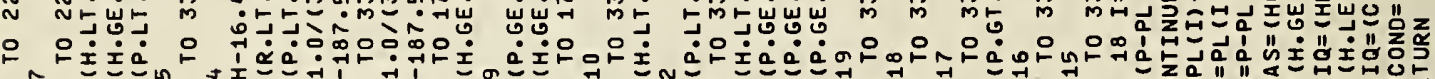

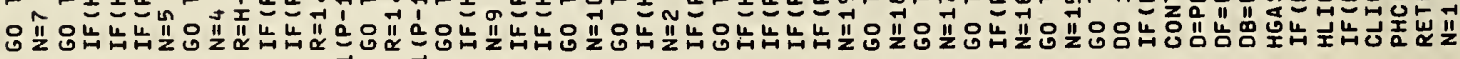
人 


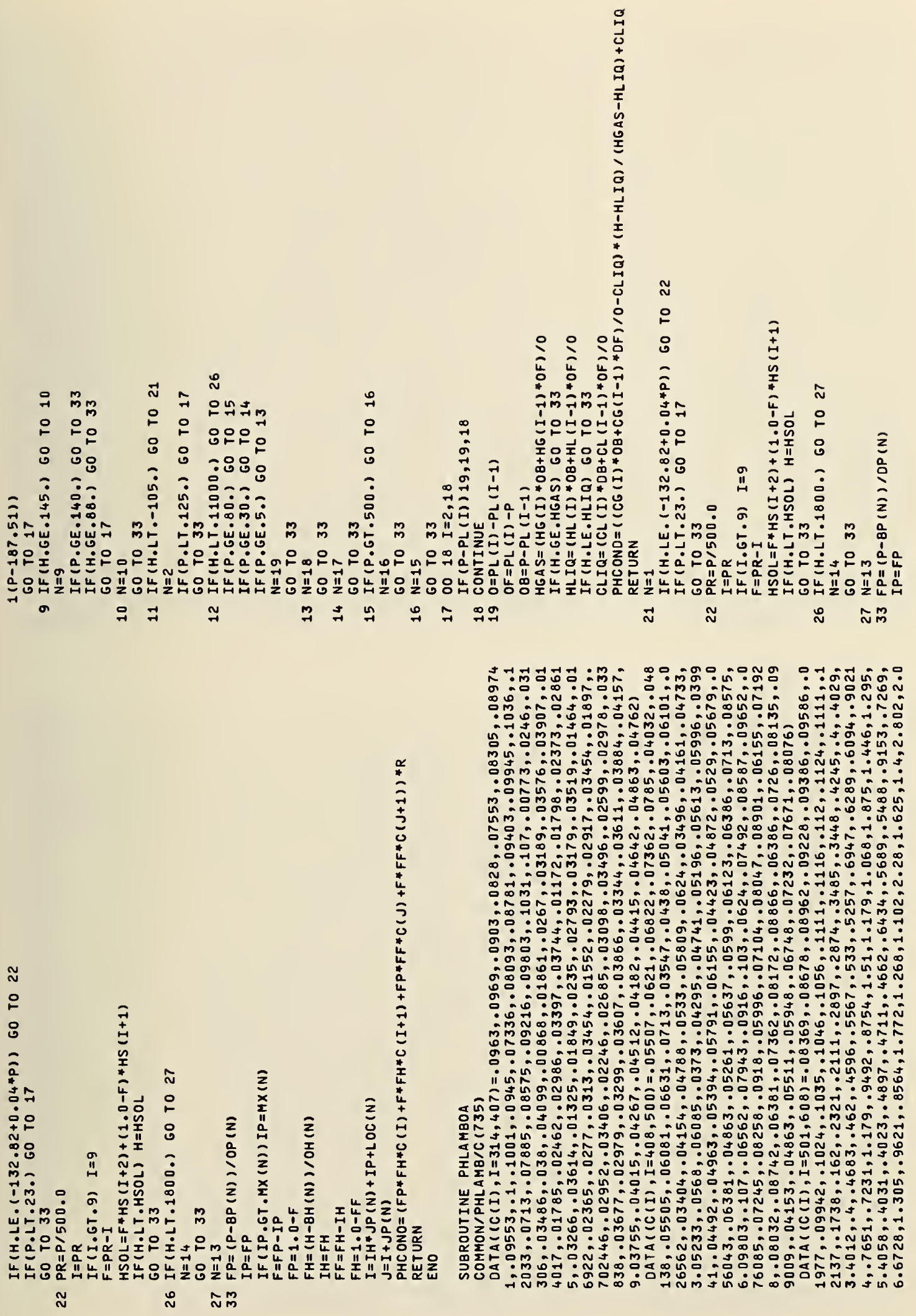




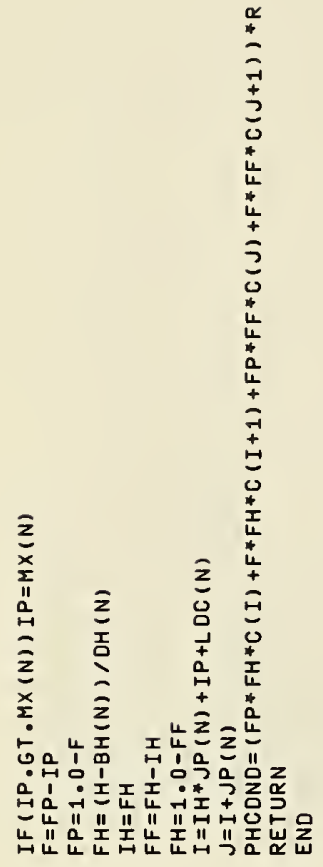

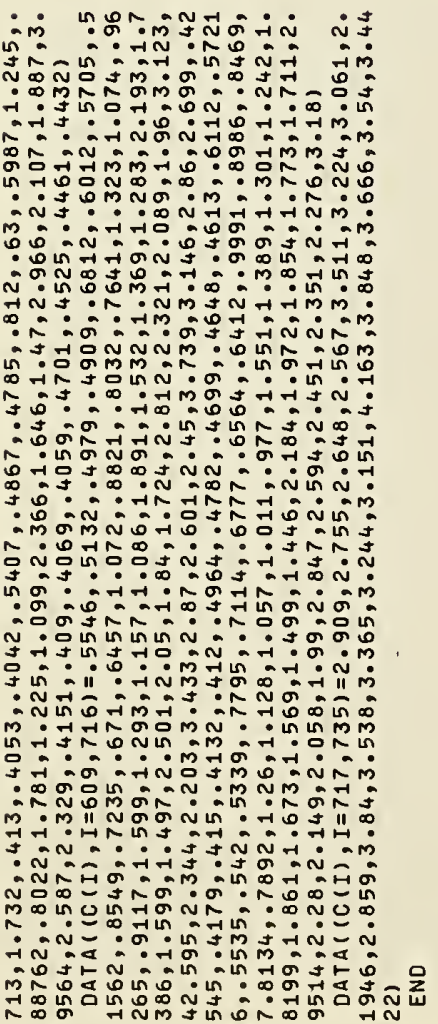

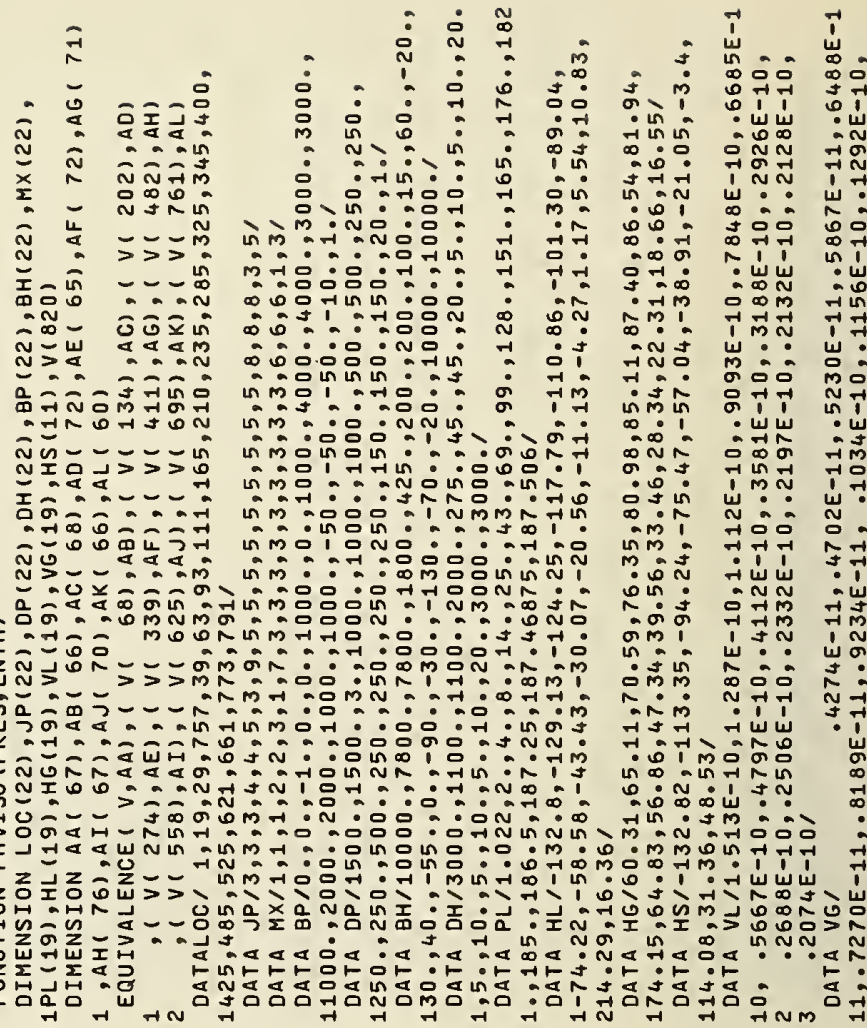

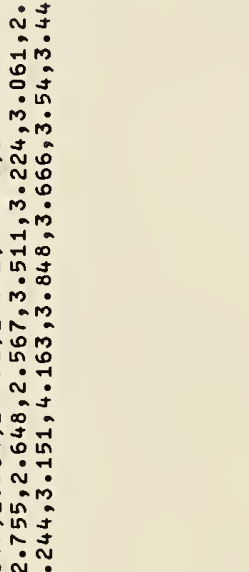

妾

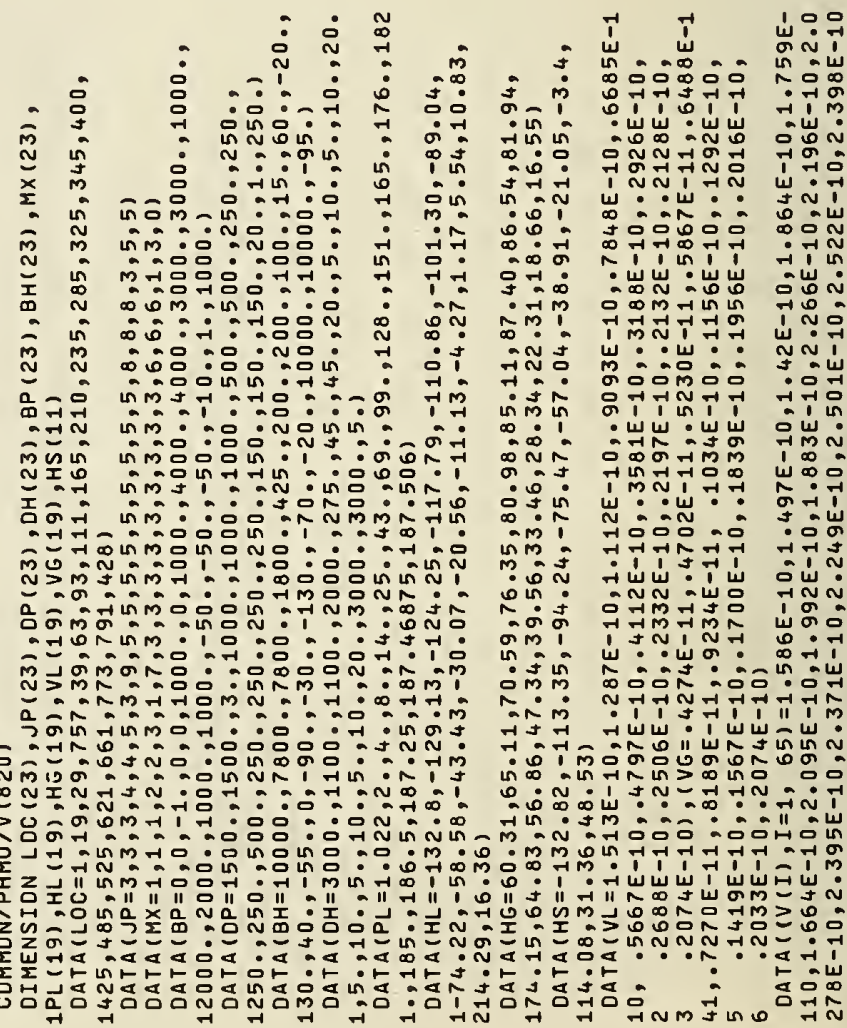




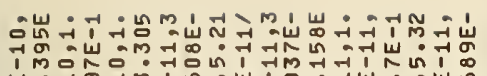

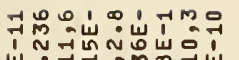

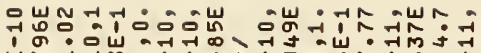

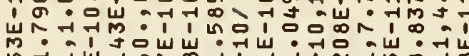

고ำ

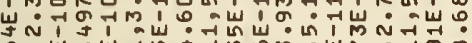

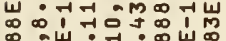

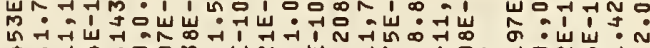

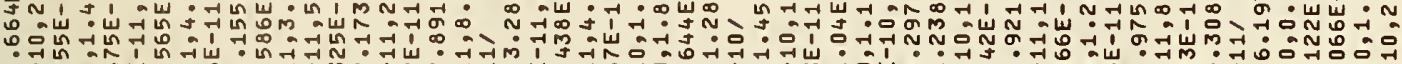

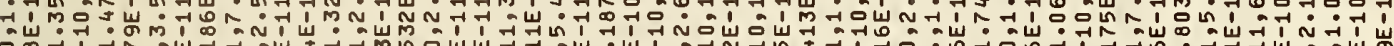

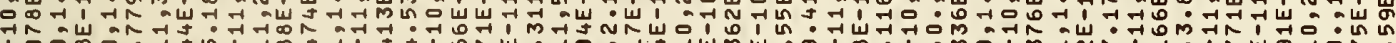
$\overrightarrow{1} 0$ 崖。. 㟶

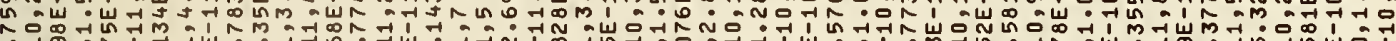

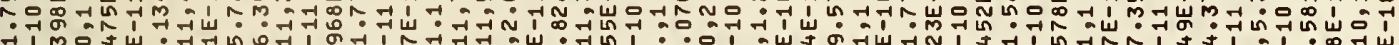

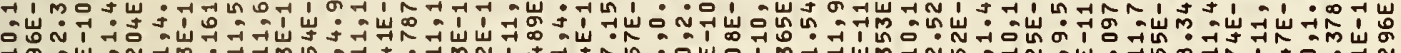
†

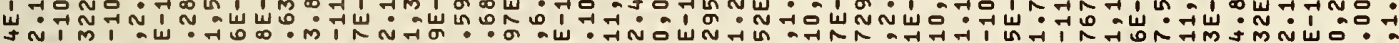

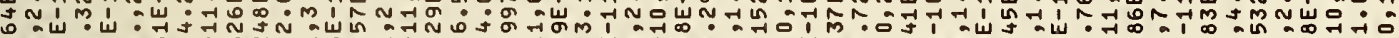
: 전

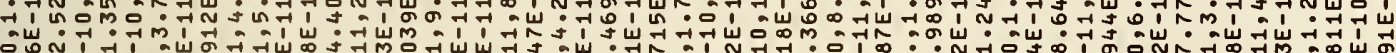
o

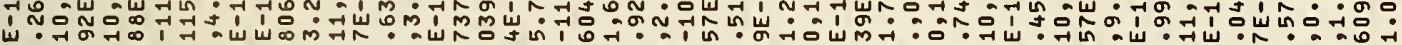

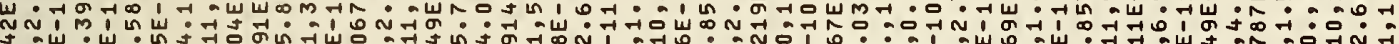

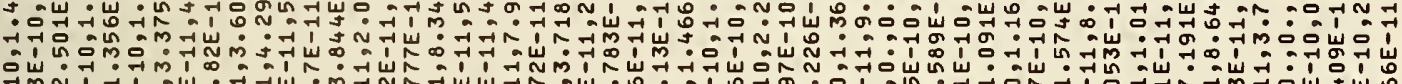

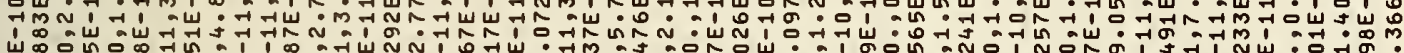

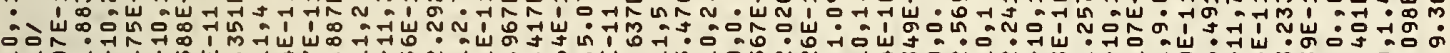

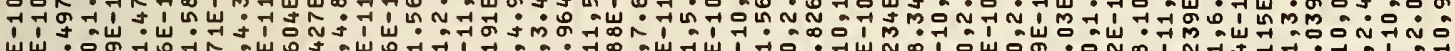
w山

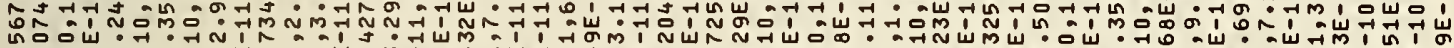

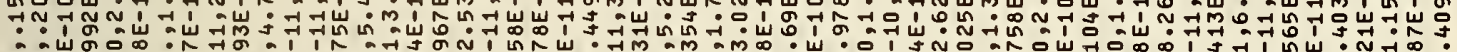
م。

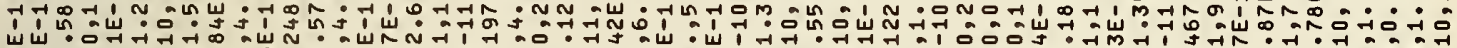
س山m

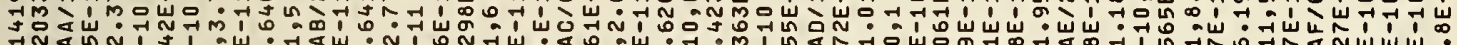

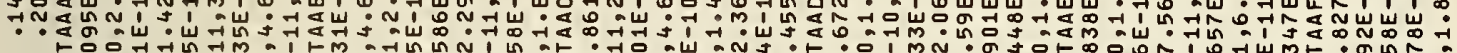

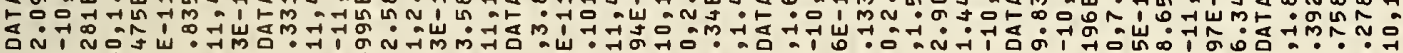

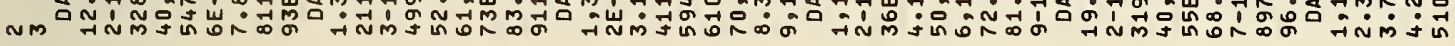

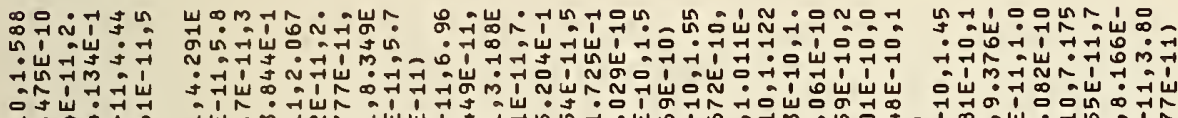
1.

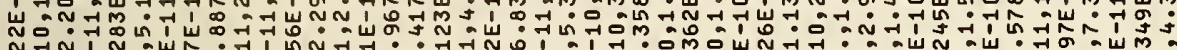
N -

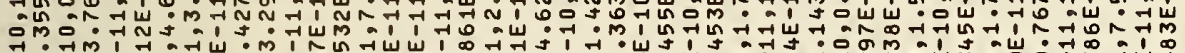
1.

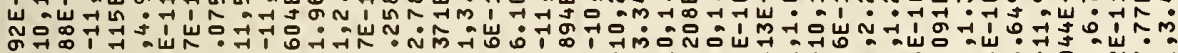

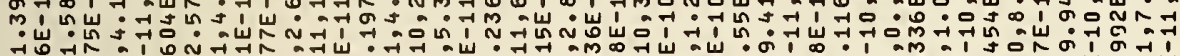

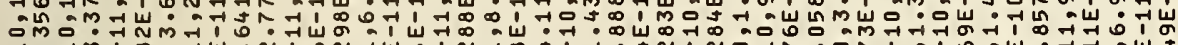

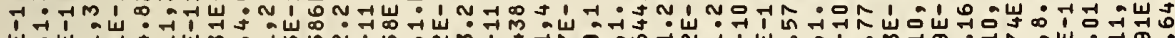
wo 于

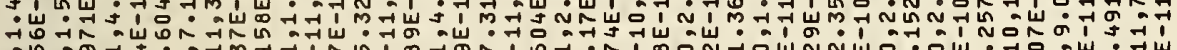

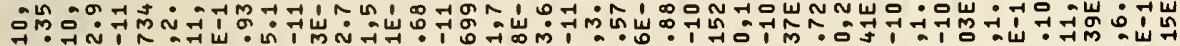

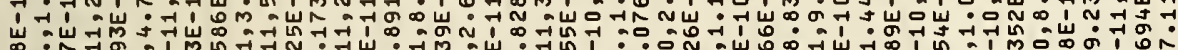

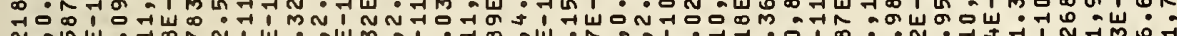

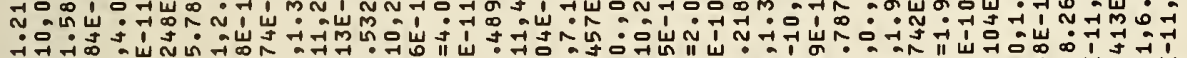

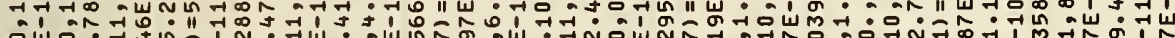
Ó 1 N

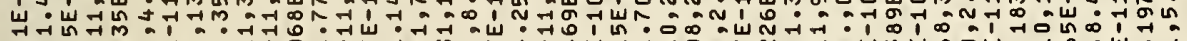

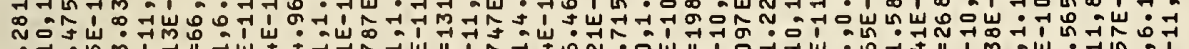

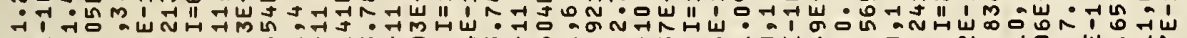

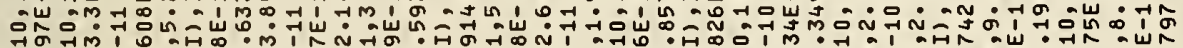

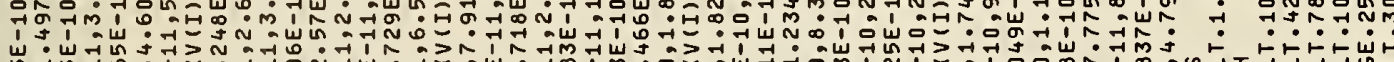
溯出

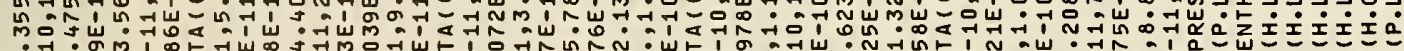

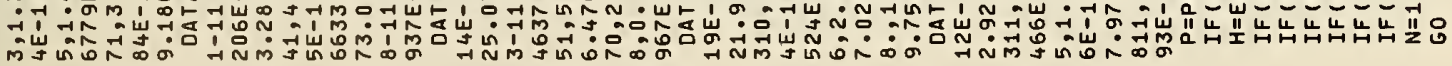




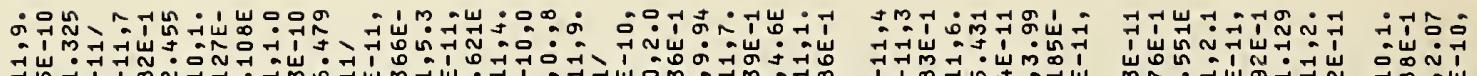

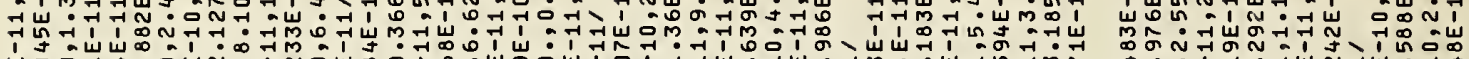

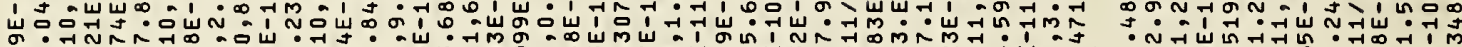
न

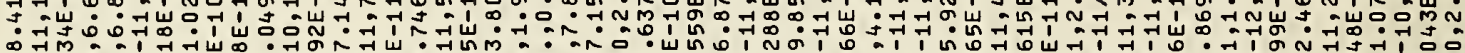
0.7

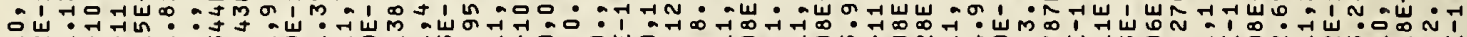

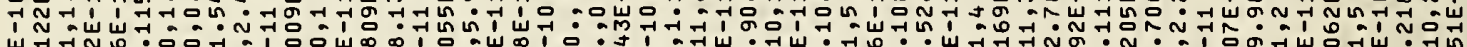

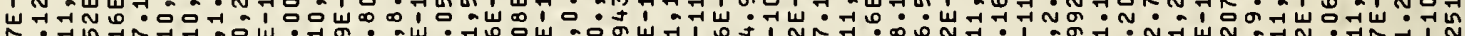

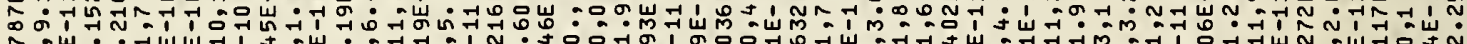

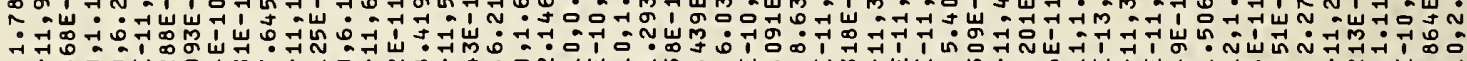

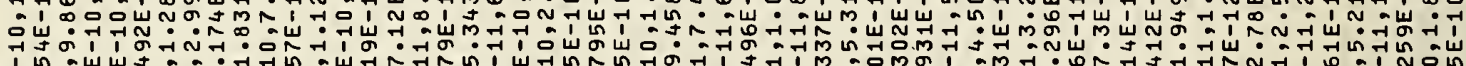

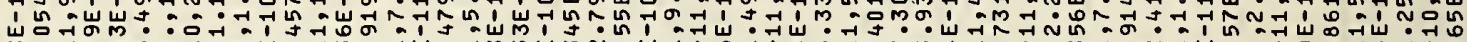
蛋

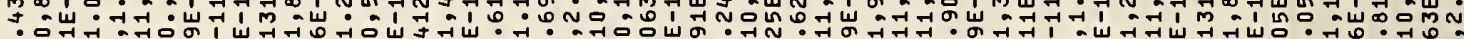

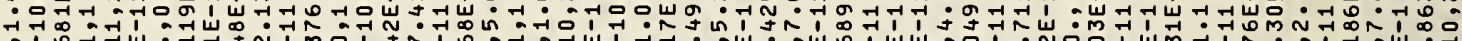

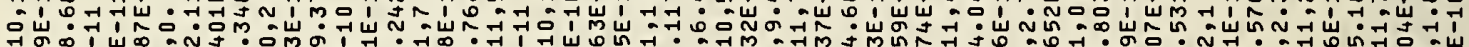
$7 \sigma \infty 1$

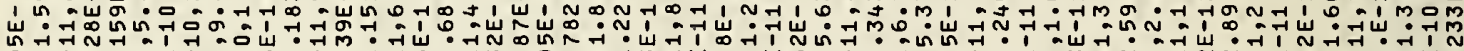

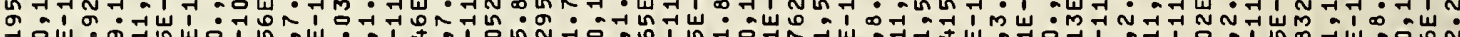

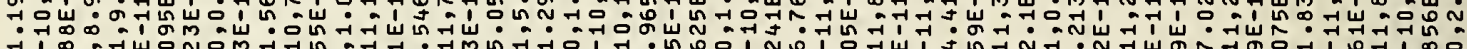
向向 न

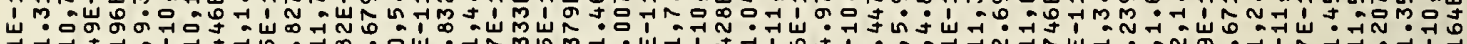

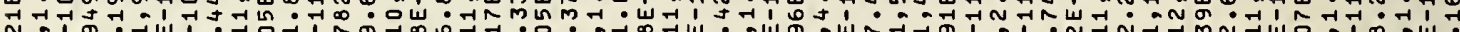

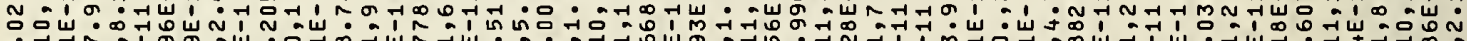

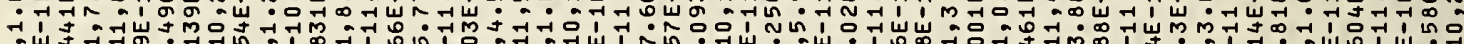

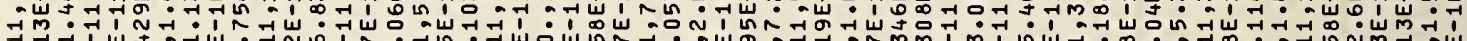

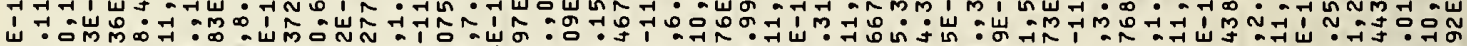

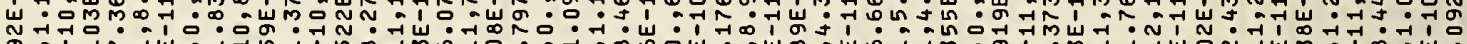

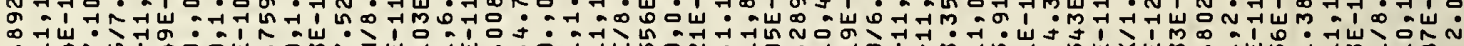
: 出

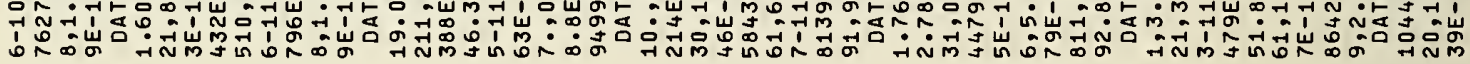

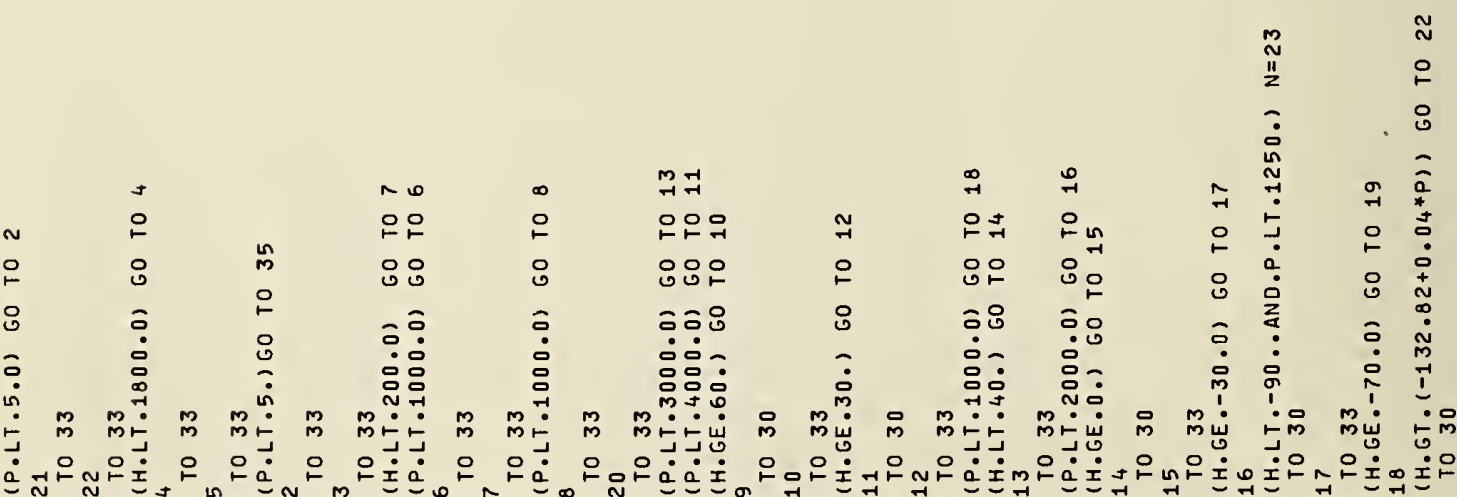

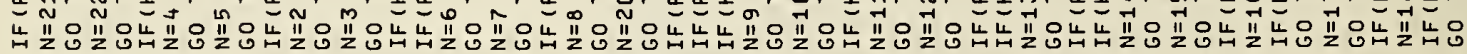

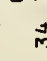

in $n$

\begin{abstract}
$\infty \sim \infty \sigma$
\end{abstract}

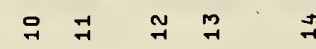

100 
岀品岂岂

क्नित

กั०ूँ

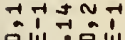

1

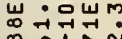

ก๊ำกับ

ㄴำㄴำ

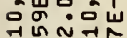

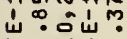

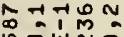

的崖

궁ำ

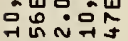

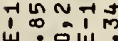

蕴

.

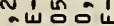

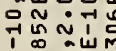

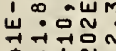

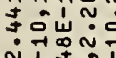

i

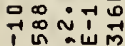

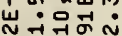

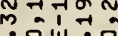

$\because \frac{1}{1} \dot{0} \div$

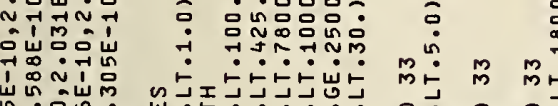

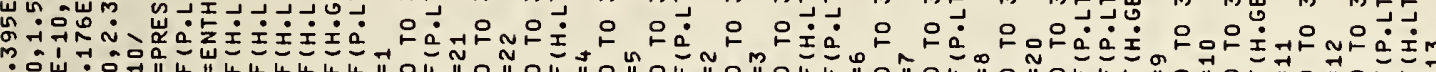

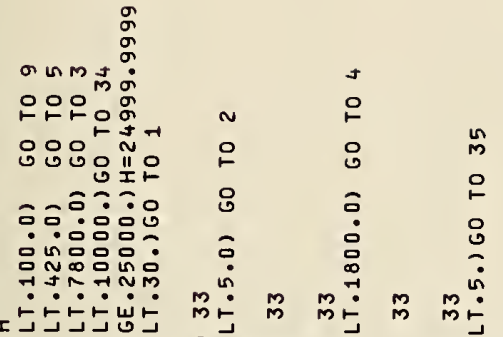

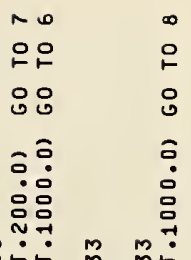

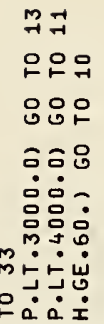

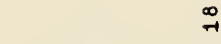

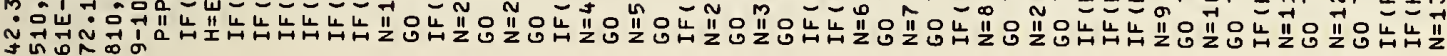
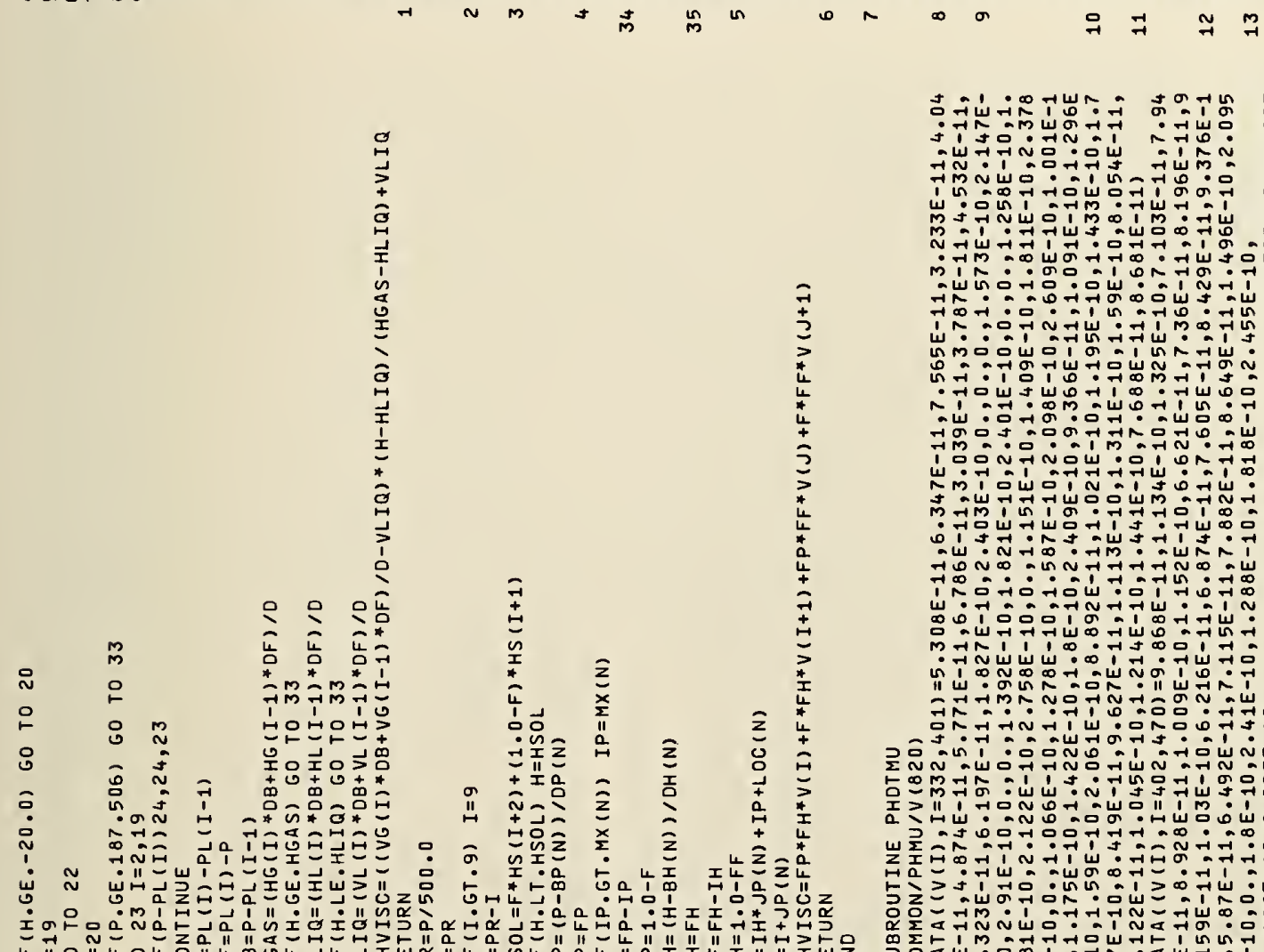

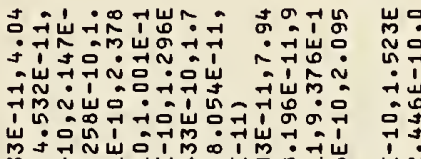

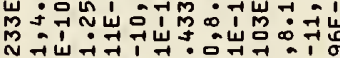
㟧 - निल्क

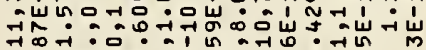
药

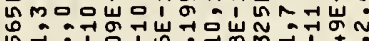
ڤ

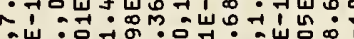

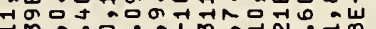

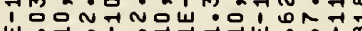

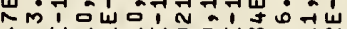
जै न जन

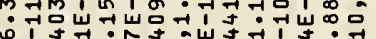

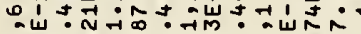

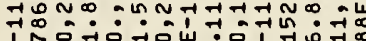

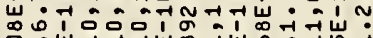

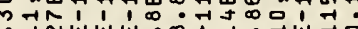
준

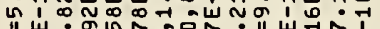

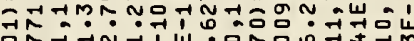

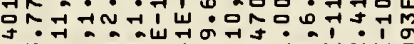

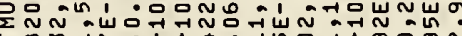

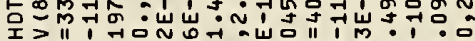

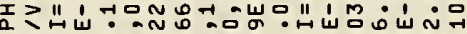

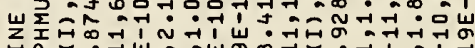

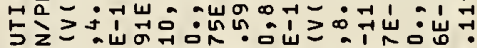

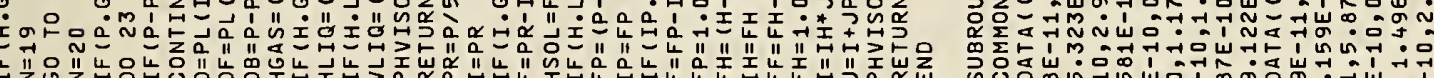

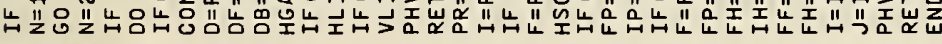

요 $\stackrel{M}{\sim}$

吕

m 


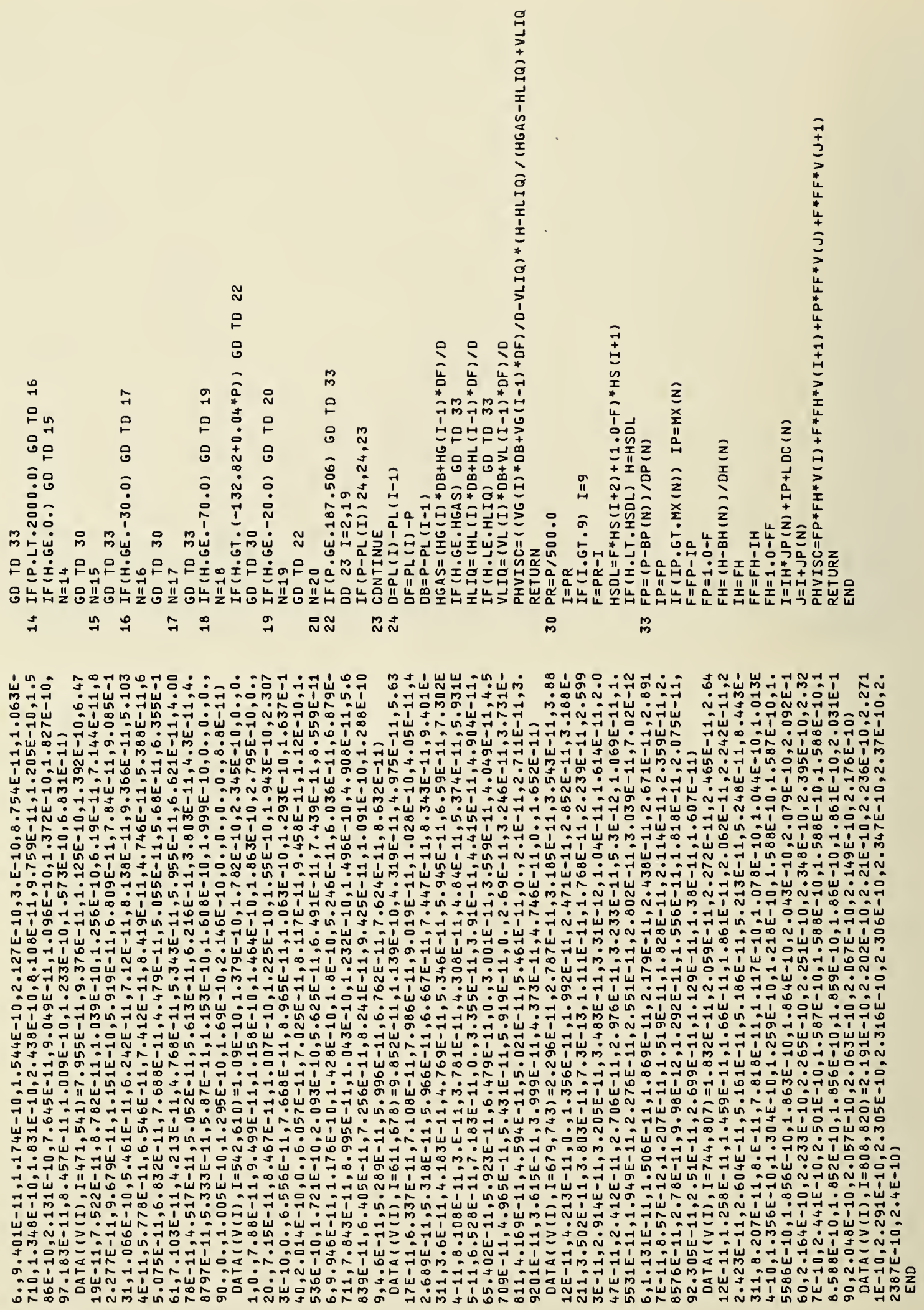



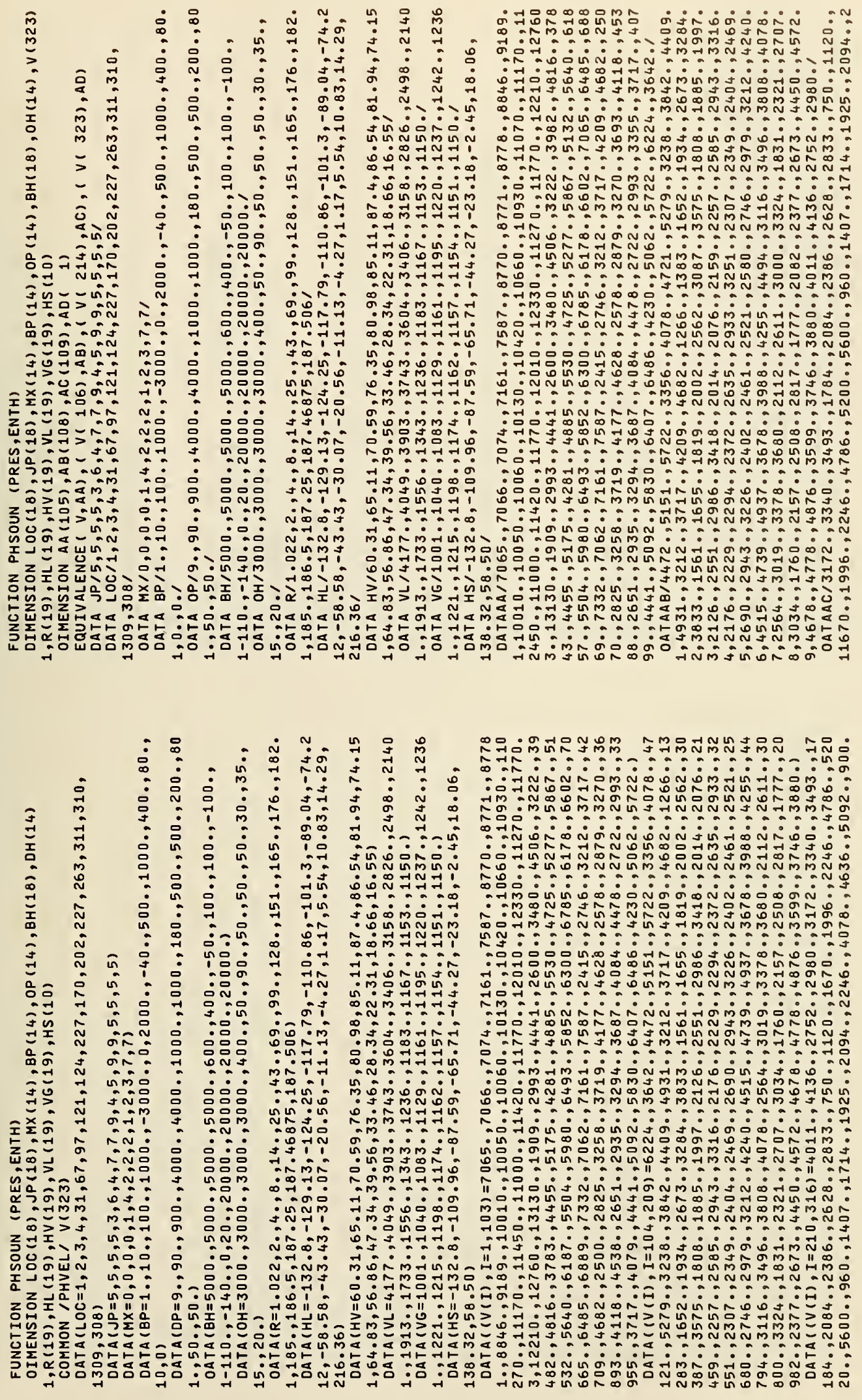


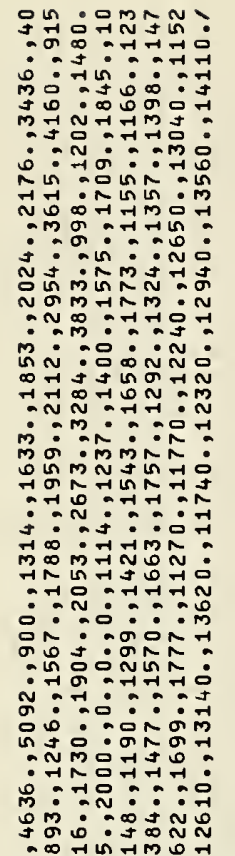

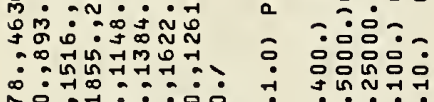

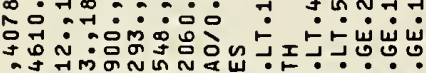

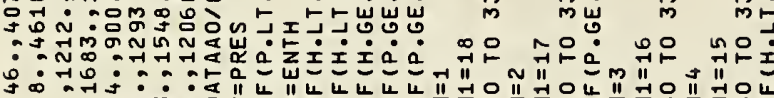
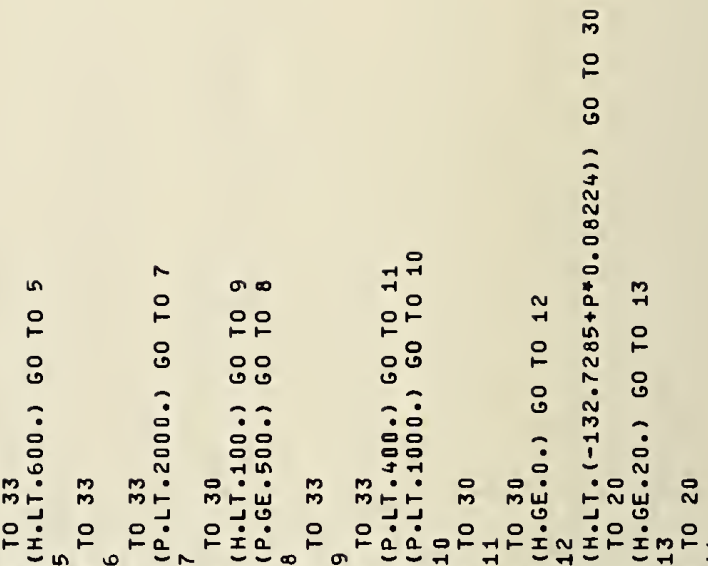

N
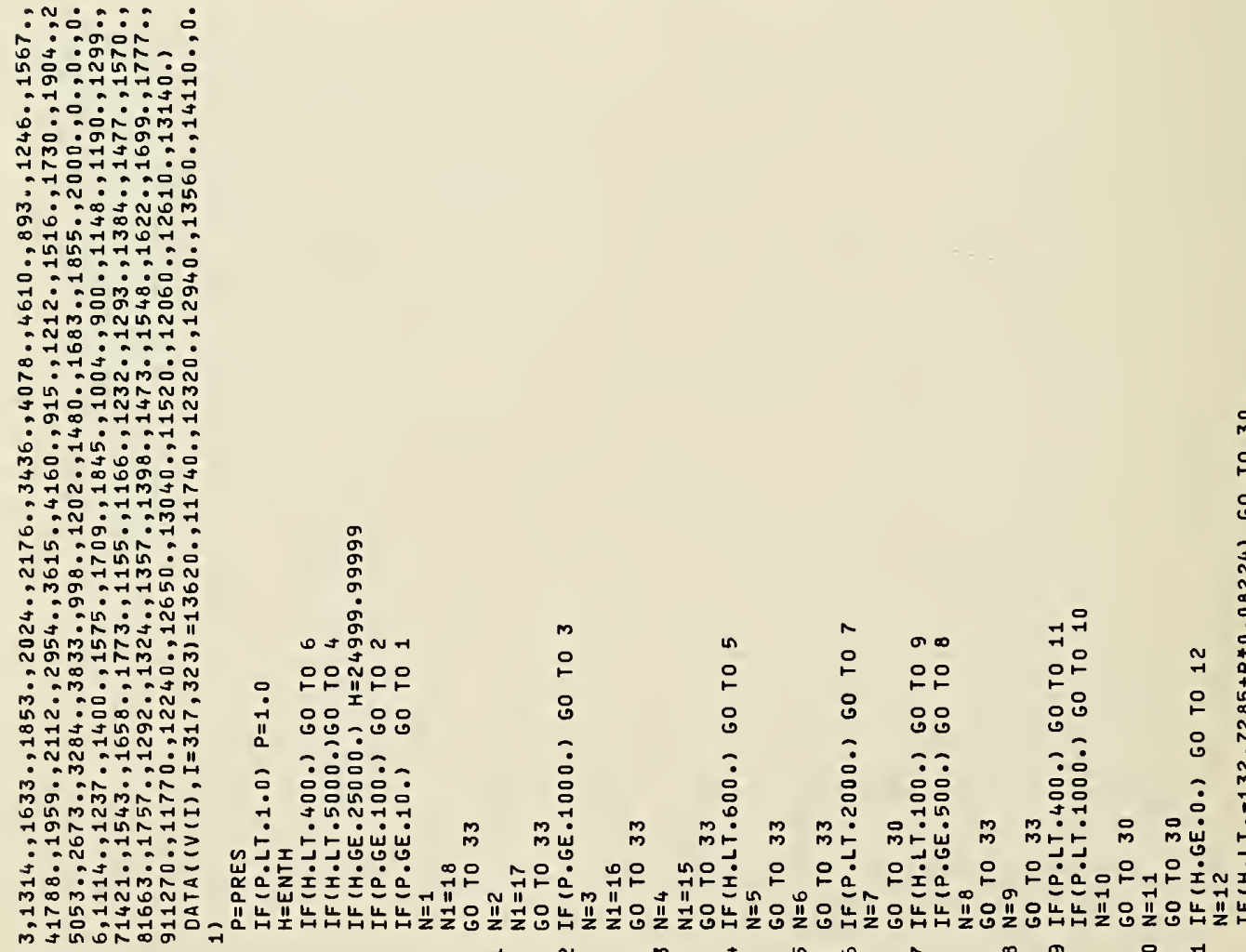

웅

웅

:

व 7 

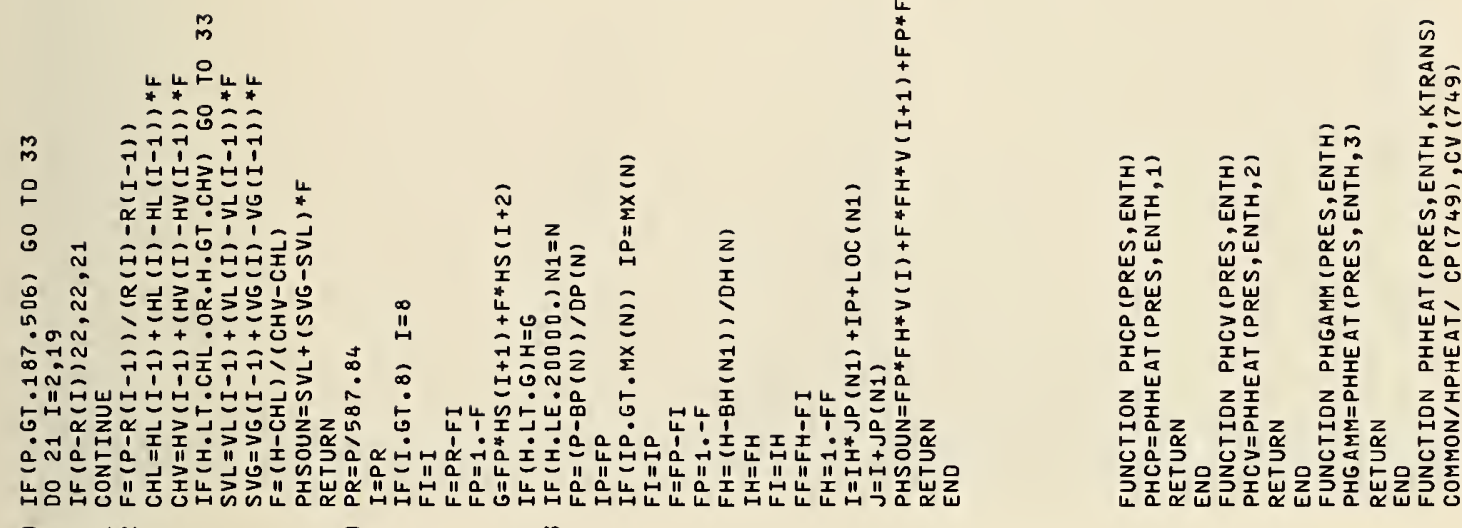

กิ $ส ก$

ले

m

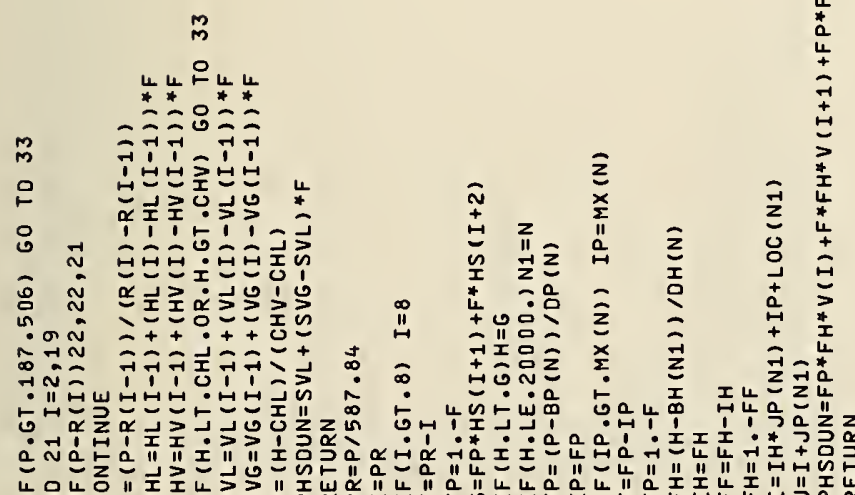

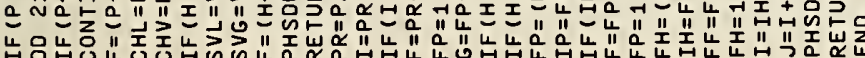

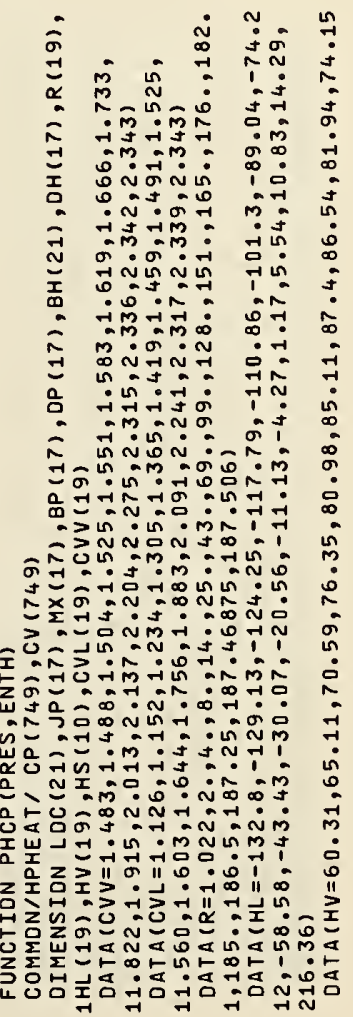

ก $ส ก$

के

in 

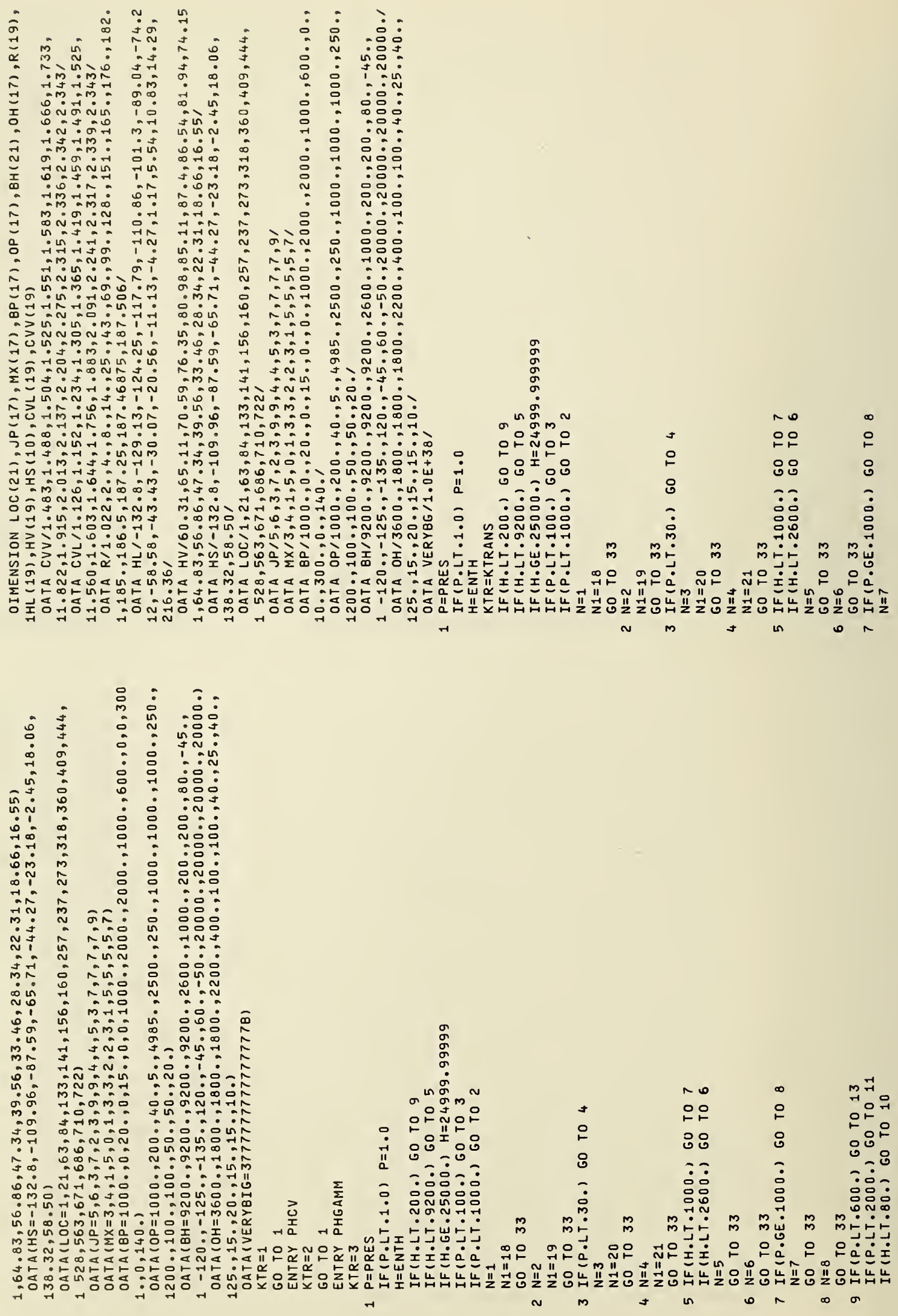

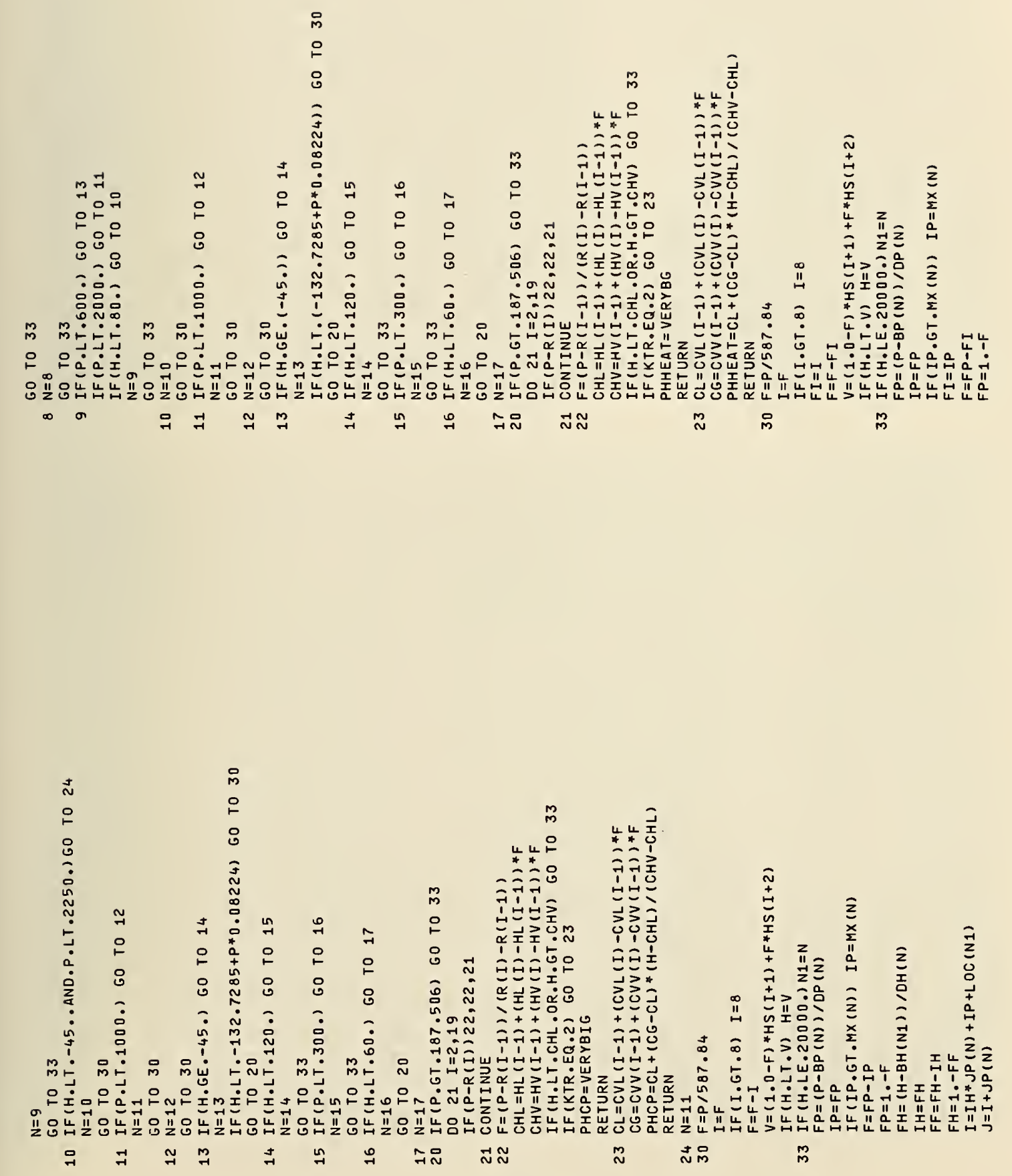


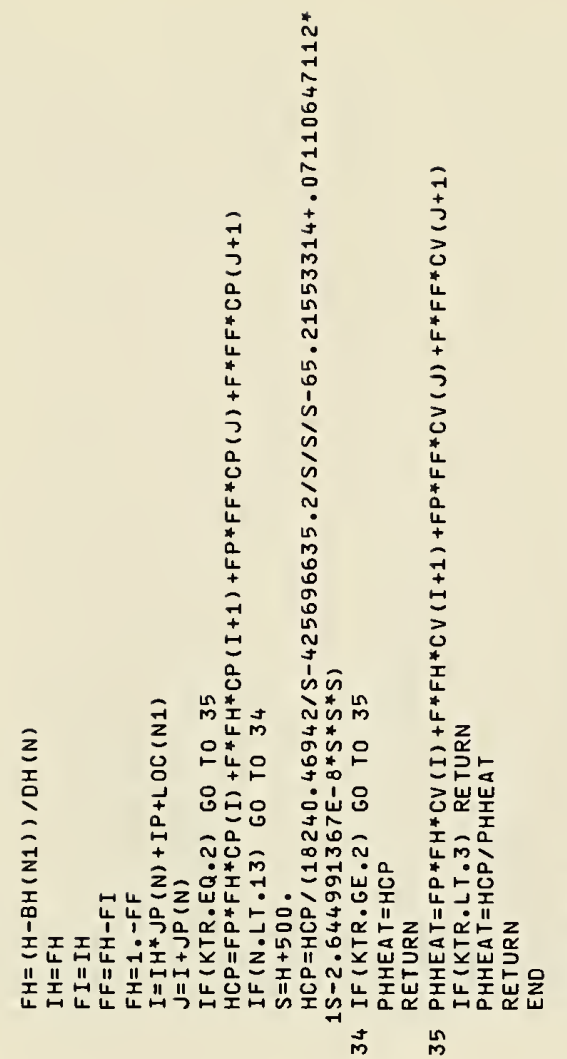

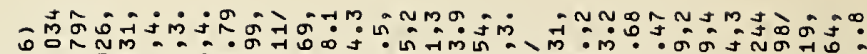
:

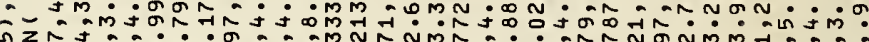

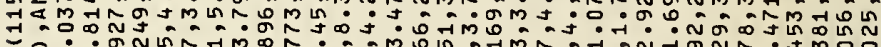

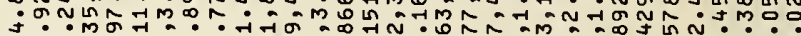
च JM

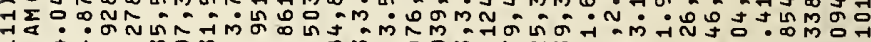
ت J Uन ר L

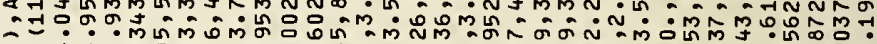

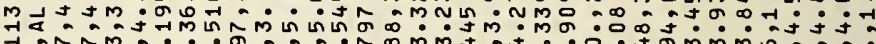

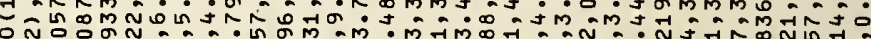

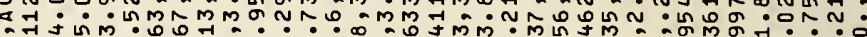
-

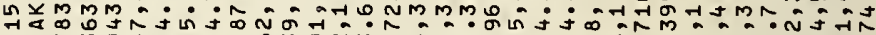

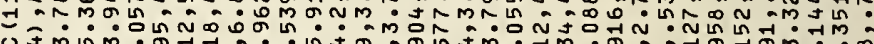
4

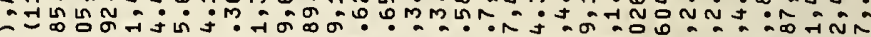
GวRM八 Hám $\dot{m}$ : us m

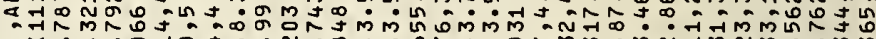

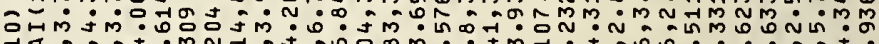
7 二

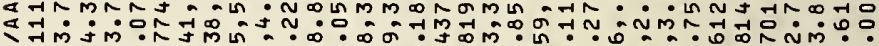
¿

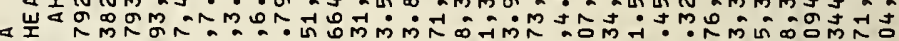

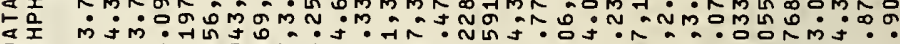

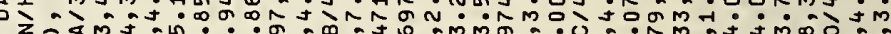

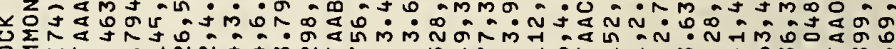

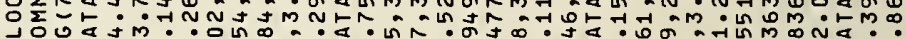

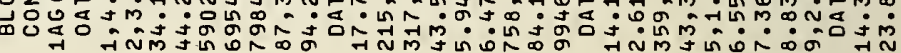
m

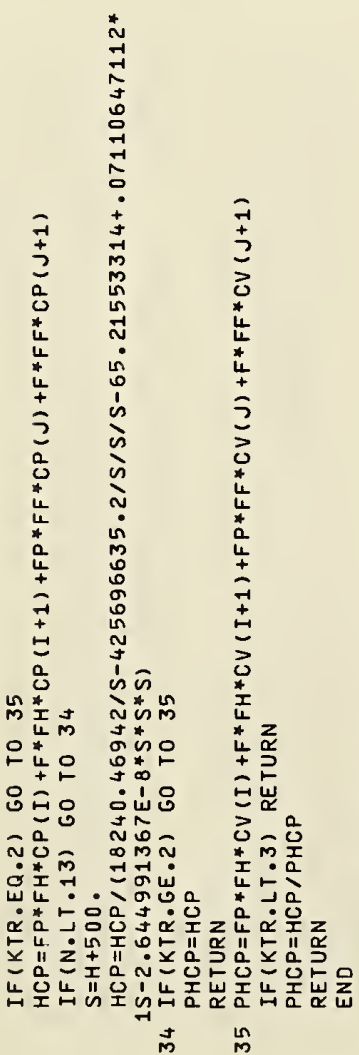

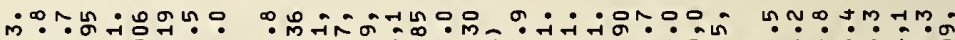
fงm:

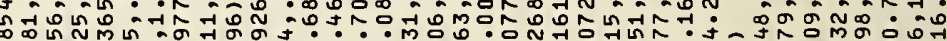

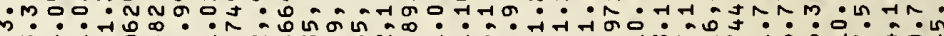
ñ.

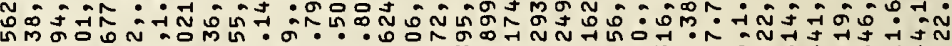
¿m. न Aงง NNก̃์

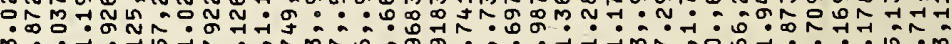
Nं: : mヘ́์: m的

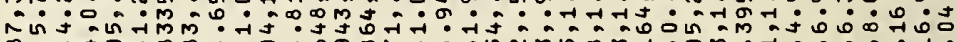

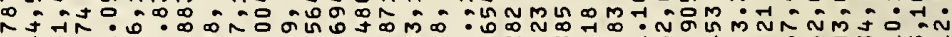

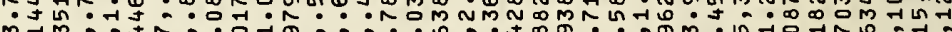
m M⿻

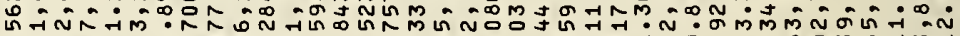
누의

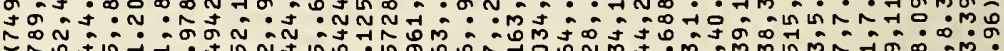

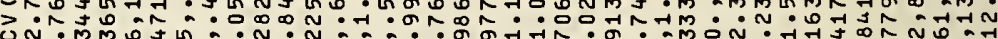

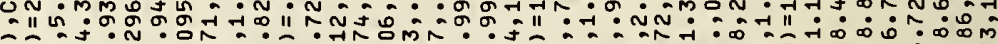

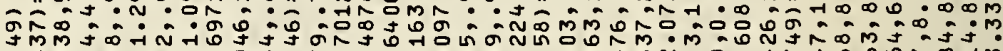

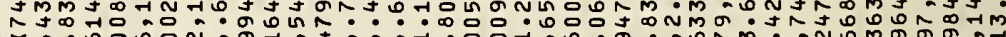

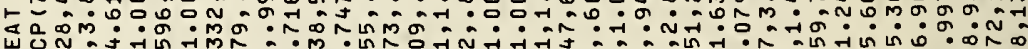

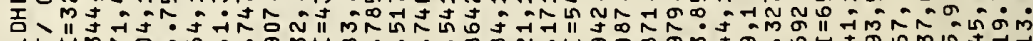

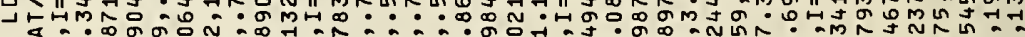

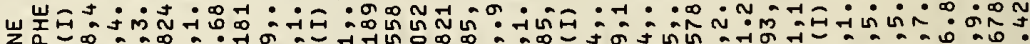

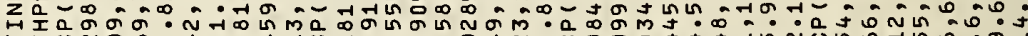

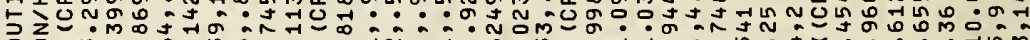
ơ

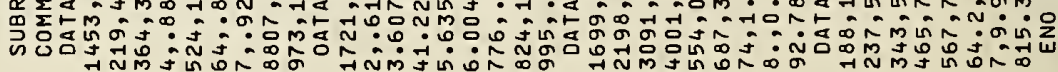


ง 品 : : 0 : N

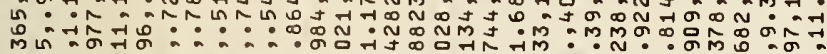

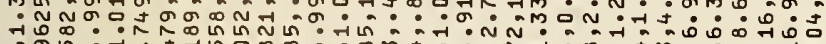

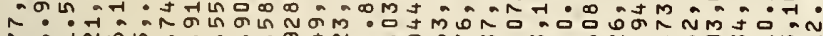
Aิ 0. กิำ:

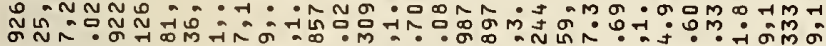

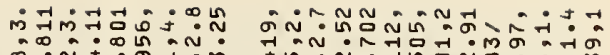

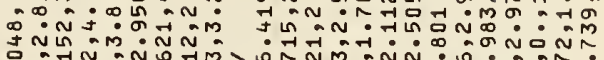

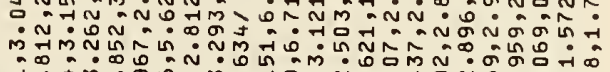
ปี MMNMNNO นกิ นnmám m N : $0-10_{0}^{\infty}$ ô : :N俞: 经 L

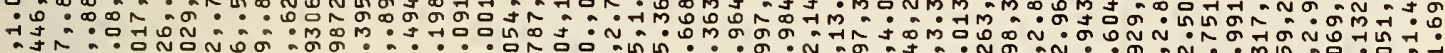
N $\because-100$

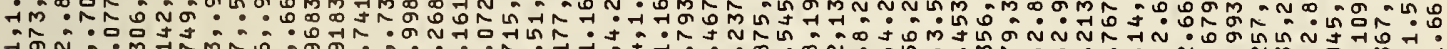
न人̃

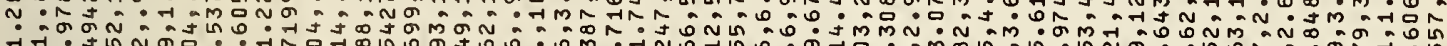

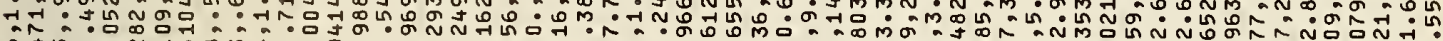

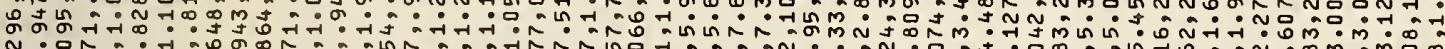
*: :

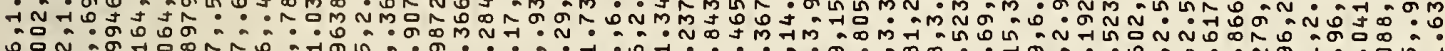

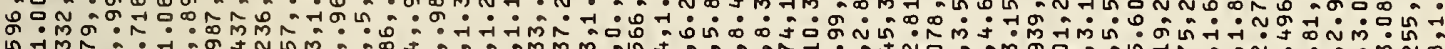

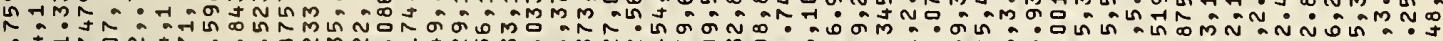

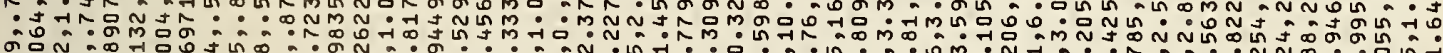
OP N

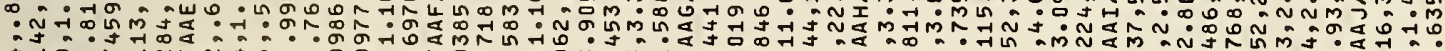
ป๋

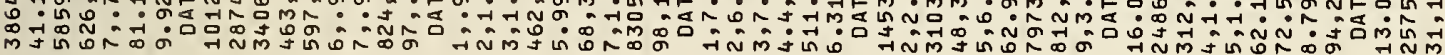

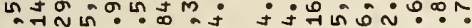
क人

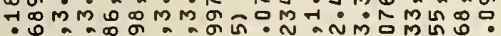

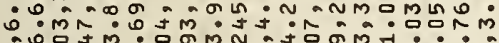
molo

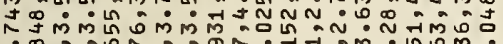

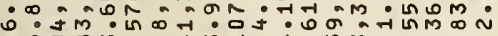

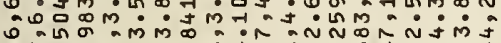

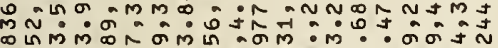

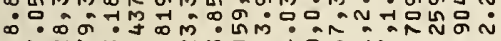
च ?MmM ษMำง N

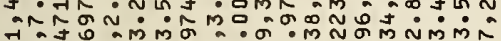

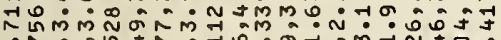

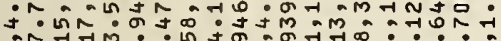
mं न 人ó:

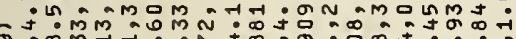

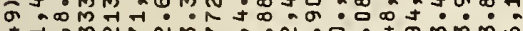

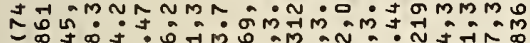
ठ -

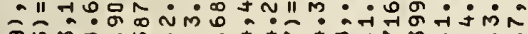

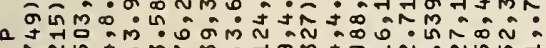
$\bumpeq N$ L

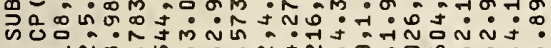
ט二

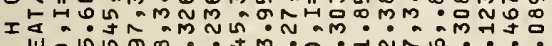

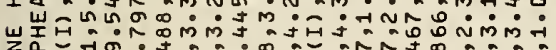

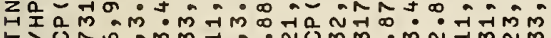

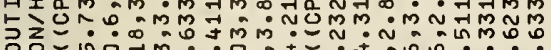

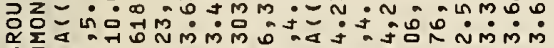

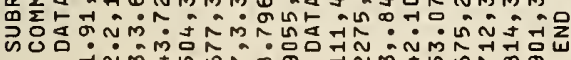

Нनம कीक

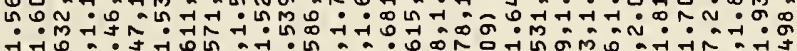
ษ

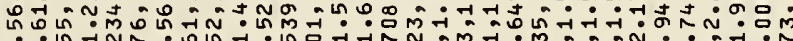

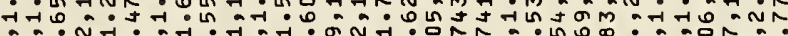

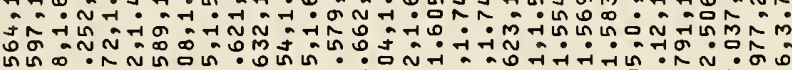

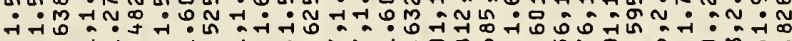

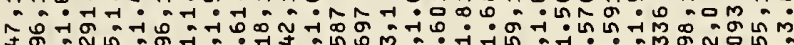
Uึ $\because-1000$

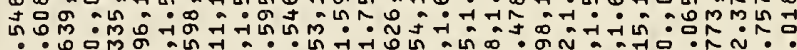

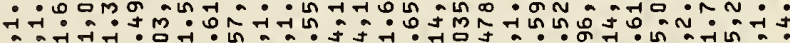

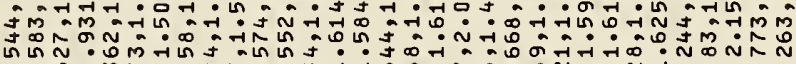

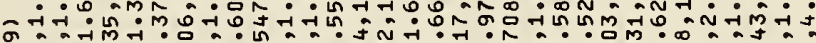
वूलं 乞 ठ A o

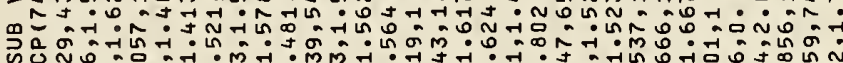
иoNo o⿰

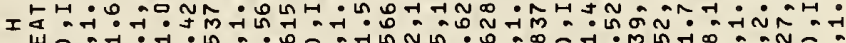

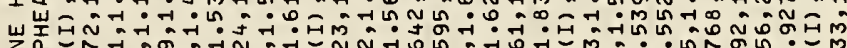

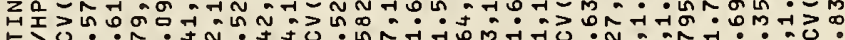

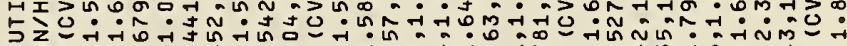
\&은

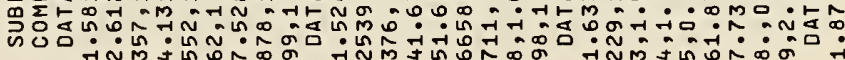




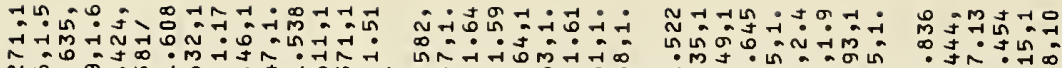

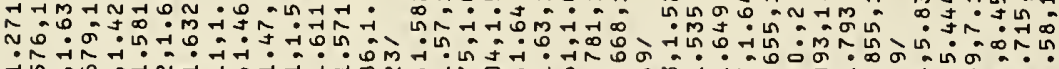

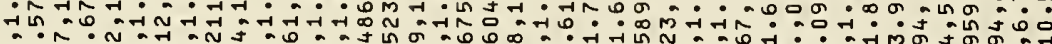

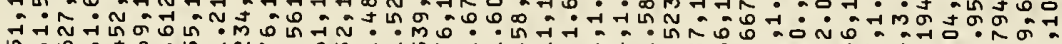

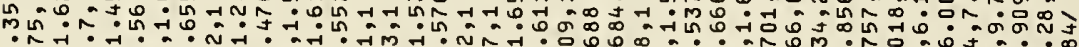
न N

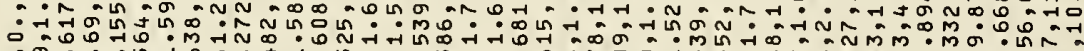

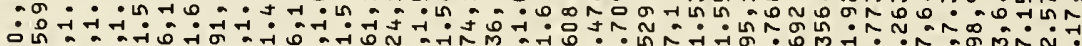

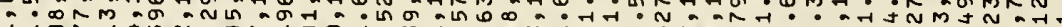

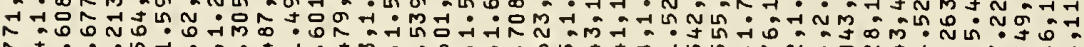

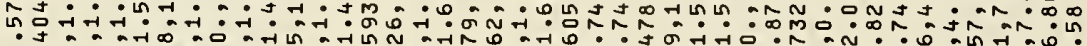
ó

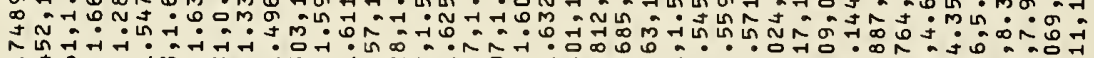
:

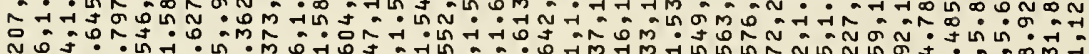
No :

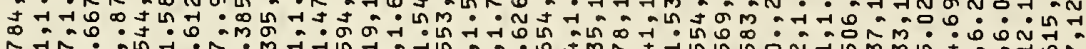

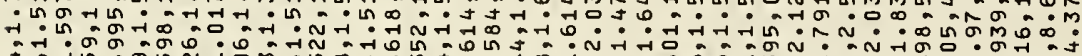
于

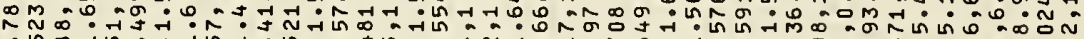
ㄴ. 人̃ón

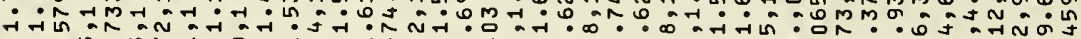
An

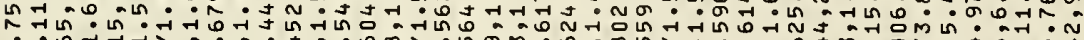
$\therefore-1000$

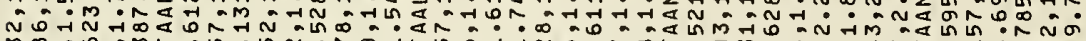

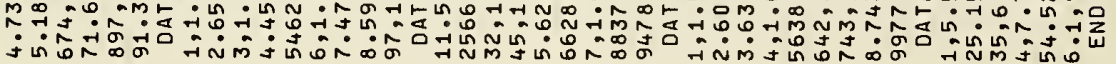

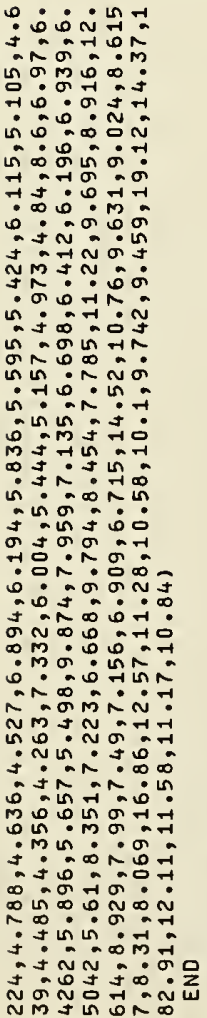

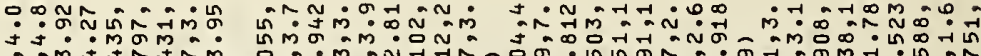
于

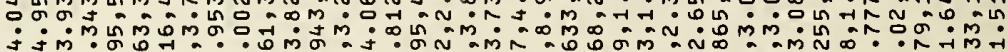
Ám

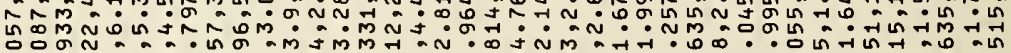

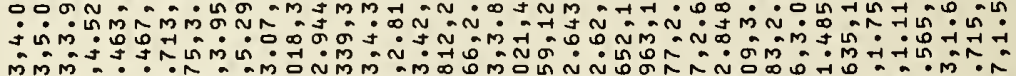

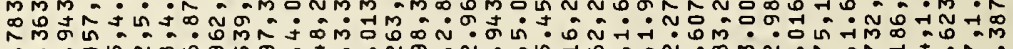

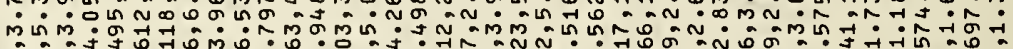

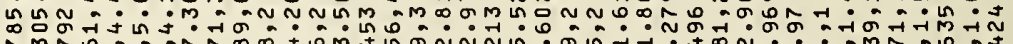
D.m.

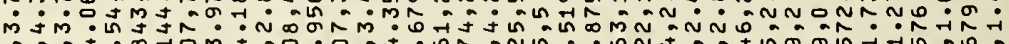

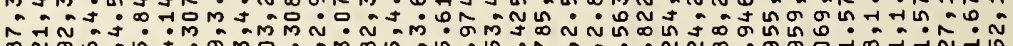
DNo00 m.?. mo. o or స̃Nm 万人oñ ớn. б"1

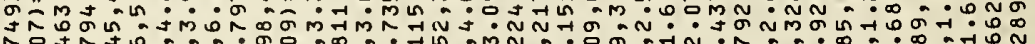
o उऐत于

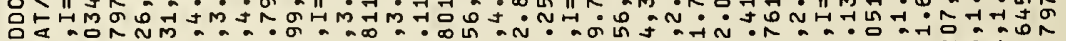

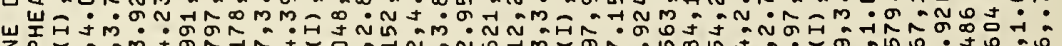

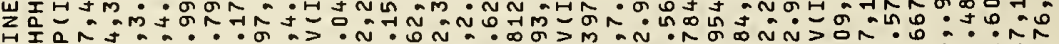

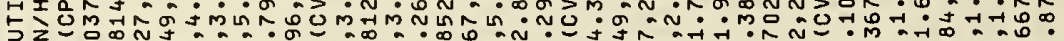

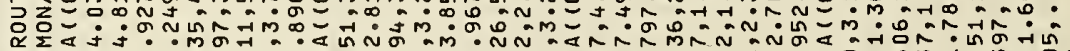

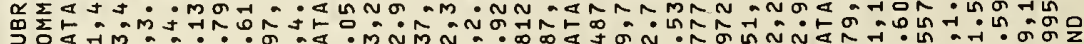

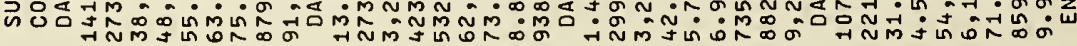




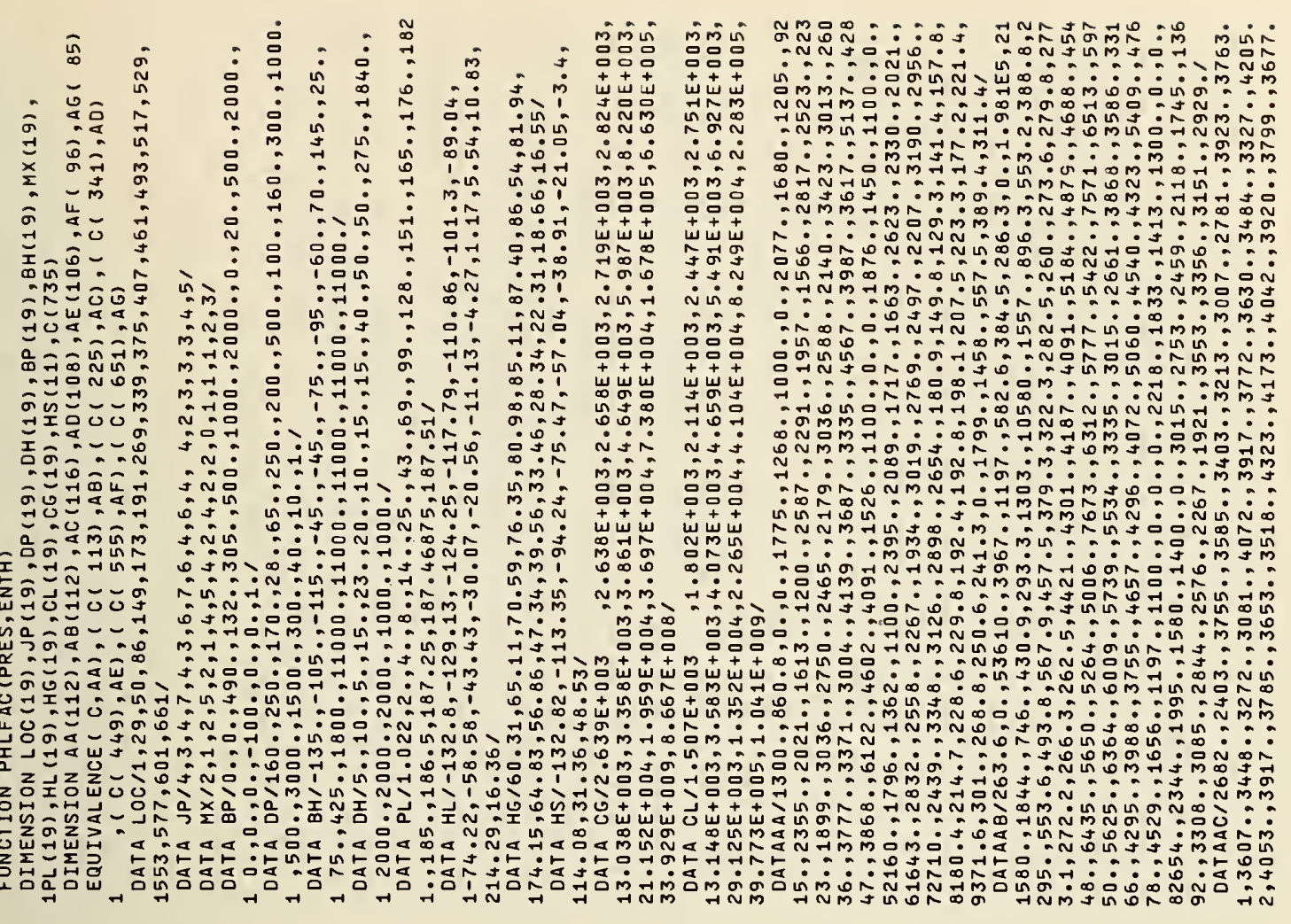

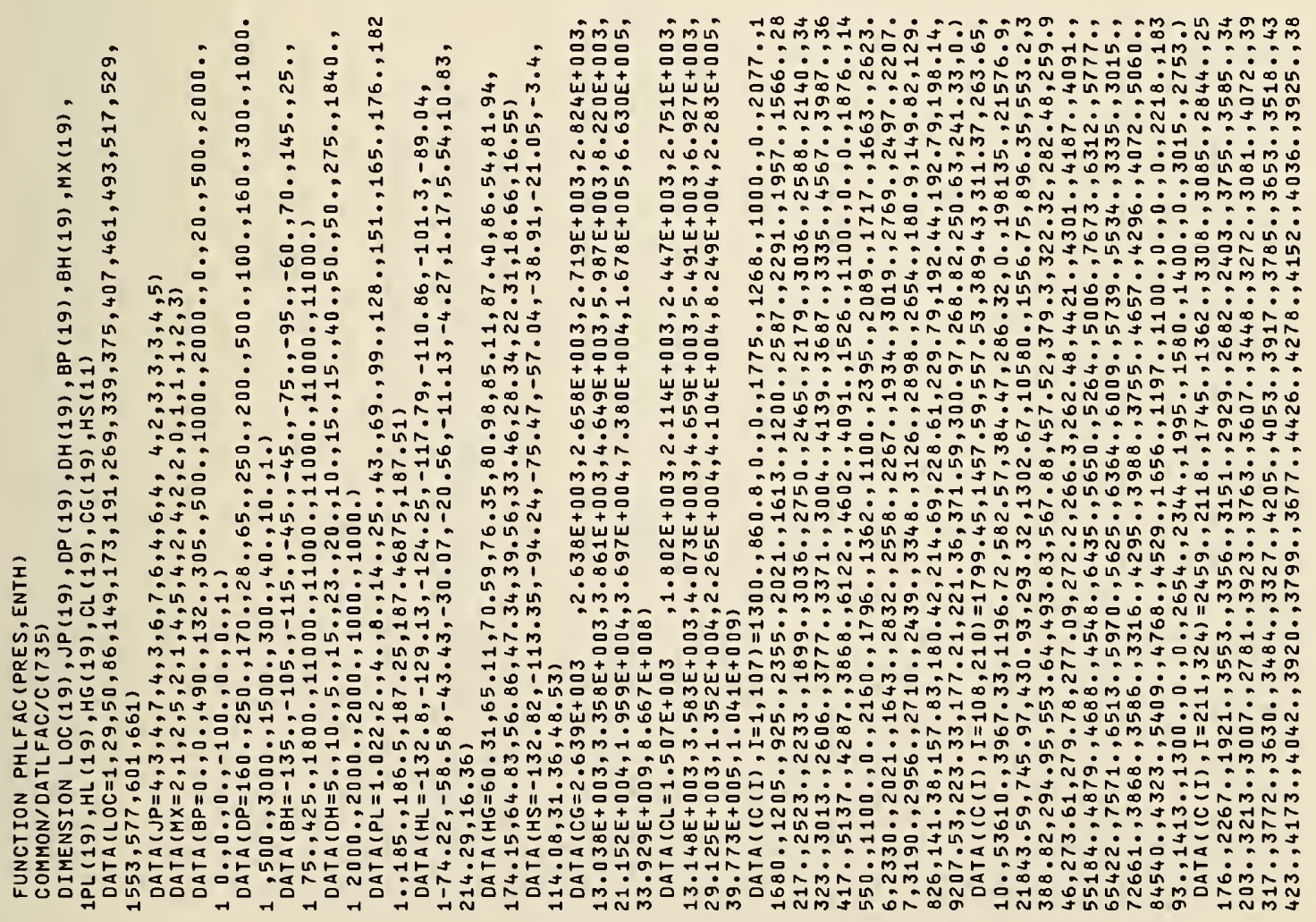




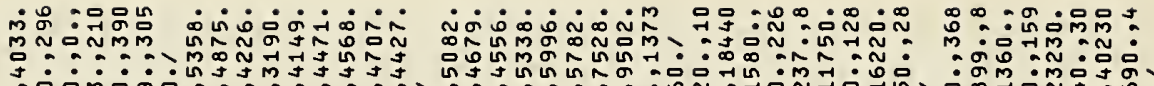

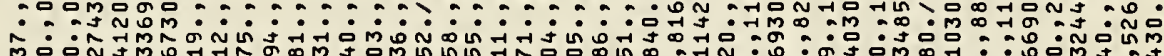
mons

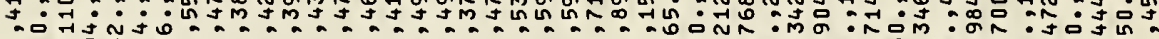

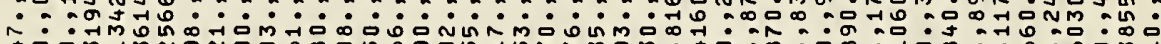
N

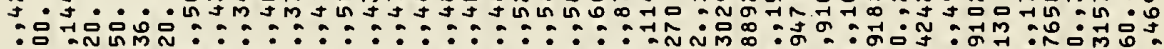

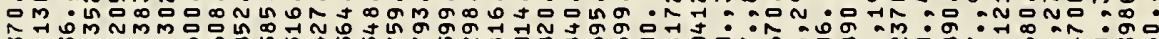
on

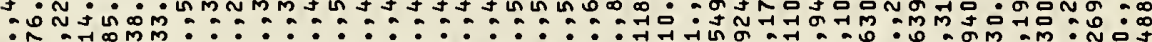
n̂n 结: : : : : Nonn 广

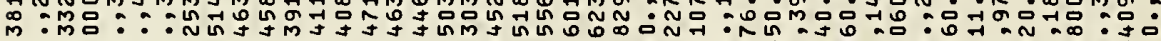
$\because \because 7 N^{2}$ N 然: : : : :

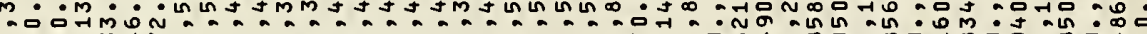

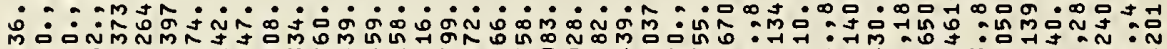
: : : : :

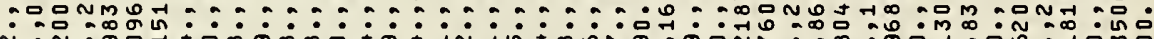
กิ

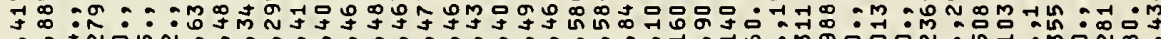

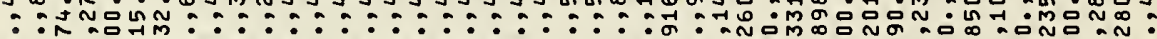

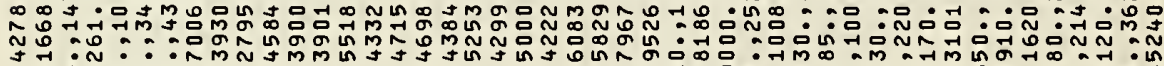

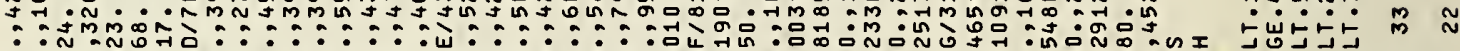

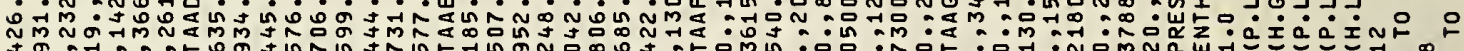

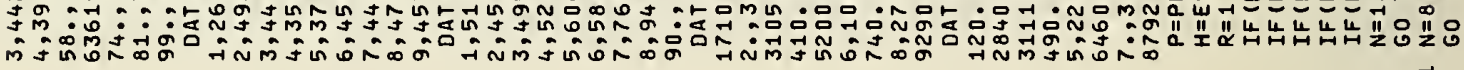

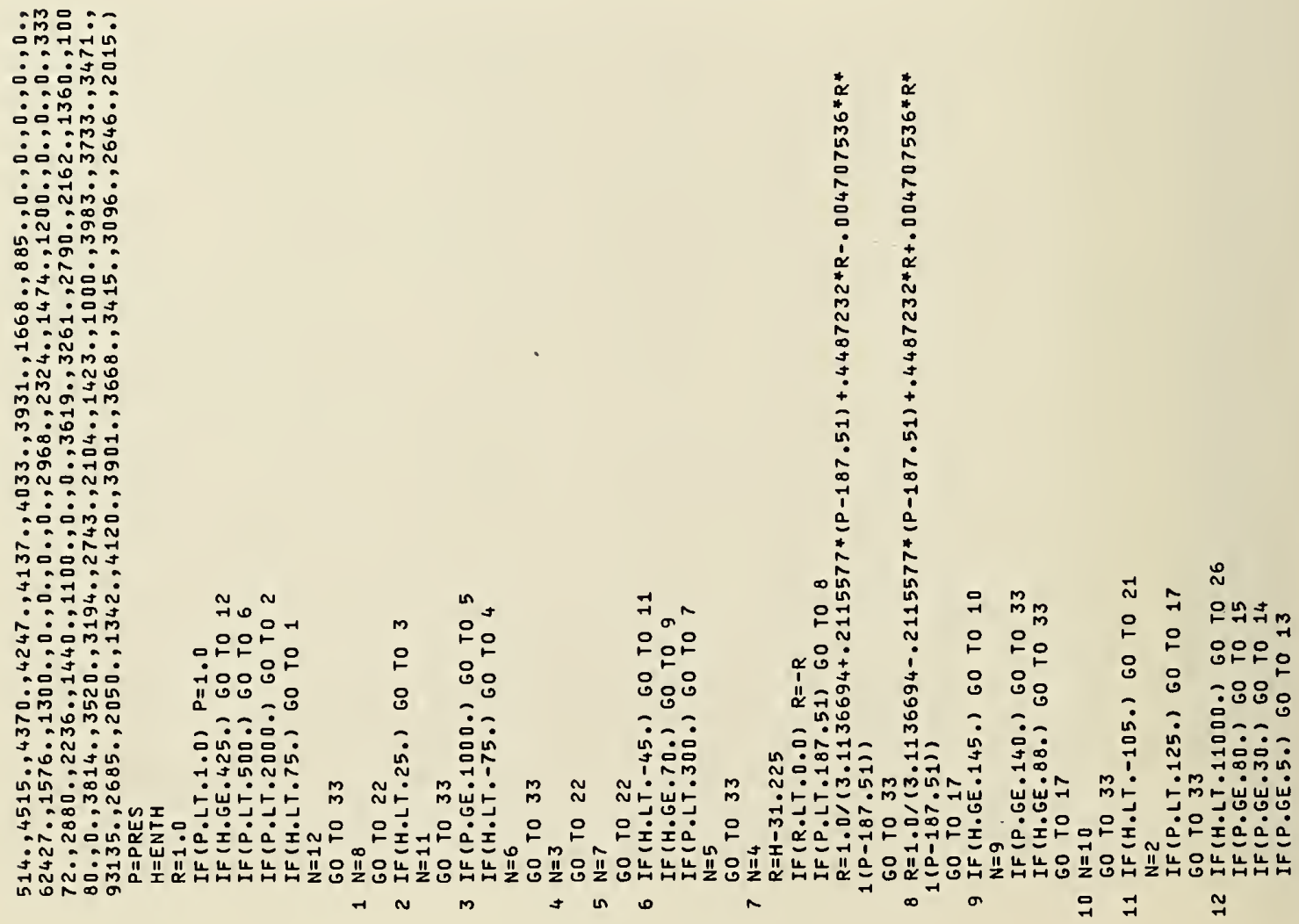




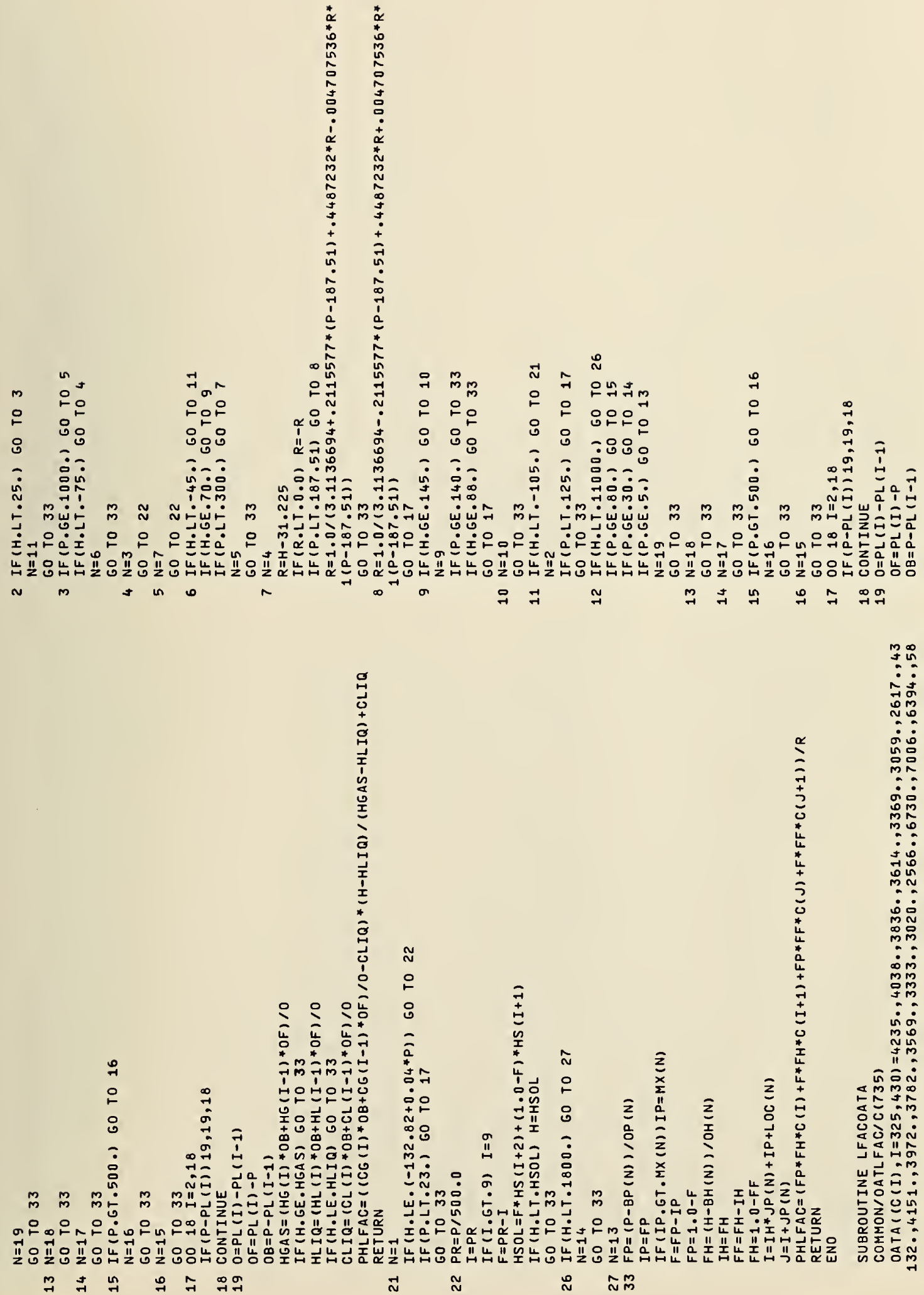



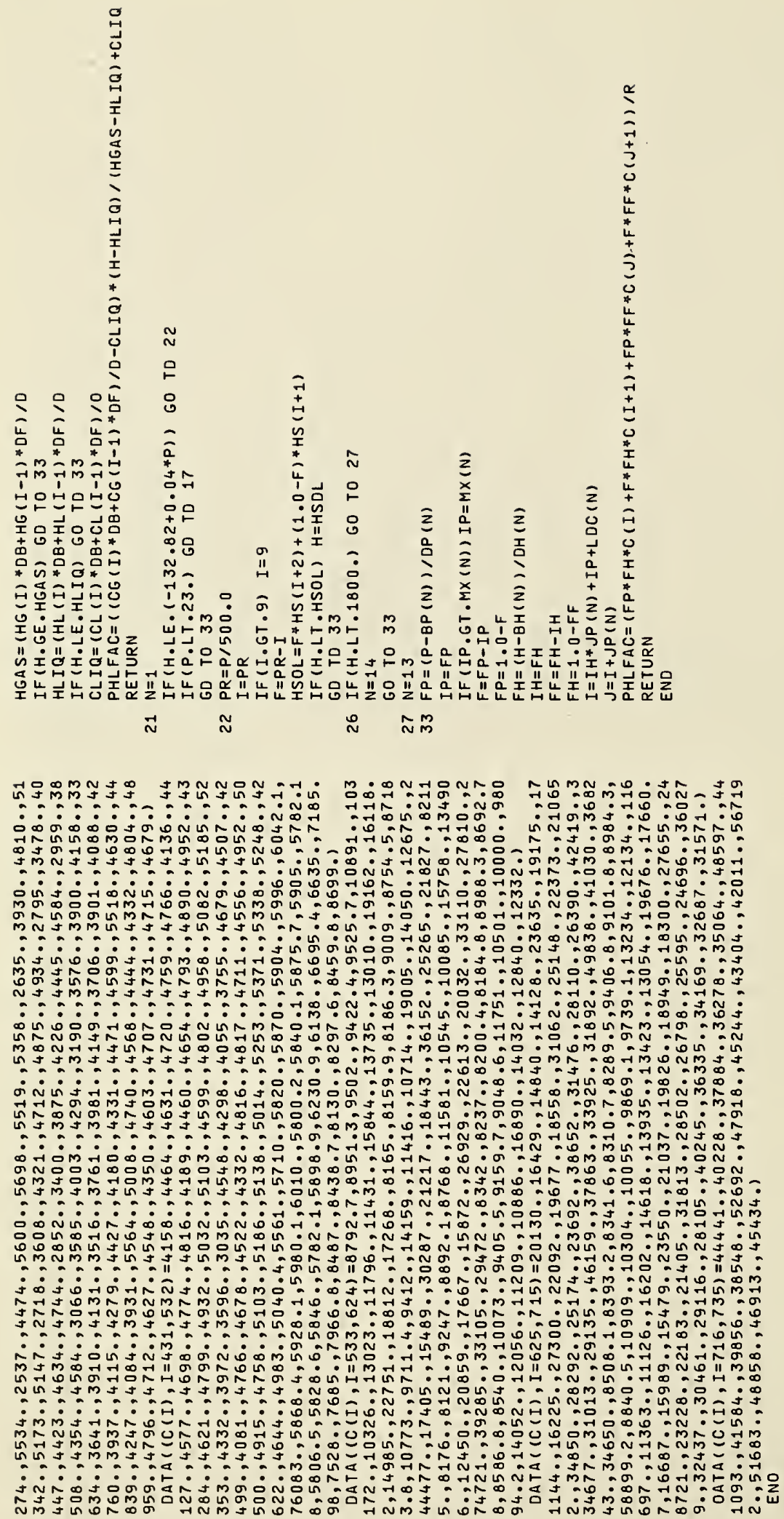


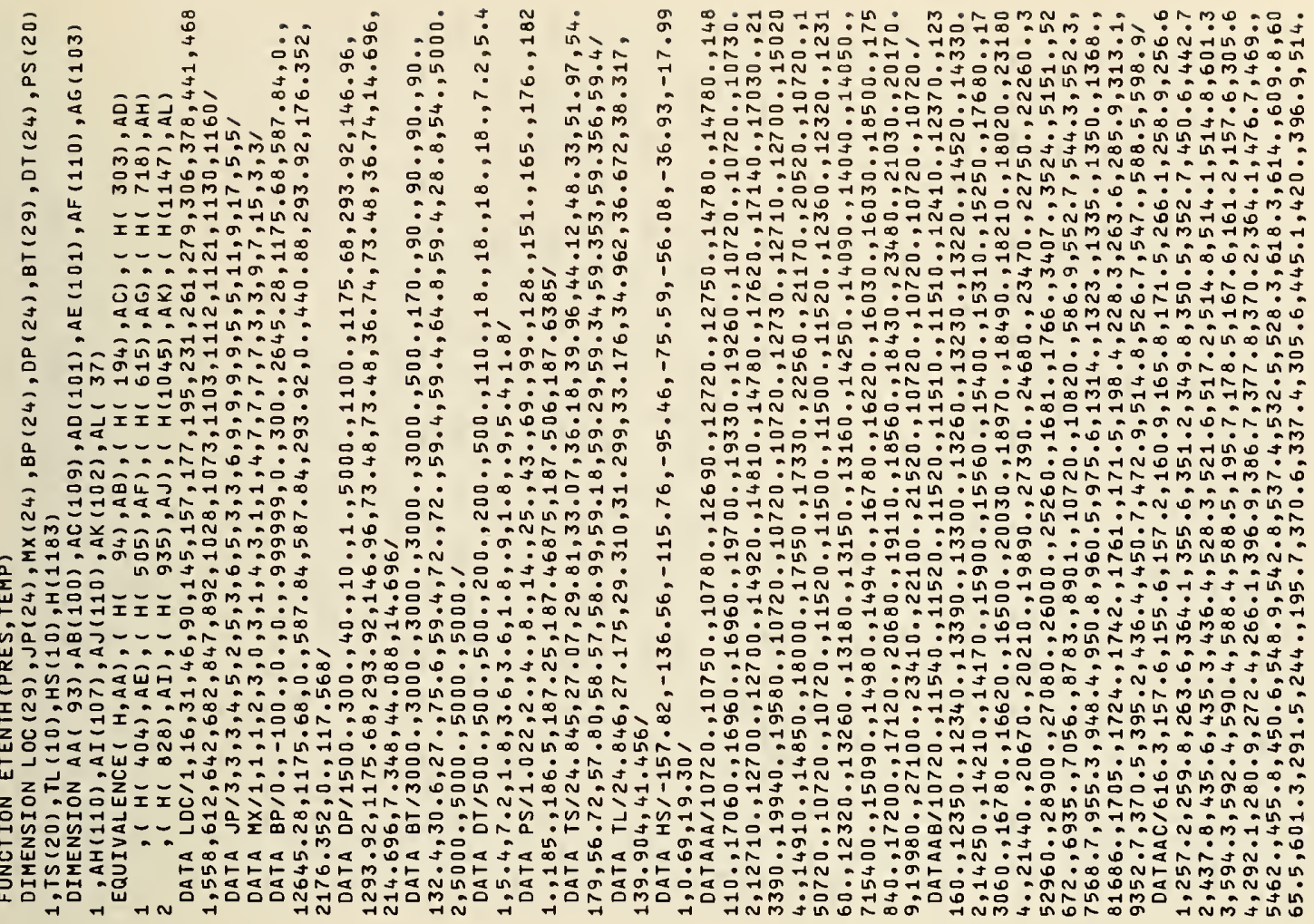

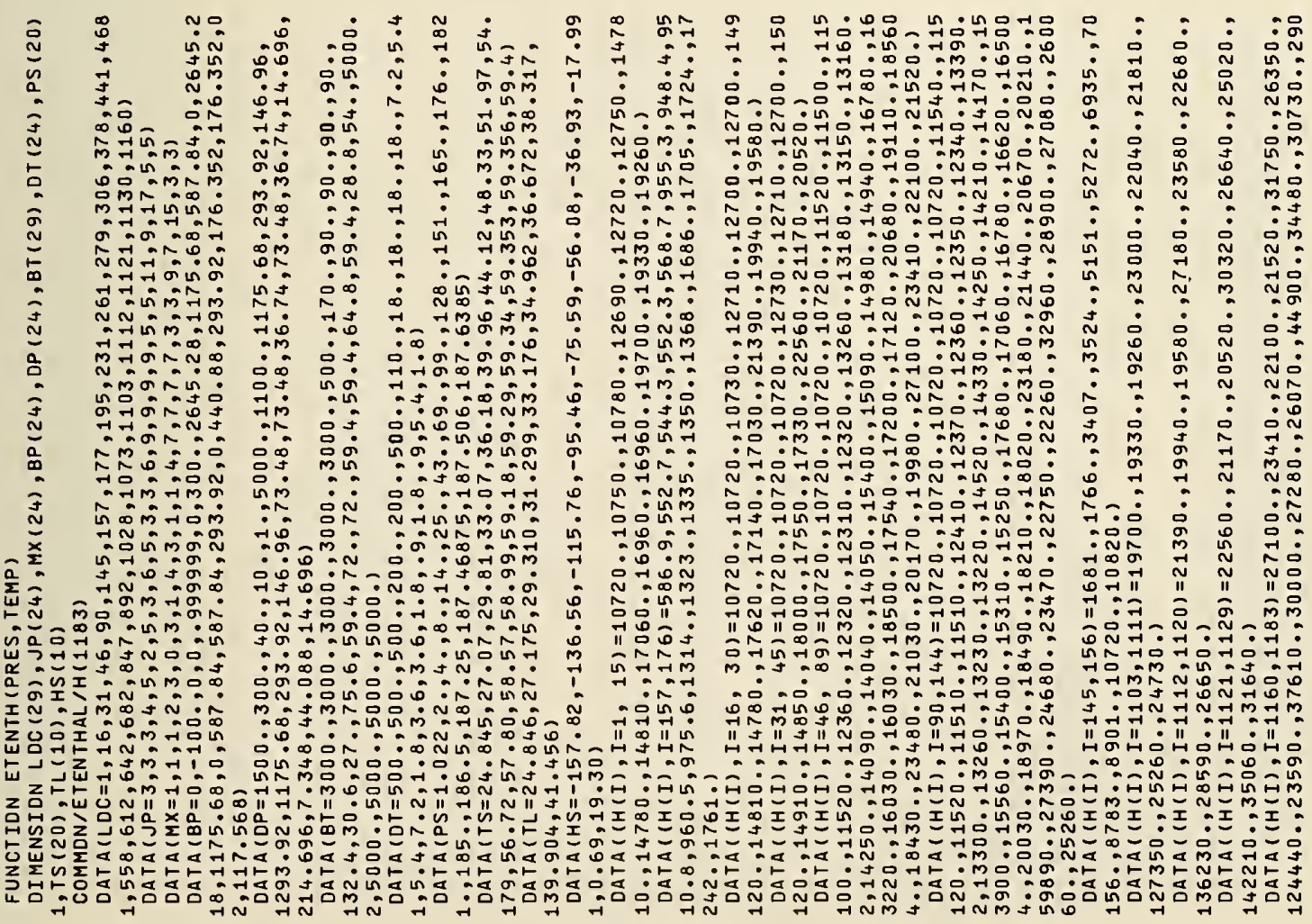




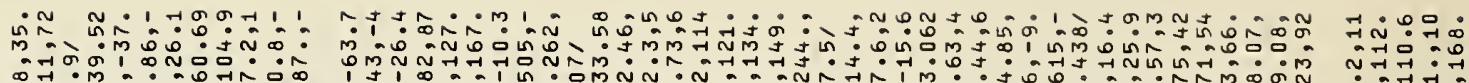

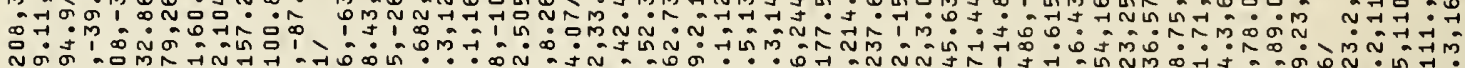
Y.?.7.

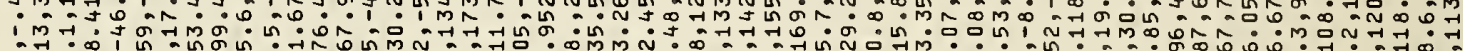

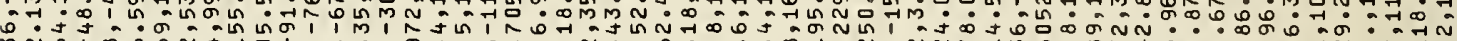
? $\mathbb{\infty}_{0}$ móf $\begin{aligned} & 0 \\ & 0\end{aligned}$ mon

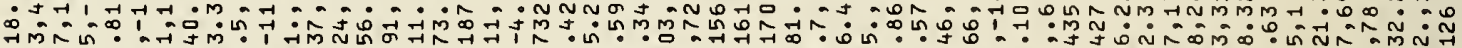
600 m. ơ

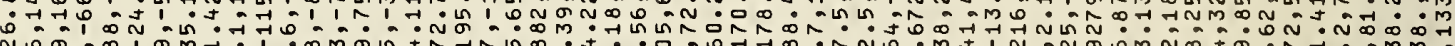
o. o.

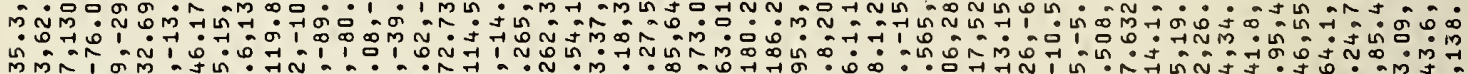

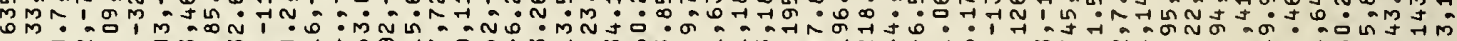

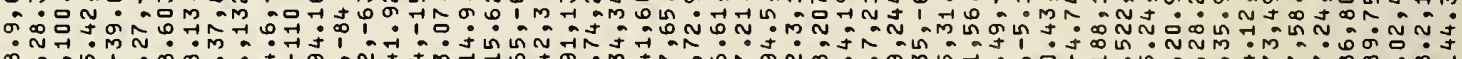
o แึ

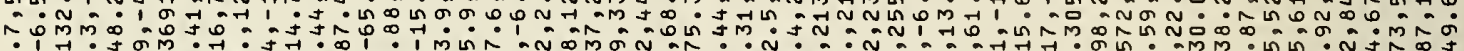
s

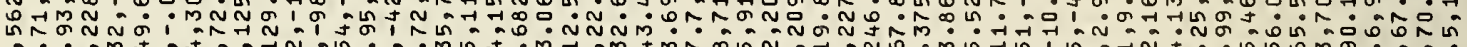

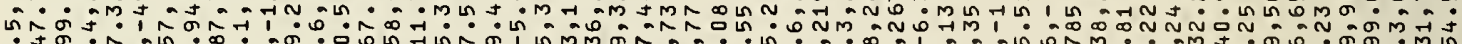
d.

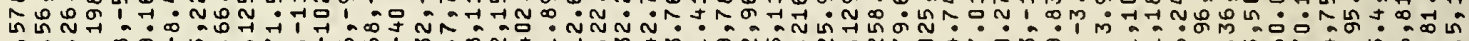

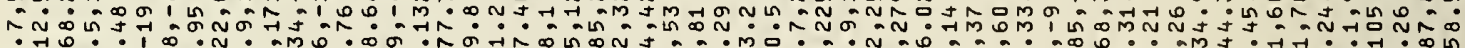

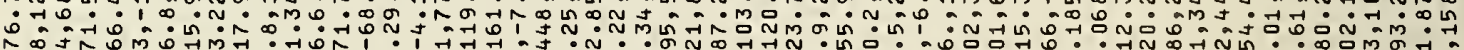

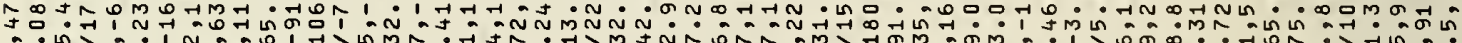
món 于 N no

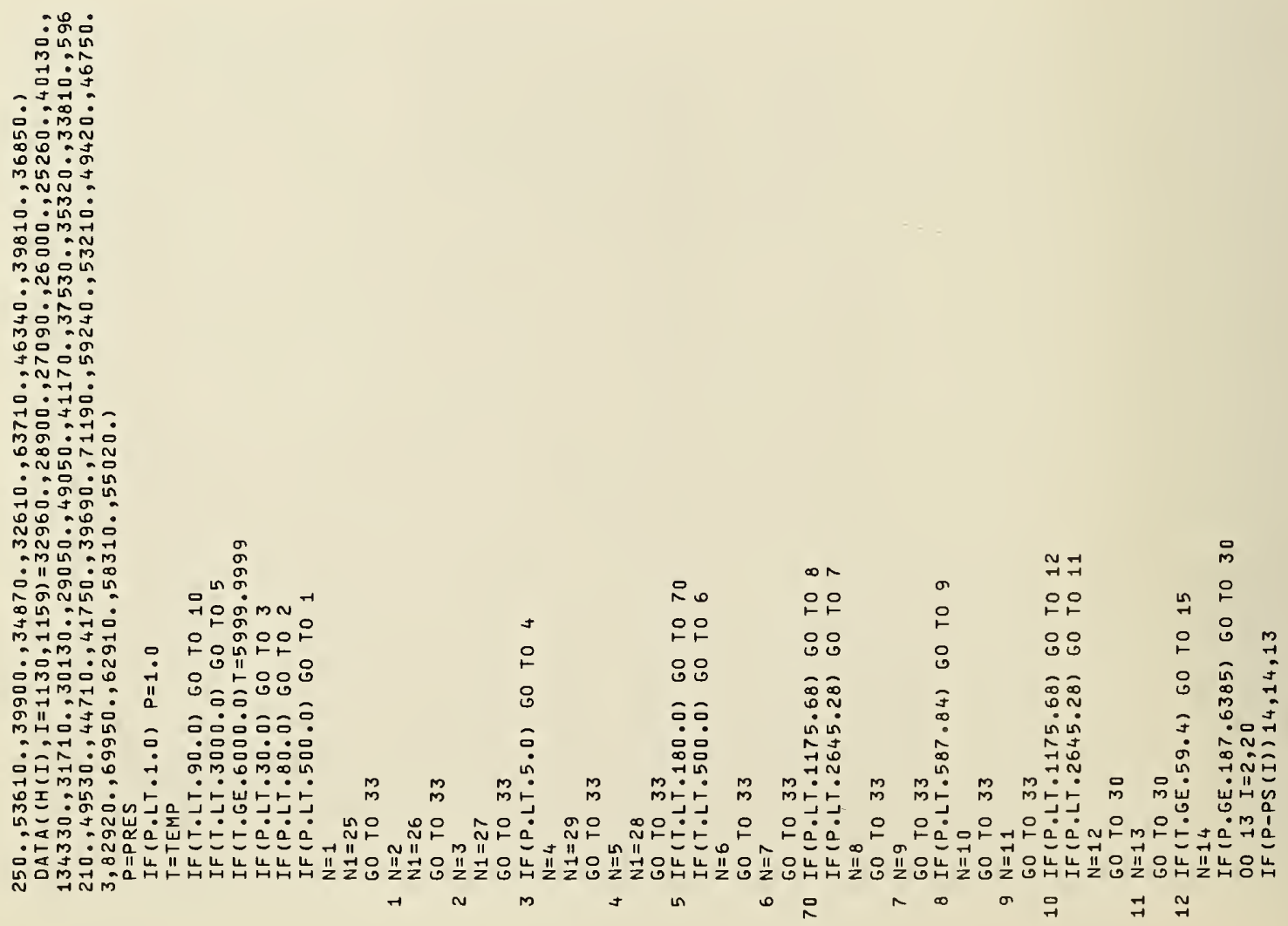




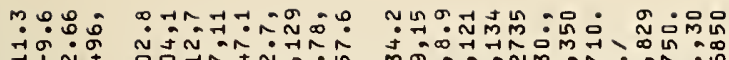

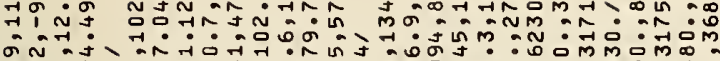

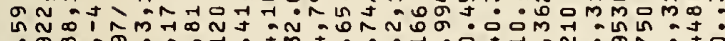

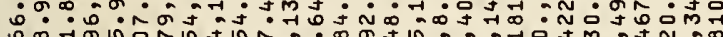

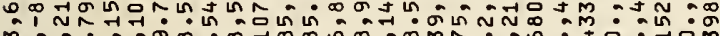

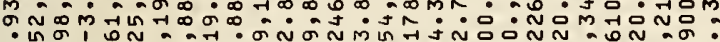

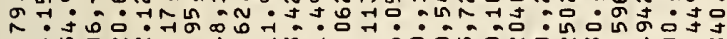
กัด N N $\mathcal{1}$ N

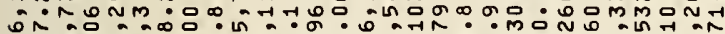

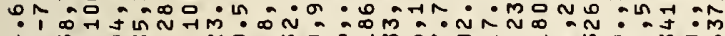

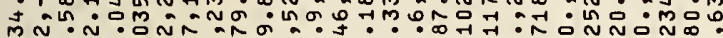
สNผ

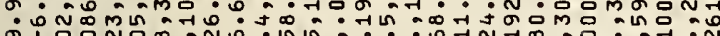

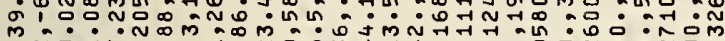

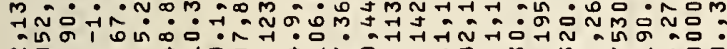

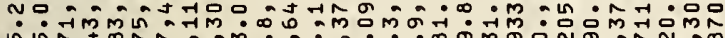

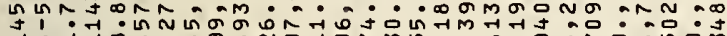

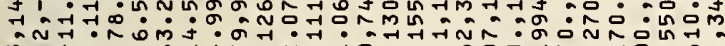
ñ.

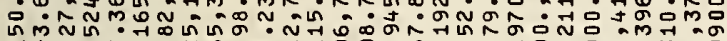

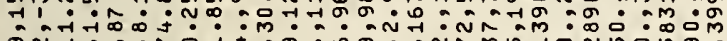
नित्र

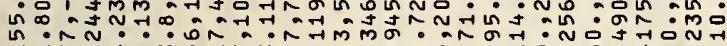

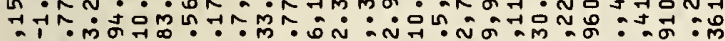

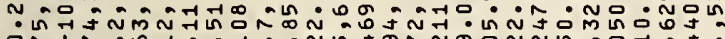

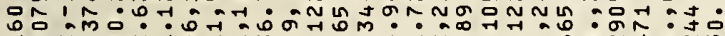

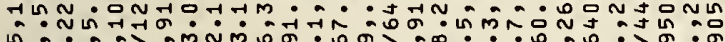

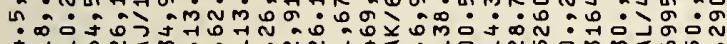

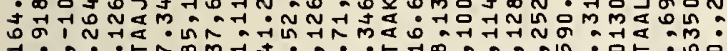
今心

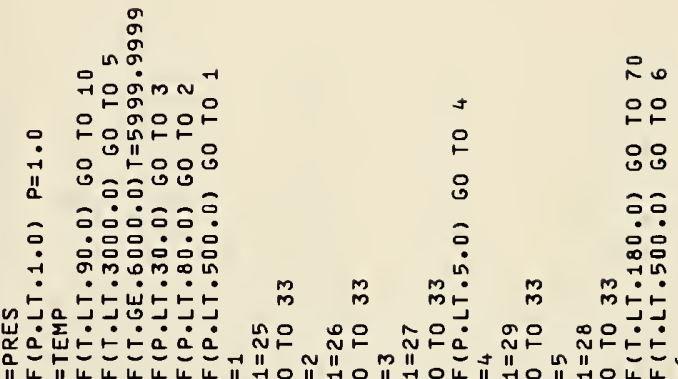

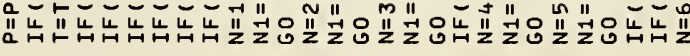

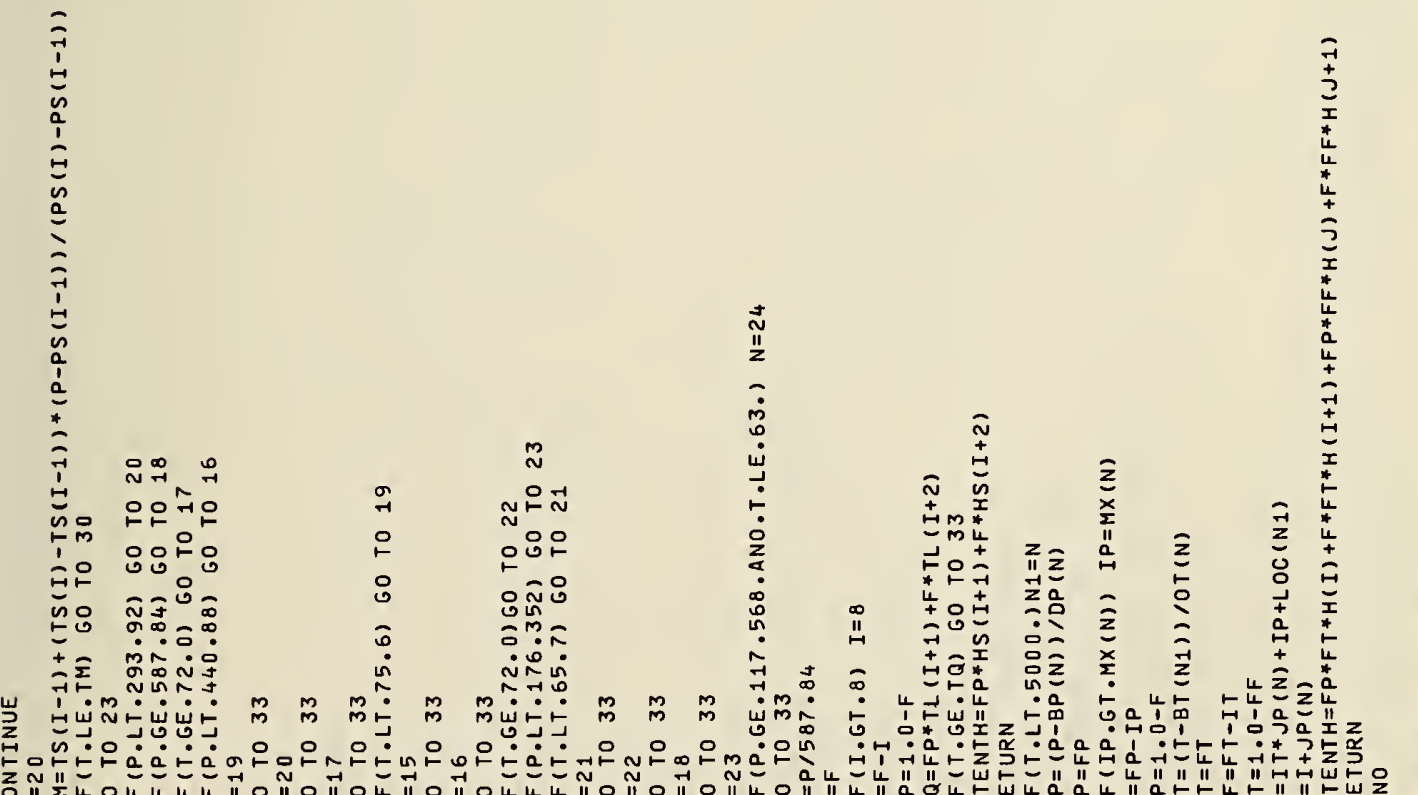

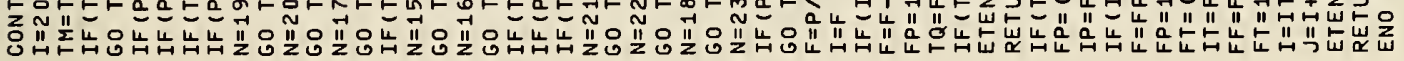

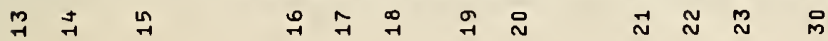




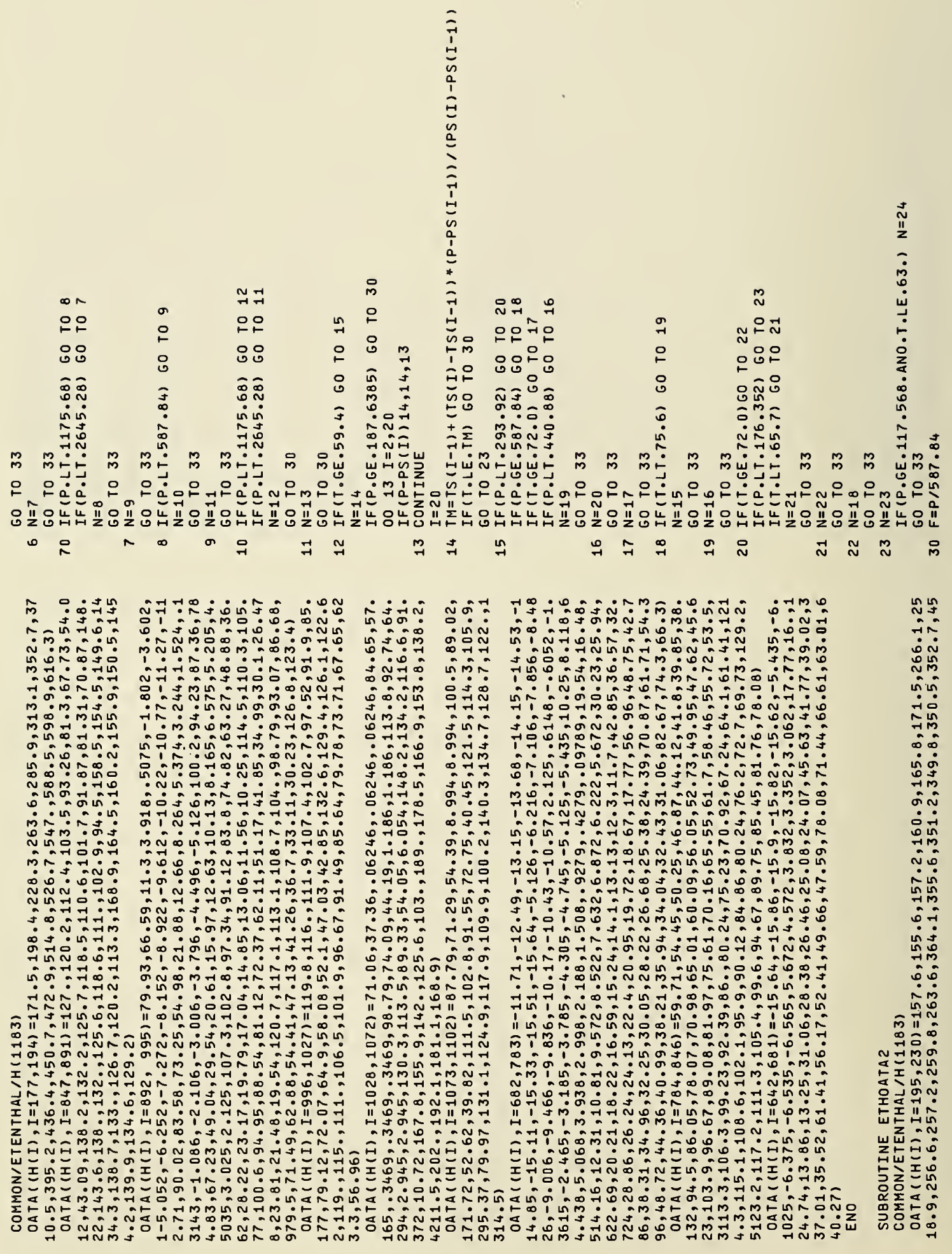



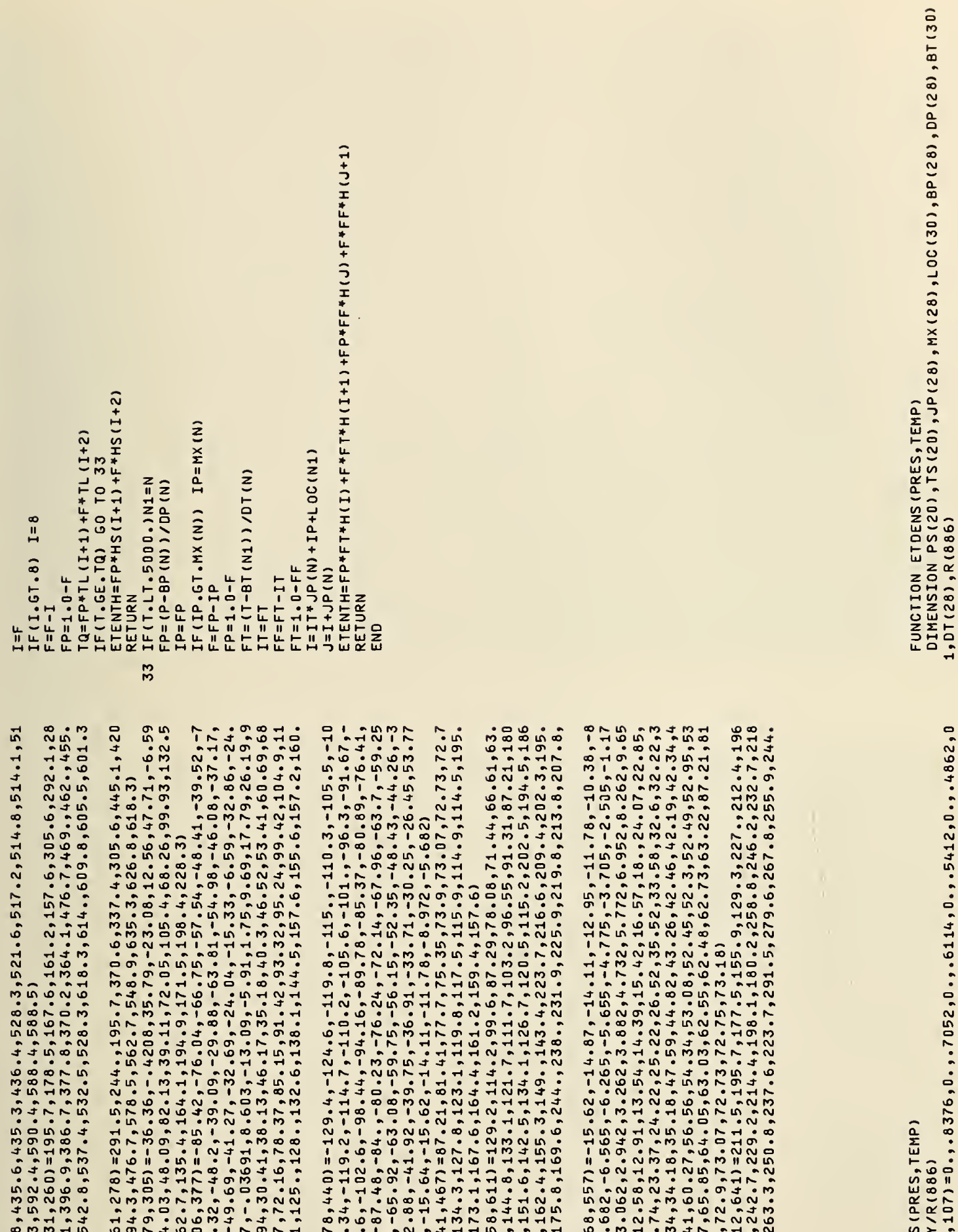

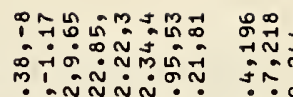
10

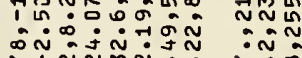

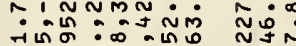
7 西:

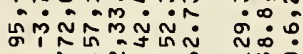
กิ่ง

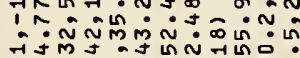

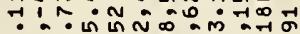

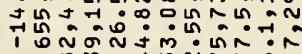

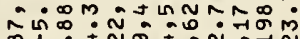
का

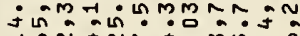
70 N fơ

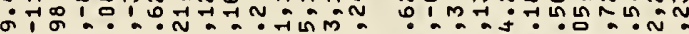

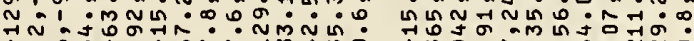
1

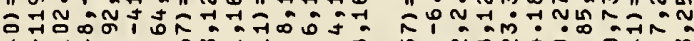

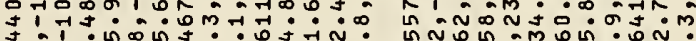
uñ

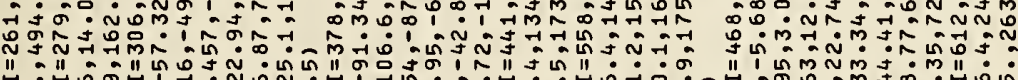

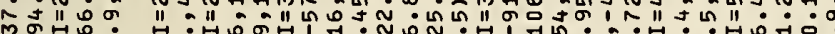
每 Un No

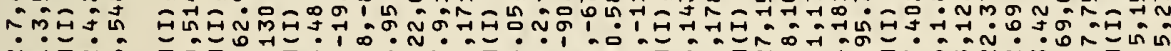

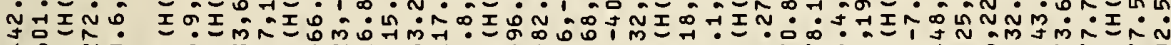
ง

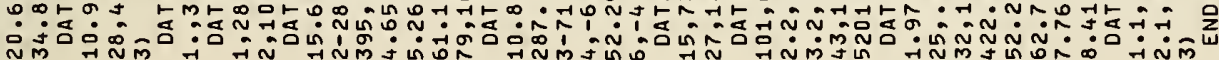



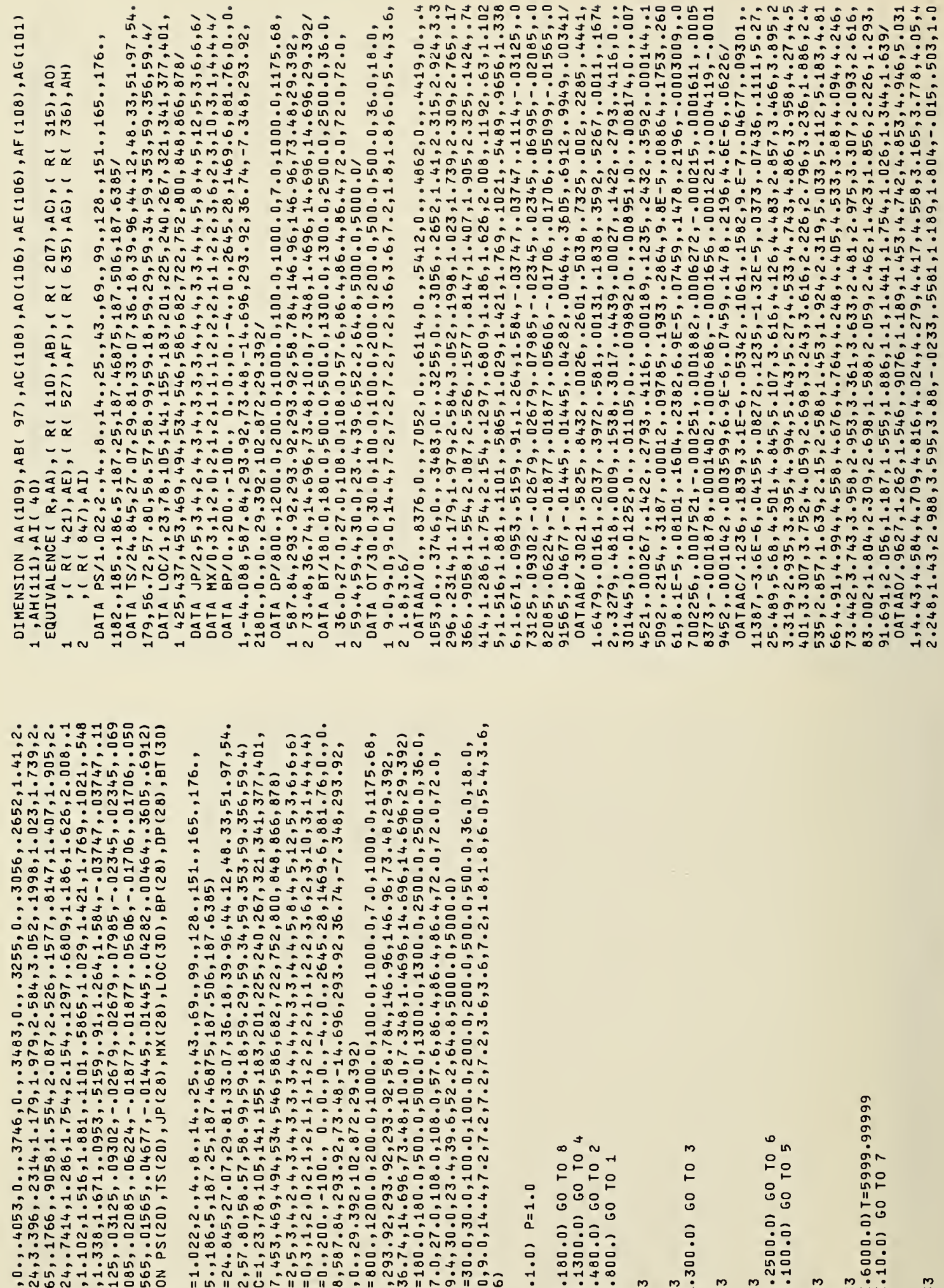

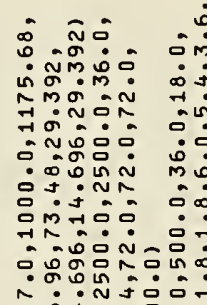

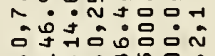

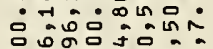
कू o

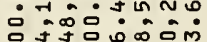
? : ñ ํำ 于क

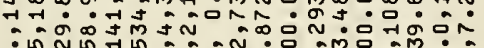
0 N is on

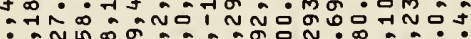
in $N$ no

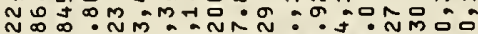

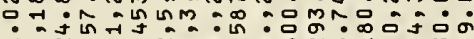

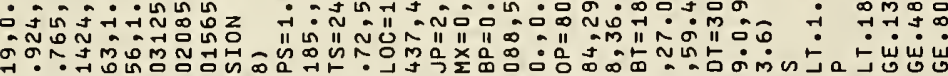

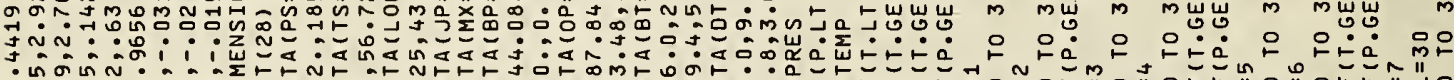

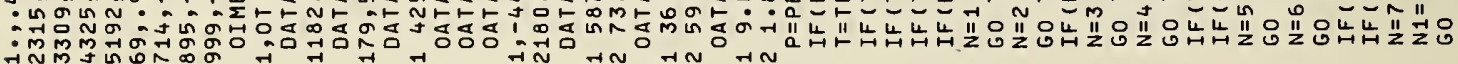


m:

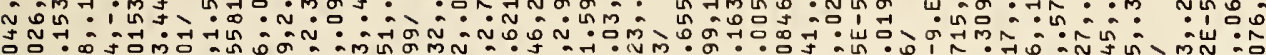

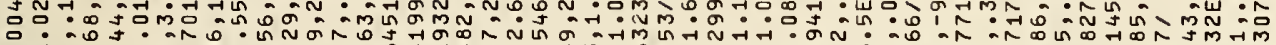
: món N $\dot{1}$ 人

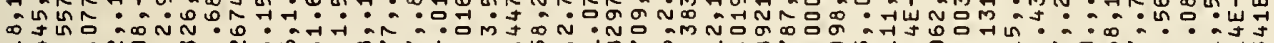
Tे : H.

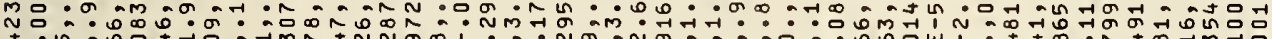

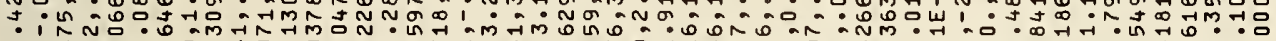

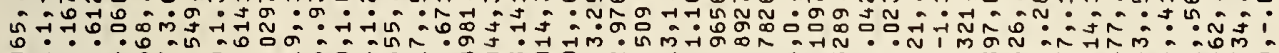
: - Goर ö :

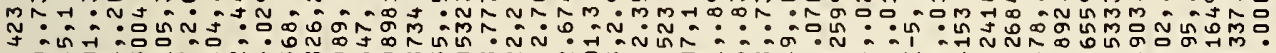

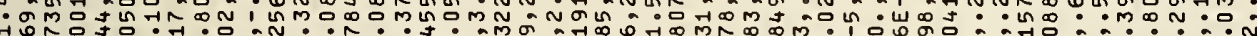

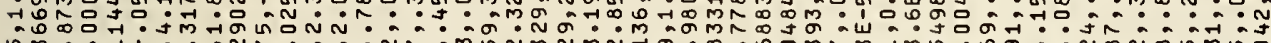

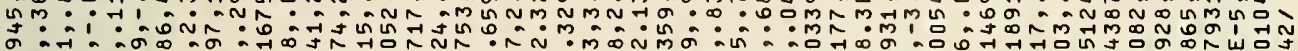
ô

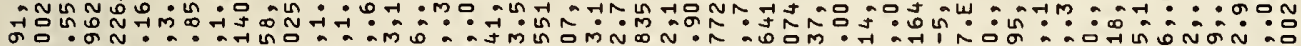

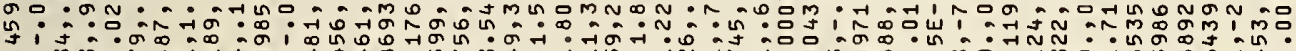

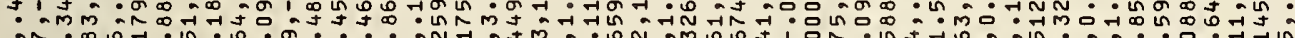
ốn

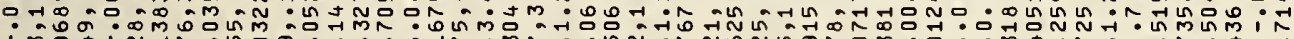

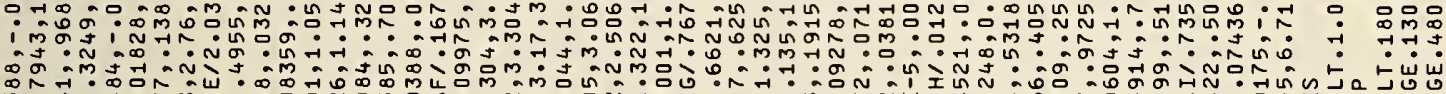

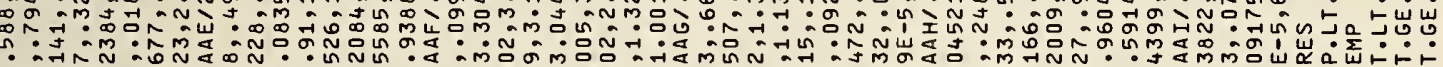

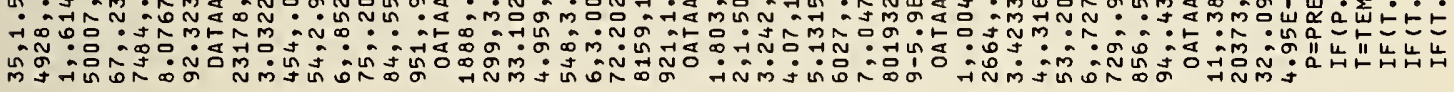




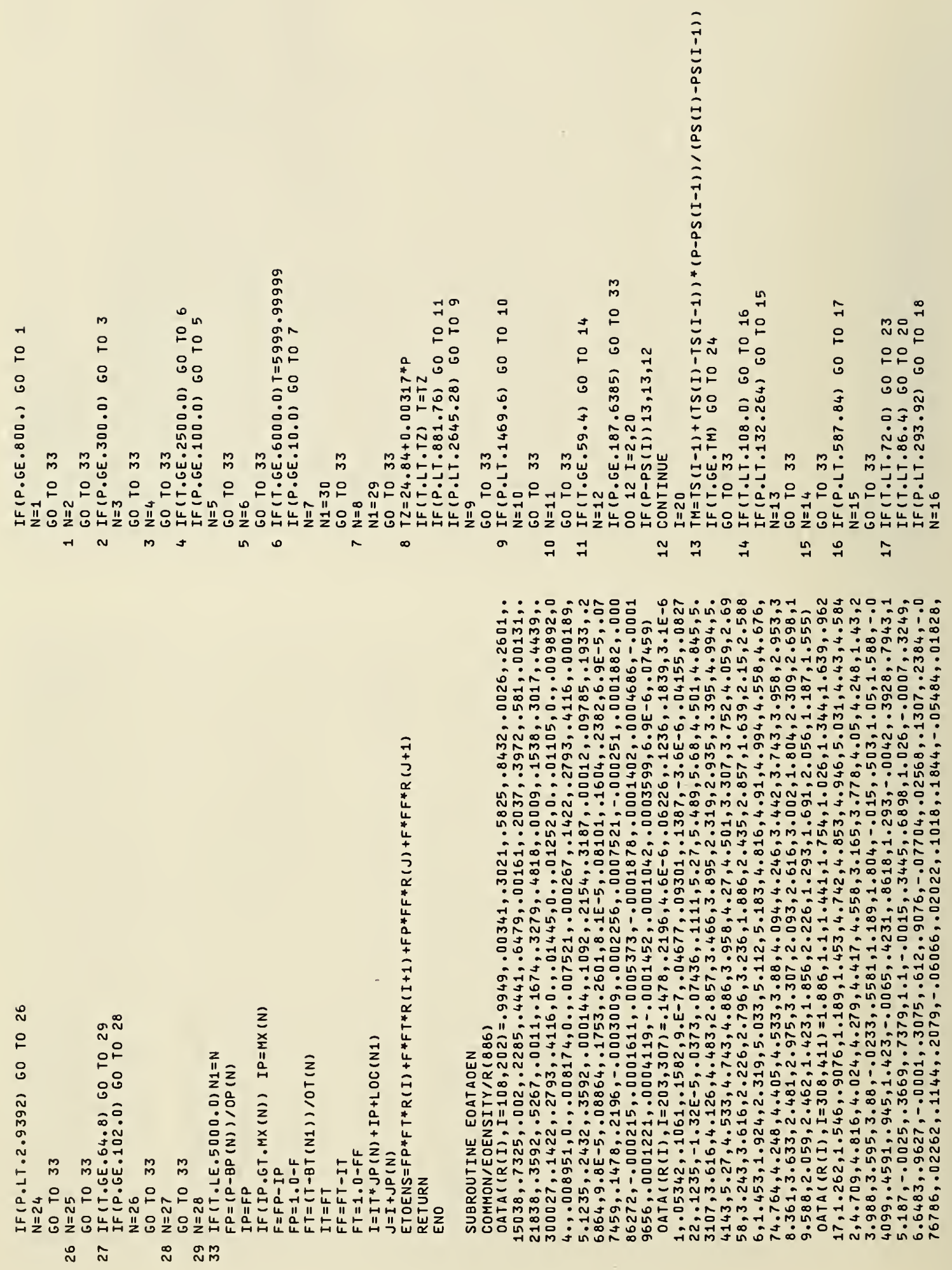




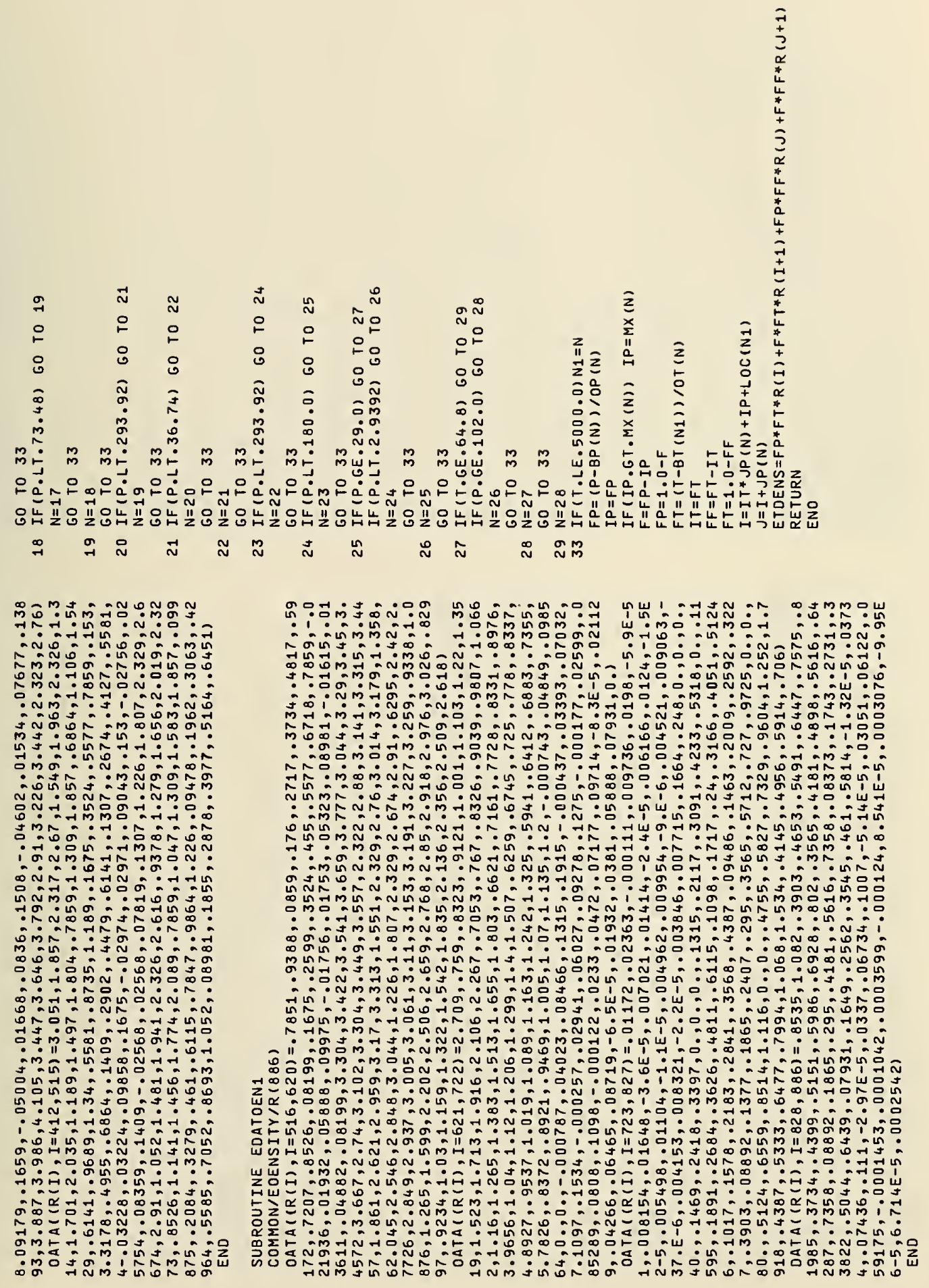



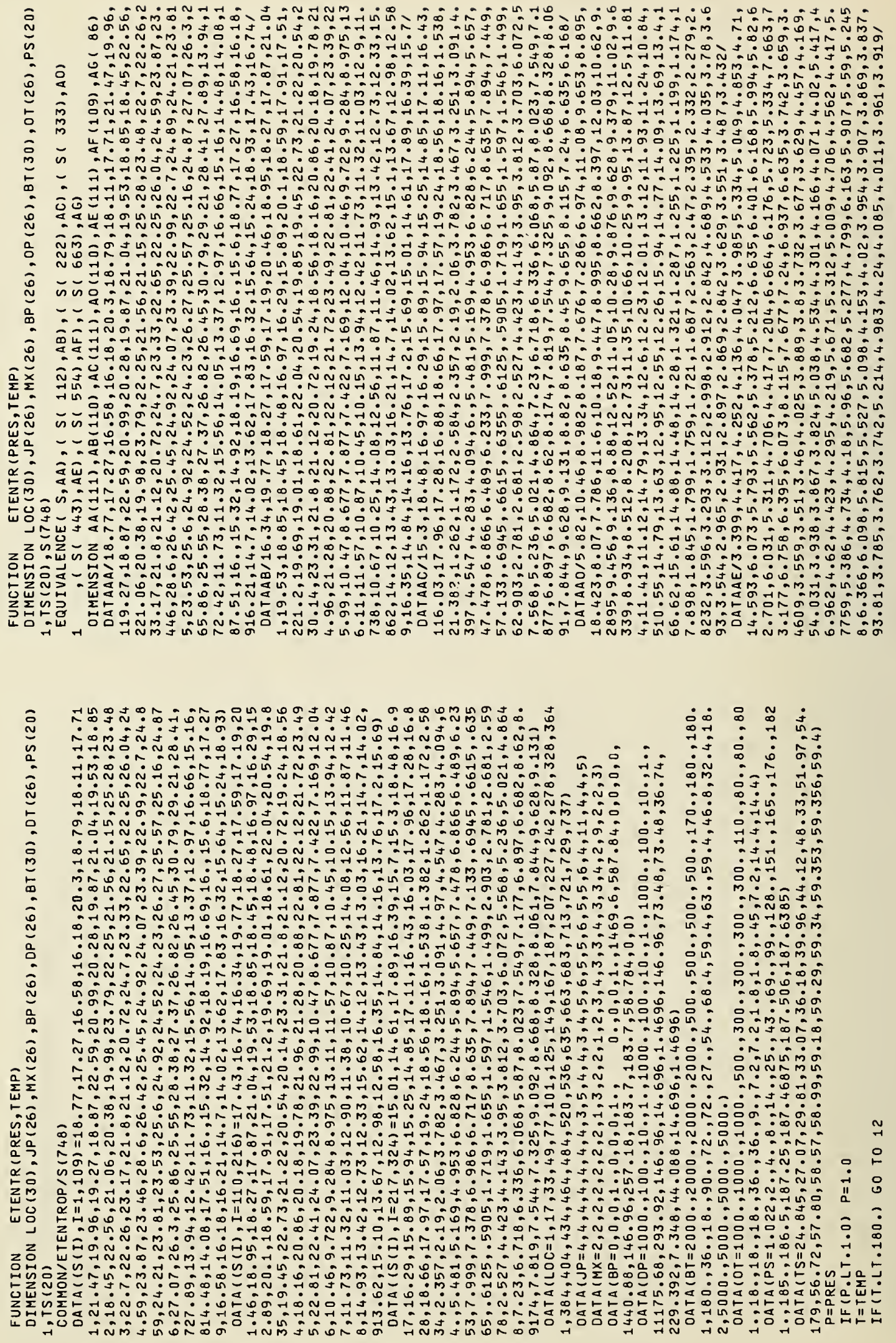


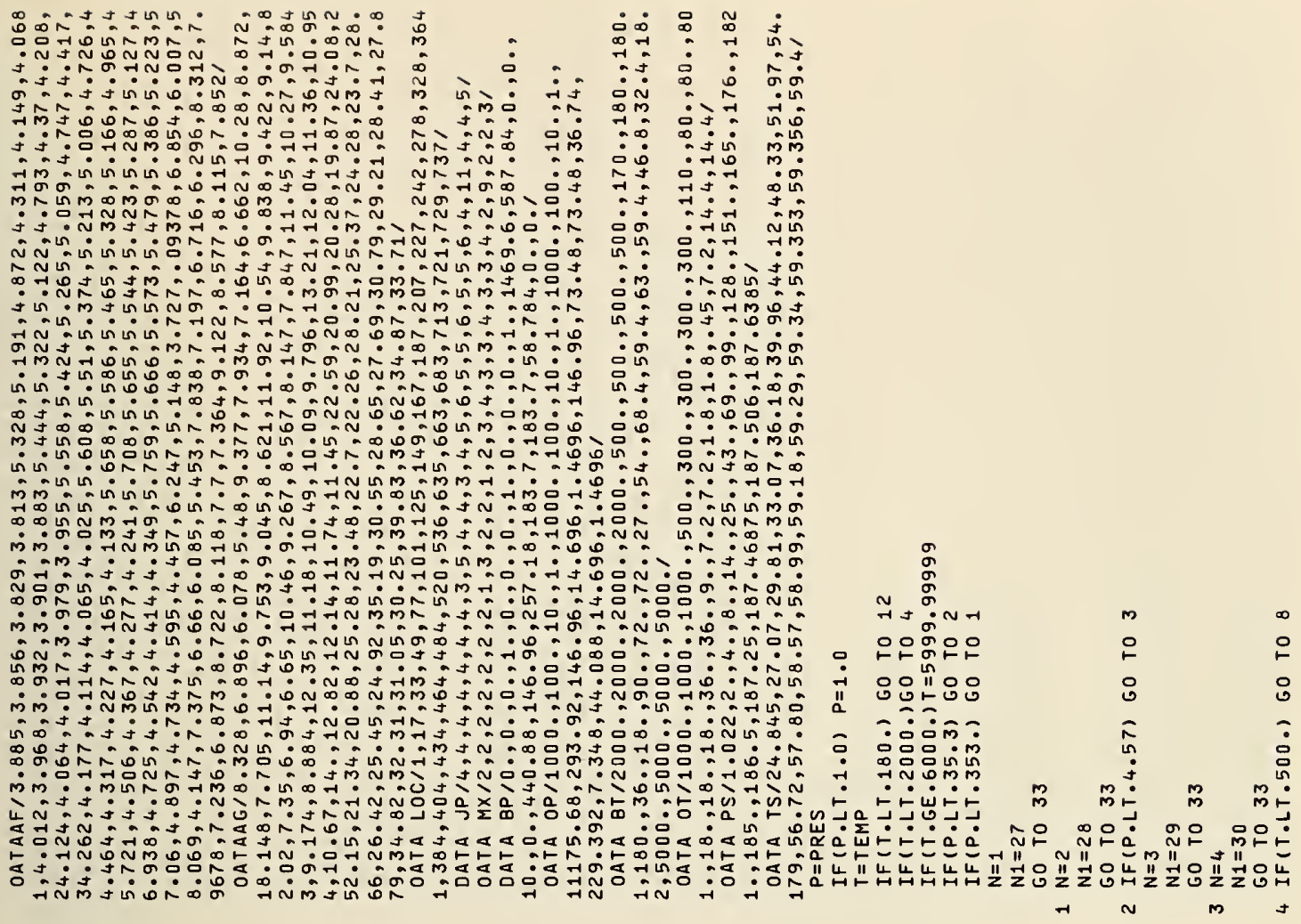

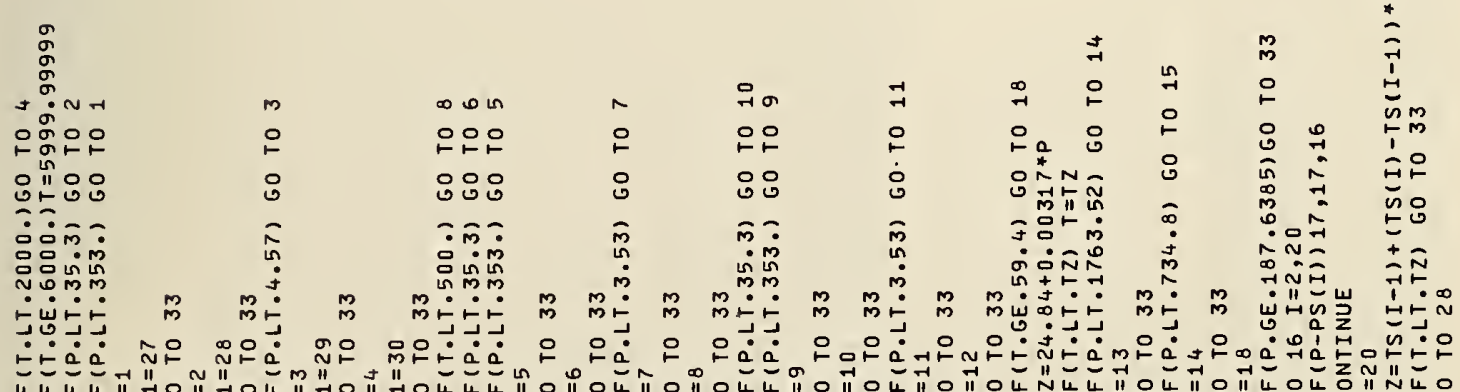

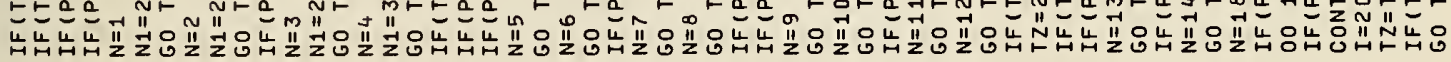
r N
m
in
a
ปี 


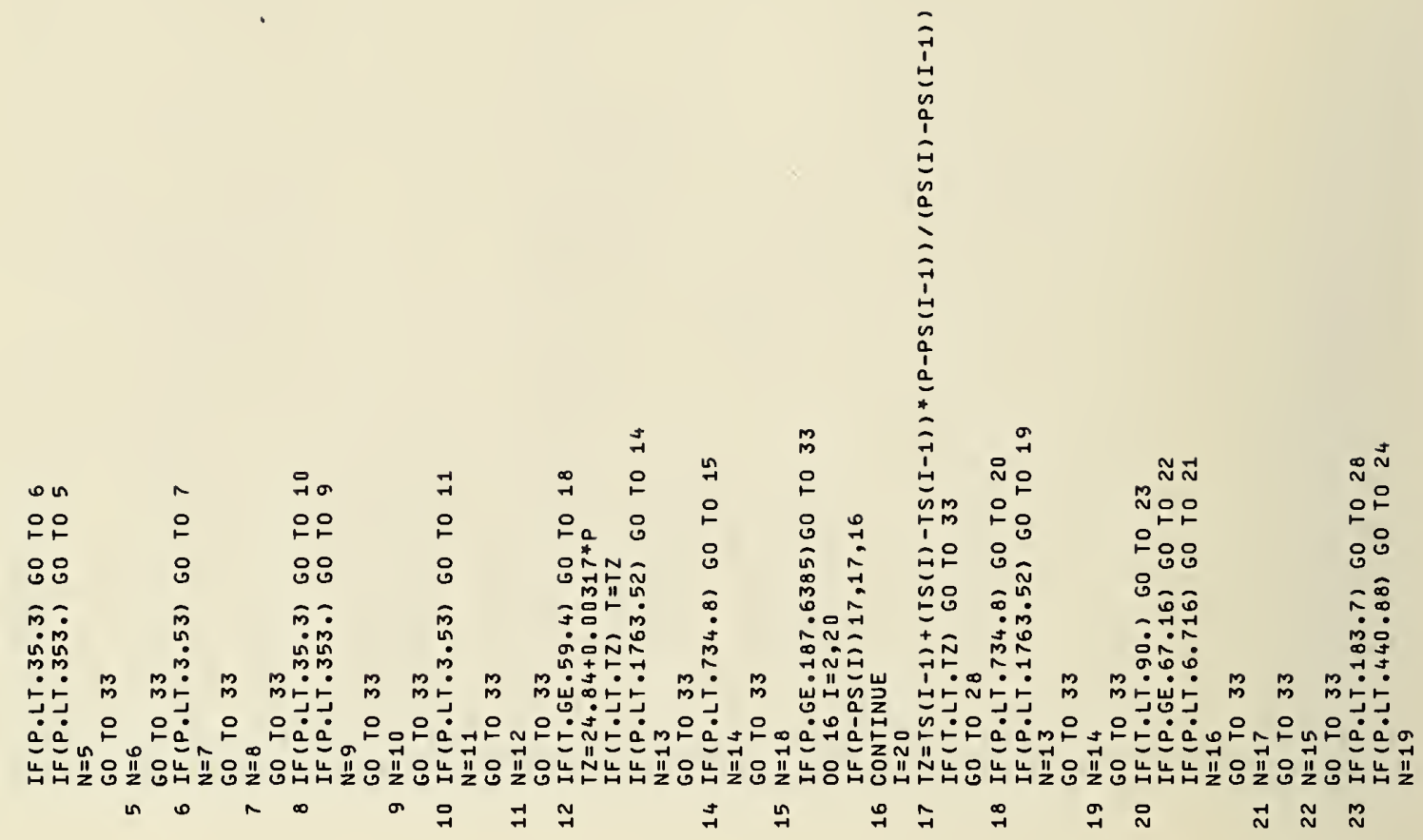

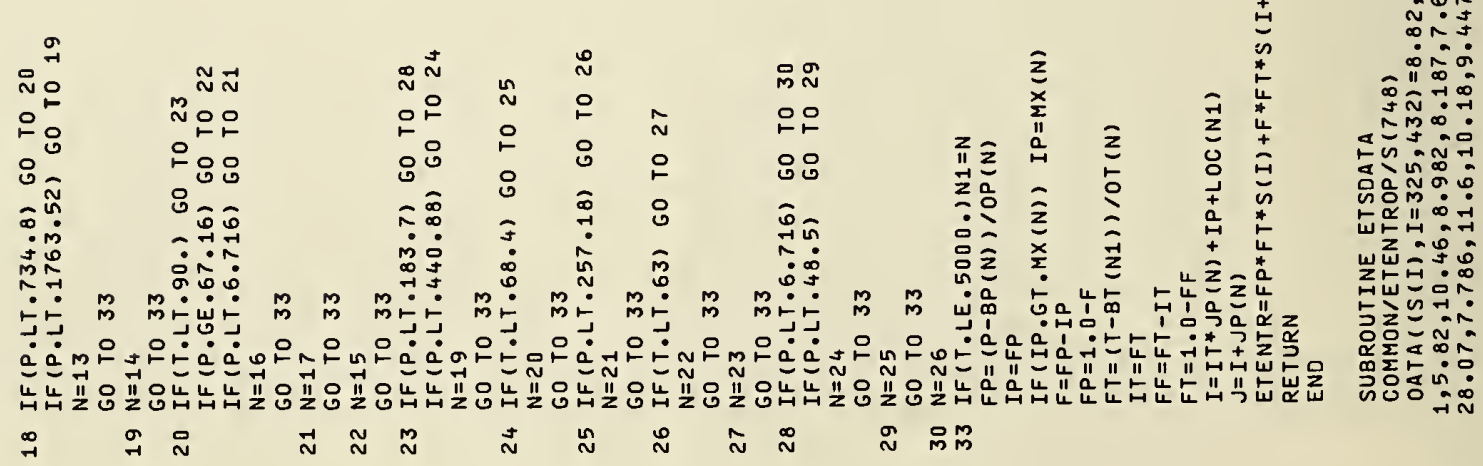




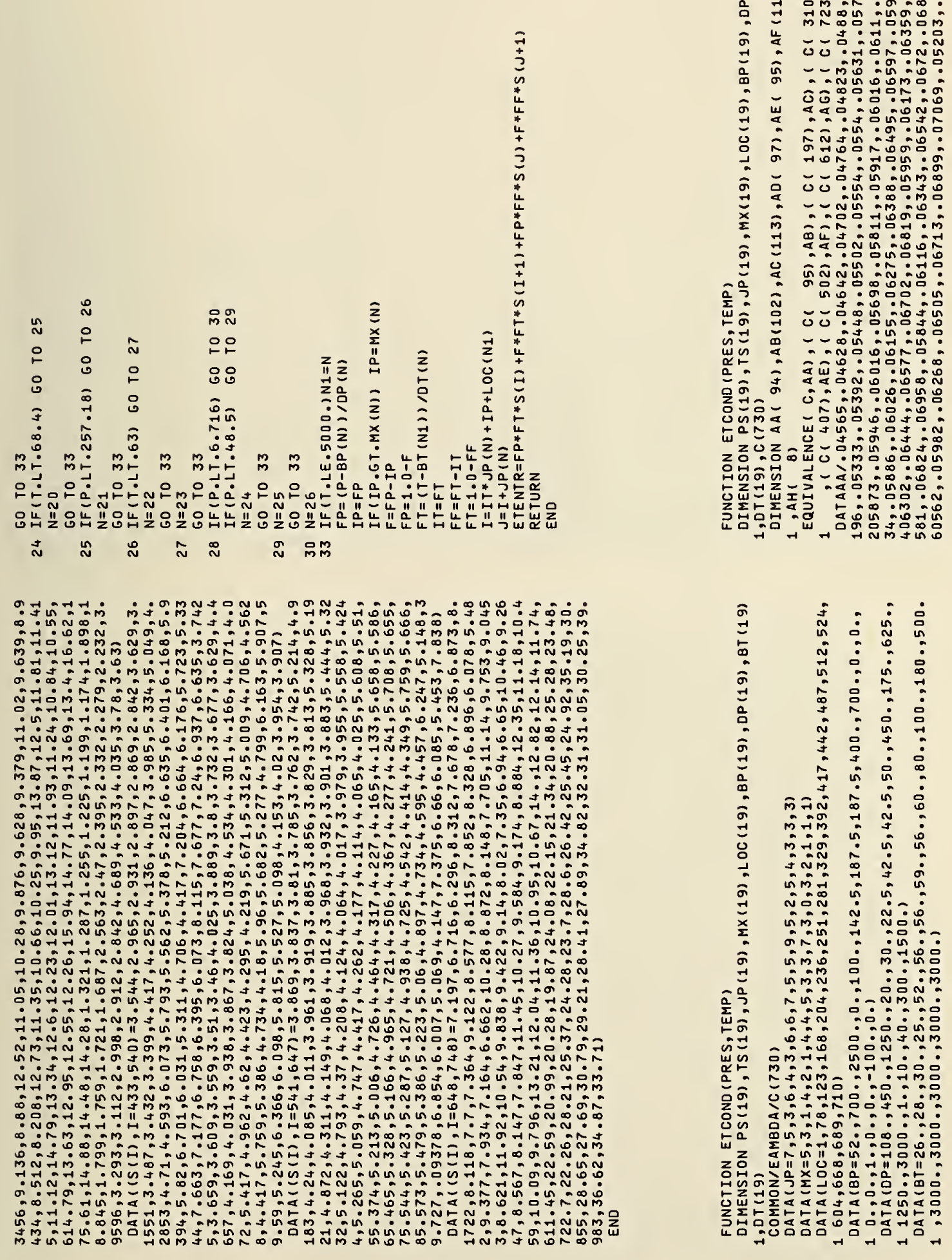




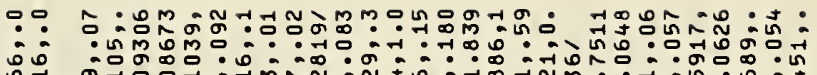

足先

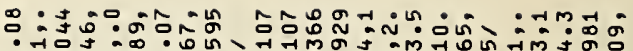
:N

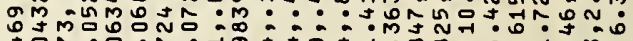

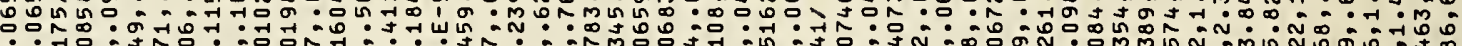
숭: : N

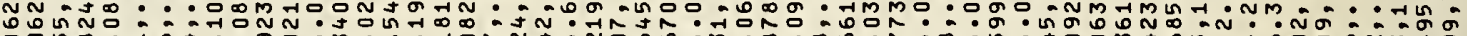

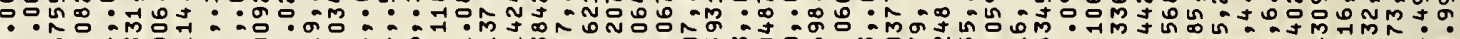
No: No

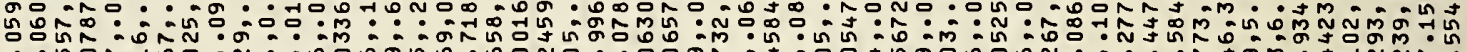

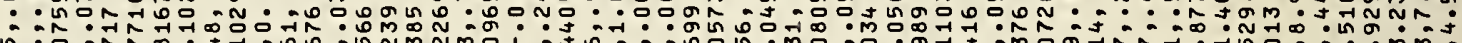

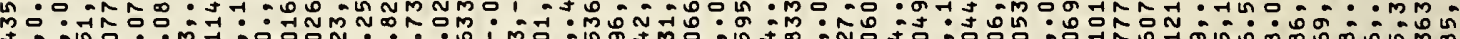
जn

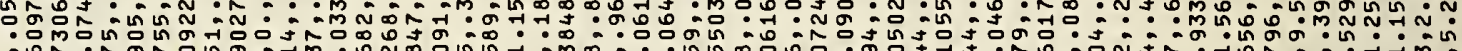
ज范: : J̃ : स:

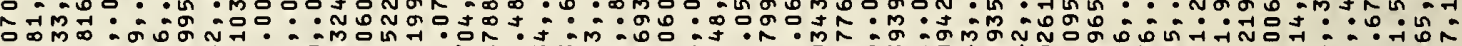
: N뮤: 位

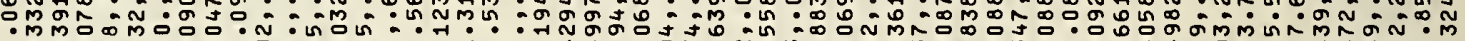

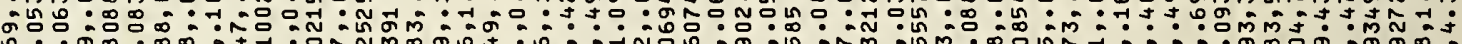
: :

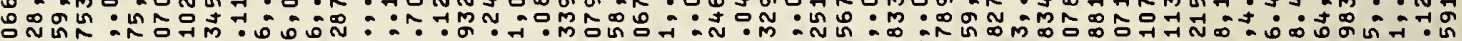

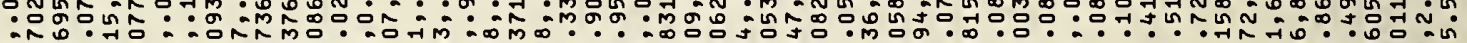

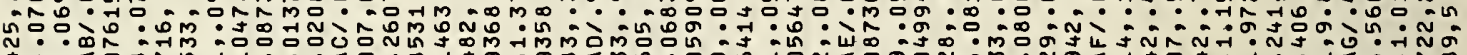

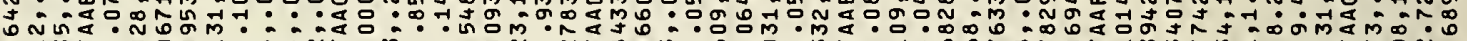

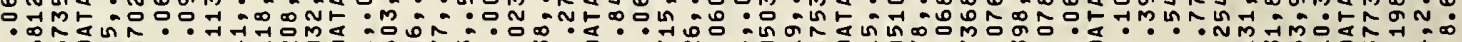

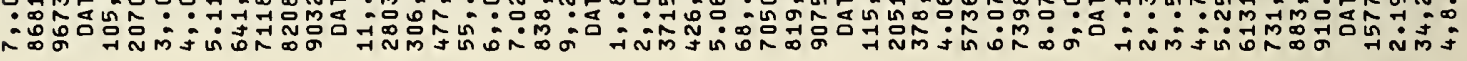

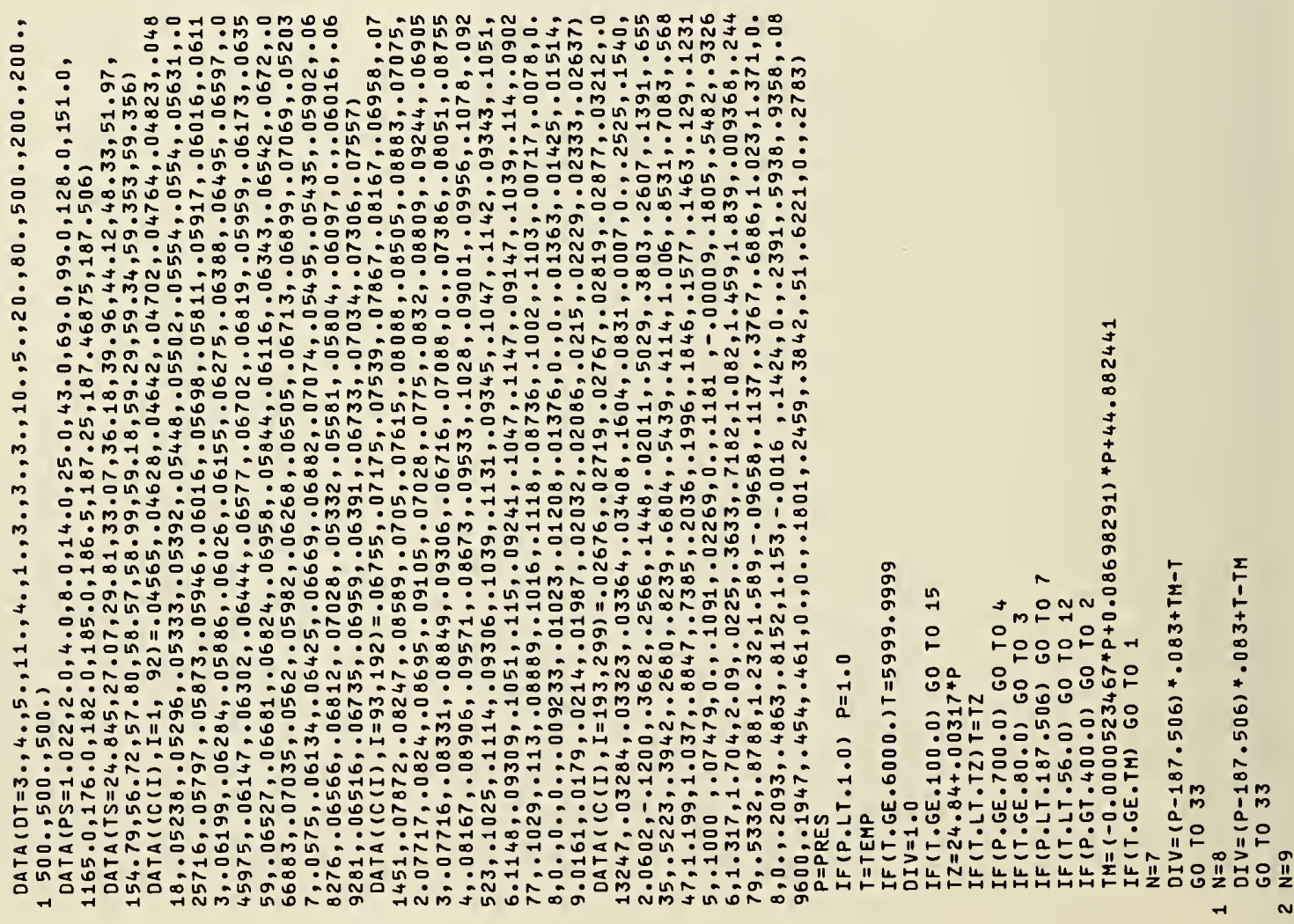



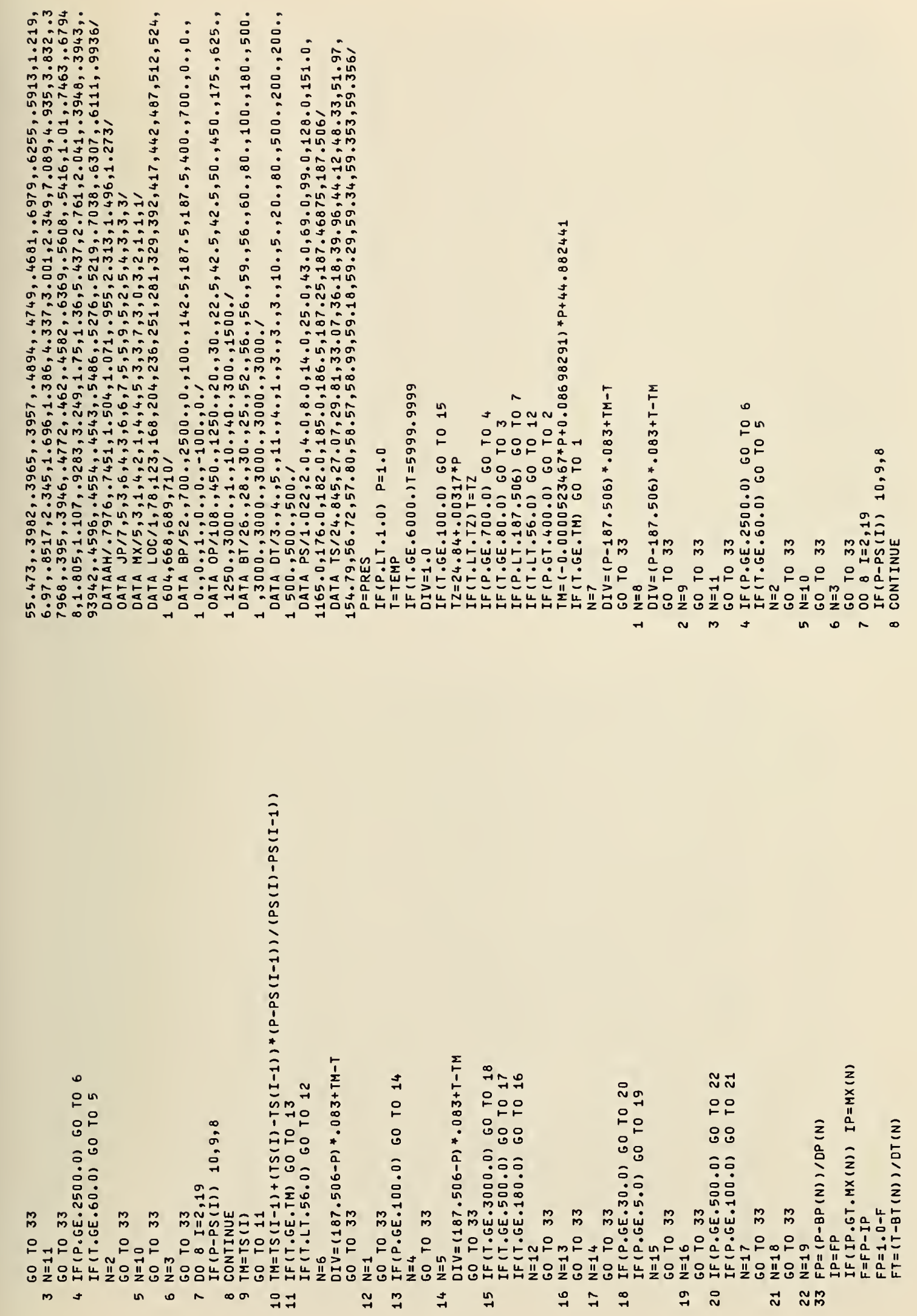

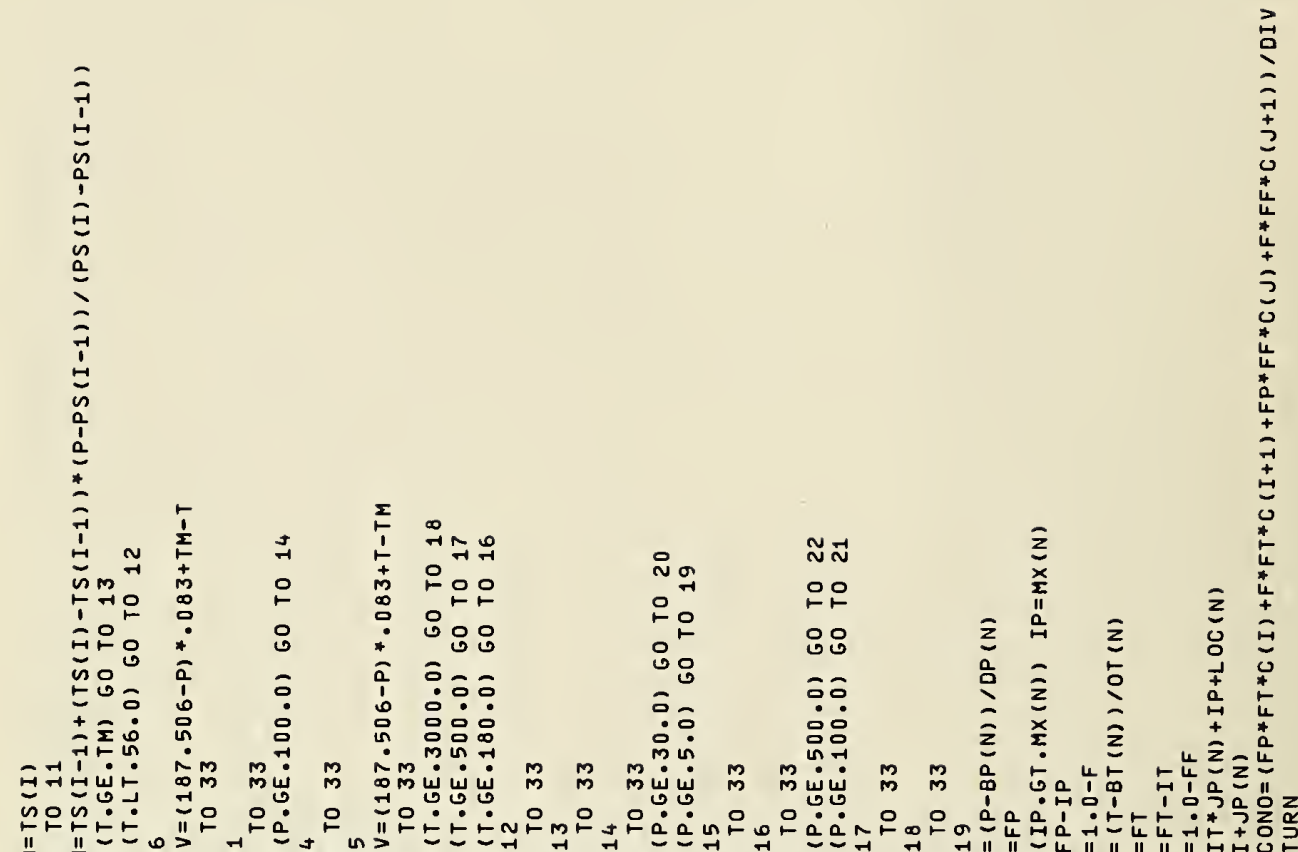

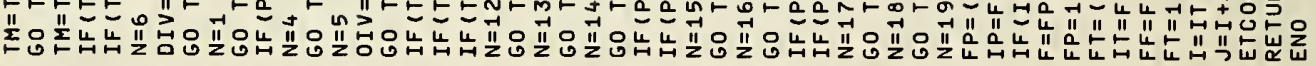

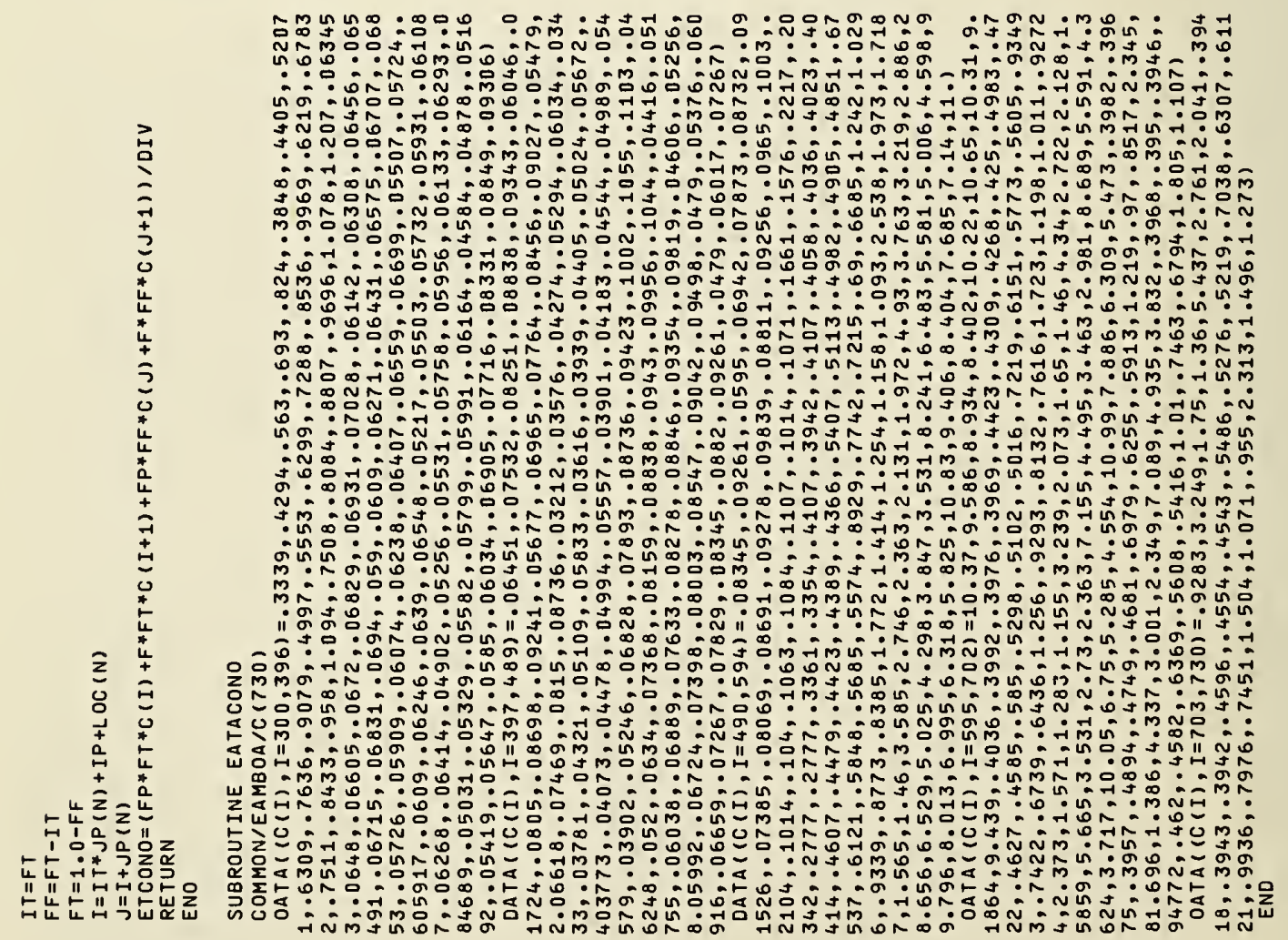




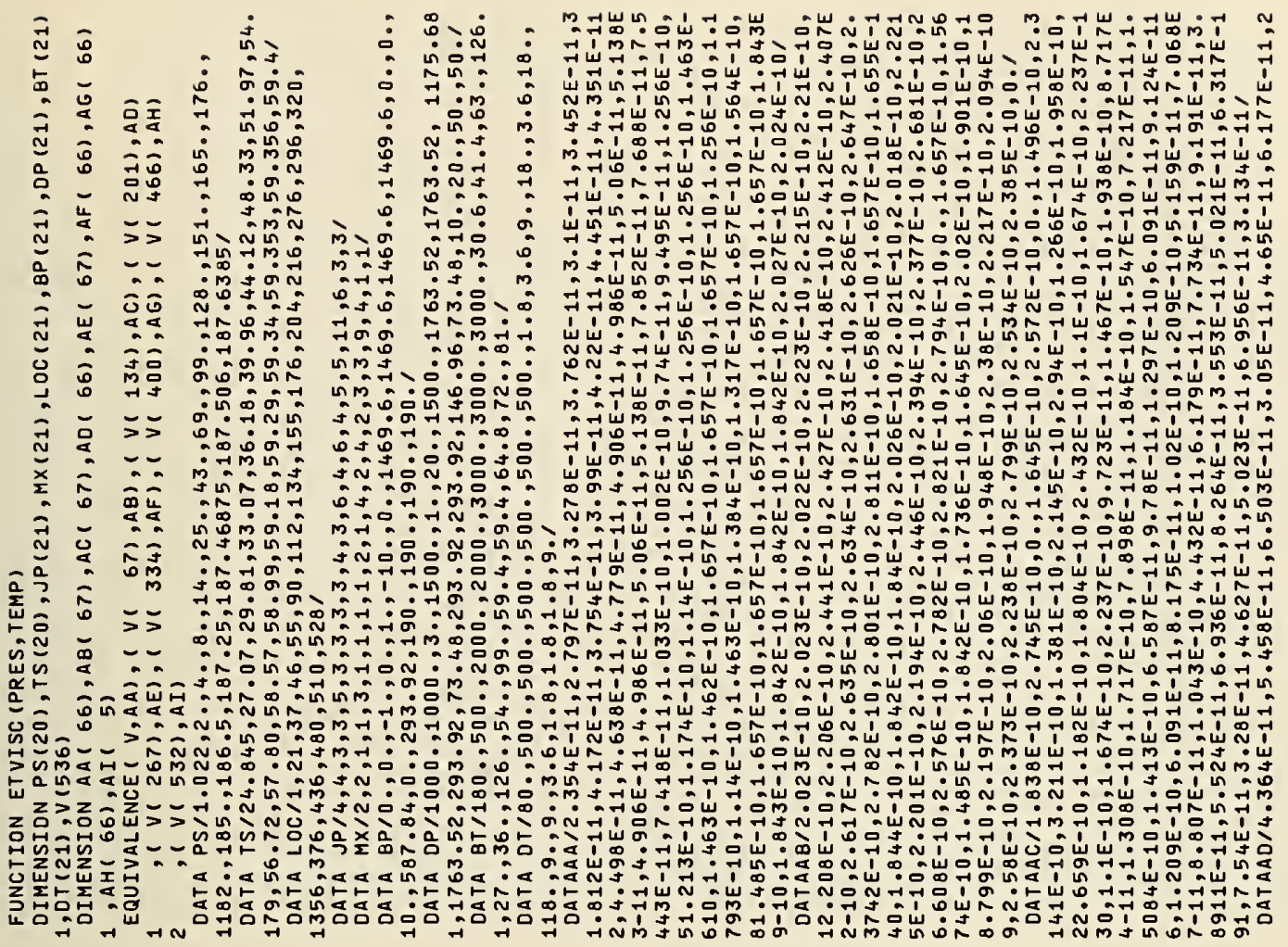

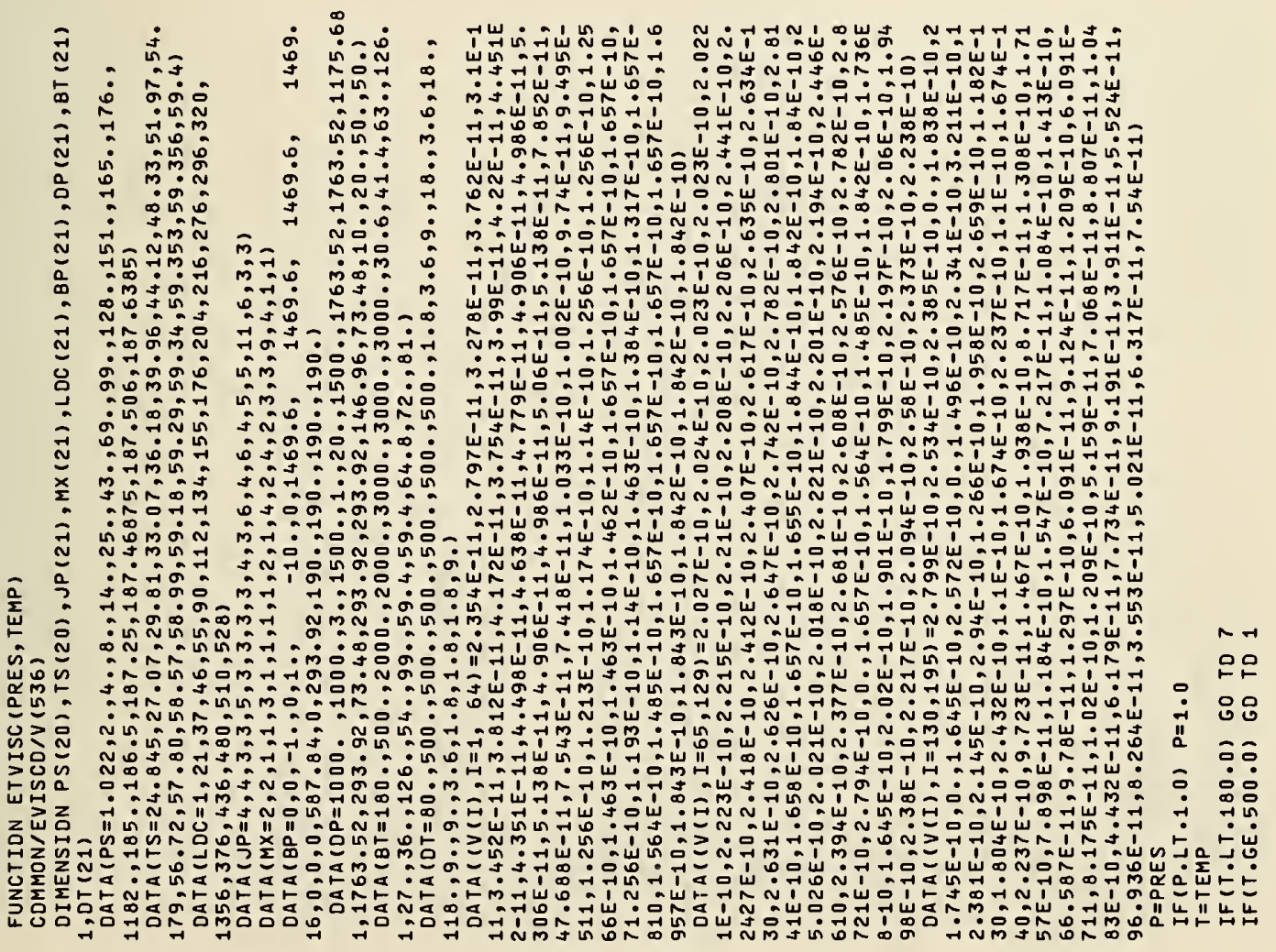



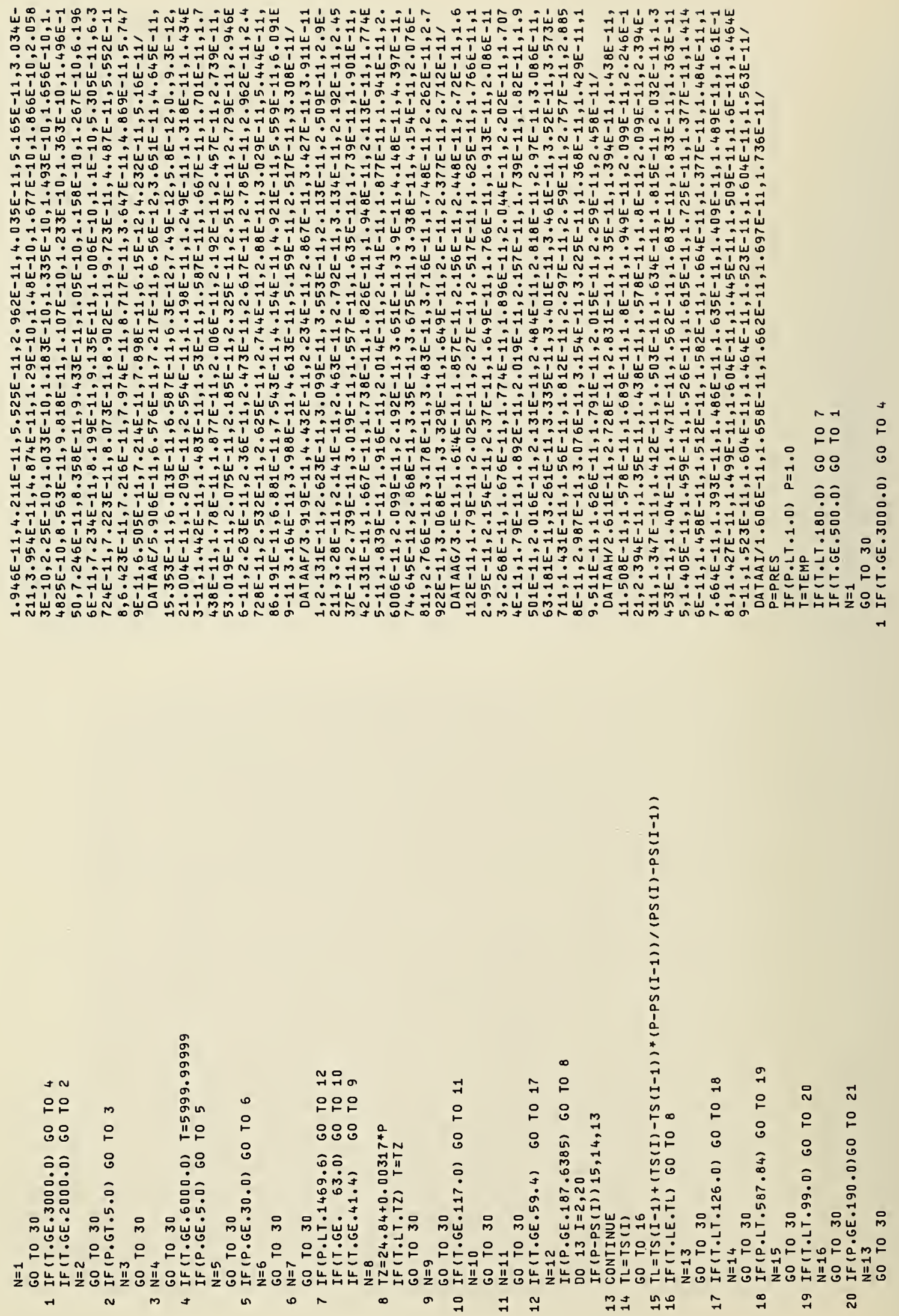


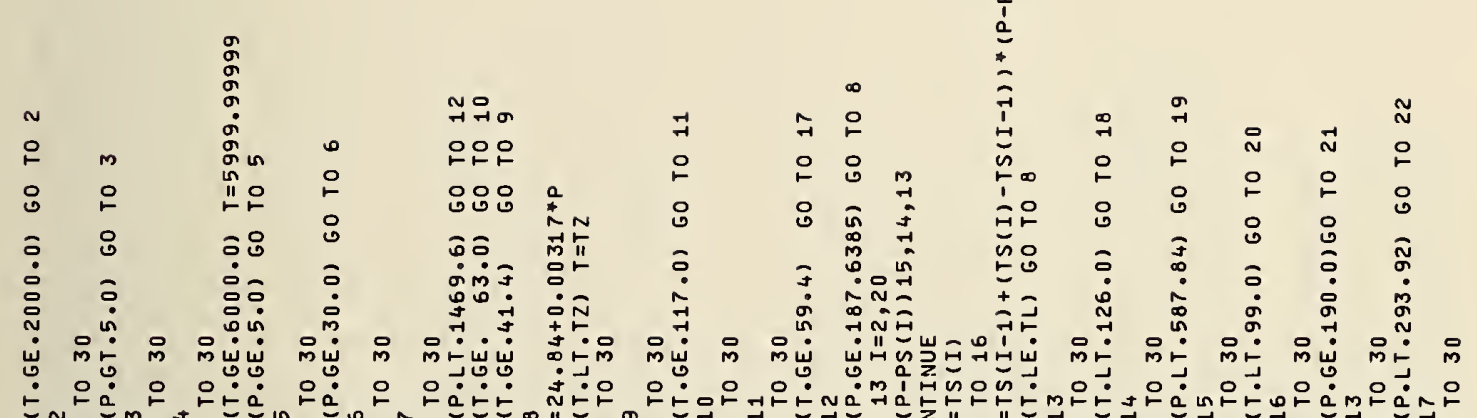

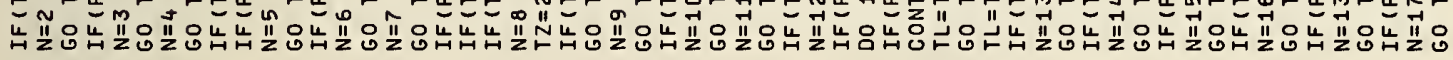

न

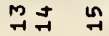

ๆ $\stackrel{\infty}{\rightarrow}$ ก

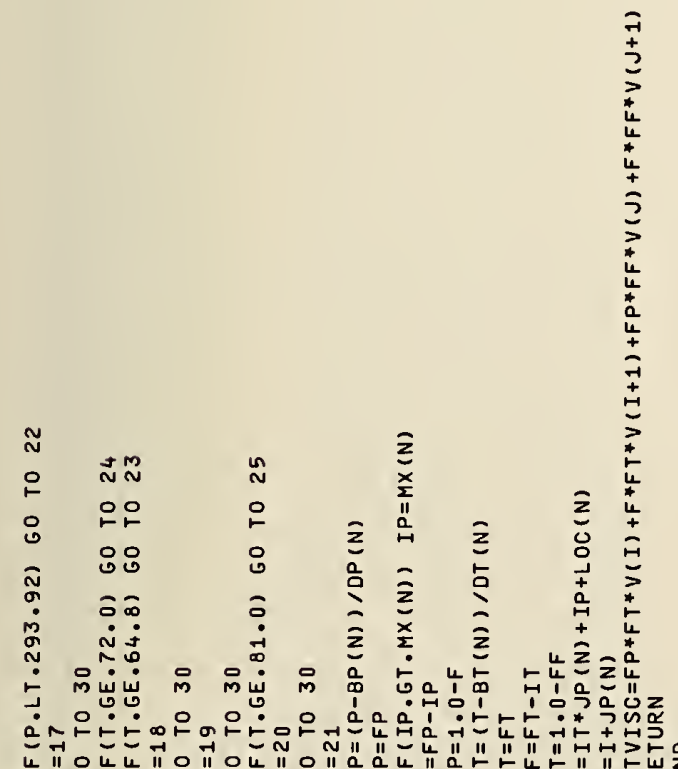

TH:

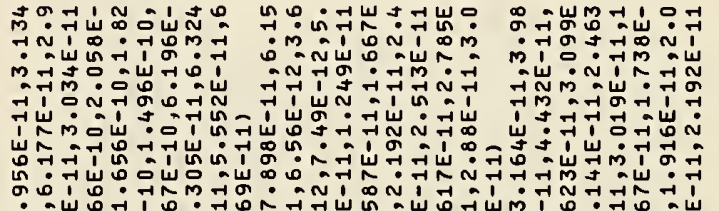
(1)

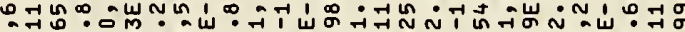
न1 山ू 出

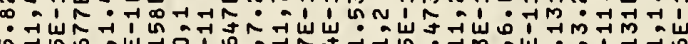
เ

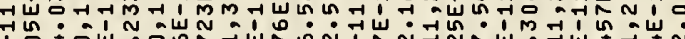

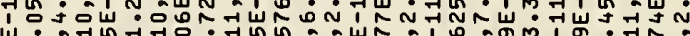

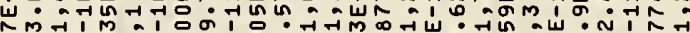
N M G

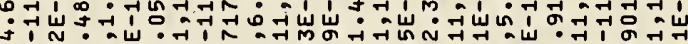
^ú

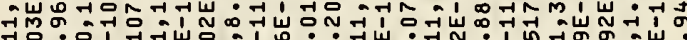
I س N iा

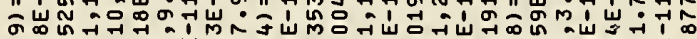
ô ט

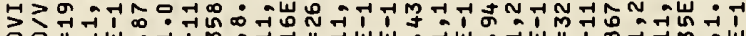

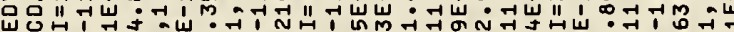

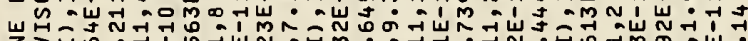

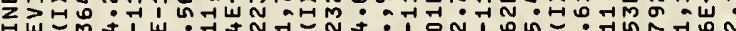

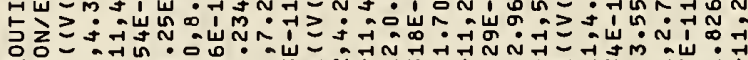

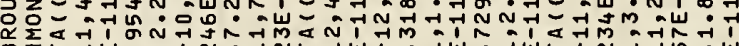

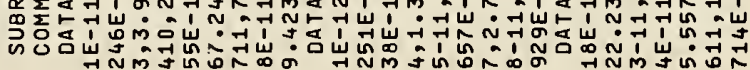




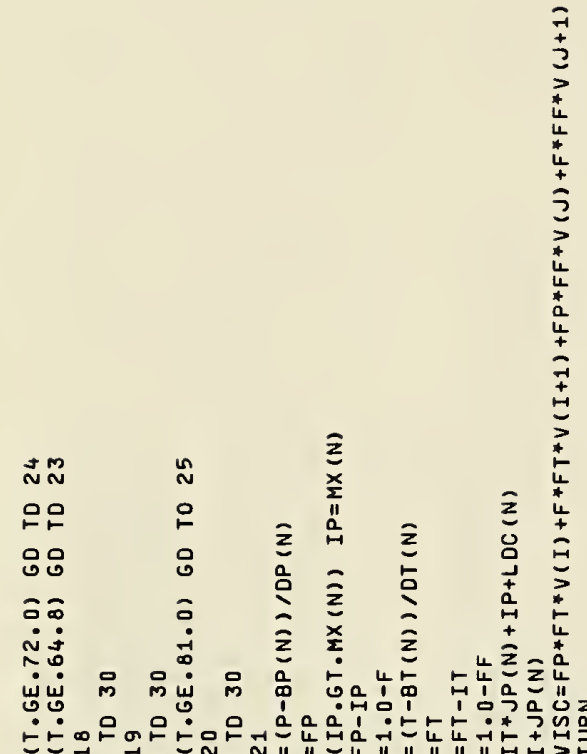

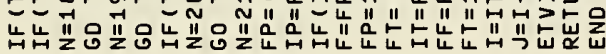
ก $\stackrel{M}{N}$ N

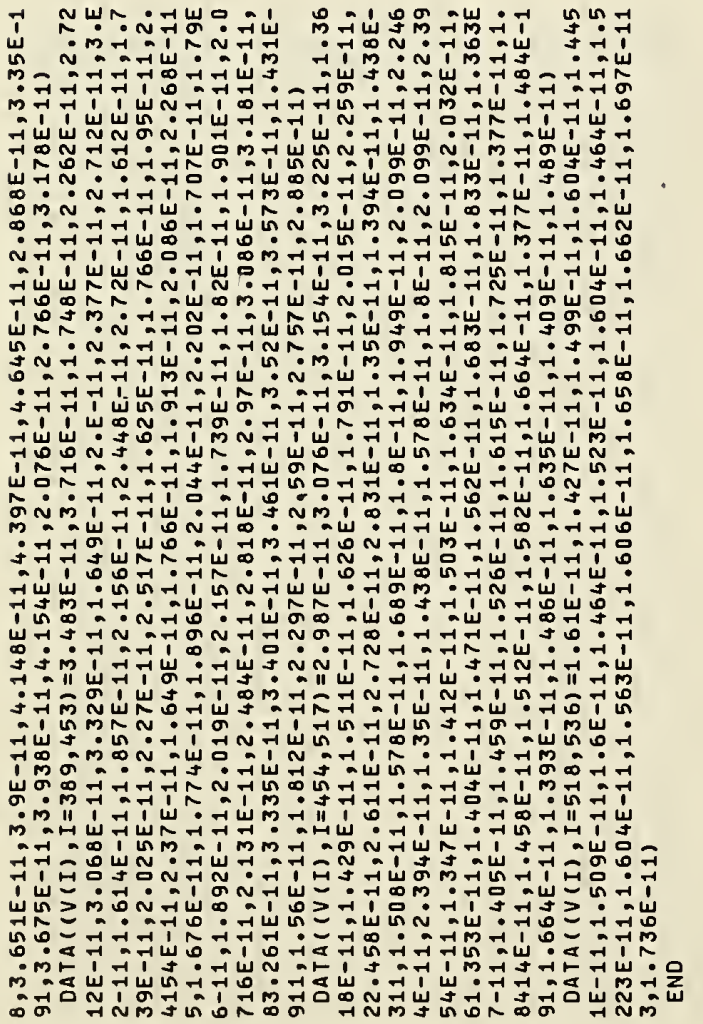

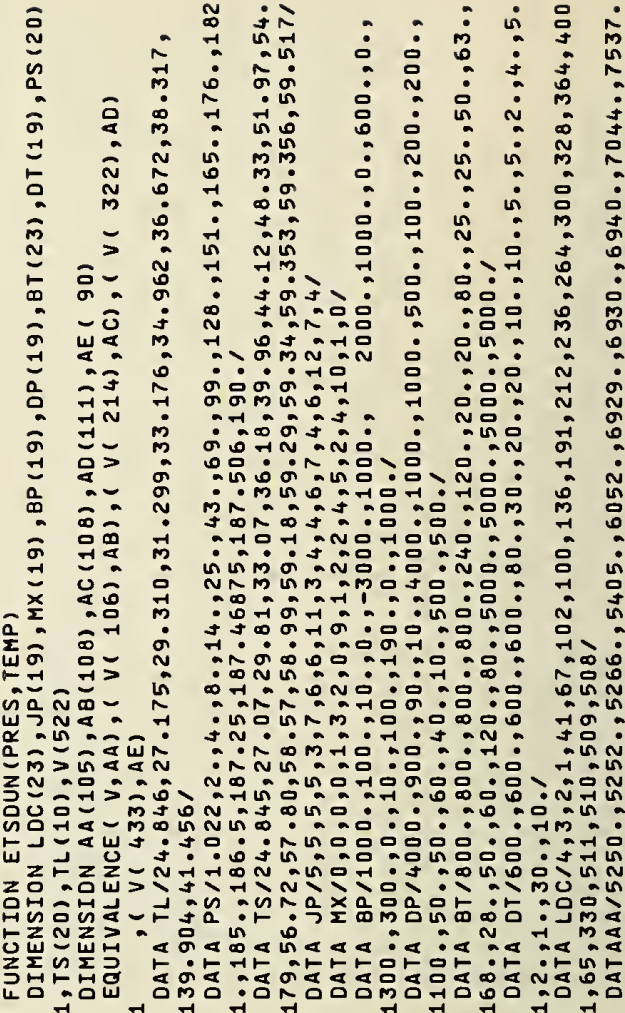

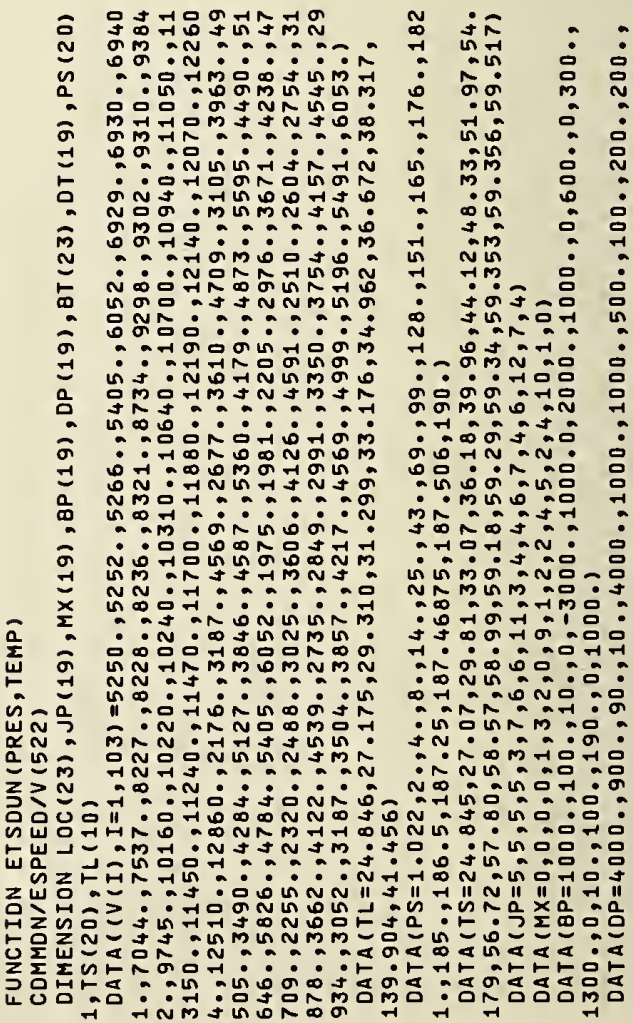




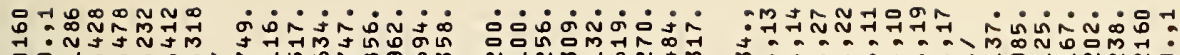

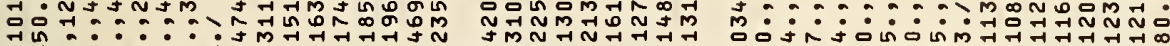

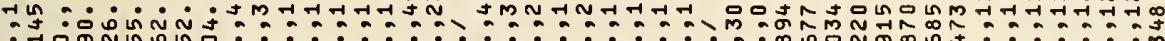

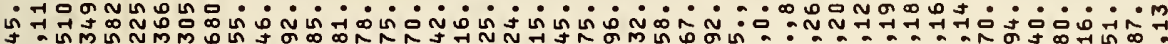

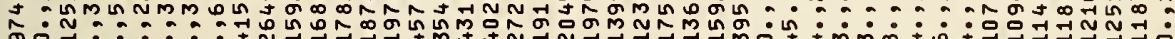
o.

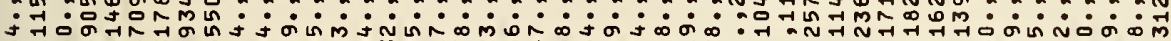

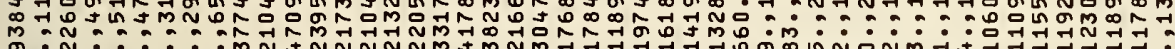

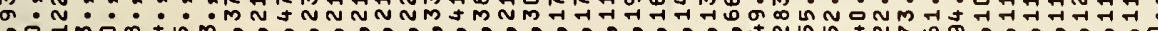

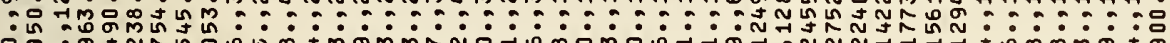

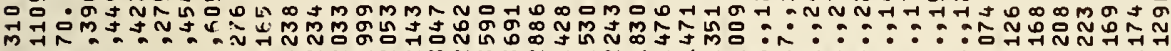

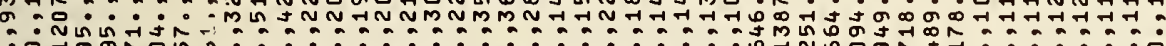

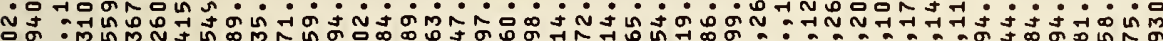
po :

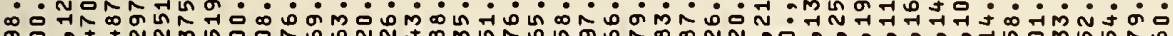

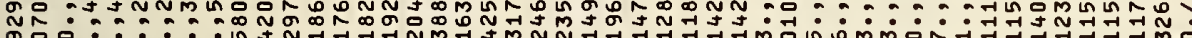
Ğ

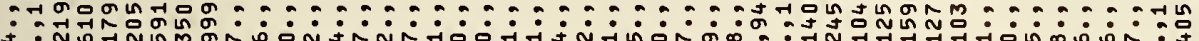
mo

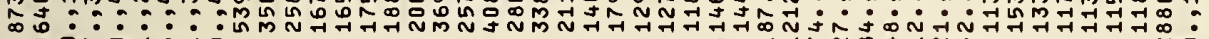

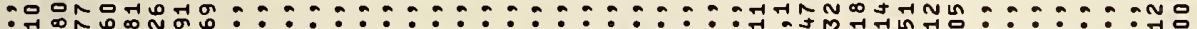

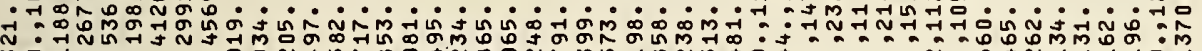

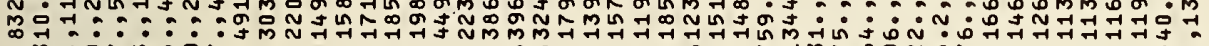

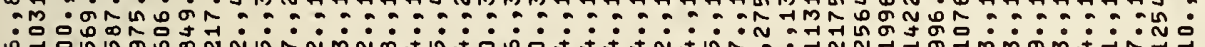

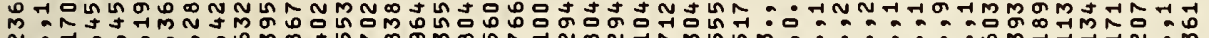
œ :

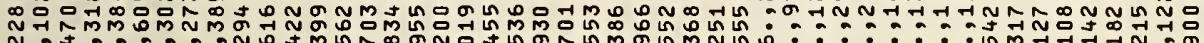

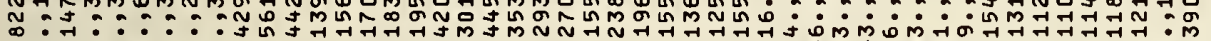
ON

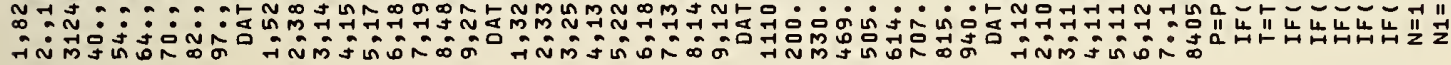
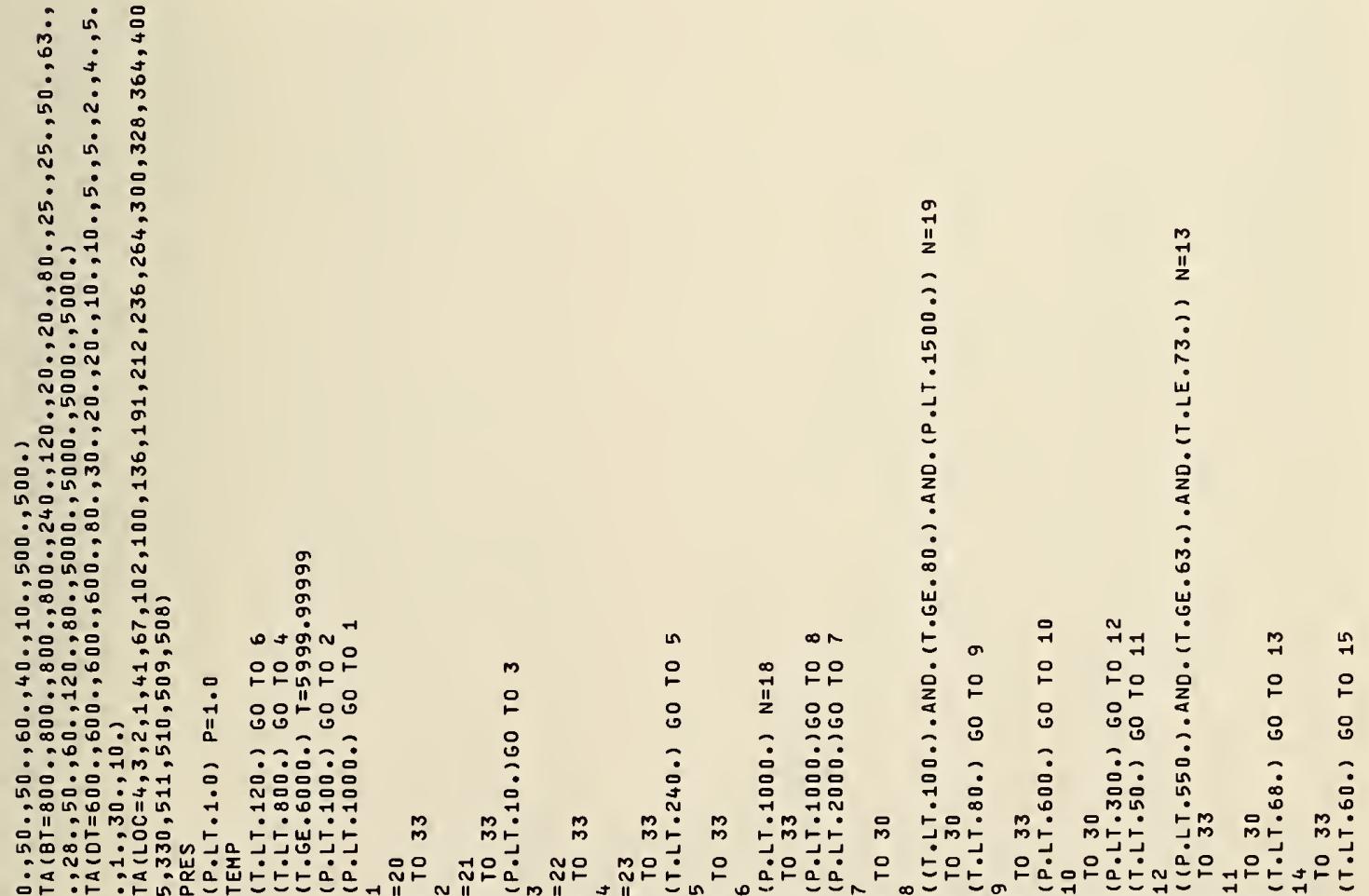

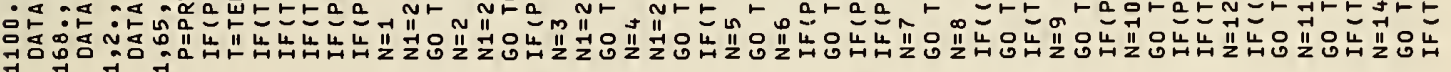




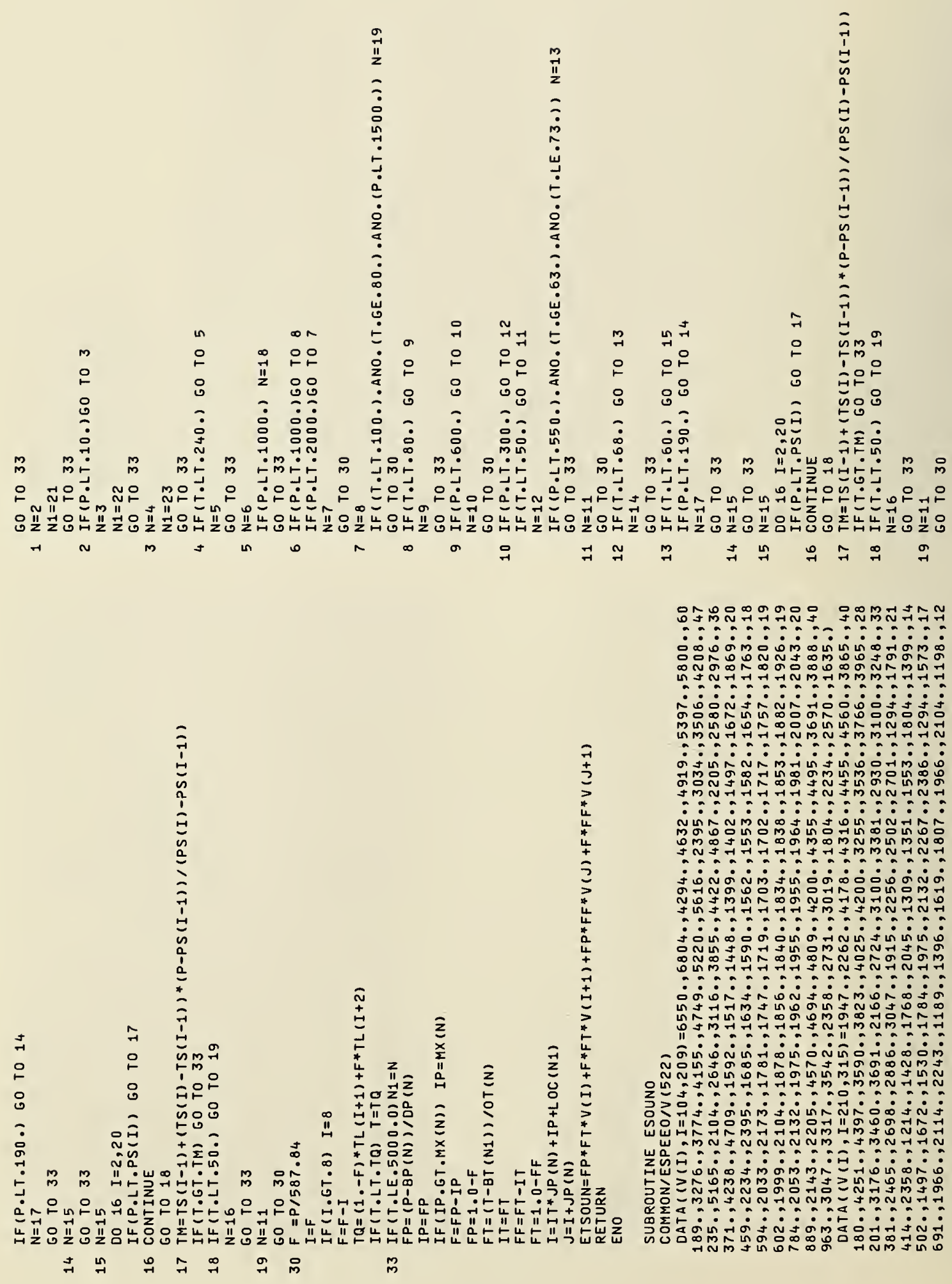




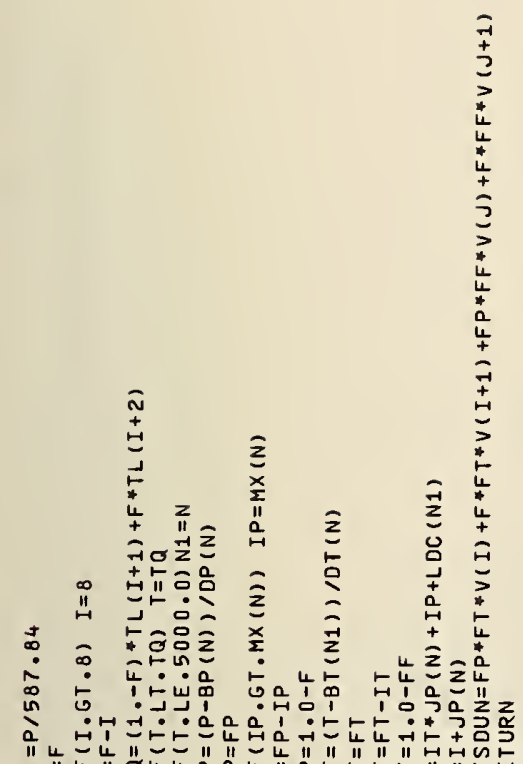

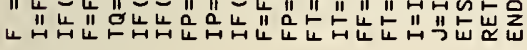

i

勇

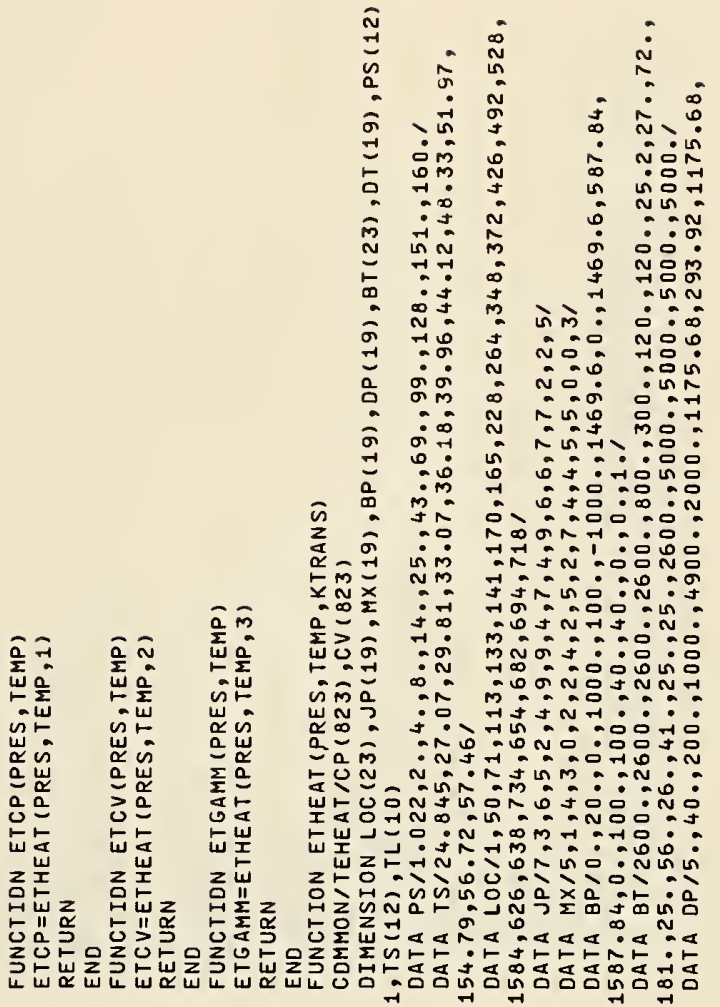

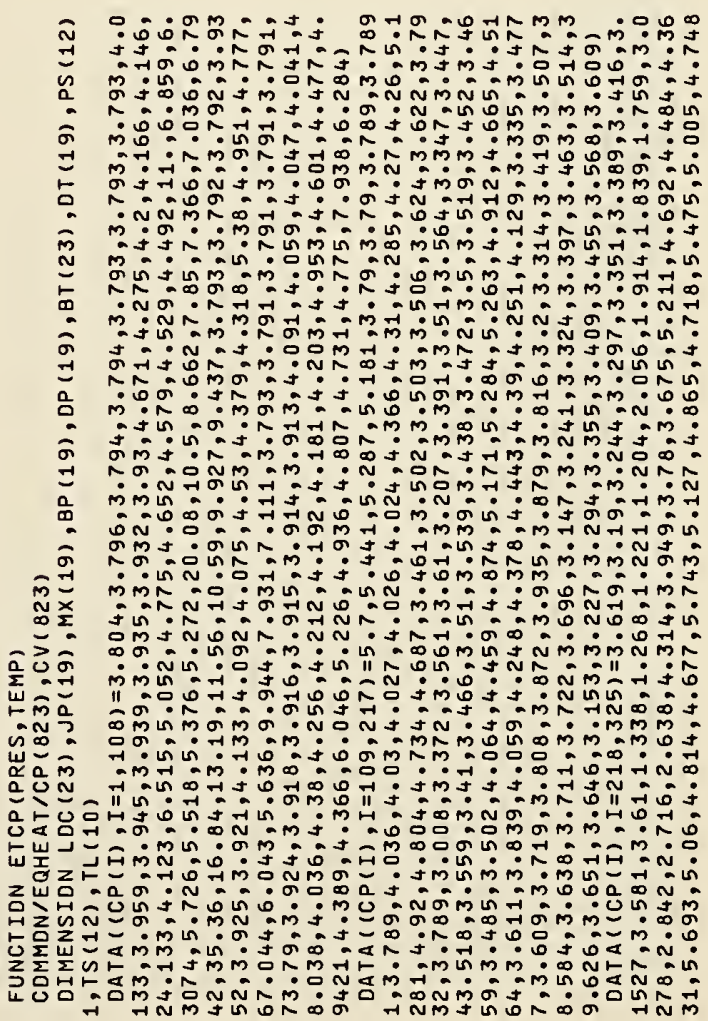



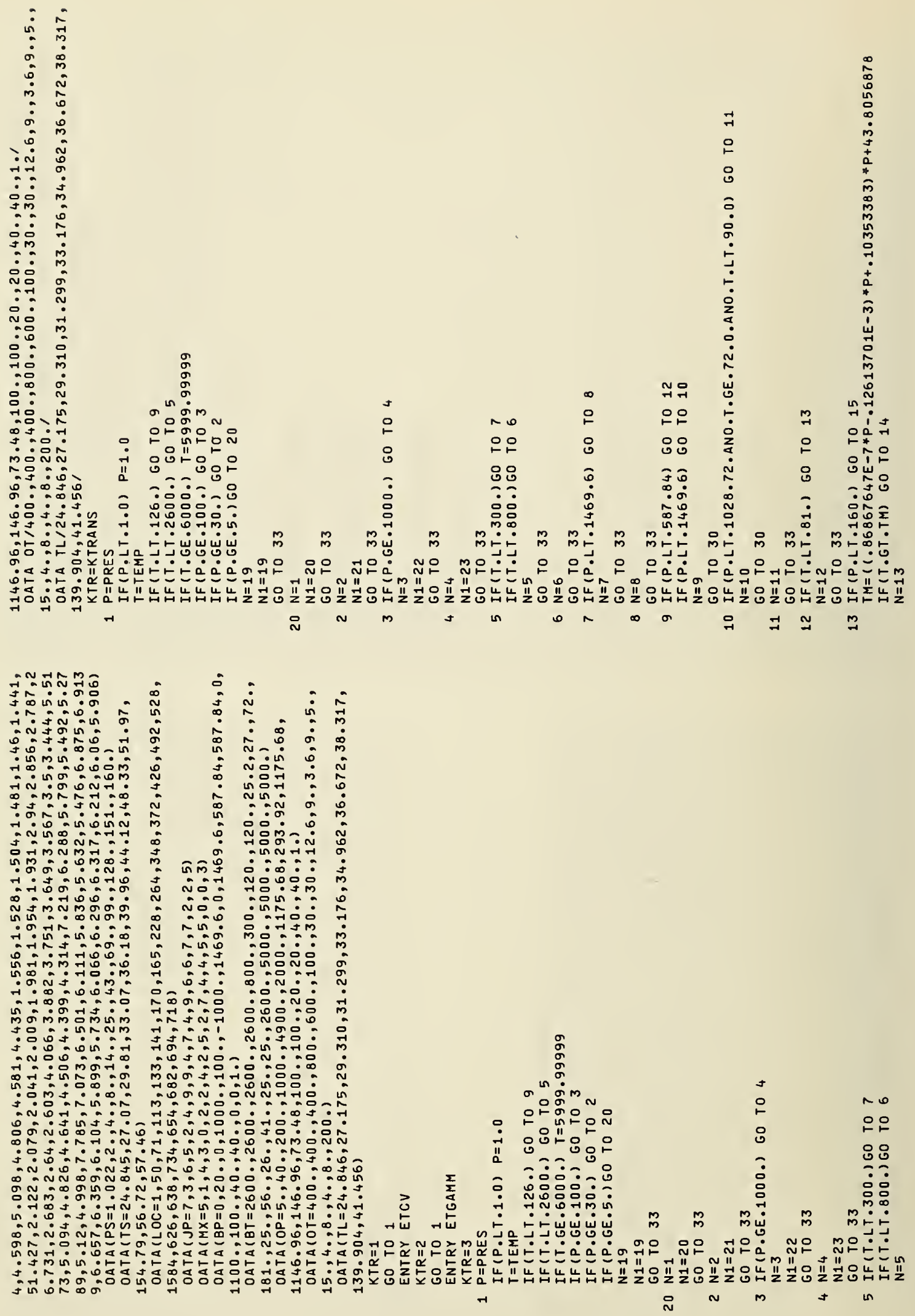

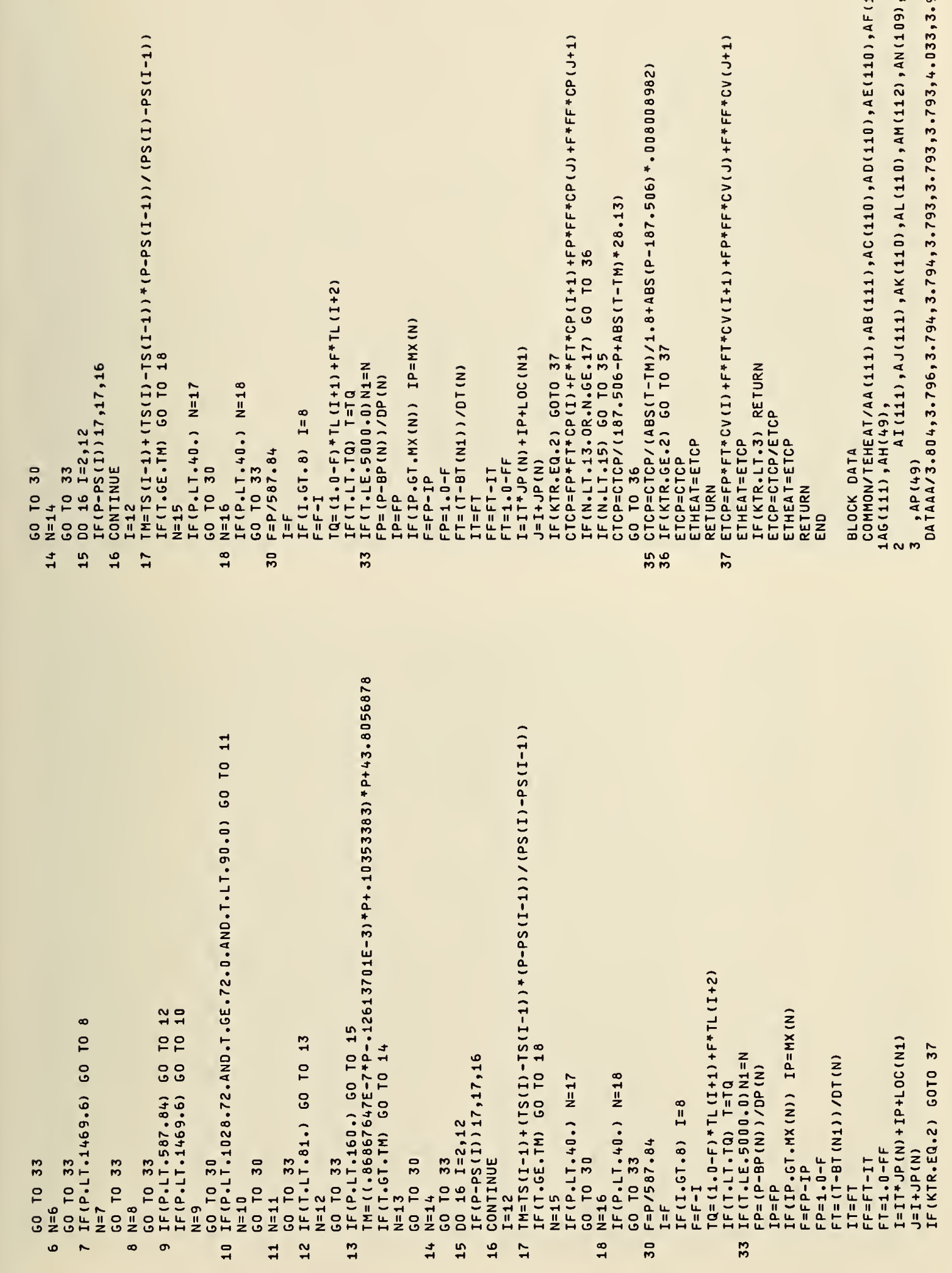


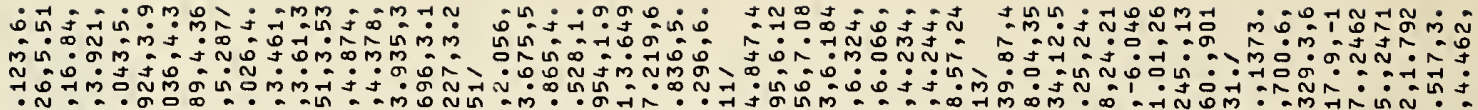
$\because N \cdots$

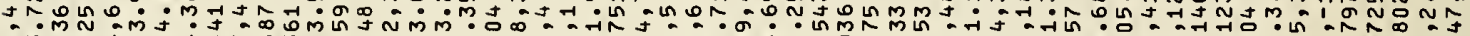
mon

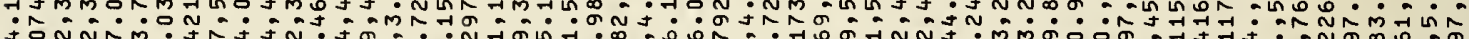
JoN

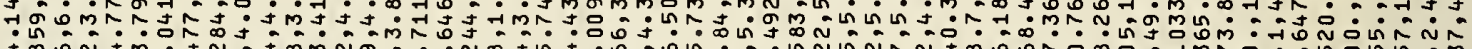

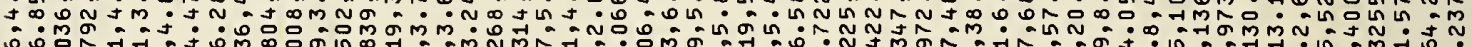

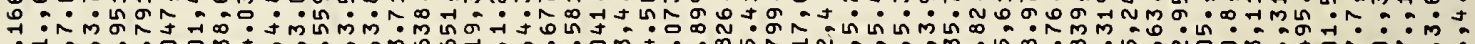
ز

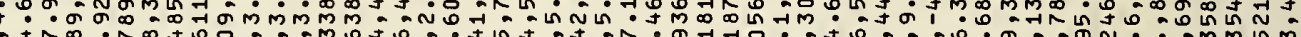

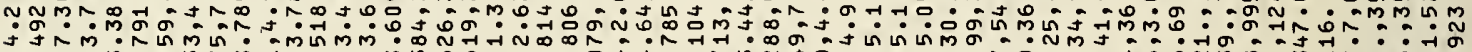

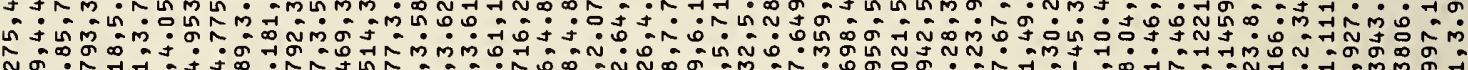

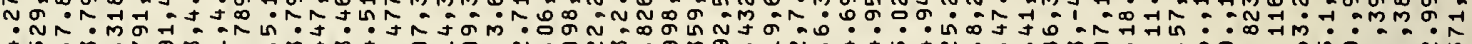

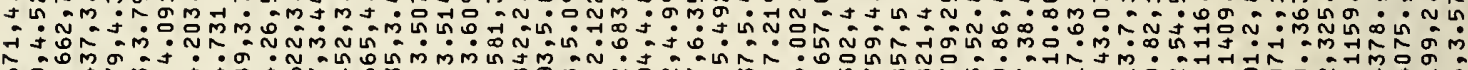

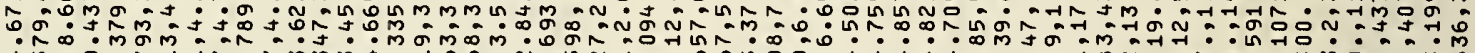

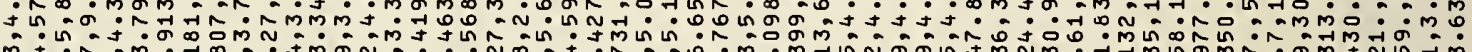
jুł m

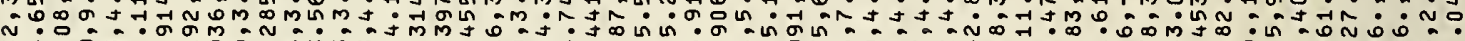
o jơñ m

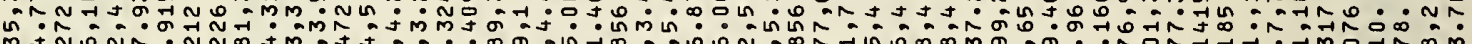

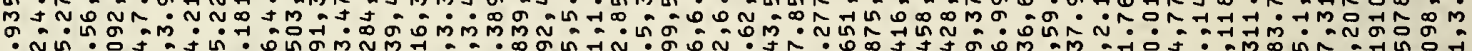
mun

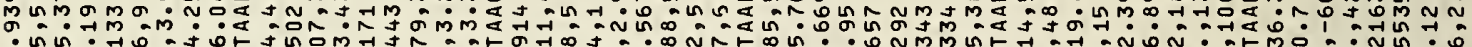
m n.

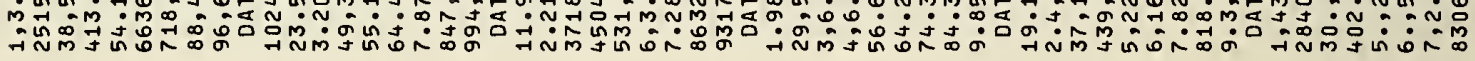

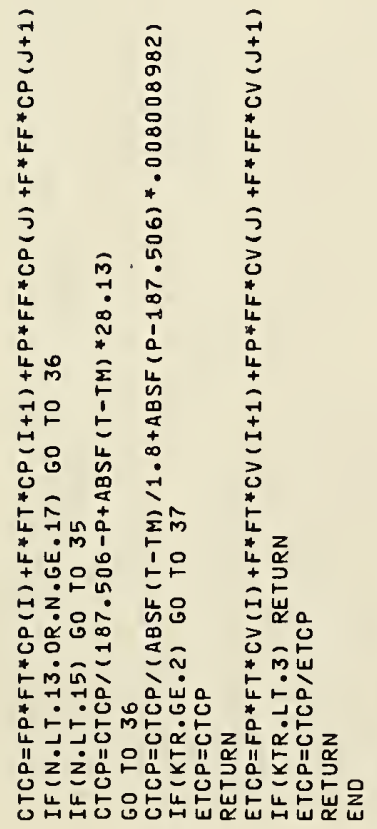

出品 向
ที大 नiñ

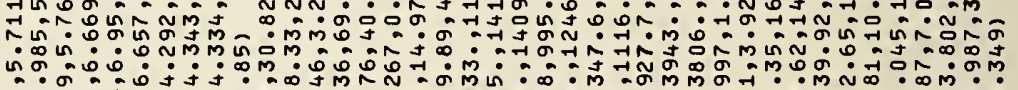

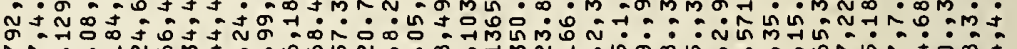

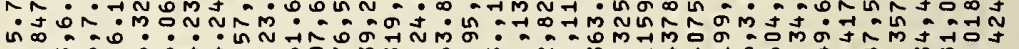
f ம0。ำ

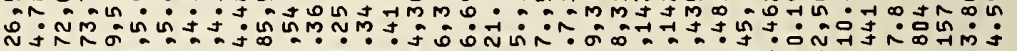
๑ñ

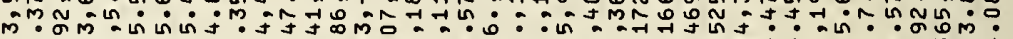
تñ

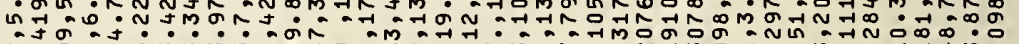

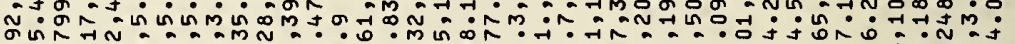

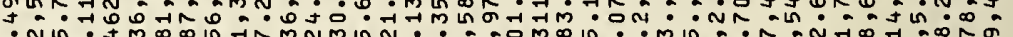

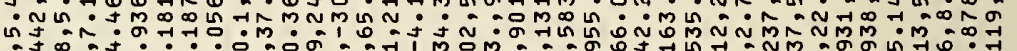

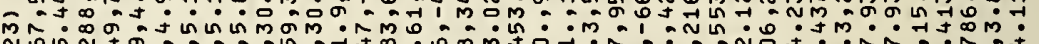

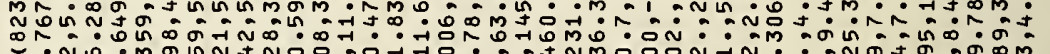
>

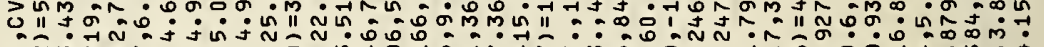

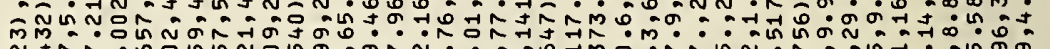

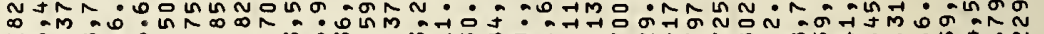

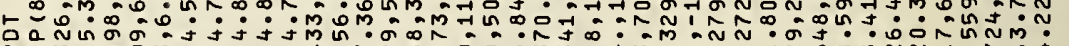

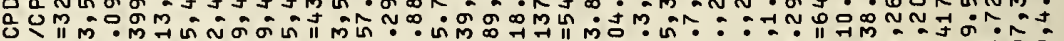

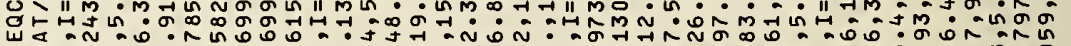

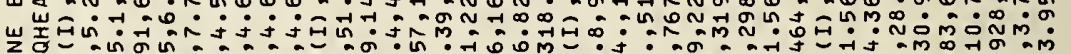

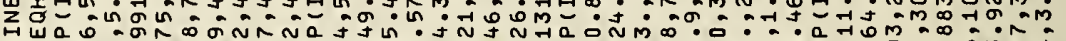

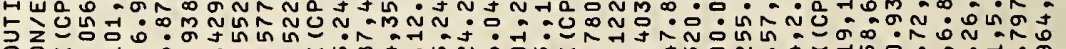

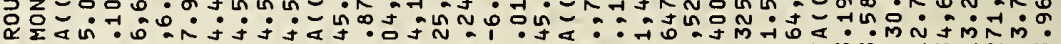

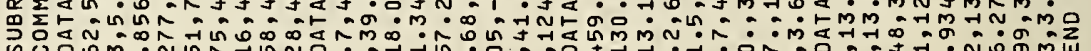

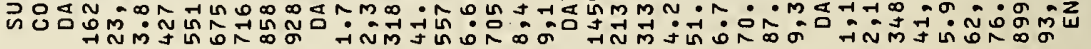




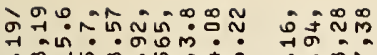

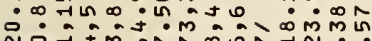
N ง $\dot{0} \dot{\sim} 0 \dot{0}=0$

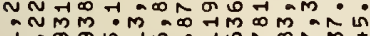
กีm. j

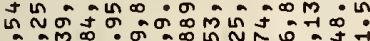

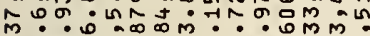
3 бำดे

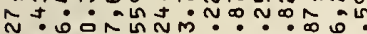

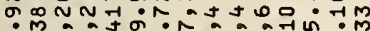
ơ

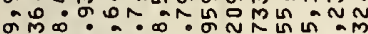

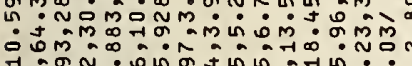
Mmómm in U. ơ

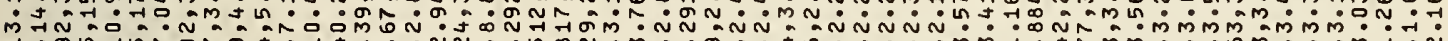
j

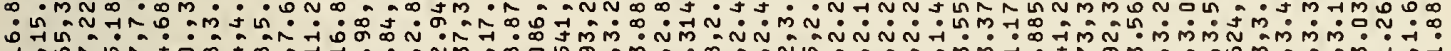

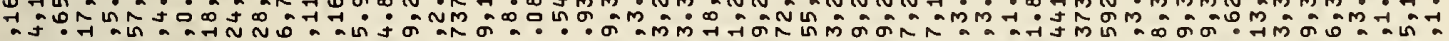

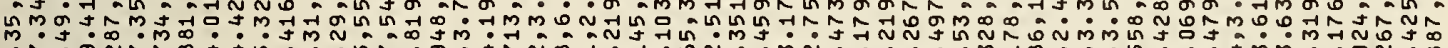

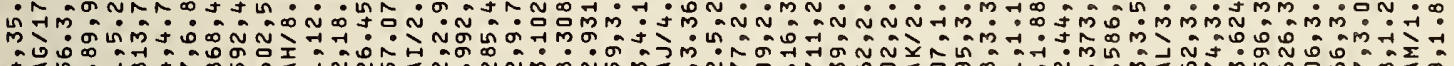

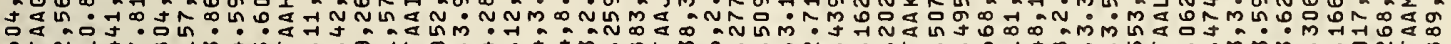
ó

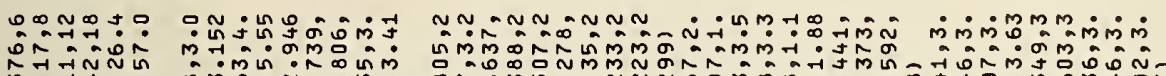
in

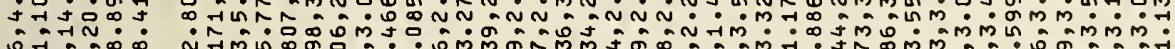

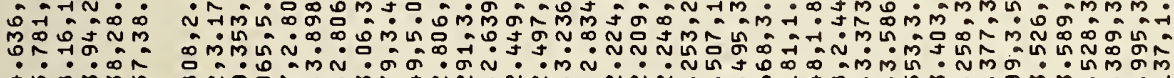
ن

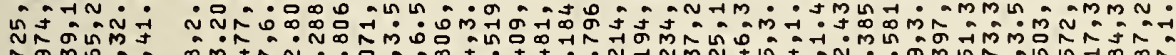

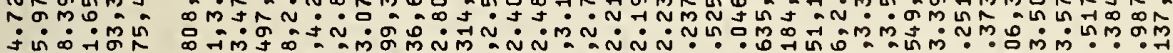
N

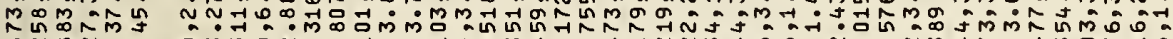
ơ

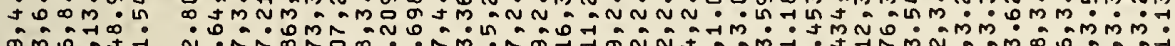
Nimón

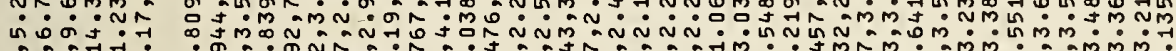

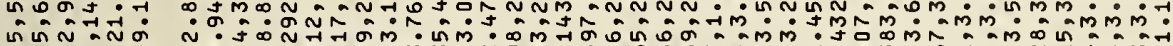

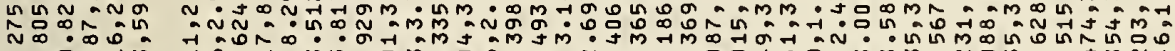

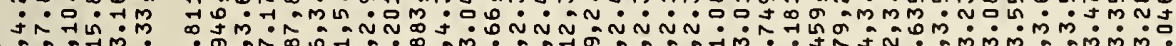

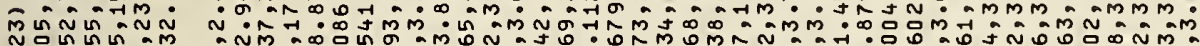

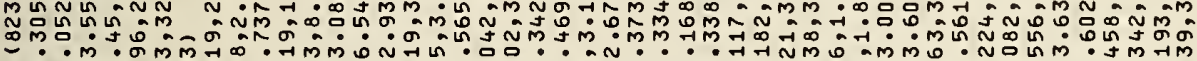

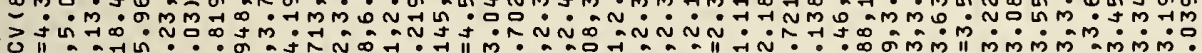
O

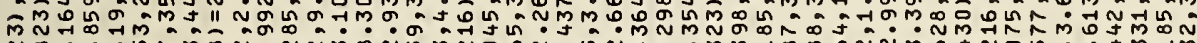
N

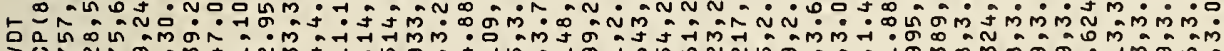

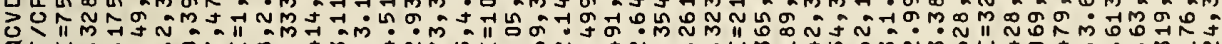

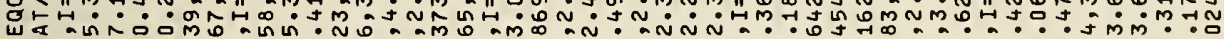

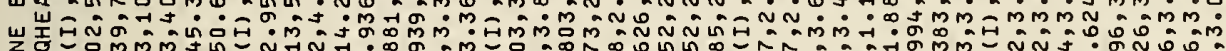

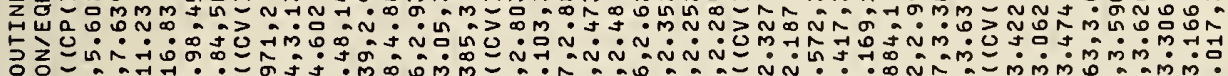
8

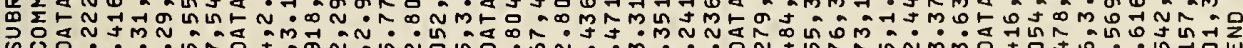

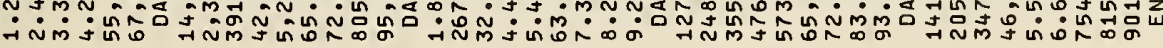

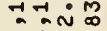

Nö̊

구 $\because$ in

i० 0 in

ำง

구응

जิनेंม

ํ.ำ

-1निं

ดิกัฒั

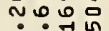

$\because \exists \dot{\sim}$

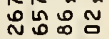

H.

野会

- $\infty$ in

-

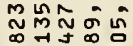

ट्र

ลू冋ूล

ल

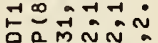

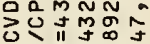

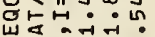

แ岌气行

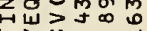

इ之नन

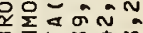

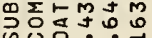
नंत 


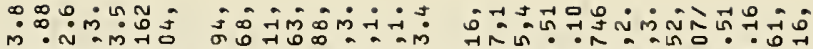

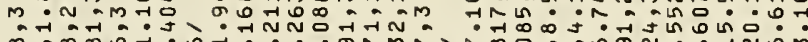
o

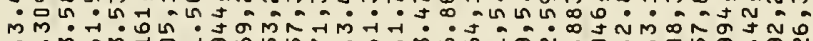

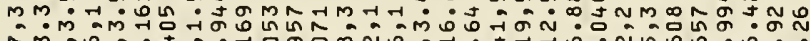

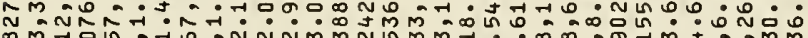
m

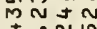
ลั०

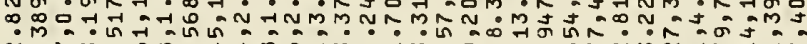

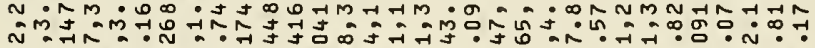

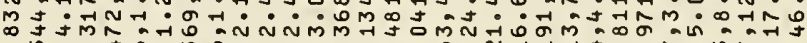

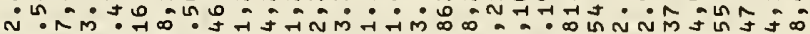

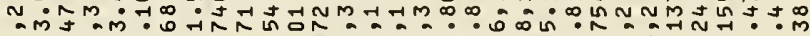
ڤ MMNM

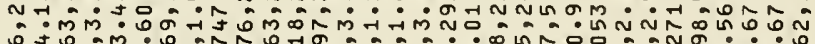

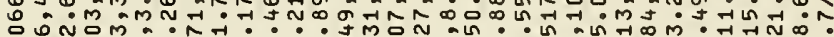
mरिभm

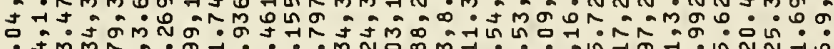
mं mm. fon

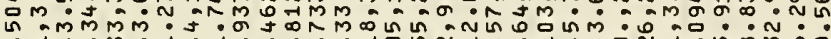
¿ वनंन กั. ڤึm

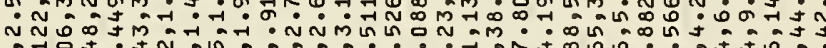

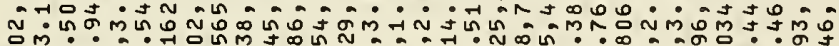

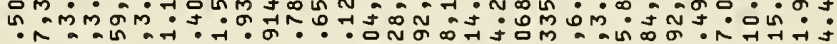

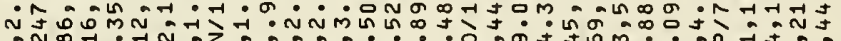
คํ.

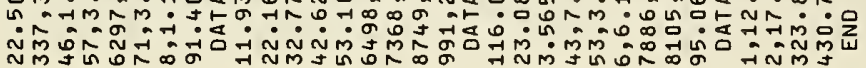

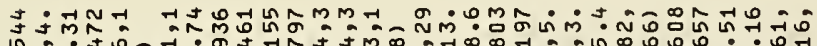

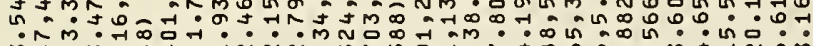

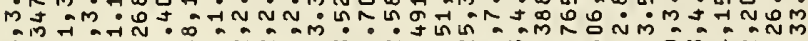

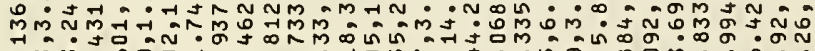
于

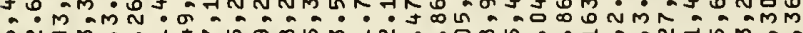

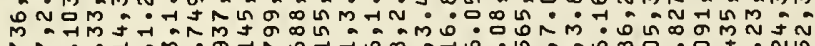

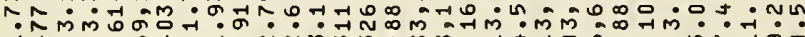
$\because$ U fmma ดें món

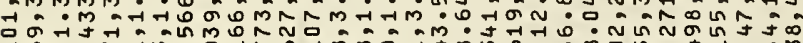
管 in

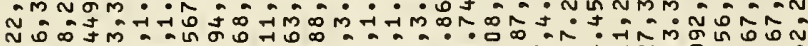

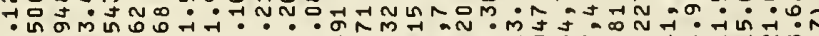
mon mmom N

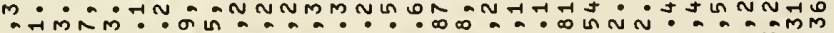

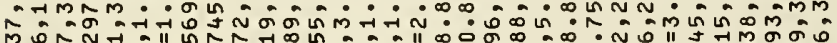

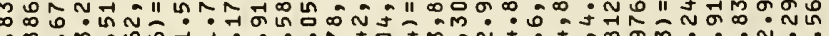

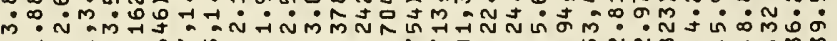
क-

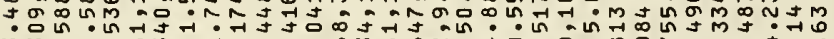
m.M. - mo ล

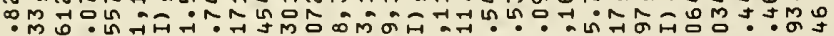
Nmpm

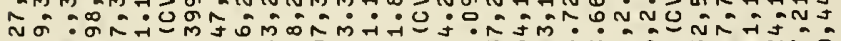
om:

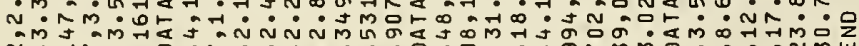

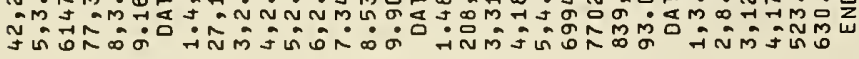

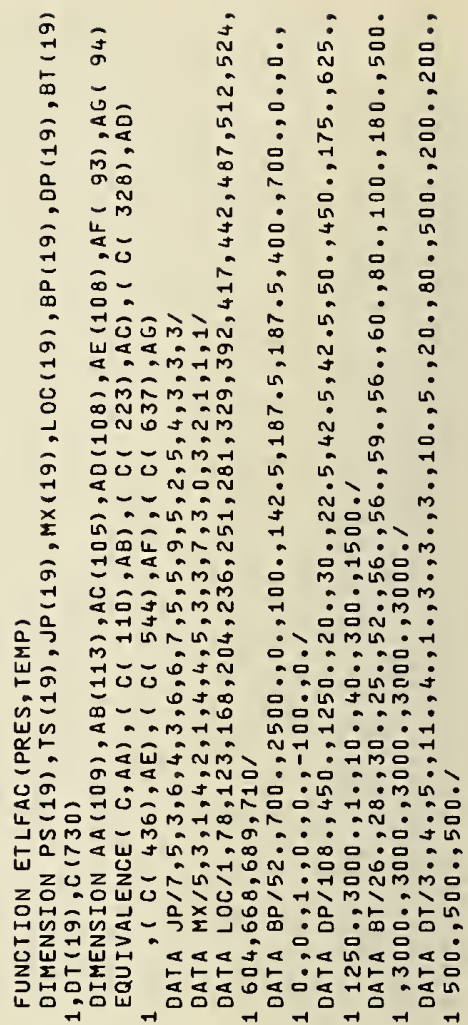

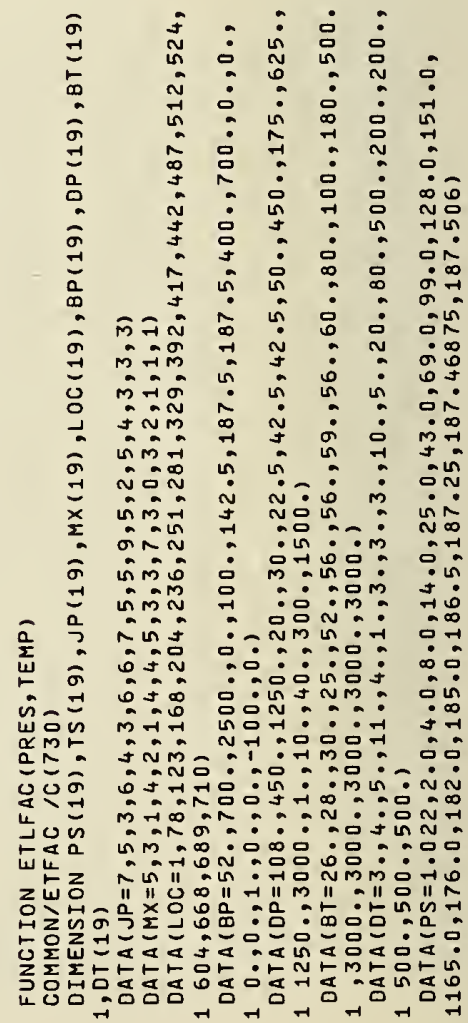




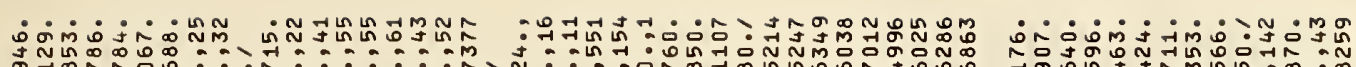
$\therefore$ Õ in

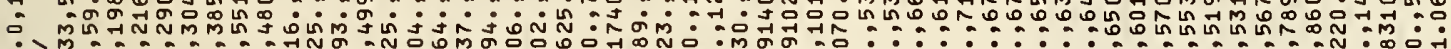

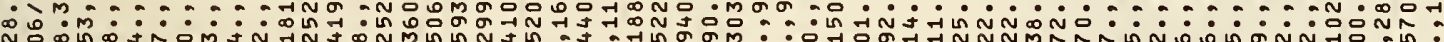
7 I ๑ก बำ

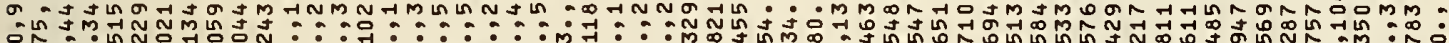

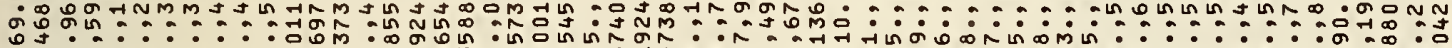

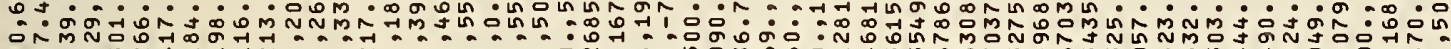

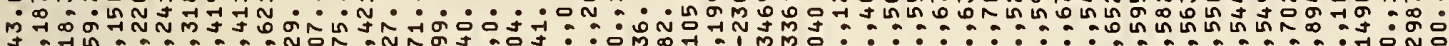
in

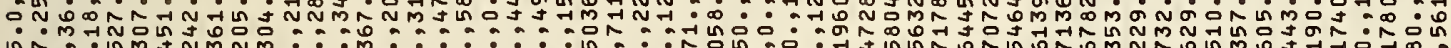
N :ڤñ J M

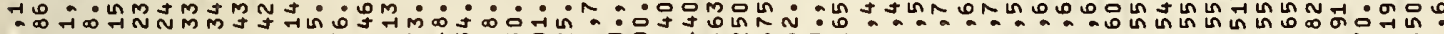

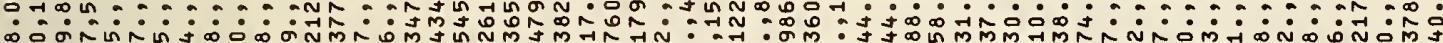

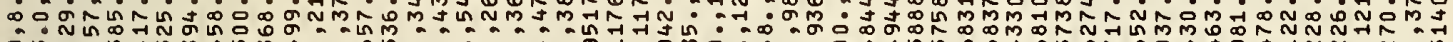

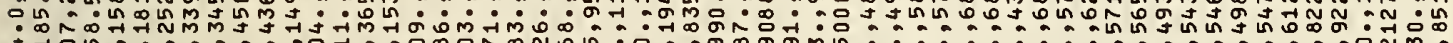

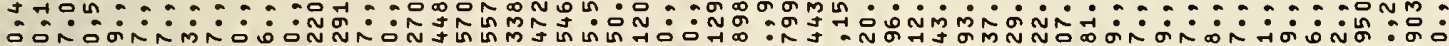
:

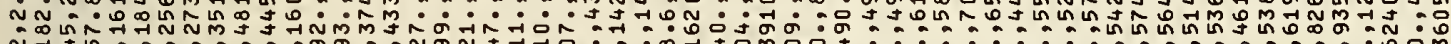

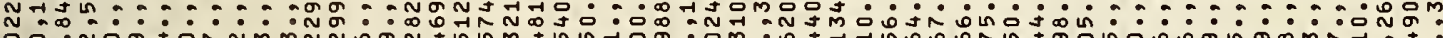

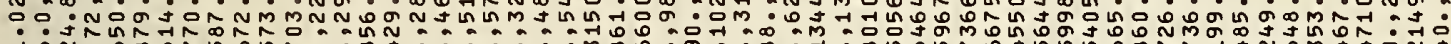

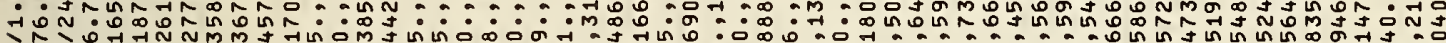
й a OA

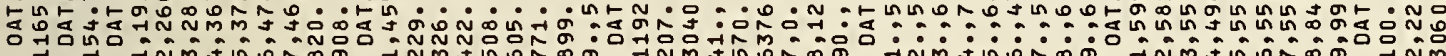

กิกี โี่

: N

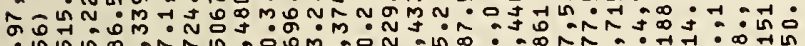
คี in in m.0ำกำ o

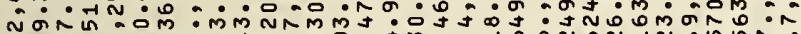

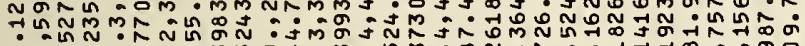
Jै? ơ⿱屮凵心夊 Si⿱⺈⿻⺕亅八 o mat-1№n

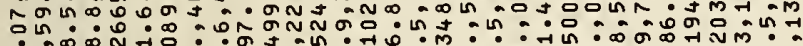

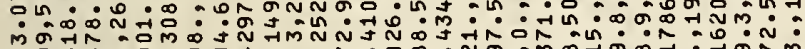
mơo -io in

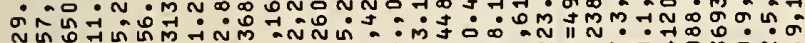
Nֻ

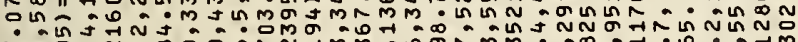

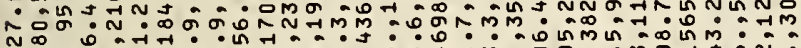
N 经

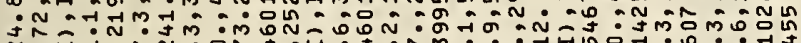
N

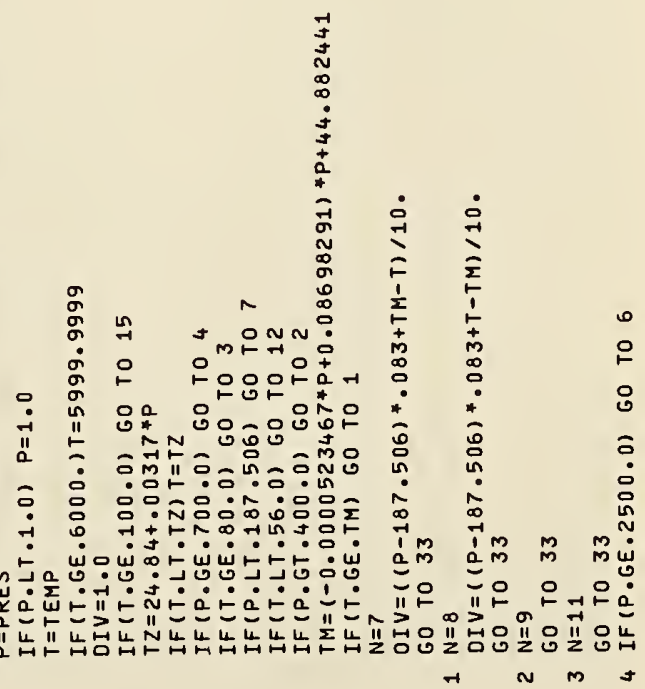



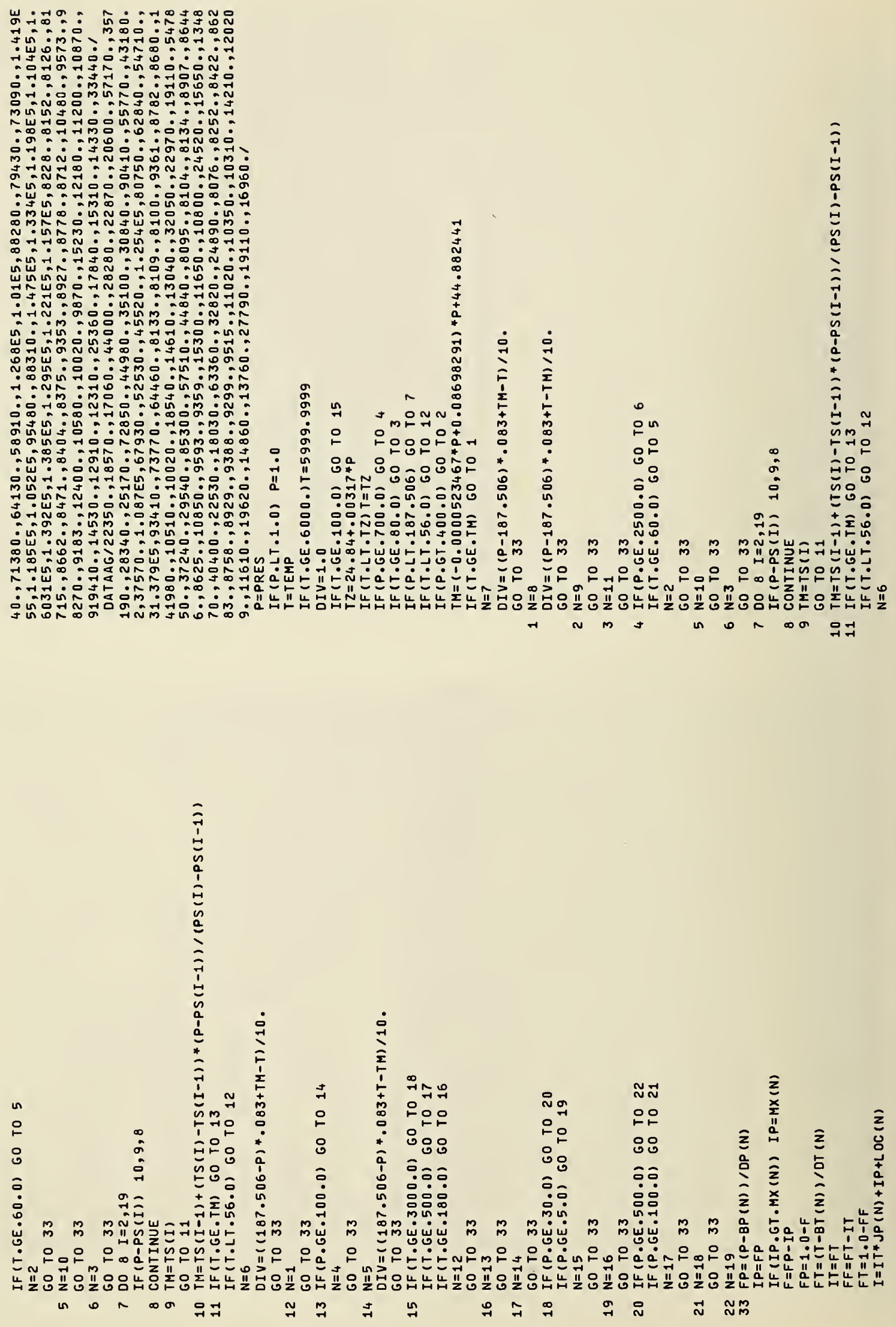

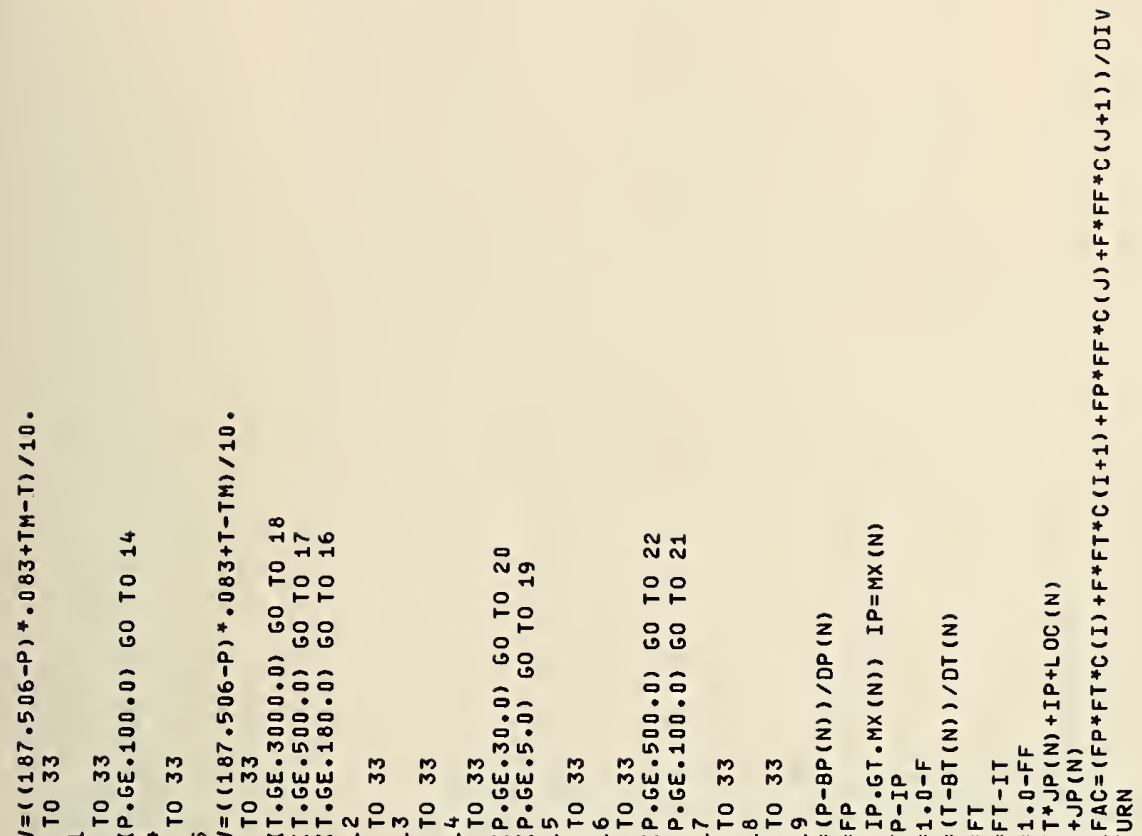

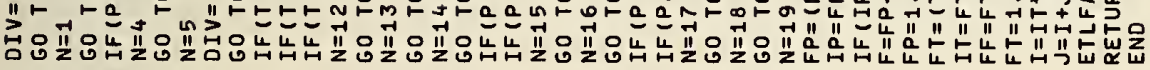

ๆ

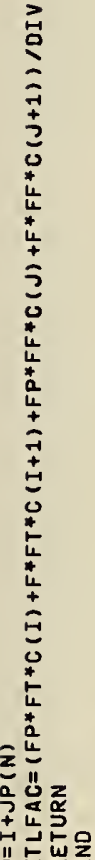

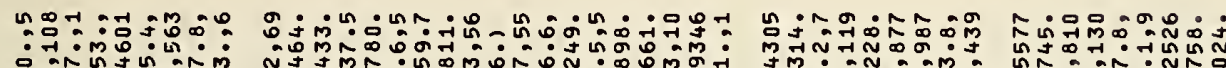
:

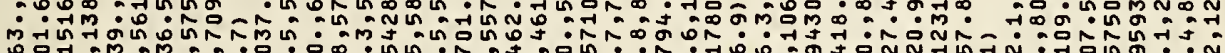
mon

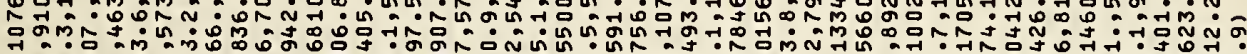

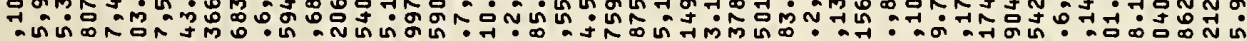
:

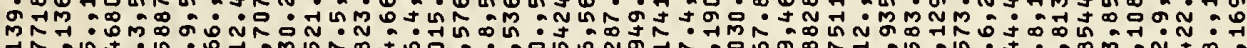

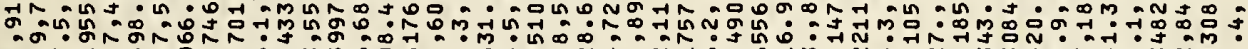

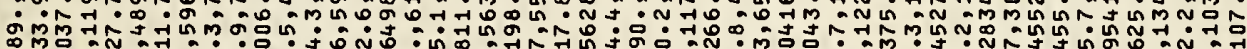

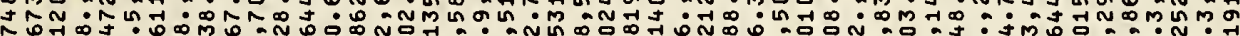

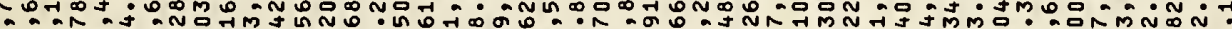
on

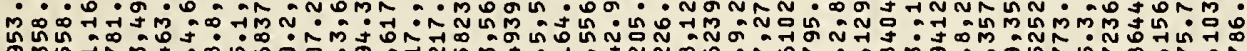

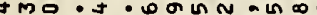

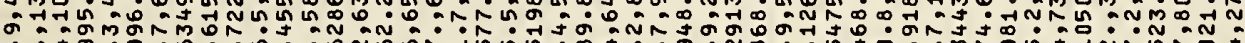

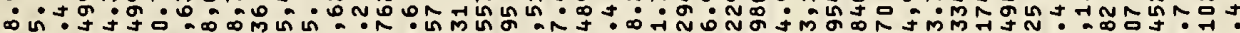
承品-

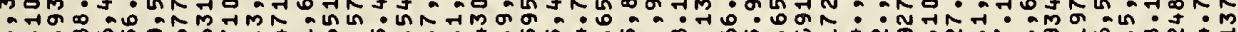

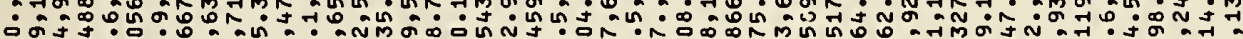

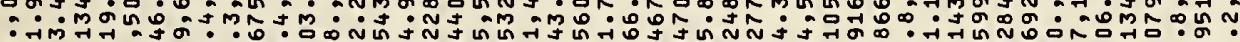
ํㅕㄹ O.

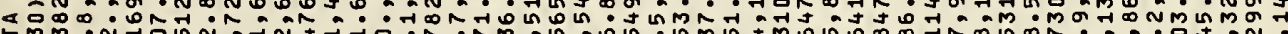

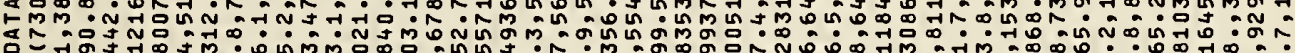

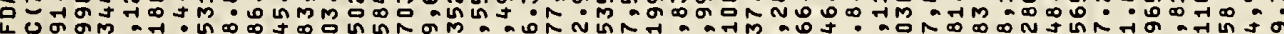

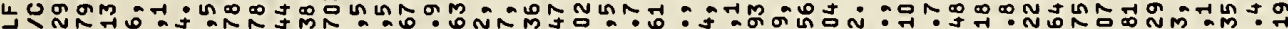
แ0"

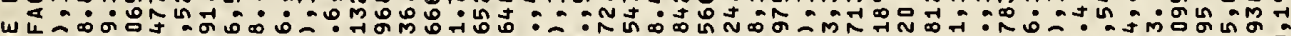

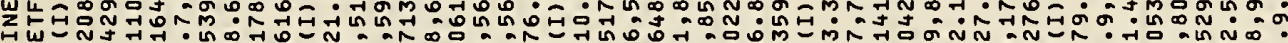

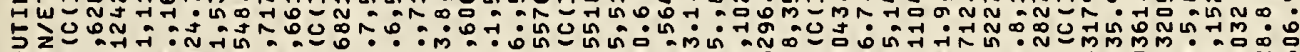

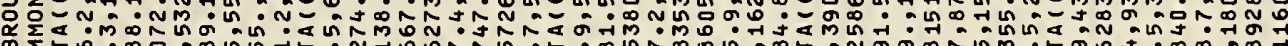

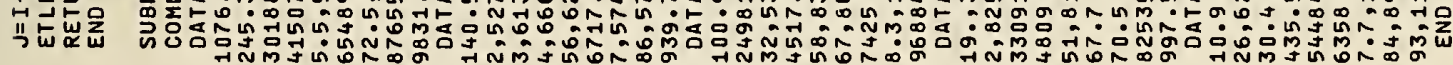



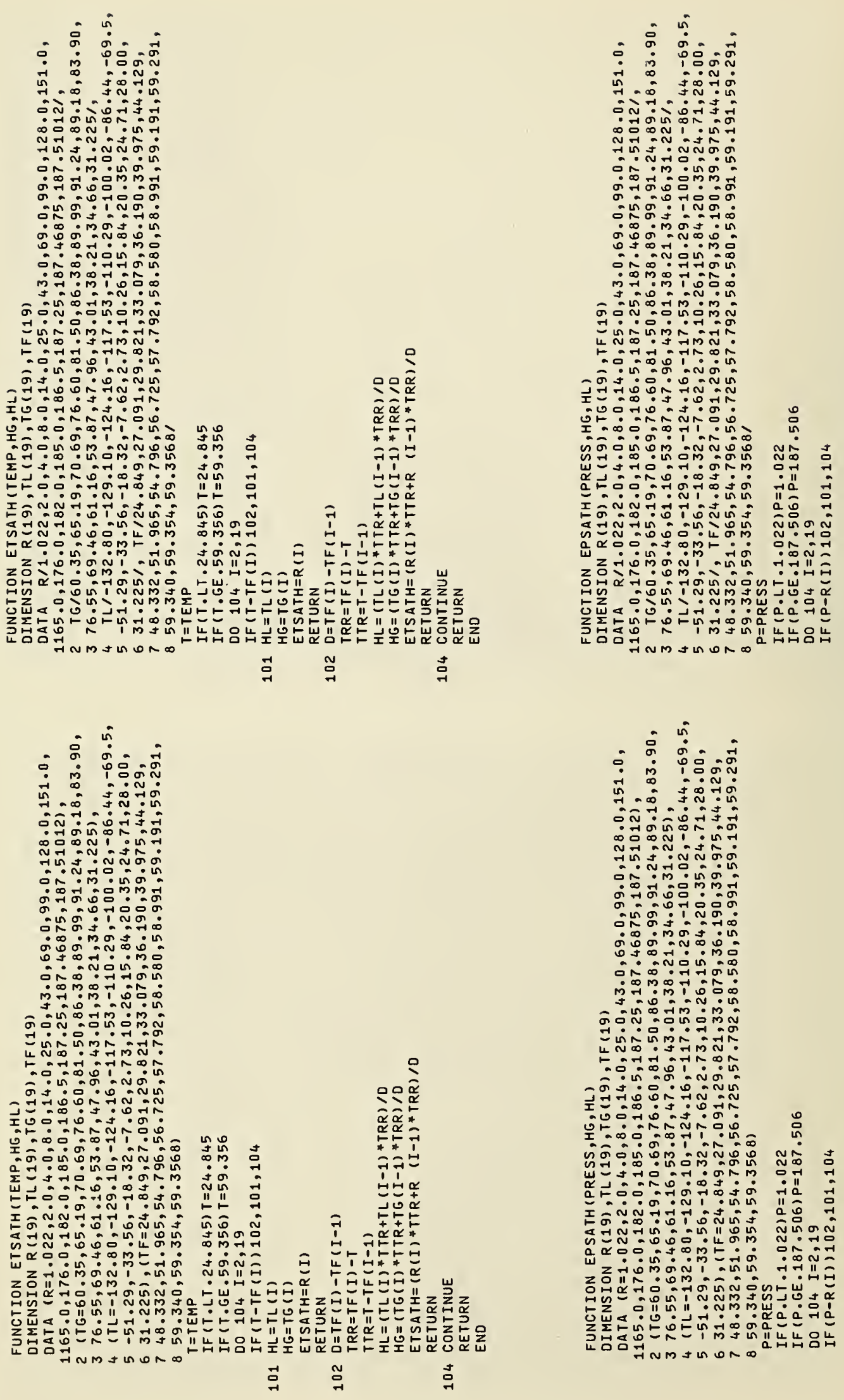

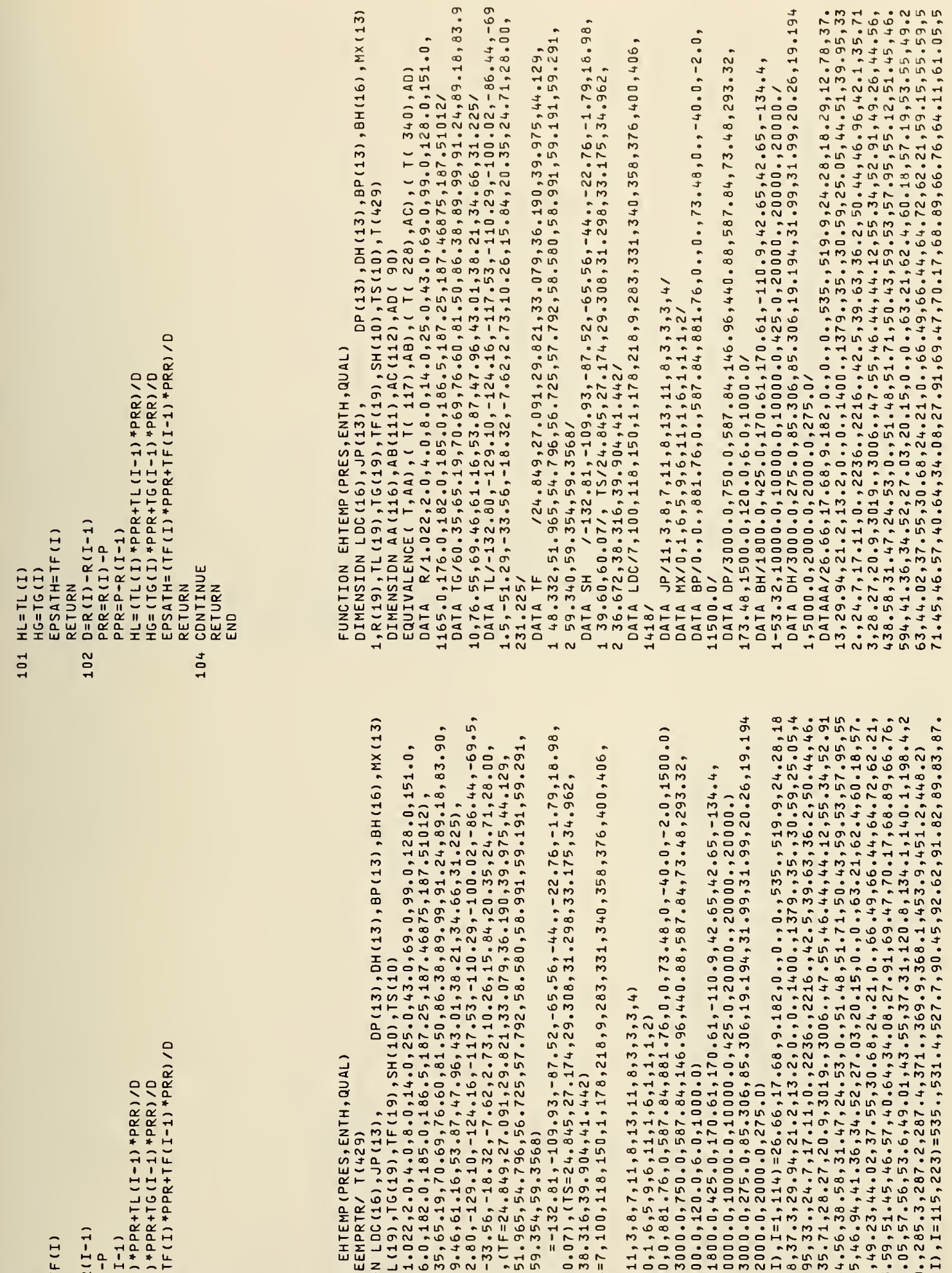

Coñ ํ.

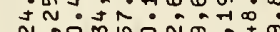
Nón on. क्ष

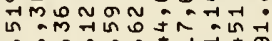
inिंक्षित? in

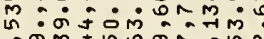

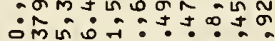

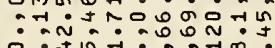
:응

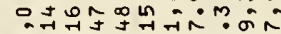
ก $:$ ก $\because \because \because 0 \cos =0$

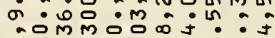
M-1

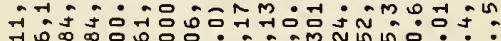
a...…의

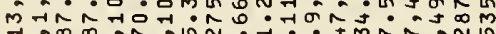
$\because$ - $\cos ^{\infty}$ in o 70.000

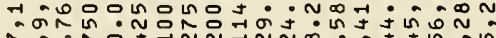
क m. न

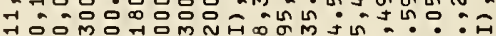
z zó

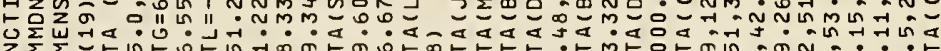

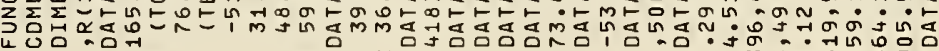

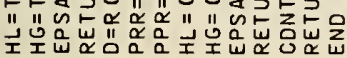
I ऽ 


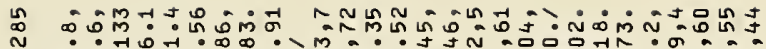

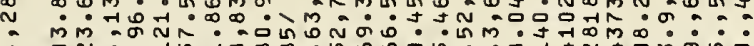

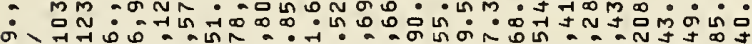

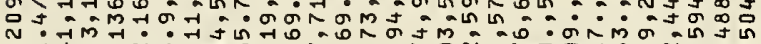
inj.

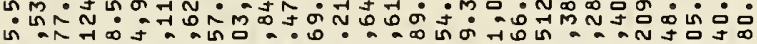
N

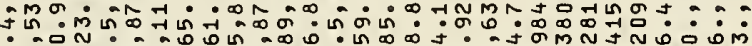
我

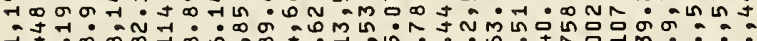

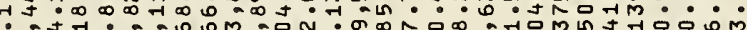
○ஸ் *

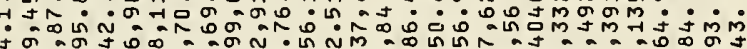

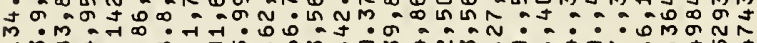

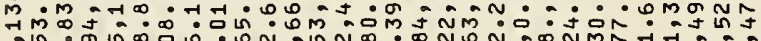
o.

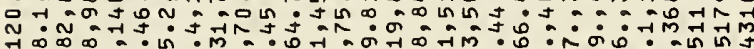

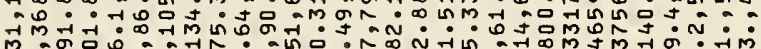
- óm.ง

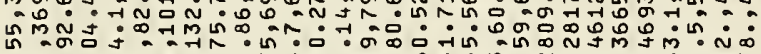
m JMU.

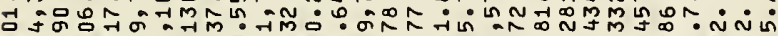

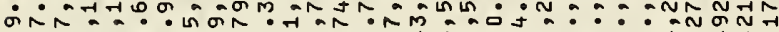

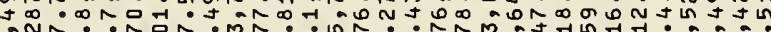

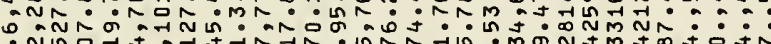

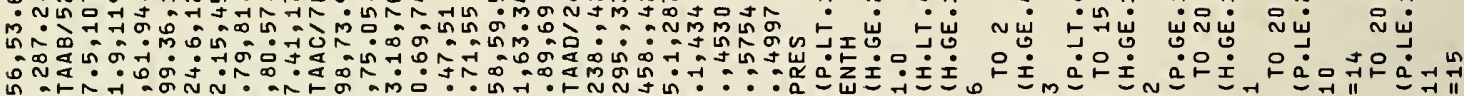

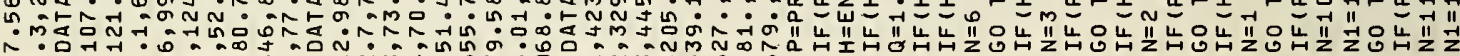
क人口

: : В on

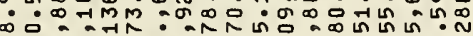

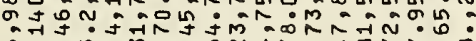

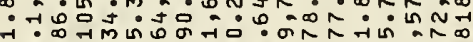

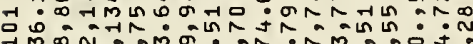

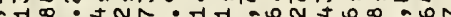
†

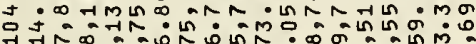

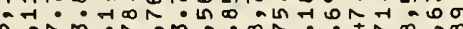
ก :

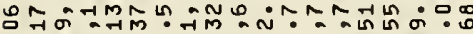

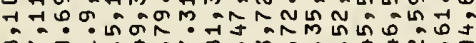

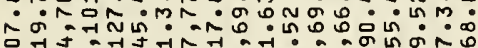

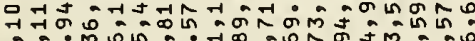

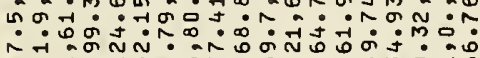
a

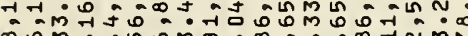
๑ M :

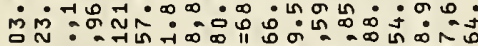

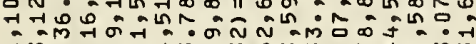
Am.

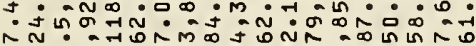

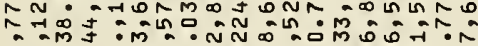
नुำ

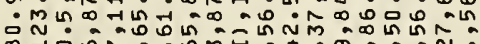

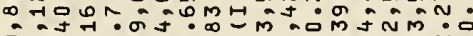
ổ.

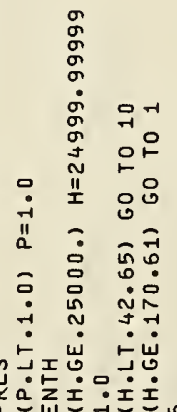

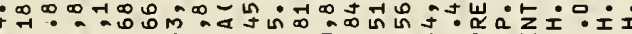

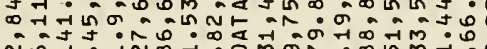

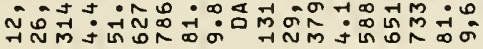

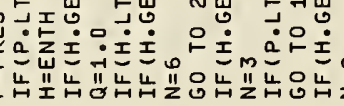

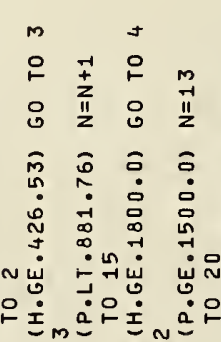

\section{而长 잉

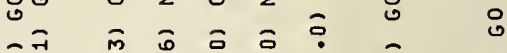

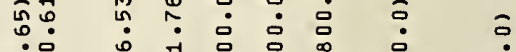 再年 $\bullet$}




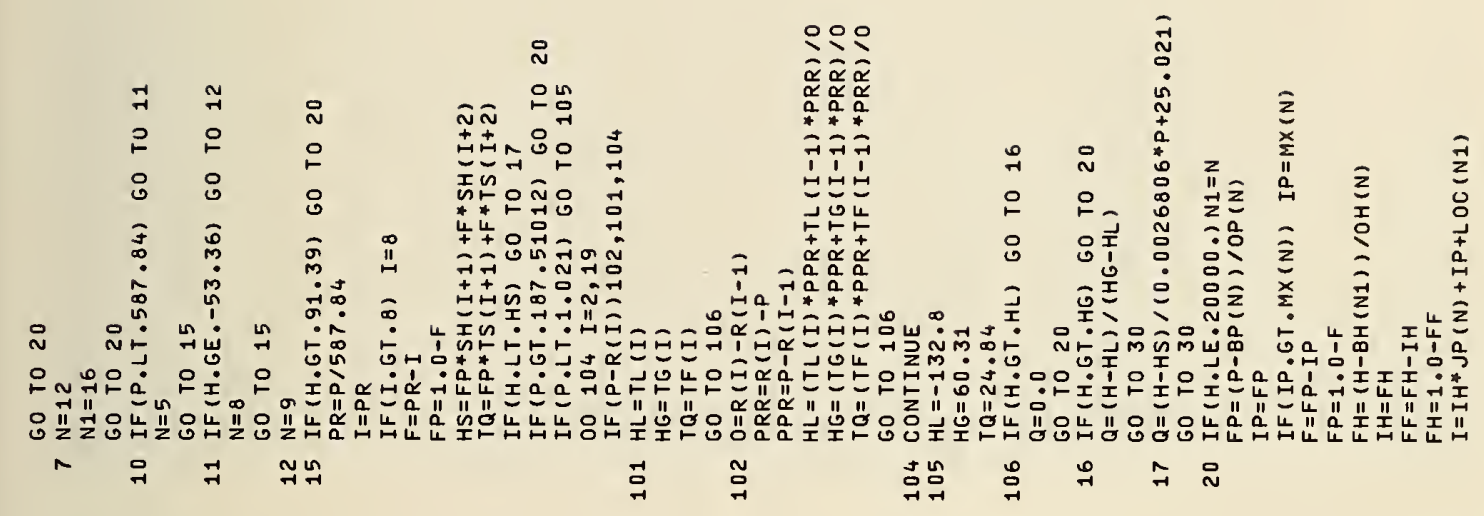

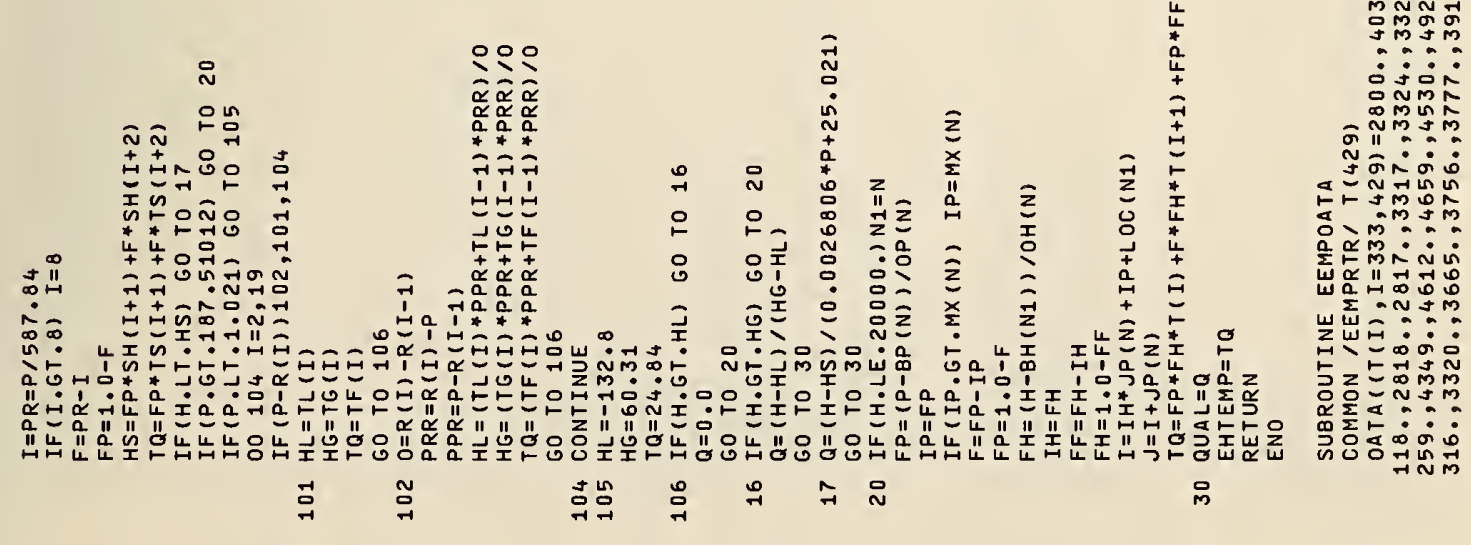




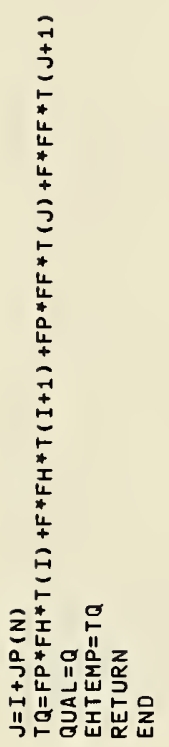

品

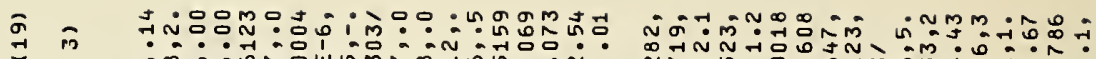

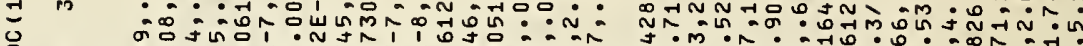

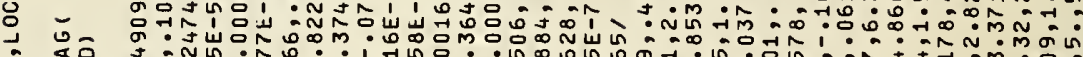

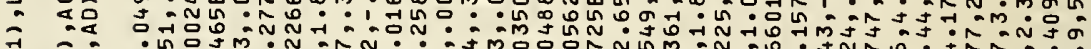

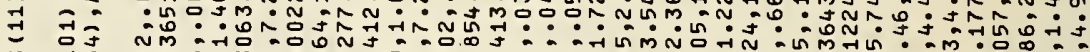
n

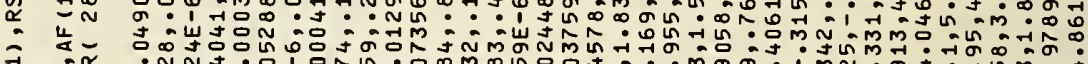
Na- iơ

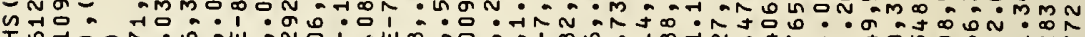

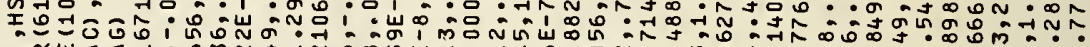

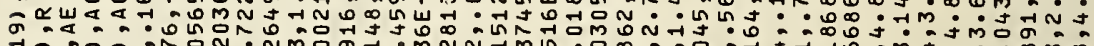

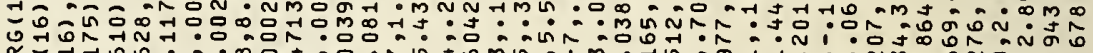

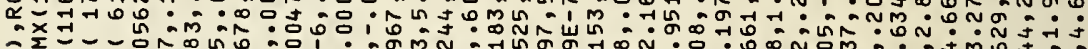

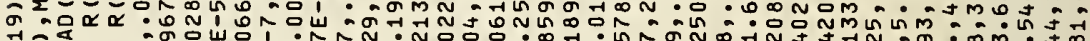

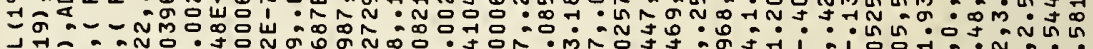

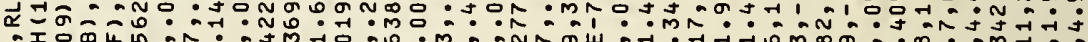

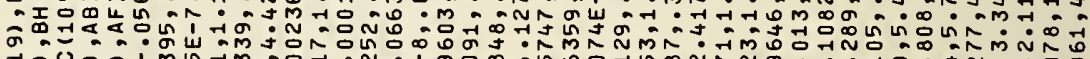

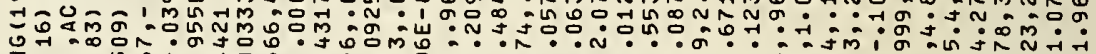

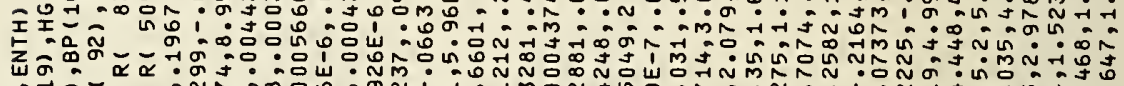

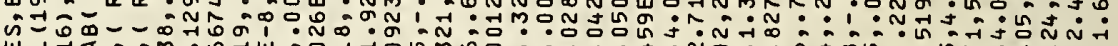
ơำ

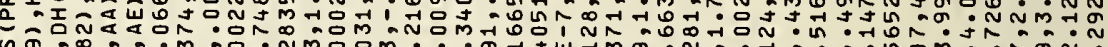

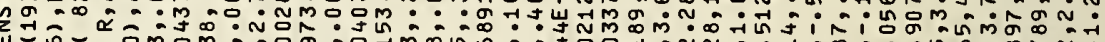

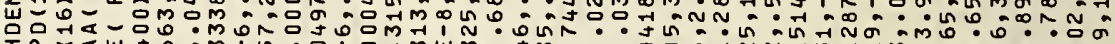

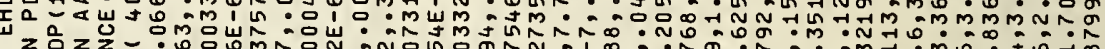

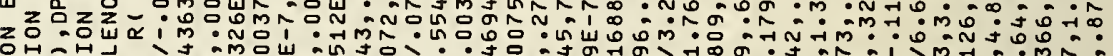

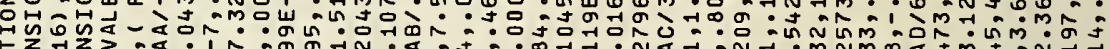

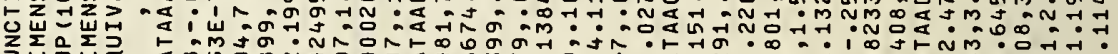

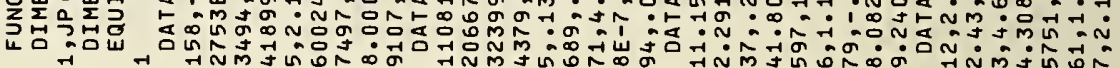

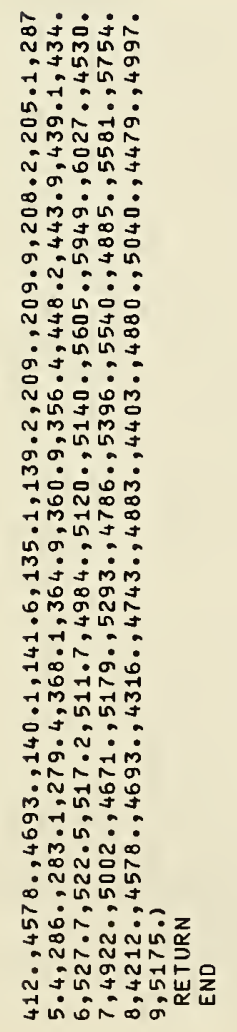

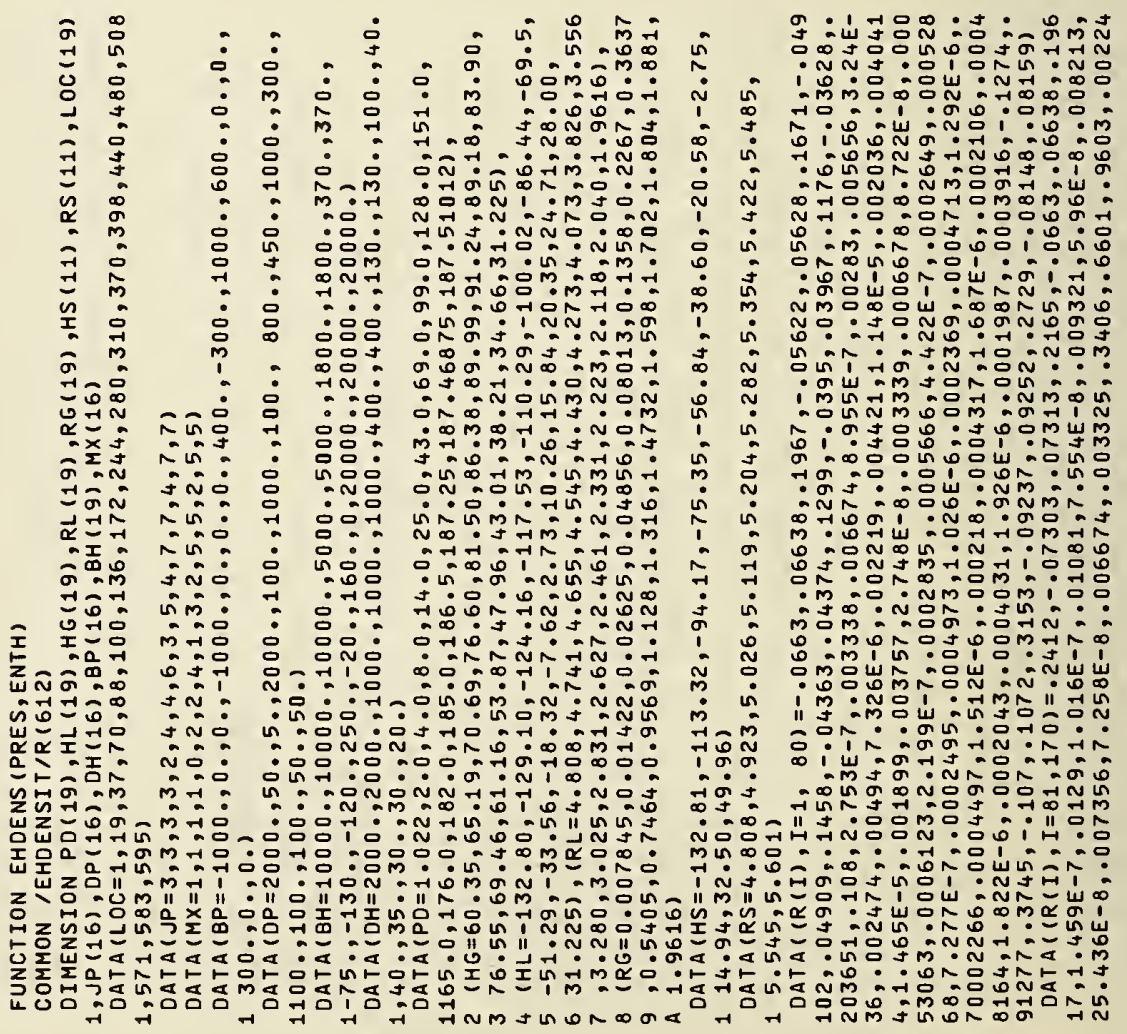




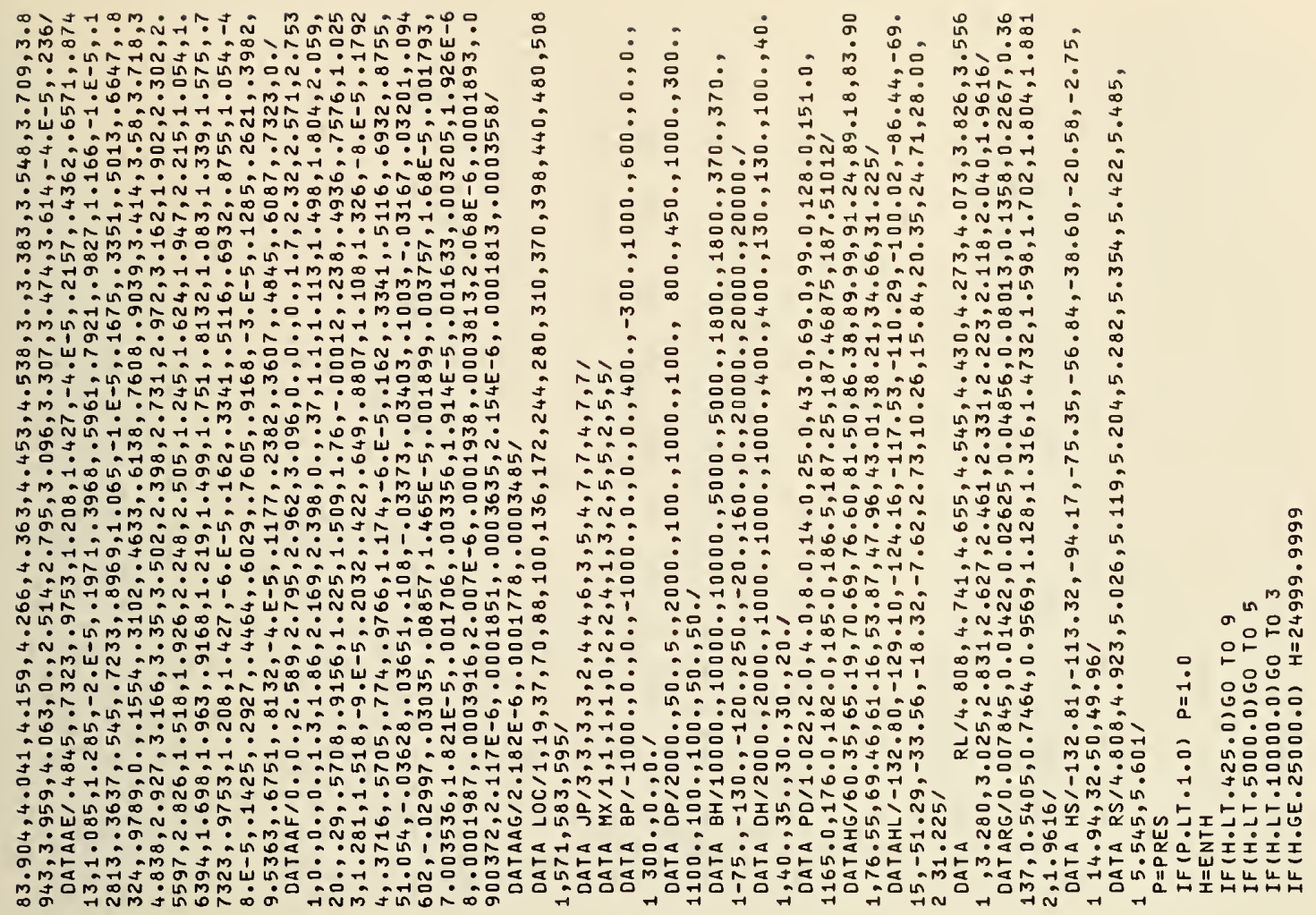

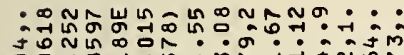

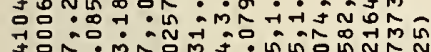

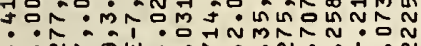

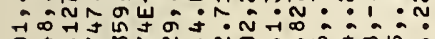

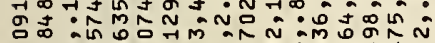

N

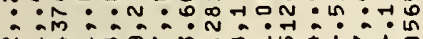

7

N

:

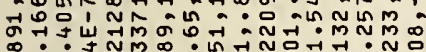

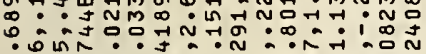

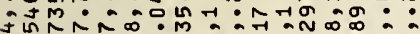
Gก N

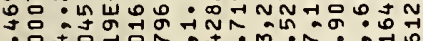

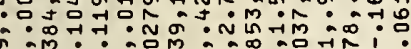

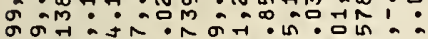

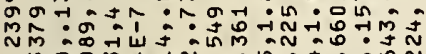

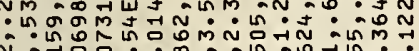

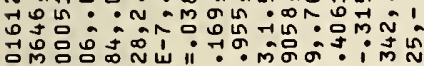

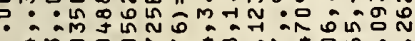

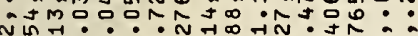

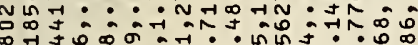

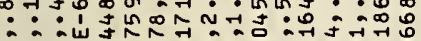

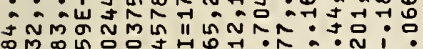

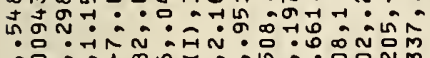
m品:

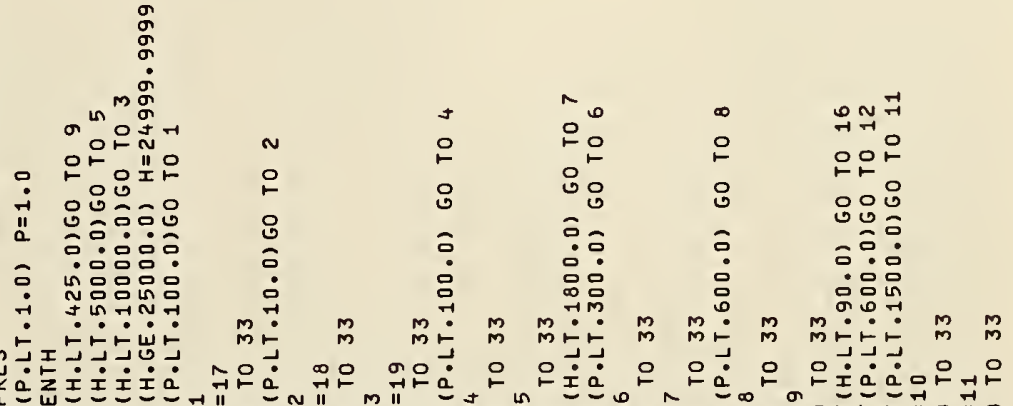
ming 

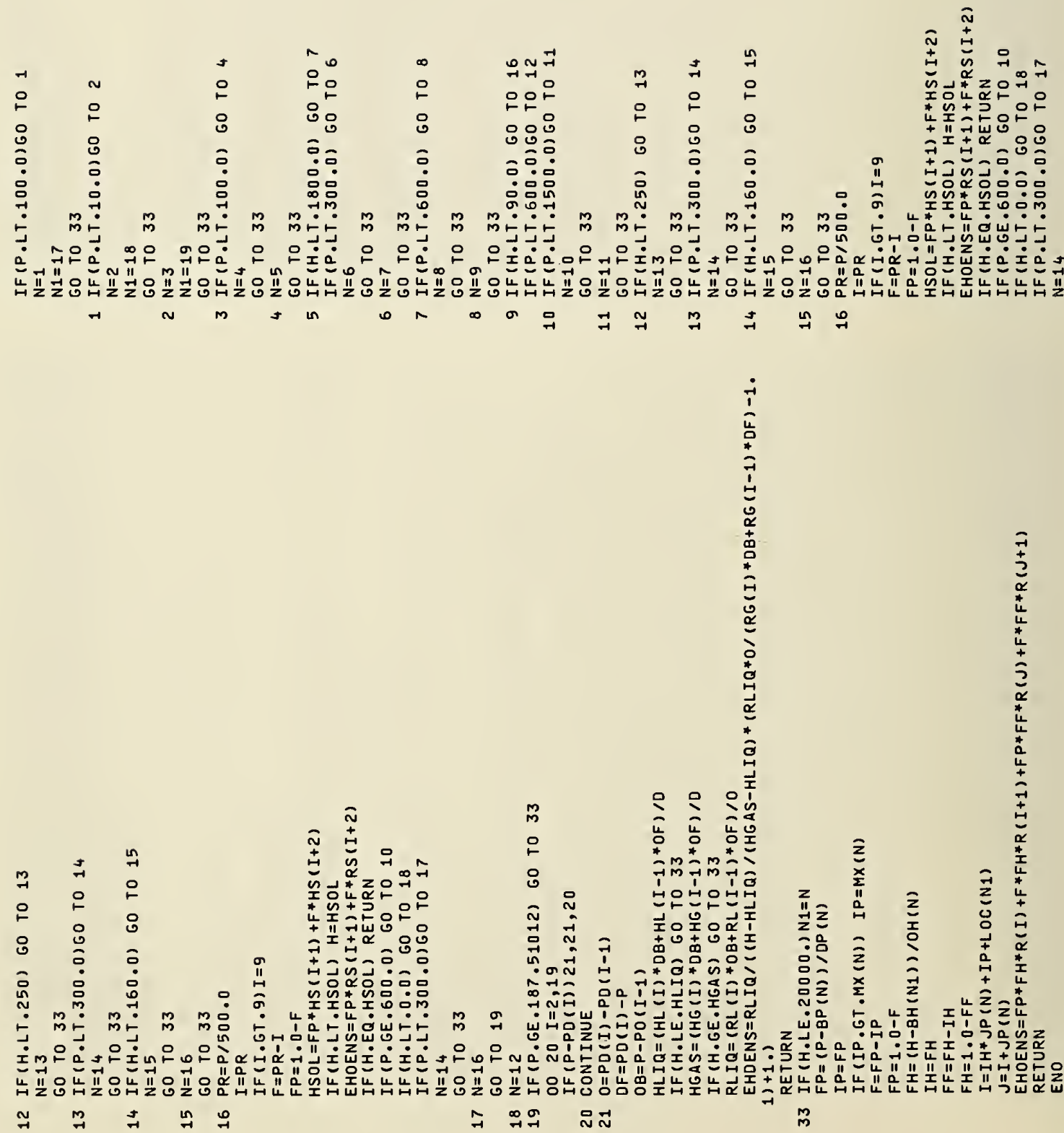


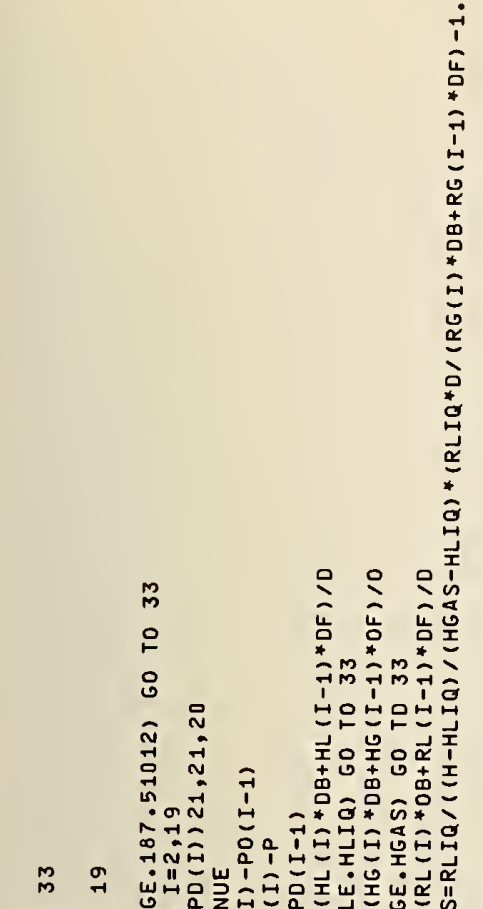

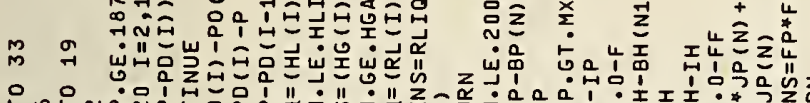

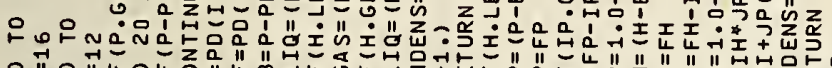

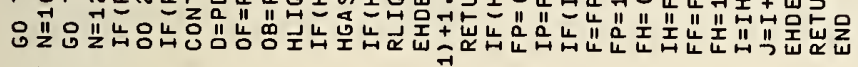

ว ศึ กี

is

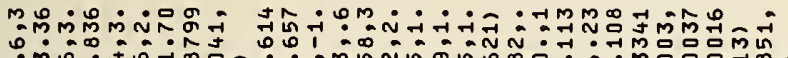

: MN

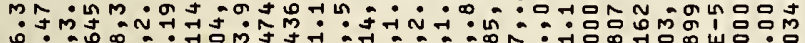
Nom ₹ مि ñ

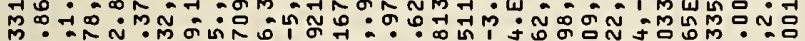

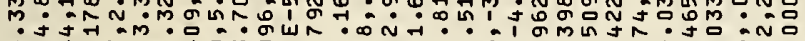

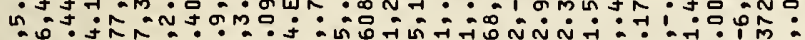
G Ф0. 0.

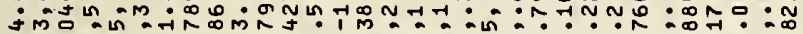
रुन

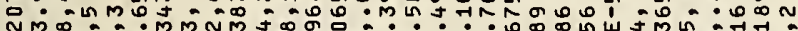

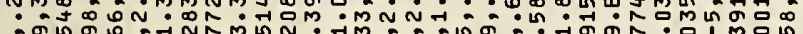
ถศึ N

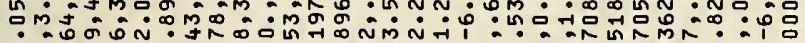
nิ

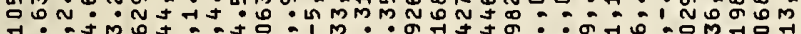

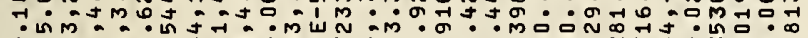
ล ไ大 ه

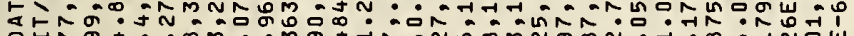
Q

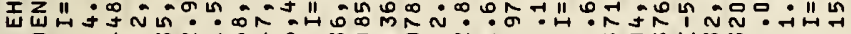

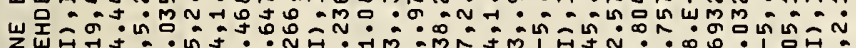
WV zỡ

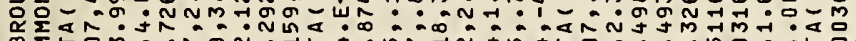

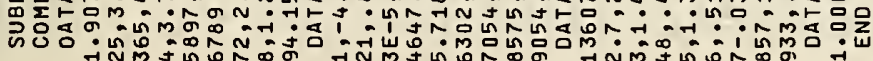

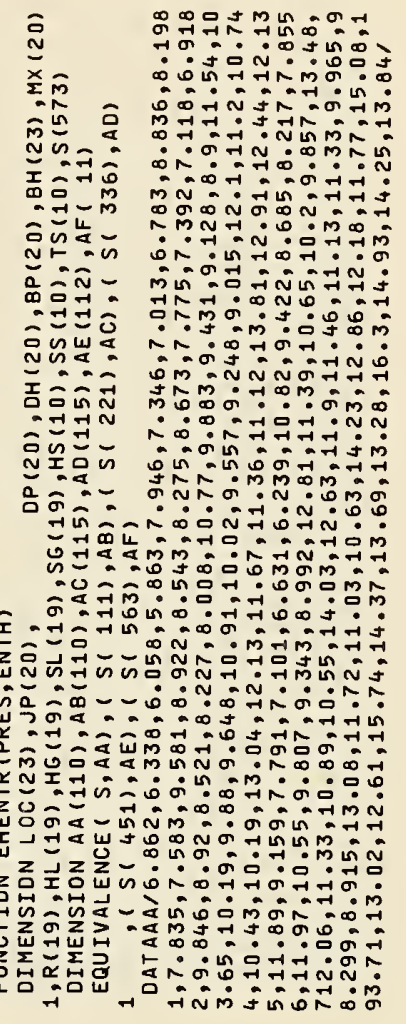

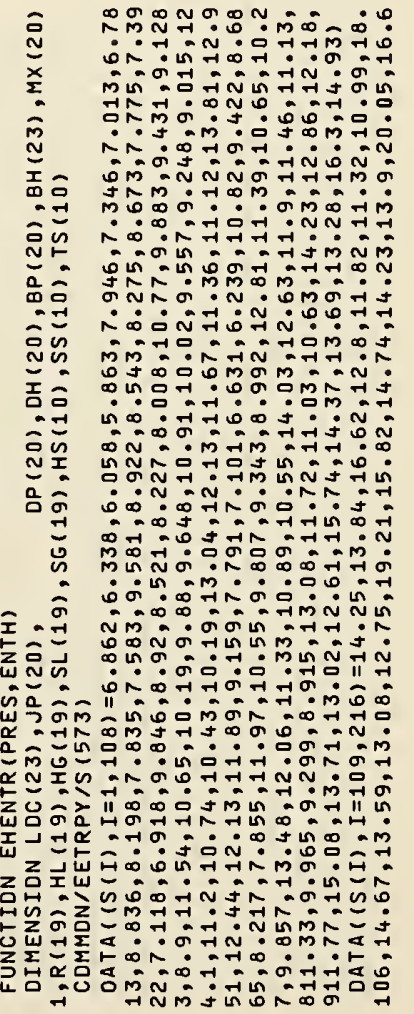




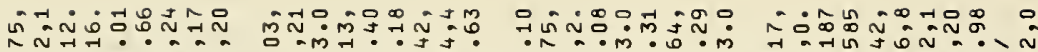
-N.a.000

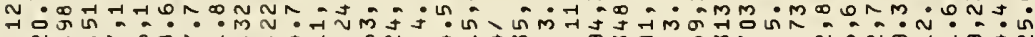

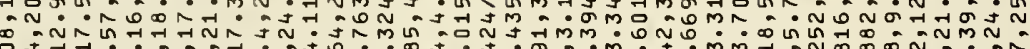

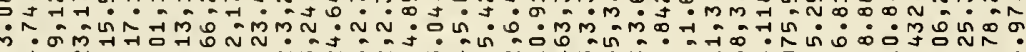
IUMN $N=0$ N

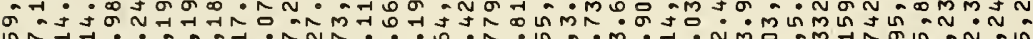
un m ôn J o-10

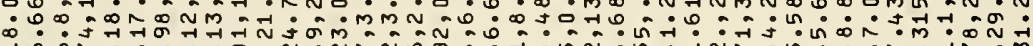

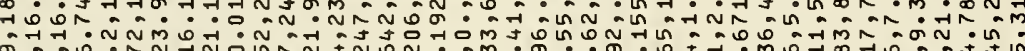

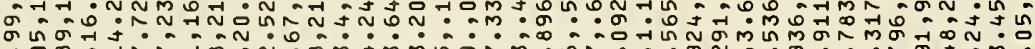
Ğó NNA N móm J J -1

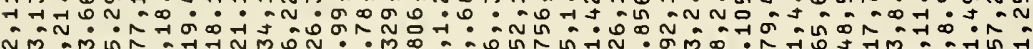

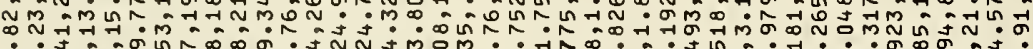
군? o.j. フ Nิ

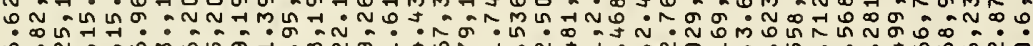

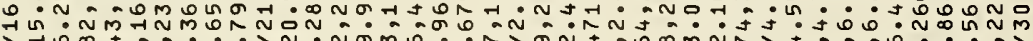
D ब añ

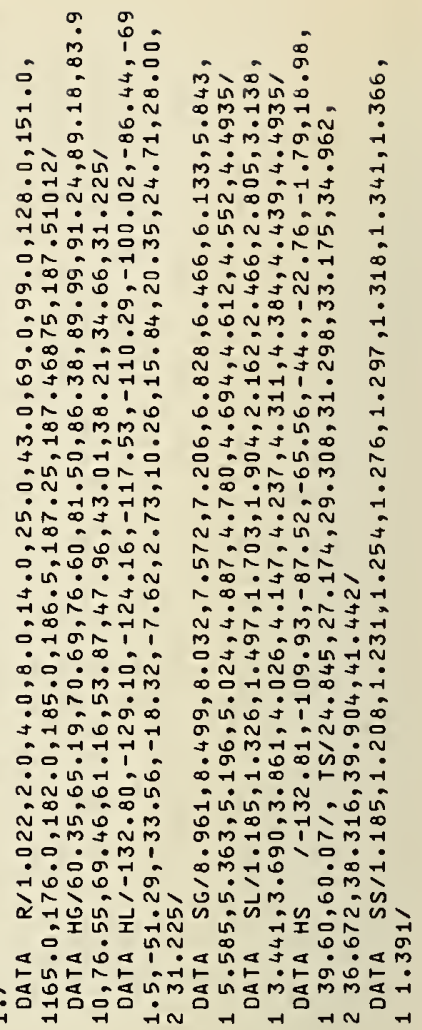

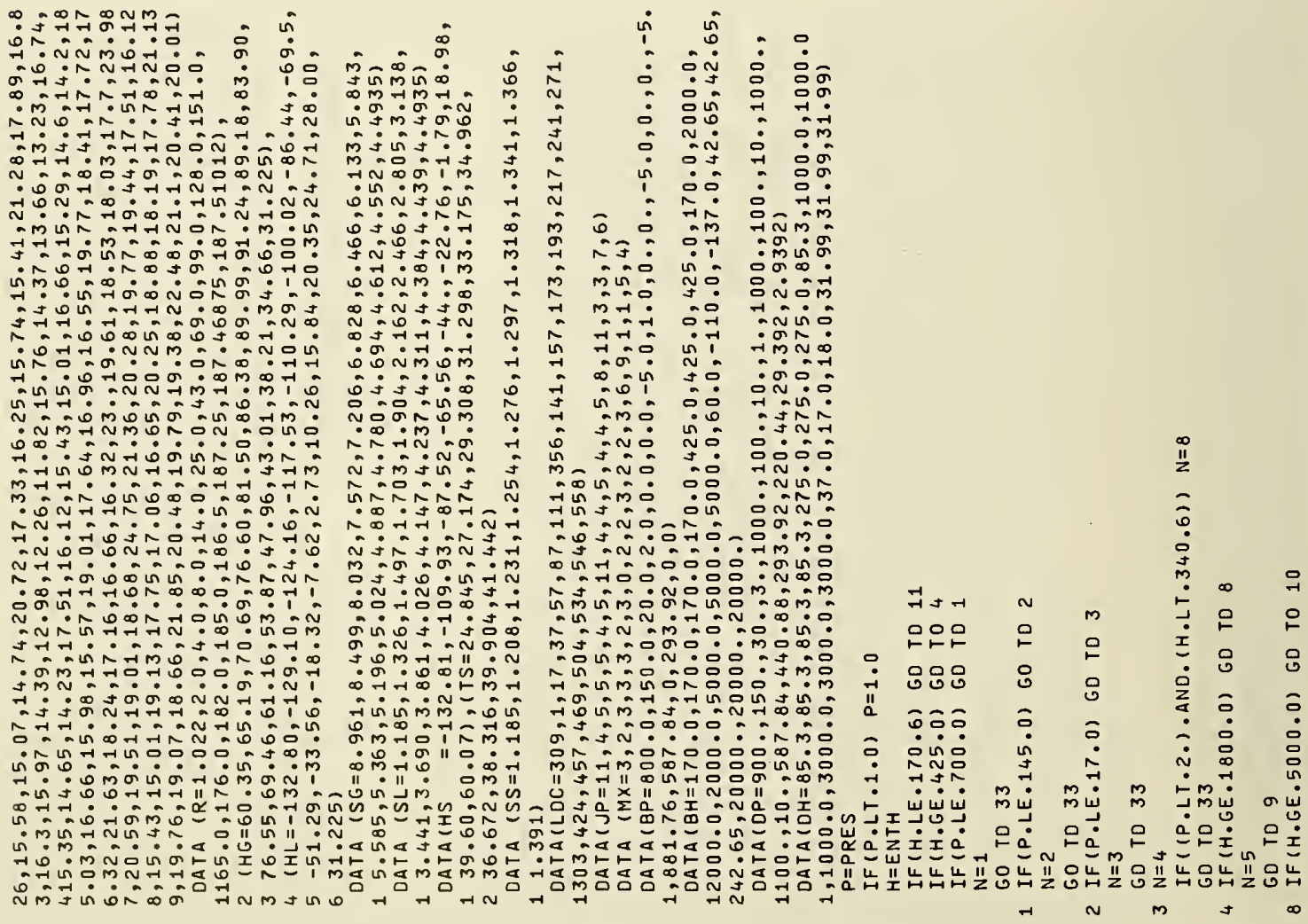




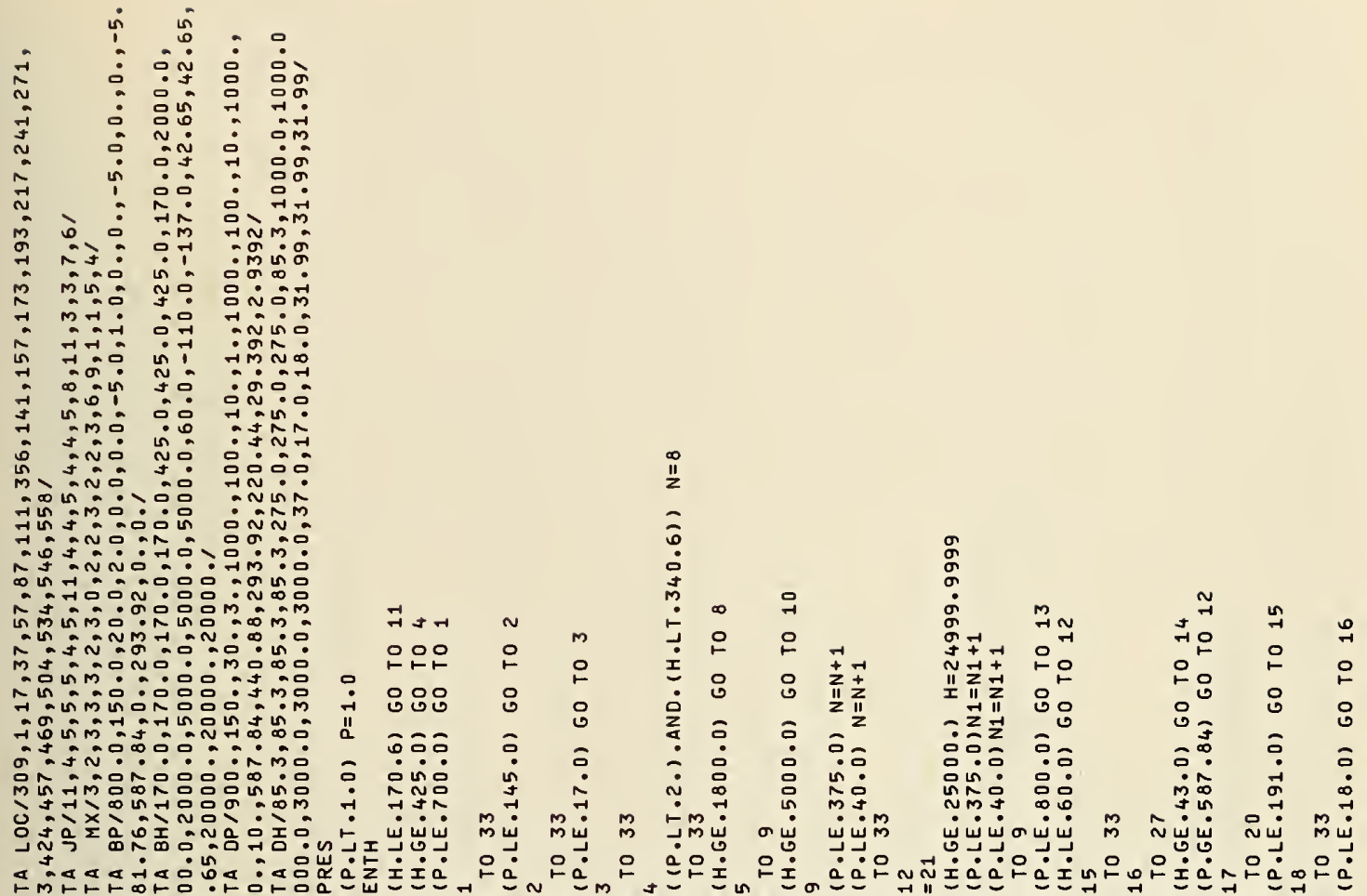

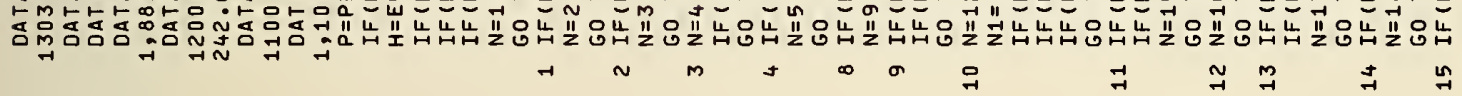

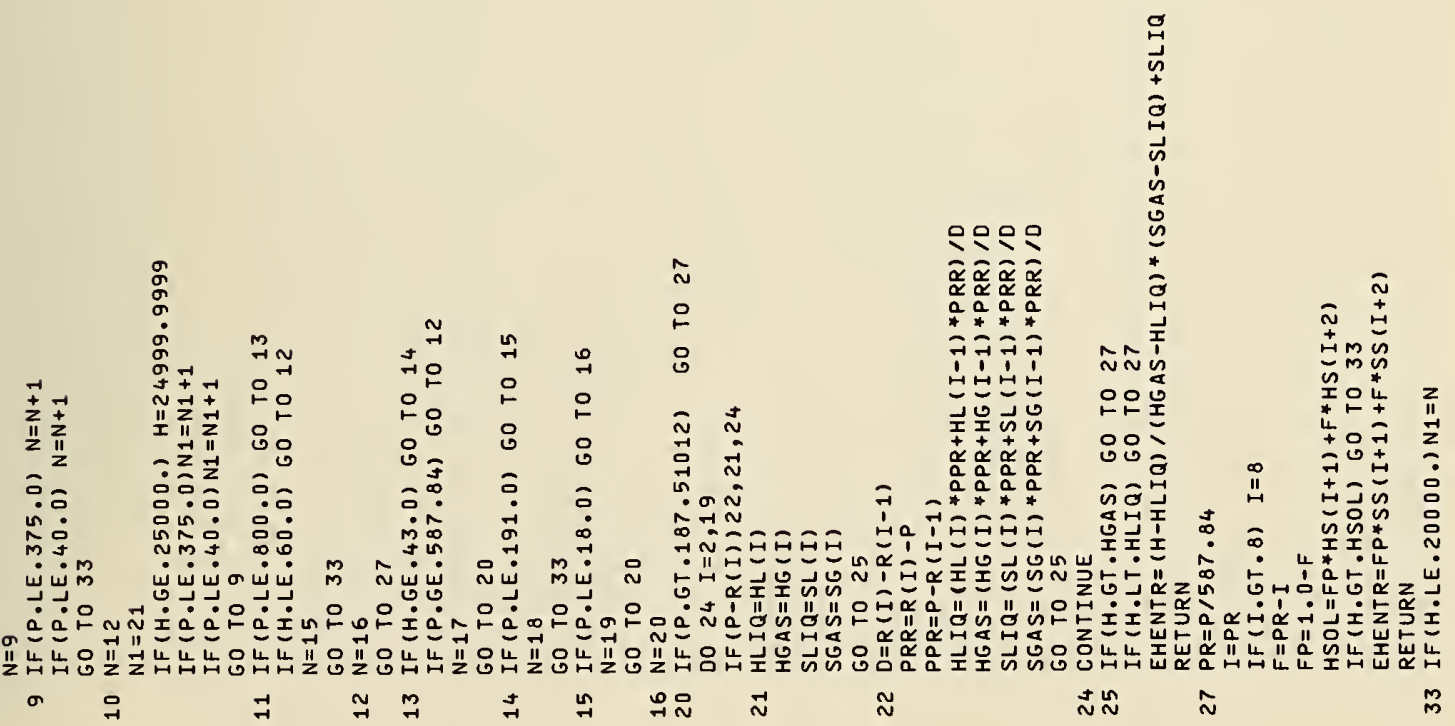




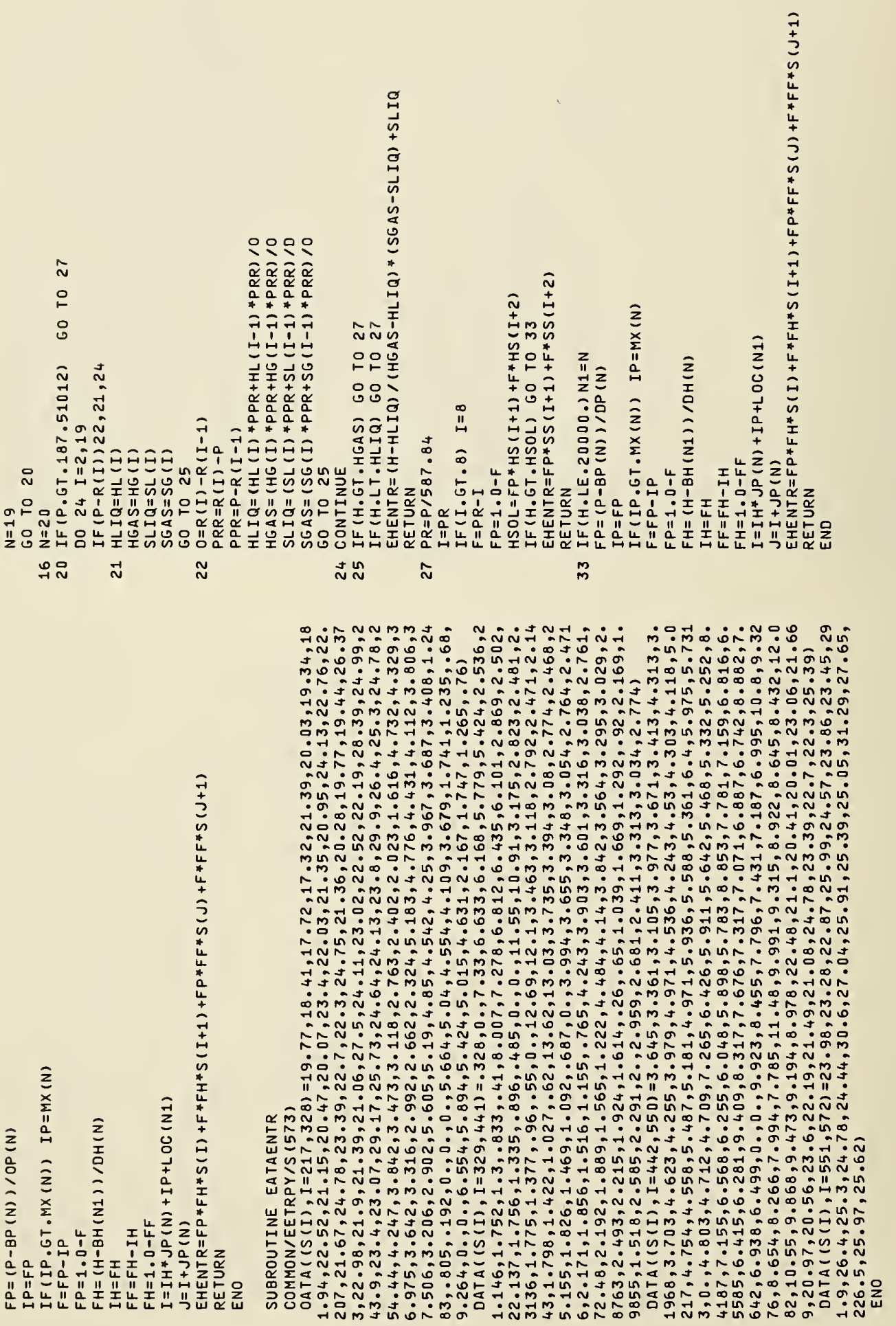




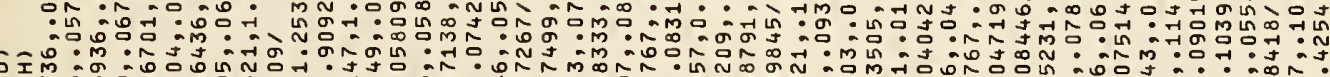

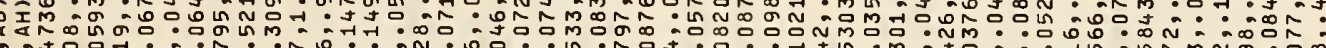

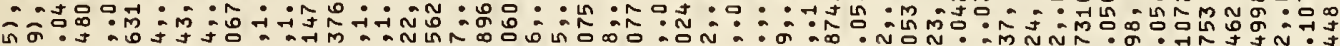

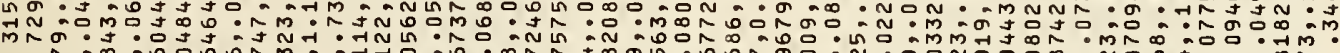

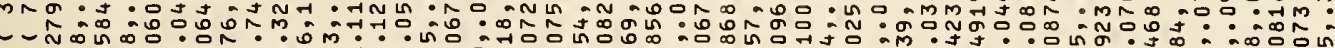

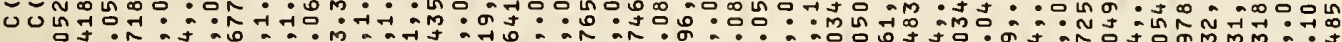

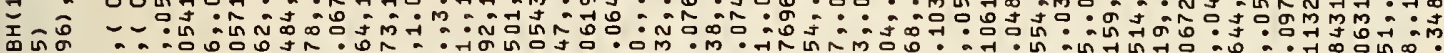

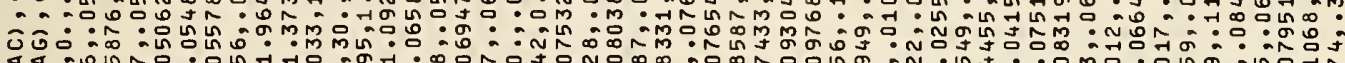

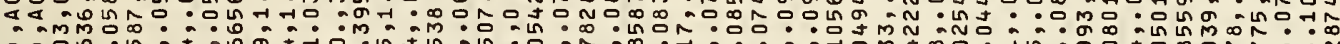

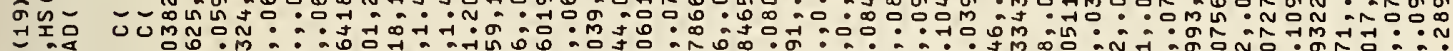

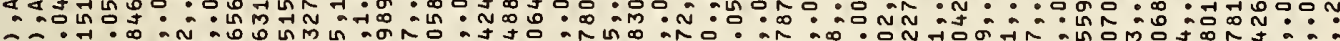

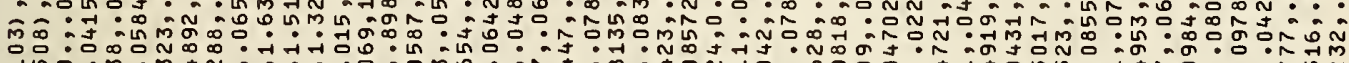

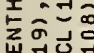

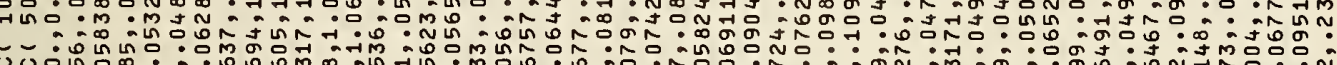
in

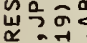

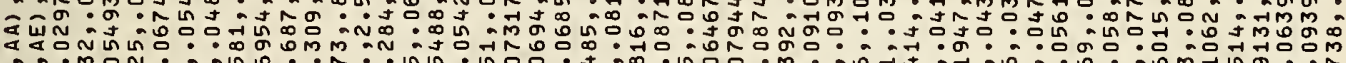

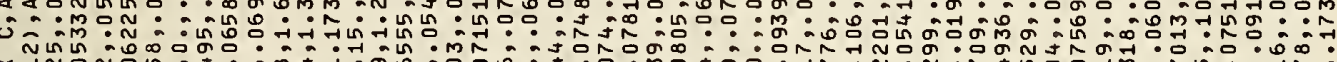

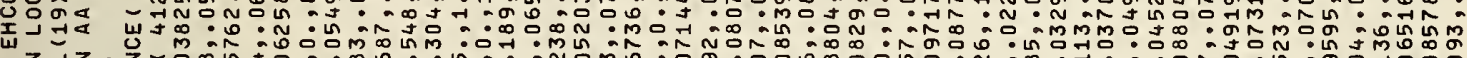

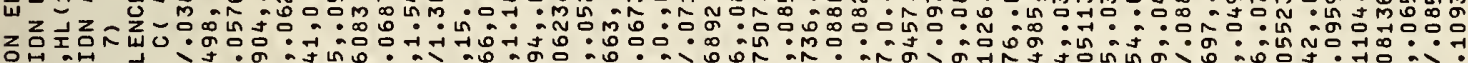

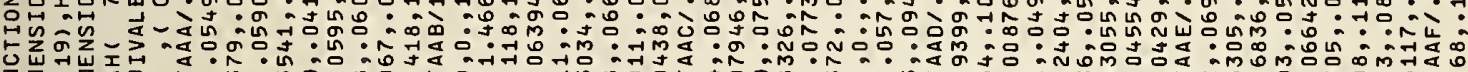

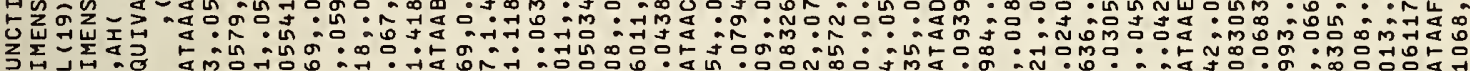

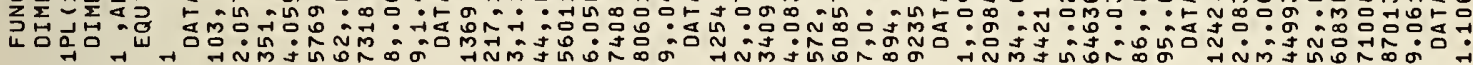

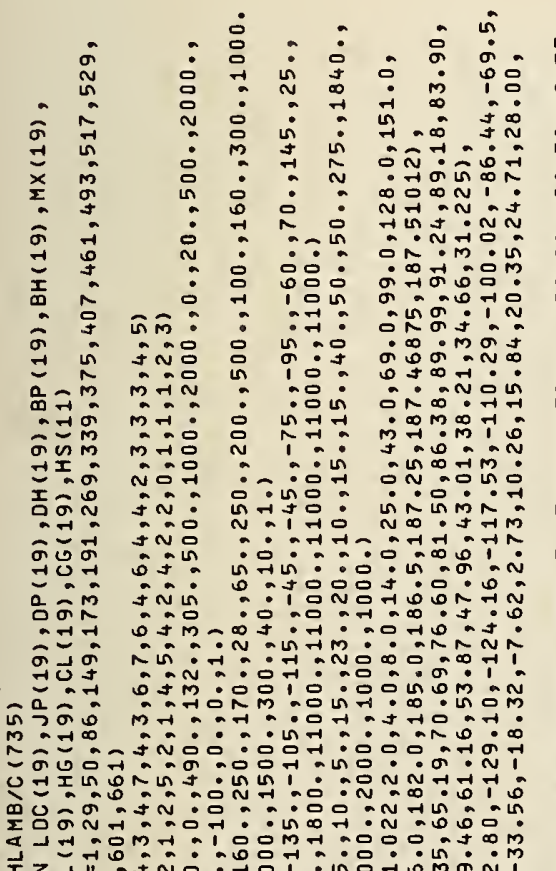

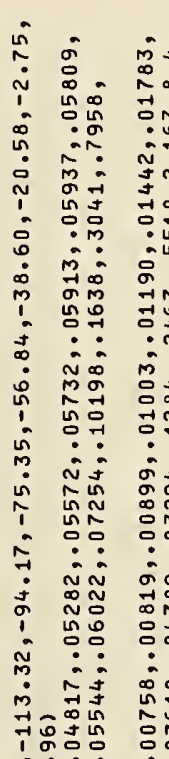

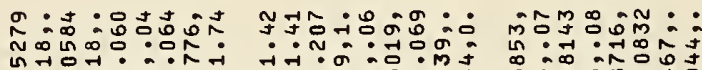

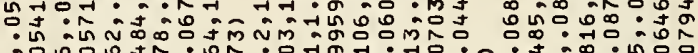
:

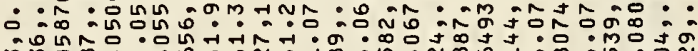

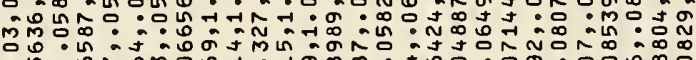
:

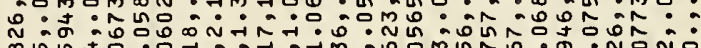

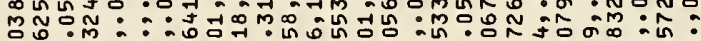
等:

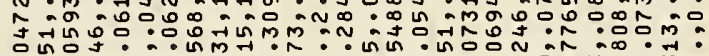

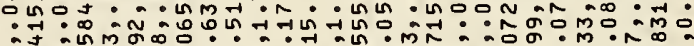

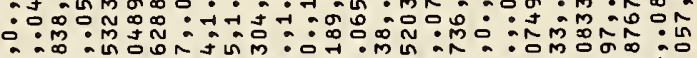

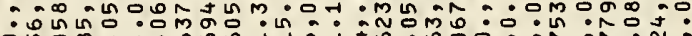

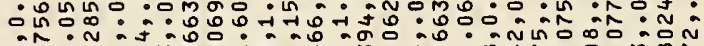

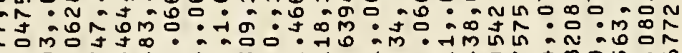

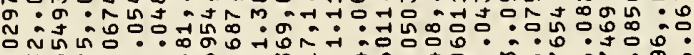
บ N

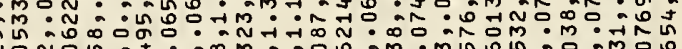

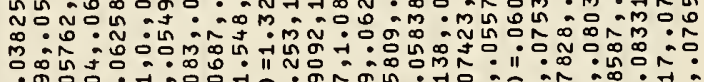

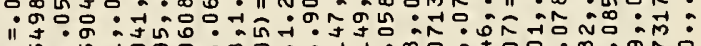

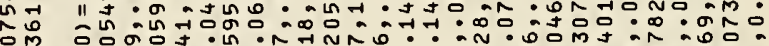

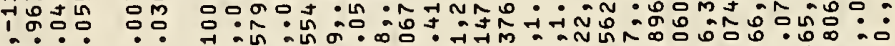
idn

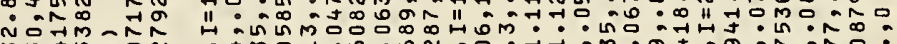

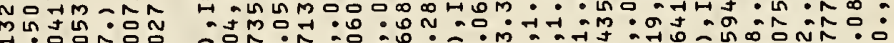

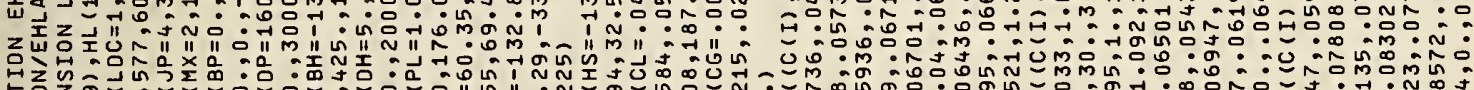

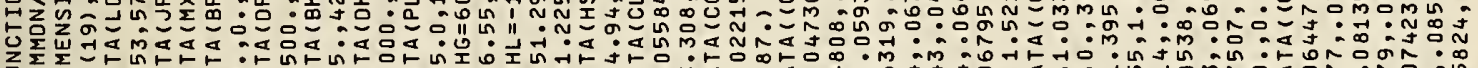

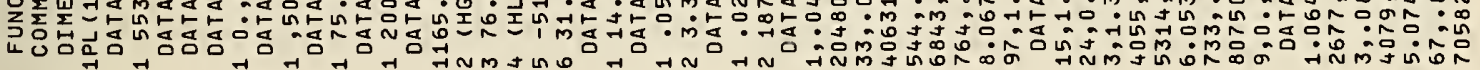



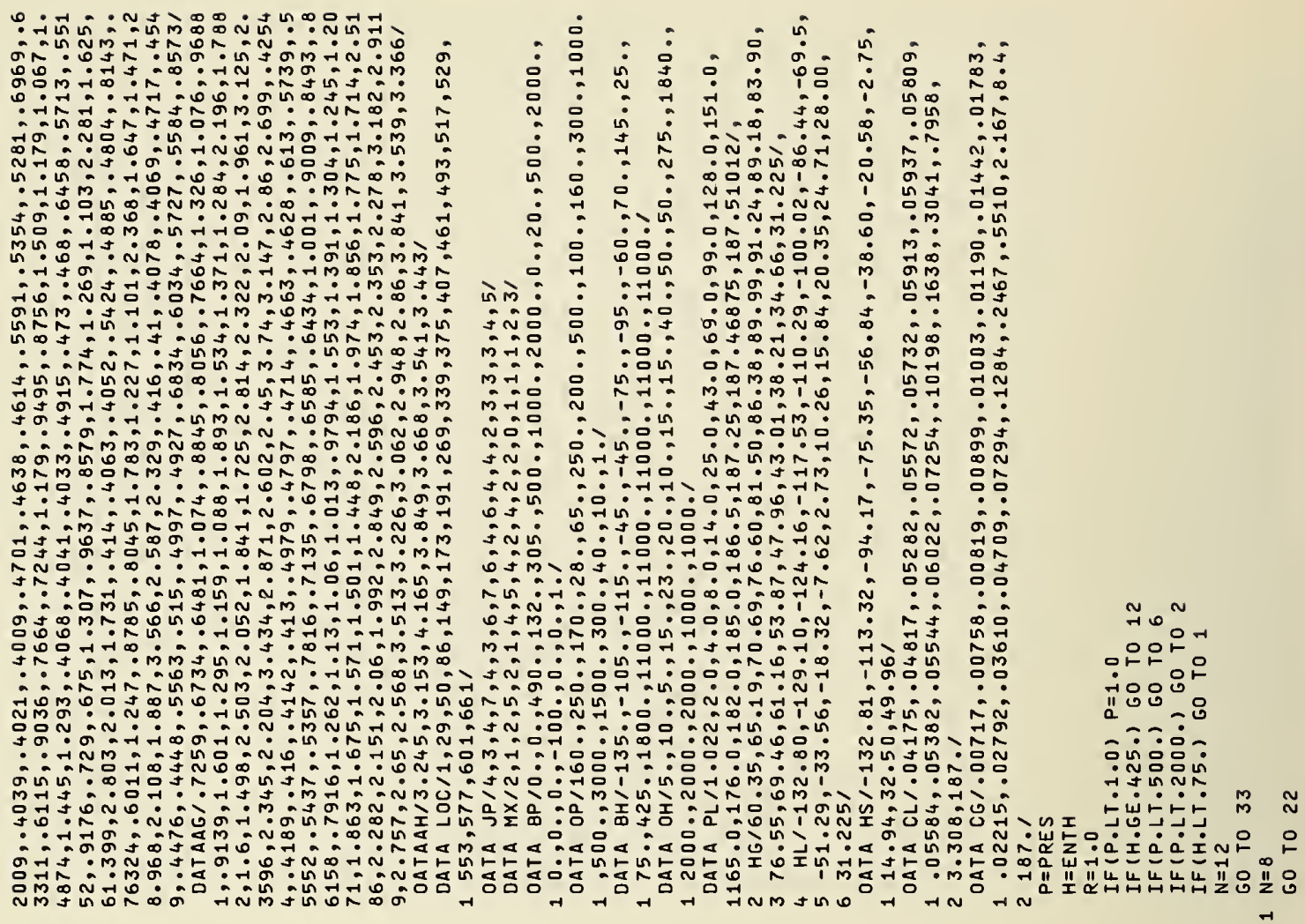

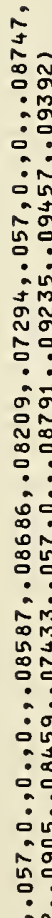

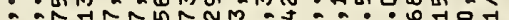

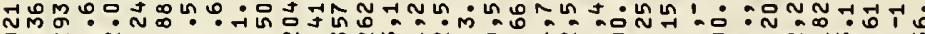

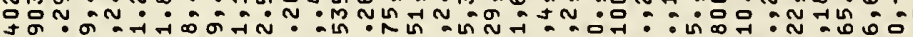
:

苟

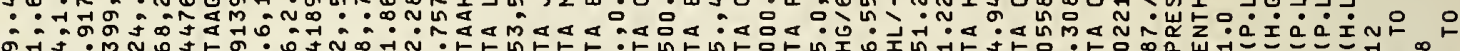

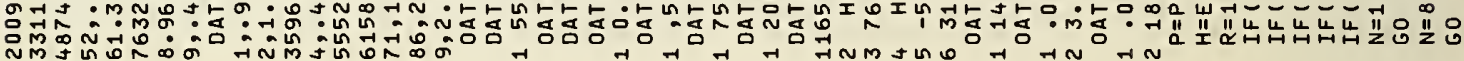
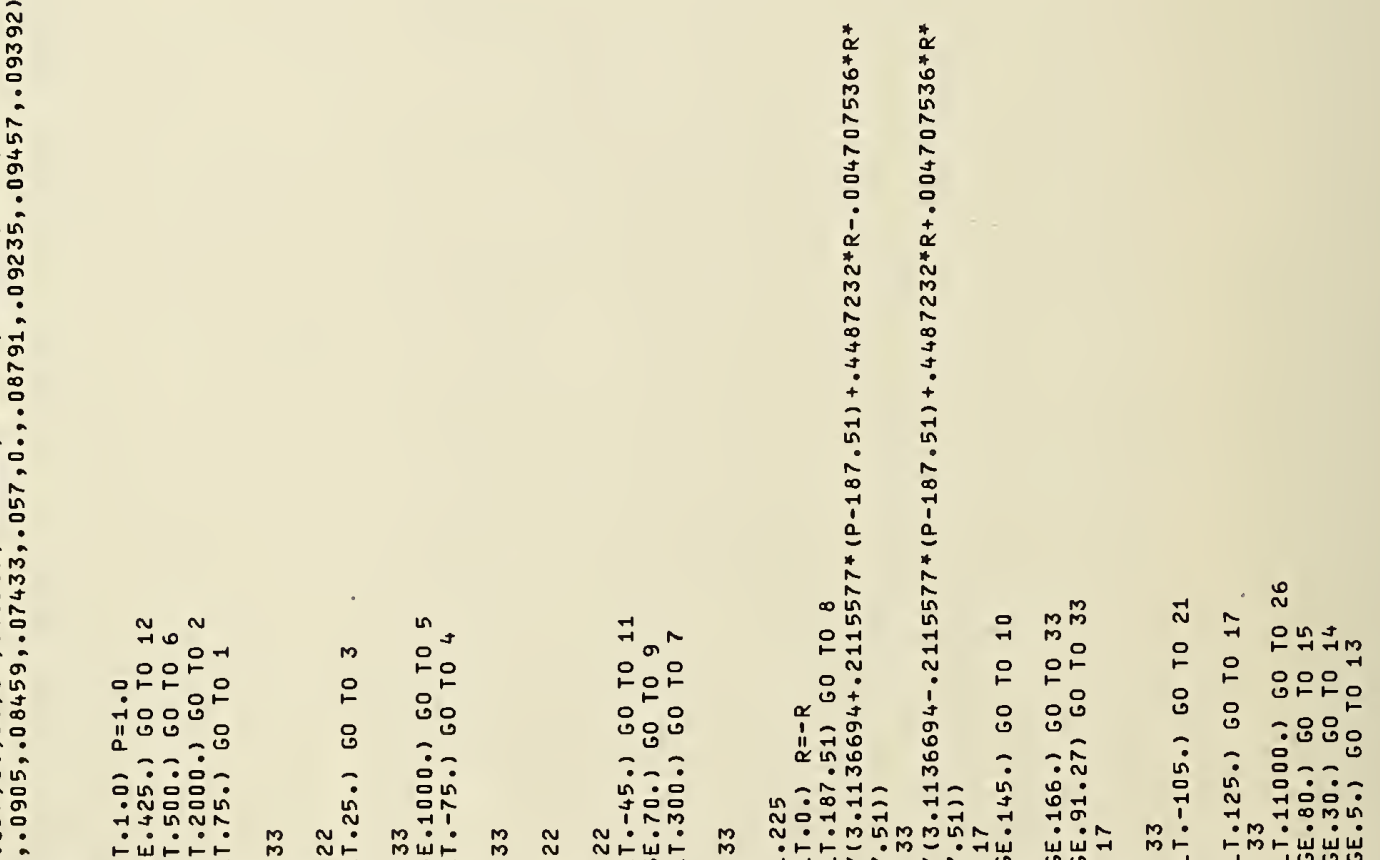

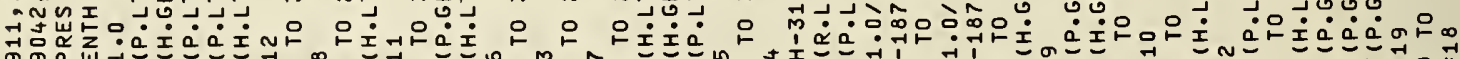

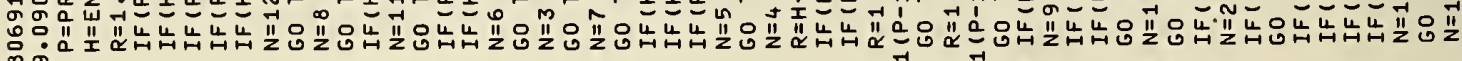

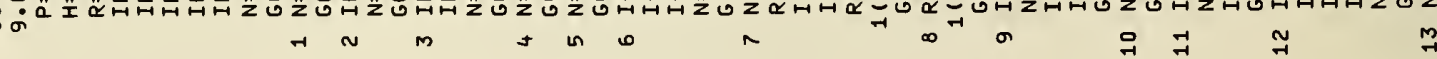



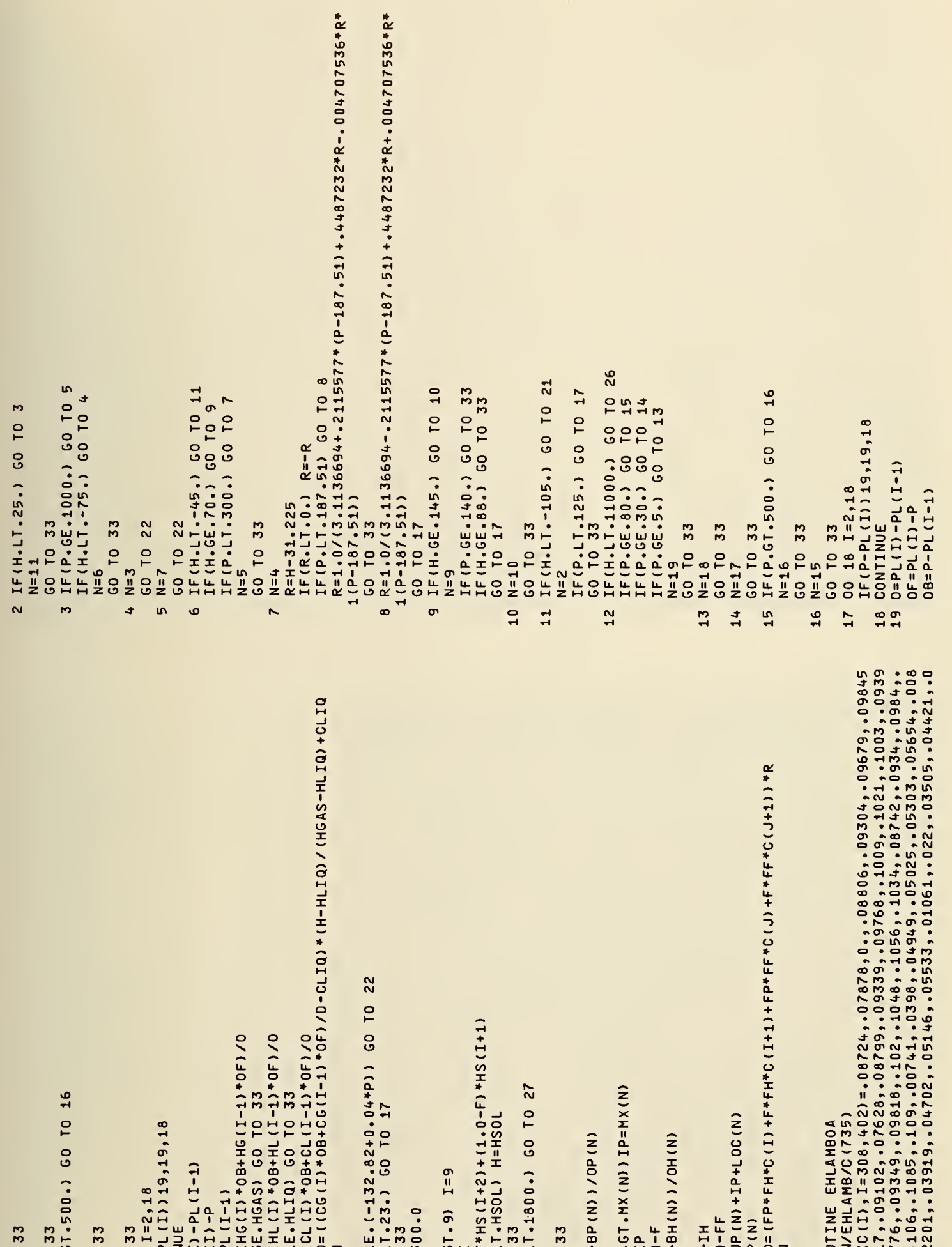

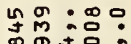

क의 :ํ워

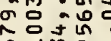

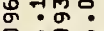

an:

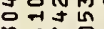
ตัต์:N

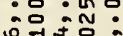
응ํㅁㅁำ 00 : ज行 काँ?

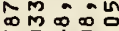
므잉 $: \because \div: 0$ ก 舟人.

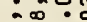

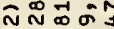

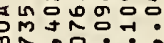
₹ $:$ : o

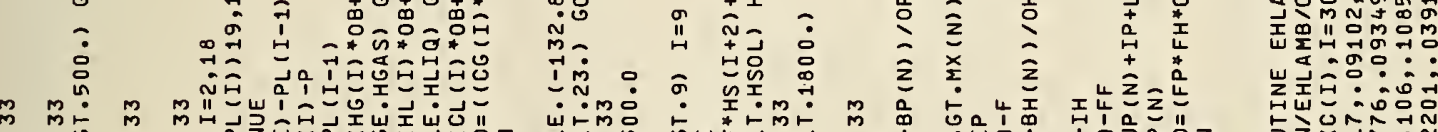

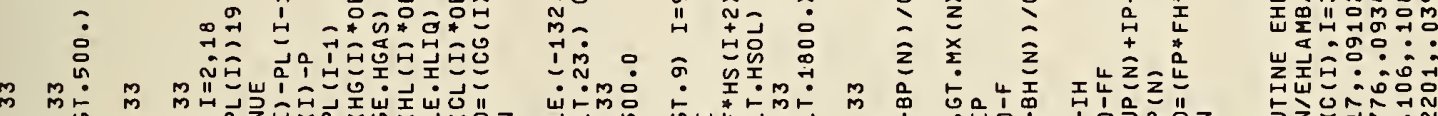
앙ㅇㅇㅇㅇㅇㅇㅢ

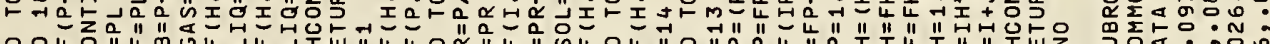

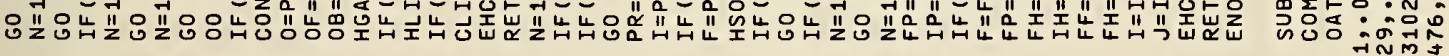

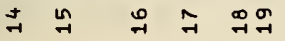

$\stackrel{\sim}{n}$

ก กิm 


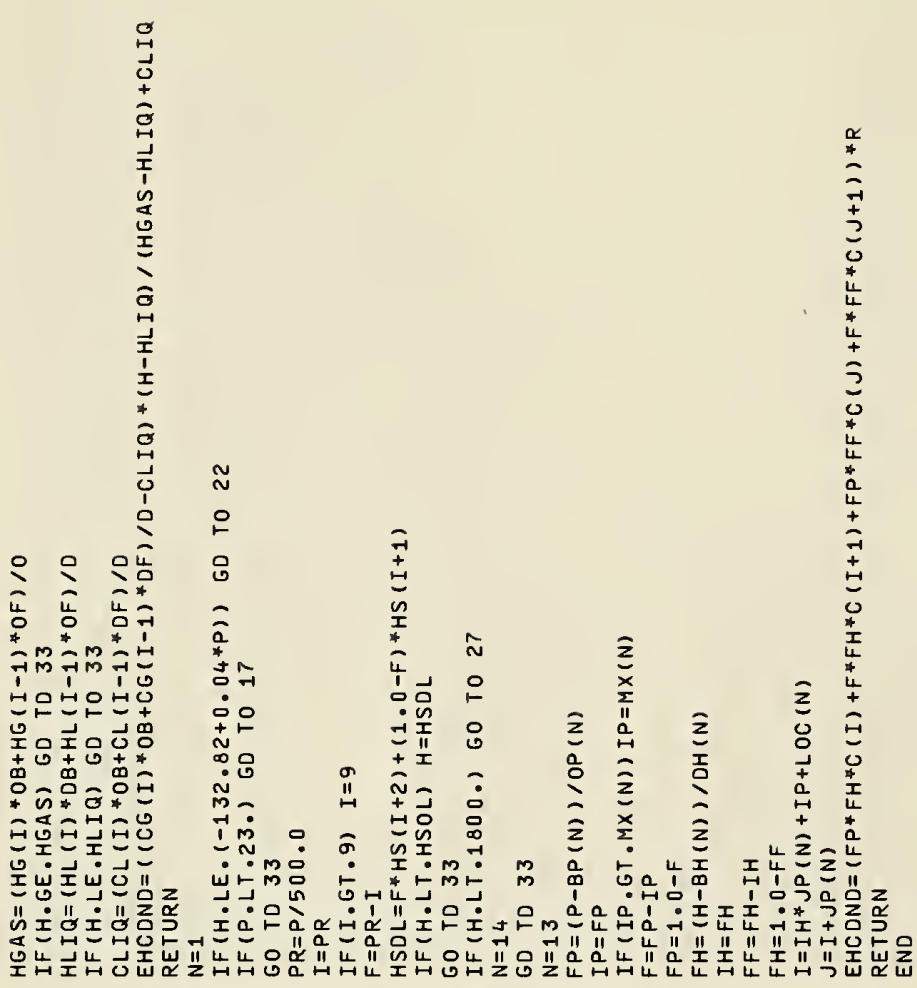

ก $\quad$ N

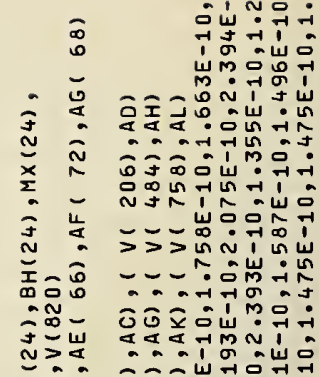

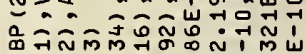

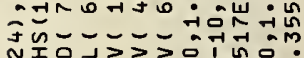

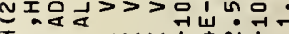

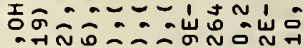

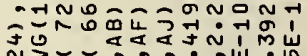

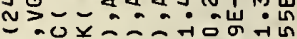

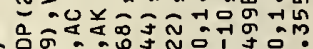

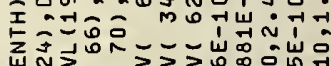

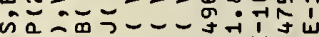
药

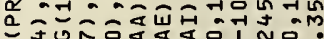

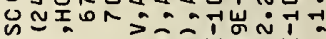

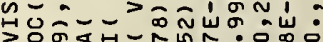

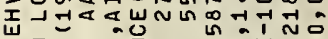

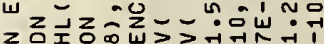

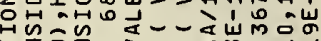

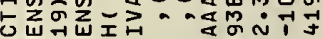

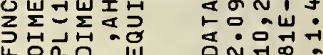

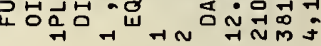

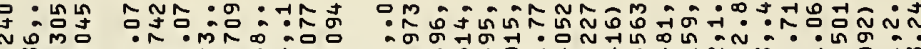

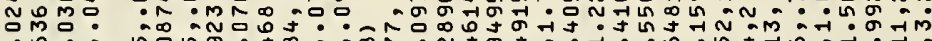
:

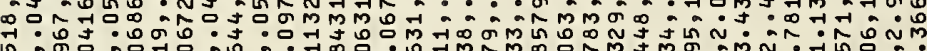
F

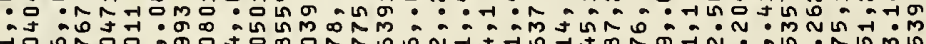

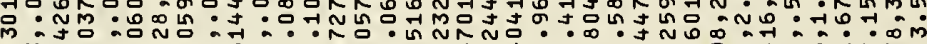
经: :

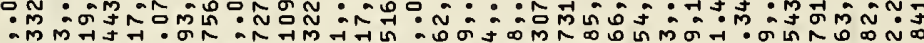

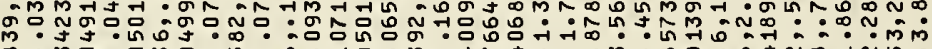

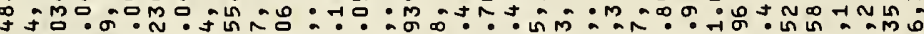

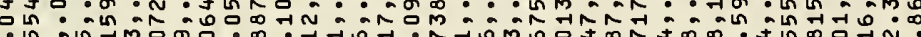
: No. N

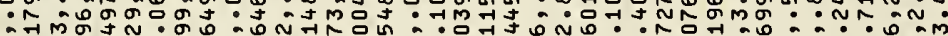
m0नmow 政: mo: :

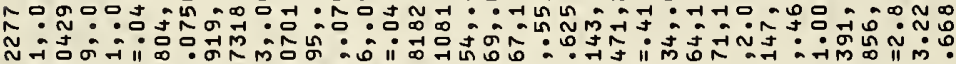

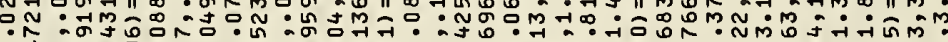

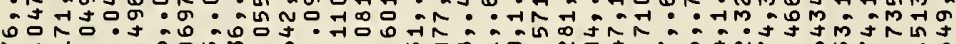

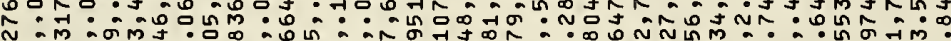

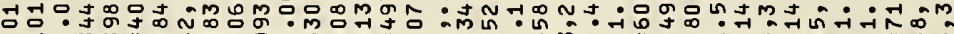

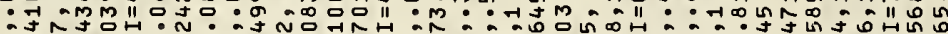

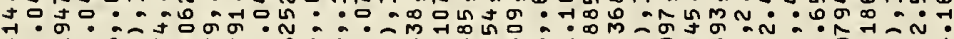

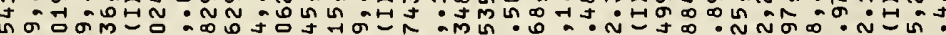
o .0丁

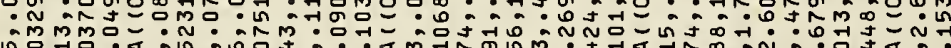

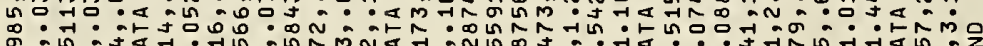

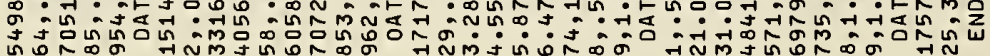

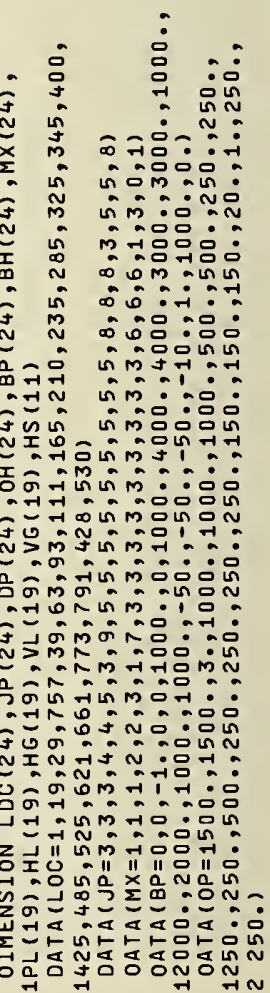




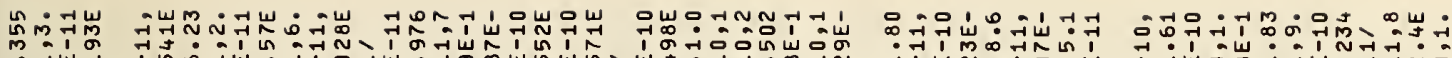
m

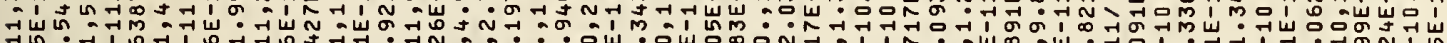

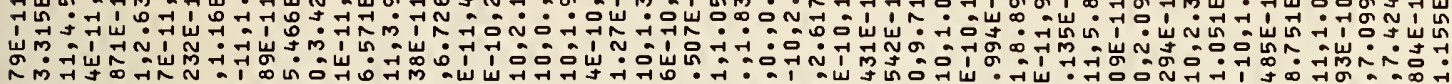
nิ

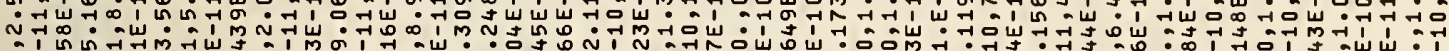

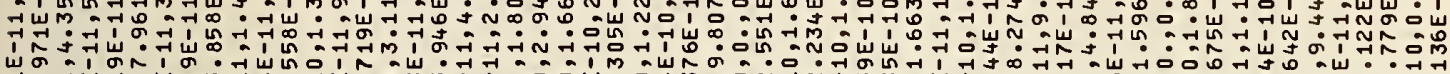

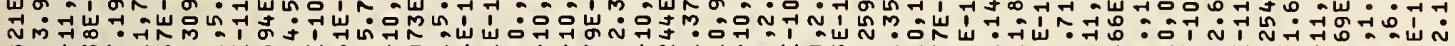

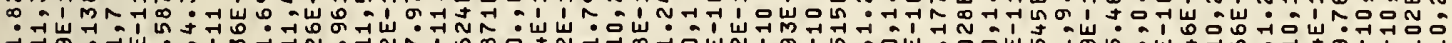
न $\sigma_{-1}$ ڤ

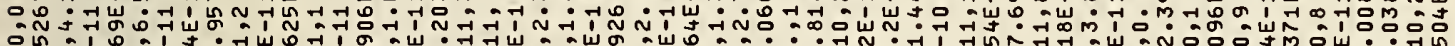
I.

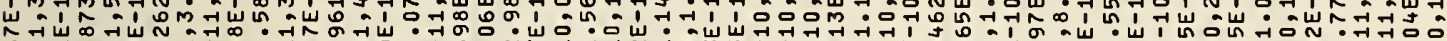

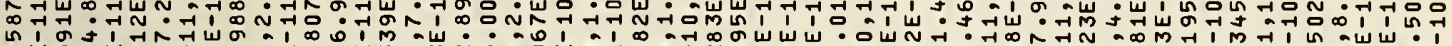

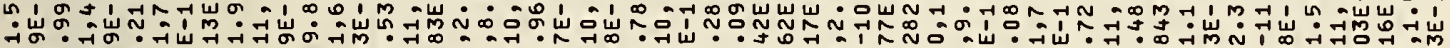

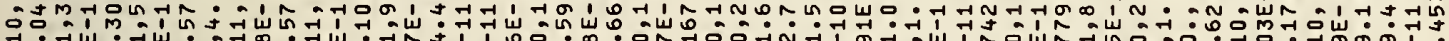
1.

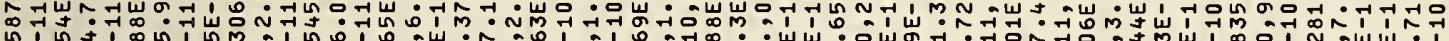

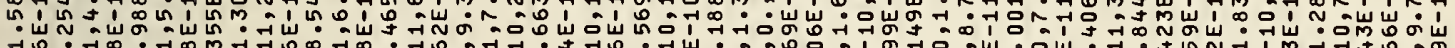

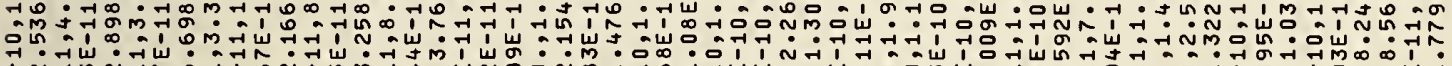
岂

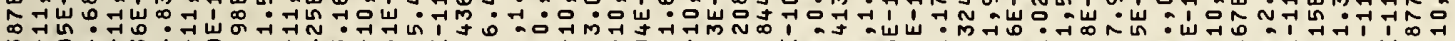

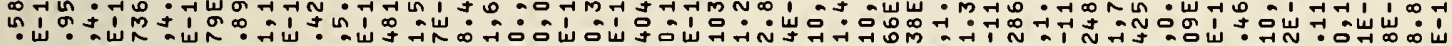

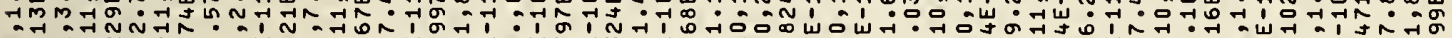

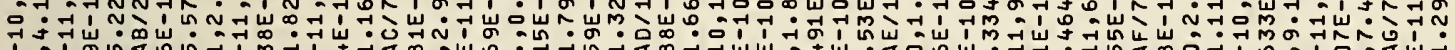

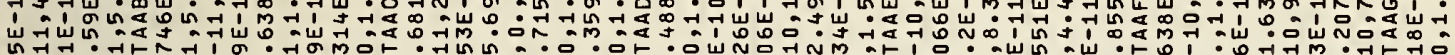

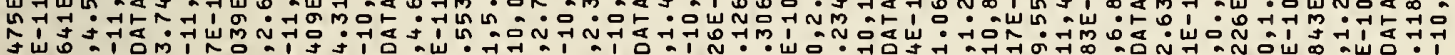

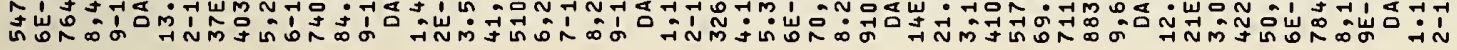
ํํ요

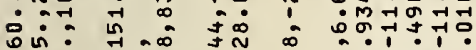

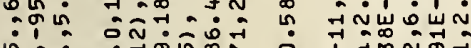
เि:-
N
品:
L.

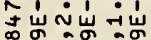
बंनें

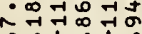

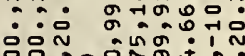

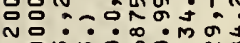

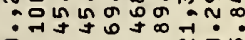

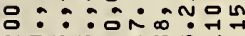

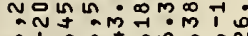
ก

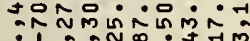

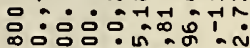

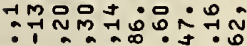
:

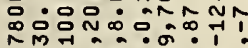
: ! : :

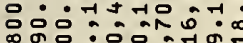

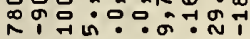
:

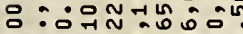

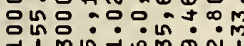

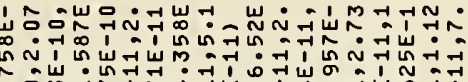

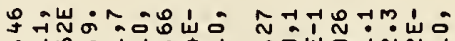

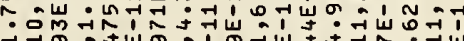

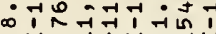

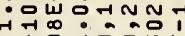

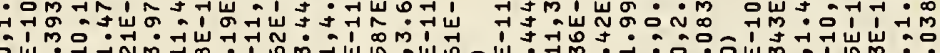

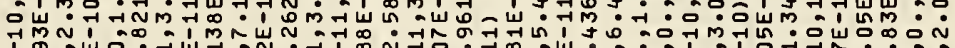 岁 œ N

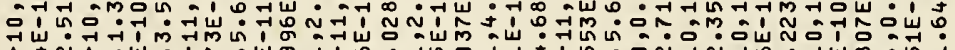

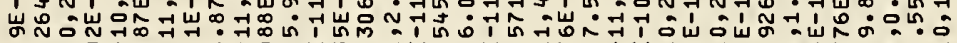

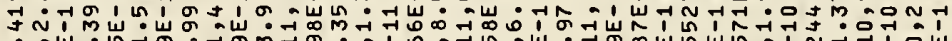 -

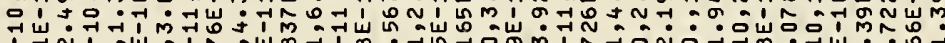

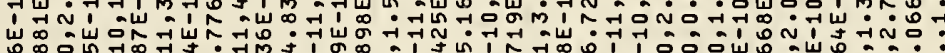

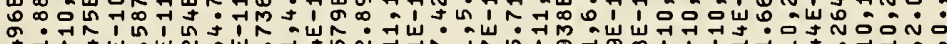 .

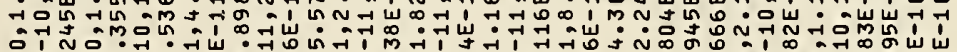

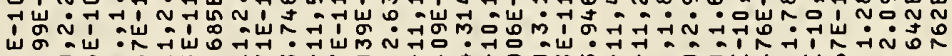

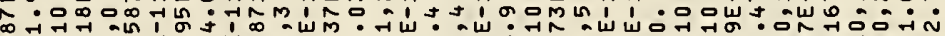 感古

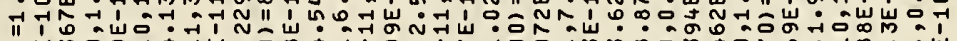 o

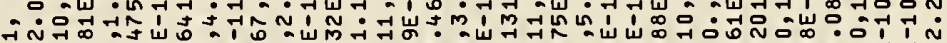 "1 10 0 w

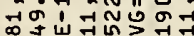 Мัญ

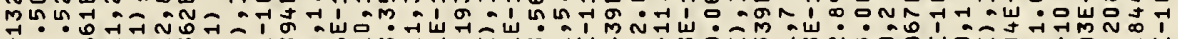 1' II : "II " "

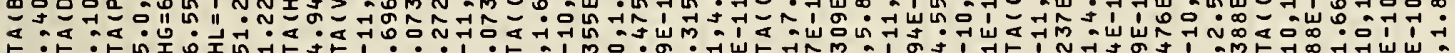

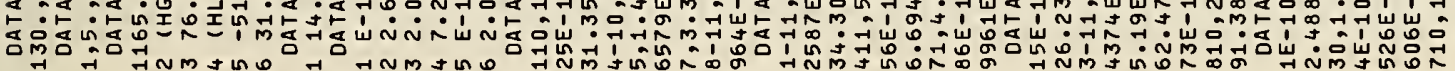




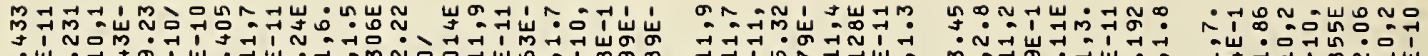

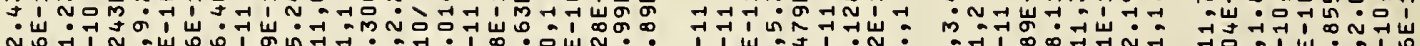

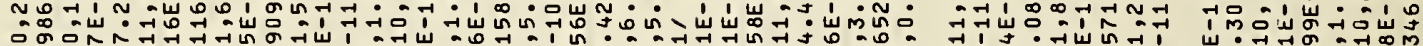

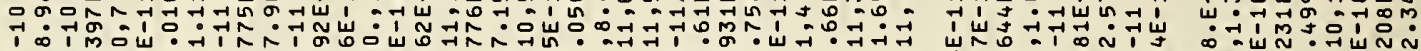

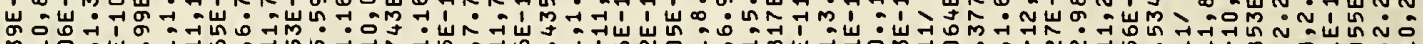
M

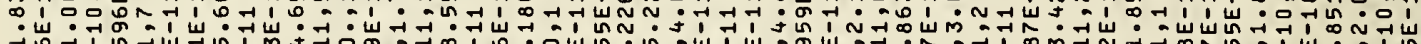

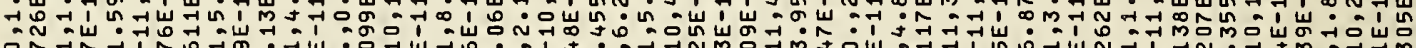

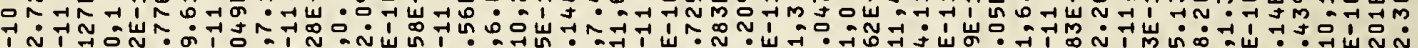

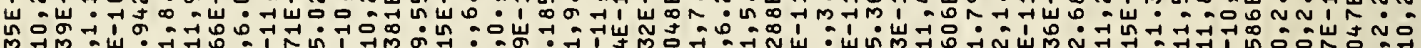

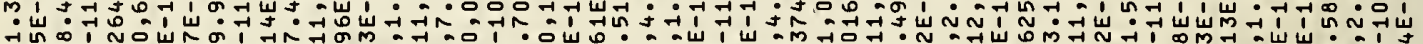

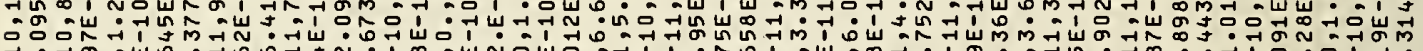

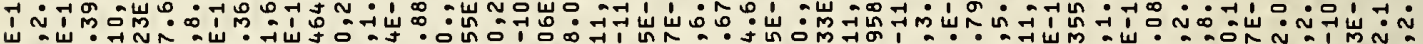

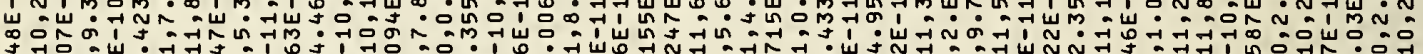

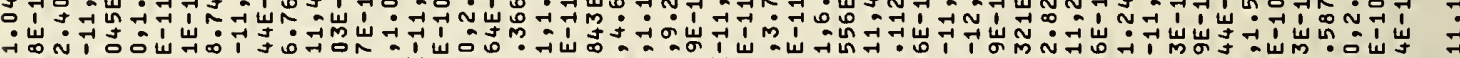

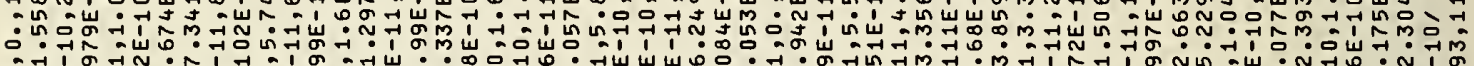

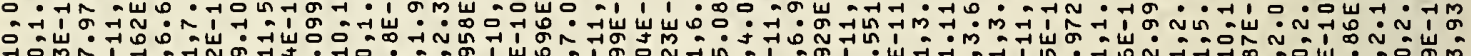

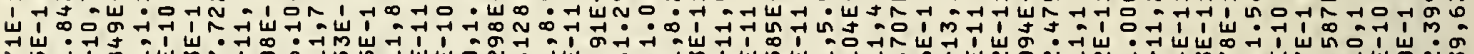

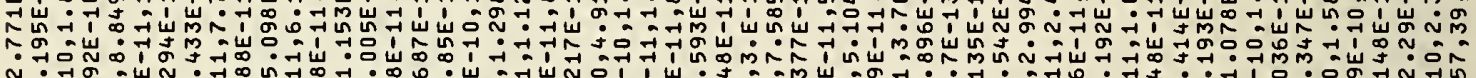
N

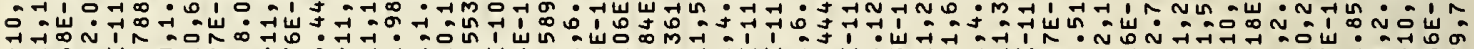

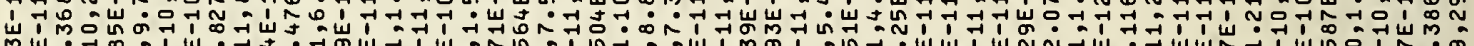

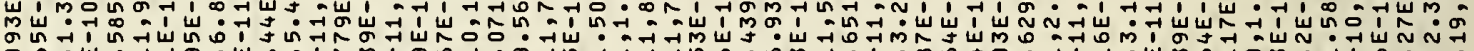

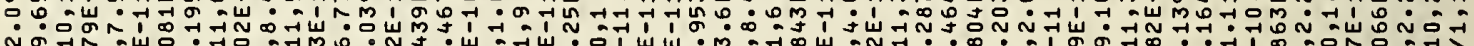
No

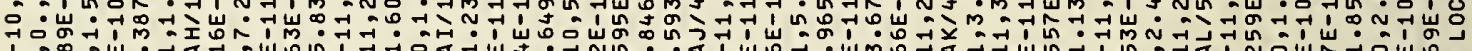

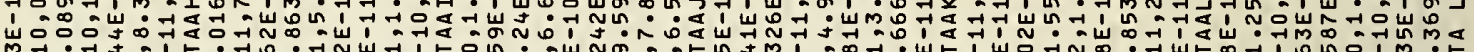

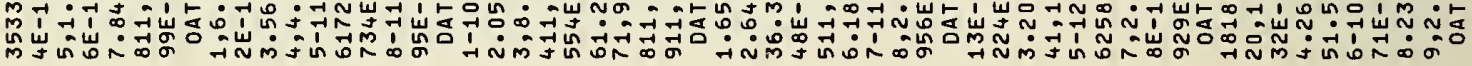

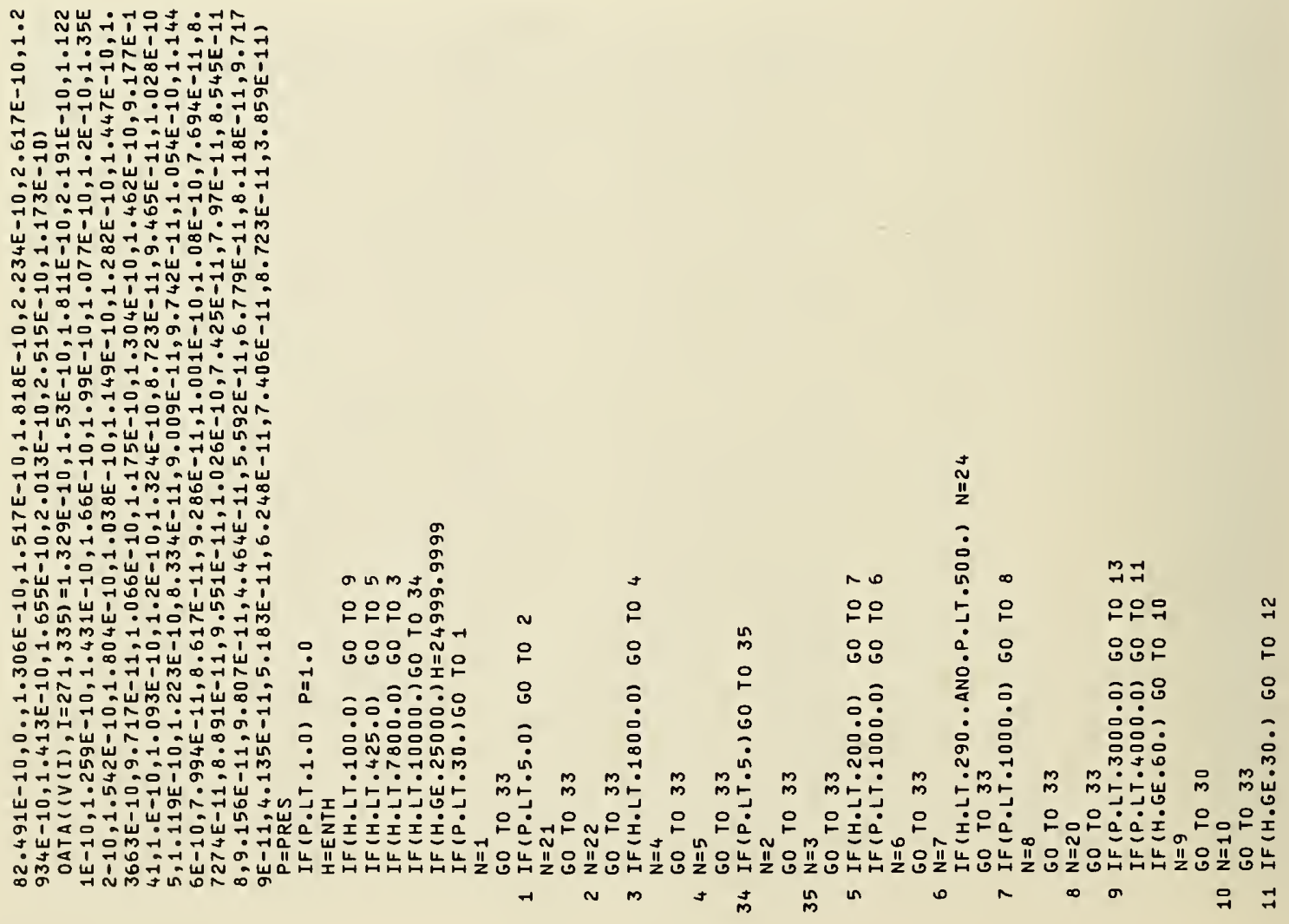




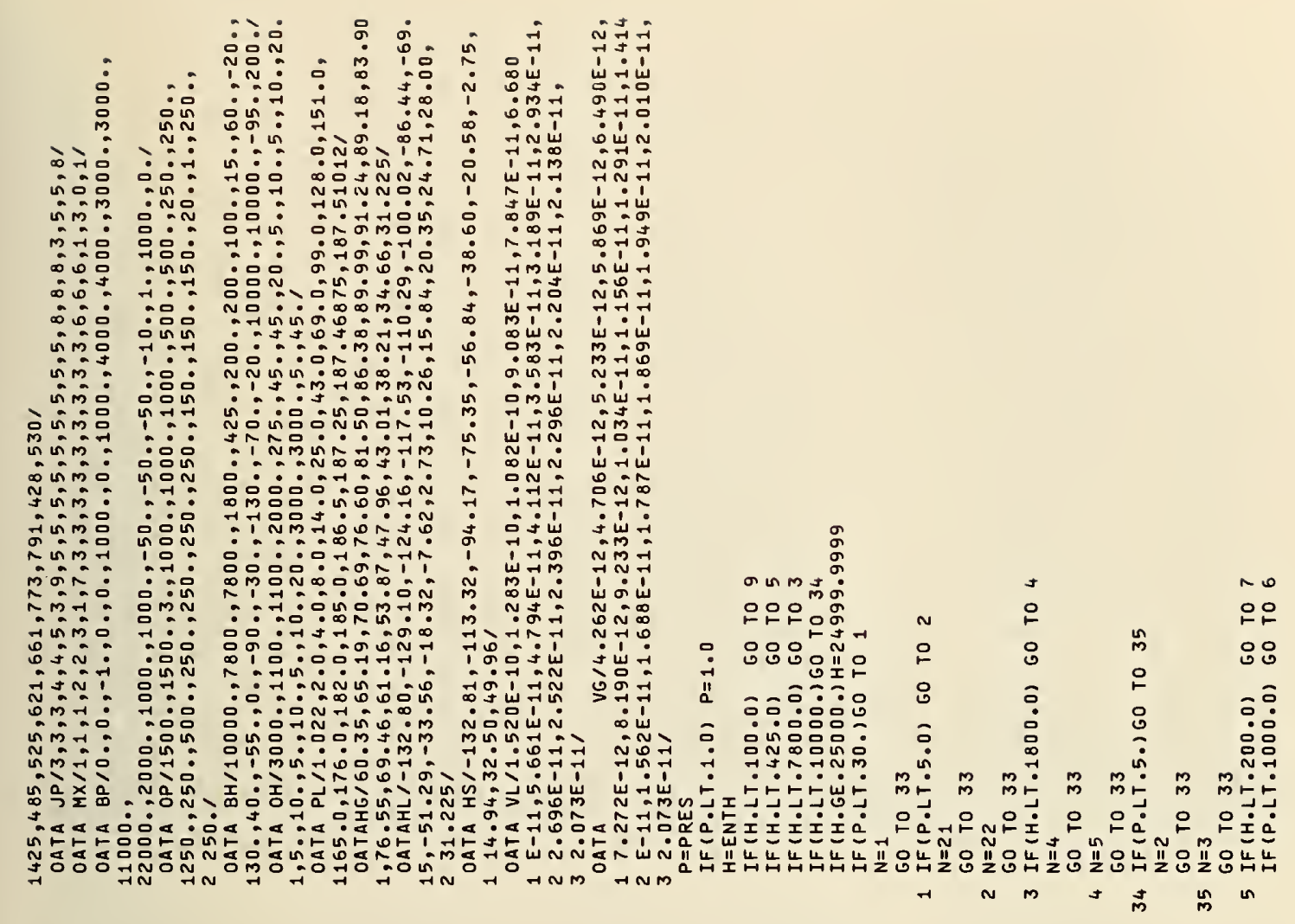

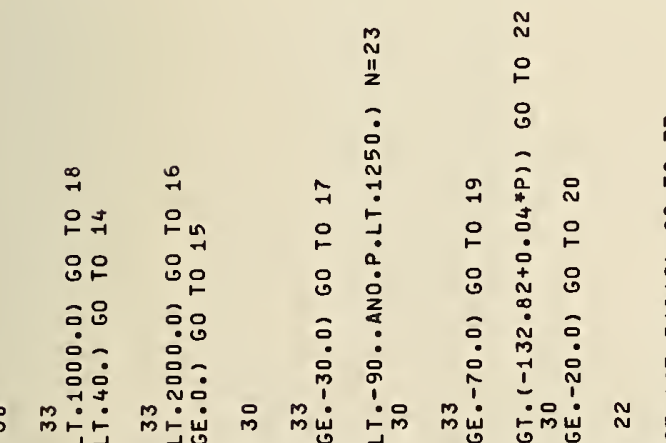

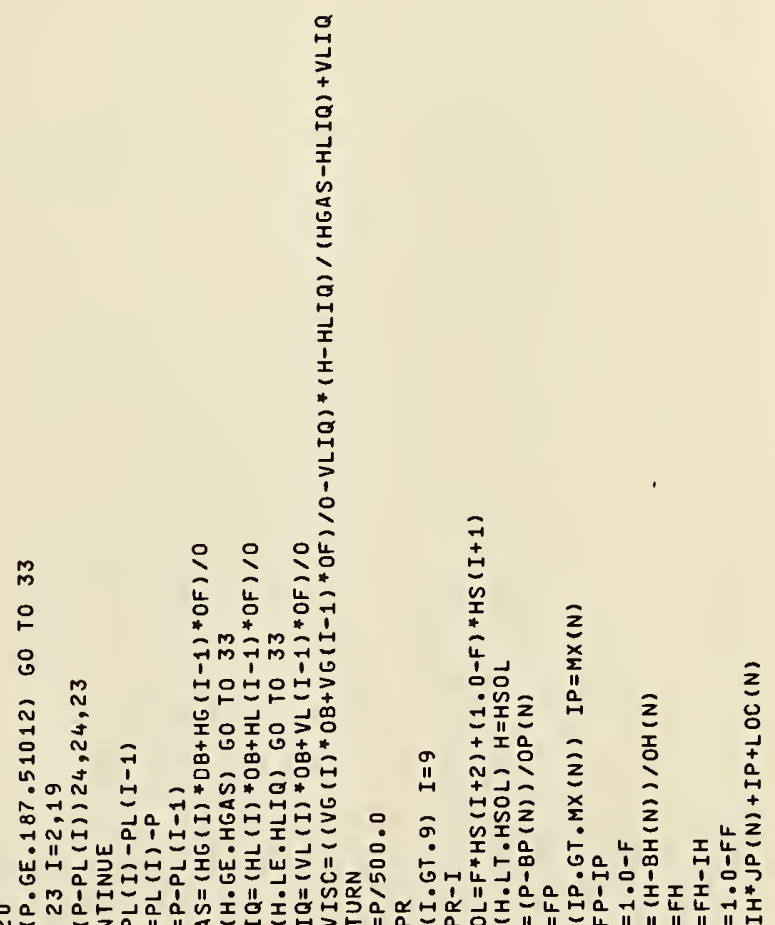

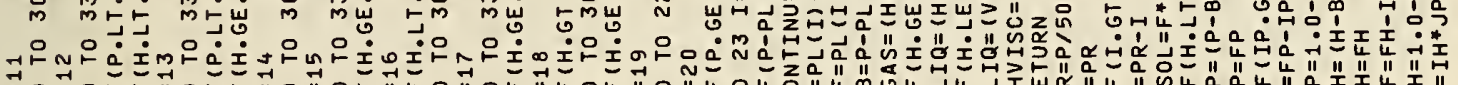

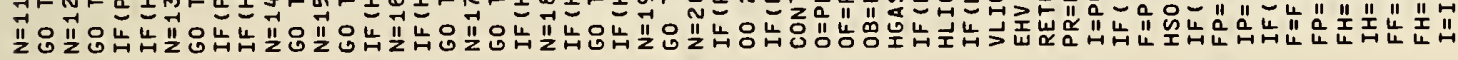
$\cong \stackrel{m}{7}$
ज
๓

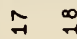
न
品
m 

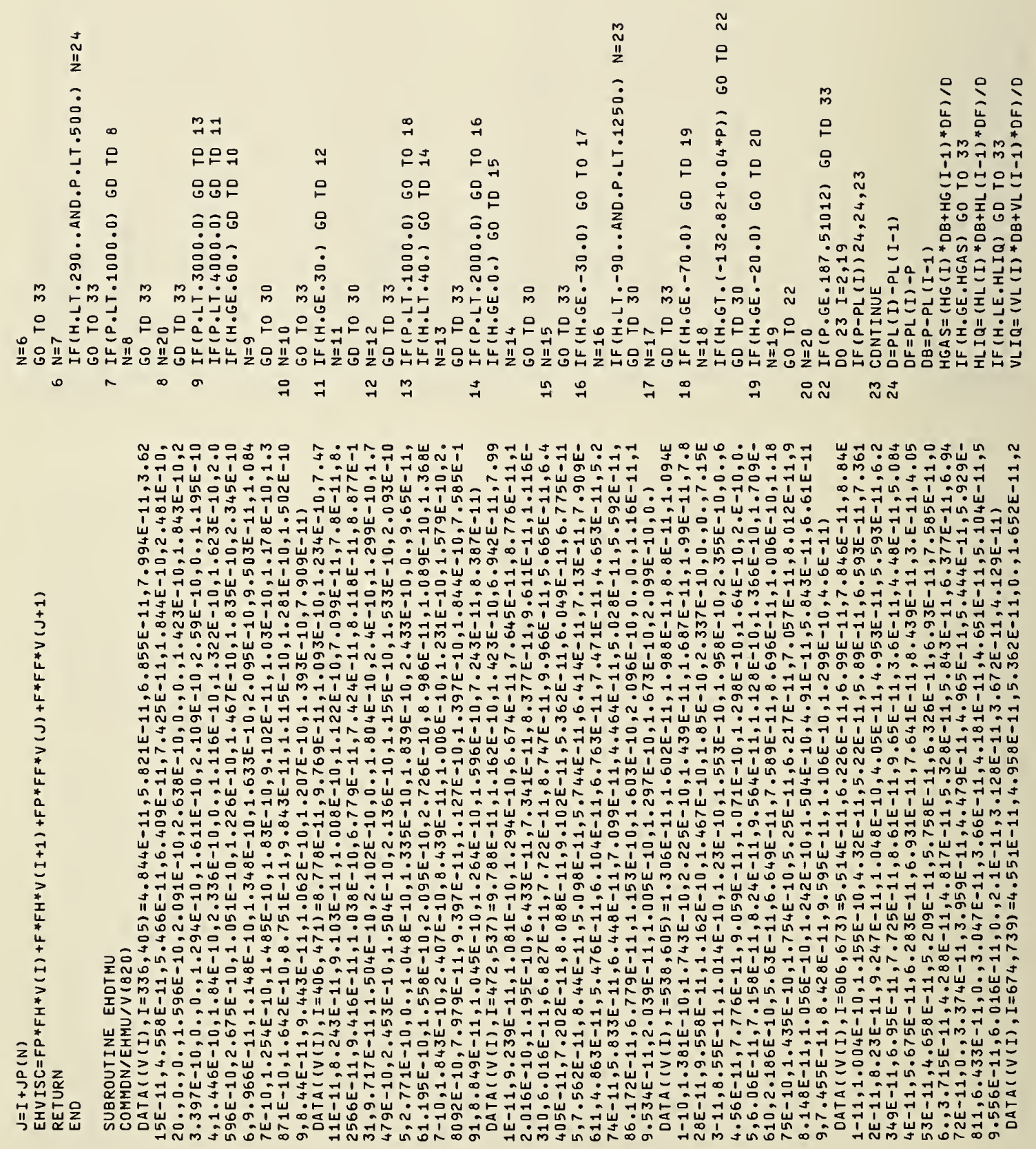


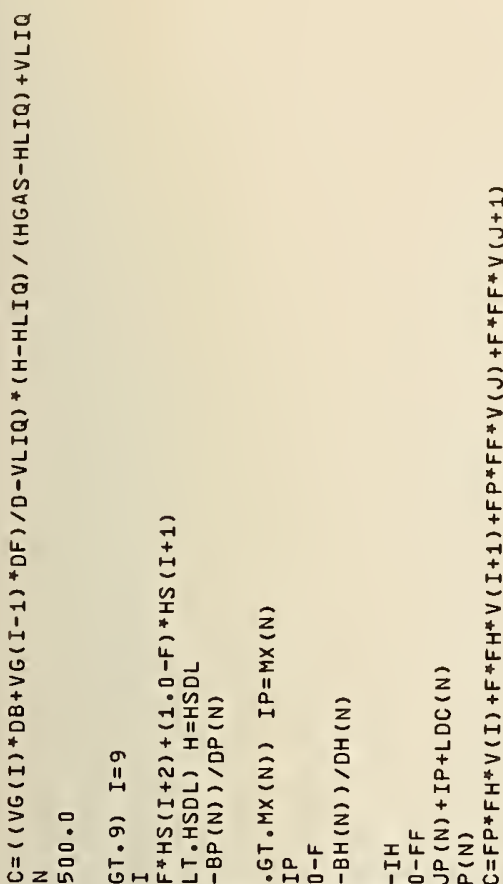

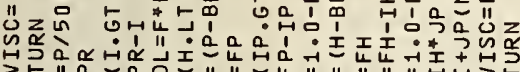

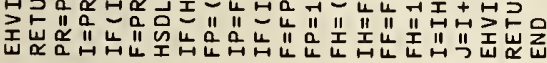
品

品

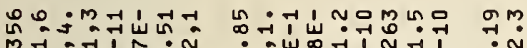

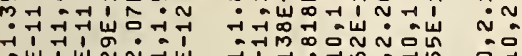

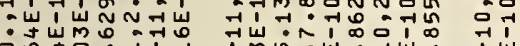

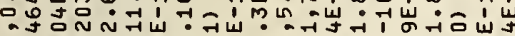

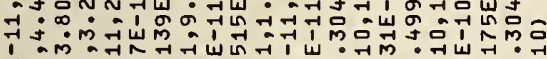

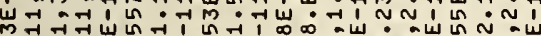

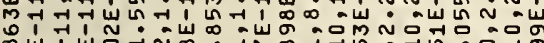

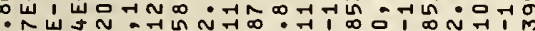

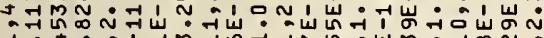

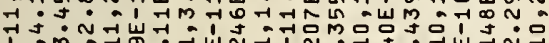

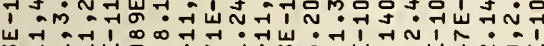

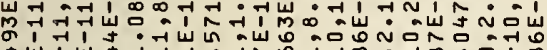

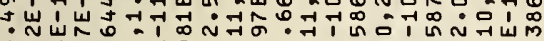

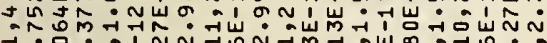

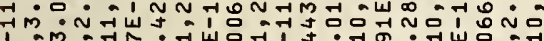
自等

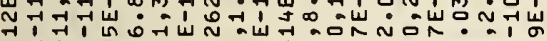
7 出出

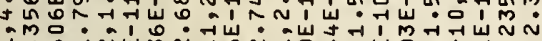

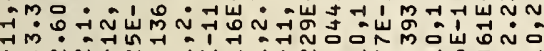

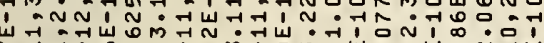
崖

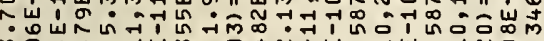

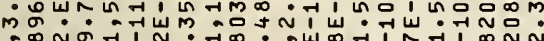

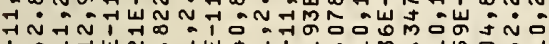

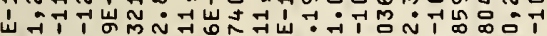

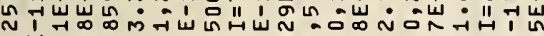

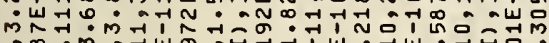

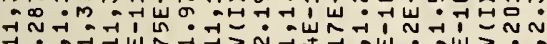

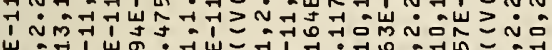

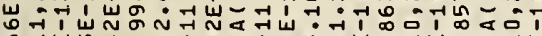

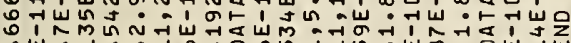

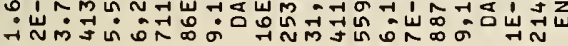
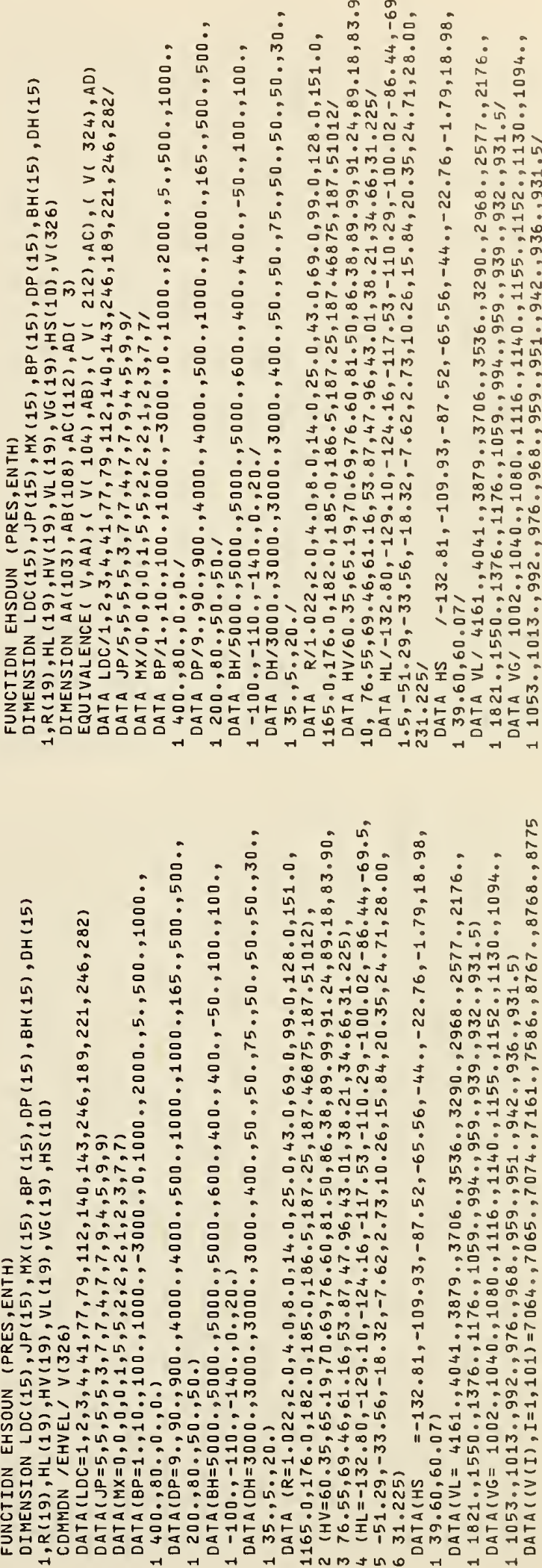

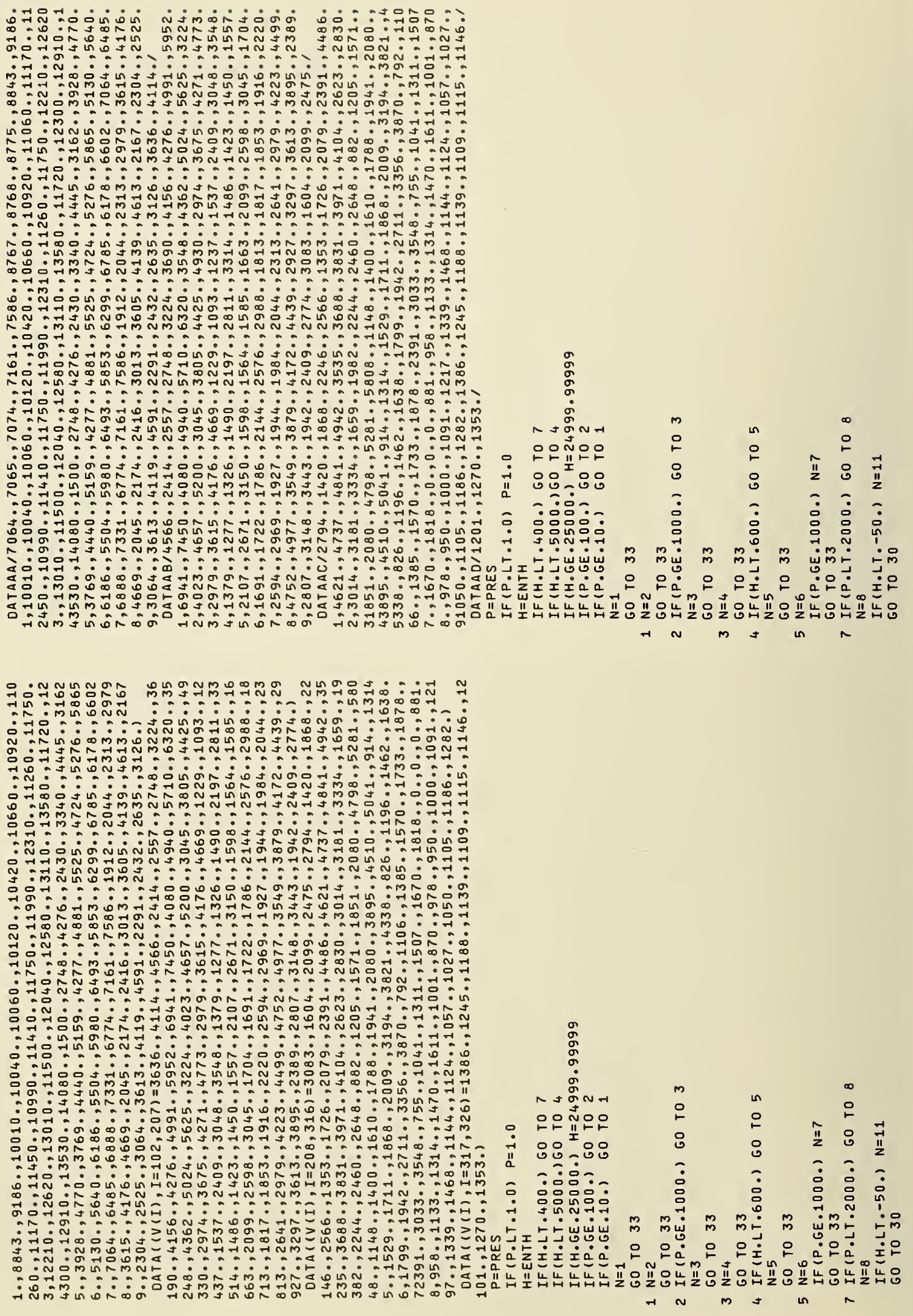

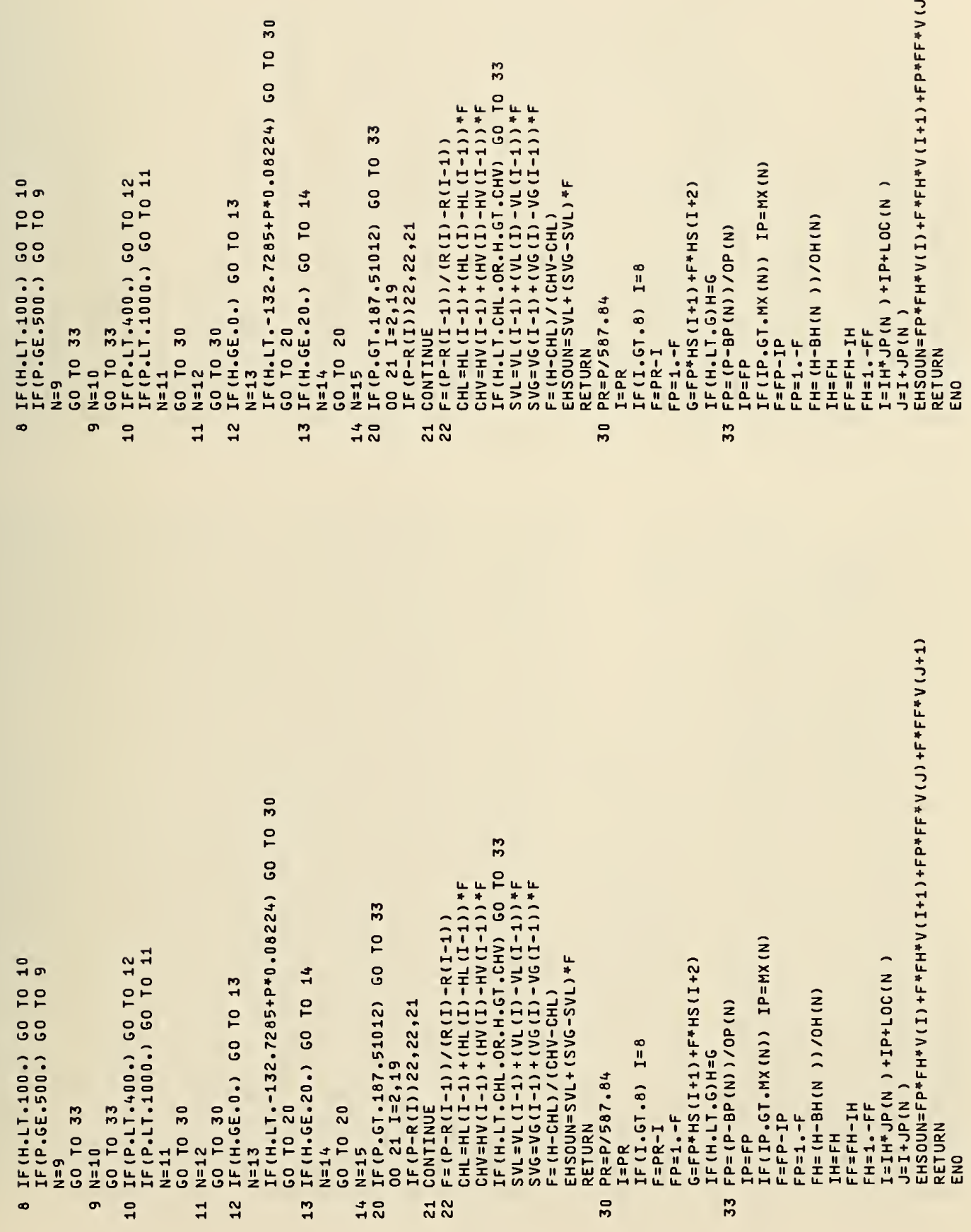


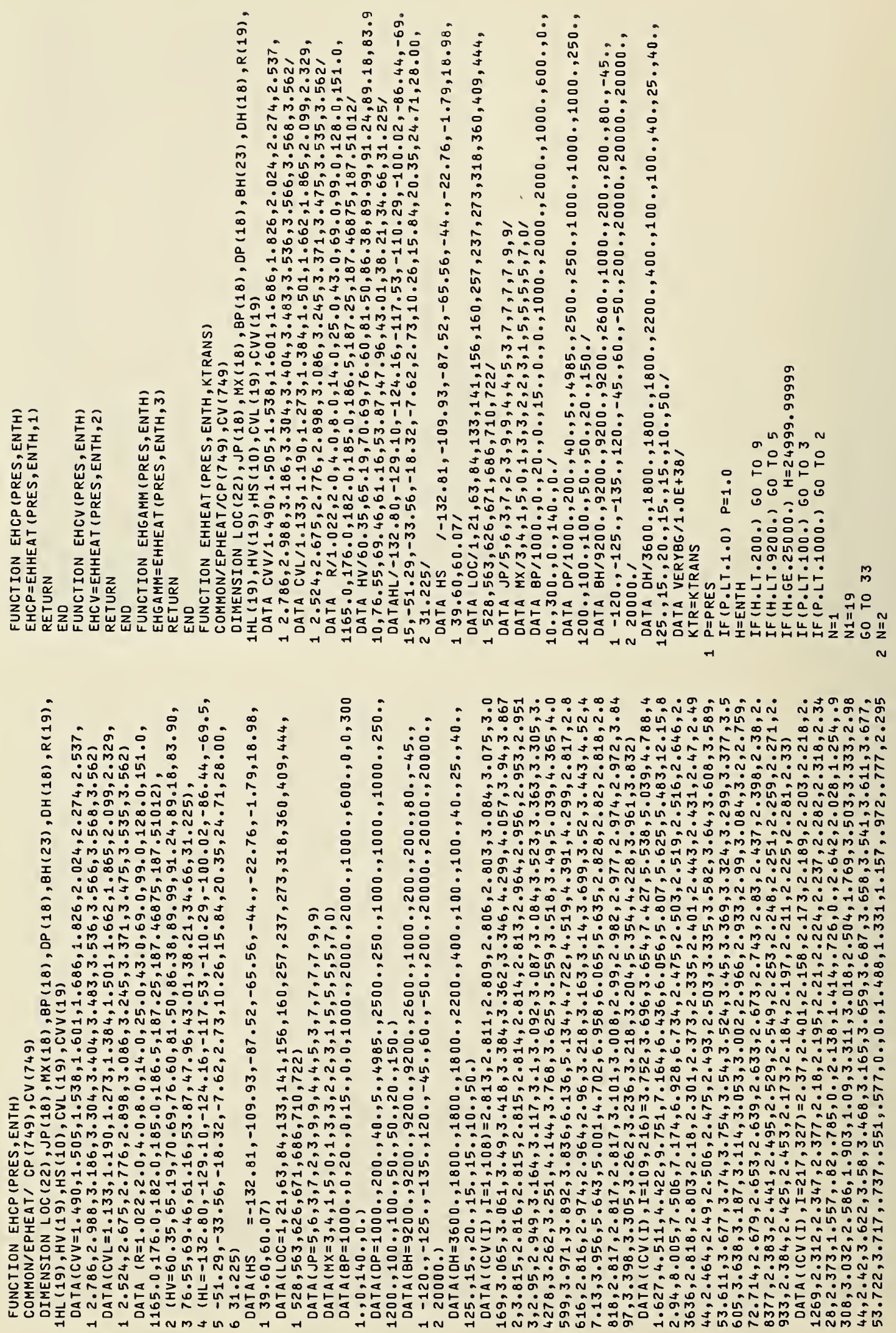




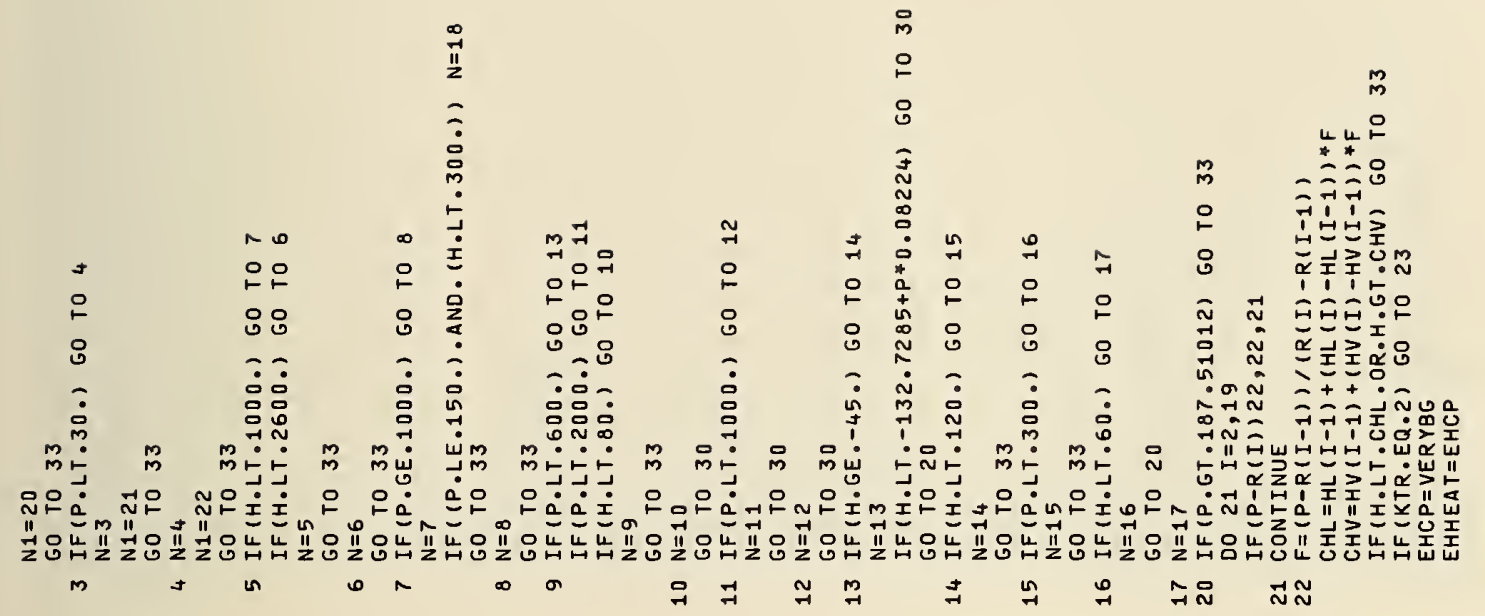

คำ

ญับิ์

min

กัด

minis

궁ำ

نंmin

मेक

ถับ

퐁

tै $\infty \begin{aligned} & 0 \\ & 0\end{aligned}$

نिं

जूञ

نिं

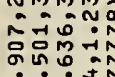

نM的出

ômin

-MM

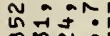

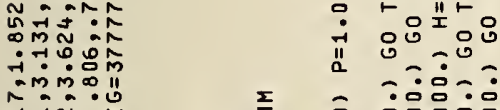

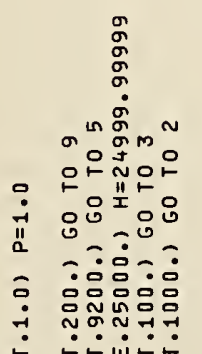

$\prod_{2}^{\infty 11}$

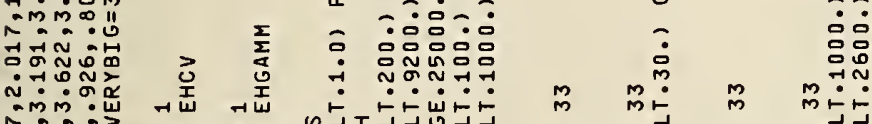



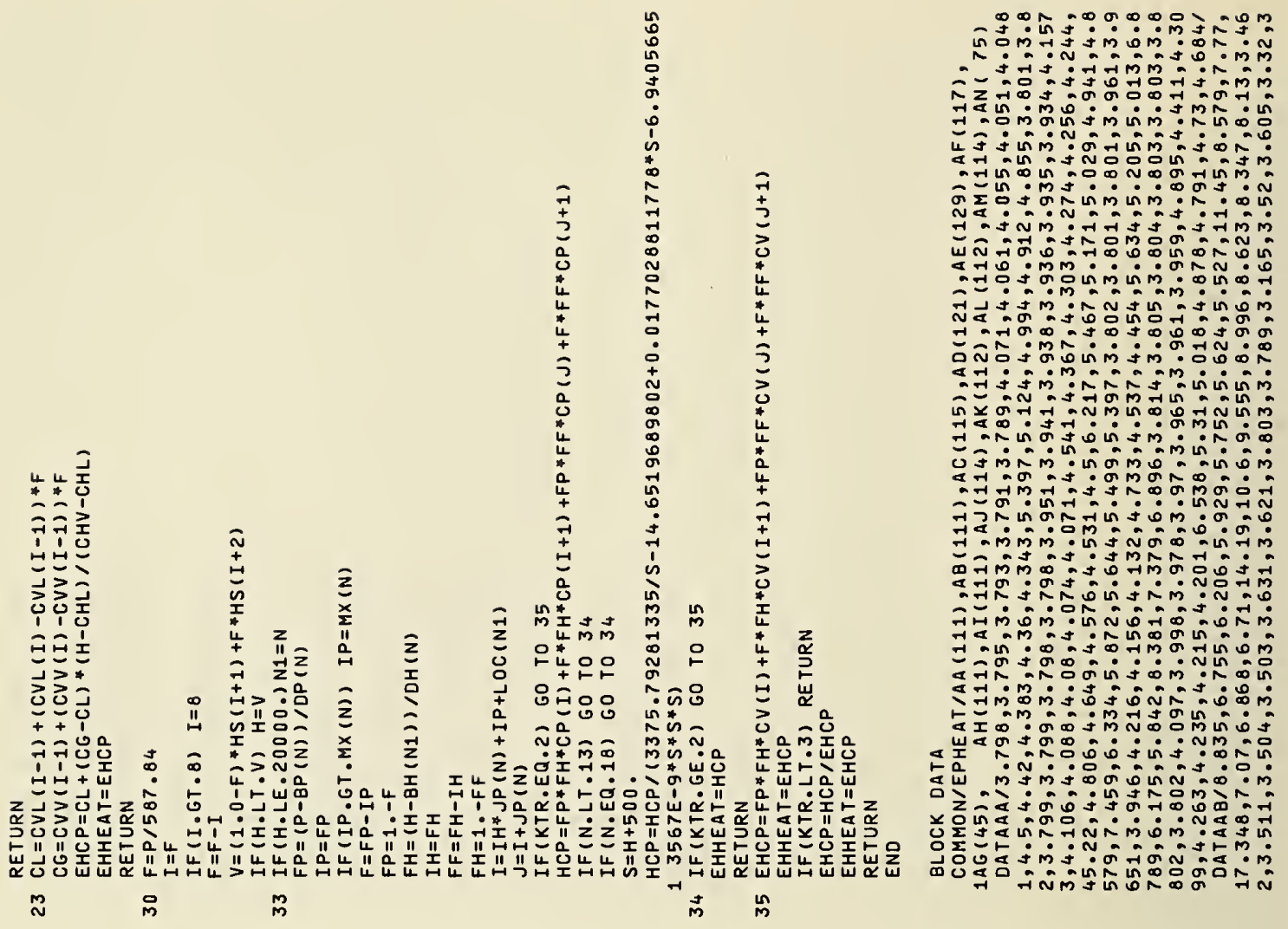

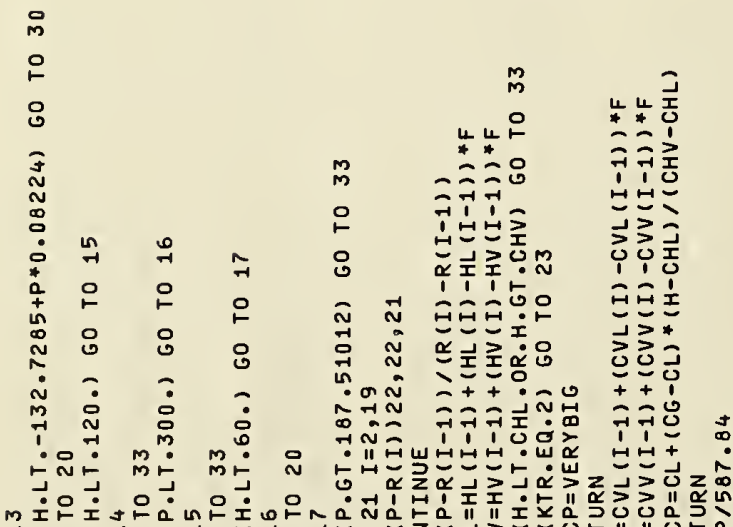

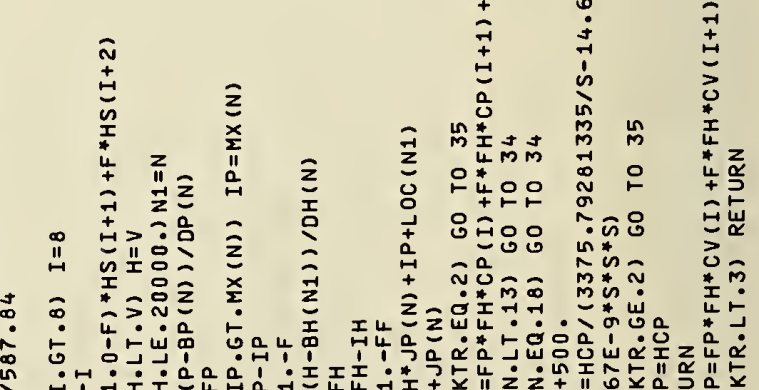
m ก 
نั

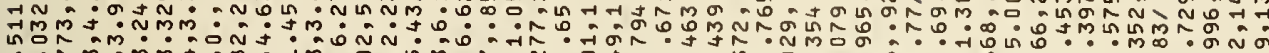
min 0 ;

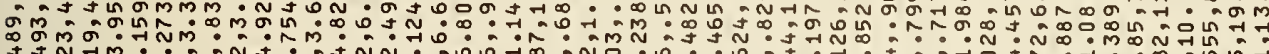
P. U N o

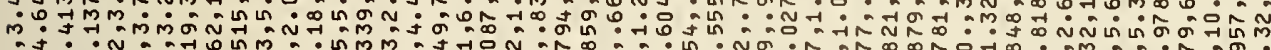
mj:

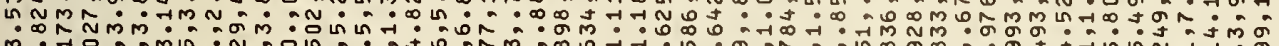

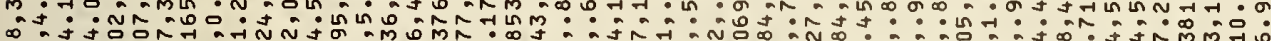

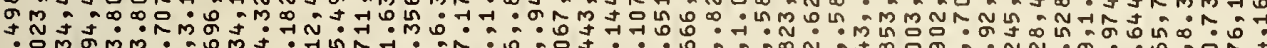

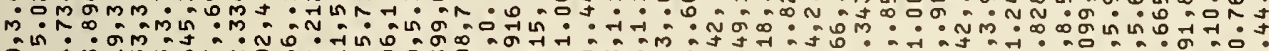

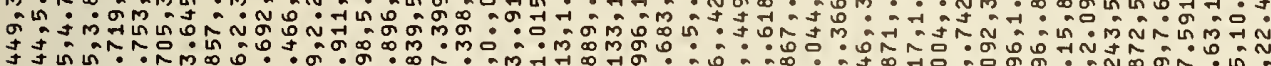
:

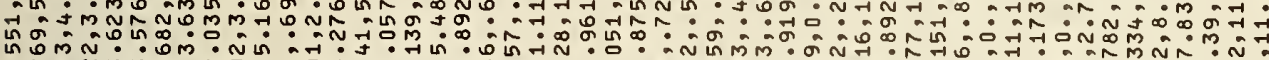

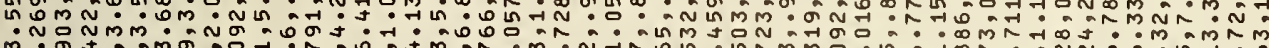
mo.

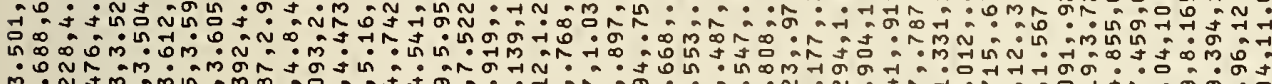

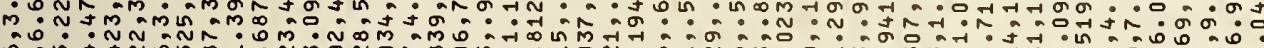
0 in $+N 0$ N

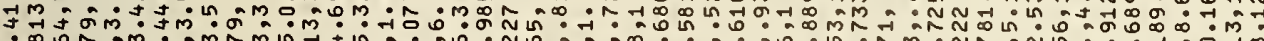
$\dot{m}: 0$ ก -

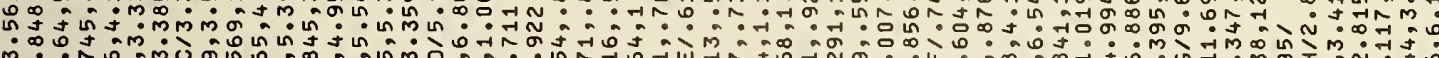
$\dot{m} \cdot 0$. on

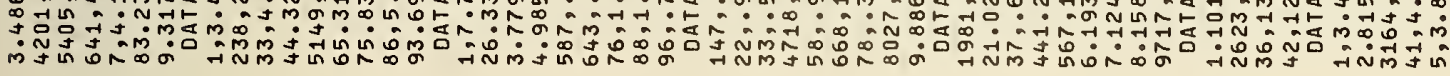

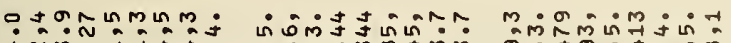

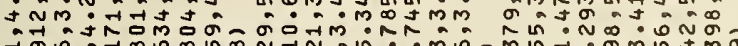

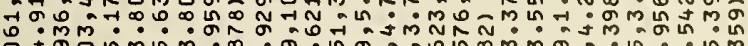

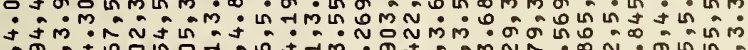
†

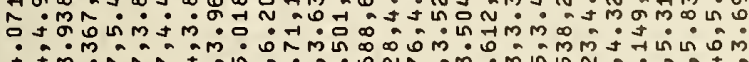

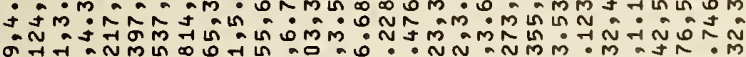
ó

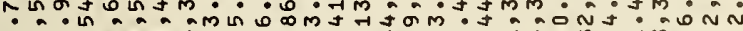

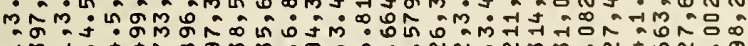

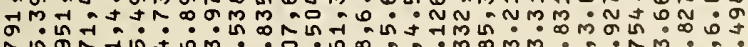
minm. in

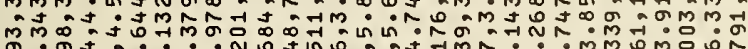

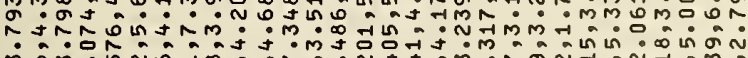

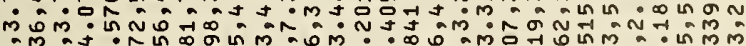

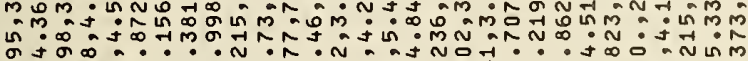
б.

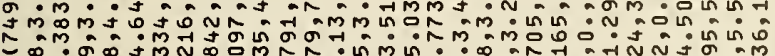

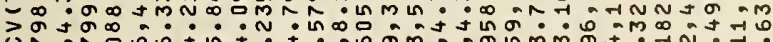

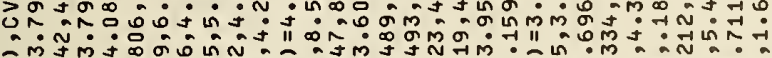
की సo

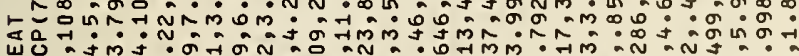

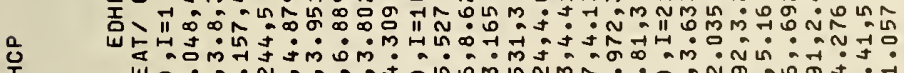
w

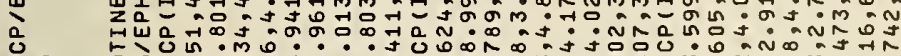
至

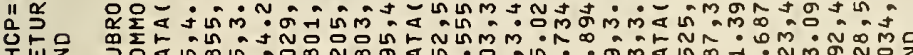

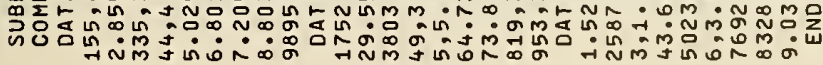

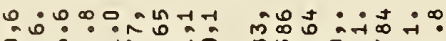
का

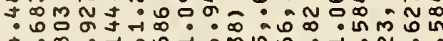
+

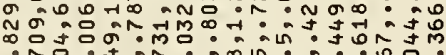
i To:

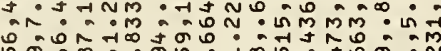
命

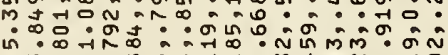
on moñmmon

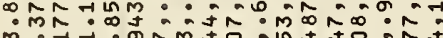

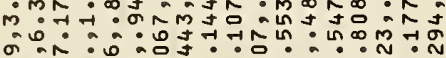
भू̆ ¡ न

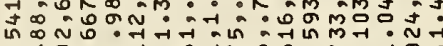

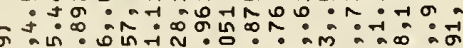

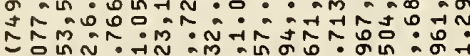

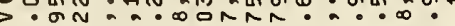
Uึं N.

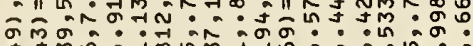
a 年疋

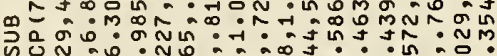
U人

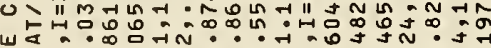

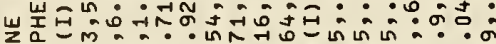

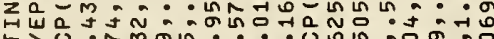

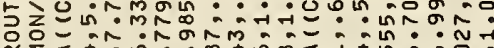

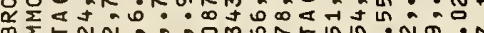

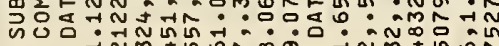




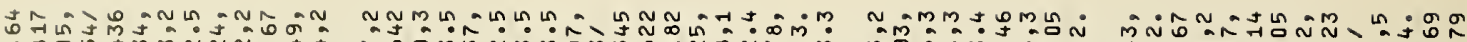

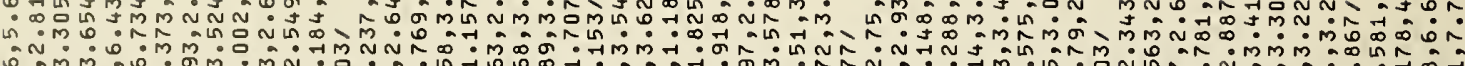
Un の.

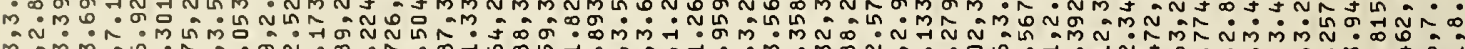

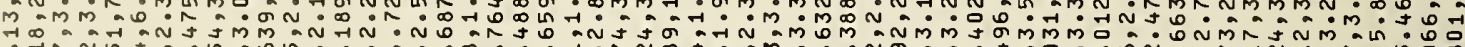

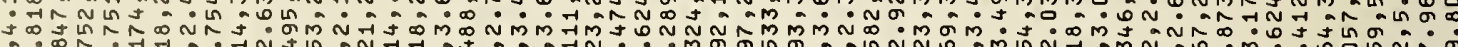
Nं*. Ůmṕñ

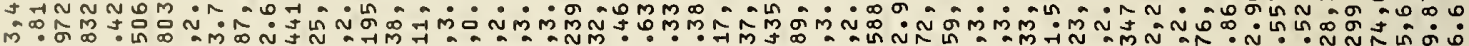

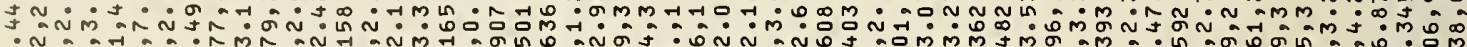

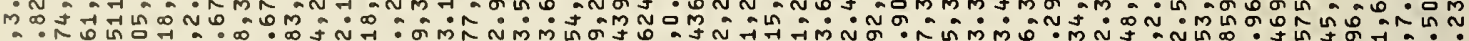
ஸ்

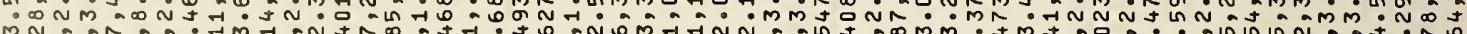
mN N ğ

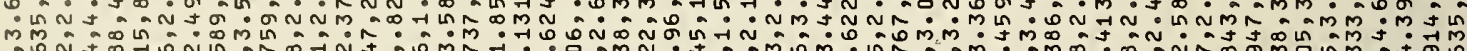

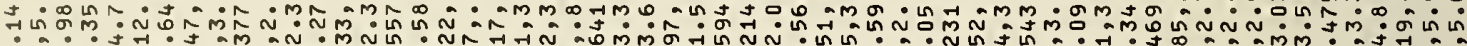

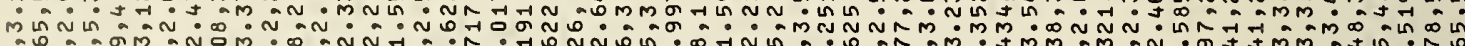

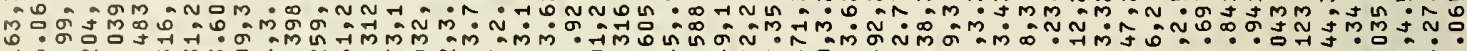

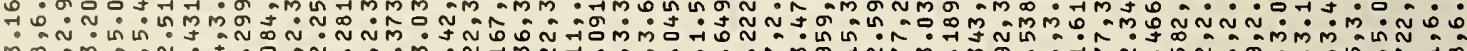
M⿻ o o.

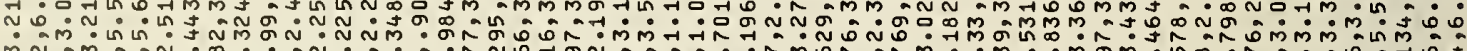

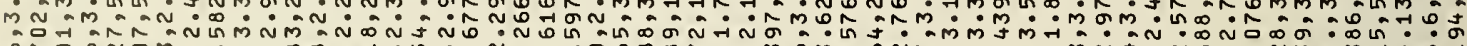

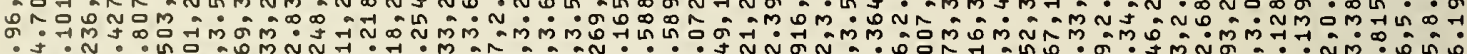

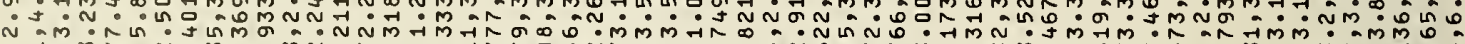

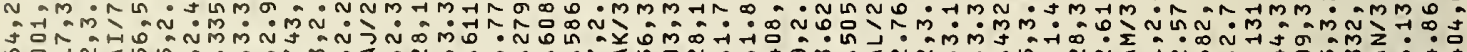
oㅁ.

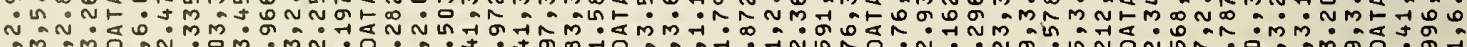

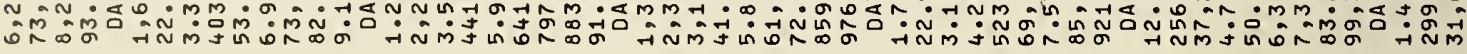

i :

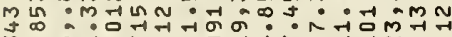
: ค. N N

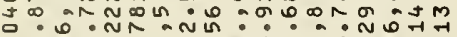

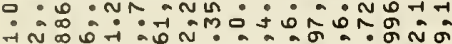
नू.

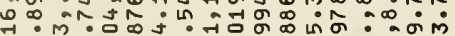
․ N 政 ง ơ : ma: m:

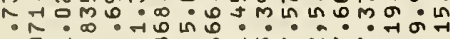

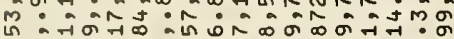

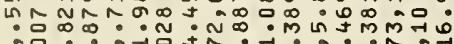
बं M

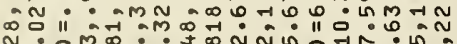
: $\because$ Ón

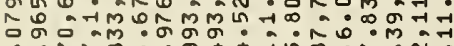
$\because \therefore$ in

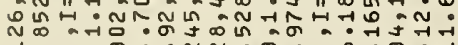

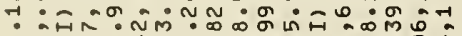

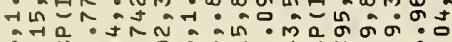

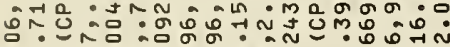

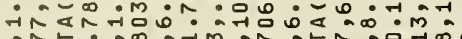

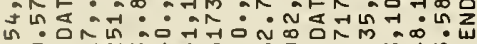

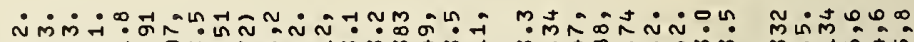
moñ ऊू N

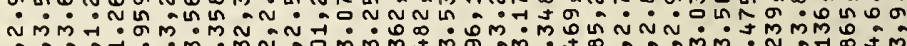

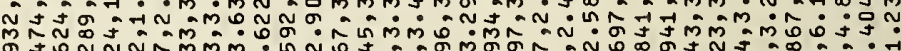

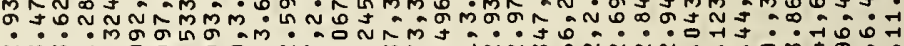
imm-1"Oa.

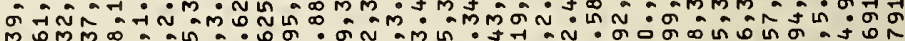

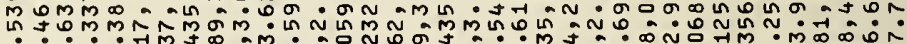

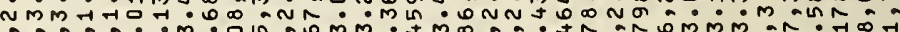

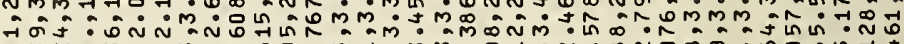

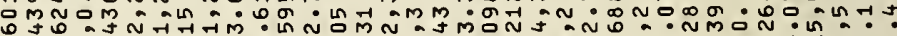

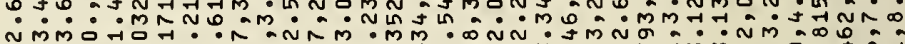
-

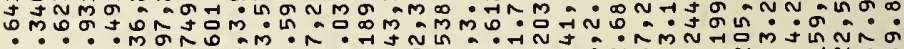

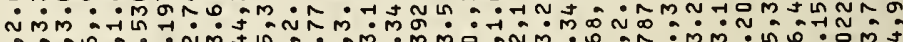
-1

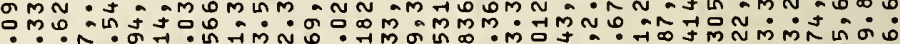

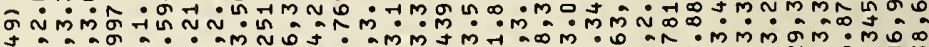
Є

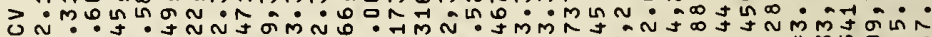

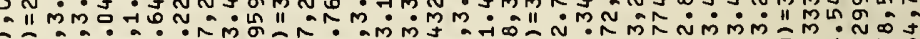
ᄀ的命乞 ง

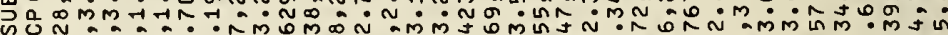
ט

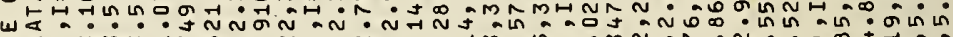
岌引MmM

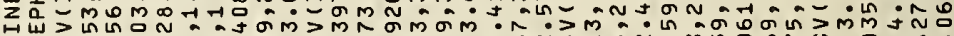

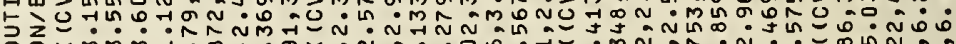
व्र

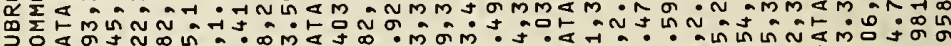

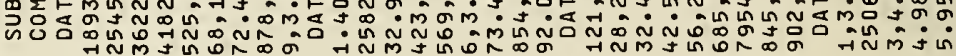




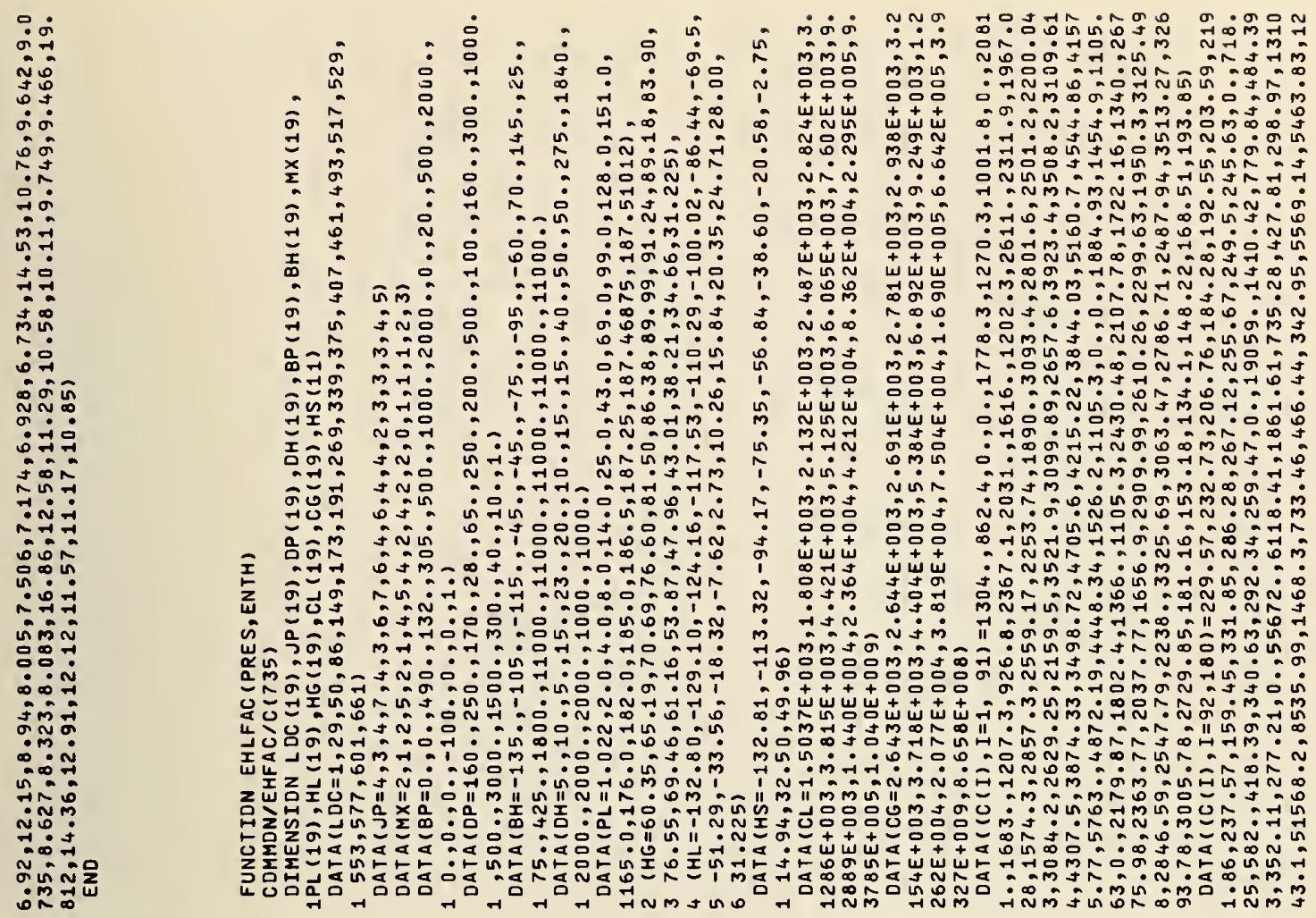

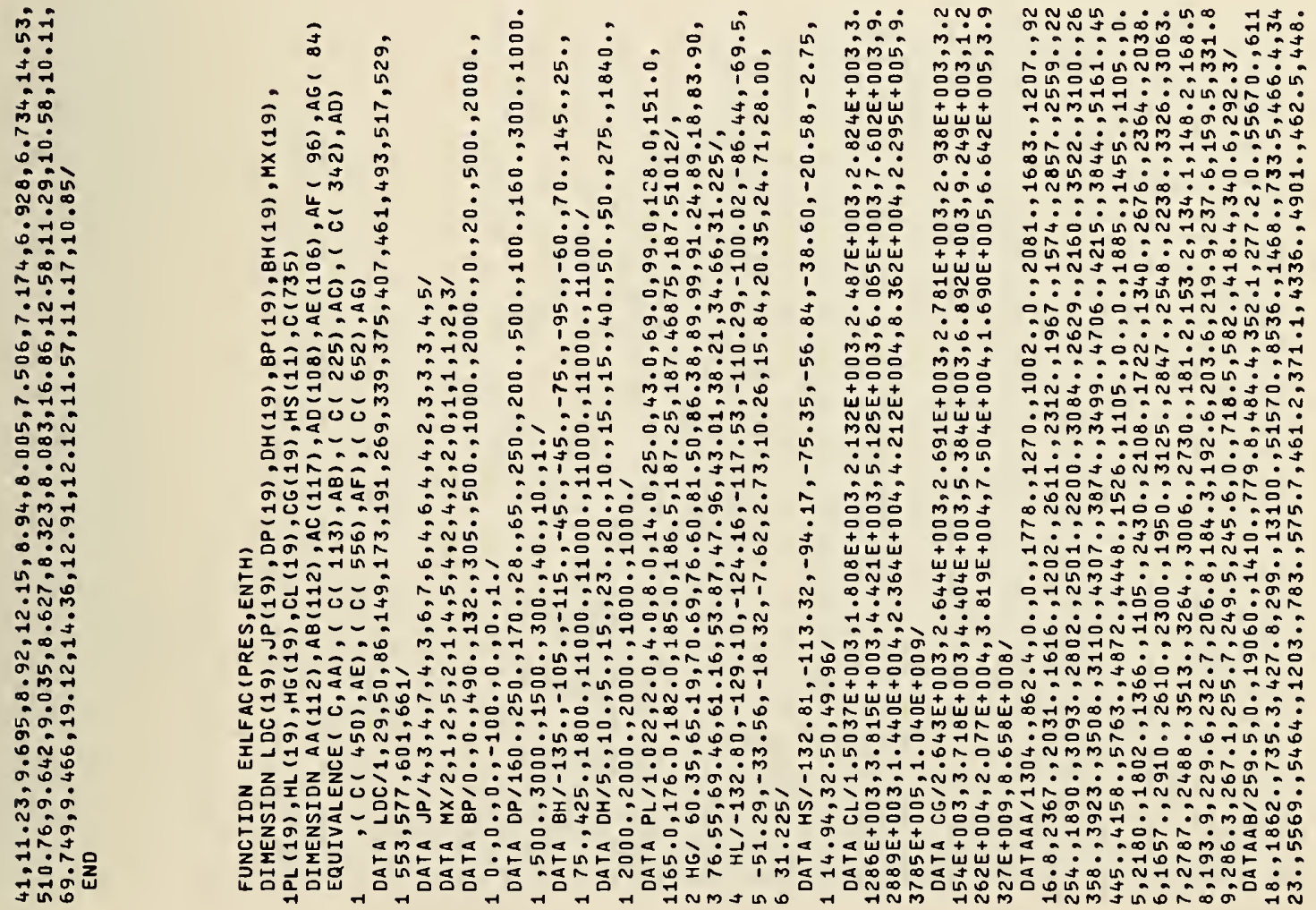




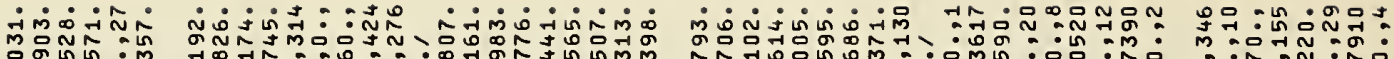

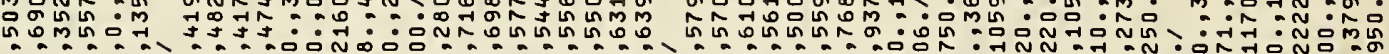

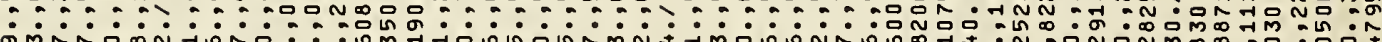

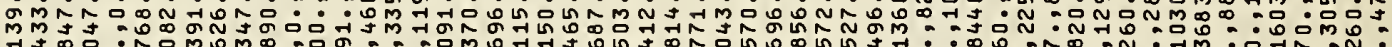

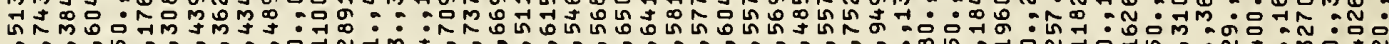
Z

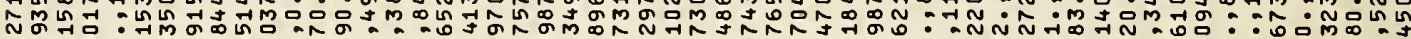
in :

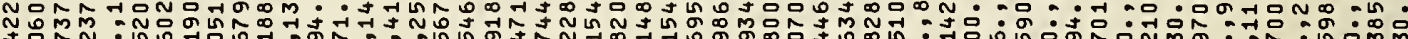

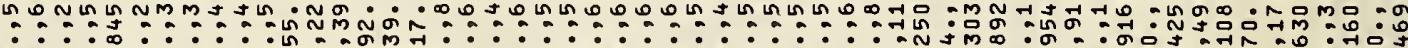

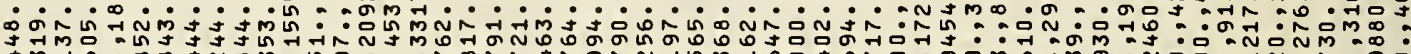

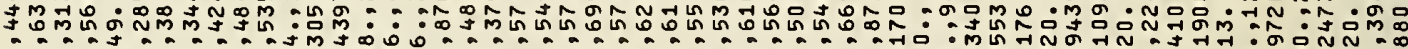

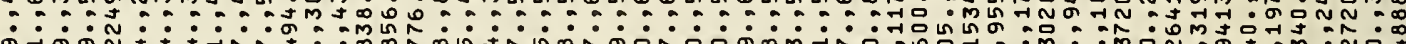

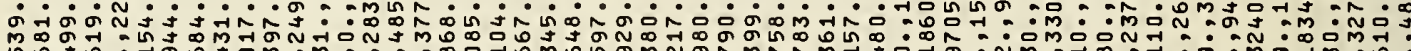
⿸尸匕

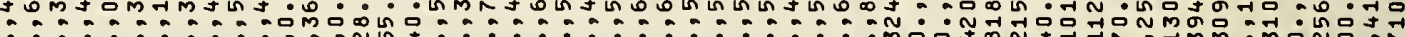

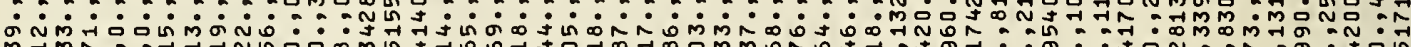

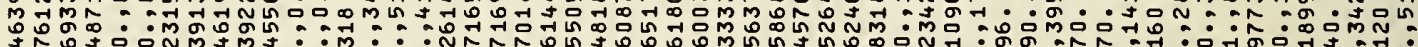

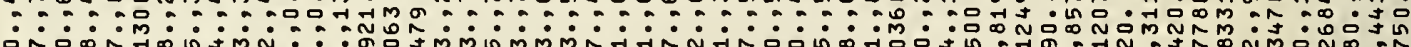

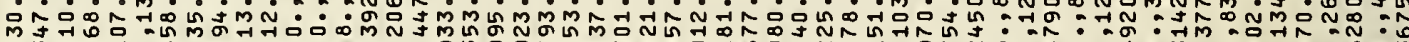

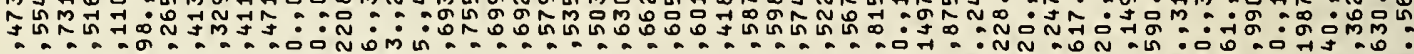
o : : :

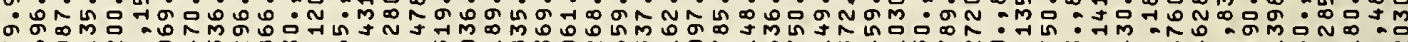

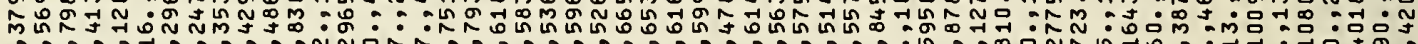

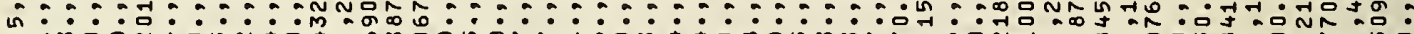

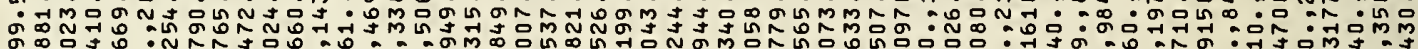

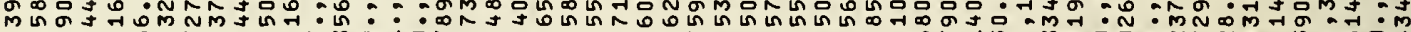
:

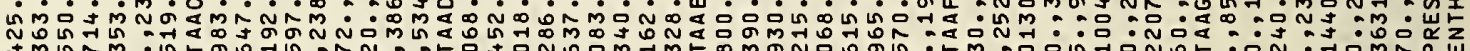

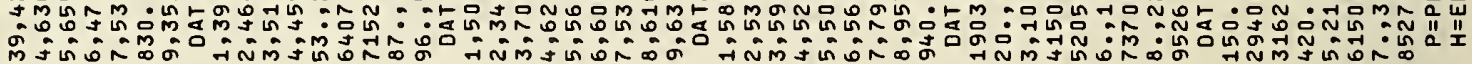

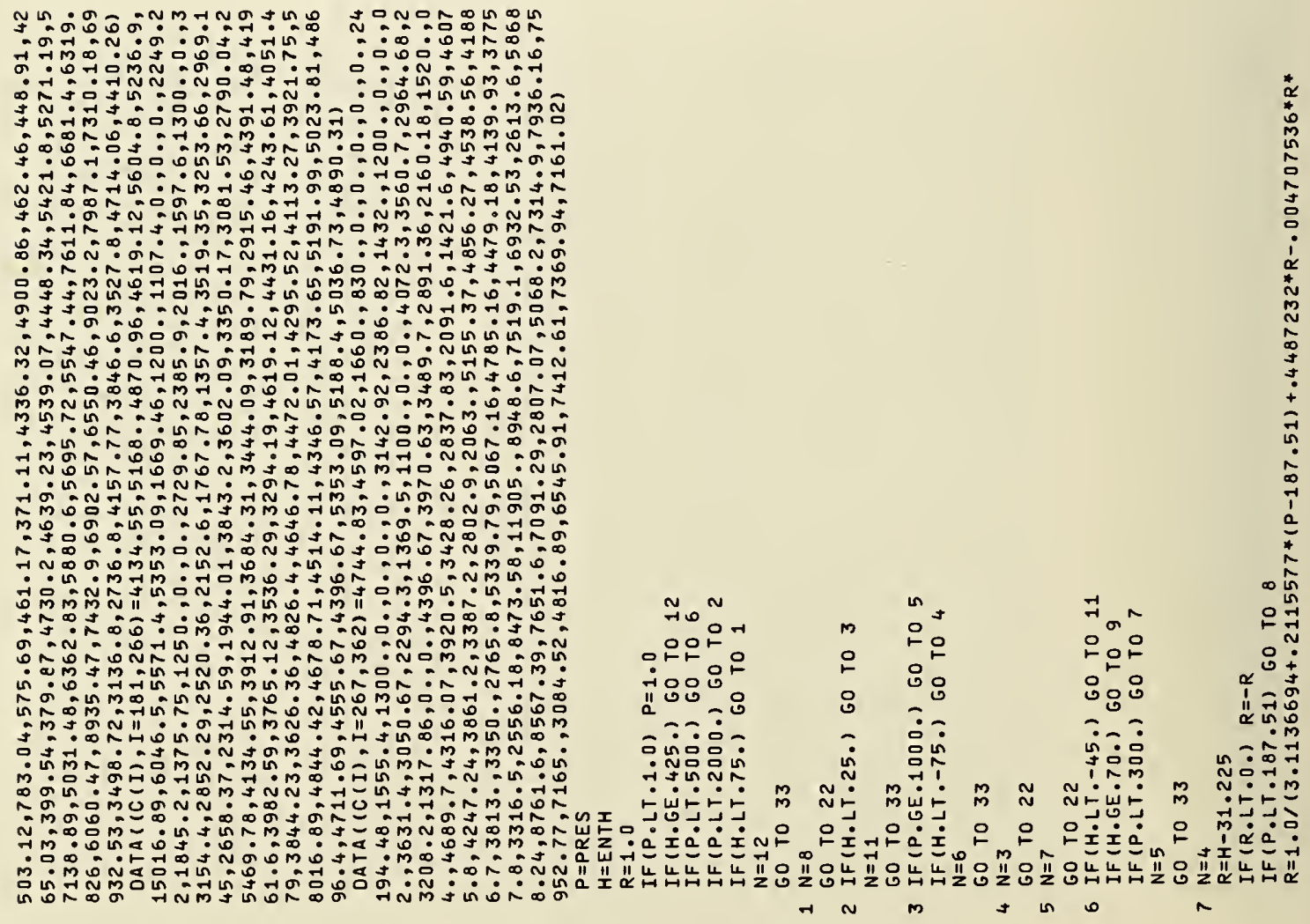




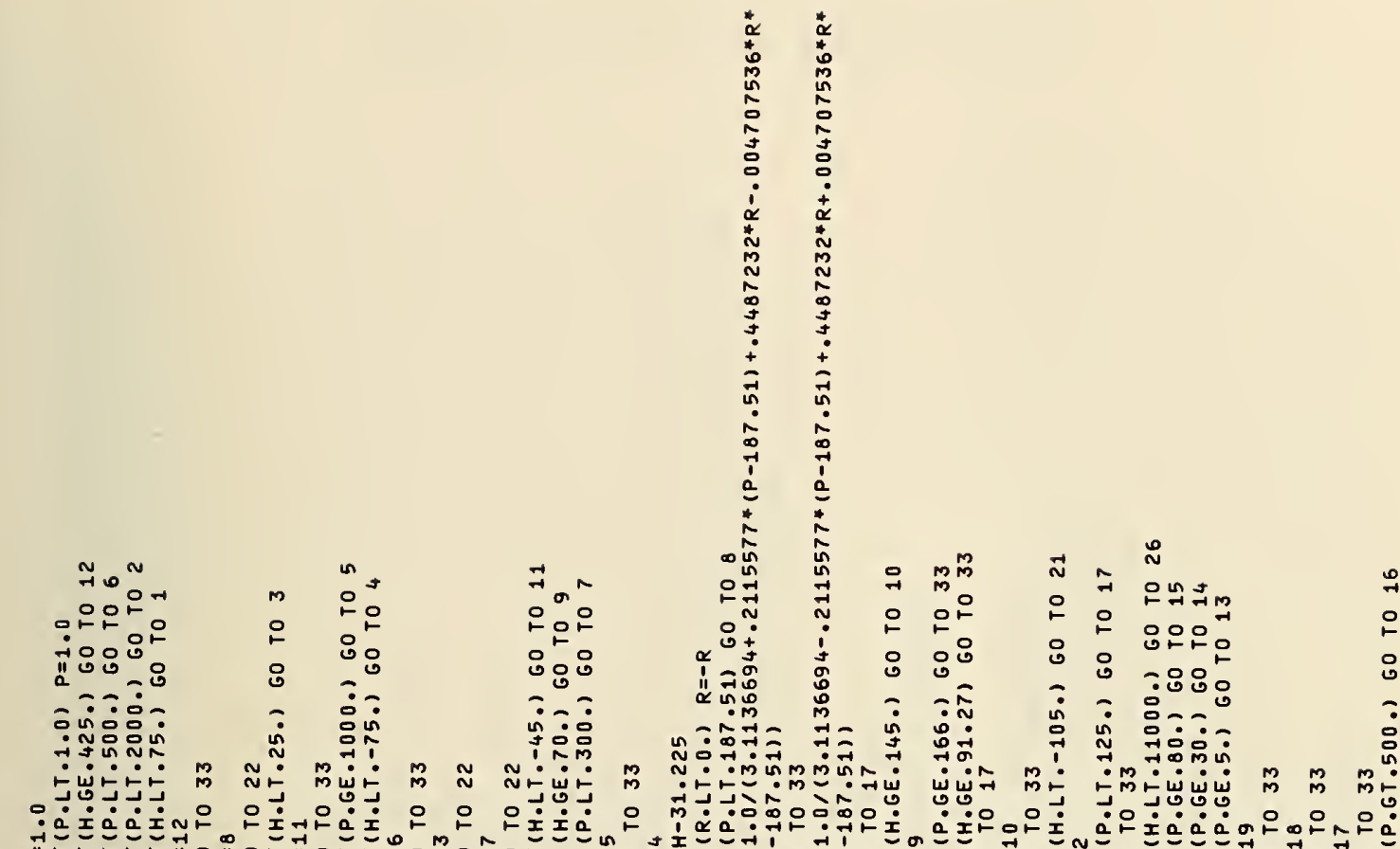

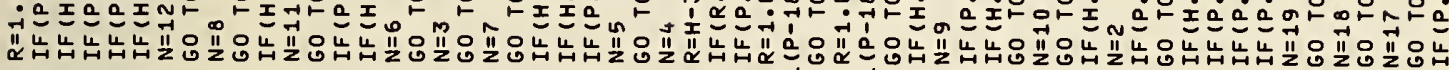

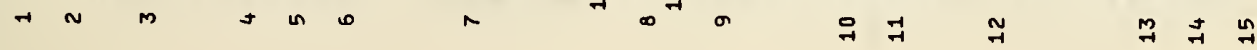

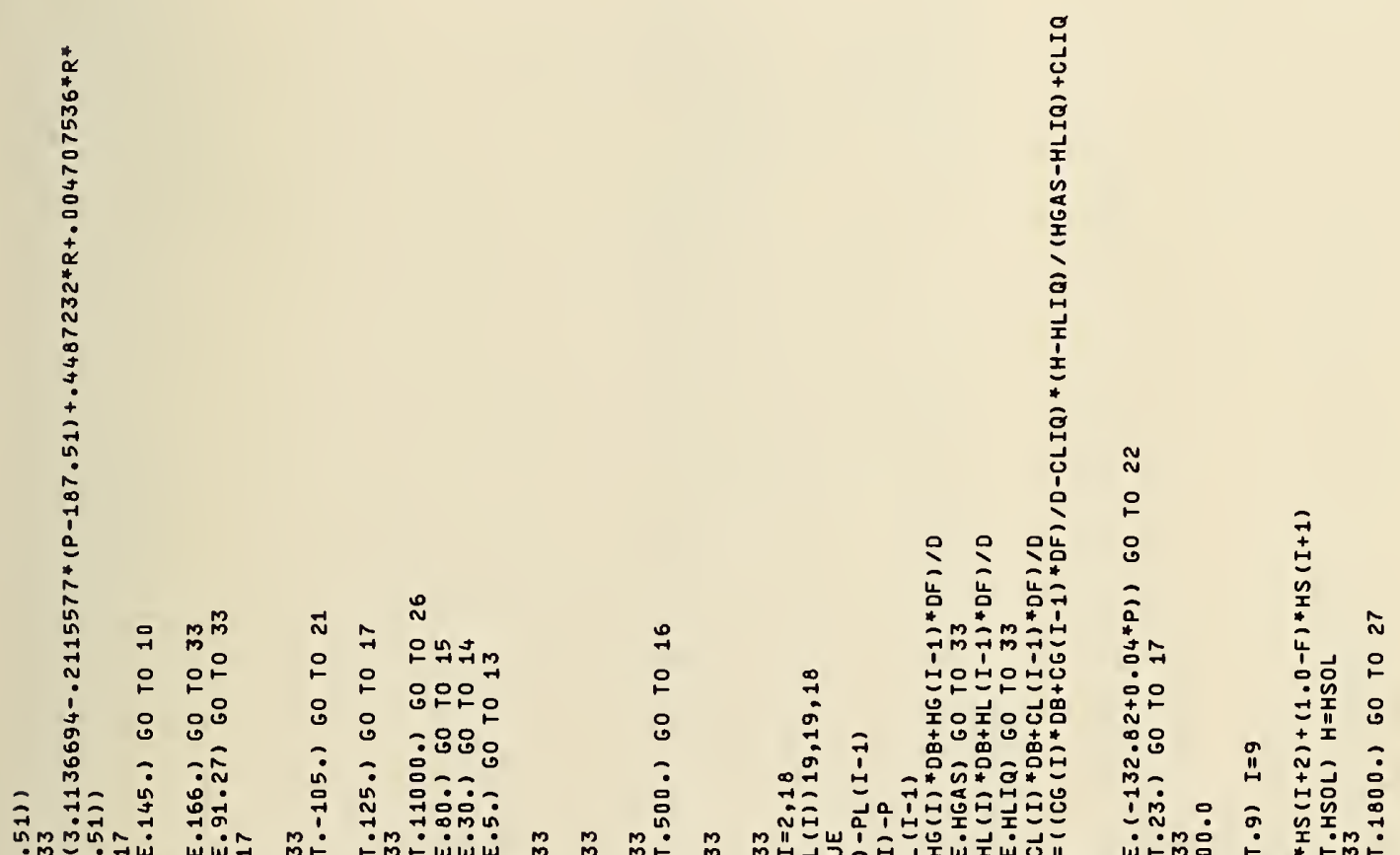
जिm

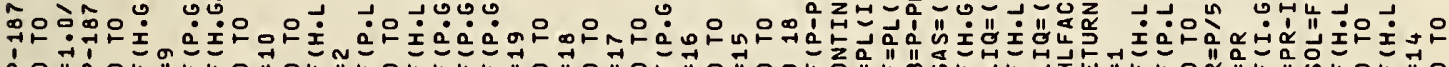

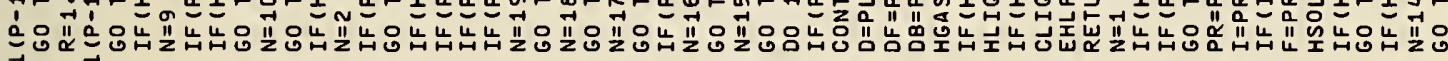
$\infty$ o
ㅇ $\Rightarrow \quad \cong$

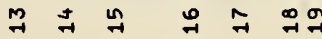
$\vec{N}$
N
ㅇ 


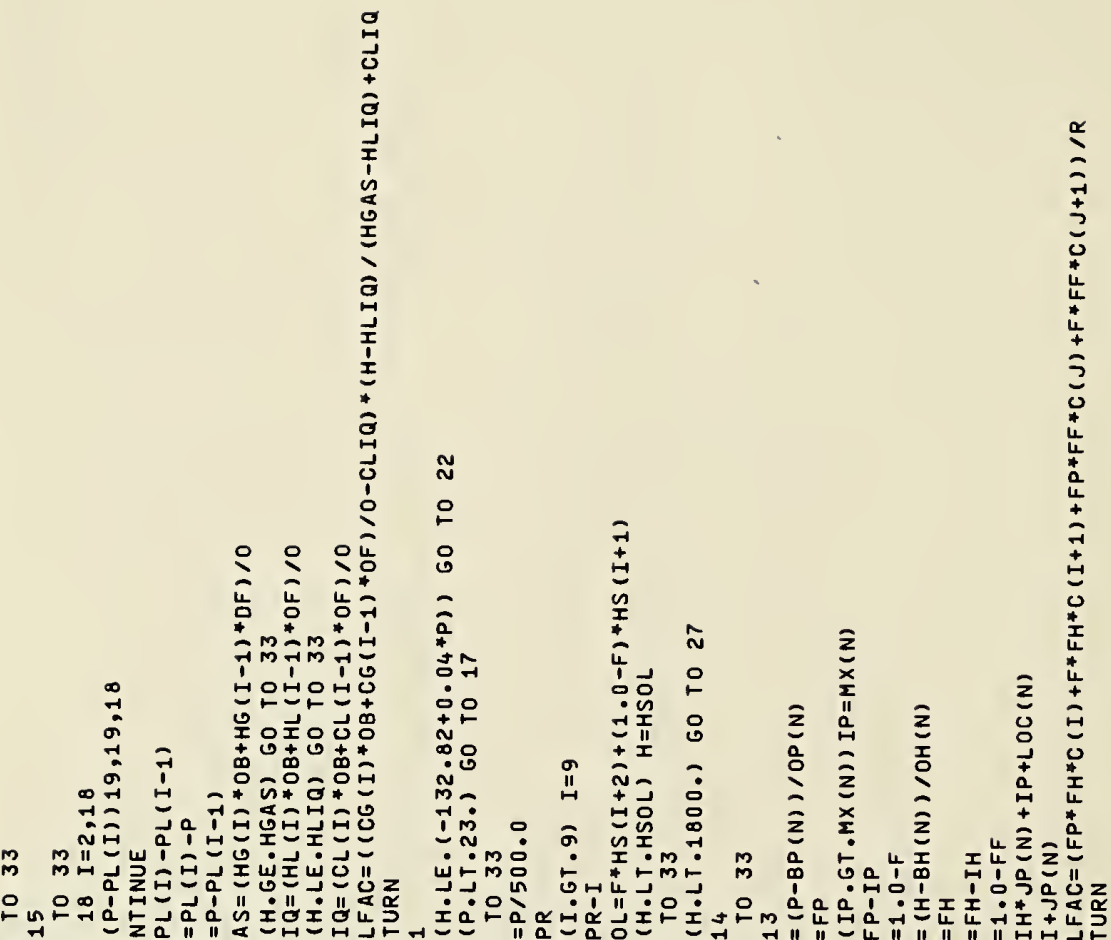

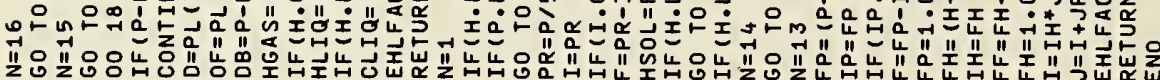

ค స

N

N

N N

ตำกำ

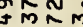

नै:

งั่ำ

न

$\sin 0$

S

它

行

S.

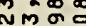

M.․า

윰ำ

जिलन

两

m.․ำ

ก⿻上丨

लित

งกำ

곡ำ

䠉?



N

11

लिख

:

이용

㘳年的

ज的

जิ둥

0ำ

更行

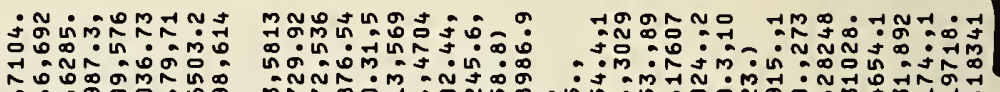

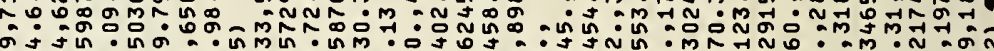

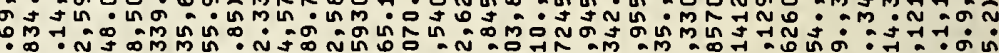

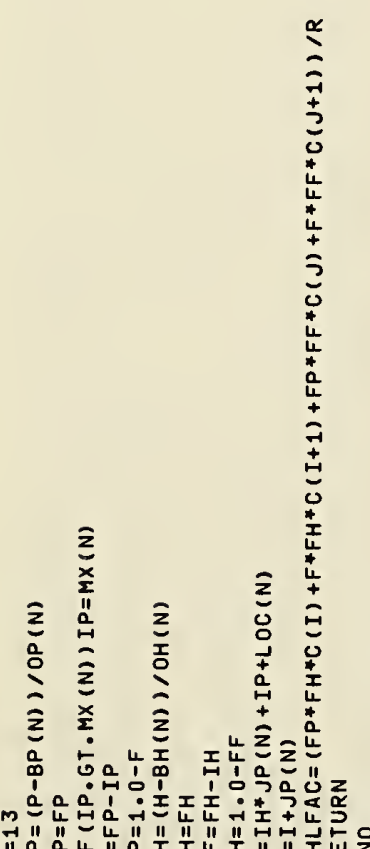

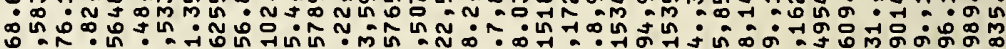

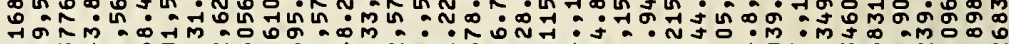

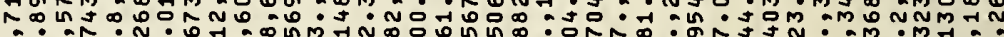

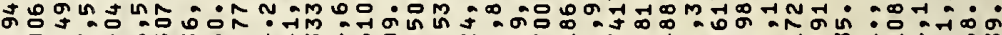

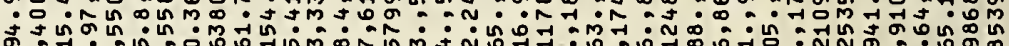

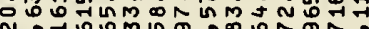

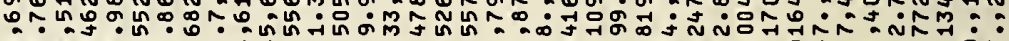
ON - o . In \$ㅅำ

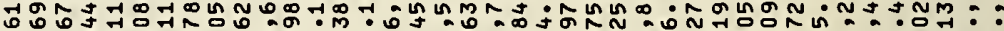
于

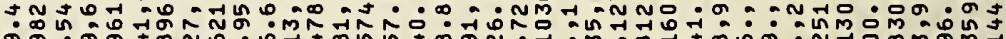
ơ

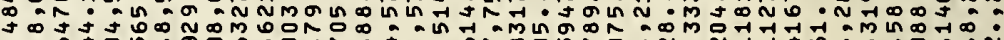

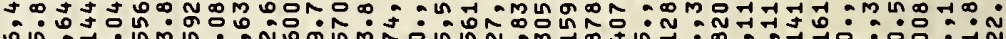
ดั : un

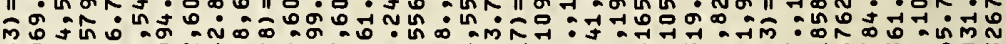
物

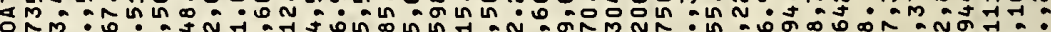

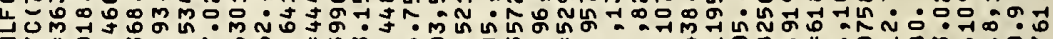

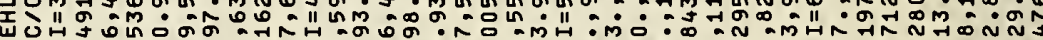

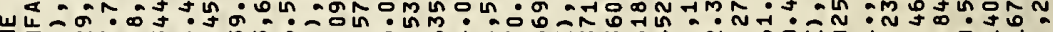

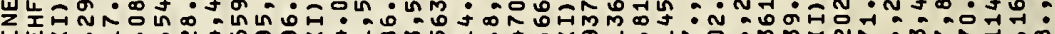

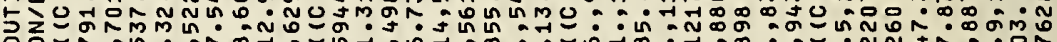
Q

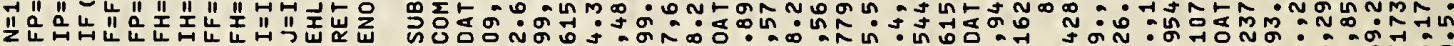
Nิm 


\section{APPENDIX C. PLOTS OF MAXIMUM INTERPOLATION \\ ERR OR AND ESTIMATED SOURCE ERROR}

Page No.

Parahydrogen

$\begin{array}{ll}\text { PTENTH } & 178 \\ \text { PTDENS } & 179 \\ \text { PTENTR } & 180 \\ \text { PTCOND } & 181 \\ \text { PTVISC } & 182 \\ \text { PTSOUN } & 183 \\ \text { PTCP } & 184 \\ \text { PTCV } & 185 \\ \text { PTGAMM } & 186 \\ \text { PTLFAC } & 187 \\ \text { PHTEMP } & 188 \\ \text { PHDENS } & 189 \\ \text { PHENTR } & 190 \\ \text { PHCOND } & 191 \\ \text { PHVISC } & 192 \\ \text { PHSOUN } & 193 \\ \text { PHCP } & 194 \\ \text { PHCV } & 195 \\ \text { PHGAMM } & 196 \\ \text { PHLFAC } & 197\end{array}$

Equilibrium-hydrogen

ET ENTH

198

ETDENS

199

ETENTR

200

ETCOND

201

ETVISC

202

ETSOUN

203

ETCP

204

ETCV

205

ETGAMM

206

ETLFAC

207

EHTEMP

208

EHDENS

209

EHENTR

210

EHCOND

211

EHVISC

212

EHSOUN

213

EHCP

214

$\mathrm{EHCV}$

215

EHGAMM

216

EHLFAC

217 

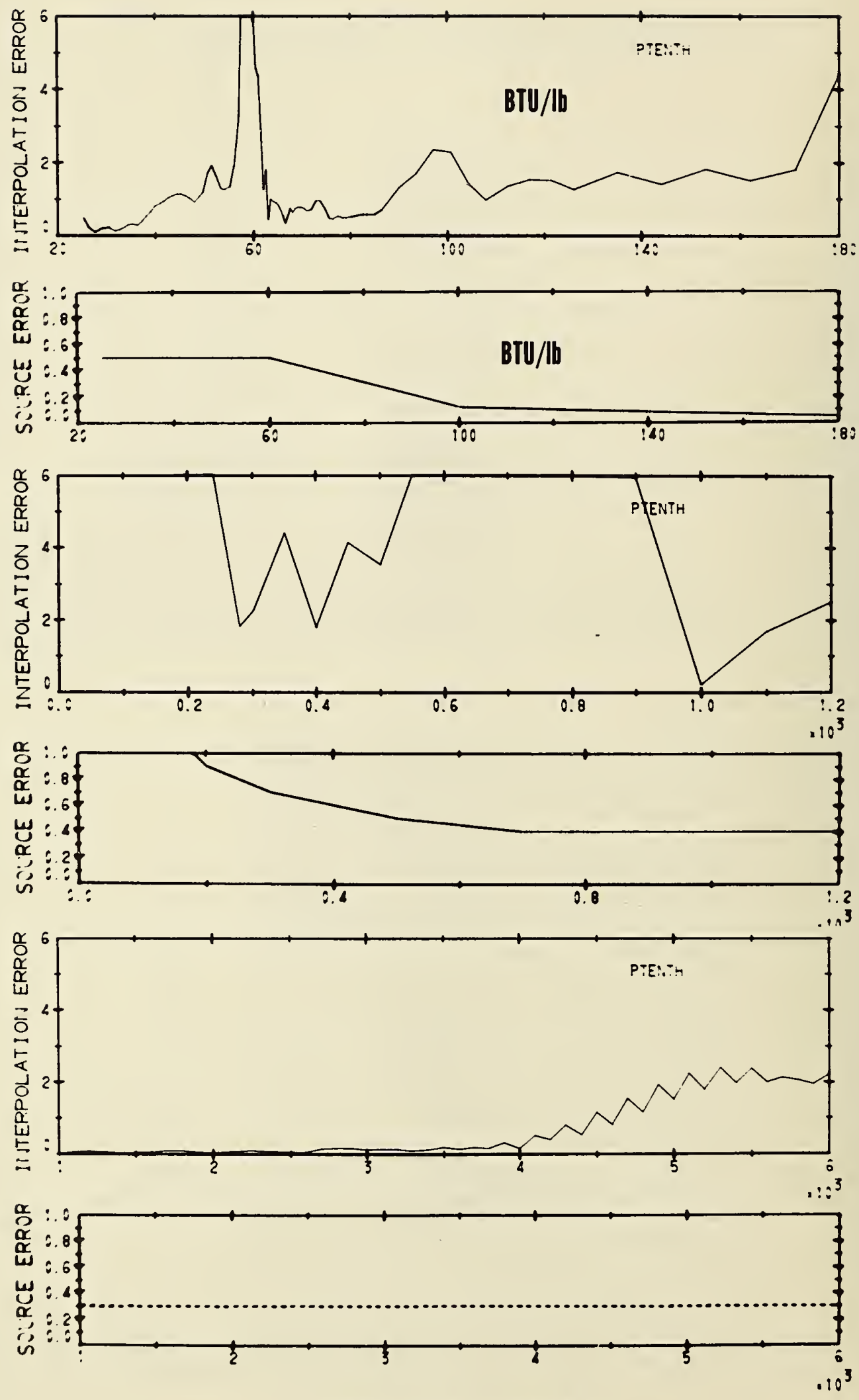

TEMPERATURE, RAIIKINE 

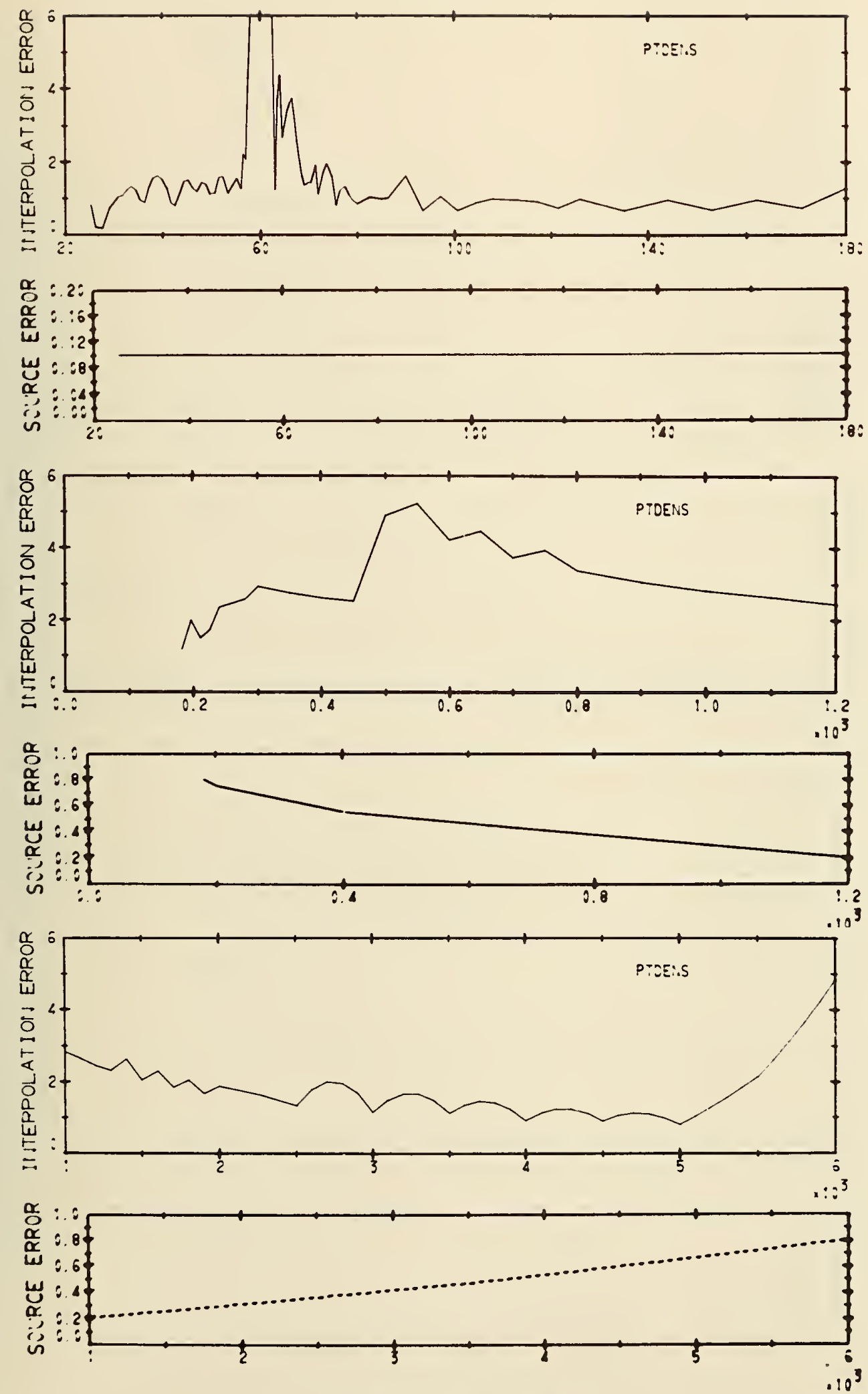

TEMPERATURE， RANKINE 

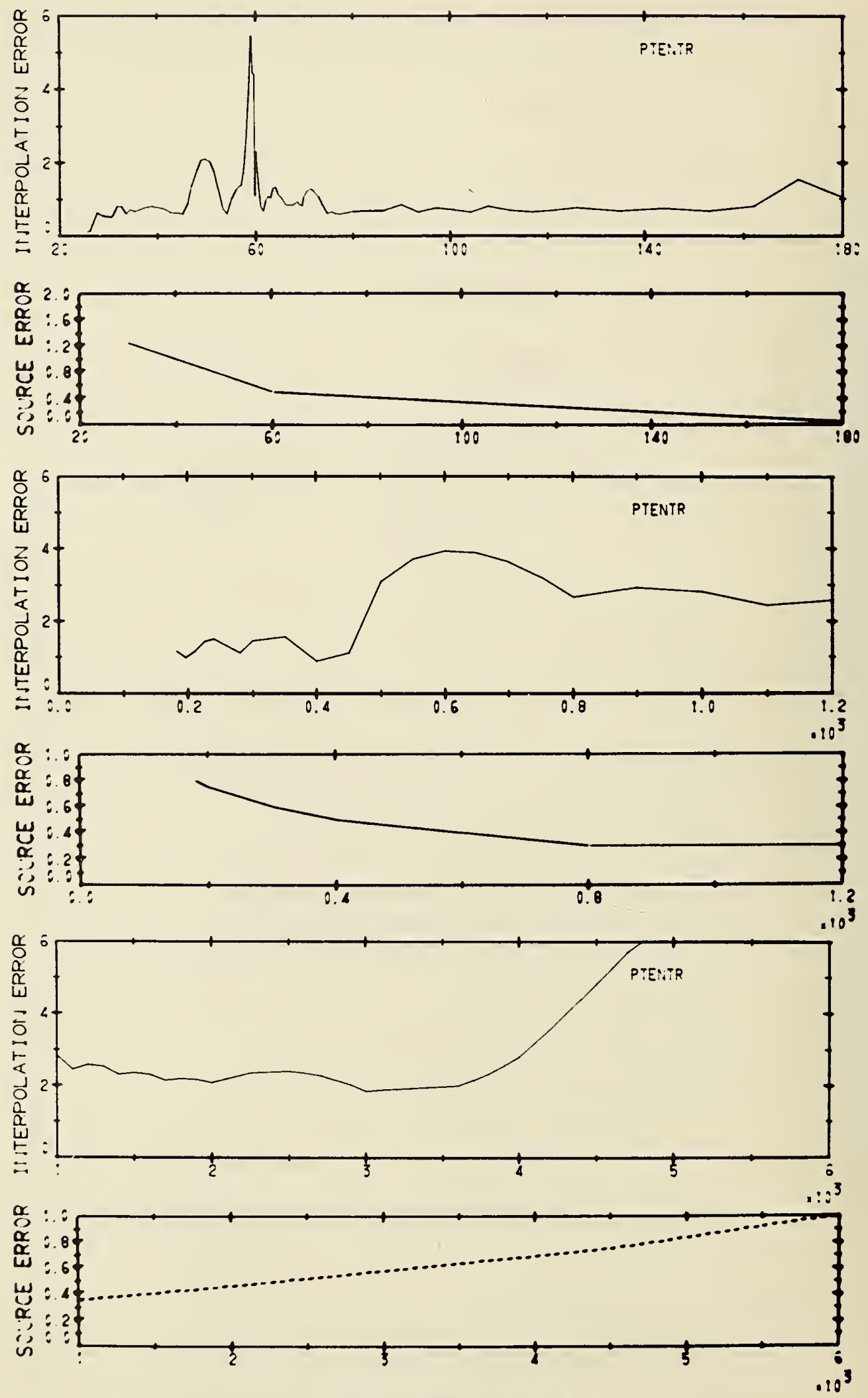

TEMPERATURE, RANIKINE 

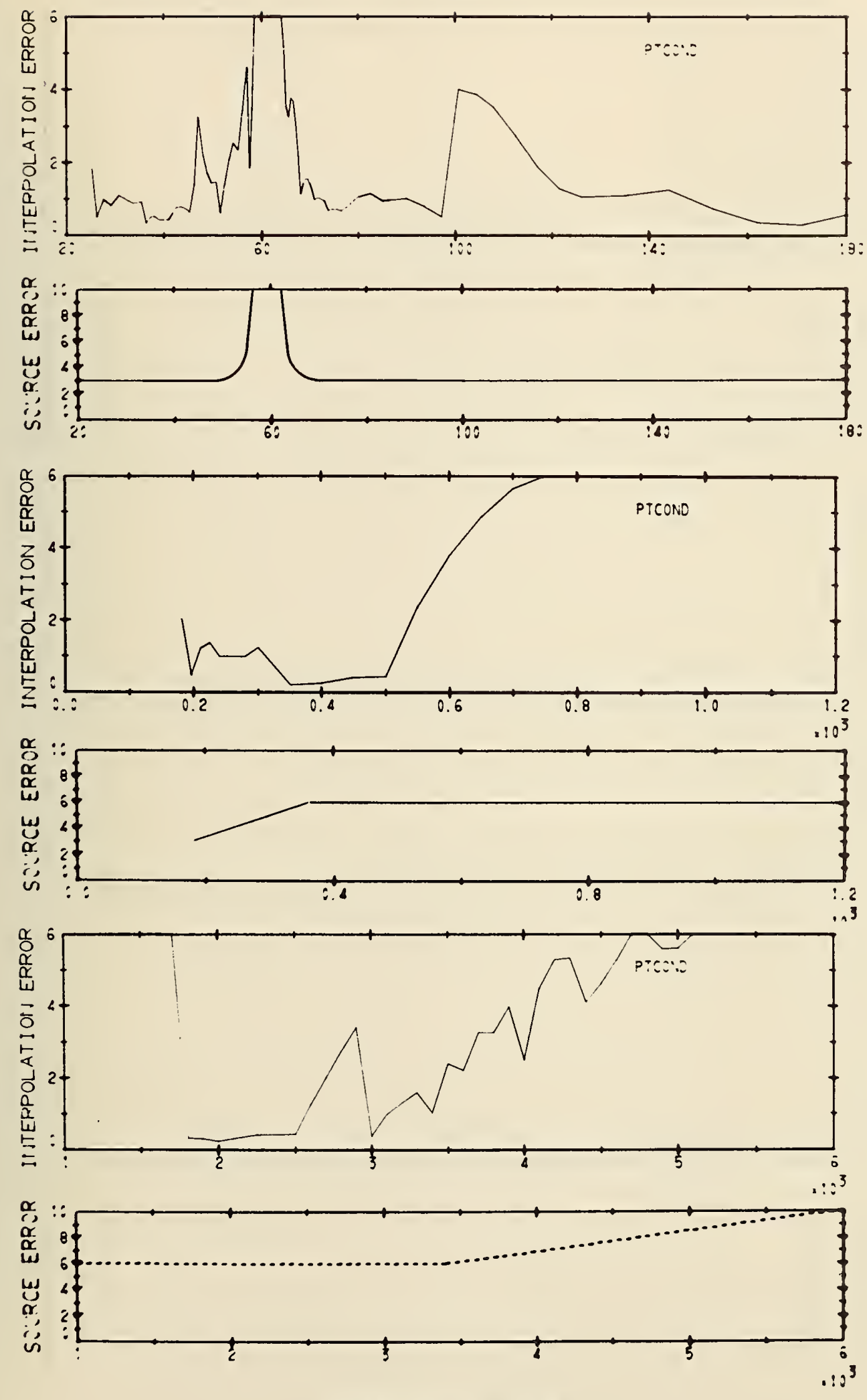

TEMPERATIURE, RAIIK!IIE 

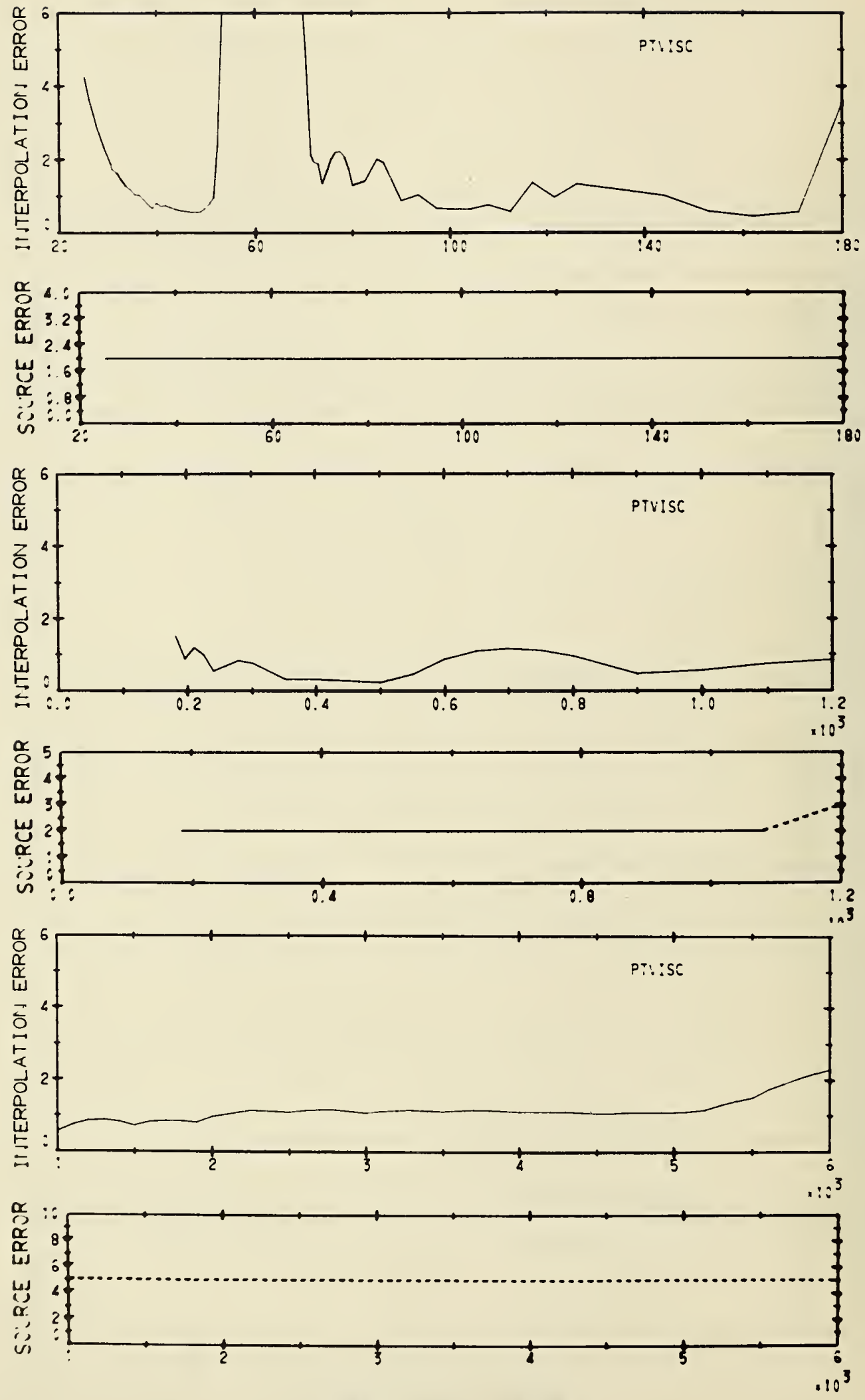

TEMPERATURE, RANKINE 

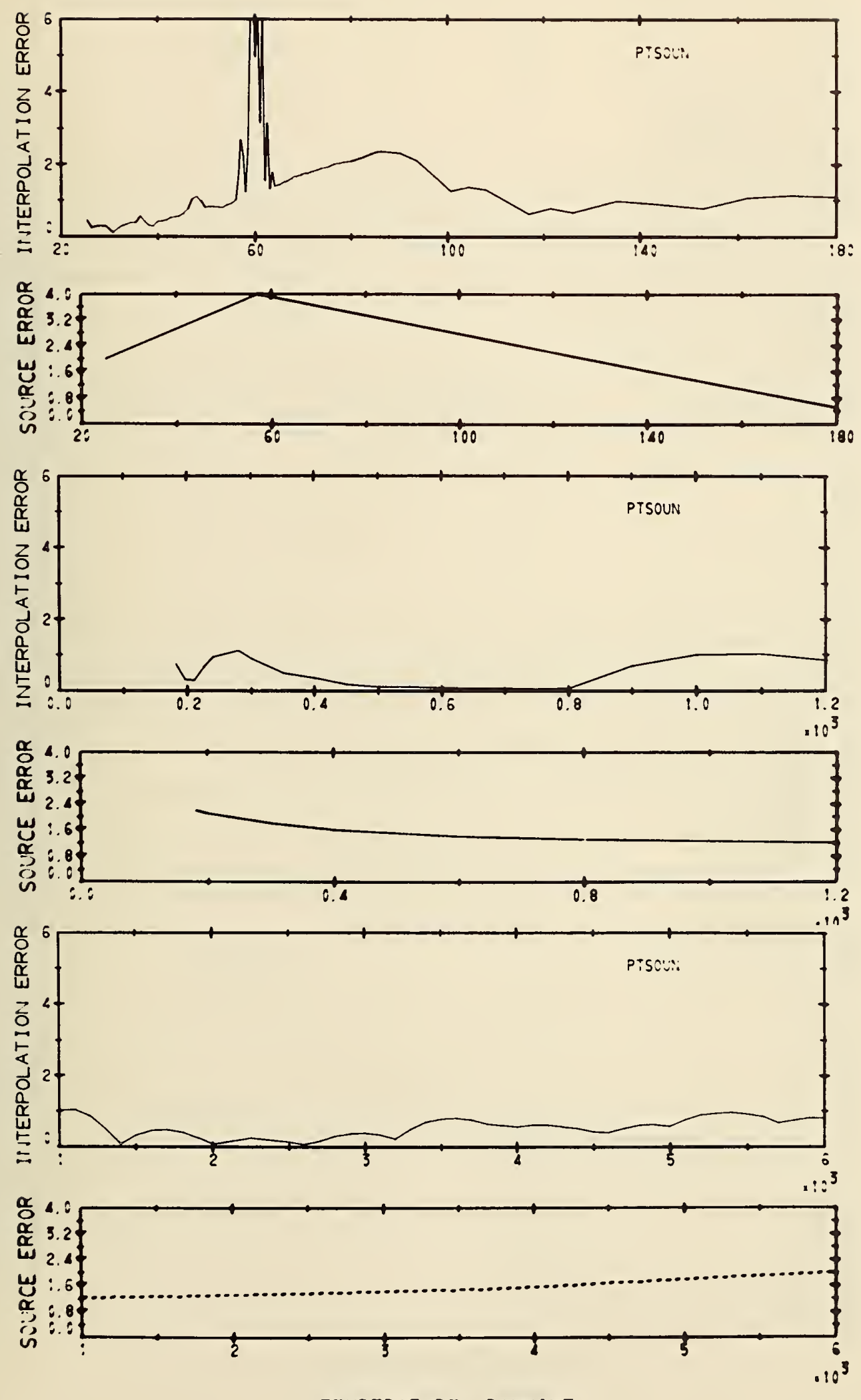

TEMPERATURE， RANIK INE 

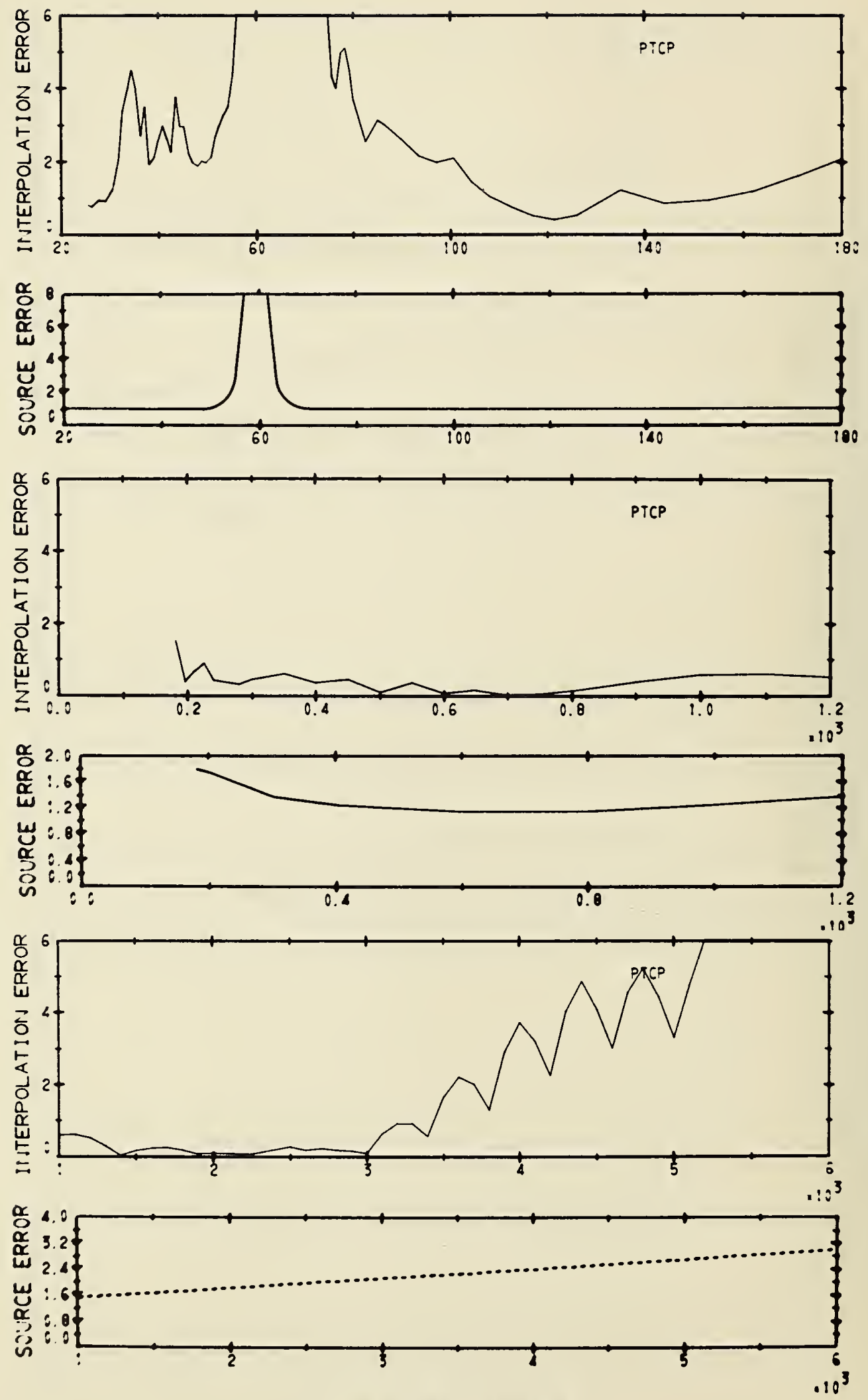

TEMPERATURE， RANKINE 

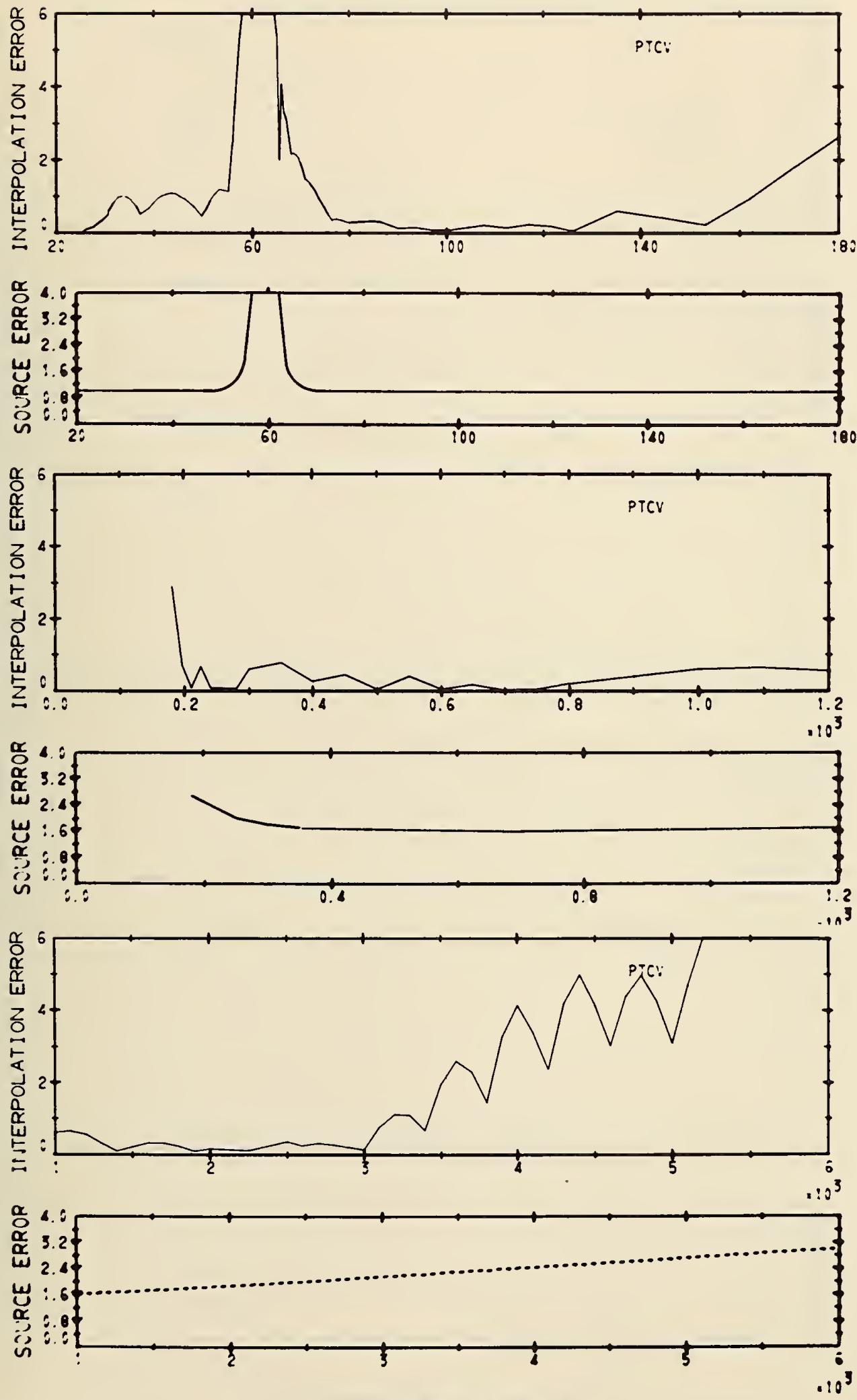

IEMPERATURE， RANK INE 

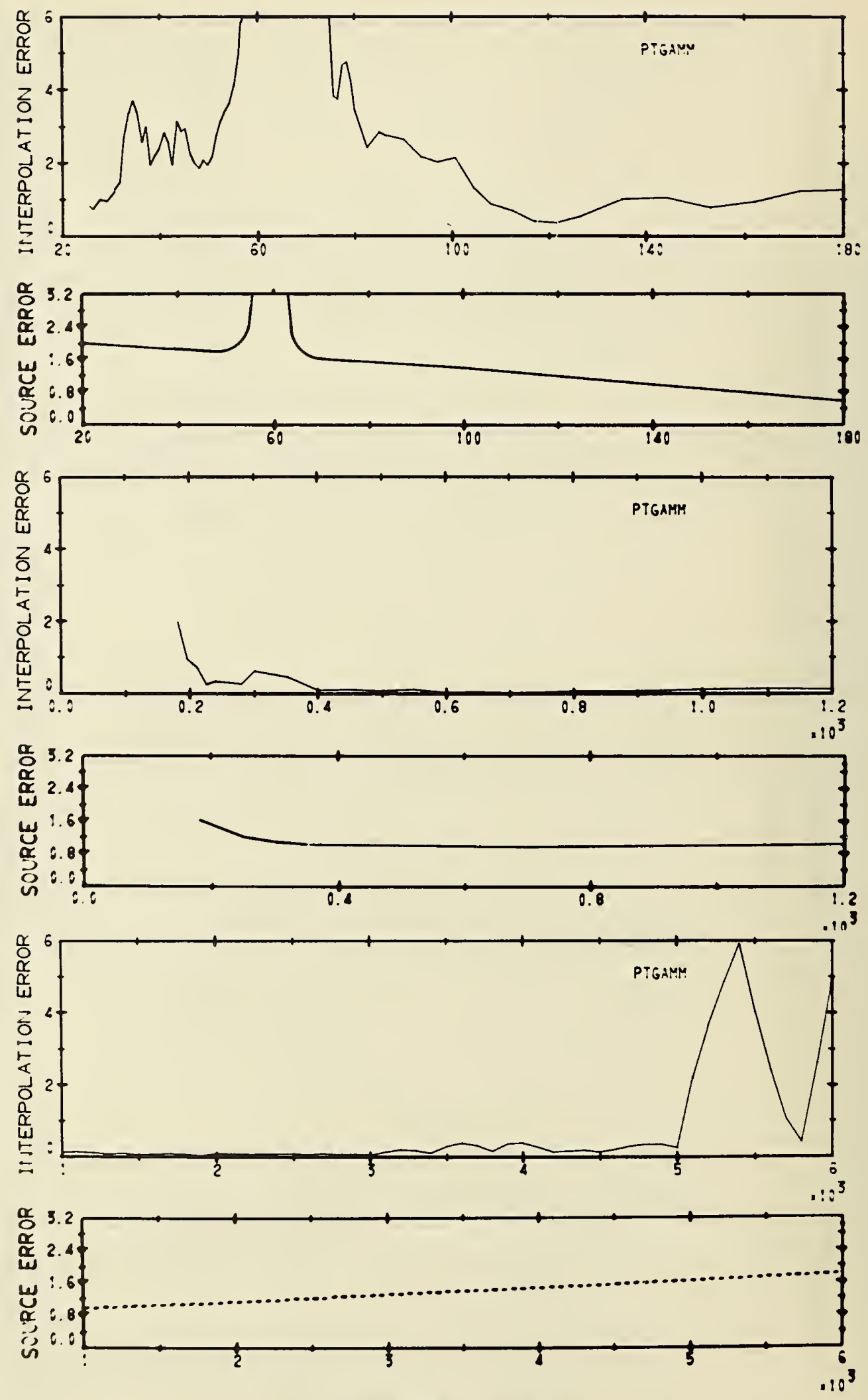

TEMPERATURE， RANKINE 

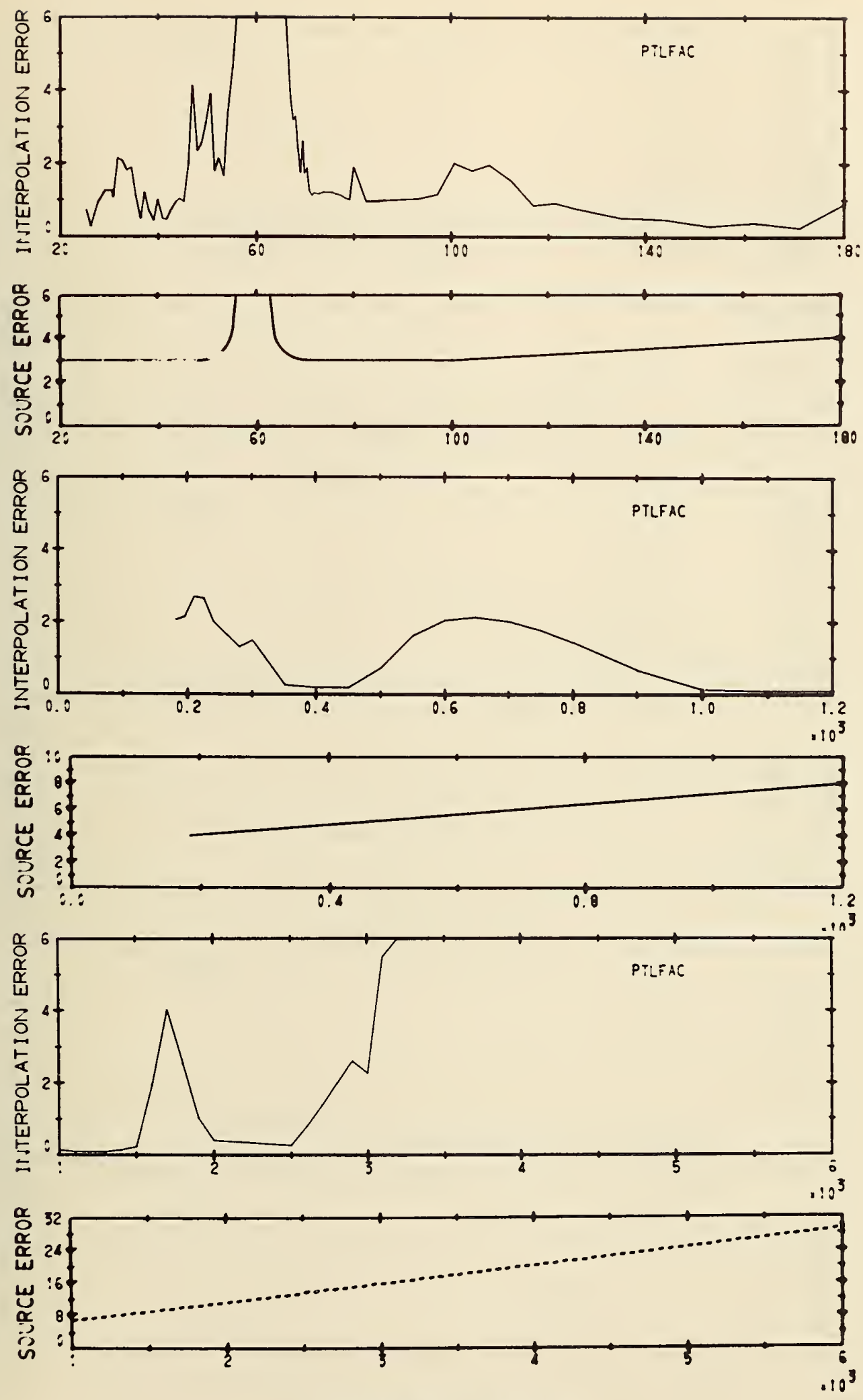

TEMPERATURE, RANKINE 

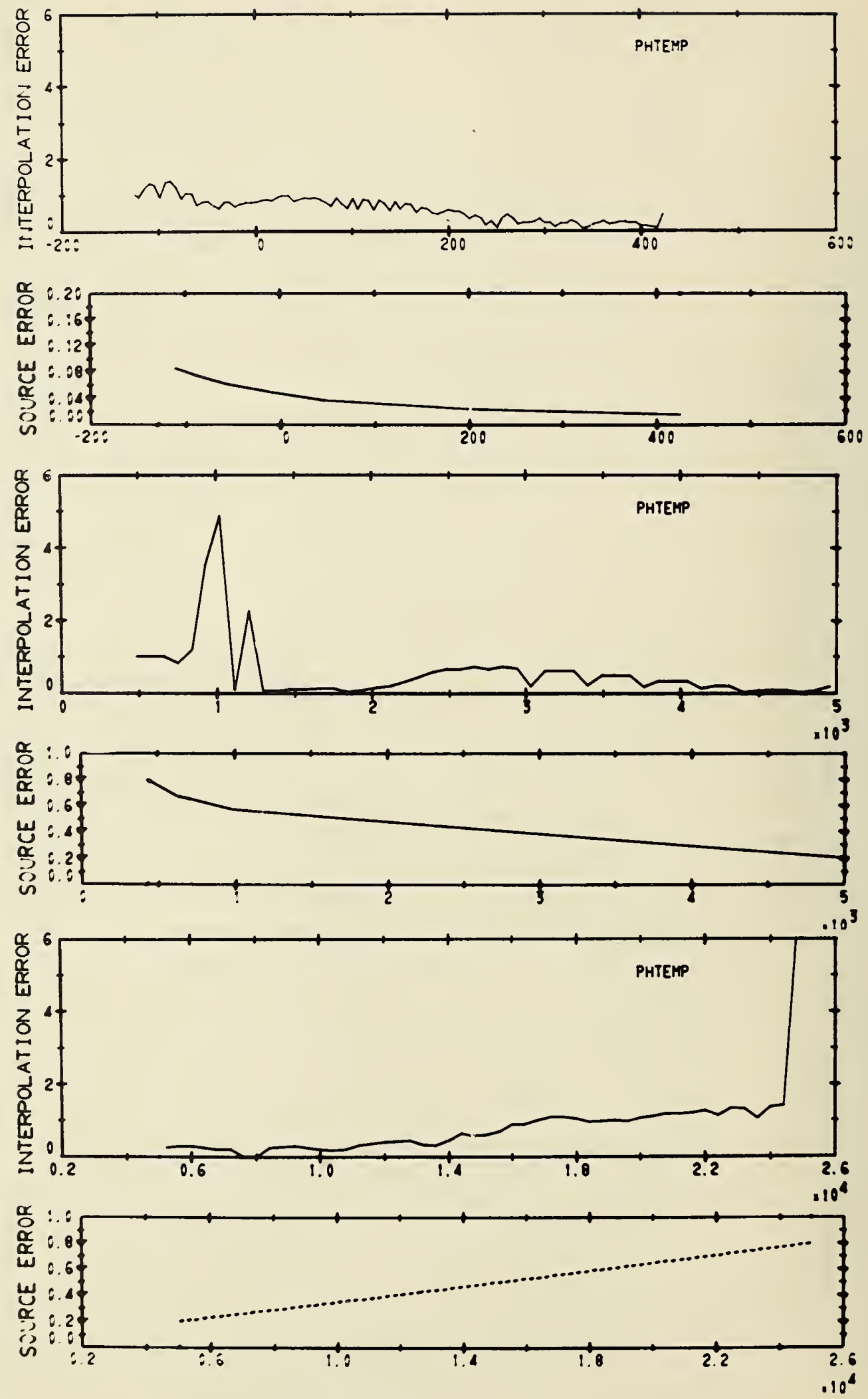

EIJTHALPY, BTU/LB. 

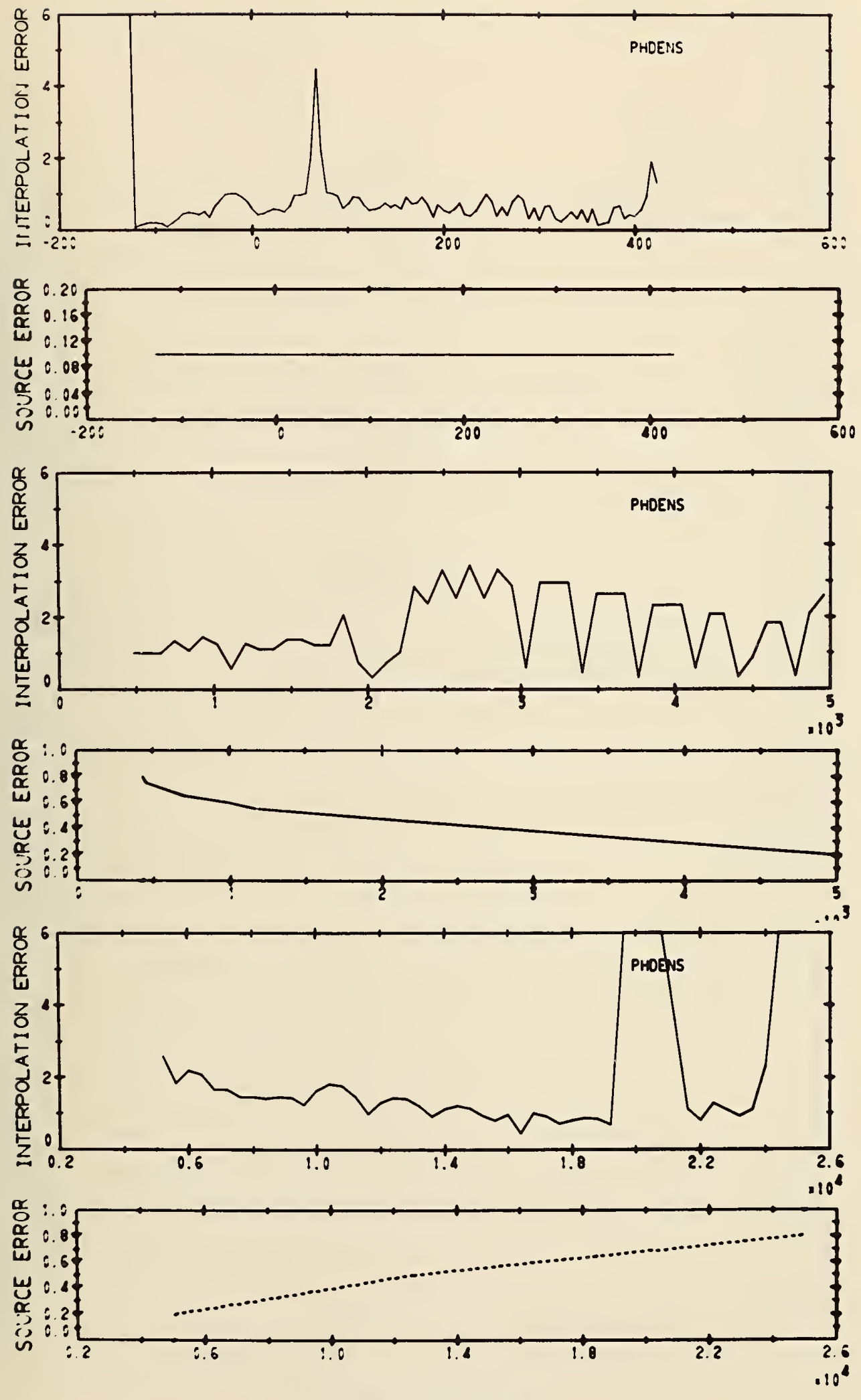

ENTHALPY, BTU/LB. 

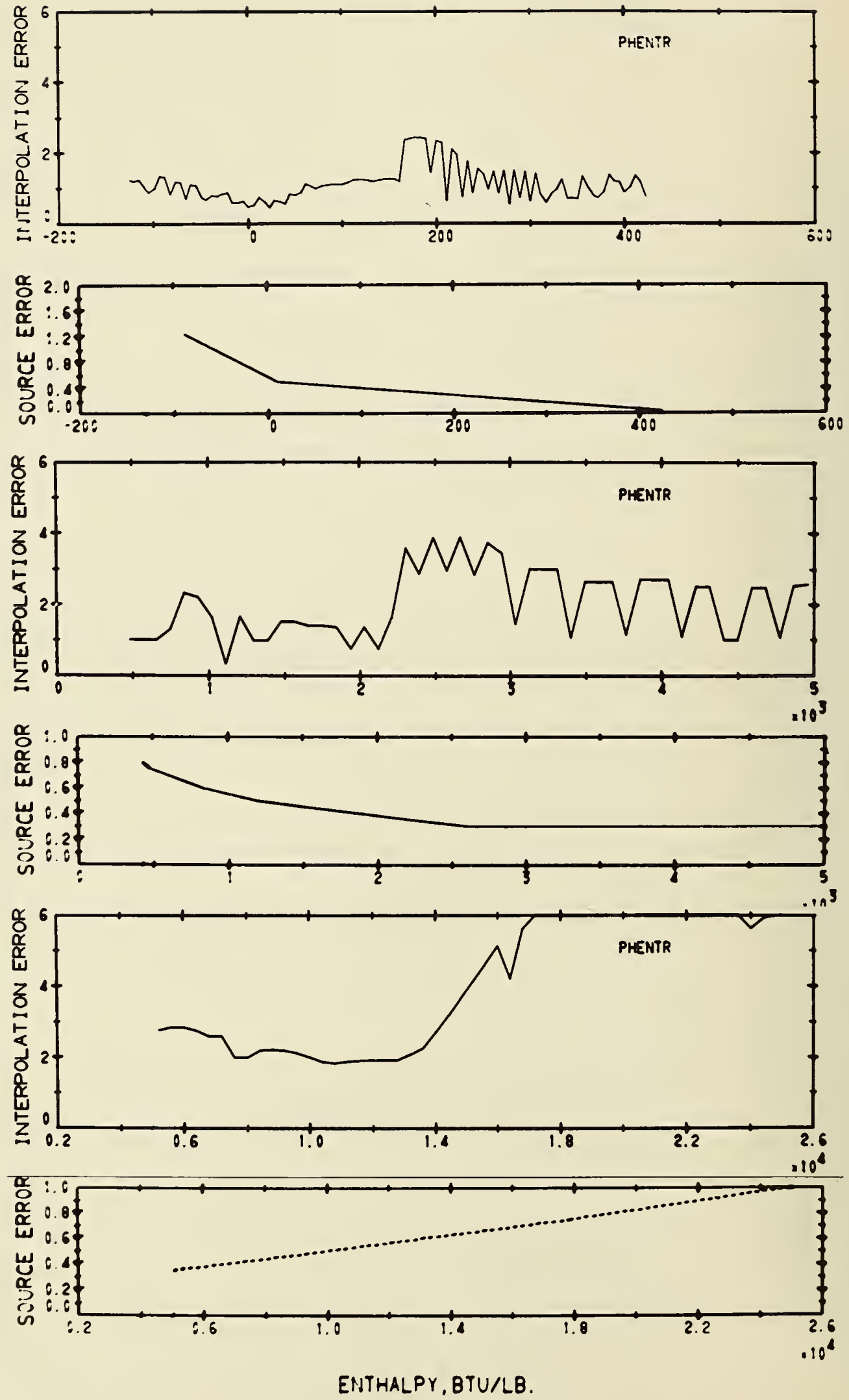

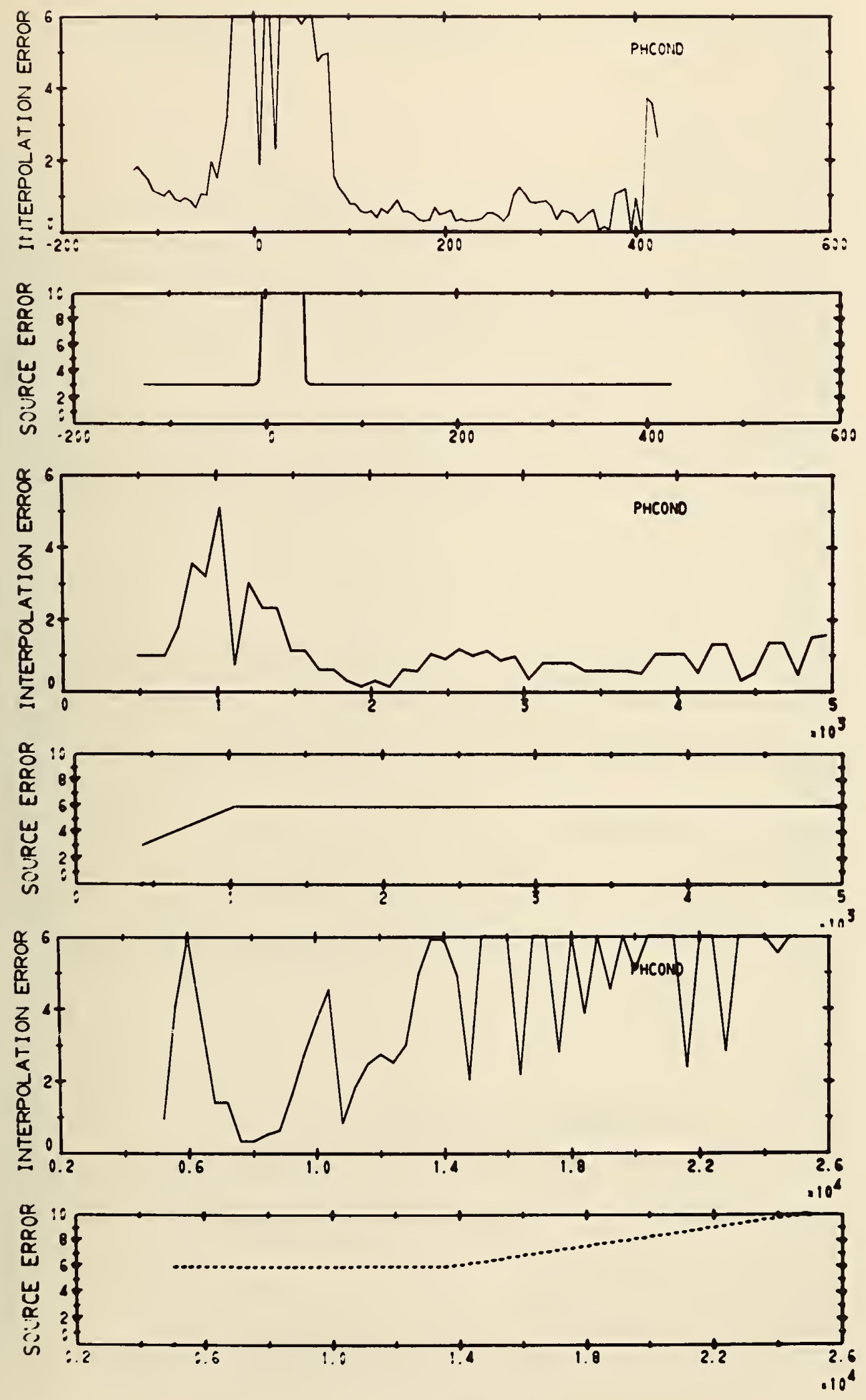

EITHALPY, BTU/LB. 

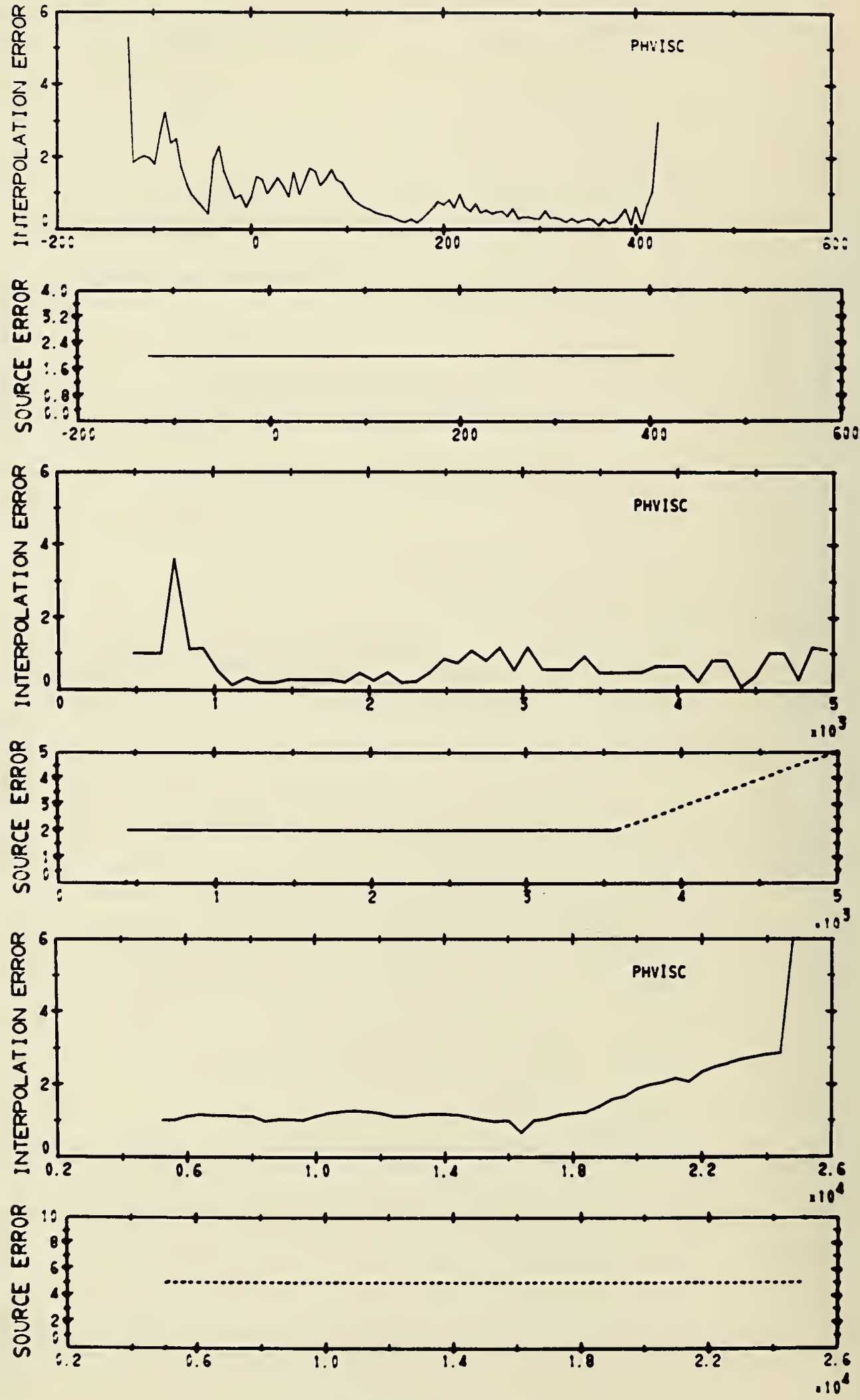

ENTHALPY, BTU/LB. 

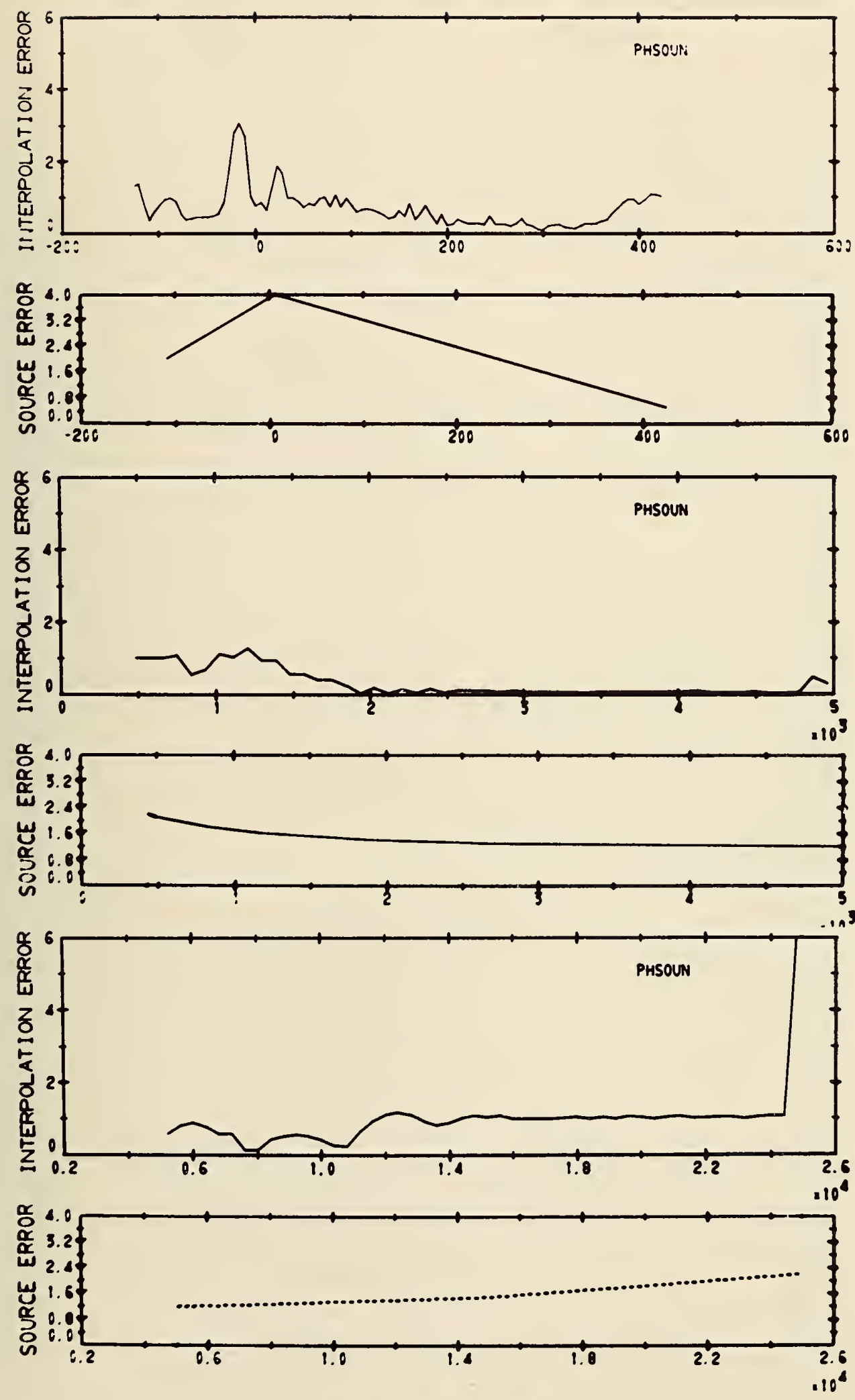

ENTHALPY, BTU/LB. 

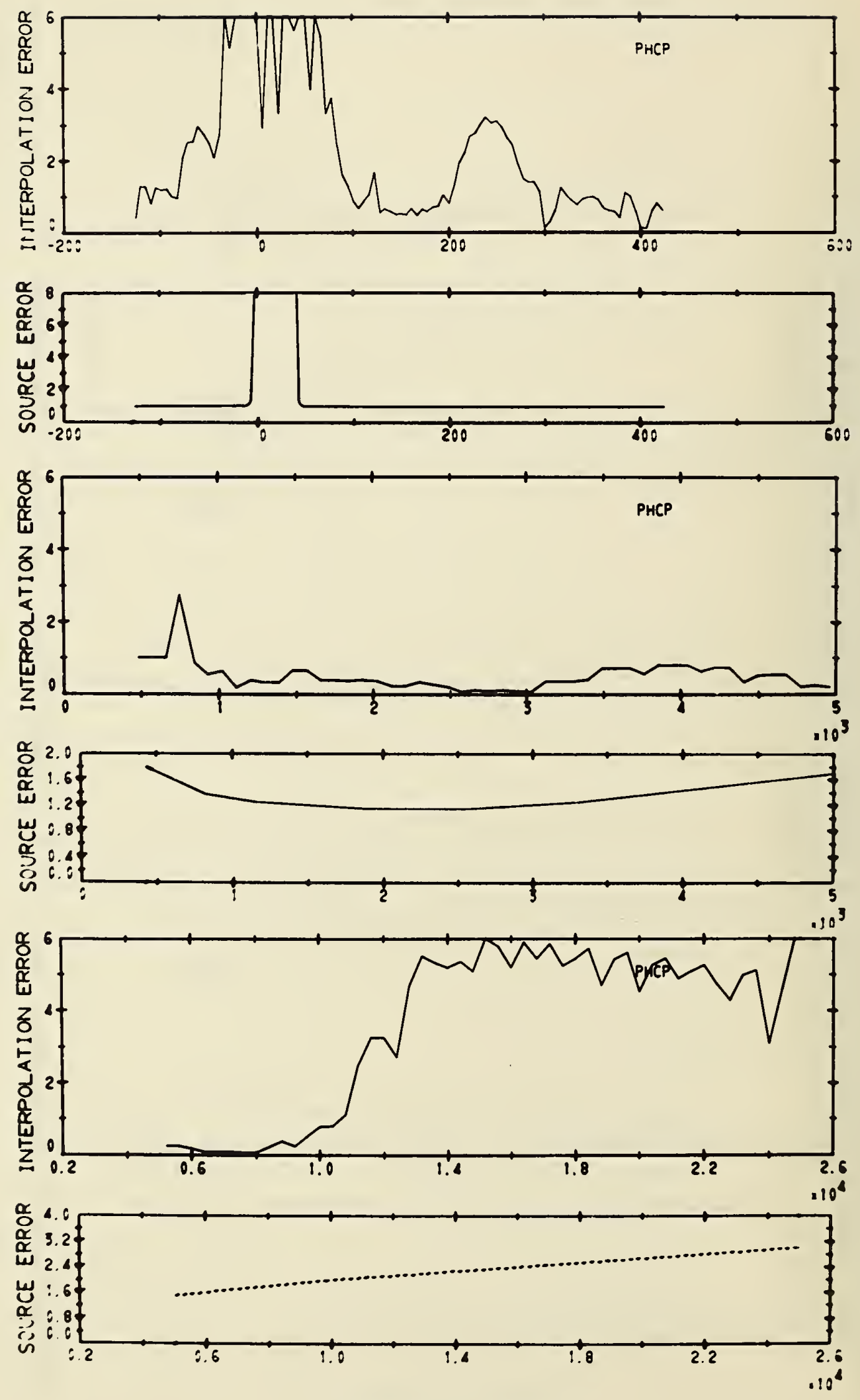

EIITHALPY, BTU/LB. 

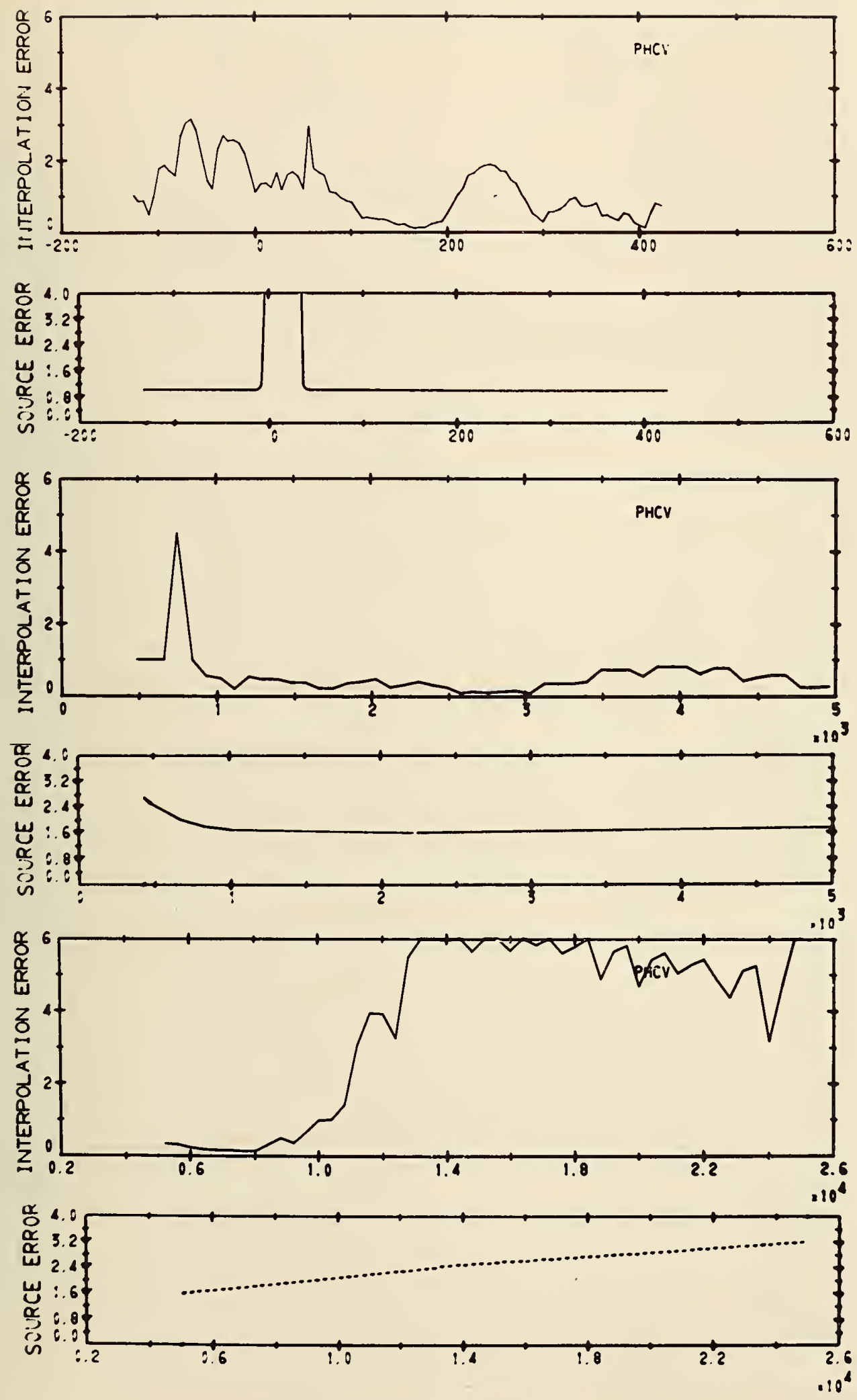

EIITHALPY, BTU/LB. 

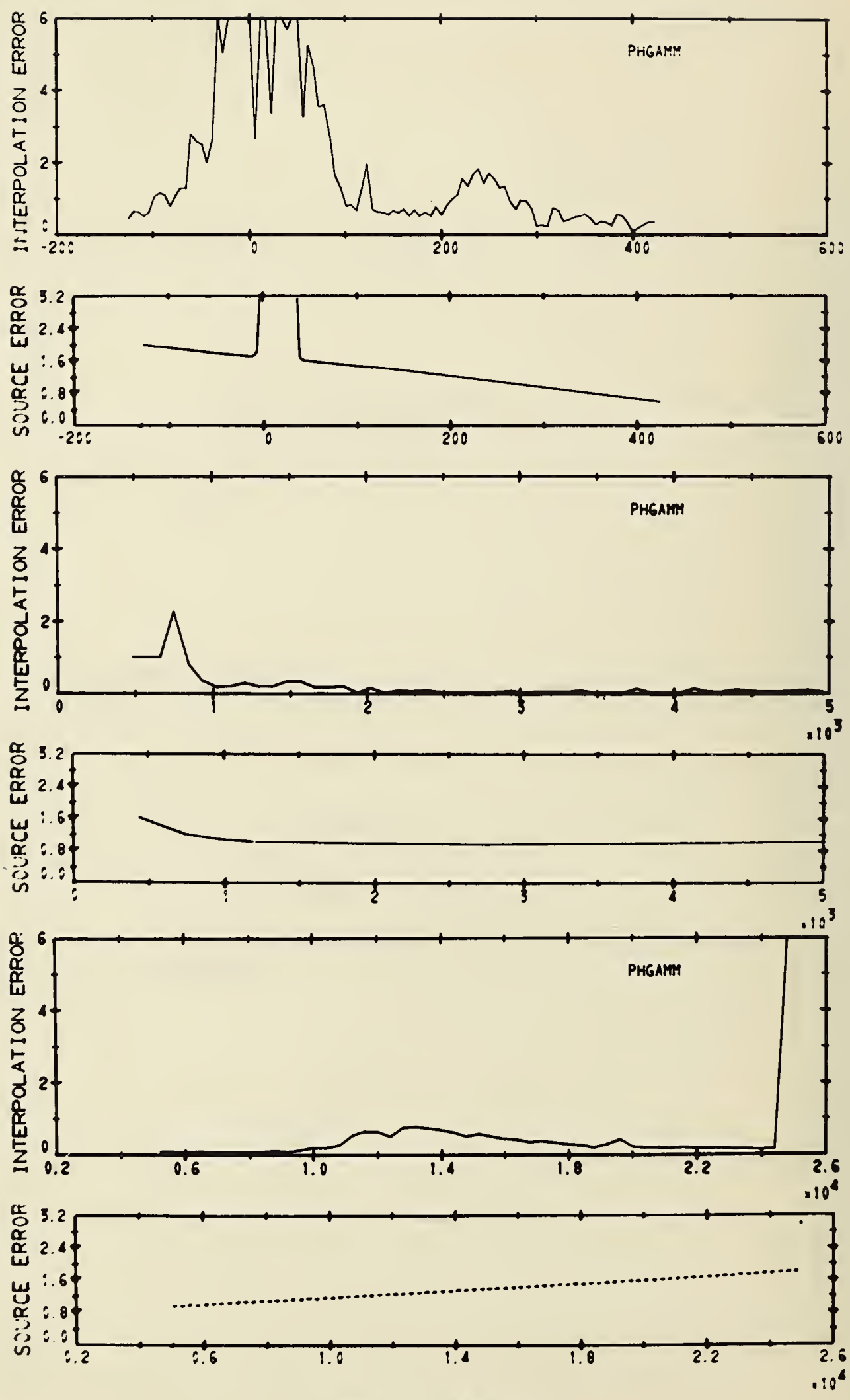

ENTHALPY, BTU/LB. 

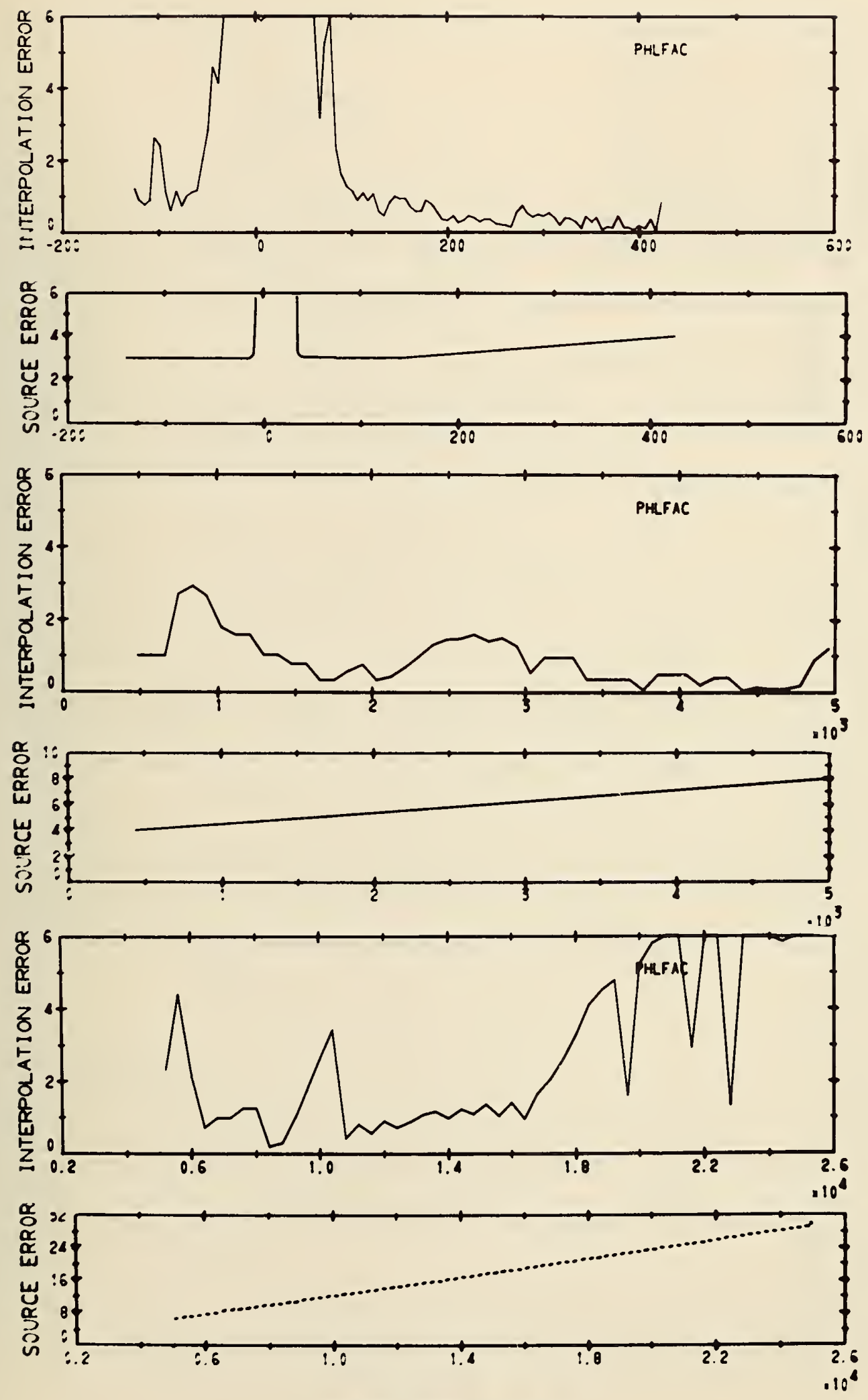

TEMPERATURE, RANIKINE 

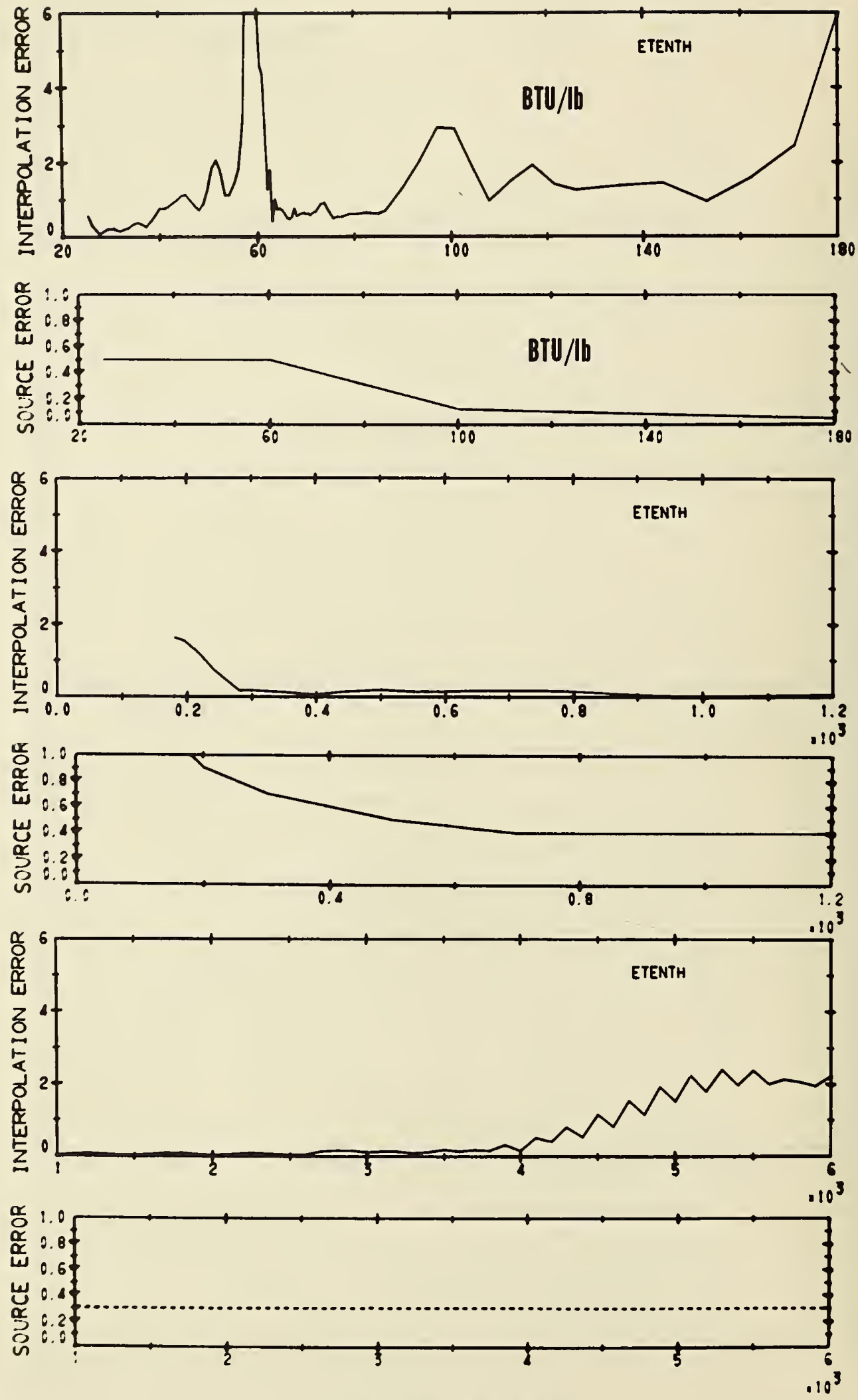

TEMPERATURE, RANKINE 
0
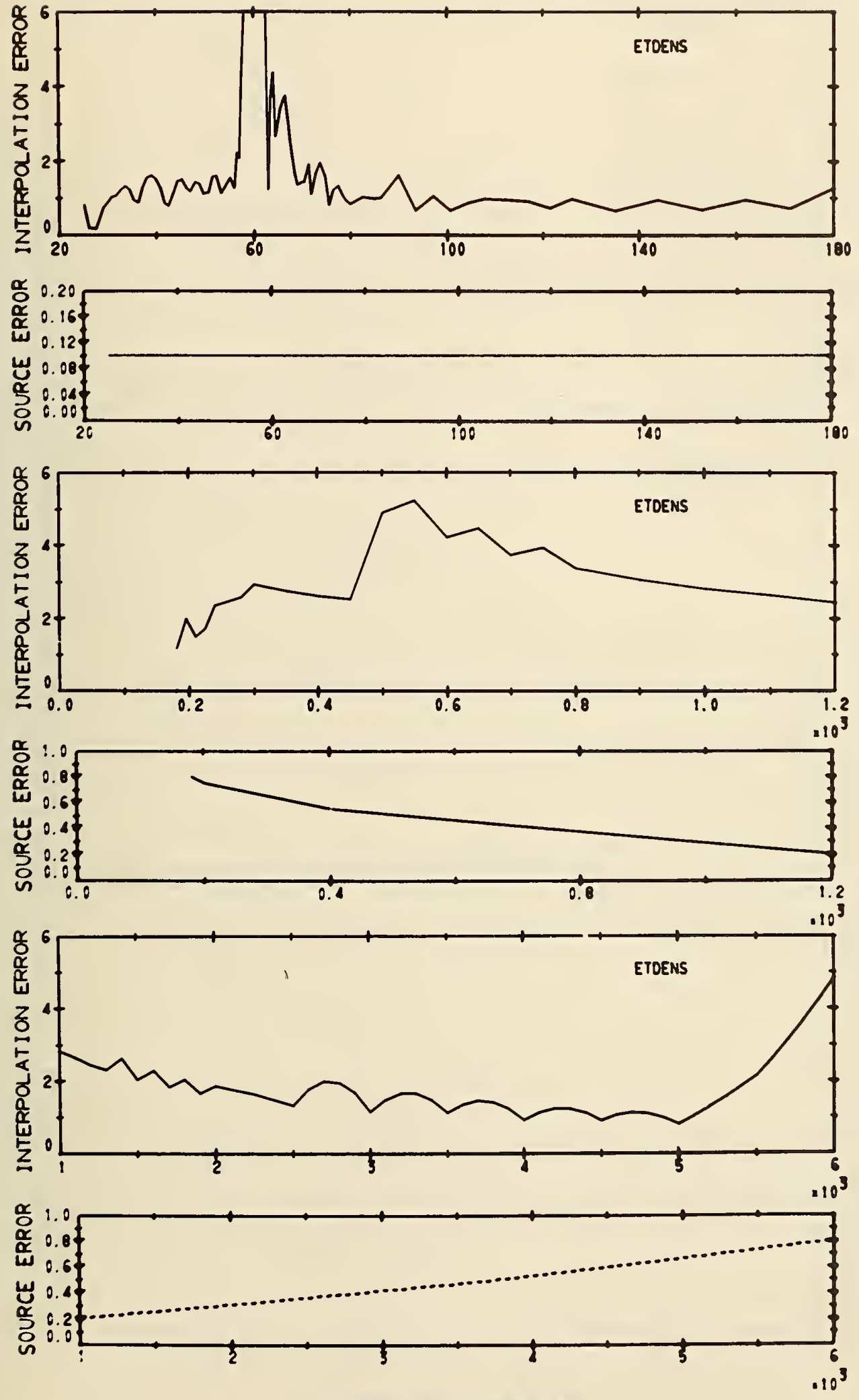

TEMPERATURE, RANKINE 

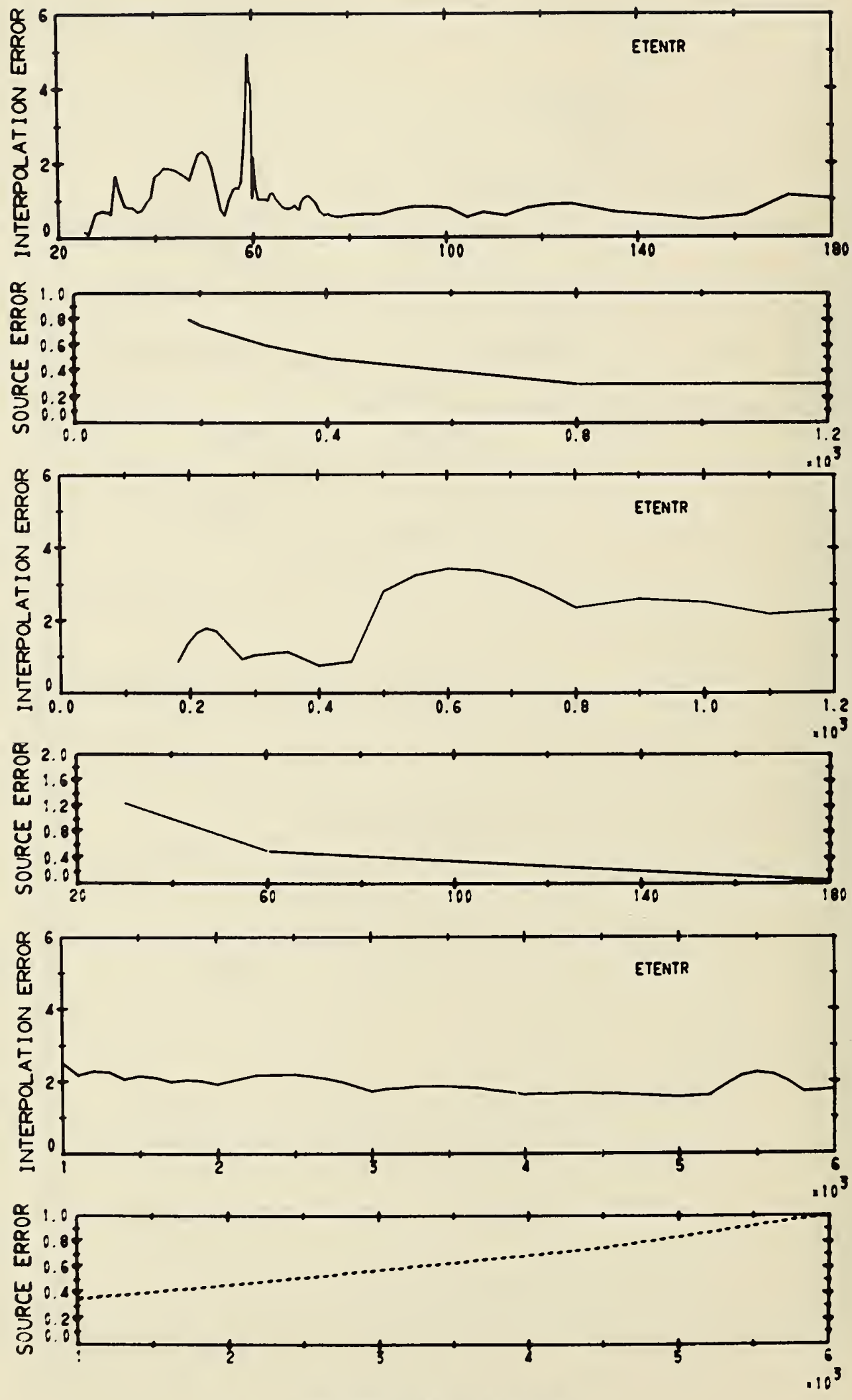

TEMPERATURE, RANKINE 

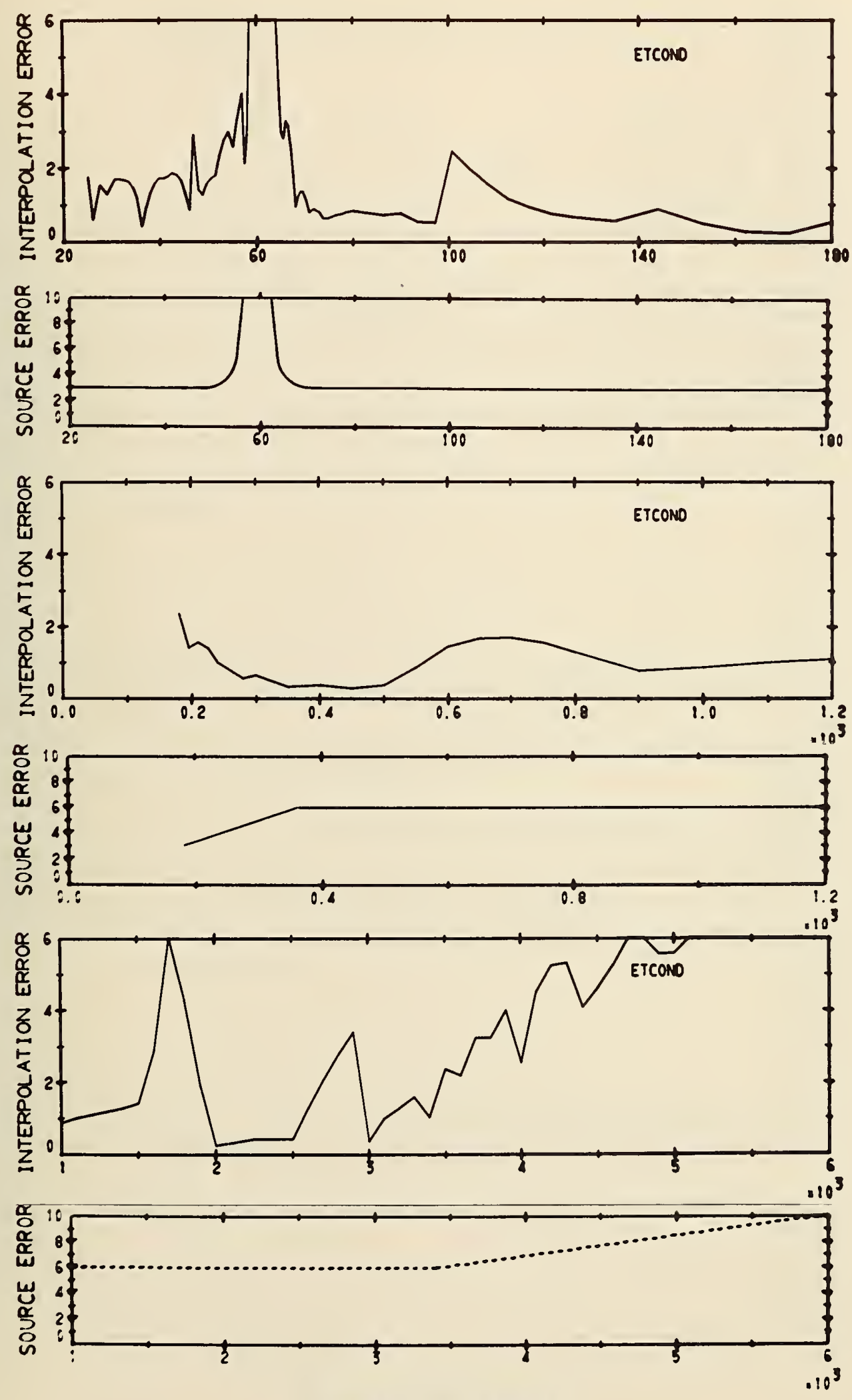

TEMPERATURE, RANKINE 

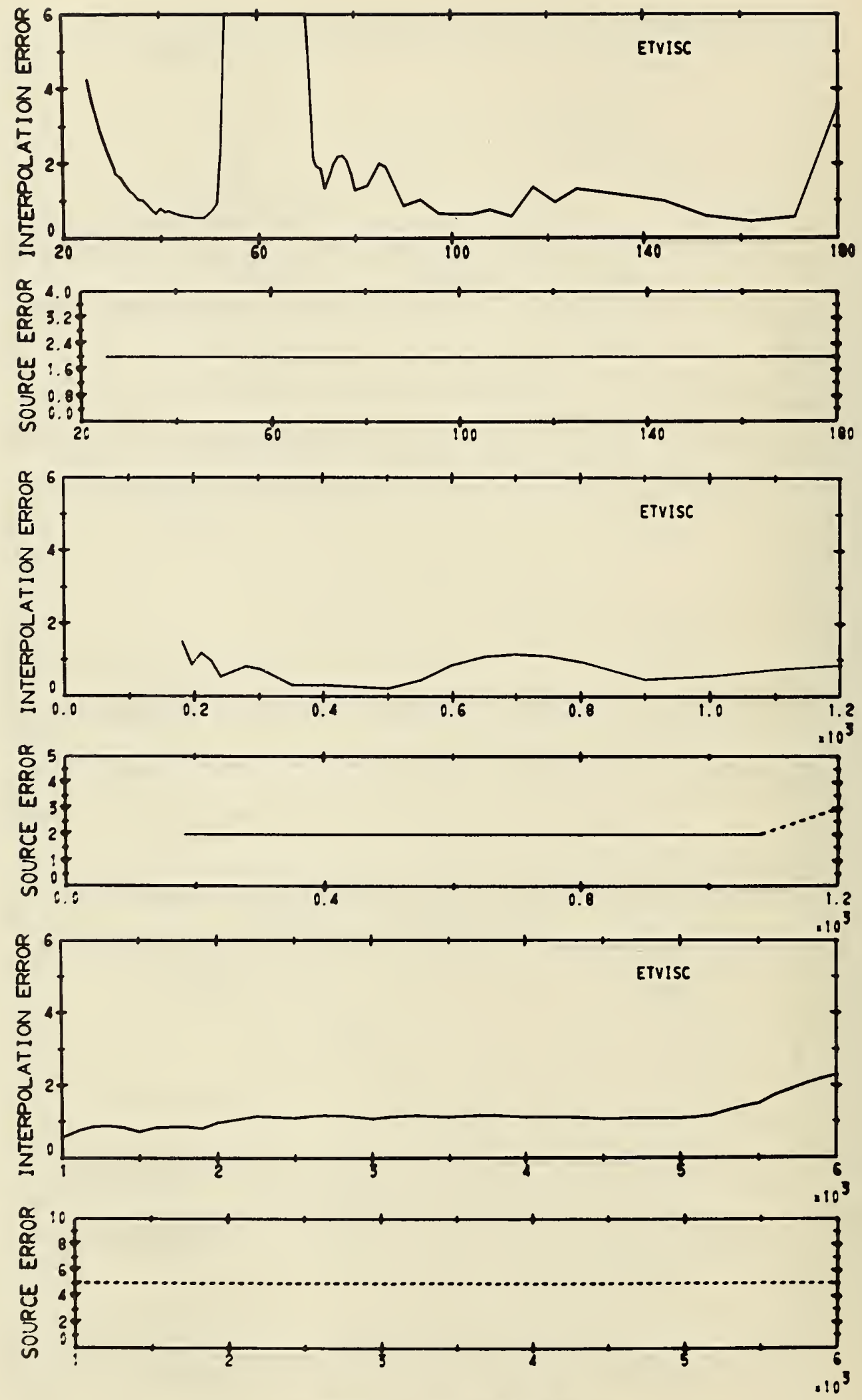

TEMPERATURE， RANKINE 

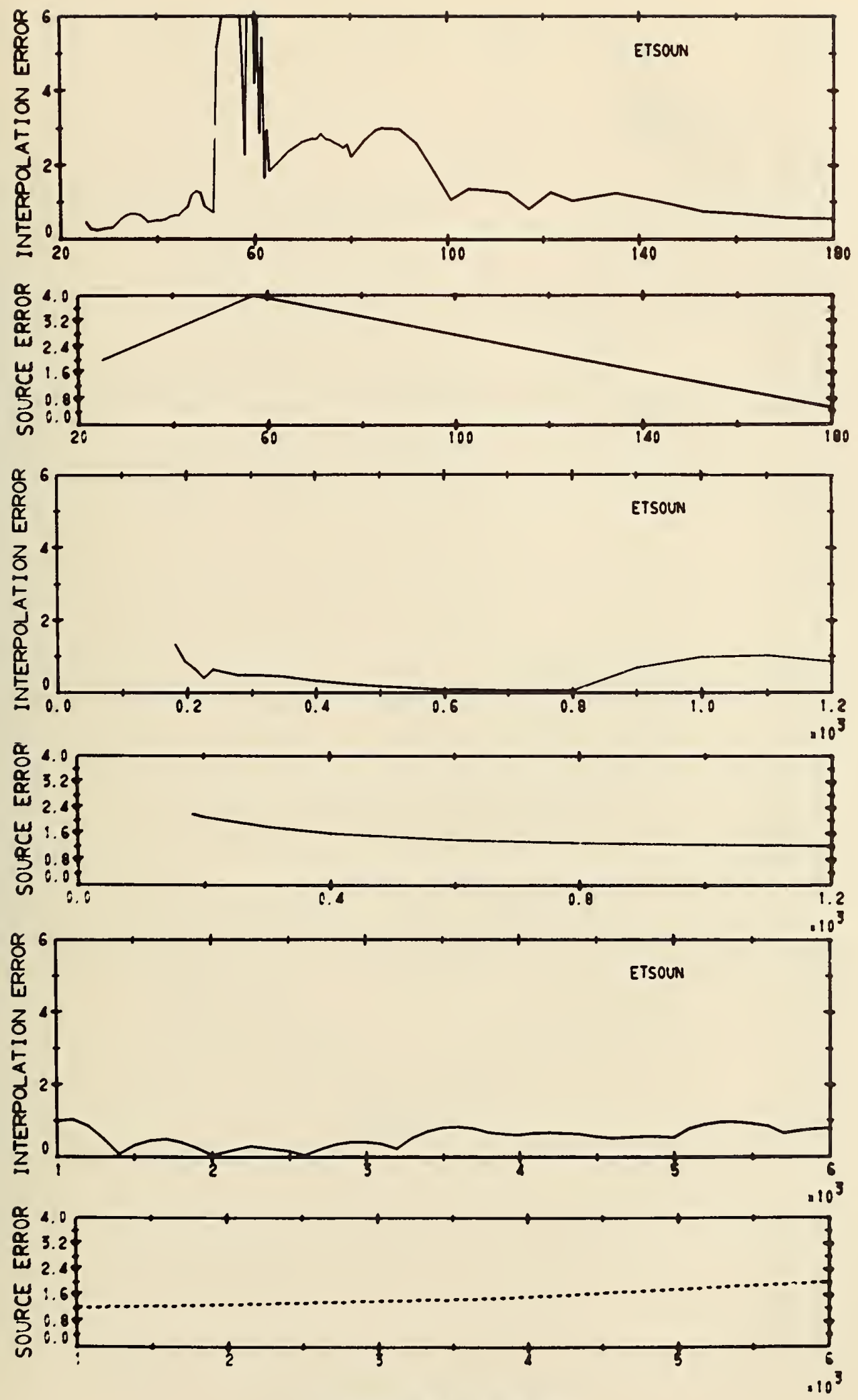

TEMPERATURE, RANK INE 

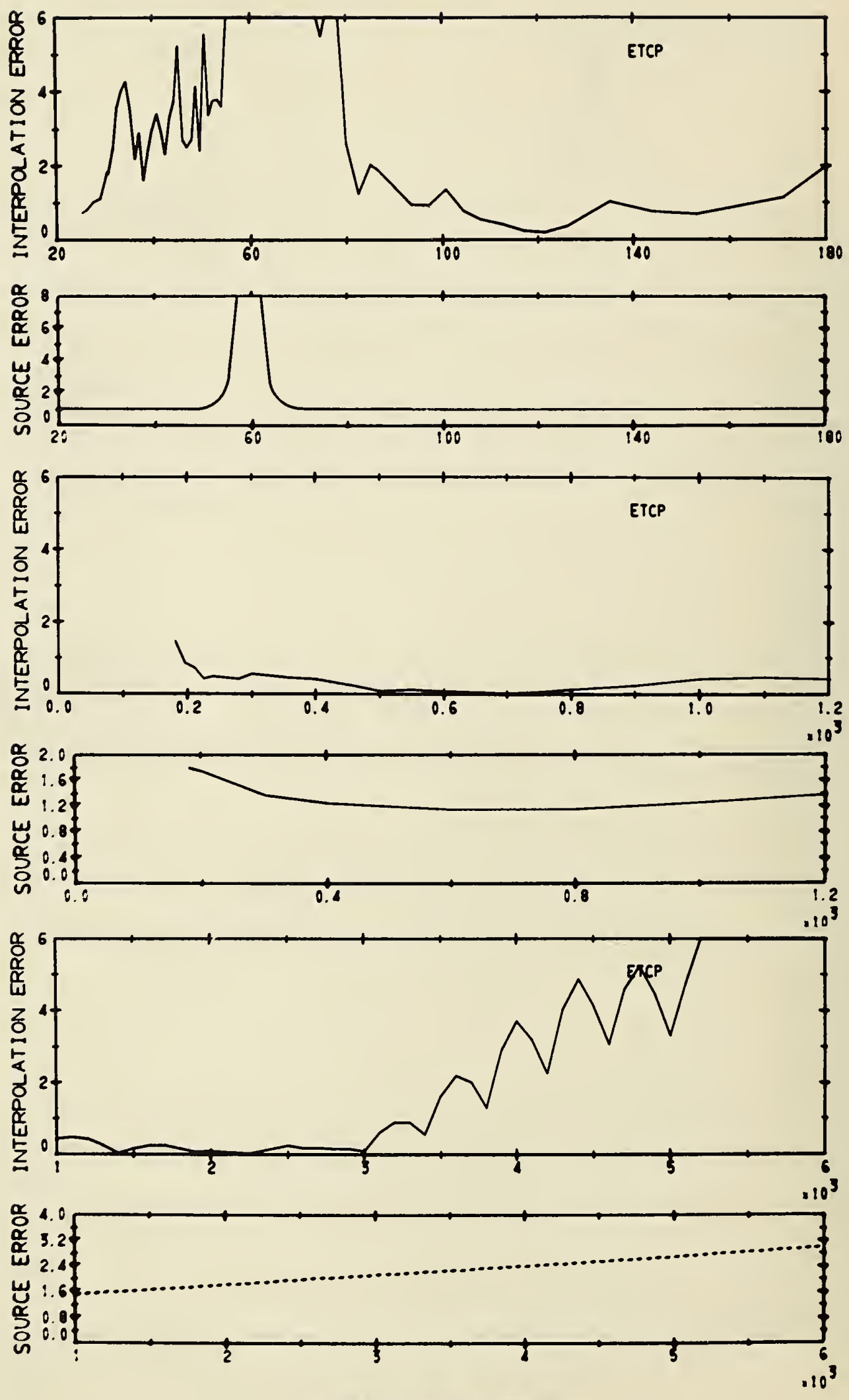

TEMPERATURE, RANKINE 

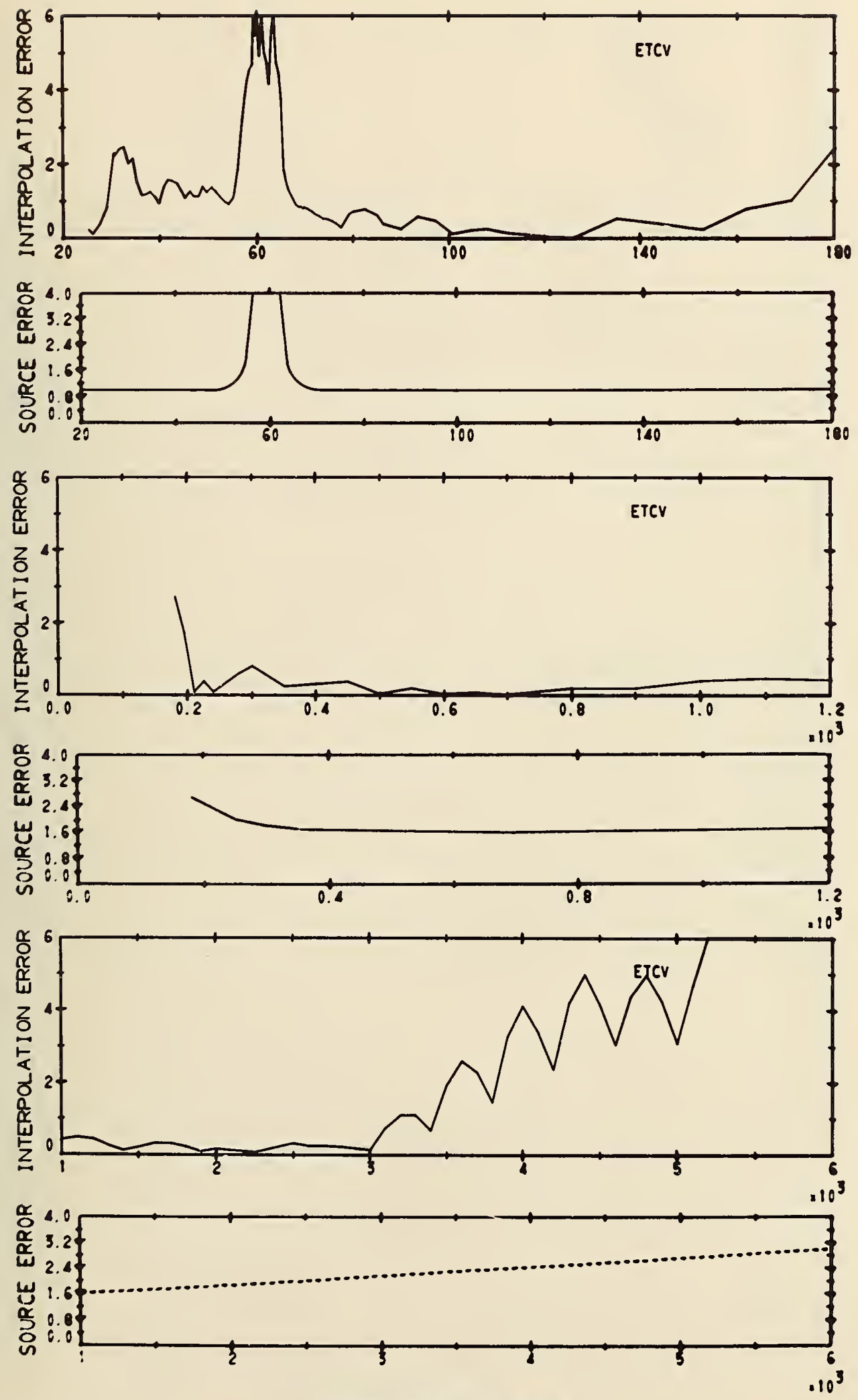

TEMPERATURE, RANKINE 

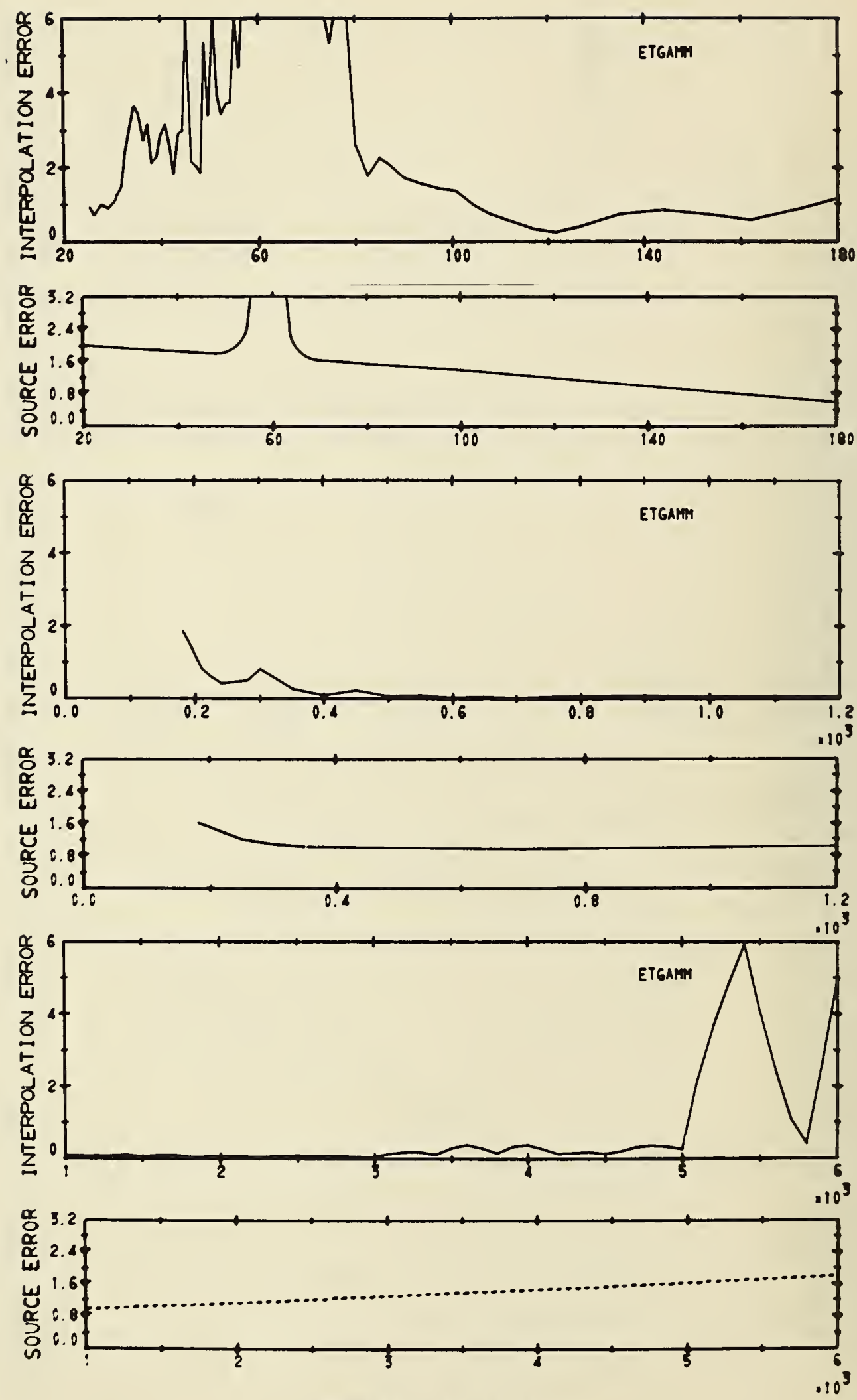

TEMPEPATURE, RANKINE 

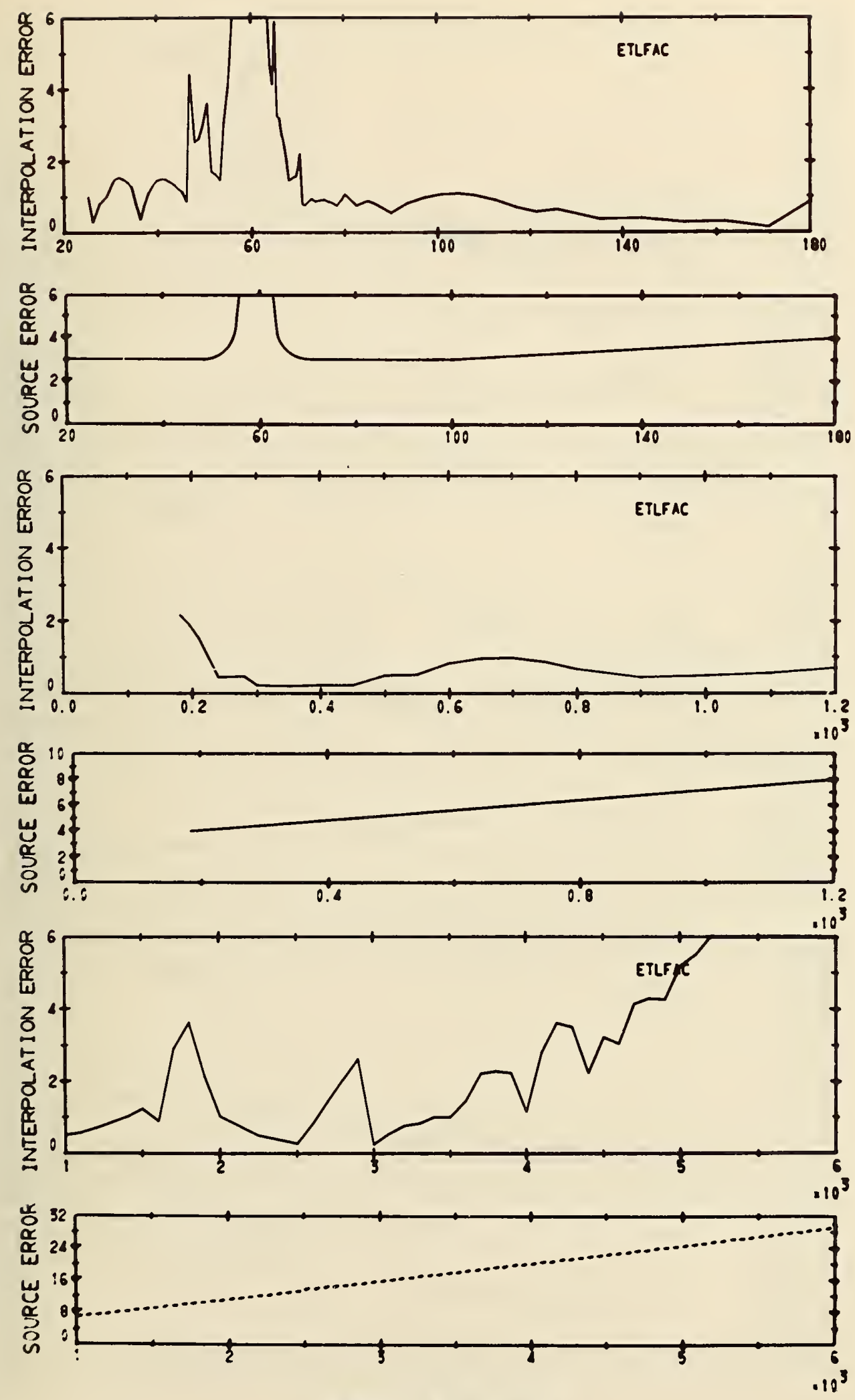

TEMPERATURE， RANK INE 

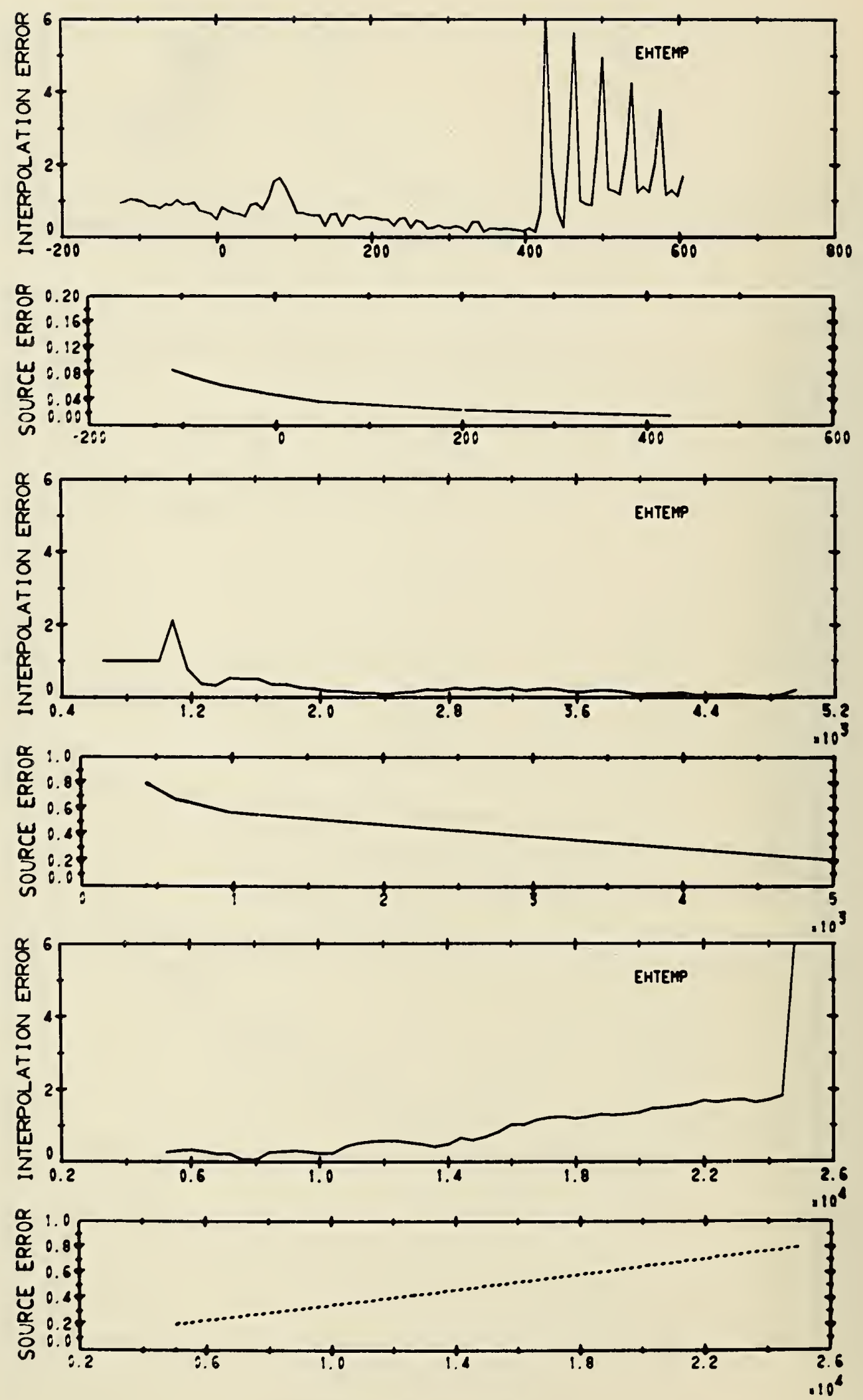

ENTHALPY, BTU/LB. 

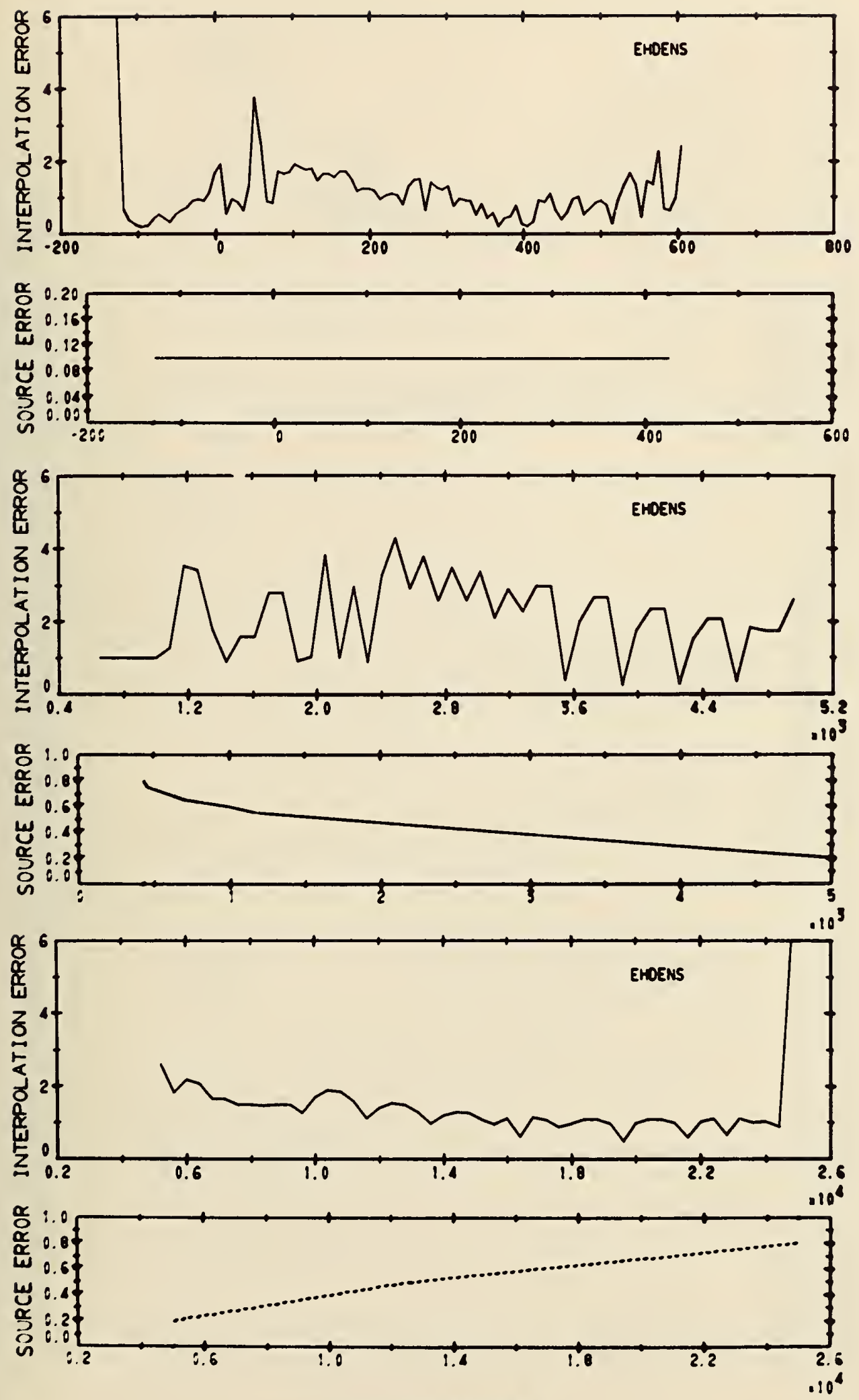

ENTHALPY, BTU/LB. 

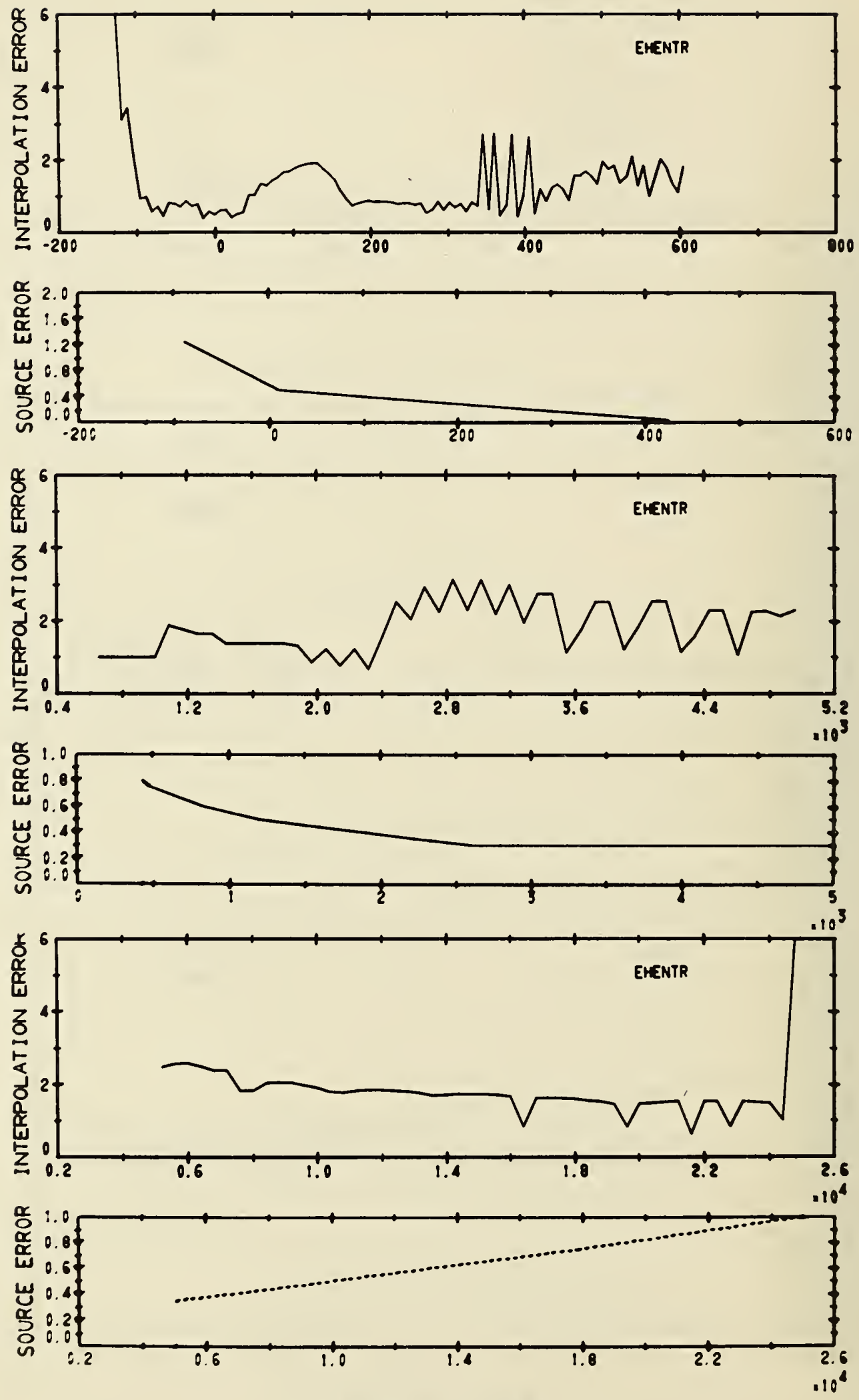

ENTHALPY, BTU/LB. 

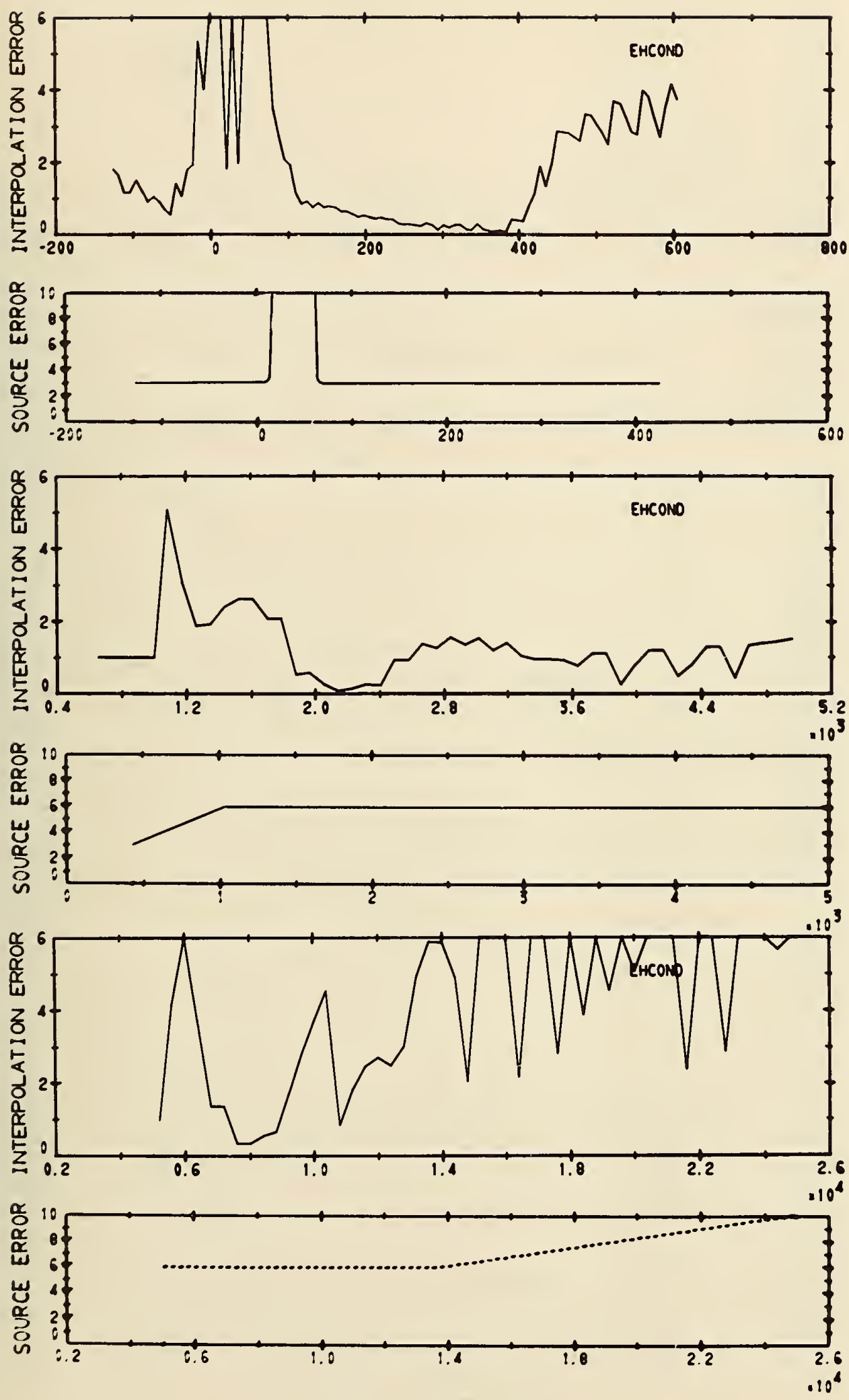

ENTHALPY, BTU/LB. 

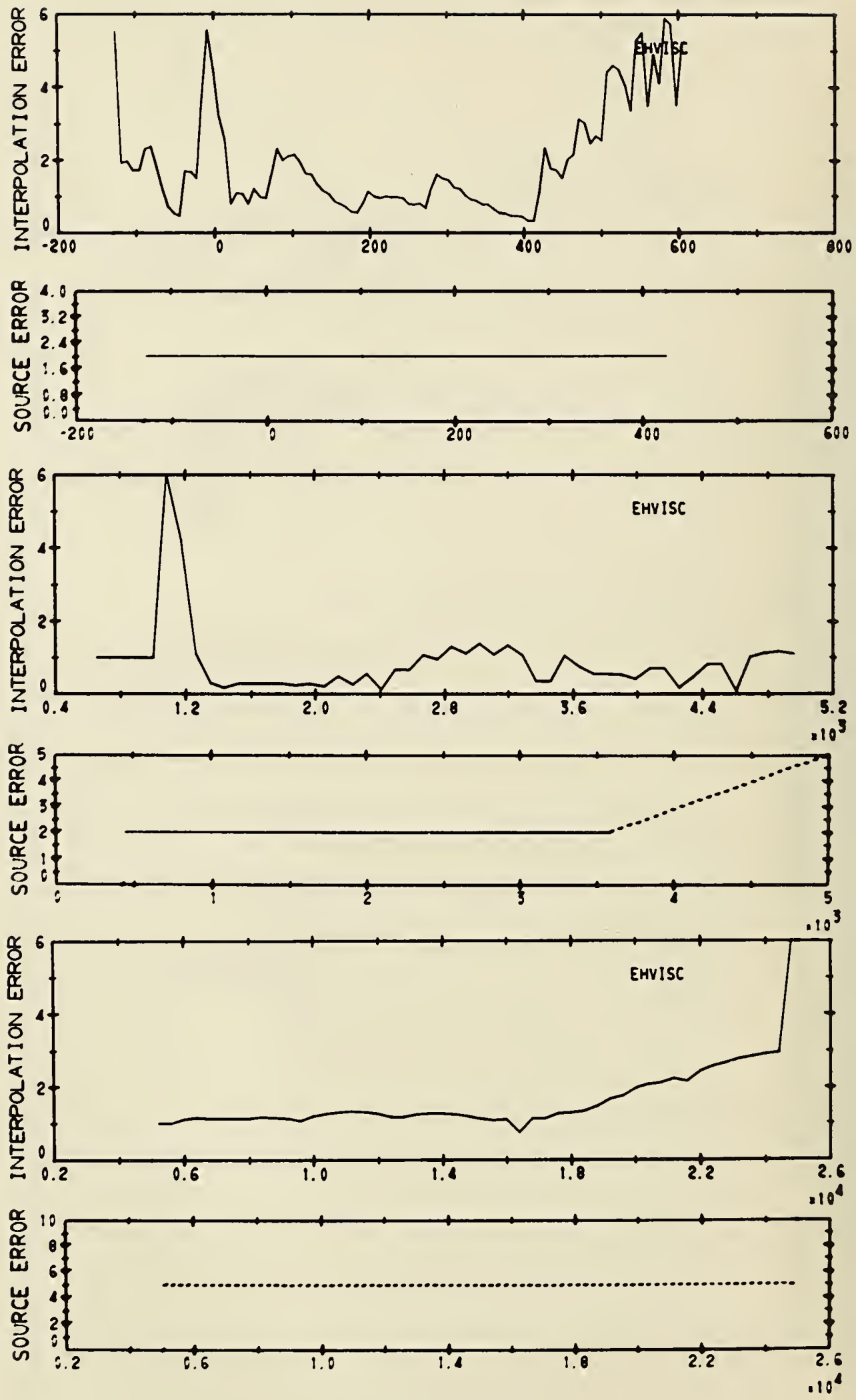

ENTHALPY, BTU/LB. 

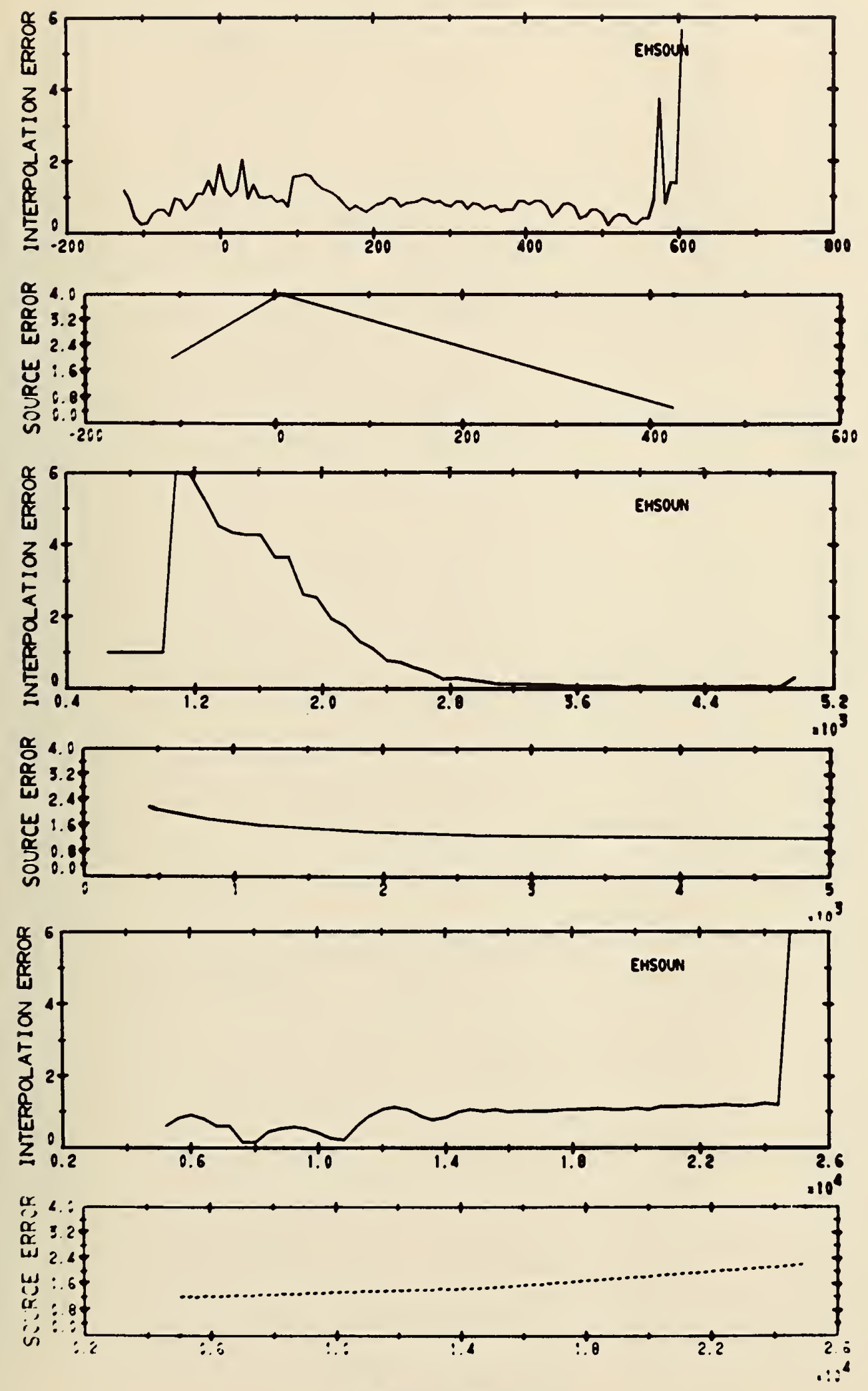

EI:THALPY, BTUJLB. 

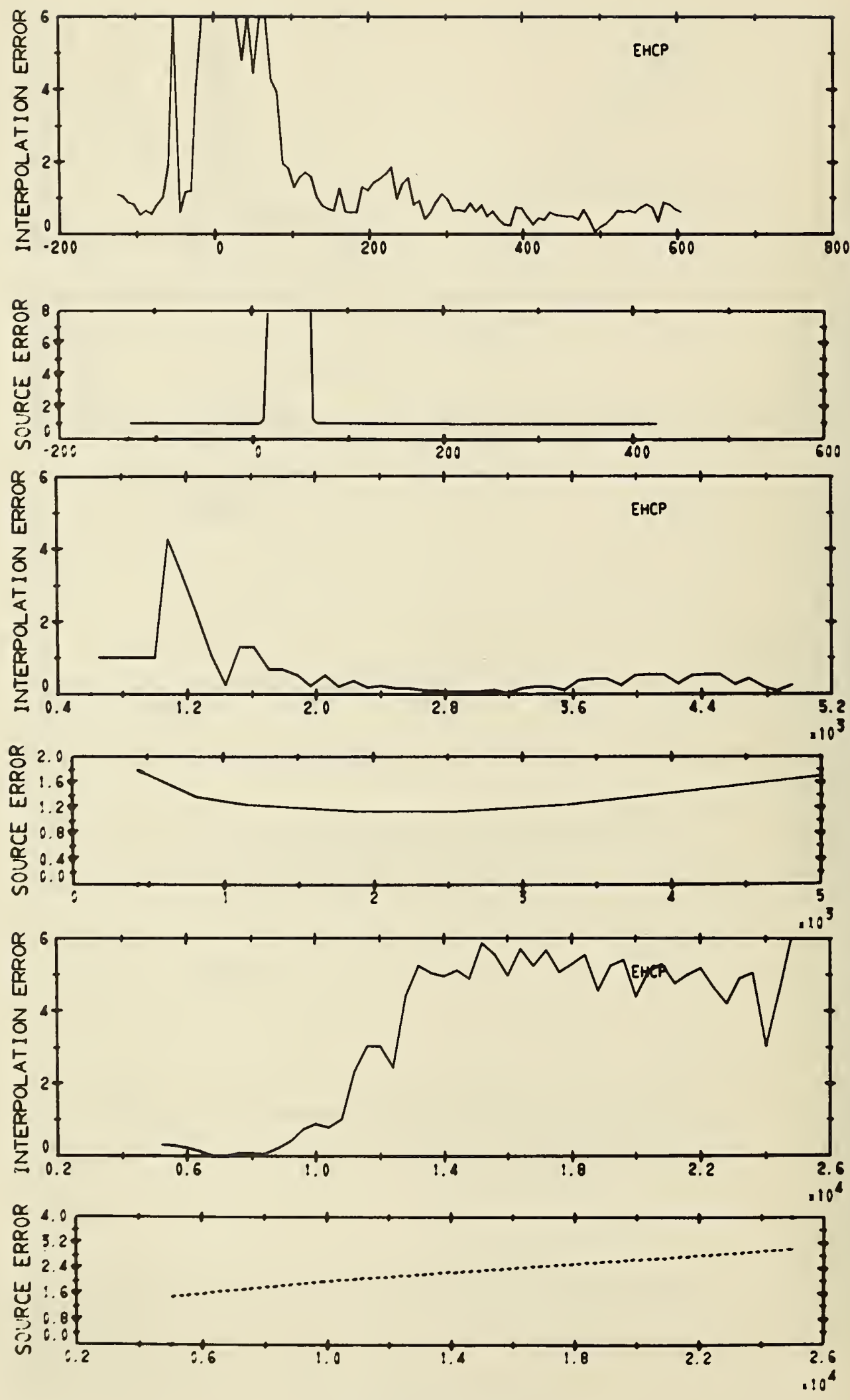

ENTHALPY, BTU/LB. 

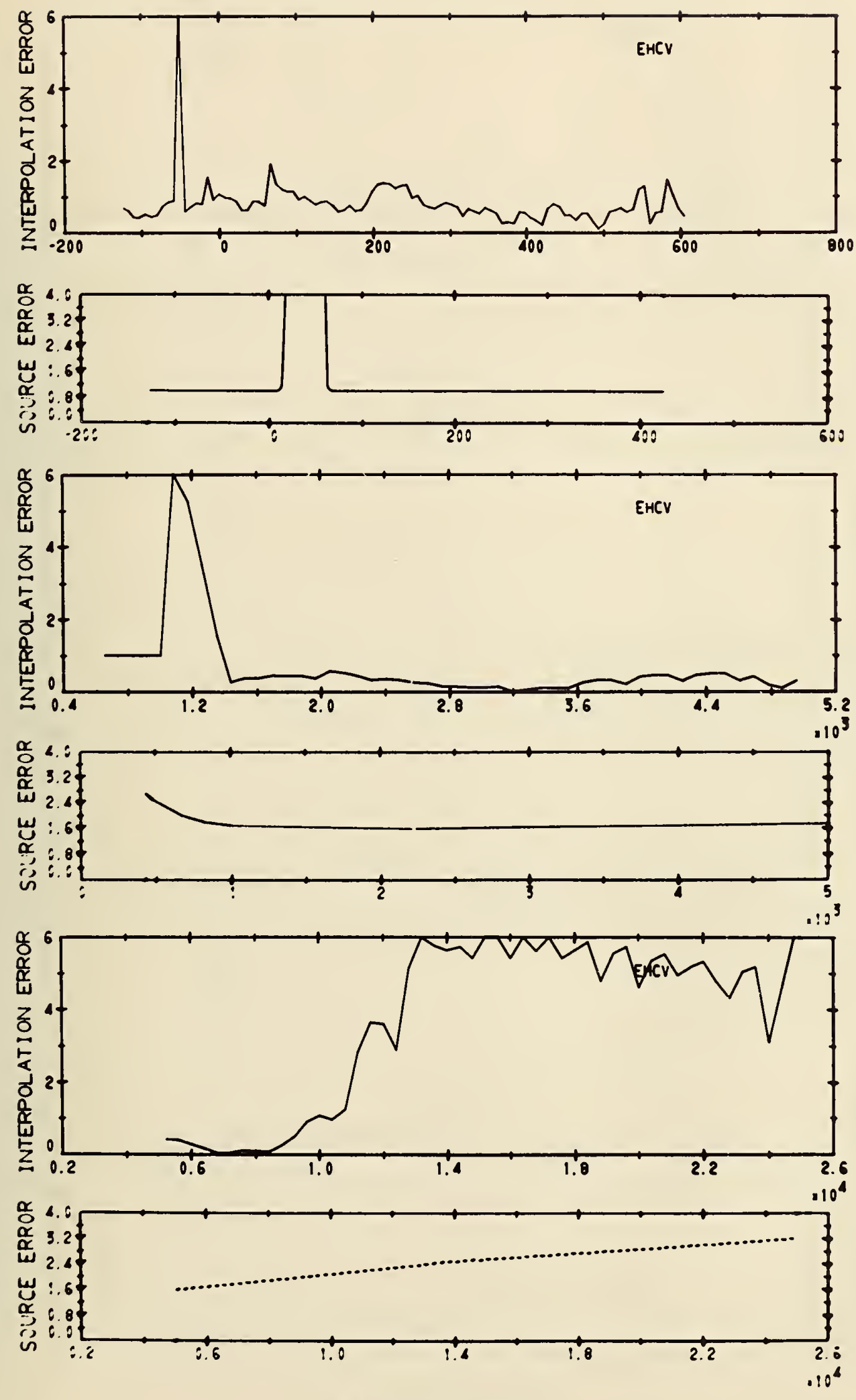

ENTHALPY, BTU/LB. 

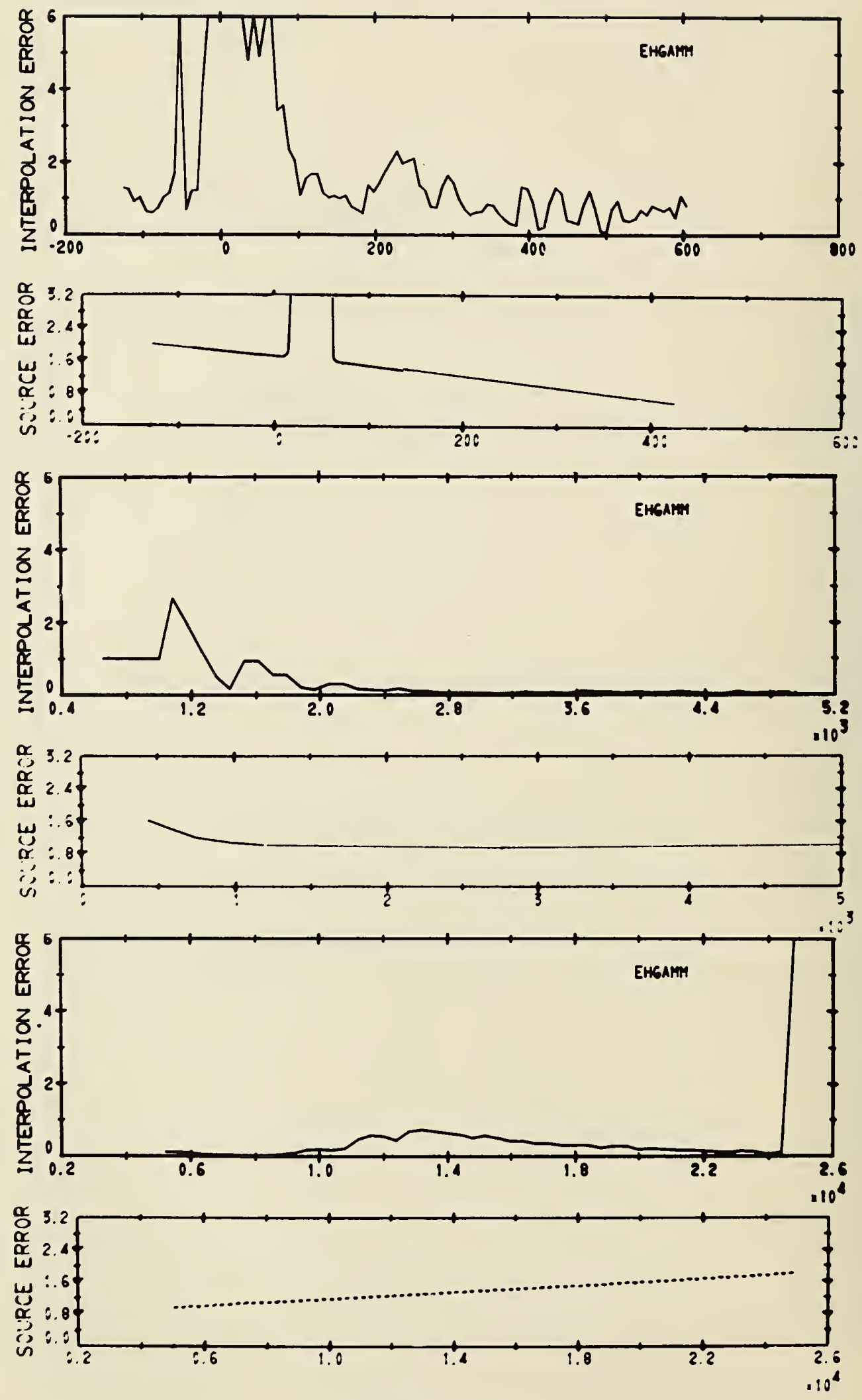

EIITHALPY, BTU/LB. 

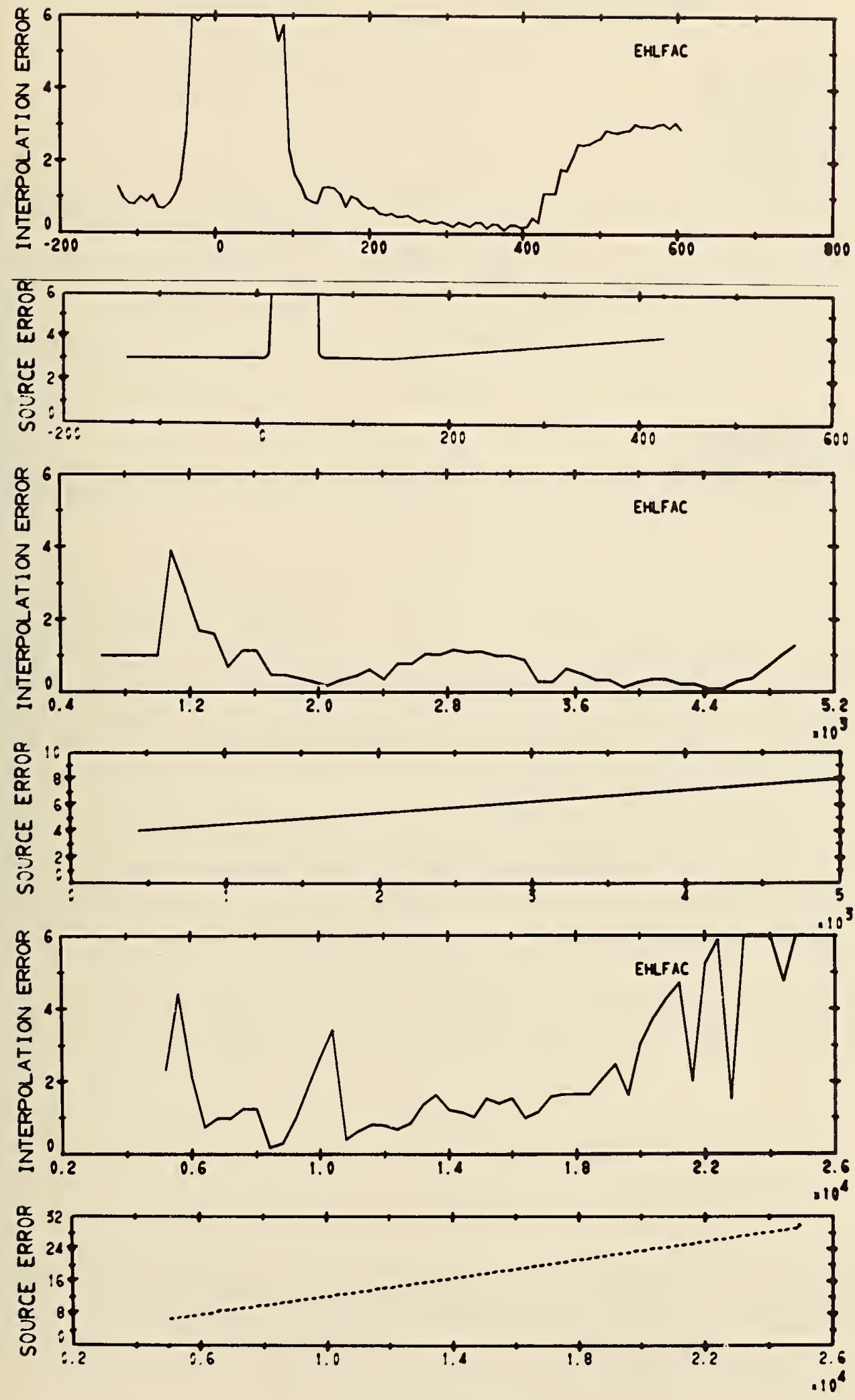

ENIHALPY, BIU/LY. 



\section{PUBLICATION OR REPORT NO. NBS TN-625}

\section{TIT LE AND SUBTITLE}

iomputer Programs for Thermodynamic and Transport 'roperties of Hydrogen (Tabcode - II)

AUTHOR(S)

- M. Roder, R. D. McCarty, and W. J. Hall

PERFORMING ORGANIZATION NAME AND ADDRESS

NATIONAL BUREAU OF STANDARDS, Boulder Labs. DEPARTMENT OF COMMERCE

Boulder, Colorado 80302

Sponsoring Organization Name and Address

ASA Headquarters

ashington, D. C.

14. Sponsoring Agency Code

5. Publication Date

October 1972

6. Performing Or ganization Code

8. Performing Organization

10. Project/Task/Work Unit No.

$$
2750400
$$

11. Contract/Grant No.

NASA Order No. $1330 \varnothing$

13. Type of Report \& Period Covered

\section{SUPPLEMENTARY NOTES}

ABSTRACT (A 200-word or less factual summary of most significant information. If document includes a significant bibliography or literature survey, mention it here.)

he thermodynamic and transport properties of para and equilibrium hydrogen have en programmed into a series of computer routines. Input variables are the pair's essure-temperature and pressure-enthalpy. The programs cover the range from to 5000 psia $\left(34 \mathrm{MN} / \mathrm{m}^{2}\right.$ ) with temperatures from the triple point to $6000^{\circ} \mathrm{R}(3300 \mathrm{~K})$ - enthalpies from $-130 \mathrm{BTU} / 1 \mathrm{~b}(-623 \mathrm{~J} / \mathrm{mol})$ to $25,000 \mathrm{BTU} / 1 \mathrm{~b}(117000 \mathrm{~J} / \mathrm{mol})$. atput variables are enthalpy or temperature, density, entropy, the rmal conductivity, scosity, velocity of sound, heat capacity at constant pressure, heat capacity at instant volume, the heat capacity ratio, and a heat transfer parameter. Property lues on the liquid and vapor boundaries a re conveniently obtained through two nall routines. The programs achieve high speed by using linear interpolation in a id of precomputed points which define the surface of the property returned. The aximum errors arising from the linear interpolation are shown on individual viation plots for each combination of variables. Error estimates for the sources data are similarly displayed.

KEY WORDS (Alphabetical order, separated by semicolons) Computer programs; density; enthalpy; entropy capacity at constant pressure; heat capacity at constant volume; heat capacity ratio; : transfer coefficient; hydrogen; pressure; saturation boundary; temperature; thermal luctivity; velocity of sound; viscosity. AVAILABILITY STATEMENT

[X] UNLIMIT ED.

FOR OFFICI AL DISTRIBUTION. DO NOT RELEASE TO NTIS.

\begin{tabular}{|c|c|}
\hline $\begin{array}{l}\text { 19. SECURITY CLASS } \\
\text { (THIS REPURT) } \\
\text { UNCL ASSIF IED }\end{array}$ & $\begin{array}{l}\text { 21. NO. OF PAGES } \\
226\end{array}$ \\
\hline $\begin{array}{l}\text { 20. SECURITY CLASS } \\
\text { (THIS P AGE) } \\
\text { UNCL ASSIFIED }\end{array}$ & $\begin{array}{l}\text { 22. Price } \\
\$ 1.75\end{array}$ \\
\hline
\end{tabular}





\section{NBS TECHNICAL PUBLICATIONS}

PERIODICALS

JOURNAL OF RESEARCH reports National Bureau of Standards research and development in physics, mathematics, and chemistry. Comprehensive scientific papers give complete details of the work, including laboratory data, experimental procedures, and theoretical and mathematical analyses. Illustrated with photographs, drawings, and charts. Includes listings of other NBS papers as issued.

\section{Published in two sections, available separately:}

\section{- Physies and Chemistry}

Papers of interest primarily to scientists working in these fields. This section covers a broad range of physical and chemical research, with major emphasis on standards of physical measurement, fundamental constants, and properties of matter. Issued six times a year. Annual subscription: Domestic, $\$ 9.50 ; \$ 2.25$ additional for foreign mailing.

\section{- Mathematical Sciences}

Studies and compilations designed mainly for the mathematician and theoretical physicist. Topics in mathematical statistics, theory of experiment design, numerical analysis, theoretical physics and chemistry, logical design and programming of computers and computer systems. Short numerical tables. Issued quarterly. Annual subscription: Domestic, \$5.00; $\$ 1.25$ additional for foreign mailing.

\section{TECHNICAL NEWS BULLETIN}

The best single source of information concerning the Bureau's measurement, research, developmental, cooperative, and publication activities, this monthly publication is designed for the industry-oriented individual whose daily work involves intimate contact with science and technology-for engineers, chemists, physicists, research managers, product-development managers, and company executives. Includes listing of all NBS papers as issued. Annual subscription: Domestic, $\$ 3.00 ; \$ 1.00$ additional for foreign mailing.

\section{Bibliographic Subscription Services}

The following current-awareness and literaturesurvey bibliographies are issued periodically by the Bureau: Cryogenic Data Center Current Awareness Service (weekly), Liquefied Natural Gas (quarterly), Superconducting Devices and Materials (quarterly), and Electromagnetic Metrology Current Awareness Service (monthly). Available only from NBS Boulder Laboratories. Ordering and cost information may be obtained from the Program Information Office, National Bureau of Standards, Boulder, Colorado 80302 .

\section{NONPERIODICALS}

Applied Mathematics Series. Mathematical tables, manuals, and studies.

Building Science Series. Research results, test methods, and performance criteria of building materials, components, systems, and structures.

Handbooks. Recommended codes of engineering and industrial practice (including safety codes) developed in cooperation with interested industries, professional organizations, and regulatory bodies.

Special Publications. Proceedings of NBS conferences, bibliographies, annual reports, wall charts, pamphlets, etc.

Monographs. Major contributions to the technical literature on various subjects related to the Bureau's scientific and technical activities.

National Standard Reference Data Series. NSRDS provides quantitative data on the physical and chemical properties of materials, compiled from the world's literature and critically evaluated.

Product Standards. Provide requirements for sizes, types, quality, and methods for testing various industrial products. These standards are developed cooperatively with interested Government and industry groups and provide the basis for common understanding of product characteristics for both buyers and sellers. Their use is voluntary.

Technical Notes. This series consists of communications and reports (covering both other-agency and NBS-sponsored work) of limited or transitory interest.

Federal Information Processing Standards Publications. This series is the official publication within the Federal Government for information on standards adopted and promulgated under the Public Law 89-306, and Bureau of the Budget Circular A-86 entitled, Standardization of Data Elements and Codes in Data Systems.

Consumer Information Series. Practical information, based on NBS research and experience, covering areas of interest to the consumer. Easily understandable language and illustrations provide useful background knowledge for shopping in today's technological marketplace.

\section{CATALOGS OF NBS PUBLICATIONS}

NBS Special Publication 305, Publications of the NBS. 1966-1967. When ordering, include Catalog No. C13.10:305. Price $\$ 2.00 ; 50$ cents additional for foreign mailing.

NBS Special Publication 305, Supplement 1, Publications of the NBS, 1968-1969. When ordering, include Catalog No. C13.10:305/Suppl. 1. Price $\$ 4.50 ; \$ 1.25$ additional for foreign mailing.

NBS Special Publication 305, Supplement 2, Publications of the NBS, 1970. When ordering, include Catalog No. C13.10:305/Suppl. 2. Price $\$ 3.25 ; 85$ cents additional for foreign mailing. 
U.S. DEPARTMENT OF COMMERCE

National Bureau of Standards

Washington, D.C. 20234

POSTAGE AND FEES PAID

OFFICIAL BUSINESS U.S. DEPARTMENT OF COMMEACE 215

Penalty for Private Use, $\$ 300$ 